Supporting Information

\title{
Radical-Mediated Distal ipso-Migration of $O / S$-Containing Heteroaryls and DFT Studies for Migratory Aptitude
}

Huihui Zhang, ${ }^{\mathrm{a}}$ Luyao Kou, ${ }^{\mathrm{a}}$ Dong Chen, ${ }^{\mathrm{a}}$ Meishan $\mathrm{Ji},{ }^{\mathrm{a}}$ Xiaoguang Bao, ${ }^{*}, \mathrm{a}$ Xinxin $\mathrm{Wu},{ }^{\mathrm{a}}$ and $\mathrm{Chen} \mathrm{Zhu}^{*, \mathrm{a}}$

${ }^{a}$ Key Laboratory of Organic Synthesis of Jiangsu Province, College of Chemistry, Chemical Engineering and Materials Science, Soochow University, 199 Ren-Ai Road, Suzhou, Jiangsu 215123, China

Email: chzhu@ suda.edu.cn, xgbao@suda.edu.cn

\section{Table of Contents}

1. General experimental details $\quad$ S2

2. Reaction conditions survey $\quad$ S2

3. General procedure for synthesis of starting materials $\quad$ S3

4. General procedure for radical difunctionalization of olefins $\quad$ S4

5. Characterization of starting materials $\quad$ S5

6. Characterization of products $\quad$ S15

7. ${ }^{1} \mathrm{H},{ }^{13} \mathrm{C},{ }^{19} \mathrm{~F}$, and HMBC NMR spectra S32

$\begin{array}{lr}\text { 8. DFT studies } & \text { S166 }\end{array}$

$\begin{array}{lr}\text { 9. Mechanism studies } & \text { S254 }\end{array}$ 


\section{General Experimental Details}

All reactions were maintained under a nitrogen atmosphere unless otherwise stated. Commercially available reagents were used without further purification. Infrared (FT-IR) spectra were recorded on a BRUKER VERTEX 70, $v_{\max }$ in $\mathrm{cm}^{-1} .{ }^{1} \mathrm{H}-\mathrm{NMR}$ spectra were recorded on a BRUKER AVANCE III HD (400 MHz) spectrometer. Chemical shifts are reported in ppm from tetramethylsilane with the solvent resonance as internal standard $\left(\mathrm{CDCl}_{3}: \delta 7.26\right.$, DMSO- $d_{6}: \delta$ 2.50, $\mathrm{CD}_{3} \mathrm{OD}: \delta 3.31$, acetone- $\left.d_{6}: \delta 2.05\right)$. Data are reported as follows: chemical shift, multiplicity $(\mathrm{s}=$ singlet, $\mathrm{d}=$ doublet, $\mathrm{t}=$ triplet, $\mathrm{q}=$ quadruplet, $\mathrm{br}=$ broad, $\mathrm{m}=$ multiplet $)$, coupling constants $(\mathrm{Hz})$ and integration. ${ }^{13} \mathrm{C}-\mathrm{NMR}$ spectra were recorded on a BRUKER AVANCE III HD (100 MHz) spectrometer with complete proton decoupling. Chemical shifts are reported in ppm from tetramethylsilane with the solvent resonance as the internal standard $\left(\mathrm{CDCl}_{3}\right.$ : $\delta$ 77.16, DMSO- $d_{6}: \delta 39.52, \mathrm{CD}_{3} \mathrm{OD}: \delta 49.00$, acetone- $\left.d_{6}: \delta 29.84,206.26\right) .{ }^{19} \mathrm{~F}-\mathrm{NMR}$ spectra were recorded on a BRUKER AVANCE III HD (376 MHz) spectrometer. Mass spectra were measured with an Agilent Technologies 6120 Quadrupole LC/MS. High resolution mass spectrometry (HRMS) were measured with a GCT Premier ${ }^{\mathrm{TM}}$ and BRUKER micrOTF-Q III. Melting points were measured using INESA WRR and values are uncorrected.

\section{Reaction conditions survey}

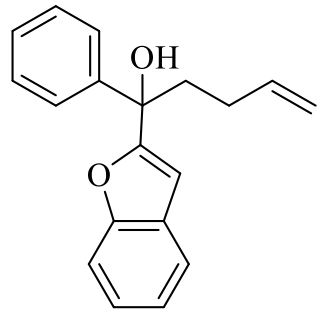

$1 \mathbf{a}$

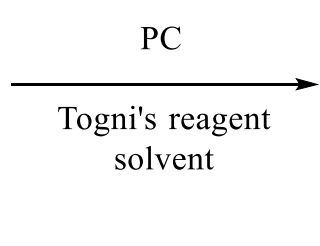<smiles>Br[Pb](Br)c1ccccc1-c1cc(-c2ccccc2)cc(-c2ccccc2)[o+]1</smiles><smiles>O=C(CCC(CC(F)(F)F)c1cc2ccccc2o1)c1ccccc1</smiles>

2a

PC 1

\begin{tabular}{|c|c|c|c|}
\hline entry & photocatalyst & solvent & yield $(\%)^{\mathrm{b}}$ \\
\hline 1 & $f a c-\operatorname{Ir}(\mathrm{ppy})_{3}$ & DMA & 75 \\
\hline 2 & {$\left[\operatorname{Ir}\left(\mathrm{dF}\left(\mathrm{CF}_{3}\right) \text { ppy }\right)_{2}(\mathrm{dtbbpy})\right] \mathrm{PF}_{6}$} & DMA & 57 \\
\hline 3 & {$\left[\operatorname{Ir}(\mathrm{ppy})_{2}(\mathrm{dtbbpy})\right] \mathrm{PF}_{6}$} & DMA & 64 \\
\hline 4 & PC 1 & DMA & 59 \\
\hline 5 & Mes-Acr-ClO 4 & DMA & 80 \\
\hline 6 & Eosin Y & DMA & 23 \\
\hline 7 & Mes-Acr-ClO 4 & $\mathrm{CH}_{2} \mathrm{Cl}_{2}$ & 47 \\
\hline 8 & Mes-Acr-ClO 4 & $\mathrm{EtOH}$ & 70 \\
\hline
\end{tabular}




\begin{tabular}{|c|c|c|}
\hline 9 & Mes-Acr-ClO 4 & EA \\
\hline 10 & Mes-Acr-ClO 4 & toluene \\
\hline 11 & Mes-Acr- $\mathrm{ClO}_{4}$ & $\mathrm{MeCN}$ \\
\hline 12 & Mes-Acr-ClO 4 & $\mathrm{Et}_{2} \mathrm{O}$ \\
\hline $13^{c}$ & Mes-Acr-ClO 4 & DMA \\
\hline 14 & - & DMA \\
\hline $15^{\mathrm{d}}$ & Mes-Acr-ClO 4 & DMA \\
\hline
\end{tabular}

Table S1. ${ }^{\text {a }}$ 1a $(0.2 \mathrm{mmol})$, Togni's reagent (II) $(0.4 \mathrm{mmol}, 2.0$ equiv), and PC (0.008 mmol, 4 $\mathrm{mol} \%)$ in solvent $(3.0 \mathrm{~mL})$ at $\mathrm{rt}, 14 \mathrm{~W}$ blue LED irradiation. ${ }^{\mathrm{b}}$ Yields of isolated products. ${ }^{\mathrm{c}}$ Tognis' reagent (II) ( $0.3 \mathrm{mmol}, 1.5$ equiv). ${ }^{\mathrm{d}}$ In dark.

\section{General procedure for synthesis of starting materials}

The starting materials 1a-1aa were prepared according to General Procedure A. The starting materials 1ab-1an were prepared according to General Procedure B.

\section{General Procedure A}

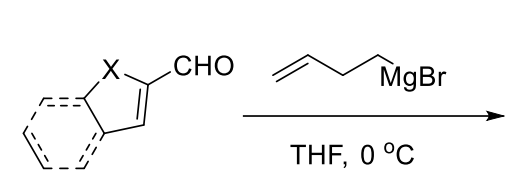

$X=0, S$

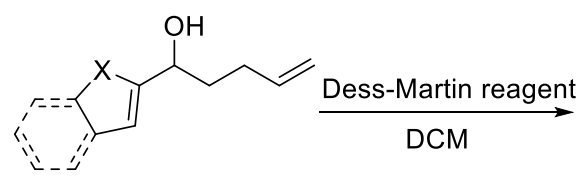

S1

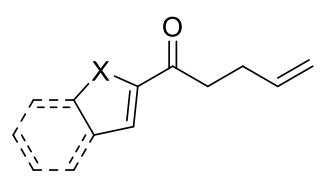

S2<smiles>C=CCCC(O)(c1[X]c2ccccc2cc1)c1ccccc1</smiles>

To a solution of freshly prepared Grignard reagent (1.2 equiv) in THF was added a solution of $\mathrm{O} / \mathrm{S}$-containing heteroaryl formaldehyde $\left(1.0\right.$ equiv) in THF $(20 \mathrm{~mL})$ dropwise at $0{ }^{\circ} \mathrm{C}$ under $\mathrm{N}_{2}$. The reaction mixture was stirred at room temperature for $2 \mathrm{~h}$ and quenched with sat. $\mathrm{NH}_{4} \mathrm{Cl}$ $(20 \mathrm{~mL})$. The reaction was extracted with ethyl acetate, and the combined organic layer was washed with brine, dried over $\mathrm{MgSO}_{4}$, filtered, concentrated in vacuum, and by flash column chromatography on silica gel (ethyl acetate/ petroleum ether) to give $\mathbf{S 1}$ with $85-95 \%$ yield as yellow oil.

Dess-Martin reagent (1.2 equiv) was added a solution of $\mathbf{S 1}$ (1.0 equiv) in DCM (20 mL) at 0 ${ }^{\circ} \mathrm{C}$. The reaction mixture was stirred at room temperature for $2 \mathrm{~h}$ and filtered with silica gel, concentrated in vacuum, and by flash column chromatography on silica gel (ethyl acetate/ petroleum ether) to give $\mathbf{S 2}$ with $90-95 \%$ yield as yellow oil or solid.

To a solution of freshly prepared phenyl Grignard reagent (1.2 equiv) in THF was added a solution of $\mathbf{S 2}$ (1.0 equiv) in THF (20 mL) dropwise at $0{ }^{\circ} \mathrm{C}$ under $\mathrm{N}_{2}$. The reaction mixture was stirred at room temperature for $2 \mathrm{~h}$ and quenched with sat. $\mathrm{NH}_{4} \mathrm{Cl}(20 \mathrm{~mL})$. The reaction was extracted with ethyl acetate, and the combined organic layer was washed with brine, dried over $\mathrm{MgSO}_{4}$, filtered, concentrated in vacuum, and by flash column chromatography on silica gel (ethyl acetate/ petroleum ether) to give $\mathbf{S 3}$ (referring to 1a-1aa) with $13-76 \%$ yield. 


\section{General Procedure B}

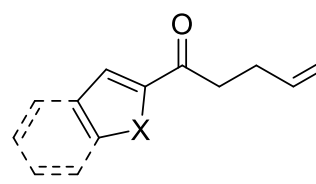

S2

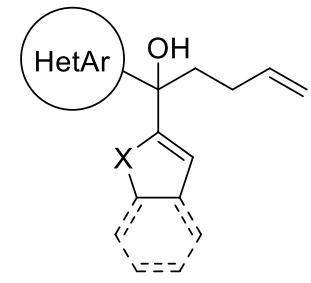

S4

$\mathrm{X}=\mathrm{O}, \mathrm{S}$

HetAr= benzothiazolyl, furyl, thiazolyl, benzothienyl, thienyl

To a solution of heteroaryls (1.0 equiv) in THF ( $3 \mathrm{~mL}$ ) was slowly added $n$-BuLi (1.5 equiv) at $-78^{\circ} \mathrm{C}$ under $\mathrm{N}_{2}$, then the mixture was stirred for $1 \mathrm{~h}$. A solution of $\mathbf{S 2}$ (1.2 equiv) in THF ( $\left.3 \mathrm{~mL}\right)$ was added to the mixture at $-78^{\circ} \mathrm{C}$. Then, the mixture was gradually warmed to $0{ }^{\circ} \mathrm{C}$. After the reaction was complete, the mixture was quenched with sat. $\mathrm{NH}_{4} \mathrm{Cl}(5 \mathrm{~mL})$. The reaction was extracted with ethyl acetate, and the combined organic layer was washed with brine, dried over $\mathrm{MgSO}_{4}$, filtered, concentrated in vacuum, and purified by flash column chromatography on silica gel (ethyl acetate/ petroleum ether) to give $\mathbf{S} 4$ (referring to $\mathbf{1 a b - 1 a n ) ~ w i t h ~} 19-82 \%$ yield.

\section{General procedure for radical difunctionalization of olefins}

\subsection{Synthesis of 2 or 3}

Tertiary alcohol 1 ( $0.2 \mathrm{mmol}, 1.0$ equiv), Togni's reagent (II) ( $0.4 \mathrm{mmol}, 2.0$ equiv), and Mes-Acr- $\mathrm{ClO}_{4}(0.008 \mathrm{mmol}, 4 \mathrm{~mol} \%)$ were loaded in a flame-dried glass reaction vial which was subjected to evacuation/ flushing with $\mathrm{N}_{2}$ three times. DMA $(3.0 \mathrm{~mL})$ was added to the mixture via syringe and the mixture was then stirred at $25{ }^{\circ} \mathrm{C}$ under $14 \mathrm{~W}$ blue LED irradiation (the distance between reaction vials and blue LED was kept in about $10 \mathrm{~cm}$ ) until the starting material had been consumed as determined by TLC. The mixture was extracted with ethyl acetate $(3 \times 10 \mathrm{~mL})$. The combined organic extracts were washed by brine, dried over $\mathrm{MgSO}_{4}$, filtered, concentrated, and purified by flash column chromatography on silica gel (ethyl acetate/ petroleum ether) to give the product 2 or 3 .

Lamp parameters: blue light, model: S-60W-ZR-A03, AC input: $110 / 240 \mathrm{~V} \pm 15 \%$, DC output: $12 \mathrm{~V} 5 \mathrm{~A}$, rated frequency: $50 / 60 \mathrm{~Hz}$, made in China, productions date: 2016 , wavelength of peak intensity: $425 \mathrm{~nm}$.

\section{5 mmol-scale synthesis:}

Tertiary alcohol 1a (1.5 mmol, 1.0 equiv), Togni's reagent (II) $(3.0 \mathrm{mmol}, 2.0$ equiv), and Mes-Acr- $\mathrm{ClO}_{4}(0.06 \mathrm{mmol}, 4 \mathrm{~mol} \%)$ were loaded in a flame-dried glass reaction vial which was subjected to evacuation/ flushing with $\mathrm{N}_{2}$ three times. DMA $(22.5 \mathrm{~mL})$ was added to the mixture via syringe and the mixture was then stirred at $25{ }^{\circ} \mathrm{C}$ under $14 \mathrm{~W}$ blue LED irradiation (the distance between reaction vials and blue LED was kept in about $10 \mathrm{~cm}$ ) until the starting material had been consumed as determined by TLC. The mixture was extracted with ethyl acetate $(3 \times 30$ $\mathrm{mL}$ ). The combined organic extracts were washed by brine, dried over $\mathrm{MgSO}_{4}$, filtered, 
concentrated, and purified by flash column chromatography on silica gel (eluent: EtOAc/Petroleum ether $=1 / 50)$ to give the product $\mathbf{2 a}(73 \%$ yield, $380.3 \mathrm{mg})$.

\subsection{Synthesis of 4}

Tertiary alcohol 1 ( $0.2 \mathrm{mmol}, 1.0$ equiv), $f a c-\operatorname{Ir}(\mathrm{ppy})_{3}(0.008 \mathrm{mmol}, 4 \mathrm{~mol} \%)$ were loaded in a flame-dried glass reaction vial which was subjected to evacuation/ flushing with $\mathrm{N}_{2}$ three times. $\mathrm{BrCF}_{2} \mathrm{COOEt}(0.4 \mathrm{mmol}, 2.0$ equiv) and $\mathrm{DMF}(2.0 \mathrm{~mL})$ was added to the mixture via syringe and the mixture was then stirred at $25{ }^{\circ} \mathrm{C}$ under $30 \mathrm{~W}$ blue LED irradiation (the distance between reaction vials and blue LED was kept in about $10 \mathrm{~cm}$ ) until the starting material had been consumed as determined by TLC. The mixture was extracted with ethyl acetate $(3 \times 10 \mathrm{~mL})$. The combined organic extracts were washed by brine, dried over $\mathrm{MgSO}_{4}$, filtered, concentrated, and purified by flash column chromatography on silica gel (ethyl acetate/ petroleum ether) to give the product 4 .

Lamp parameters: blue light, model: WD-SX-2, rated voltage: DC220V, rated frequency: $50 / 60 \mathrm{~Hz}$, executive standard: GB700.1-2007, GB700.202-2008, quality inspection number: QC-H02, productions date: 2017.07, wavelength of peak intensity: $450 \mathrm{~nm}$.

\subsection{Synthesis of 5}

Tertiary alcohol 1 ( $0.2 \mathrm{mmol}, 1.0$ equiv), $f a c-\operatorname{Ir}(\mathrm{ppy})_{3}(0.006 \mathrm{mmol}, 3 \mathrm{~mol} \%)$ were loaded in a flame-dried glass reaction vial which was subjected to evacuation/ flushing with $\mathrm{N}_{2}$ three times. BrCHFCOOEt ( $0.3 \mathrm{mmol}, 1.5$ equiv) and ethyl acetate $(2.0 \mathrm{~mL})$ was added to the mixture via syringe and the mixture was then stirred at $25{ }^{\circ} \mathrm{C}$ under $30 \mathrm{~W}$ blue LED irradiation (the distance between reaction vials and blue LED was kept in about $10 \mathrm{~cm}$ ) until the starting material had been consumed as determined by TLC. The resultant organic solvent was removed under vacuum and the residue was purified by flash column chromatography on silica gel (ethyl acetate/ petroleum ether) to give the product 5 .

Lamp parameters: blue light, model: WD-SX-2, rated voltage: DC220V, rated frequency: $50 / 60 \mathrm{~Hz}$, executive standard: GB700.1-2007, GB700.202-2008, quality inspection number: QC-H02, productions date: 2017.07, wavelength of peak intensity: $450 \mathrm{~nm}$.

\section{Characterization of starting materials}

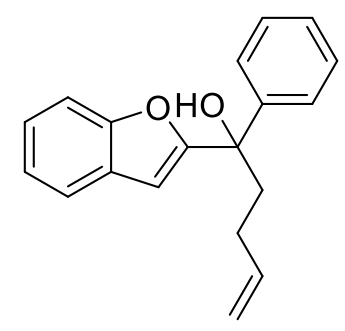

1a: $0.485 \mathrm{~g}, 60 \%$ yield, yellow oil. Purification by flash column chromatography (eluent: EtOAc/Petroleum ether $=1 / 50) .{ }^{1} \mathrm{H}$ NMR $(400$ $\left.\mathrm{MHz}, \mathrm{CDCl}_{3}\right) \delta$ 7.57-7.53 (m, 1H), 7.52-7.50 (m, 1H), 7.49-7.47 (m, 1H), 7.45-7.40 (m, 1H), 7.39-7.31 (m, 2H), 7.31-7.27 (m, 1H), 7.24-7.19 (m, $1 \mathrm{H}), 6.67(\mathrm{~d}, J=0.8 \mathrm{~Hz}, 1 \mathrm{H}), 5.94-5.75(\mathrm{~m}, 1 \mathrm{H}), 5.10-4.89(\mathrm{~m}, 2 \mathrm{H})$, 2.53-2.43 (m, 1H), 2.39-2.28 (m, 1H), 2.26-2.14 (m, 1H), 2.10-1.99 (m, $1 \mathrm{H}) ;{ }^{13} \mathrm{C}$ NMR $\left(100 \mathrm{MHz}, \mathrm{CDCl}_{3}\right) \delta 160.6,154.3,143.2,137.8,127.8$, 127.6, 127.0, 125.2, 123.7, 122.4, 120.6, 114.4, 110.9, 102.7, 75.4, 39.5, 27.5. FT-IR: $v\left(\mathrm{~cm}^{-1}\right)$ 3676, 3065, 2974, 1638, 1541, 1474, 1454, 1305, 1251. HRMS [ESI] m/z: [M+Na] ${ }^{+}$calcd for $\mathrm{C}_{19} \mathrm{H}_{18} \mathrm{NaO}_{2}$ 301.1199; found 301.1196. 


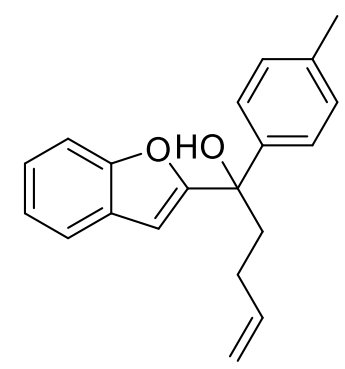

1b: $0.280 \mathrm{~g}, 76 \%$ yield, yellow oil. Purification by flash column chromatography (eluent: EtOAc/Petroleum ether $=1 / 50) .{ }^{1} \mathrm{H}$ NMR $(400$ MHz, DMSO) $\delta$ 7.61-7.55 (m, 1H), 7.50-7.44 (m, 1H), 7.39-7.33 (m, 2H), 7.26-7.16 (m, 2H), 7.15-7.09 (m, 2H), $6.76(\mathrm{~d}, J=0.8 \mathrm{~Hz}, 1 \mathrm{H}), 5.95(\mathrm{~s}$, $1 \mathrm{H}), 5.87-5.71(\mathrm{~m}, 1 \mathrm{H}), 5.03-4.84(\mathrm{~m}, 2 \mathrm{H}), 2.38-2.18(\mathrm{~m}, 2 \mathrm{H}), 2.27(\mathrm{~s}$, $3 \mathrm{H}), 2.09-1.97(\mathrm{~m}, 1 \mathrm{H}), 1.93-1.80(\mathrm{~m}, 1 \mathrm{H}) ;{ }^{13} \mathrm{C} \mathrm{NMR}$ (100 MHz, DMSO) $\delta 163.4,154.5,142.1,139.0,136.3,128.9,128.4,126.0,124.3,123.2$, 121.5, 115.0, 111.5, 102.6, 74.3, 28.0, 21.1. FT-IR: $v\left(\mathrm{~cm}^{-1}\right)$ 3676, 3065, 2974, 2923, 1558, 1489 , 1437, 1375, 1305, 1251. HRMS [ESI] m/z: $[\mathrm{M}+\mathrm{Na}]^{+}$calcd for $\mathrm{C}_{20} \mathrm{H}_{20} \mathrm{NaO}_{2}$ 315.1356; found 315.1360 .

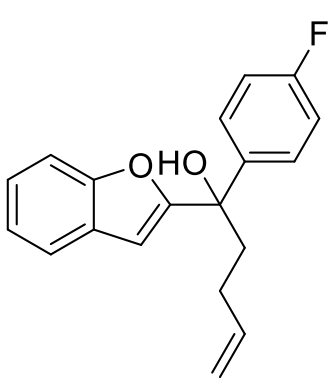

1c: $0.195 \mathrm{~g}, 53 \%$ yield, yellow oil. Purification by flash column chromatography (eluent: EtOAc/Petroleum ether $=1 / 50) .{ }^{1} \mathrm{H}$ NMR $(400$ $\mathrm{MHz}, \mathrm{DMSO}) \delta$ 7.63-7.57 (m, 1H), 7.56-7.44 (m, 3H), 7.30-7.10 (m, $4 \mathrm{H}), 6.83-6.78(\mathrm{~m}, 1 \mathrm{H}), 6.10(\mathrm{~s}, 1 \mathrm{H}), 5.92-5.71(\mathrm{~m}, 1 \mathrm{H}), 5.04-4.83(\mathrm{~m}$, $2 \mathrm{H}), 2.40-2.19(\mathrm{~m}, 2 \mathrm{H}), 2.08-1.97(\mathrm{~m}, 1 \mathrm{H}), 1.92-1.80(\mathrm{~m}, 1 \mathrm{H}) ;{ }^{13} \mathrm{C}$ NMR $(100 \mathrm{MHz}, \mathrm{DMSO}) \delta 162.9,161.6\left(\mathrm{~d}, J_{\mathrm{C}-\mathrm{F}}=241.3 \mathrm{~Hz}\right), 154.6,141.2(\mathrm{~d}$, $\left.J_{\mathrm{C}-\mathrm{F}}=2.9 \mathrm{~Hz}\right), 138.9,128.4,128.2\left(\mathrm{~d}, J_{\mathrm{C}-\mathrm{F}}=8.0 \mathrm{~Hz}\right), 124.4,123.2,121.6$, $115.2,115.1\left(\mathrm{~d}, J_{\mathrm{C}-\mathrm{F}}=9.9 \mathrm{~Hz}\right), 111.5,102.9,74.1,27.9 .{ }^{19} \mathrm{~F}$ NMR $(376$ $\left.\mathrm{MHz}, \mathrm{CDCl}_{3}\right) \delta-115.3$ (s). FT-IR: $v\left(\mathrm{~cm}^{-1}\right) 3649,3070,2953,2925,2857,1647,1558,1508,1437$, 1396, 1228. HRMS [ESI] m/z: [M+Na] $]^{+}$calcd for $\mathrm{C}_{19} \mathrm{H}_{17} \mathrm{FNaO}_{2}$ 319.1105; found 319.1113 .

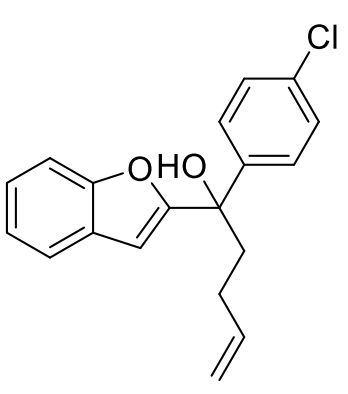

1d: $0.280 \mathrm{~g}, 72 \%$ yield, yellow oil. Purification by flash column chromatography (eluent: EtOAc/Petroleum ether $=1 / 50) .{ }^{1} \mathrm{H}$ NMR $(400$ $\left.\mathrm{MHz}, \mathrm{CDCl}_{3}\right) \delta$ 7.48-7.42 (m, 1H), 7.37-7.29 (m, 3H), 7.23-7.18 (m, $2 \mathrm{H}), 7.17-7.09(\mathrm{~m}, 2 \mathrm{H}), 6.55(\mathrm{~s}, 1 \mathrm{H}), 5.82-5.65(\mathrm{~m}, 1 \mathrm{H}), 4.98-4.83(\mathrm{~m}$, $2 \mathrm{H}), 2.67(\mathrm{~s}, 1 \mathrm{H}), 2.40-2.30(\mathrm{~m}, 1 \mathrm{H}), 2.23-2.04(\mathrm{~m}, 2 \mathrm{H}), 1.97-1.85(\mathrm{~m}$, $1 \mathrm{H}) ;{ }^{13} \mathrm{C}$ NMR (100 MHz, $\left.\mathrm{CDCl}_{3}\right) \delta 160.6,154.8,142.1,138.0,133.4$, $128.4,127.9,127.2,124.4,123.0,121.2,115.2,111.4,103.3,75.6,39.9$, 27.9. FT-IR: $v\left(\mathrm{~cm}^{-1}\right) 3530,3071,2951,2851,1641,1490,1454,1305$, 1251. HRMS [ESI] m/z: [M+Na] ${ }^{+}$calcd for $\mathrm{C}_{19} \mathrm{H}_{17} \mathrm{ClNaO}_{2} 335.0809$; found 335.0803 .

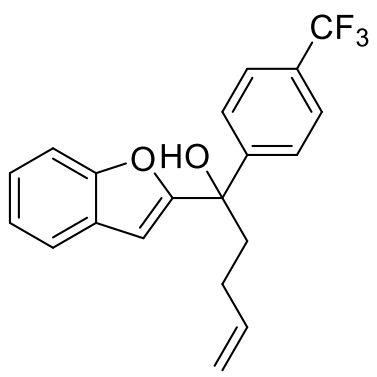

1e: $0.178 \mathrm{~g}, 41 \%$ yield, yellow oil. Purification by flash column chromatography (eluent: EtOAc/Petroleum ether $=1 / 50$ ). ${ }^{1} \mathrm{H}$ NMR (400 MHz, DMSO) $\delta$ 7.74-7.67 (m, 3H), 7.63-7.58 (m, 1H), 7.52-7.46 (m, 1H), 7.29-7.18 (m, 2H), 6.88-6.84 (m, 1H), $6.28(\mathrm{~s}, 1 \mathrm{H}), 5.89-5.72$ (m, 1H), 5.05-4.86 (m, 2H), 2.45-2.25 (m, 2H), 2.13-2.02 (m, 1H), 2.12-2.04 (m, 2H), 1.88-1.74 (m, 1H); ${ }^{13} \mathrm{C}$ NMR (100 MHz, $\left.\mathrm{CDCl}_{3}\right) \delta$ $159.7,154.3,147.1,137.4,129.2\left(\mathrm{q}, J_{\mathrm{C}-\mathrm{F}}=32.2 \mathrm{~Hz}\right), 127.4,125.6$, $124.8\left(\mathrm{q}, J_{\mathrm{C}-\mathrm{F}}=3.7 \mathrm{~Hz}\right), 124.1,123.6\left(\mathrm{q}, J_{\mathrm{C}-\mathrm{F}}=270.4 \mathrm{~Hz}\right), 122.6,120.8,114.8,110.9,103.0,75.2$, 39.5, 27.4. ${ }^{19} \mathrm{~F}$ NMR $\left(376 \mathrm{MHz}, \mathrm{CDCl}_{3}\right) \delta-62.5(\mathrm{~s})$. FT-IR: $v\left(\mathrm{~cm}^{-1}\right) 3676,2973,2925,1558,1489$, 1411, 1363, 1251, 1166. HRMS [ESI] m/z: $[\mathrm{M}+\mathrm{Na}]^{+}$calcd for $\mathrm{C}_{20} \mathrm{H}_{17} \mathrm{~F}_{3} \mathrm{NaO}_{2}$ 369.1073; found 364.1064 . 


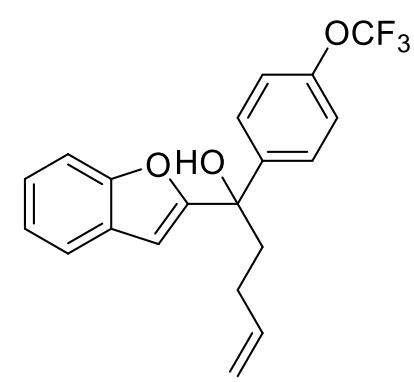

1f: $0.260 \mathrm{~g}, 59 \%$ yield, yellow oil. Purification by flash column chromatography (eluent: EtOAc/Petroleum ether $=1 / 50$ ). ${ }^{1} \mathrm{H}$ NMR (400 MHz, DMSO) $\delta$ 7.66-7.55 (m, 3H), 7.52-7.46 (m, 1H), 7.36-7.29 (m, 2H), 7.28-7.17 (m, 2H), $6.83(\mathrm{~d}, J=0.8 \mathrm{~Hz}, 1 \mathrm{H}), 6.20$ (s, 1H), 5.88-5.72 (m, 1H), 5.08-4.83 (m, 2H), 2.42-2.22 (m, 2H), 2.10-1.98 (m, 1H), 1.90-1.79 (m, 1H); ${ }^{13} \mathrm{C}$ NMR (100 MHz, DMSO) $\delta 162.4,154.6,147.6\left(\mathrm{q}, J_{\mathrm{C}-\mathrm{F}}=1.7 \mathrm{~Hz}\right), 144.4,138.7,128.3,128.1$, $124.5,123.3,121.6,120.9,120.5\left(\mathrm{q}, J_{\mathrm{C}-\mathrm{F}}=254.4 \mathrm{~Hz}\right), 115.1,111.5$, 103.1, 74.1, 39.7, 27.8. ${ }^{19} \mathrm{~F}$ NMR (376 MHz, DMSO) $\delta ~-56.8$ (s). FT-IR: $v\left(\mathrm{~cm}^{-1}\right) 3071,2979$, 2901, 1706, 1645, 1507, 1455, 1417, 1363, 1257, 1221. HRMS [ESI] m/z: $[\mathrm{M}+\mathrm{Na}]^{+}$calcd for $\mathrm{C}_{20} \mathrm{H}_{17} \mathrm{~F}_{3} \mathrm{NaO}_{3}$ 385.1022; found 385.1021.

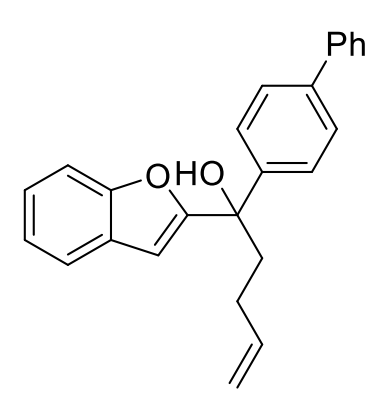

1g: $0.290 \mathrm{~g}, 66 \%$ yield, white solid. m.p. $94-95{ }^{\circ} \mathrm{C}$. Purification by flash column chromatography (eluent: EtOAc/Petroleum ether $=1 / 50) .{ }^{1} \mathrm{H}$ NMR $\left(400 \mathrm{MHz}, \mathrm{CD}_{3} \mathrm{OD}\right) \delta$ 7.63-7.46 $(\mathrm{m}, 7 \mathrm{H}), 7.42-7.31(\mathrm{~m}, 3 \mathrm{H})$, 7.29-7.22 (m, 1H), 7.21-7.12 (m, 2H), 6.74-6.66 (m, 1H), 5.91-5.73 (m, $1 \mathrm{H})$, 5.02-4.89 (m, 2H), 2.52-2.41 (m, $1 \mathrm{H}), 2.38-2.28(\mathrm{~m}, 1 \mathrm{H})$, 2.21-2.10 (m, 1H), 2.07-1.95 (m, 1H); ${ }^{13} \mathrm{C}$ NMR $\left(100 \mathrm{MHz}, \mathrm{CD}_{3} \mathrm{OD}\right) \delta$ $162.1,154.9,143.3,140.6,139.9,138.3,128.4,128.3,126.9,126.6$, $126.3,126.1,123.7,122.4,120.7,113.6,110.6,102.5,74.8,39.9,27.8$.

FT-IR: $v\left(\mathrm{~cm}^{-1}\right) 3676,3567,2973,2901,1792,1648,1508,1436,1375,1339,1243$. HRMS [ESI] $\mathrm{m} / \mathrm{z}$ : $[\mathrm{M}+\mathrm{Na}]^{+}$calcd for $\mathrm{C}_{25} \mathrm{H}_{22} \mathrm{NaO}_{2}$ 377.1512; found 377.1508.

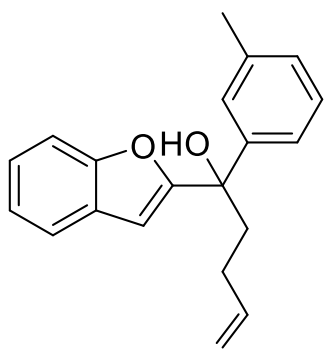

1h: $0.323 \mathrm{~g}, 61 \%$ yield, yellow oil. Purification by flash column chromatography (eluent: EtOAc/Petroleum ether $=1 / 50) .{ }^{1} \mathrm{H}$ NMR $(400$ $\left.\mathrm{MHz}, \mathrm{CDCl}_{3}\right) \delta$ 7.57-7.53 (m, 1H), 7.45-7.41 (m, 1H), $7.33(\mathrm{~s}, 1 \mathrm{H})$, 7.30-7.26 (m, 2H), 7.25-7.20 (m, 2H), 7.13-7.09 (m, 1H), $6.68(\mathrm{~d}, J=0.8$ $\mathrm{Hz}, 1 \mathrm{H}), 5.95-5.77(\mathrm{~m}, 1 \mathrm{H}), 5.11-4.91(\mathrm{~m}, 2 \mathrm{H}), 2.64(\mathrm{~s}, 1 \mathrm{H}), 2.52-2.42$ $(\mathrm{m}, 1 \mathrm{H}), 2.36(\mathrm{~s}, 3 \mathrm{H}), 2.34-2.27(\mathrm{~m}, 1 \mathrm{H}), 2.25-2.14(\mathrm{~m}, 1 \mathrm{H}), 2.11-2.01(\mathrm{~m}$ $1 \mathrm{H}) ;{ }^{13} \mathrm{C}$ NMR $\left(100 \mathrm{MHz}, \mathrm{CDCl}_{3}\right) \delta 161.2,154.8,143.6,138.4,137.9$, 128.3, 128.1, 128.1, 126.2, 124.1, 122.8, 122.7, 121.1, 114.9, 111.4, 103.1, 75.9, 39.9, 28.0, 21.7. FT-IR: $v\left(\mathrm{~cm}^{-1}\right) 3649,3066,2973,2921,1638,1558,1489,1437,1396,1251$. HRMS [ESI] m/z: $[\mathrm{M}+\mathrm{Na}]^{+}$calcd for $\mathrm{C}_{20} \mathrm{H}_{20} \mathrm{NaO}_{2}$ 315.1356; found 315.1370.

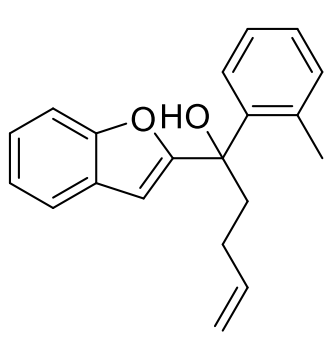

1i: $0.307 \mathrm{~g}, 57 \%$ yield, yellow oil. Purification by flash column chromatography (eluent: EtOAc/Petroleum ether $=1 / 50) .{ }^{1} \mathrm{H}$ NMR $(400$ $\left.\mathrm{MHz},\left(\mathrm{CD}_{3}\right)_{2} \mathrm{CO}\right) \delta$ 7.68-7.63 (m, 1H), 7.59-7.54 (m, $\left.1 \mathrm{H}\right), 7.37-7.31(\mathrm{~m}$, $1 \mathrm{H}), 7.26-7.14(\mathrm{~m}, 4 \mathrm{H}), 7.10-7.06(\mathrm{~m}, 1 \mathrm{H}), 6.75(\mathrm{~d}, J=1.2 \mathrm{~Hz}, 1 \mathrm{H})$, 5.92-5.70 (m, 1H), 5.02-4.82 (m, 2H), $3.74(\mathrm{~s}, 1 \mathrm{H}), 2.49-2.36(\mathrm{~m}, 2 \mathrm{H})$, $2.04(\mathrm{~s}, 3 \mathrm{H}), 1.91-1.89(\mathrm{~m}, 1 \mathrm{H}), 1.82-1.72(\mathrm{~m}, 1 \mathrm{H}) ;{ }^{13} \mathrm{C} \mathrm{NMR}(100 \mathrm{MHz}$, $\left.\left(\mathrm{CD}_{3}\right)_{2} \mathrm{CO}\right) \delta 162.5,154.9,141.7,139.2,136.2,132.5,128.7,128.1,127.6$, 126.0, 124.8, 123.5, 121.8, 114.5, 111.5, 103.6, 75.1, 38.2, 28.3, 20.7. FT-IR: $v\left(\mathrm{~cm}^{-1}\right) 3067,2955$, 2924, 1637, 1558, 1489, 1455, 1376, 1253. HRMS [ESI] m/z: $[\mathrm{M}+\mathrm{Na}]^{+}$calcd for $\mathrm{C}_{20} \mathrm{H}_{20} \mathrm{NaO}_{2}$ 


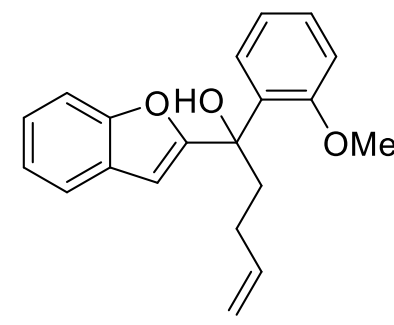

1j: $0.261 \mathrm{~g}$, $68 \%$ yield, white solid. m.p. $97-98{ }^{\circ} \mathrm{C}$. Purification by flash column chromatography (eluent: EtOAc/Petroleum ether = 1/50). ${ }^{1} \mathrm{H}$ NMR $\left(400 \mathrm{MHz},\left(\mathrm{CD}_{3}\right)_{2} \mathrm{CO}\right) \delta 7.68-7.63(\mathrm{~m}, 1 \mathrm{H})$, 7.59-7.54 (m, 1H), 7.37-7.32 (m, 1H), 7.31-7.24 (m, 1H), 7.21-7.11 $(\mathrm{m}, 2 \mathrm{H}), 7.02-6.93(\mathrm{~m}, 2 \mathrm{H}), 6.60(\mathrm{~s}, 1 \mathrm{H}), 5.91-5.71(\mathrm{~m}, 1 \mathrm{H})$, 5.00-4.77 (m, 2H), $4.94(\mathrm{~s}, 1 \mathrm{H}), 3.62(\mathrm{~s}, 3 \mathrm{H}), 2.70-2.59(\mathrm{~m}, 1 \mathrm{H})$, 2.46-2.36 (m, 1H), 2.21-2.09 (m, 1H), 1.97-1.84 (m, 1H); ${ }^{13} \mathrm{C}$ NMR $\left(100 \mathrm{MHz},\left(\mathrm{CD}_{3}\right)_{2} \mathrm{CO}\right) \delta 162.8,157.0,154.4,139.0,131.7,128.8,128.7,127.7,123.5,122.5$, $120.9,120.4,113.6,111.8,110.8,101.8,73.8,55.1,37.2,28.0 .{ }^{19} \mathrm{~F} \mathrm{NMR}\left(376 \mathrm{MHz}, \mathrm{CDCl}_{3}\right) \delta$ -115.0 (s). FT-IR: $v\left(\mathrm{~cm}^{-1}\right) 3649,2965,2925,1717,1647,1558,1507,1437,1289,1236$. HRMS [ESI] m/z: $[\mathrm{M}+\mathrm{Na}]^{+}$calcd for $\mathrm{C}_{20} \mathrm{H}_{20} \mathrm{NaO}_{3} 331.1305$; found 331.1312 .

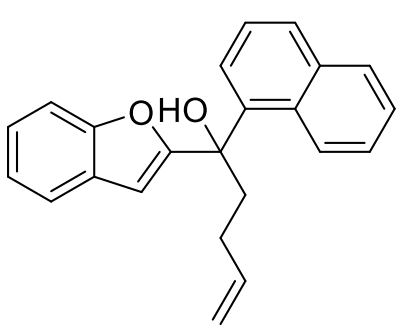

1k: $0.161 \mathrm{~g}, 40 \%$ yield, yellow oil. Purification by flash column chromatography (eluent: EtOAc/Petroleum ether $=1 / 50) .{ }^{1} \mathrm{H}$ NMR (400 MHz, DMSO) $\delta 8.19(\mathrm{~d}, J=8.8 \mathrm{~Hz}, 1 \mathrm{H}), 7.94-7.82(\mathrm{~m}, 3 \mathrm{H})$, 7.66-7.59 (m, 1H), 7.57-7.51 (t, $J=8.0 \mathrm{~Hz}, 1 \mathrm{H}), 7.43-7.35(\mathrm{~m}, 2 \mathrm{H})$, 7.32-7.26 (m, 1H), 7.23-7.16 (m, 2H), $6.98(\mathrm{~s}, 1 \mathrm{H}), 6.21(\mathrm{~s}, 1 \mathrm{H})$, 5.82-5.67 (m, 1H), 4.97-4.82 (m, 2H), 2.70-2.55 (m, 2H), 2.13-2.00 (m, 1H), 1.77-1.64 (m, 1H); ${ }^{13} \mathrm{C}$ NMR (100 MHz, DMSO) $\delta$ 163.2, 154.3, 139.4, 138.9, 134.5, 130.8, 129.3, 129.0, 128.5, 125.8, 125.7, 125.5, 125.4, 125.3, 124.4, 123.2, 121.6, 115.0, 111.6, 103.2, 74.4, 38.5, 28.1. FT-IR: $v\left(\mathrm{~cm}^{-1}\right)$ 3058, 2974, 2929, 1647, 1508, 1396, 1362, 1252, 1243. HRMS [ESI] m/z: $[\mathrm{M}+\mathrm{Na}]^{+}$calcd for $\mathrm{C}_{23} \mathrm{H}_{20} \mathrm{NaO}_{2} 351.1356$; found 351.1363.

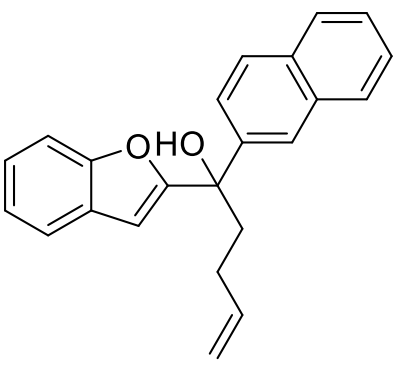

11: $0.218 \mathrm{~g}, 54 \%$ yield, yellow oil. Purification by flash column chromatography (eluent: EtOAc/Petroleum ether $=1 / 50) .{ }^{1} \mathrm{H}$ NMR $\left(400 \mathrm{MHz}, \mathrm{CDCl}_{3}\right) \delta 7.93(\mathrm{~s}, 1 \mathrm{H}), 7.81-7.67(\mathrm{~m}, 3 \mathrm{H}), 7.49-7.43(\mathrm{~m}$, 2H), 7.41-7.35 (m, 2H), 7.34-7.28 (m, 1H), 7.19-7.09 (m, 3H), 6.63-6.56 (m, 1H), 5.84-5.68 (m, 1H), 4.98-4.83 (m, 2H), $2.71(\mathrm{br}$, $1 \mathrm{H}), 2.53-2.43(\mathrm{~m}, 1 \mathrm{H}), 2.39-2.28(\mathrm{~m}, 1 \mathrm{H}), 2.21-2.10(\mathrm{~m}, 1 \mathrm{H})$, 2.01-1.90 (m, $1 \mathrm{H}) ;{ }^{13} \mathrm{C}$ NMR $\left(100 \mathrm{MHz}, \mathrm{CDCl}_{3}\right) \delta 160.6,154.4$, $140.4,137.8,132.6,132.2,127.9,127.6,127.6,127.1,125.8,125.7,124.0,123.8,123.5,122.4$, 120.7, 114.5, 110.9, 102.9, 75.5, 39.3, 27.5. FT-IR: $v\left(\mathrm{~cm}^{-1}\right)$ 3060, 2973, 2926, 1869, 1772, 1507, 1455, 1362, 1305, 1250. HRMS [ESI] m/z: $[\mathrm{M}+\mathrm{Na}]^{+}$calcd for $\mathrm{C}_{23} \mathrm{H}_{20} \mathrm{NaO}_{2}$ 351.1356; found 351.1351 .

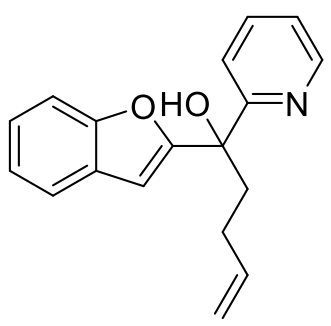

1m: 69mg, 13\% yield, yellow oil. Purification by flash column chromatography (eluent: EtOAc/Petroleum ether $=1 / 50) .{ }^{1} \mathrm{H}$ NMR $(400$ $\left.\mathrm{MHz},\left(\mathrm{CD}_{3}\right)_{2} \mathrm{CO}\right) \delta 8.55-8.49(\mathrm{~m}, 1 \mathrm{H}), 7.77(\mathrm{ddd}, J=8.0,8.0,2.0 \mathrm{~Hz}$, $1 \mathrm{H}), 7.63-7.56(\mathrm{~m}, 1 \mathrm{H}), 7.55-7.49(\mathrm{~m}, 1 \mathrm{H}), 7.40-7.34(\mathrm{~m}, 1 \mathrm{H}), 7.31-7.25$ $(\mathrm{m}, 1 \mathrm{H}), 7.24-7.13(\mathrm{~m}, 2 \mathrm{H}), 6.75(\mathrm{~d}, J=0.8 \mathrm{~Hz}, 1 \mathrm{H}), 5.88-5.72(\mathrm{~m}, 1 \mathrm{H})$, 5.77 (br, $1 \mathrm{H}), 5.00-4.82(\mathrm{~m}, 2 \mathrm{H}), 2.49-2.38(\mathrm{~m}, 1 \mathrm{H}), 2.35-2.25(\mathrm{~m}, 1 \mathrm{H})$, 
2.11-2.06 (m, 1H), 1.86-1.75 (m, 1H); ${ }^{13} \mathrm{C}$ NMR $\left(100 \mathrm{MHz},\left(\mathrm{CD}_{3}\right)_{2} \mathrm{CO}\right) \delta 162.0,161.0,155.3$, 148.1, 139.0, 138.1, 128.8, 124.7, 123.6, 123.4, 121.7, 121.4, 114.5, 111.4, 103.4, 75.1, 39.3, 28.0.FT-IR: $v\left(\mathrm{~cm}^{-1}\right) 3346,3070,2974,2924,1641,1593,1455,1396,1298,1250$. HRMS [ESI] $\mathrm{m} / \mathrm{z}:[\mathrm{M}+\mathrm{Na}]^{+}$calcd for $\mathrm{C}_{18} \mathrm{H}_{17} \mathrm{NNaO}_{2} 302.1151$; found 302.1151 .

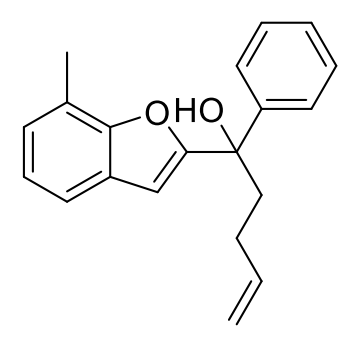

1n: $0.267 \mathrm{~g}, 60 \%$ yield, yellow oil. Purification by flash column chromatography (eluent: EtOAc/Petroleum ether $=1 / 50) .{ }^{1} \mathrm{H}$ NMR $(400$ $\left.\mathrm{MHz}, \mathrm{CDCl}_{3}\right) \delta$ 7.54-7.48 (m, 2H), 7.41-7.31 (m, 3H), 7.27-7.21 (m, 1H), 7.12-7.02 (m, 2H), $6.72(\mathrm{~s}, 1 \mathrm{H}), 6.01(\mathrm{~s}, 1 \mathrm{H}), 5.87-4.73(\mathrm{~m}, 1 \mathrm{H})$, 5.01-4.87 (m, 2H), $2.40(\mathrm{~s}, 3 \mathrm{H}), 2.38-2.31(\mathrm{~m}, 1 \mathrm{H}), 2.30-2.21(\mathrm{~m}, 1 \mathrm{H})$, 2.10-1.99 (m, 1H), 1.94-1.82 (m, 1H); ${ }^{13} \mathrm{C}$ NMR (100 MHz, DMSO) $\delta$ $162.8,153.5,145.2,139.0,128.4,127.9,127.3,126.1,125.1,123.2$, 121.0, 118.9, 115.0, 103.1, 74.5, 28.1, 15.2. FT-IR: $v\left(\mathrm{~cm}^{-1}\right)$ 3676, 3064, 2973, 2923, 1647, 1558, $1508,1489,1448,1340,1266$. HRMS [ESI] m/z: $[\mathrm{M}+\mathrm{Na}]^{+}$calcd for $\mathrm{C}_{20} \mathrm{H}_{20} \mathrm{NaO}_{2} 315.1356$; found 315.1357.

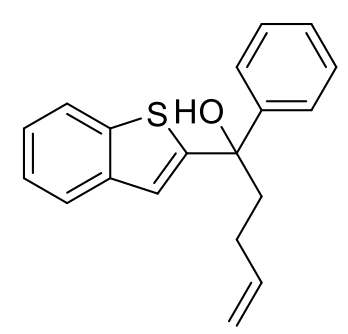

1o: $0.374 \mathrm{~g}, 32 \%$ yield, yellow oil. Purification by flash column chromatography (eluent: EtOAc/Petroleum ether $=1 / 50) .{ }^{1} \mathrm{H}$ NMR $(400$ $\left.\mathrm{MHz}, \mathrm{CDCl}_{3}\right) \delta$ 7.80-7.74 (m, 1H), 7.73-7.68 (m, 1H), 7.60-7.50 (m, 2H), 7.42-7.32 (m, 3H), 7.32-7.27 (m, 2H), 7.20-7.16 (m, 1H), 5.96-5.81 (m, $1 \mathrm{H}), 5.10-4.95(\mathrm{~m}, 2 \mathrm{H}), 2.58(\mathrm{br}, 1 \mathrm{H}), 2.52-2.45(\mathrm{~m}, 2 \mathrm{H}), 2.32-2.19(\mathrm{~m}$, $1 \mathrm{H}), 2.15-2.02(\mathrm{~m}, 1 \mathrm{H}) ;{ }^{13} \mathrm{C} \mathrm{NMR}\left(100 \mathrm{MHz}, \mathrm{CDCl}_{3}\right) \delta 152.9,144.6$, $139.2,139.1,137.8,127.8,126.9,125.2,123.8,123.7,123.1,121.8$, 119.9, 114.7, 77.1, 41.7, 27.9. FT-IR: $v\left(\mathrm{~cm}^{-1}\right)$ 3060, 2926, 2865, 1869, 1684, 1558, 1458, 1362 , 1306. HRMS [ESI] m/z: [M+Na] $]^{+}$calcd for $\mathrm{C}_{19} \mathrm{H}_{18} \mathrm{NaOS} 317.0971$; found 317.0984.

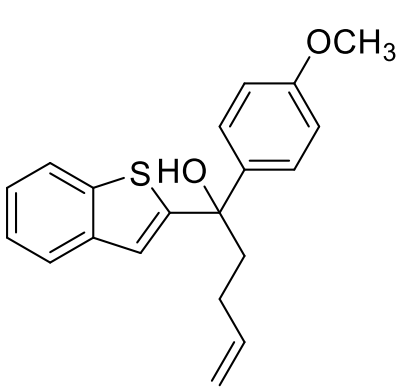

1p: $0.230 \mathrm{~g}, 57 \%$ yield, yellow solid. m.p. $67-68^{\circ} \mathrm{C}$. Purification by flash column chromatography (eluent: EtOAc/Petroleum ether $=$ 1/50). ${ }^{1} \mathrm{H}$ NMR $\left(400 \mathrm{MHz},\left(\mathrm{CD}_{3}\right)_{2} \mathrm{CO}\right) \delta 7.69(\mathrm{~d}, J=8.0 \mathrm{~Hz}, 1 \mathrm{H})$, $7.63(\mathrm{~d}, J=7.6 \mathrm{~Hz}, 1 \mathrm{H}), 7.38-7.32(\mathrm{~m}, 2 \mathrm{H}), 7.26-7.17(\mathrm{~m}, 2 \mathrm{H}), 7.16$ (s, $1 \mathrm{H}), 6.82-6.76(\mathrm{~m}, 2 \mathrm{H}), 5.85-5.70(\mathrm{~m}, 1 \mathrm{H}), 4.98-4.80(\mathrm{~m}, 2 \mathrm{H})$, $3.85(\mathrm{~s}, 1 \mathrm{H}), 3.67(\mathrm{~s}, 3 \mathrm{H}), 2.40-2.25(\mathrm{~m}, 2 \mathrm{H}), 2.12-2.05(\mathrm{~m}, 1 \mathrm{H})$, 1.94-1.86 (m, 1H); ${ }^{13} \mathrm{C}$ NMR (100 MHz, $\left.\left(\mathrm{CD}_{3}\right)_{2} \mathrm{CO}\right) \delta 159.2,155.7$, 140.4, 139.9, 139.2, 138.7, 127.4, 124.8, 124.6, 124.0, 122.7, 120.0, 114.6, 113.8, 76.9, 55.4, 42.3, 28.6. FT-IR: $v\left(\mathrm{~cm}^{-1}\right)$ 3676, 3503, 2968, 2930, 1684, 1509, 1473, 1374, 1292, 1241. HRMS [ESI] m/z: [M+Na] ${ }^{+}$calcd for $\mathrm{C}_{20} \mathrm{H}_{20} \mathrm{NaO}_{2} \mathrm{~S}$ 347.1076; found 347.1082.

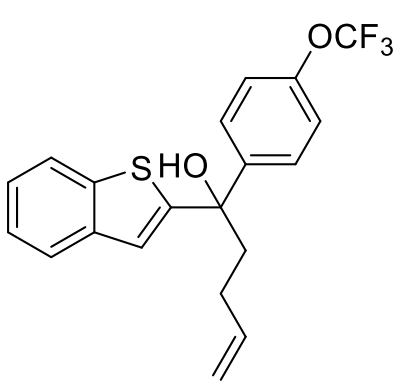

1q: $0.301 \mathrm{~g}, 64 \%$ yield, white solid. m.p. $79-80{ }^{\circ} \mathrm{C}$. Purification by flash column chromatography (eluent: EtOAc/Petroleum ether = 1/50). ${ }^{1} \mathrm{H}$ NMR (400 MHz, $\left.\left(\mathrm{CD}_{3}\right)_{2} \mathrm{CO}\right) \delta 7.82(\mathrm{~d}, J=7.6 \mathrm{~Hz}, 1 \mathrm{H})$, 7.79-7.71 (m, 3H), $7.40(\mathrm{~s}, 1 \mathrm{H}), 7.35-7.25(\mathrm{~m}, 4 \mathrm{H}), 5.96-5.78(\mathrm{~m}$, $1 \mathrm{H}), 5.29$ (s, 1H), 5.06-4.87 (m, 2H), 2.61-2.47 (m, 2H), 2.33-2.21 (m, 1H), 2.03-1.92 (m, 1H); ${ }^{13} \mathrm{C}$ NMR (100 MHz, DMSO) $\delta 155.4$, $147.4\left(\mathrm{q}, J_{\mathrm{C}-\mathrm{F}}=1.6 \mathrm{~Hz}\right), 146.3,140.0,139.2,138.8,127.8,124.6$, 
124.4, 124.0, 122.7, 120.9, $120.5\left(\mathrm{q}, J_{\mathrm{C}-\mathrm{F}}=254.4 \mathrm{~Hz}\right), 119.7,115.1,75.8,41.3,28.2 .{ }^{19} \mathrm{~F}$ NMR $\left(376 \mathrm{MHz},\left(\mathrm{CD}_{3}\right)_{2} \mathrm{CO}\right) \delta-58.6$ (s). FT-IR: $v\left(\mathrm{~cm}^{-1}\right)$ 2959, 2926, 1717, 1541, 1507, 1457, 1258, 1220. HRMS [ESI] m/z: [M+Na] ${ }^{+}$calcd for $\mathrm{C}_{20} \mathrm{H}_{17} \mathrm{~F}_{3} \mathrm{NaO}_{2} \mathrm{~S} 401.0794$; found 401.0784 .

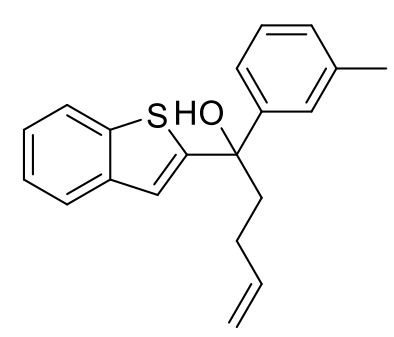

1r: $0.272 \mathrm{~g}, 71 \%$ yield, yellow oil. Purification by flash column chromatography (eluent: EtOAc/Petroleum ether $=1 / 50$ ). ${ }^{1} \mathrm{H}$ NMR $\left(400 \mathrm{MHz},\left(\mathrm{CD}_{3}\right)_{2} \mathrm{CO}\right) \delta$ 7.78-7.66 (m, 2H), $7.34(\mathrm{~s}, 1 \mathrm{H}), 7.31-7.15(\mathrm{~m}$, $5 \mathrm{H}), 7.07-6.99(\mathrm{~m}, 1 \mathrm{H}), 5.92-5.76(\mathrm{~m}, 1 \mathrm{H}), 5.03-4.87(\mathrm{~m}, 2 \mathrm{H}), 3.95(\mathrm{~s}$, $1 \mathrm{H}), 2.47-2.34(\mathrm{~m}, 2 \mathrm{H}), 2.28(\mathrm{~s}, 3 \mathrm{H}), 2.20-2.12(\mathrm{~m}, 1 \mathrm{H}), 1.97-1.90(\mathrm{~m}$, $1 \mathrm{H}) ;{ }^{13} \mathrm{C}$ NMR $\left(100 \mathrm{MHz},\left(\mathrm{CD}_{3}\right)_{2} \mathrm{CO}\right) \delta 155.4,146.3,140.0,139.6$, $138.7,137.3,127.9,127.5,126.3,124.1,123.9,123.5,122.8,122.1$, 119.5, 114.0, 76.4, 42.2, 28.2, 21.0. FT-IR: $v\left(\mathrm{~cm}^{-1}\right)$ 3649, 3062, 2972, 2923, 1684, 1508, 1457, 1375, 1250, 1229. HRMS [ESI] m/z: [M+Na] ${ }^{+}$calcd for $\mathrm{C}_{20} \mathrm{H}_{20} \mathrm{NaOS} 331.1127$; found 331.1132 .

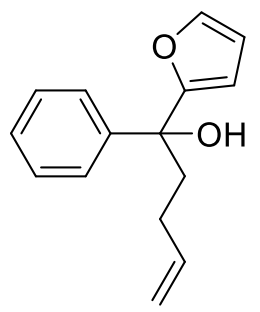

1s: $0.221 \mathrm{~g}, \quad 44 \%$ yield, yellow oil. Purification by flash column chromatography (eluent: EtOAc/Petroleum ether $=1 / 50) .{ }^{1} \mathrm{H}$ NMR $(400 \mathrm{MHz}$, $\left.\mathrm{CD}_{3} \mathrm{OD}\right) \delta$ 7.45-7.36 (m, 3H), 7.34-7.27 (m, 2H), 7.25-7.19 (m, 1H), $6.35(\mathrm{dd}$, $J=3.2,2.0 \mathrm{~Hz}, 1 \mathrm{H}), 6.24(\mathrm{dd}, J=3.2,0.8 \mathrm{~Hz}, 1 \mathrm{H}), 5.85-5.72(\mathrm{~m}, 1 \mathrm{H})$, 4.98-4.87 (m, 2H), 2.37-2.27 (m, 1H), 2.23-2.14 (m, 1H), 2.12-2.01 (m, 1H), 1.94-1.83 (m, 1H); ${ }^{13} \mathrm{C}$ NMR (100 MHz, $\left.\mathrm{CDCl}_{3}\right) \delta 163.3,148.7,145.4,142.3$, 131.4, 130.5, 129.5, 117.2, 113.5, 109.6, 78.6, 44.2, 31.7. FT-IR: $v\left(\mathrm{~cm}^{-1}\right) 3063,2951,2930,2857$, 1641, 1494, 1448, 1319, 1225. HRMS [ESI] m/z: [M+Na] ${ }^{+}$calcd for $\mathrm{C}_{15} \mathrm{H}_{16} \mathrm{NaO}_{2} 251.1043$; found 251.1043

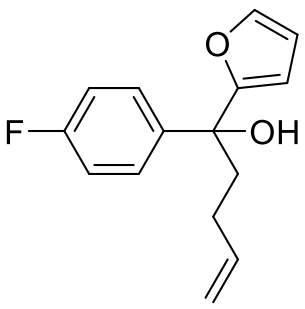

1t: $0.145 \mathrm{~g}, 45 \%$ yield, yellow oil. Purification by flash column chromatography (eluent: EtOAc/Petroleum ether $=1 / 50) .{ }^{1} \mathrm{H}$ NMR $(400$ MHz, DMSO) $\delta 7.54(\mathrm{dd}, J=1.6,0.8 \mathrm{~Hz}, 1 \mathrm{H}), 7.44-7.35(\mathrm{~m}, 2 \mathrm{H})$, 7.16-7.08 (m, 2H), $6.38(\mathrm{dd}, J=3.2,1.6 \mathrm{~Hz}, 1 \mathrm{H}), 6.28(\mathrm{dd}, J=3.2,0.8 \mathrm{~Hz}$, $1 \mathrm{H}), 5.82(\mathrm{~s}, 1 \mathrm{H}), 5.80-5.70(\mathrm{~m}, 1 \mathrm{H}), 4.98-4.85(\mathrm{~m}, 2 \mathrm{H}), 2.28-2.16(\mathrm{~m}, 1 \mathrm{H})$, 2.15-2.07 (m, 1H), 2.03-1.93 (m, 1H), 1.82-1.72 (m, 1H); ${ }^{13} \mathrm{C}$ NMR $(100$ $\left.\mathrm{MHz}, \mathrm{CD}_{3} \mathrm{OD}\right) \delta 163.0\left(\mathrm{~d}, J_{\mathrm{C}-\mathrm{F}}=242.4 \mathrm{~Hz}\right), 159.1,141.6,140.8\left(\mathrm{~d}, J_{\mathrm{C}-\mathrm{F}}=3.2 \mathrm{~Hz}\right), 138.3,127.5(\mathrm{~d}$, $\left.J_{\mathrm{C}-\mathrm{F}}=8.0 \mathrm{~Hz}\right), 114.1\left(\mathrm{~d}, J_{\mathrm{C}-\mathrm{F}}=21.4 \mathrm{~Hz}\right), 113.4,109.7,105.7,74.3,40.3,27.7 .{ }^{19} \mathrm{~F}$ NMR $(376 \mathrm{MHz}$, DMSO) $\delta$-116.8 (s). FT-IR: $v\left(\mathrm{~cm}^{-1}\right)$ 3649, 3079, 2977, 2872, 1734, 1684, 1603, 1508, 1457, 1302, 1225. HRMS [ESI] m/z: [M+Na] ${ }^{+}$calcd for $\mathrm{C}_{15} \mathrm{H}_{15} \mathrm{FNaO}_{2}$ 269.0948; found 269.0938.

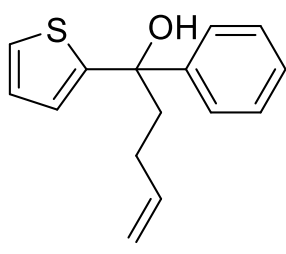

1u: $0.998 \mathrm{~g}, 61 \%$ yield, yellow oil. Purification by flash column chromatography (eluent: EtOAc/Petroleum ether $=1 / 50) .{ }^{1} \mathrm{H}$ NMR $(400$ $\left.\mathrm{MHz}, \mathrm{CDCl}_{3}\right) \delta$ 7.59-7.57 (m, 1H), 7.57-7.55 (m, 1H), 7.44-7.38 (m, 2H), 7.36-7.30 (m, 1H), 7.29-7.26 (m, 1H), 7.02-6.95 (m, 2H), 6.00-5.85 (m, 1H), 5.16-4.98 (m, 2H), $2.70(\mathrm{~s}, 1 \mathrm{H}), 2.57-2.41(\mathrm{~m}, 2 \mathrm{H}), 2.36-2.22(\mathrm{~m}, 1 \mathrm{H})$, 2.15-2.01 (m, 1H); ${ }^{13} \mathrm{C}$ NMR $\left(100 \mathrm{MHz}, \mathrm{CDCl}_{3}\right) \delta 152.7,145.2,138.0,127.8,126.8,126.2,125.3$, 124.4, 123.6, 114.5, 76.7, 42.4, 28.0. FT-IR: $v\left(\mathrm{~cm}^{-1}\right)$ 3070, 2975, 2924, 2853, 1638, 1492, 1447 , 1362, 1316, 1235. HRMS [ESI] m/z: [M+Na] $]^{+}$calcd for $\mathrm{C}_{15} \mathrm{H}_{16} \mathrm{NaOS} 267.0814$; found 267.0828. 


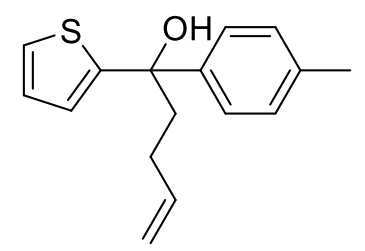

1v: $0.485 \mathrm{~g}, 60 \%$ yield, yellow oil. $66-67{ }^{\circ} \mathrm{C}$. Purification by flash column chromatography (eluent: EtOAc/Petroleum ether $=1 / 50$ ). ${ }^{1} \mathrm{H}$ NMR $\left(400 \mathrm{MHz}, \mathrm{CD}_{3} \mathrm{OD}\right) \delta$ 7.39-7.31 (m, 2H), 7.25-7.17 (m,1H), 7.14-7.08 (m, 2H), 6.94-6.86 (m, 2H), 5.88-5.73 (m, 1H), 4.99-4.87 (m, $2 \mathrm{H}), 2.38-2.31(\mathrm{~m}, 2 \mathrm{H}), 2.30(\mathrm{~s}, 3 \mathrm{H}), 2.21-2.09(\mathrm{~m}, 1 \mathrm{H}), 1.97-1.85(\mathrm{~m}$, 1H); ${ }^{13} \mathrm{C}$ NMR (100 MHz, DMSO) $\delta$ 155.5, 144.6, 139.2, 135.8, 128.8, 126.9, 125.8, 124.6, 123.1, 114.9, 75.7, 42.3, 28.4, 21.0. FT-IR: $v\left(\mathrm{~cm}^{-1}\right)$ 3547, 3074, 2921, 1640, 1511, 1436, 1352, 1311, 1234. HRMS [ESI] m/z: [M+Na] ${ }^{+}$calcd for $\mathrm{C}_{16} \mathrm{H}_{18} \mathrm{NaOS} 281.0971$; found 281.0976.

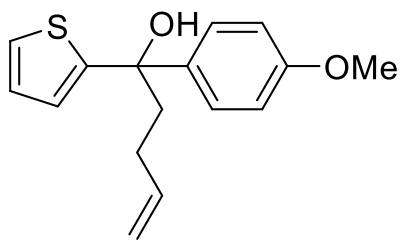

1w: $0.263 \mathrm{~g}, 76 \%$ yield, yellow oil. Purification by flash column chromatography (eluent: EtOAc/Petroleum ether $=1 / 50$ ). ${ }^{1} \mathrm{H}$ NMR $\left(400 \mathrm{MHz}, \mathrm{CD}_{3} \mathrm{OD}\right) \delta$ 7.41-7.33 (m, 2H), 7.26-7.20 (m, 1H), 6.93-6.88 (m, 2H), 6.87-6.82 (m, 2H), 5.88-5.75 (m, 1H), 5.00-4.88 $(\mathrm{m}, 2 \mathrm{H}), 3.76(\mathrm{~s}, 3 \mathrm{H}), 2.38-2.28(\mathrm{~m}, 2 \mathrm{H}), 2.19-2.08(\mathrm{~m}, 1 \mathrm{H})$, 1.99-1.86 (m, 1H); ${ }^{13} \mathrm{C}$ NMR (100 MHz, $\left.\mathrm{CD}_{3} \mathrm{OD}\right) \delta 158.5,153.9,138.5,138.5,126.7,125.8$, 123.8, 123.2, 113.3, 112.8, 75.9, 54.3, 42.8, 28.2. FT-IR: $v\left(\mathrm{~cm}^{-1}\right)$ 3676, 3074, 2971, 2931, 1638, 1541, 1417, 1395, 1340, 1248. HRMS [ESI] m/z: $[\mathrm{M}+\mathrm{Na}]^{+}$calcd for $\mathrm{C}_{16} \mathrm{H}_{18} \mathrm{NaO}_{2} \mathrm{~S}$ 297.0920; found 297.0919.

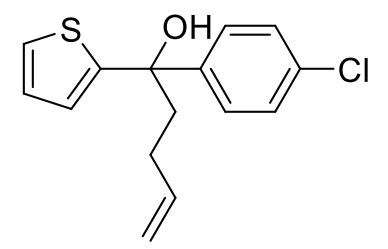

1x: $0.180 \mathrm{~g}, 52 \%$ yield, colorless oil. Purification by flash column chromatography (eluent: EtOAc/Petroleum ether $=1 / 50$ ). ${ }^{1} \mathrm{H}$ NMR $\left(400 \mathrm{MHz},\left(\mathrm{CD}_{3}\right)_{2} \mathrm{CO}\right) \delta$ 7.40-7.34 (m, 2H), 7.26-7.20 (m, 2H), 7.16 $(\mathrm{dd}, J=5.2,1.2 \mathrm{~Hz}, 1 \mathrm{H}), 6.88(\mathrm{dd}, J=3.2,1.2 \mathrm{~Hz}, 1 \mathrm{H}), 6.86-6.82(\mathrm{~m}$, $1 \mathrm{H}), 5.82-5.68(\mathrm{~m}, 1 \mathrm{H}), 4.94-4.80(\mathrm{~m}, 2 \mathrm{H}), 3.85(\mathrm{~s}, 1 \mathrm{H}), 2.33-2.20(\mathrm{~m}$, $2 \mathrm{H}), 2.05-1.97(\mathrm{~m}, 1 \mathrm{H}), 1.82-1.71(\mathrm{~m}, 1 \mathrm{H}) ;{ }^{13} \mathrm{C} \mathrm{NMR}\left(100 \mathrm{MHz},\left(\mathrm{CD}_{3}\right)_{2} \mathrm{CO}\right) \delta 154.1,146.2,139.0$, 132.6, 128.5, 127.9, 127.2, 125.3, 124.0, 114.6, 76.6, 42.6, 28.5. FT-IR: $v\left(\mathrm{~cm}^{-1}\right) 3649,3078,2973$, 2923, 1638, 1521, 1418, 1375, 1318, 1235. HRMS [ESI] m/z: [M+Na] ${ }^{+}$calcd for $\mathrm{C}_{15} \mathrm{H}_{15} \mathrm{ClNaOS}$ 301.0424; found 301.0428 .

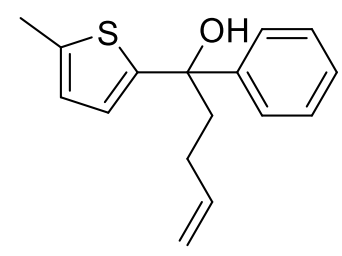

1y: $0.297 \mathrm{~g}, 61 \%$ yield, yellow oil. Purification by flash column chromatography (eluent: EtOAc/Petroleum ether $=1 / 50) .{ }^{1} \mathrm{H}$ NMR $(400$ $\left.\mathrm{MHz}, \mathrm{CDCl}_{3}\right) \delta$ 7.49-7.43 (m, 2H), 7.35-7.30 (m, 2H), 7.26-7.22 (m, 1H), 6.72-6.64 (m, 1H), 6.60-6.52 (m, 1H), 5.89-5.75 (m, 1H), 5.05-4.90 (m, $2 \mathrm{H}), 2.43-2.39(\mathrm{~m}, 3 \mathrm{H}), 2.38-2.32(\mathrm{~m}, 2 \mathrm{H}), 2.34(\mathrm{~s}, 1 \mathrm{H}), 2.25-2.13(\mathrm{~m}$, 1H), 2.00-1.89 (m, 1H); ${ }^{13} \mathrm{C}$ NMR (100 MHz, $\left.\mathrm{CDCl}_{3}\right) \delta 150.5,145.6,139.5,138.5,128.1,127.1$, 125.7, 124.6, 123.8, 114.8, 42.5, 28.4, 15.3. FT-IR: $v\left(\mathrm{~cm}^{-1}\right)$ 3675, 3061, 2973, 2920, 1640, 1493, 1446, 1311, 1256, 1228. HRMS [ESI] $\mathrm{m} / \mathrm{z}$ : $[\mathrm{M}+\mathrm{Na}]^{+}$calcd for $\mathrm{C}_{16} \mathrm{H}_{18} \mathrm{NaOS} 281.0971$; found 281.0965 .

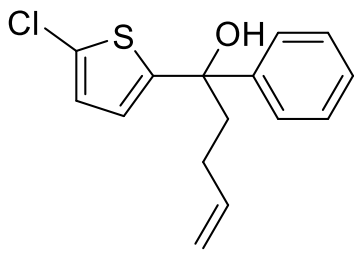

1z: $0.247 \mathrm{~g}, 44 \%$ yield, yellow oil. Purification by flash column chromatography (eluent: EtOAc/Petroleum ether $=1 / 50$ ). ${ }^{1} \mathrm{H}$ NMR $\left(400 \mathrm{MHz}, \mathrm{CDCl}_{3}\right) \delta$ 7.56-7.49 (m, 2H), 7.45-7.38 (m, 2H), 7.37-7.29 $(\mathrm{m}, 1 \mathrm{H}), 6.82-6.76(\mathrm{~m}, 1 \mathrm{H}), 6.75-6.70(\mathrm{~m}, 1 \mathrm{H}), 5.98-5.78(\mathrm{~m}, 1 \mathrm{H})$, 5.15-4.94 (m, 2H), $2.54(\mathrm{br}, 1 \mathrm{H}), 2.48-2.33(\mathrm{~m}, 2 \mathrm{H}), 2.30-2.18(\mathrm{~m}, 1 \mathrm{H})$, 
2.12-2.00 (m, 1H); ${ }^{13} \mathrm{C}$ NMR $\left(100 \mathrm{MHz}, \mathrm{CDCl}_{3}\right) \delta 151.1,144.4,137.7,129.0,127.8,127.0,125.1$, 125.0, 122.6, 114.7, 76.8, 41.7, 27.8. FT-IR: $v\left(\mathrm{~cm}^{-1}\right)$ 3475, 2923, 1640, 1491, 1447, 1362, 1260 , 1216. HRMS [ESI] m/z: [M+Na] ${ }^{+}$calcd for $\mathrm{C}_{15} \mathrm{H}_{15} \mathrm{ClNaOS} 301.0424$; found 301.0429 .

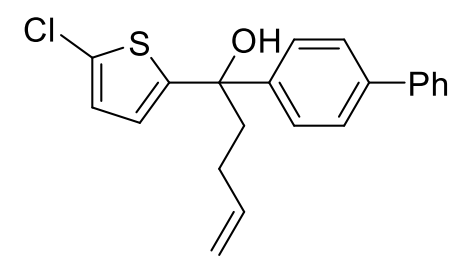

1aa: 99mg, 28\% yield, yellow oil. Purification by flash column chromatography (eluent: EtOAc/Petroleum ether $=1 / 50) . \quad{ }^{1} \mathrm{H}$ NMR (400 MHz, $\left.\left(\mathrm{CD}_{3}\right)_{2} \mathrm{CO}\right) \delta$ 7.57-7.49 (m, 4H), 7.47-7.43 (m, $2 \mathrm{H}), 7.38-7.32(\mathrm{~m}, 2 \mathrm{H}), 7.28-7.23(\mathrm{~m}, 1 \mathrm{H}), 6.76-6.68(\mathrm{~m}, 2 \mathrm{H})$, 5.83-5.68 (m, 1H), 4.96-4.81 (m, 2H), $3.98(\mathrm{br}, 1 \mathrm{H}), 2.35-2.19(\mathrm{~m}$,

2H), 1.89-1.84 (m, 2H); ${ }^{13} \mathrm{C}$ NMR $\left(100 \mathrm{MHz},\left(\mathrm{CD}_{3}\right)_{2} \mathrm{CO}\right) \delta 153.8,145.8,140.9,140.1,138.9$, 129.5, 128.6, 128.0, 127.4, 127.2, 126.7, 126.5, 123.2, 114.7, 77.0, 42.1, 28.5. FT-IR: $v\left(\mathrm{~cm}^{-1}\right)$ 3546, 3064, 2925, 2854, 1640, 1487, 1448, 1403, 1216. HRMS [ESI] $\mathrm{m} / \mathrm{z}:[\mathrm{M}+\mathrm{Na}]^{+}$calcd for $\mathrm{C}_{21} \mathrm{H}_{19} \mathrm{ClNaOS} 377.0737$; found 377.0730.

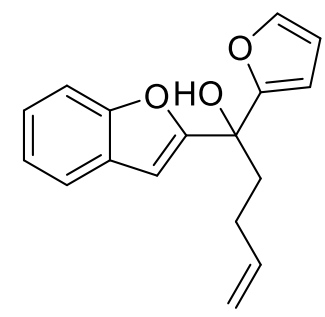

1ab: $0.232 \mathrm{~g}, \quad 47 \%$ yield, red oil. Purification by flash column chromatography (eluent: EtOAc/Petroleum ether $=1 / 50) .{ }^{1} \mathrm{H}$ NMR $(400$ MHz, DMSO) $\delta$ 7.63-7.56 (m, 2H), 7.53-7.48 (m, 1H), 7.28-7.18 (m, 2H), 6.79-6.74 (m, 1H), 6.45-6.40 (m, 1H), 6.38-6.34 (m, 1H), $6.23(\mathrm{~s}, 1 \mathrm{H})$, 5.86-5.71 (m, 1H), 5.03-4.86 (m, 2H), 2.35-2.20 (m, 2H), 2.07-1.86 (m, 2H); ${ }^{13} \mathrm{C}$ NMR (100 MHz, DMSO) $\delta 160.7,157.1,154.6,142.7,138.7$, 128.3, 124.4, 123.3, 121.6, 115.1, 111.5, 110.8, 106.9, 103.4, 71.6, 37.8, 27.9. FT-IR: $v\left(\mathrm{~cm}^{-1}\right)$ 3073, 2956, 2928, 2855, 1641, 1582, 1557, 1454, 1372, 1252. HRMS [ESI] m/z: [M+Na] ${ }^{+}$calcd for $\mathrm{C}_{17} \mathrm{H}_{16} \mathrm{NaO}_{3} 291.0992$; found 291.0993 .

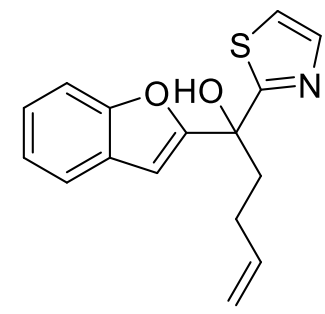

1ac: $0.200 \mathrm{~g}, 56 \%$ yield, white solid. m.p. $76-77^{\circ} \mathrm{C}$. Purification by flash column chromatography (eluent: EtOAc/Petroleum ether $=1 / 50) .{ }^{1} \mathrm{H}$ NMR $\left(400 \mathrm{MHz},\left(\mathrm{CD}_{3}\right)_{2} \mathrm{CO}\right) \delta$ 7.80-7.73 (m, $\left.1 \mathrm{H}\right), 7.70-7.64(\mathrm{~m}, 1 \mathrm{H}), 7.62-7.57$ (m, $1 \mathrm{H}), 7.54-7.48(\mathrm{~m}, 1 \mathrm{H}), 7.29-7.18(\mathrm{~m}, 2 \mathrm{H}), 6.89(\mathrm{~s}, 1 \mathrm{H}), 6.86-6.80(\mathrm{~m}$, $1 \mathrm{H}), 5.87-5.71(\mathrm{~m}, 1 \mathrm{H}), 5.02-4.88(\mathrm{~m}, 2 \mathrm{H}), 2.49-2.40(\mathrm{~m}, 2 \mathrm{H}), 2.18-2.07$ $(\mathrm{m}, 1 \mathrm{H}), 1.96-1.84(\mathrm{~m}, 1 \mathrm{H}) ;{ }^{13} \mathrm{C}$ NMR $(100 \mathrm{MHz}, \mathrm{DMSO}) \delta 175.7,160.5$, 154.6, 143.1, 138.5, 128.2, 124.7, 123.3, 121.7, 120.8, 115.2, 111.6, 103.4, 75.1, 39.1, 27.7. FT-IR: $v\left(\mathrm{~cm}^{-1}\right) 3649,2983,2918,1684,1653,1577,1474,1418,1363,1248$. HRMS [ESI] m/z: $[\mathrm{M}+\mathrm{Na}]^{+}$calcd for $\mathrm{C}_{16} \mathrm{H}_{15} \mathrm{NNaO}_{2} \mathrm{~S} 308.0716$; found 308.0708 .

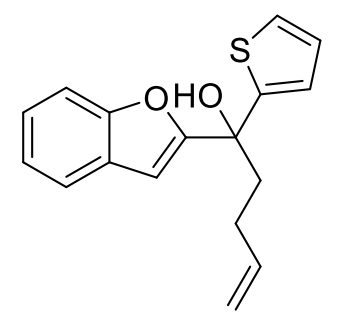

1ad: $0.438 \mathrm{~g}, 82 \%$ yield, brown oil. Purification by flash column chromatography (eluent: EtOAc/Petroleum ether $=1 / 50) .{ }^{1} \mathrm{H}$ NMR $(400$ $\left.\mathrm{MHz},\left(\mathrm{CD}_{3}\right)_{2} \mathrm{CO}\right) \delta$ 7.58-7.52 (m, 1H), 7.43-7.38 (m, $\left.1 \mathrm{H}\right)$, 7.29-7.17 (m, $3 \mathrm{H}), 7.02-6.98(\mathrm{~m}, 1 \mathrm{H}), 6.97-6.91(\mathrm{~m}, 1 \mathrm{H}), 6.73(\mathrm{~d}, J=0.8 \mathrm{~Hz}, 1 \mathrm{H})$, 5.88-5.75 (m, 1H), 5.01-4.86 (m, 2H), $4.24(\mathrm{~s}, 1 \mathrm{H}), 2.47-2.38(\mathrm{~m}, 1 \mathrm{H})$, 2.35-2.26 (m, 1H), 2.11-2.06 (m, 1H), 2.05-1.96 (m, 1H); ${ }^{13} \mathrm{C}$ NMR $(100$ $\left.\mathrm{MHz},\left(\mathrm{CD}_{3}\right)_{2} \mathrm{CO}\right) \delta 161.7,155.2,150.2,138.8,128.7,127.4,125.4,124.8,124.5,123.5,121.8$, 114.7, 111.5, 103.1, 74.5, 41.3, 28.4. FT-IR: $v\left(\mathrm{~cm}^{-1}\right)$ 3676, 3072, 2922, 1702, 1638, 1521, 1489 , 1436, 1363, 1305, 1250. HRMS [ESI] m/z: $[\mathrm{M}+\mathrm{Na}]^{+}$calcd for $\mathrm{C}_{17} \mathrm{H}_{16} \mathrm{NaO}_{2} \mathrm{~S}$ 307.0763; found 307.0760 . 


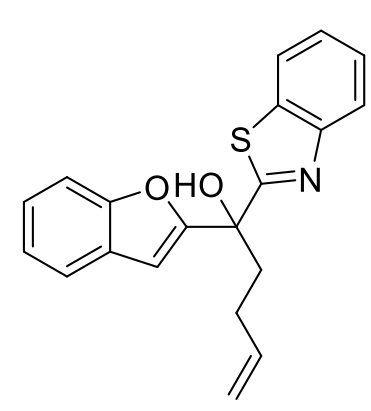

1ae: $0.312 \mathrm{~g}, 50 \%$ yield, yellow solid. m.p. $104-105^{\circ} \mathrm{C}$. Purification by flash column chromatography (eluent: EtOAc/Petroleum ether $=1 / 50$ ). ${ }^{1} \mathrm{H}$ NMR (400 MHz, CD $\left.{ }_{3} \mathrm{OD}\right) \delta 8.02-7.92(\mathrm{~m}, 2 \mathrm{H}), 7.59-7.53(\mathrm{~m}, 1 \mathrm{H})$, 7.52-7.46 (m, 1H), 7.46-7.38 (m, 2H), 7.28-7.15 (m, 2H), 6.88-6.80 (m, $1 \mathrm{H})$, 5.92-5.77 (m, 1H), 5.04-4.88 (m, 2H), 2.71-2.55 (m, 2H), 2.32-2.20 (m, 1H) , 2.13-2.00 (m, 1H); ${ }^{13} \mathrm{C}$ NMR (100 MHz, DMSO) $\delta$ $177.4,159.8,154.7,153.6,138.4,135.3,128.2,126.5,125.5,124.8$, 123.4, 123.3, 122.7, 121.8, 115.3, 111.7, 103.8, 75.4, 38.8, 27.7. FT-IR: $v\left(\mathrm{~cm}^{-1}\right) 2956,2928,1507,1453,1439,1258$. HRMS [ESI] $\mathrm{m} / \mathrm{z}:[\mathrm{M}+\mathrm{Na}]^{+}$calcd for $\mathrm{C}_{20} \mathrm{H}_{17} \mathrm{NNaO}_{2} \mathrm{~S}$ 358.0872; found 358.0870.

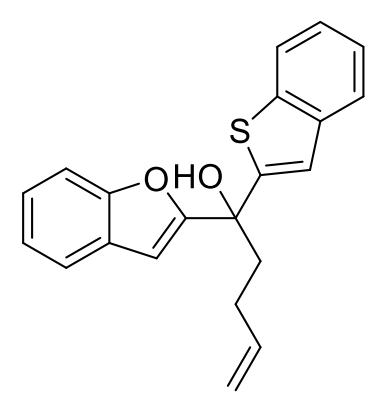

1af: $0.180 \mathrm{~g}, 27 \%$ yield, yellow oil. Purification by flash column chromatography (eluent: EtOAc/Petroleum ether $=1 / 50) .{ }^{1} \mathrm{H}$ NMR $(400$ $\left.\mathrm{MHz}, \mathrm{CDCl}_{3}\right) \delta$ 7.65-7.59 (m, 2H), 7.55-7.48 (m, 2H), 7.29-7.20 (m, $4 \mathrm{H}), 6.89(\mathrm{~d}, J=0.8 \mathrm{~Hz}, 2 \mathrm{H}), 6.50(\mathrm{~s}, 1 \mathrm{H}), 5.88-5.74(\mathrm{~m}, 1 \mathrm{H})$, 5.05-4.87 (m, 2H), 2.45-2.36 (m, 2H), 2.08-1.98 (m, 2H); ${ }^{13} \mathrm{C}$ NMR (100 MHz, DMSO) $\delta 160.0,154.6,138.5,128.3,124.6,123.4,121.7$, 115.3, 111.6, 103.8, 71.9, 37.5, 27.8. FT-IR: $v\left(\mathrm{~cm}^{-1}\right) 3064,2951,2853$, 1640, 1454, 1435, 1305, 1251. HRMS [ESI] m/z: $[\mathrm{M}+\mathrm{Na}]^{+}$calcd for $\mathrm{C}_{21} \mathrm{H}_{18} \mathrm{NaO}_{2} \mathrm{~S}$ 357.0920; found 357.0921.

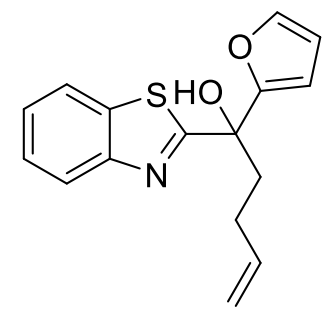

1ag: $0.415 \mathrm{~g}, 58 \%$ yield, yellow solid, m.p. $107-108{ }^{\circ} \mathrm{C}$. Purification by flash column chromatography (eluent: EtOAc/Petroleum ether $=1 / 50$ ). ${ }^{1} \mathrm{H}$ NMR $\left(400 \mathrm{MHz}, \mathrm{CDCl}_{3}\right) \delta 8.04(\mathrm{~d}, J=8.0 \mathrm{~Hz}, 1 \mathrm{H}), 7.87(\mathrm{~d}, J=8.0 \mathrm{~Hz}$, $1 \mathrm{H}), 7.49$ (ddd, $J=8.4,7.2,1.2 \mathrm{~Hz}, 1 \mathrm{H}), 7.42-7.37(\mathrm{~m}, 2 \mathrm{H}), 6.44$ (dd, $J=$ $3.2,0.8 \mathrm{~Hz}, 1 \mathrm{H}), 6.36(\mathrm{dd}, J=3.2,2.0 \mathrm{~Hz}, 1 \mathrm{H}), 5.90-5.79(\mathrm{~m}, 1 \mathrm{H})$, 5.06-5.00 (m, 1H), 4.98-4.94 (m, 1H), 4.19 (br, 1H), 2.60-2.43 (m, 2H), 2.34-2.20 (m, 1H), 2.14-2.04 (m, 1H); ${ }^{13} \mathrm{C}$ NMR (100 MHz, $\left.\mathrm{CDCl}_{3}\right) \delta$ 174.6, 155.0, 151.7, 142.1, 137.3, 135.3, 125.7, 124.8, 122.7, 121.3, 114.6, 110.1, 106.5, 75.3, 39.5, 27.2. FT-IR: $v\left(\mathrm{~cm}^{-1}\right)$ $3675,3169,2972,2921,1638,1508,1433,1375,1262$. HRMS [ESI] $\mathrm{m} / \mathrm{z}:[\mathrm{M}+\mathrm{H}]^{+}$calcd for $\mathrm{C}_{16} \mathrm{H}_{16} \mathrm{NO}_{2} \mathrm{~S} 286.0902$; found 286.0898 .

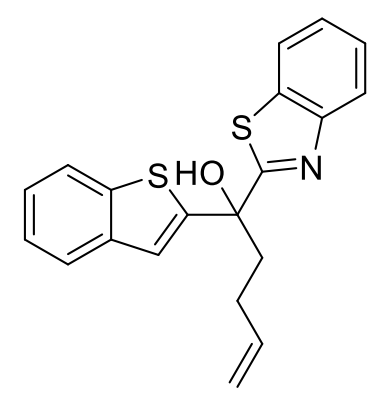

1ah: $0.300 \mathrm{~g}, 34 \%$ yield, yellow oil. Purification by flash column chromatography (eluent: EtOAc/Petroleum ether $=1 / 50) .{ }^{1} \mathrm{H}$ NMR $(400$ MHz, DMSO) $\delta$ 8.09-8.04 (m, 1H), 8.01-7.97 (m, 1H), 7.90-7.85 (m, $1 \mathrm{H})$, 7.82-7.77 $(\mathrm{m}, 1 \mathrm{H}), 7.52-7.46(\mathrm{~m}, 2 \mathrm{H}), 7.43-7.38(\mathrm{~m}, 1 \mathrm{H})$, 7.35-7.26 (m, 2H), $7.32(\mathrm{~s}, 1 \mathrm{H}), 5.91-5.77(\mathrm{~m}, 1 \mathrm{H}), 5.05-4.90(\mathrm{~m}, 2 \mathrm{H})$, 2.69-2.52 (m, 2H), 2.25-2.13 (m, 1H), 2.10-1.99 (m, 1H); ${ }^{13} \mathrm{C}$ NMR (100 MHz, DMSO) $\delta 179.3,153.6,151.2,139.8,139.4,138.4,135.2$, $126.5,125.5,124.8,124.6,124.2,123.2,122.8,122.7,120.7,115.4$, 77.4, 41.6, 28.0. FT-IR: $v\left(\mathrm{~cm}^{-1}\right)$ 3065, 2925, 2958, 1703, 1638, 1508, 1457, 1436, 1363, 1314. HRMS [ESI] m/z: [M+Na] ${ }^{+}$calcd for $\mathrm{C}_{20} \mathrm{H}_{17} \mathrm{NNaOS}_{2} 374.0644$; found 374.0640. 


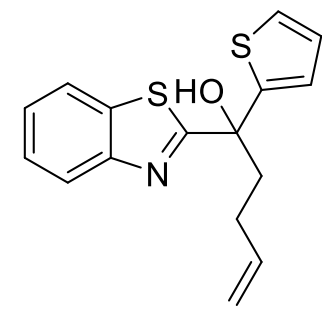

1ai: $0.326 \mathrm{~g}, 58 \%$ yield, yellow solid, m.p. $64-65{ }^{\circ} \mathrm{C}$. Purification by flash column chromatography (eluent: EtOAc/Petroleum ether $=1 / 50) .{ }^{1} \mathrm{H}$ NMR $\left(400 \mathrm{MHz}, \mathrm{CDCl}_{3}\right) \delta 8.03(\mathrm{~d}, J=8.0 \mathrm{~Hz}, 1 \mathrm{H}), 7.86(\mathrm{~d}, J=8.0 \mathrm{~Hz}, 1 \mathrm{H})$, 7.49 (ddd, $J=8.0,6.8,1.2 \mathrm{~Hz}, 1 \mathrm{H}), 7.38$ (ddd, $J=8.0,7.2,0.8 \mathrm{~Hz}, 1 \mathrm{H}$ ), $7.27(\mathrm{dd}, J=5.2,1.2 \mathrm{~Hz}, 1 \mathrm{H}), 7.18(\mathrm{dd}, J=3.6,1.2 \mathrm{~Hz}, 1 \mathrm{H}), 7.00(\mathrm{dd}, J=$ $4.8,3.6 \mathrm{~Hz}, 1 \mathrm{H}), 5.94-5.82(\mathrm{~m}, 1 \mathrm{H}), 5.09-5.03(\mathrm{~m}, 1 \mathrm{H}), 5.02-4.97(\mathrm{~m}, 1 \mathrm{H})$, $4.55(\mathrm{br}, 1 \mathrm{H}), 2.67-2.53(\mathrm{~m}, 2 \mathrm{H}), 2.40-2.29(\mathrm{~m}, 1 \mathrm{H}), 2.23-2.12(\mathrm{~m}, 1 \mathrm{H}) ;{ }^{13} \mathrm{C}$ NMR $(100 \mathrm{MHz}$, $\left.\mathrm{CDCl}_{3}\right) \delta 176.7,151.9,148.9,137.4,135.3,126.6,125.7,124.9,124.8,123.7,122.7,121.4,114.9$, 77.4, 42.4, 27.7. FT-IR: $v\left(\mathrm{~cm}^{-1}\right) 3675,2988,2902,1637,1508,1434,1352,1242$. HRMS [ESI] $\mathrm{m} / \mathrm{z}:[\mathrm{M}+\mathrm{H}]^{+}$calcd for $\mathrm{C}_{16} \mathrm{H}_{16} \mathrm{NOS}_{2}$ 302.0673; found 302.0674 .

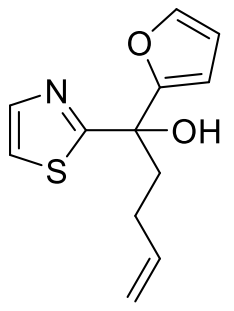

1aj: $0.240 \mathrm{~g}, 41 \%$ yield, green solid, m.p. $84-85{ }^{\circ} \mathrm{C}$. Purification by flash column chromatography (eluent: EtOAc/Petroleum ether $=1 / 50) .{ }^{1} \mathrm{H}$ NMR $(400$ MHz, DMSO) $\delta 7.72(\mathrm{~d}, J=3.2 \mathrm{~Hz}, 1 \mathrm{H}), 7.62(\mathrm{~d}, J=3.2 \mathrm{~Hz}, 1 \mathrm{H}), 7.58-7.53(\mathrm{~m}$, $1 \mathrm{H}), 6.59(\mathrm{~s}, 1 \mathrm{H}), 6.41-6.36(\mathrm{~m}, 1 \mathrm{H}), 6.33-6.28(\mathrm{~m}, 1 \mathrm{H}), 5.83-5.71(\mathrm{~m}, 1 \mathrm{H})$, 5.01-4.86 (m, 2H), 2.41-2.27 (m, 2H), 2.13-2.02 (m, 1H), 1.87-1.75 (m, $1 \mathrm{H}) ;{ }^{13} \mathrm{C}$ NMR (100 MHz, DMSO) $\delta 176.3,157.6,142.9,142.7,138.7,120.5,115.1$, 110.7, 106.5, 74.7, 39.3, 27.7. FT-IR: $v\left(\mathrm{~cm}^{-1}\right)$ 3119, 2956, 2856, 1793, 1686, 1558, 1437, 1323, 1255. HRMS [ESI] m/z: [M+Na] ${ }^{+}$calcd for $\mathrm{C}_{12} \mathrm{H}_{13} \mathrm{NNaO}_{2} \mathrm{~S} 258.0559$; found 258.0549.

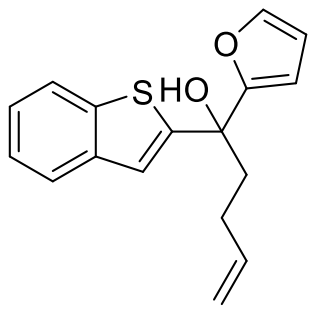

1ak: $0.258 \mathrm{~g}, 49 \%$ yield, red oil. Purification by flash column chromatography (eluent: EtOAc/Petroleum ether $=1 / 50) .{ }^{1} \mathrm{H}$ NMR $(400$ MHz, DMSO) $\delta$ 7.90-7.84 (m, 1H), 7.79-7.74 (m, 1H), 7.62-7.58 (m, 1H), 7.35-7.25 (m, 2H), $7.24(\mathrm{~s}, 1 \mathrm{H}), 6.44-6.40(\mathrm{~m}, 1 \mathrm{H}), 6.40-6.38(\mathrm{~m}, 1 \mathrm{H})$, $6.36(\mathrm{br}, 1 \mathrm{H}), 5.88-5.75(\mathrm{~m}, 1 \mathrm{H}), 5.02-4.88(\mathrm{~m}, 2 \mathrm{H}), 2.40-2.20(\mathrm{~m}, 2 \mathrm{H})$, 2.10-1.97 (m, 2H); ${ }^{13} \mathrm{C}$ NMR (100 MHz, DMSO) $\delta 158.5,152.3,142.6$, 140.0, 139.1, 138.7, 124.6, 124.4, 123.9, 122.7, 120.1, 115.1, 110.7, 106.3, 73.5, 40.9, 28.2. FT-IR: $v\left(\mathrm{~cm}^{-1}\right) 3649,3073,2973,2922,1638,1541,1489,1435,1375,1250,1224$. HRMS [ESI] m/z: $[\mathrm{M}+\mathrm{Na}]^{+}$calcd for $\mathrm{C}_{17} \mathrm{H}_{16} \mathrm{NaO}_{2} \mathrm{~S} 307.0763$; found 307.0763 .

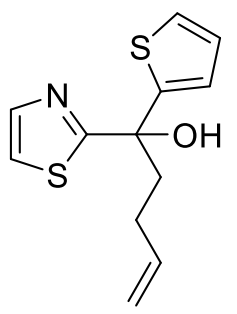

1al: $0.295 \mathrm{~g}$, $67 \%$ yield, red solid, m.p. $80-81{ }^{\circ} \mathrm{C}$. Purification by flash column chromatography (eluent: EtOAc/Petroleum ether $=1 / 50) .{ }^{1} \mathrm{H}$ NMR $(400 \mathrm{MHz}$, DMSO) $\delta 7.74(\mathrm{~d}, J=3.2 \mathrm{~Hz}, 1 \mathrm{H}), 7.58(\mathrm{~d}, J=3.2 \mathrm{~Hz}, 1 \mathrm{H}), 7.37(\mathrm{dd}, J=4.8$, $0.8 \mathrm{~Hz}, 1 \mathrm{H}), 7.05(\mathrm{dd}, J=3.6,1.2 \mathrm{~Hz}, 1 \mathrm{H}), 6.94(\mathrm{dd}, J=5.2,3.6 \mathrm{~Hz}, 1 \mathrm{H}), 6.88$ (s, 1H), 5.83-5.70 (m, 1H), 4.99-4.87 (m, 2H), 2.48-2.39 (m, 1H), 2.37-2.28 (m, $1 \mathrm{H}), 2.13-2.02(\mathrm{~m}, 1 \mathrm{H}), 1.93-1.82(\mathrm{~m}, 1 \mathrm{H}) ;{ }^{13} \mathrm{C}$ NMR (100 MHz, DMSO) $\delta$ 178.3, 151.2, 143.0, 138.6, 127.1, 125.3, 123.9, 120.2, 115.1, 77.0, 42.7, 28.1. FT-IR: $v\left(\mathrm{~cm}^{-1}\right)$ 3676, 3567, 2988, 2901, 1648, 1576, 1498, 1436, 1419, 1251. HRMS [ESI] m/z: $[\mathrm{M}+\mathrm{Na}]^{+}$calcd for $\mathrm{C}_{12} \mathrm{H}_{13} \mathrm{NNaOS}_{2} 274.0331$; found 274.0334 .

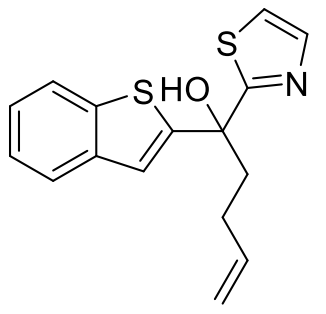

1am: $0.394 \mathrm{~g}, 70 \%$ yield, yellow solid. m.p. $99-100{ }^{\circ} \mathrm{C}$. Purification by flash column chromatography (eluent: EtOAc/Petroleum ether $=1 / 50) .{ }^{1} \mathrm{H}$ NMR $\left(400 \mathrm{MHz}, \mathrm{CDCl}_{3}\right) \delta$ 7.81-7.74 $(\mathrm{m}, 2 \mathrm{H}), 7.74-7.70(\mathrm{~m}, 1 \mathrm{H})$, 7.36-7.27 (m, 4H), 5.94-5.79 (m, 1H), 5.08-4.95 (m, 2H), 4.23 (br, 1H), 
2.63-2.51 (m, 2H), 2.33-2.22 (m, 1H), 2.22-2.11 (m, 1H); ${ }^{13} \mathrm{C}$ NMR (100 MHz, DMSO) $\delta$ 177.7, 152.0, 143.1, 139.8, 139.3, 138.5, 124.7, 124.5, 124.1, 122.7, 120.6, 120.3, 115.2, 77.2, 42.2, 28.0. FT-IR: $v\left(\mathrm{~cm}^{-1}\right) 3109,2974,2928,2857,1646,1558,1473,1435,1304,1218$. HRMS [ESI] m/z: $[\mathrm{M}+\mathrm{Na}]^{+}$calcd for $\mathrm{C}_{16} \mathrm{H}_{15} \mathrm{NNaOS}_{2} 324.0487$; found 324.0488 .

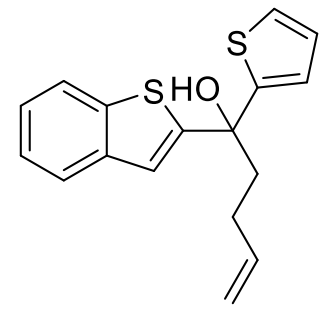

1an: $0.110 \mathrm{~g}, 19 \%$ yield, yellow solid. m.p. $64-65^{\circ} \mathrm{C}$. Purification by flash column chromatography (eluent: EtOAc/Petroleum ether $=1 / 50) .{ }^{1} \mathrm{H}$ NMR (400 MHz, DMSO) $\delta$ 7.90-7.83 (m, 1H), 7.80-7.73 (m, 1H), 7.41-7.36 (m, $1 \mathrm{H}), 7.35-7.25(\mathrm{~m}, 2 \mathrm{H}), 7.33(\mathrm{~s}, 1 \mathrm{H}), 7.08(\mathrm{dd}, J=3.6,1.2 \mathrm{~Hz}, 1 \mathrm{H}), 6.97$ (dd, $J=4.8,3.2 \mathrm{~Hz}, 1 \mathrm{H}), 5.90-5.79(\mathrm{~m}, 1 \mathrm{H}), 5.05-4.92(\mathrm{~m}, 2 \mathrm{H}), 2.46-2.36$ (m, 2H), 2.15-2.03 (m, 2H); ${ }^{13} \mathrm{C}$ NMR (100 MHz, DMSO) $\delta 154.7,153.1$, 140.0, 139.1, 138.8, 127.2, 125.1, 124.6, 124.4, 123.9, 123.6, 122.7, 119.7, 115.2, 75.2, 43.0, 28.4. FT-IR: $v\left(\mathrm{~cm}^{-1}\right) 3503,3065,2931,2864,1637,1559,1456,1419,1362,1243$. HRMS [ESI] m/z: $[\mathrm{M}+\mathrm{Na}]^{+}$calcd for $\mathrm{C}_{17} \mathrm{H}_{16} \mathrm{NaOS}_{2}$ 323.0535; found 323.0526.

\section{Characterization of products}<smiles>O=C(CCC(CC(F)(F)F)c1cc2ccccc2o1)c1ccccc1</smiles>

2a: $55.1 \mathrm{mg}, 80 \%$, white solid, m.p. $94-95{ }^{\circ} \mathrm{C}$. Purification by flash column chromatography (eluent: EtOAc/Petroleum ether $=1 / 30$ ). ${ }^{1} \mathrm{H}$ NMR (400 MHz, $\left.\mathrm{CDCl}_{3}\right) \delta$ 7.90-7.80 (m, 2H), 7.57-7.48 (m, 2H), 7.47-7.37 (m, 3H), 7.28-7.19 (m, 2H), $6.51(\mathrm{~s}, 1 \mathrm{H}), 3.44-3.32(\mathrm{~m}$, $1 \mathrm{H}), 2.93(\mathrm{t}, J=7.2 \mathrm{~Hz}, 2 \mathrm{H}), 2.82-2.67(\mathrm{~m}, 1 \mathrm{H}), 2.60-2.46(\mathrm{~m}, 1 \mathrm{H})$, 2.37-2.27 (m, 1H), 2.26-2.16 (m, 1H); ${ }^{13} \mathrm{C}$ NMR (100 MHz, $\left.\mathrm{CDCl}_{3}\right)$ $\delta 199.0,157.3,154.8,136.7,133.2,128.6,128.2,128.0,126.2(\mathrm{q}$, $\left.J_{\mathrm{C}-\mathrm{F}}=275.6 \mathrm{~Hz}\right), 123.9,122.8,120.8,111.1,104.0,38.2\left(\mathrm{q}, J_{\mathrm{C}-\mathrm{F}}=28.0 \mathrm{~Hz}\right), 35.6,33.2\left(\mathrm{q}, J_{\mathrm{C}-\mathrm{F}}=\right.$ $2.6 \mathrm{~Hz}$ ), 27.9; ${ }^{19} \mathrm{~F}$ NMR $\left(376 \mathrm{MHz}, \mathrm{CDCl}_{3}\right) \delta-64.3$ (s). FT-IR: $v\left(\mathrm{~cm}^{-1}\right)$ 2968, 2922, 2855, 1692, 1597, 1541, 1423, 1386, 1341, 1261, 1206. HRMS [ESI] m/z: $[\mathrm{M}+\mathrm{Na}]+$ calcd for $\mathrm{C}_{20} \mathrm{H}_{17} \mathrm{~F}_{3} \mathrm{NaO}_{2}$ 369.1073; found 369.1080 .

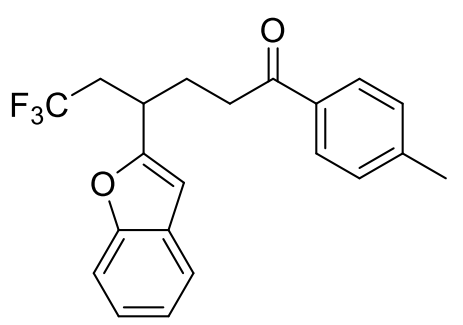

2b: $62.9 \mathrm{mg}, 88 \%$, white solid, m.p. $62-63{ }^{\circ} \mathrm{C}$. Purification by flash column chromatography (eluent: EtOAc/Petroleum ether = 1/30). ${ }^{1} \mathrm{H}$ NMR (400 MHz, $\left.\mathrm{CDCl}_{3}\right) \delta$ 7.79-7.72 (m, 2H), 7.53-7.48 $(\mathrm{m}, 1 \mathrm{H}), 7.46-7.41(\mathrm{~m}, 1 \mathrm{H}), 7.28-7.24(\mathrm{~m}, 1 \mathrm{H})$, 7.24-7.18 (m, 3H), $6.51(\mathrm{~d}, J=0.4 \mathrm{~Hz}, 1 \mathrm{H}), 3.42-3.31(\mathrm{~m}, 1 \mathrm{H})$, $2.90(\mathrm{t}, J=7.2 \mathrm{~Hz}, 2 \mathrm{H}), 2.79-2.66(\mathrm{~m}, 1 \mathrm{H}), 2.59-2.47(\mathrm{~m}, 1 \mathrm{H})$, $2.39(\mathrm{~s}, 3 \mathrm{H}), 2.35-2.26(\mathrm{~m}, 1 \mathrm{H}), 2.25-2.15(\mathrm{~m}, 1 \mathrm{H}) ;{ }^{13} \mathrm{C}$ NMR $\left(100 \mathrm{MHz}, \mathrm{CDCl}_{3}\right) \delta 198.6,157.4,154.8,144.0,134.2,129.3,128.3,128.1,126.2\left(\mathrm{q}, J_{\mathrm{C}-\mathrm{F}}=275.6\right.$ $\mathrm{Hz}), 123.9,122.8,120.7,111.1,104.0,38.1\left(\mathrm{q}, J_{\mathrm{C}-\mathrm{F}}=27.9 \mathrm{~Hz}\right), 35.5,33.2\left(\mathrm{q}, J_{\mathrm{C}-\mathrm{F}}=2.6 \mathrm{~Hz}\right), 28.0$, 21.6; ${ }^{19} \mathrm{~F}$ NMR (376 MHz, $\left.\mathrm{CDCl}_{3}\right) \delta-64.3$ (s). FT-IR: $v\left(\mathrm{~cm}^{-1}\right)$ 2972, 2902, 1780, 1606, 1474 , 1406, 1325, 1286, $1208 \mathrm{HRMS}$ [ESI] m/z: [M+Na]+ calcd for $\mathrm{C}_{21} \mathrm{H}_{19} \mathrm{~F}_{3} \mathrm{NaO}_{2}$ 383.1229; found 383.1231 . 


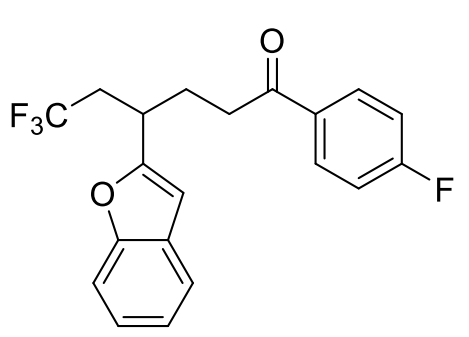

2c: $43.2 \mathrm{mg}, 60 \%$, yellow solid, m.p. $76-77{ }^{\circ} \mathrm{C}$. Purification by flash column chromatography (eluent: EtOAc/Petroleum ether $=$ 1/30). ${ }^{1} \mathrm{H}$ NMR $\left(400 \mathrm{MHz}, \mathrm{CDCl}_{3}\right) \delta$ 7.91-7.81 (m, 2H), 7.53-7.47 (m, 1H), $7.43(\mathrm{~d}, J=8.0 \mathrm{~Hz}, 1 \mathrm{H}), 7.28-7.19(\mathrm{~m}, 2 \mathrm{H})$, 7.12-6.99 (m, 2H), $6.50(\mathrm{~s}, 1 \mathrm{H}), 3.42-3.30(\mathrm{~m}, 1 \mathrm{H}), 2.88(\mathrm{t}, J=$ $7.6 \mathrm{~Hz}, 2 \mathrm{H}), 2.80-2.66(\mathrm{~m}, 1 \mathrm{H}), 2.59-2.45(\mathrm{~m}, 1 \mathrm{H}), 2.35-2.26(\mathrm{~m}$, 1H), 2.25-2.15 (m, 1H); ${ }^{13} \mathrm{C}$ NMR $\left(100 \mathrm{MHz}, \mathrm{CDCl}_{3}\right) \delta 197.3$, $165.7\left(\mathrm{~d}, J_{\mathrm{C}-\mathrm{F}}=253.3 \mathrm{~Hz}\right), 157.2,154.8,133.1\left(\mathrm{~d}, J_{\mathrm{C}-\mathrm{F}}=3.0 \mathrm{~Hz}\right), 130.6\left(\mathrm{~d}, J_{\mathrm{C}-\mathrm{F}}=9.2 \mathrm{~Hz}\right), 128.2$, $126.2\left(\mathrm{q}, J_{\mathrm{C}-\mathrm{F}}=275.5 \mathrm{~Hz}\right), 124.0,122.8,120.8,115.7\left(\mathrm{~d}, J_{\mathrm{C}-\mathrm{F}}=21.7 \mathrm{~Hz}\right), 111.1,104.0,38.1(\mathrm{q}$, $\left.J_{\mathrm{C}-\mathrm{F}}=28.0 \mathrm{~Hz}\right), 35.5,33.2\left(\mathrm{q}, J_{\mathrm{C}-\mathrm{F}}=2.6 \mathrm{~Hz}\right), 27.9 ;{ }^{19} \mathrm{~F} \mathrm{NMR}\left(376 \mathrm{MHz}, \mathrm{CDCl}_{3}\right) \delta-64.3(\mathrm{~s}),-105.1$ (s). FT-IR: $v\left(\mathrm{~cm}^{-1}\right) 3052,2927,1890,1684,1576,1508,1457,1436,1374,1253$. HRMS [ESI] $\mathrm{m} / \mathrm{z}$ : $[\mathrm{M}+\mathrm{Na}]^{+}$calcd for $\mathrm{C}_{20} \mathrm{H}_{16} \mathrm{~F}_{4} \mathrm{NaO}_{2}$ 387.0979; found 387.0975.

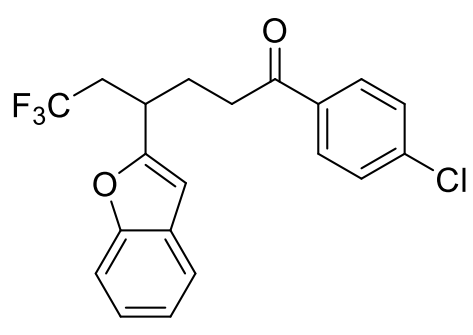

2d: $54.1 \mathrm{mg}, 72 \%$, white solid, m.p. $72-73^{\circ} \mathrm{C}$. Purification by flash column chromatography (eluent: EtOAc/Petroleum ether $=1 / 30) .{ }^{1} \mathrm{H}$ NMR $\left(400 \mathrm{MHz}, \mathrm{CDCl}_{3}\right) \delta$ 7.81-7.73 (m, 2H), 7.54-7.47 (m, 1H), 7.46-7.41 (m, 1H), 7.40-7.34 (m, 2H), 7.29-7.20 (m, 2H), $6.50(\mathrm{~s}, 1 \mathrm{H}), 3.41-3.30(\mathrm{~m}, 1 \mathrm{H}), 2.88(\mathrm{t}, J=$ $7.2 \mathrm{~Hz}, 2 \mathrm{H}), 2.79-2.66(\mathrm{~m}, 1 \mathrm{H}), 2.59-2.46(\mathrm{~m}, 1 \mathrm{H}), 2.35-2.26$ (m, 1H), 2.25-2.15 (m, 1H); ${ }^{13} \mathrm{C}$ NMR $\left(100 \mathrm{MHz}, \mathrm{CDCl}_{3}\right) \delta$ 197.7, 157.2, 154.8, 139.6, 134.9, 130.3 (q, $J_{\mathrm{C}-\mathrm{F}}=275.6 \mathrm{~Hz}$ ), 129.4, 128.9, 128.2, 124.0, 122.9 , $120.8,111.1,104.1,38.1\left(\mathrm{q}, J_{\mathrm{C}-\mathrm{F}}=27.9 \mathrm{~Hz}\right), 35.6,33.1\left(\mathrm{q}, J_{\mathrm{C}-\mathrm{F}}=2.6 \mathrm{~Hz}\right), 27.8 ;{ }^{19} \mathrm{~F}$ NMR $(376$ $\left.\mathrm{MHz}, \mathrm{CDCl}_{3}\right) \delta-64.3$ (s). FT-IR: $v\left(\mathrm{~cm}^{-1}\right)$ 2959, 2921, 2852, 1687, 1589, 1521, 1456, 1384, 1241. HRMS [ESI] m/z: [M+H] ${ }^{+}$calcd for $\mathrm{C}_{20} \mathrm{H}_{16} \mathrm{ClF}_{3} \mathrm{HO}_{2}$ 403.0683; found 403.0675 .

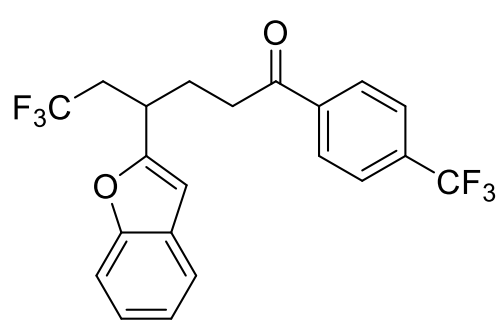

2e: $64.8 \mathrm{mg}, 79 \%$, yellow oil. Purification by flash column chromatography (eluent: EtOAc/Petroleum ether $=1 / 30$ ). ${ }^{1} \mathrm{H}$ NMR $\left(400 \mathrm{MHz}, \mathrm{CDCl}_{3}\right) \delta 7.83(\mathrm{~d}, J=8.0 \mathrm{~Hz}, 2 \mathrm{H}), 7.57(\mathrm{~d}, J$ $=8.0 \mathrm{~Hz}, 2 \mathrm{H}), 7.42-7.37(\mathrm{~m}, 1 \mathrm{H}), 7.36-7.30(\mathrm{~m}, 1 \mathrm{H})$, 7.19-7.10 (m, 2H), 6.41(s, 1H), 3.33-3.23 (m, 1H), $2.84(\mathrm{t}, J=$ $7.2 \mathrm{~Hz}, 2 \mathrm{H}), 2.72-2.58(\mathrm{~m}, 1 \mathrm{H}), 2.50-2.36(\mathrm{~m}, 1 \mathrm{H}), 2.28-2.19$ $(\mathrm{m}, 1 \mathrm{H}), 2.18-2.08(\mathrm{~m}, 1 \mathrm{H}) ;{ }^{13} \mathrm{C}$ NMR $\left(100 \mathrm{MHz}, \mathrm{CDCl}_{3}\right) \delta$ 198.0, 157.1, 154.8, 139.3, $134.4\left(\mathrm{q}, J_{\mathrm{C}-\mathrm{F}}=32.4 \mathrm{~Hz}\right), 128.3,128.2,126.2\left(\mathrm{q}, J_{\mathrm{C}-\mathrm{F}}=275.7 \mathrm{~Hz}\right)$, $125.7\left(\mathrm{q}, J_{\mathrm{C}-\mathrm{F}}=3.6 \mathrm{~Hz}\right), 123.6\left(\mathrm{q}, J_{\mathrm{C}-\mathrm{F}}=271.2 \mathrm{~Hz}\right), 124.0,122.9,120.8,111.1,104.1,38.1\left(\mathrm{q}, J_{\mathrm{C}-\mathrm{F}}\right.$ $=28.0 \mathrm{~Hz}), 35.9,33.1\left(\mathrm{q}, J_{\mathrm{C}-\mathrm{F}}=2.5 \mathrm{~Hz}\right), 27.7 ;{ }^{19} \mathrm{~F} \mathrm{NMR}\left(376 \mathrm{MHz}, \mathrm{CDCl}_{3}\right) \delta-63.2(\mathrm{~s}),-64.3(\mathrm{~s})$. FT-IR: $v\left(\mathrm{~cm}^{-1}\right) 3038,2917,2831,1694,1584,1456,1410,1325,1252$. HRMS [ESI] $\mathrm{m} / \mathrm{z}$ : $[\mathrm{M}+\mathrm{Na}]^{+}$calcd for $\mathrm{C}_{21} \mathrm{H}_{16} \mathrm{~F}_{6} \mathrm{NaO}_{2}$ 437.0947; found 437.0956.

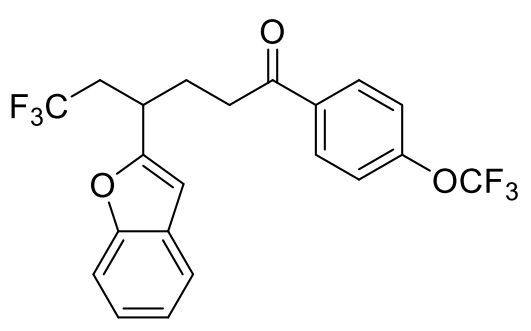

2f: $68.5 \mathrm{mg}, 80 \%$, yellow oil. Purification by flash column chromatography (eluent: EtOAc/Petroleum ether $=1 / 30) .{ }^{1} \mathrm{H}$ NMR (400 MHz, $\left.\mathrm{CDCl}_{3}\right) \delta$ 7.83-7.73 (m, 2H), 7.43-7.38 (m, $1 \mathrm{H}), 7.36-7.30(\mathrm{~m}, 1 \mathrm{H}), 7.19-7.10(\mathrm{~m}, 4 \mathrm{H}), 6.41(\mathrm{~d}, J=0.4$ $\mathrm{Hz}, 1 \mathrm{H}), 3.32-3.22(\mathrm{~m}, 1 \mathrm{H}), 2.80(\mathrm{t}, J=6.8 \mathrm{~Hz}, 2 \mathrm{H})$, 2.72-2.57 (m, 1H), 2.50-2.36 (m, 1H), 2.27-2.18 (m, 1H), 
2.16-2.06 (m, 1H); ${ }^{13} \mathrm{C}$ NMR $\left(100 \mathrm{MHz}, \mathrm{CDCl}_{3}\right) \delta 197.4,157.2,154.8,152.6\left(\mathrm{q}, J_{\mathrm{C}-\mathrm{F}}=1.7 \mathrm{~Hz}\right)$, $134.9,130.0,128.2,126.2\left(\mathrm{q}, J_{\mathrm{C}-\mathrm{F}}=275.6 \mathrm{~Hz}\right), 124.0,122.9,120.8,120.4,120.3\left(\mathrm{q}, J_{\mathrm{C}-\mathrm{F}}=257.2\right.$ $\mathrm{Hz}), 111.1,104.1,38.1\left(\mathrm{q}, J_{\mathrm{C}-\mathrm{F}}=27.9 \mathrm{~Hz}\right), 35.6,33.1\left(\mathrm{q}, J_{\mathrm{C}-\mathrm{F}}=2.5 \mathrm{~Hz}\right), 27.8 ;{ }^{19} \mathrm{~F}$ NMR $(376 \mathrm{MHz}$, $\left.\mathrm{CDCl}_{3}\right) \delta$-57.6 (s), -64.4 (s). FT-IR: $v\left(\mathrm{~cm}^{-1}\right)$ 2953, 2902, 1689, 1558, 1417, 1382, 1254, 1210. HRMS [ESI] m/z: [M+Na] ${ }^{+}$calcd for $\mathrm{C}_{21} \mathrm{H}_{16} \mathrm{~F}_{6} \mathrm{NaO}_{3}$ 453.0896; found 453.0906.

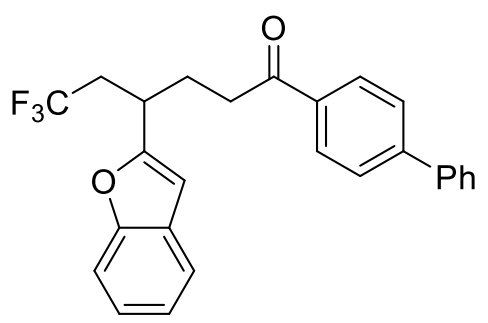

2g: $60.6 \mathrm{mg}, 72 \%$, white solid, m.p. $87-88^{\circ} \mathrm{C}$. Purification by flash column chromatography (eluent: EtOAc/Petroleum ether $=1 / 30) .{ }^{1} \mathrm{H}$ NMR $\left(400 \mathrm{MHz}, \mathrm{CDCl}_{3}\right) \delta 7.96-7.88(\mathrm{~m}, 2 \mathrm{H})$, 7.66-7.58 $(\mathrm{m}, 4 \mathrm{H}), 7.52-7.38(\mathrm{~m}, 5 \mathrm{H}), 7.26-7.18(\mathrm{~m}, 2 \mathrm{H})$, 6.55-6.49 (m, $1 \mathrm{H}), 3.43-3.33(\mathrm{~m}, 1 \mathrm{H}), 2.95(\mathrm{t}, J=7.2 \mathrm{~Hz}, 2 \mathrm{H})$, 2.80-2.68 (m, 1H), 2.59-2.47 (m, 1H), 2.39-2.30 (m, $1 \mathrm{H})$, 2.27-2.17 (m, 1H); ${ }^{13} \mathrm{C}$ NMR $\left(100 \mathrm{MHz}, \mathrm{CDCl}_{3}\right) \delta$ 198.6, 157.3, $154.8,145.8,139.8,135.4,129.0,128.6,128.3,127.3,127.2,126.2\left(\mathrm{q}, J_{\mathrm{C}-\mathrm{F}}=275.3 \mathrm{~Hz}\right), 123.9$, $122.8,120.8,111.1,104.1,38.2\left(\mathrm{q}, J_{\mathrm{C}-\mathrm{F}}=27.7 \mathrm{~Hz}\right), 35.6,33.2\left(\mathrm{q}, J_{\mathrm{C}-\mathrm{F}}=2.6 \mathrm{~Hz}\right), 28.0 ;{ }^{19} \mathrm{~F}$ NMR $\left(376 \mathrm{MHz}, \mathrm{CDCl}_{3}\right) \delta-64.3$ (s). FT-IR: $v\left(\mathrm{~cm}^{-1}\right)$ 2988, 2901, 1684, 1521, 1474, 1395, 1251, 1232. HRMS [ESI] m/z: [M+Na] $]^{+}$calcd for $\mathrm{C}_{26} \mathrm{H}_{21} \mathrm{~F}_{3} \mathrm{NaO}_{2}$ 445.1386; found 445.1391.<smiles>Cc1cccc(C(=O)CCC(CC(F)(F)F)c2cc3ccccc3o2)c1</smiles>

2h: $63.2 \mathrm{mg}, 88 \%$, white solid, m.p. $62-63{ }^{\circ} \mathrm{C}$. Purification by flash column chromatography (eluent: EtOAc/Petroleum ether $=$ 1/30). ${ }^{1} \mathrm{H}$ NMR (400 MHz, $\left.\mathrm{CDCl}_{3}\right) \delta 7.57-7.51(\mathrm{~m}, 2 \mathrm{H})$, 7.43-7.38 (m, 1H), 7.36-7.32 (m, 1H), 7.27-7.22 (m, $1 \mathrm{H})$, 7.22-7.18 (m, 1H), 7.18-7.09 (m, 2H), $6.41(\mathrm{~d}, J=0.4 \mathrm{~Hz}, 1 \mathrm{H})$, $3.32-3.22(\mathrm{~m}, 1 \mathrm{H}), 2.81(\mathrm{t}, J=7.2 \mathrm{~Hz}, 2 \mathrm{H}), 2.71-2.56(\mathrm{~m}, 1 \mathrm{H})$, 2.49-2.35 (m, 1H), 2.26(s, 3H), 2.24-2.05 (m, 2H); ${ }^{13} \mathrm{C}$ NMR $\left(100 \mathrm{MHz}, \mathrm{CDCl}_{3}\right) \delta 199.2,157.4,154.9,138.4,136.7,133.9,128.5,128.5,128.3,126.3\left(\mathrm{q}, J_{\mathrm{C}-\mathrm{F}}=\right.$ $275.6 \mathrm{~Hz}), 125.2,123.9,122.8,120.8,111.1,104.0,38.2\left(\mathrm{q}, J_{\mathrm{C}-\mathrm{F}}=27.9 \mathrm{~Hz}\right), 35.6,33.2\left(\mathrm{q}, J_{\mathrm{C}-\mathrm{F}}=\right.$ $2.6 \mathrm{~Hz}), 28.0,21.3 ;{ }^{19} \mathrm{~F}$ NMR $\left(376 \mathrm{MHz}, \mathrm{CDCl}_{3}\right) \delta-64.3(\mathrm{~s})$. FT-IR: $v\left(\mathrm{~cm}^{-1}\right) 3060,2928,1792$, 1684, 1576, 1507, 1436, 1375, 1339. HRMS [ESI] m/z: $[\mathrm{M}+\mathrm{Na}]^{+}$calcd for $\mathrm{C}_{21} \mathrm{H}_{19} \mathrm{~F}_{3} \mathrm{NaO}_{2}$ 383.1229; found 383.1229.

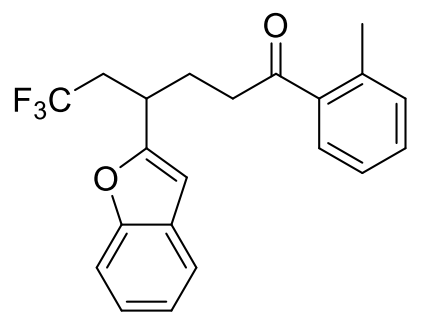

2i: $46 \mathrm{mg}, 64 \%$, white solid, m.p. $50-51{ }^{\circ} \mathrm{C}$. Purification by flash column chromatography (eluent: EtOAc/Petroleum ether $=1 / 30$ ). ${ }^{1} \mathrm{H}$ NMR $\left(400 \mathrm{MHz}, \mathrm{CDCl}_{3}\right) \delta$ 7.44-7.36 (m, 2H), 7.36-7.31 (m, 1H), 7.28-7.22 (m, 1H), 7.19-7.13 (m, 2H), 7.13-7.08 (m, 2H), $6.41(\mathrm{~d}, J$ $=0.4 \mathrm{~Hz}, 1 \mathrm{H}), 3.32-3.21(\mathrm{~m}, 1 \mathrm{H}), 2.80-2.70(\mathrm{~m}, 2 \mathrm{H}), 2.70-2.57(\mathrm{~m}$, $1 \mathrm{H}), 2.49-2.38(\mathrm{~m}, 1 \mathrm{H}), 2.37(\mathrm{~s}, 3 \mathrm{H}), 2.24-2.15(\mathrm{~m}, 1 \mathrm{H}), 2.13-2.02$ $(\mathrm{m}, 1 \mathrm{H}) ;{ }^{13} \mathrm{C}$ NMR $\left(100 \mathrm{MHz}, \mathrm{CDCl}_{3}\right) \delta 202.9,157.3,154.8,138.1$, $137.5,132.0,131.4,128.4,128.2,126.2\left(\mathrm{q}, J_{\mathrm{C}-\mathrm{F}}=275.6 \mathrm{~Hz}\right), 125.7,123.9,122.8,120.7,111.1$, $103.9,38.4,38.1\left(\mathrm{q}, J_{\mathrm{C}-\mathrm{F}}=27.9 \mathrm{~Hz}\right), 33.2\left(\mathrm{q}, J_{\mathrm{C}-\mathrm{F}}=2.6 \mathrm{~Hz}\right), 28.1,21.3 ;{ }^{19} \mathrm{~F}$ NMR $(376 \mathrm{MHz}$, $\left.\mathrm{CDCl}_{3}\right) \delta-64.3$ (s). FT-IR: $v\left(\mathrm{~cm}^{-1}\right)$ 2960, 2921, 2853, 1869, 1698, 1603, 1570, 1521, 1436, 1375, 1258. HRMS [ESI] m/z: [M+Na] ${ }^{+}$calcd for $\mathrm{C}_{21} \mathrm{H}_{19} \mathrm{~F}_{3} \mathrm{NaO}_{2} 383.1229$; found 383.1231 . 
<smiles>COc1ccccc1C(=O)CCC(CC(F)(F)F)c1cc2ccccc2o1</smiles>

2j: $68.5 \mathrm{mg}, 91 \%$, yellow oil. Purification by flash column chromatography (eluent: EtOAc/Petroleum ether $=1 / 30$ ). ${ }^{1} \mathrm{H}$ NMR $\left(400 \mathrm{MHz}, \mathrm{CDCl}_{3}\right) \delta 7.52(\mathrm{dd}, J=7.6,1.6 \mathrm{~Hz}, 1 \mathrm{H}), 7.41-7.37(\mathrm{~m}$, $1 \mathrm{H})$, 7.35-7.28 (m, 2H), 7.16-7.12 (m, 1H), 7.12-7.07 (m, 1H), 6.86 (ddd, $J=7.6,7.6,0.8 \mathrm{~Hz}, 1 \mathrm{H}), 6.78(\mathrm{~d}, J=8.4 \mathrm{~Hz}, 1 \mathrm{H}), 6.38$ (d, $J=0.4 \mathrm{~Hz}, 1 \mathrm{H}), 3.60$ (s, 3H), 3.28-3.19 (m, 1H), 2.84 (t, $J=7.2$ $\mathrm{Hz}, 2 \mathrm{H}), 2.67-2.55(\mathrm{~m}, 1 \mathrm{H}), 2.46-2.33(\mathrm{~m}, 1 \mathrm{H}), 2.18-2.09(\mathrm{~m}, 1 \mathrm{H})$, 2.09-1.98 (m, 1H); ${ }^{13} \mathrm{C}$ NMR $\left(100 \mathrm{MHz}, \mathrm{CDCl}_{3}\right) \delta 201.5,158.5,157.8,154.8,133.5,130.2,128.4$, $128.0,126.3\left(\mathrm{q}, J_{\mathrm{C}-\mathrm{F}}=275.6 \mathrm{~Hz}\right), 123.8,122.7,120.7,120.6,111.5,111.0,103.8,55.3,40.9,38.0$ $\left(\mathrm{q}, J_{\mathrm{C}-\mathrm{F}}=27.8 \mathrm{~Hz}\right), 33.3\left(\mathrm{q}, J_{\mathrm{C}-\mathrm{F}}=2.6 \mathrm{~Hz}\right), 28.4 .{ }^{19} \mathrm{~F} \mathrm{NMR}\left(376 \mathrm{MHz}, \mathrm{CDCl}_{3}\right) \delta-64.3(\mathrm{~s})$. FT-IR: $v$ $\left(\mathrm{cm}^{-1}\right)$ 3014, 2947, 2841, 1672, 1598, 1507, 1456, 1380, 1249. HRMS [ESI] m/z: [M+Na] ${ }^{+}$calcd for $\mathrm{C}_{21} \mathrm{H}_{19} \mathrm{~F}_{3} \mathrm{NaO}_{3}$ 399.1179; found 399.1187 .<smiles>O=C(CCC(CC(F)(F)F)c1cc2ccccc2o1)c1cccc2ccccc12</smiles>

2k: $32.7 \mathrm{mg}, 42 \%$, yellow solid, m.p. $114-115{ }^{\circ} \mathrm{C}$. Purification by flash column chromatography (eluent: EtOAc/Petroleum ether $=$ 1/30). ${ }^{1} \mathrm{H}$ NMR (400 MHz, $\left.\mathrm{CDCl}_{3}\right) \delta 8.57-8.50(\mathrm{~m}, 1 \mathrm{H}), 7.96(\mathrm{~d}, J=$ $8.0 \mathrm{~Hz}, 1 \mathrm{H}), 7.89-7.83(\mathrm{~m}, 1 \mathrm{H}), 7.70(\mathrm{dd}, J=7.6,1.2 \mathrm{~Hz}, 1 \mathrm{H})$, 7.58-7.48 (m, 3H), 7.45-7.38 (m, 2H), 7.26-7.18 (m, 2H), $6.52(\mathrm{~s}$, $1 \mathrm{H}), 3.46-3.37(\mathrm{~m}, 1 \mathrm{H}), 3.06-2.96(\mathrm{~m}, 2 \mathrm{H}), 2.81-2.70(\mathrm{~m}, 1 \mathrm{H})$, 2.61-2.49 (m, 1H), 2.43-2.34 (m, 1H) , 2.31-2.21 (m, 1H); ${ }^{13} \mathrm{C}$ NMR $\left(101 \mathrm{MHz}, \mathrm{CDCl}_{3}\right) \delta 203.1,157.3,154.9,135.6,133.9,132.8,130.0,128.4,128.2,128.0,127.5$, $126.5,126.2\left(\mathrm{q}, J_{\mathrm{C}-\mathrm{F}}=275.6 \mathrm{~Hz}\right), 125.7,124.3,123.9,122.8,120.8,111.1,104.0,39.0,38.2(\mathrm{q}$, $\left.J_{\mathrm{C}-\mathrm{F}}=275.9 \mathrm{~Hz}\right), 33.2\left(\mathrm{q}, J_{\mathrm{C}-\mathrm{F}}=2.7 \mathrm{~Hz}\right), 28.3 ;{ }^{19} \mathrm{~F} \mathrm{NMR}\left(376 \mathrm{MHz}, \mathrm{CDCl}_{3}\right) \delta-64.3(\mathrm{~s})$. FT-IR: $v$ $\left(\mathrm{cm}^{-1}\right) 3750,3675,2925,2853,2372,1717,1647,1560,1488,1437,1383,1269,1250$. HRMS [ESI] m/z: [M+Na] $]^{+}$calcd for $\mathrm{C}_{24} \mathrm{H}_{19} \mathrm{~F}_{3} \mathrm{NaO}_{2} 419.1229$; found 419.1223 .<smiles>O=C(CCC(CC(F)(F)F)c1cc2ccccc2o1)c1ccc2ccccc2c1</smiles>

2I: $30 \mathrm{mg}, 38 \%$, white solid, m.p. $96-97{ }^{\circ} \mathrm{C}$. Purification by flash column chromatography (eluent: EtOAc/Petroleum ether $=1 / 30$ ). ${ }^{1} \mathrm{H}$ NMR $\left(400 \mathrm{MHz}, \mathrm{CDCl}_{3}\right) \delta 8.31(\mathrm{~s}, 1 \mathrm{H}), 7.95(\mathrm{dd}, J=8.8,1.6$ $\mathrm{Hz}, 1 \mathrm{H}), 7.89-7.81(\mathrm{~m}, 3 \mathrm{H}), 7.62-7.56(\mathrm{~m}, 1 \mathrm{H}), 7.56-7.48(\mathrm{~m}$, $2 \mathrm{H})$, 7.47-7.42 (m, 1H), 7.29-7.20 (m, 2H), $6.54(\mathrm{~s}, 1 \mathrm{H})$, 3.48-3.34 (m, 1H), $3.06(\mathrm{t}, J=7.6 \mathrm{~Hz}, 2 \mathrm{H}), 2.83-2.69(\mathrm{~m}, 1 \mathrm{H})$, $2.82-2.48(\mathrm{~m}, 1 \mathrm{H}), 2.43-2.33(\mathrm{~m}, 1 \mathrm{H}), 2.32-2.21(\mathrm{~m}, 1 \mathrm{H}) ;{ }^{13} \mathrm{C}$ NMR $\left(100 \mathrm{MHz}, \mathrm{CDCl}_{3}\right) \delta 199.0,157.4,154.9,135.6,134.0,132.4,129.7,129.6,128.5,128.5$, $128.3,127.8,126.8,126.2\left(\mathrm{q}, J_{\mathrm{C}-\mathrm{F}}=275.6 \mathrm{~Hz}\right), 123.9,123.7,122.8,120.8,111.1,104.1,38.2(\mathrm{q}$, $\left.J_{\mathrm{C}-\mathrm{F}}=28.0 \mathrm{~Hz}\right), 35.7,33.3\left(\mathrm{q}, J_{\mathrm{C}-\mathrm{F}}=2.6 \mathrm{~Hz}\right), 28.1 ;{ }^{19} \mathrm{~F} \mathrm{NMR}\left(376 \mathrm{MHz}, \mathrm{CDCl}_{3}\right) \delta-64.3(\mathrm{~s})$. FT-IR: $v\left(\mathrm{~cm}^{-1}\right)$ 2970, 1678, 1626, 1472, 1415, 1383, 1323, 1276, 1250. HRMS [ESI] m/z: [M+Na] ${ }^{+}$calcd for $\mathrm{C}_{24} \mathrm{H}_{19} \mathrm{~F}_{3} \mathrm{NaO}_{2} 419.1229$; found 419.1237 .

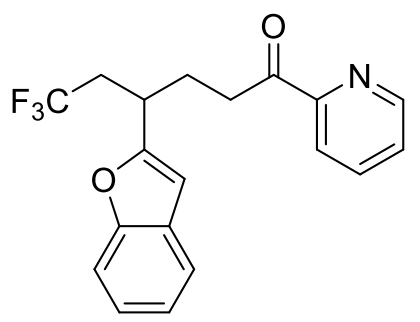

2m: $59.7 \mathrm{mg}$, 86\%, white solid, m.p. $77-78{ }^{\circ} \mathrm{C}$. Purification by flash column chromatography (eluent: EtOAc/Petroleum ether $=1 / 30$ ). ${ }^{1} \mathrm{H} \mathrm{NMR}\left(400 \mathrm{MHz}, \mathrm{CDCl}_{3}\right) \delta 8.64-8.55(\mathrm{~m}, 1 \mathrm{H}), 7.99-7.92(\mathrm{~m}, 1 \mathrm{H})$, 7.78 (ddd, $J=7.6,7.6,2.0 \mathrm{~Hz}, 1 \mathrm{H}), 7.53-7.36(\mathrm{~m}, 3 \mathrm{H}), 7.26-7.15$ $(\mathrm{m}, 2 \mathrm{H}), 6.51(\mathrm{~s}, 1 \mathrm{H}), 3.42-3.32(\mathrm{~m}, 1 \mathrm{H}), 3.30-3.14(\mathrm{~m}, 2 \mathrm{H})$, 
2.81-2.67 (m, 1H), 2.61-2.48 (m, 1H), 2.34-2.21 (m, 2H); ${ }^{13} \mathrm{C}$ NMR (100 MHz, $\left.\mathrm{CDCl}_{3}\right) \delta 200.8$, 157.5, 154.8, 153.1, 148.9, 136.9, 128.4, 127.2, $126.3\left(\mathrm{q}, J_{\mathrm{C}-\mathrm{F}}=275.5 \mathrm{~Hz}\right), 123.8,122.7,121.7$, 120.7, 111.0, 103.9, $38.0\left(\mathrm{q}, J_{\mathrm{C}-\mathrm{F}}=28.0 \mathrm{~Hz}\right), 35.0,33.3\left(\mathrm{q}, J_{\mathrm{C}-\mathrm{F}}=2.7 \mathrm{~Hz}\right), 27.8 ;{ }^{19} \mathrm{~F}$ NMR $(376$ $\left.\mathrm{MHz}, \mathrm{CDCl}_{3}\right) \delta-64.3$ (s). FT-IR: $v\left(\mathrm{~cm}^{-1}\right)$ 2359, 2342, 1729, 1687, 1551, 1449, 1392, 1258. HRMS [ESI] m/z: [M+Na] $]^{+}$calcd for $\mathrm{C}_{19} \mathrm{H}_{16} \mathrm{~F}_{3} \mathrm{NNaO}_{2}$ 370.1025; found 370.1026.

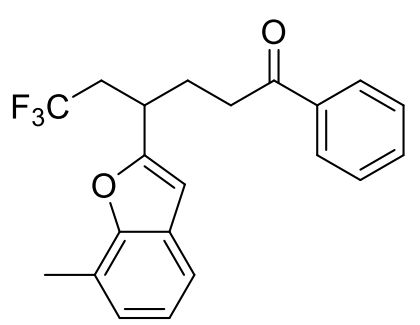

2n: $55.1 \mathrm{mg}, \quad 77 \%$, red oil. Purification by flash column chromatography (eluent: EtOAc/Petroleum ether $=1 / 30$ ). ${ }^{1} \mathrm{H}$ NMR $\left(400 \mathrm{MHz}, \mathrm{CDCl}_{3}\right) \delta$ 7.90-7.80 (m, 2H), 7.56-7.51 (m, $\left.1 \mathrm{H}\right)$, 7.45-7.37 (m, 2H), 7.36-7.30 (m, 1H), 7.14-7.08 (m, 1H), 7.08-7.03 $(\mathrm{m}, 1 \mathrm{H}), 6.49(\mathrm{~s}, 1 \mathrm{H}), 3.42-3.32(\mathrm{~m}, 1 \mathrm{H}), 2.98-2.89(\mathrm{~m}, 2 \mathrm{H})$, 2.80-2.67 (m, 1H), 2.60-2.51 (m, 1H), $2.49(\mathrm{~s}, 3 \mathrm{H}), 2.37-2.28(\mathrm{~m}$, 1H), 2.27-2.17 (m, 1H); $\left.{ }^{13} \mathrm{C} \mathrm{NMR} \mathrm{(100} \mathrm{MHz,} \mathrm{CDCl}_{3}\right) \delta$ 199.1, 157.1, $153.9,136.7,133.2,128.6,128.0,127.7,126.3\left(\mathrm{q}, J_{\mathrm{C}-\mathrm{F}}=275.5 \mathrm{~Hz}\right), 124.9,122.8,121.3,118.2$, $104.1,38.2\left(\mathrm{q}, J_{\mathrm{C}-\mathrm{F}}=27.7 \mathrm{~Hz}\right), 35.7,33.2\left(\mathrm{q}, J_{\mathrm{C}-\mathrm{F}}=2.7 \mathrm{~Hz}\right), 28.0,15.0 ;{ }^{19} \mathrm{~F}$ NMR $(376 \mathrm{MHz}$, $\left.\mathrm{CDCl}_{3}\right) \delta-64.3$ (s). FT-IR: $v\left(\mathrm{~cm}^{-1}\right)$ 2967, 1733, 1716, 1674, 1558, 1507, 1457, 1388, 1240. HRMS [ESI] m/z: [M+Na] $]^{+}$calcd for $\mathrm{C}_{21} \mathrm{H}_{19} \mathrm{~F}_{3} \mathrm{NaO}_{2}$ 383.1229; found 383.1242 .

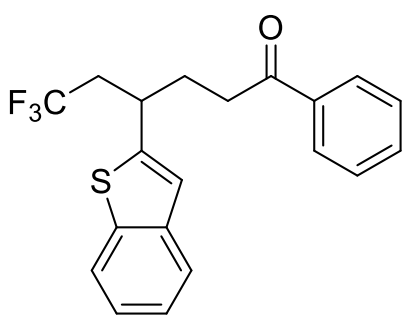

2o: $45 \mathrm{mg}, 63 \%$, white solid, m.p. $96-97{ }^{\circ} \mathrm{C}$. Purification by flash column chromatography (eluent: EtOAc/Petroleum ether $=1 / 30$ ). ${ }^{1} \mathrm{H}$ NMR $\left(400 \mathrm{MHz}, \mathrm{CDCl}_{3}\right) \delta$ 7.87-7.82 (m, 2H), 7.82-7.78 (m, 1H), 7.71-7.67 (m, 1H), 7.55-7.49 (m, 1H), 7.43-7.37 (m, 2H), 7.37-7.28 $(\mathrm{m}, 2 \mathrm{H}), 7.11(\mathrm{~s}, 1 \mathrm{H}), 3.59-3.46(\mathrm{~m}, 1 \mathrm{H}), 2.94(\mathrm{t}, J=7.2 \mathrm{~Hz}, 2 \mathrm{H})$, 2.68-2.51 (m, 2H), 2.46-2.35 (m, 1H), 2.14-2.02 (m, 1H); ${ }^{13} \mathrm{C}$ NMR $\left(100 \mathrm{MHz}, \mathrm{CDCl}_{3}\right) \delta 199.1,146.8,139.5,139.1,136.7,133.2,128.6$, $128.0,126.1\left(\mathrm{q}, J_{\mathrm{C}-\mathrm{F}}=275.8 \mathrm{~Hz}\right), 124.4,124.2,123.3,122.4,122.0,41.6\left(\mathrm{q}, J_{\mathrm{C}-\mathrm{F}}=27.5 \mathrm{~Hz}\right), 35.8$, $35.6\left(\mathrm{q}, J_{\mathrm{C}-\mathrm{F}}=2.6 \mathrm{~Hz}\right), 31.1 ;{ }^{19} \mathrm{~F}$ NMR $\left(376 \mathrm{MHz}, \mathrm{CDCl}_{3}\right) \delta-63.9$ (s). FT-IR: $v\left(\mathrm{~cm}^{-1}\right) 3056,2956$, 2855, 1681, 1617, 1541, 1412, 1380, 1246. HRMS [ESI] m/z: [M+Na] calcd for $\mathrm{C}_{20} \mathrm{H}_{17} \mathrm{~F}_{3} \mathrm{NaOS}$ 385.0844; found 385.0842 .

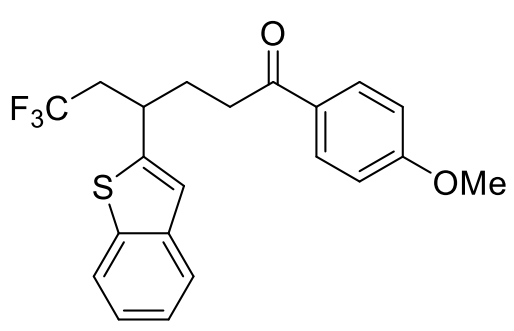

2p: $46.5 \mathrm{mg}, 60 \%$, white solid, m.p. $81-82{ }^{\circ} \mathrm{C}$. Purification by flash column chromatography (eluent: EtOAc/Petroleum ether $=1 / 30) .{ }^{1} \mathrm{H}$ NMR $\left(400 \mathrm{MHz}, \mathrm{CDCl}_{3}\right) \delta 7.86-7.77(\mathrm{~m}$, $3 \mathrm{H}), 7.71-7.66(\mathrm{~m}, 1 \mathrm{H}), 7.37-7.27(\mathrm{~m}, 2 \mathrm{H}), 7.10(\mathrm{~s}, 1 \mathrm{H})$, 6.90-6.83 (m, 2H), $3.83(\mathrm{~s}, 3 \mathrm{H}), 3.56-3.45(\mathrm{~m}, 1 \mathrm{H}), 2.88(\mathrm{t}, J$ $=7.4 \mathrm{~Hz}, 2 \mathrm{H}), 2.66-2.51(\mathrm{~m}, 2 \mathrm{H}), 2.42-2.33(\mathrm{~m}, 1 \mathrm{H})$, 2.11-2.01 (m, 1H); ${ }^{13} \mathrm{C}$ NMR $\left(100 \mathrm{MHz}, \mathrm{CDCl}_{3}\right) \delta$ 197.6, $163.5,146.9,139.6,139.1,130.2,129.8,126.1\left(\mathrm{q}, J_{\mathrm{C}-\mathrm{F}}=275.9 \mathrm{~Hz}\right), 124.4,124.1,123.3,122.4$, $122.0,113.7,55.4,41.6\left(\mathrm{q}, J_{\mathrm{C}-\mathrm{F}}=27.5 \mathrm{~Hz}\right), 35.7\left(\mathrm{q}, J_{\mathrm{C}-\mathrm{F}}=2.7 \mathrm{~Hz}\right), 35.4,31.3 ;{ }^{19} \mathrm{~F}$ NMR $(376$ $\left.\mathrm{MHz}, \mathrm{CDCl}_{3}\right) \delta-63.8$ (s). FT-IR: $v\left(\mathrm{~cm}^{-1}\right) 3034,2939,2931,1869,1698,1602,1521,1457,1397$, 1339, 1261. HRMS [ESI] m/z: [M+Na] ${ }^{+}$calcd for $\mathrm{C}_{21} \mathrm{H}_{19} \mathrm{~F}_{3} \mathrm{NaO}_{2} \mathrm{~S} 415.0950$; found 415.0956. 


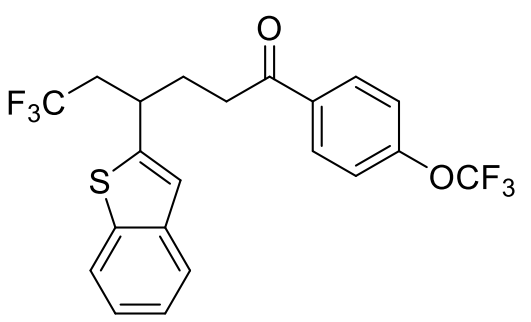

2q: $49.5 \mathrm{mg}, 56 \%$, yellow oil. Purification by flash column chromatography (eluent: EtOAc/Petroleum ether $=1 / 30) .{ }^{1} \mathrm{H}$ NMR $\left(400 \mathrm{MHz}, \mathrm{CDCl}_{3}\right) \delta$ 7.91-7.85 (m, 2H), 7.82-7.76 (m, $1 \mathrm{H}), 7.71-7.65(\mathrm{~m}, 1 \mathrm{H}), 7.38-7.28(\mathrm{~m}, 2 \mathrm{H}), 7.25-7.19(\mathrm{~m}$, $2 \mathrm{H}), 7.09(\mathrm{~s}, 1 \mathrm{H}), 3.56-3.46(\mathrm{~m}, 1 \mathrm{H}), 2.91(\mathrm{t}, J=7.2 \mathrm{~Hz}$, $2 \mathrm{H}), 2.68-2.50(\mathrm{~m}, 2 \mathrm{H}), 2.45-2.35(\mathrm{~m}, 1 \mathrm{H}), 2.14-2.00(\mathrm{~m}$, $1 \mathrm{H}) ;{ }^{13} \mathrm{C}$ NMR $\left(100 \mathrm{MHz}, \mathrm{CDCl}_{3}\right) \delta 197.5,152.6,146.6$, $139.5,139.1,134.9,130.0,126.1\left(\mathrm{q}, J_{\mathrm{C}-\mathrm{F}}=275.9 \mathrm{~Hz}\right), 124.5,124.3,123.3,122.5,122.1,120.4$, $120.3\left(\mathrm{q}, J_{\mathrm{C}-\mathrm{F}}=257.1 \mathrm{~Hz}\right), 41.5\left(\mathrm{q}, J_{\mathrm{C}-\mathrm{F}}=27.6 \mathrm{~Hz}\right), 35.8,35.6\left(\mathrm{q}, J_{\mathrm{C}-\mathrm{F}}=2.8 \mathrm{~Hz}\right), 30.9 ;{ }^{19} \mathrm{~F}$ NMR $\left(376 \mathrm{MHz}, \mathrm{CDCl}_{3}\right) \delta-57.6(\mathrm{~s}),-63.9(\mathrm{~s})$. FT-IR: $v\left(\mathrm{~cm}^{-1}\right)$ 2917, 2849, 2362, 1689, 1603, 1505, 1381, 1256, 1210. HRMS [ESI] $\mathrm{m} / \mathrm{z}$ : $[\mathrm{M}+\mathrm{Na}]^{+}$calcd for $\mathrm{C}_{21} \mathrm{H}_{16} \mathrm{~F}_{6} \mathrm{NaO}_{2} \mathrm{~S}$ 469.0667; found 469.0660<smiles>Cc1cccc(C(=O)CCC(CC(F)(F)F)c2cc3ccccc3s2)c1</smiles>

2r: $38.8 \mathrm{mg}, 52 \%$, white solid, m.p. $64-65{ }^{\circ} \mathrm{C}$. Purification by flash column chromatography (eluent: EtOAc/Petroleum ether $=$ 1/30). ${ }^{1} \mathrm{H}$ NMR (400 MHz, $\left.\mathrm{CDCl}_{3}\right) \delta$ 7.74-7.68 (m, 1H), 7.63-7.58 (m, 1H), 7.58-7.50 (m, 2H), 7.26-7.17 (m, 4H), $7.02(\mathrm{~s}$, $1 \mathrm{H}), 3.48-3.37(\mathrm{~m}, 1 \mathrm{H}), 2.83$ (t, $J=7.6 \mathrm{~Hz}, 2 \mathrm{H}), 2.58-2.42(\mathrm{~m}$, $2 \mathrm{H}), 2.35-2.28(\mathrm{~m}, 1 \mathrm{H}), 2.27(\mathrm{~s}, 3 \mathrm{H}), 2.04-1.93(\mathrm{~m}, 1 \mathrm{H}) ;{ }^{13} \mathrm{C}$ NMR $\left(100 \mathrm{MHz}, \mathrm{CDCl}_{3}\right) \delta 199.3,146.8,139.6,139.2,138.4$, $136.7,133.9,128.5,128.4,127.5$ (q, $\left.J_{\mathrm{C}-\mathrm{F}}=275.9 \mathrm{~Hz}\right), 125.2,124.4,124.2,123.3,122.4,122.0$, $41.7\left(\mathrm{q}, J_{\mathrm{C}-\mathrm{F}}=27.5 \mathrm{~Hz}\right), 35.8,35.7\left(\mathrm{q}, J_{\mathrm{C}-\mathrm{F}}=2.7 \mathrm{~Hz}\right), 31.2,21.3 ;{ }^{19} \mathrm{~F} \mathrm{NMR}\left(376 \mathrm{MHz}, \mathrm{CDCl}_{3}\right) \delta$ -63.8 (s). FT-IR: $v\left(\mathrm{~cm}^{-1}\right)$ 2988, 2901, 1717, 1681, 1521, 1437, 1379, 1272, 1248. HRMS [ESI] $\mathrm{m} / \mathrm{z}:[\mathrm{M}+\mathrm{Na}]^{+}$calcd for C21H19F3NaOS 399.1001; found 399.0994.<smiles>O=C(CCC(CC(F)(F)F)c1ccco1)c1ccccc1</smiles>

2s: $23.1 \mathrm{mg}, 39 \%$, yellow solid, m.p. $72-73{ }^{\circ} \mathrm{C}$. Purification by flash column chromatography (eluent: EtOAc/Petroleum ether $=1 / 30$ ). ${ }^{1} \mathrm{H}$ NMR $\left(400 \mathrm{MHz}, \mathrm{CDCl}_{3}\right) \delta$ 7.91-7.83 (m, 2H), 7.58-7.52 (m, 1H), 7.48-7.40 (m, 2H), 7.38-7.32 (m, $1 \mathrm{H}), 6.29(\mathrm{dd}, J=3.2,2.0 \mathrm{~Hz}, 1 \mathrm{H})$ $6.09(\mathrm{~d}, J=3.2 \mathrm{~Hz}, 1 \mathrm{H}), 3.27-3.18(\mathrm{~m}, 1 \mathrm{H}), 2.89-2.82(\mathrm{~m}, 2 \mathrm{H})$, 2.67-2.52 (m, 1H), 2.49-2.35 (m, 1H), 2.25-2.16 (m, 1H), 2.13-2.03 (m, 1H); ${ }^{13} \mathrm{C}$ NMR (100 MHz, $\left.\mathrm{CDCl}_{3}\right) \delta 199.2,154.5,141.8,136.8,133.1,128.6,128.0,126.3\left(\mathrm{q}, J_{\mathrm{C}-\mathrm{F}}=275.6 \mathrm{~Hz}\right), 110.2,106.7$, $38.3\left(\mathrm{q}, J_{\mathrm{C}-\mathrm{F}}=27.6 \mathrm{~Hz}\right), 35.6,32.7\left(\mathrm{q}, J_{\mathrm{C}-\mathrm{F}}=2.7 \mathrm{~Hz}\right), 28.3 ;{ }^{19} \mathrm{~F} \mathrm{NMR}\left(376 \mathrm{MHz}, \mathrm{CDCl}_{3}\right) \delta-64.5$ (s). FT-IR: $v\left(\mathrm{~cm}^{-1}\right)$ 2960, 2874, 2362, 1721, 1685, 1502, 1449, 1375, 1257, 1216. HRMS [ESI] $\mathrm{m} / \mathrm{z}$ : $[\mathrm{M}+\mathrm{Na}]^{+}$calcd for $\mathrm{C}_{16} \mathrm{H}_{15} \mathrm{~F}_{3} \mathrm{NaO}_{2}$ 319.0916; found 319.0923 .<smiles>O=C(CCC(CC(F)(F)F)c1ccco1)c1ccc(F)cc1</smiles>

2t: $17 \mathrm{mg}, 27 \%$, red oil. Purification by flash column chromatography (eluent: EtOAc/Petroleum ether $=1 / 30$ ). ${ }^{1} \mathrm{H}$ NMR (400 MHz, $\left.\mathrm{CDCl}_{3}\right) \delta$ 7.95-7.82 (m, 2H), $7.35(\mathrm{~d}, J=1.2$ $\mathrm{Hz}, 1 \mathrm{H}), 7.15-7.06(\mathrm{~m}, 2 \mathrm{H}), 6.33-6.24(\mathrm{~m}, 1 \mathrm{H}), 6.08(\mathrm{~d}, J=3.6$ $\mathrm{Hz}, 1 \mathrm{H}), 3.26-3.16(\mathrm{~m}, 1 \mathrm{H}), 2.82(\mathrm{t}, J=7.4 \mathrm{~Hz}, 2 \mathrm{H}), 2.66-2.52$ $(\mathrm{m}, 1 \mathrm{H}), 2.48-2.36(\mathrm{~m}, 1 \mathrm{H}), 2.25-2.16(\mathrm{~m}, 1 \mathrm{H}), 2.12-2.02(\mathrm{~m}, 1 \mathrm{H}) ;{ }^{13} \mathrm{C} \mathrm{NMR}\left(100 \mathrm{MHz}, \mathrm{CDCl}_{3}\right) \delta$ $197.5,165.7\left(\mathrm{~d}, J_{\mathrm{C}-\mathrm{F}}=253.3 \mathrm{~Hz}\right), 154.4,141.8,133.2\left(\mathrm{~d}, J_{\mathrm{C}-\mathrm{F}}=3.1 \mathrm{~Hz}\right), 130.6\left(\mathrm{~d}, J_{\mathrm{C}-\mathrm{F}}=9.1 \mathrm{~Hz}\right)$, $126.3\left(\mathrm{q}, J_{\mathrm{C}-\mathrm{F}}=275.4 \mathrm{~Hz}\right), 115.7\left(\mathrm{~d}, J_{\mathrm{C}-\mathrm{F}}=21.7 \mathrm{~Hz}\right), 110.2,106.8,38.3\left(\mathrm{q}, J_{\mathrm{C}-\mathrm{F}}=27.6 \mathrm{~Hz}\right), 35.5$, 
$32.6\left(\mathrm{q}, J_{\mathrm{C}-\mathrm{F}}=2.7 \mathrm{~Hz}\right), 28.2 ;{ }^{19} \mathrm{~F}$ NMR $\left(376 \mathrm{MHz}, \mathrm{CDCl}_{3}\right) \delta-64.5(\mathrm{~s}),-105.2(\mathrm{~s})$. FT-IR: $v\left(\mathrm{~cm}^{-1}\right)$ 2959, 2930, 1794, 1685, 1599, 1508, 1410, 1376, 1259, 1236, 1211. HRMS [ESI] m/z: [M+Na] ${ }^{+}$ calcd for $\mathrm{C}_{16} \mathrm{H}_{14} \mathrm{~F}_{4} \mathrm{NaO}_{2}$ 337.0822; found 337.0814.

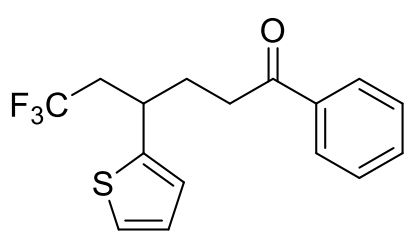

2u: $34.2 \mathrm{mg}, 56 \%$, white solid, m.p. $87-88^{\circ} \mathrm{C}$. Purification by flash column chromatography (eluent: EtOAc/Petroleum ether $=1 / 30$ ). ${ }^{1} \mathrm{H} \mathrm{NMR}\left(400 \mathrm{MHz}, \mathrm{CDCl}_{3}\right) \delta$ 7.90-7.81 (m, 2H), 7.58-7.49 (m, 1H), 7.47-7.38 $(\mathrm{m}, 2 \mathrm{H}), 7.21(\mathrm{~d}, J=5.2 \mathrm{~Hz}, 1 \mathrm{H}), 6.97-6.91(\mathrm{~m}, 1 \mathrm{H})$, 6.88-6.83 (m, 1H), 3.50-3.38 (m, 1H), 2.95-2.81 (m, 2H), 2.58-2.37 (m, 2H), 2.39-2.29 (m, 1H), 2.06-1.95 (m, 1H); $\left.{ }^{13} \mathrm{C} \mathrm{NMR} \mathrm{(100} \mathrm{MHz,} \mathrm{CDCl}_{3}\right) \delta 199.2,146.0$, $136.8,133.1,128.6,127.9,126.8,126.2\left(\mathrm{q}, J_{\mathrm{C}-\mathrm{F}}=275.9 \mathrm{~Hz}\right), 125.0,124.0,42.0\left(\mathrm{q}, J_{\mathrm{C}-\mathrm{F}}=27.3 \mathrm{~Hz}\right)$, $35.8,34.8\left(\mathrm{q}, J_{\mathrm{C}-\mathrm{F}}=2.7 \mathrm{~Hz}\right), 31.6 ;{ }^{19} \mathrm{~F}$ NMR $\left(376 \mathrm{MHz}, \mathrm{CDCl}_{3}\right) \delta-63.9(\mathrm{~s})$. FT-IR: $v\left(\mathrm{~cm}^{-1}\right) 2988$, 2901, 1772, 1685, 1576, 1541, 1420, 1386, 1314, 1260, 1242. HRMS [ESI] m/z: $[\mathrm{M}+\mathrm{Na}]^{+}$calcd for $\mathrm{C}_{16} \mathrm{H}_{15} \mathrm{~F}_{3} \mathrm{NaOS}$ 335.0688; found 335.0693.

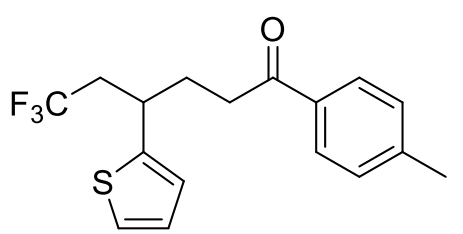

2v: $37.1 \mathrm{mg}, 59 \%$, yellow solid, m.p. $48-49{ }^{\circ} \mathrm{C}$. Purification by flash column chromatography (eluent: EtOAc/Petroleum ether $=$ 1/30). ${ }^{1} \mathrm{H}$ NMR $\left(400 \mathrm{MHz}, \mathrm{CDCl}_{3}\right) \delta$ 7.87-7.80 (m, 2H), $7.20(\mathrm{dd}$ $J=5.2,0.4 \mathrm{~Hz}, 1 \mathrm{H}), 6.95-6.82(\mathrm{~m}, 4 \mathrm{H}), 3.85(\mathrm{~s}, 3 \mathrm{H}), 3.47-3.37$ $(\mathrm{m}, 1 \mathrm{H}), 2.88-2.76(\mathrm{~m}, 2 \mathrm{H}), 2.57-2.47(\mathrm{~m}, 2 \mathrm{H}), 2.37-2.27(\mathrm{~m}$, 1H), 2.04-1.93 (m, 1H); ${ }^{13} \mathrm{C}$ NMR (100 MHz, $\left.\mathrm{CDCl}_{3}\right) \delta 197.8,163.5,146.0,130.2,129.8,126.8$, $126.2\left(\mathrm{q}, J_{\mathrm{C}-\mathrm{F}}=275.9 \mathrm{~Hz}\right), 125.0,123.9,113.7,55.5,42.0\left(\mathrm{q}, J_{\mathrm{C}-\mathrm{F}}=27.5 \mathrm{~Hz}\right), 35.4,34.8\left(\mathrm{q}, J_{\mathrm{C}-\mathrm{F}}=\right.$ $2.6 \mathrm{~Hz}), 31.8 ;{ }^{19} \mathrm{~F}$ NMR $\left(376 \mathrm{MHz}, \mathrm{CDCl}_{3}\right) \delta-63.9$ (s). FT-IR: $v\left(\mathrm{~cm}^{-1}\right) 2954,2931,2358,1673$, 1602, 1577, 1458, 1377, 1273, 1250, 1214. HRMS [ESI] m/z: $[\mathrm{M}+\mathrm{Na}]^{+}$calcd for $\mathrm{C}_{17} \mathrm{H}_{18} \mathrm{~F}_{3} \mathrm{OS}$ 327.1025; found 327.1025.

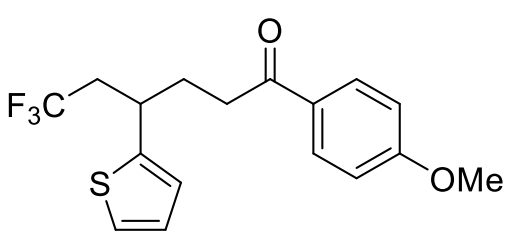

2w: $36.5 \mathrm{mg}, 54 \%$, white solid, m.p. $66-67{ }^{\circ} \mathrm{C}$. Purification by flash column chromatography (eluent: EtOAc/Petroleum ether $=1 / 30) .{ }^{1} \mathrm{H}$ NMR $\left(400 \mathrm{MHz}, \mathrm{CDCl}_{3}\right) \delta$ 7.78-7.73 $(\mathrm{m}$, $2 \mathrm{H}), 7.25-7.17(\mathrm{~m}, 3 \mathrm{H}), 6.93(\mathrm{dd}, J=5.2,3.6 \mathrm{~Hz}, 1 \mathrm{H})$, 6.87-6.84 (m, $1 \mathrm{H}), 3.48-3.38(\mathrm{~m}, 1 \mathrm{H}), 2.90-2.80(\mathrm{~m}, 2 \mathrm{H})$, 2.57-2.47 (m, 2H), 2.39 (s, 3H), 2.36-2.28 (m, 1H), 2.04-1.94 (m, 1H); ${ }^{13} \mathrm{C}$ NMR (100 MHz, $\left.\mathrm{CDCl}_{3}\right) \delta 198.9,146.0,143.9,134.3,129.2,128.1,126.8,126.2\left(\mathrm{q}, J_{\mathrm{C}-\mathrm{F}}=275.9 \mathrm{~Hz}\right), 125.0,124.0$, $42.0\left(\mathrm{q}, J_{\mathrm{C}-\mathrm{F}}=27.2 \mathrm{~Hz}\right), 35.7,34.8\left(\mathrm{q}, J_{\mathrm{C}-\mathrm{F}}=2.6 \mathrm{~Hz}\right), 31.7,21.6 ;{ }^{19} \mathrm{~F} \mathrm{NMR}\left(376 \mathrm{MHz}, \mathrm{CDCl}_{3}\right) \delta$ -63.9 (s). FT-IR: $v\left(\mathrm{~cm}^{-1}\right)$ 2948, 2868, 1717, 1653, 1520, 1435, 1380, 1286, 1219. HRMS [ESI] $\mathrm{m} / \mathrm{z}:[\mathrm{M}+\mathrm{H}]^{+}$calcd for $\mathrm{C}_{17} \mathrm{H}_{18} \mathrm{~F}_{3} \mathrm{O}_{2} \mathrm{~S} 343.0974$; found 343.0964 .<smiles>O=C(CCC(CC(F)(F)F)c1cccs1)c1ccc(Cl)cc1</smiles>

2x: $34.0 \mathrm{mg}, 50 \%$, white solid, m.p. $109-110^{\circ} \mathrm{C}$. Purification by flash column chromatography (eluent: EtOAc/Petroleum ether $=$ 1/30). ${ }^{1} \mathrm{H}$ NMR $\left(400 \mathrm{MHz}, \mathrm{CDCl}_{3}\right) \delta$ 7.82-7.74 (m, 2H), 7.44-7.35 (m, 2H), $7.21(\mathrm{dd}, J=5.2,0.4 \mathrm{~Hz}, 1 \mathrm{H}), 6.94(\mathrm{dd}, J=$ 5.2, $3.6 \mathrm{~Hz}, 1 \mathrm{H}), 6.88-6.82(\mathrm{~m}, 1 \mathrm{H}), 3.47-3.37(\mathrm{~m}, 1 \mathrm{H})$, 2.89-2.79 (m, 2H), 2.58-2.46 (m, 2H), 2.38-2.29 (m, 1H), 2.04-1.94 (m, 1H); ${ }^{13} \mathrm{C}$ NMR (100 MHz, $\left.\mathrm{CDCl}_{3}\right) \delta 197.9,145.8,139.6,135.0,129.4,128.9,126.9,126.1\left(\mathrm{q}, J_{\mathrm{C}-\mathrm{F}}=275.9 \mathrm{~Hz}\right), 125.0,124.1$, 
$42.0\left(\mathrm{q}, J_{\mathrm{C}-\mathrm{F}}=27.3 \mathrm{~Hz}\right), 35.8,34.7\left(\mathrm{q}, J_{\mathrm{C}-\mathrm{F}}=2.6 \mathrm{~Hz}\right), 31.4 ;{ }^{19} \mathrm{~F}$ NMR $\left(376 \mathrm{MHz}, \mathrm{CDCl}_{3}\right) \delta-63.9$ (s). FT-IR: $v\left(\mathrm{~cm}^{-1}\right)$ 3649, 2962, 2926, 2856, 1682, 1653, 1571, 1488, 1435, 1375, 1250. HRMS [ESI] m/z: [M+Na] ${ }^{+}$calcd for $\mathrm{C}_{16} \mathrm{H}_{14} \mathrm{ClF}_{3} \mathrm{NaOS} 369.0298$; found 369.0300 .

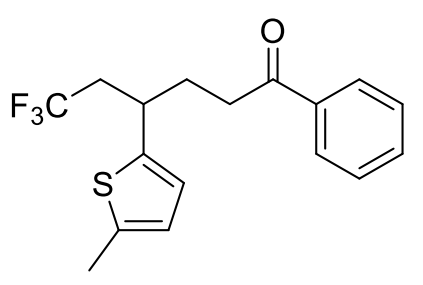

2y: $38.7 \mathrm{mg}, 60 \%$, yellow solid, m.p. $94-95^{\circ} \mathrm{C}$. Purification by flash column chromatography (eluent: EtOAc/Petroleum ether $=1 / 30$ ). ${ }^{1} \mathrm{H}$ NMR $\left(400 \mathrm{MHz}, \mathrm{CDCl}_{3}\right)$ $\delta$ 7.93-7.82 (m, 2H), 7.58-7.50 (m, 1H), 7.47-7.39 (m, 2H), 6.64-6.59 (m, 1H), 6.57-6.53 (m, 1H), 3.36-3.26 (m, 1H), 2.95-2.85 (m, 2H), 2.55-2.45 (m, 2H), 2.45-2.41 (m, 3H), 2.33-2.25 (m, 1H), 2.00-1.90 (m, 1H); ${ }^{13} \mathrm{C}$ NMR $\left(100 \mathrm{MHz}, \mathrm{CDCl}_{3}\right)$ $\delta 199.3,143.5,138.5,136.8,133.1,128.6,128.0,126.2\left(\mathrm{q}, J_{\mathrm{C}-\mathrm{F}}=275.9 \mathrm{~Hz}\right), 124.9,124.7,41.9(\mathrm{q}$, $\left.J_{\mathrm{C}-\mathrm{F}}=27.1 \mathrm{~Hz}\right), 35.9,35.0\left(\mathrm{q}, J_{\mathrm{C}-\mathrm{F}}=2.6 \mathrm{~Hz}\right), 31.4,15.4 ;{ }^{19} \mathrm{~F} \mathrm{NMR}\left(376 \mathrm{MHz}, \mathrm{CDCl}_{3}\right) \delta-63.8(\mathrm{~s})$. FT-IR: $v\left(\mathrm{~cm}^{-1}\right)$ 2988, 2901, 1683, 1521, 1437, 1379, 1319, 1272, 1207. HRMS [ESI] m/z: $[\mathrm{M}+\mathrm{Na}]^{+}$calcd for $\mathrm{C}_{17} \mathrm{H}_{17} \mathrm{~F}_{3} \mathrm{NaOS} 349.0844$; found 349.0832.<smiles>O=C(CCC(CC(F)(F)F)c1ccc(Cl)s1)c1ccccc1</smiles>

2z: $57.8 \mathrm{mg}, 84 \%$, white solid, m.p. $95-96{ }^{\circ} \mathrm{C}$. Purification by flash column chromatography (eluent: EtOAc/Petroleum ether $=1 / 30$ ). ${ }^{1} \mathrm{H}$ NMR (400 MHz, $\left.\mathrm{CDCl}_{3}\right)$ $\delta$ 7.90-7.84 (m, 2H), 7.59-7.52 (m, 1H), 7.48-7.41 (m, 2H), 6.75-6.71 (m, 1H), 6.65-6.61 (m, 1H), 3.37-3.27 (m, 1H), $2.91(\mathrm{t}, J=7.2 \mathrm{~Hz}, 2 \mathrm{H}), 2.53-2.42(\mathrm{~m}, 2 \mathrm{H}), 2.35-2.26(\mathrm{~m}$, 1H), 1.99-1.88 (m, 1H); ${ }^{13} \mathrm{C}$ NMR $\left(100 \mathrm{MHz}, \mathrm{CDCl}_{3}\right) \delta 198.9,144.7$, $136.7,133.2,128.6,128.4,128.0\left(\mathrm{q}, J_{\mathrm{C}-\mathrm{F}}=105.9 \mathrm{~Hz}\right), 127.9,125.8,124.6,41.7\left(\mathrm{q}, J_{\mathrm{C}-\mathrm{F}}=27.4 \mathrm{~Hz}\right)$, 35.6, $35.3\left(\mathrm{q}, J_{\mathrm{C}-\mathrm{F}}=2.7 \mathrm{~Hz}\right), 31.3 .{ }^{19} \mathrm{~F}$ NMR $\left(376 \mathrm{MHz}, \mathrm{CDCl}_{3}\right) \delta-63.8(\mathrm{~s})$. FT-IR: $v\left(\mathrm{~cm}^{-1}\right) 3057$, 2926, 2855, 1792, 1681, 1596, 1508, 1445, 1338, 1268, 1207. HRMS [ESI] m/z: [M+Na] ${ }^{+}$calcd for $\mathrm{C}_{16} \mathrm{H}_{14} \mathrm{ClF}_{3} \mathrm{NaOS}$ 369.0298; found 369.0295.

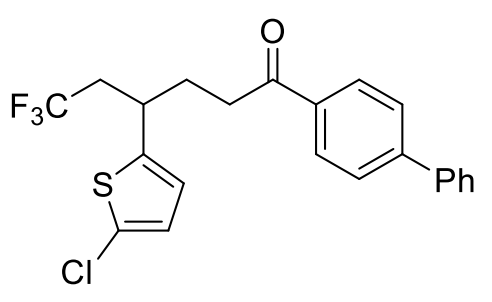

2aa: $57.8 \mathrm{mg}, 65 \%$, white solid, m.p. $148-149^{\circ} \mathrm{C}$. Purification by flash column chromatography (eluent: EtOAc/Petroleum ether $=1 / 30) .{ }^{1} \mathrm{H}$ NMR $\left(400 \mathrm{MHz}, \mathrm{CDCl}_{3}\right) \delta$ 7.99-7.91 (m, 2H), 7.69-7.64 (m, 2H), 7.64-7.59 (m, 2H), 7.50-7.44 (m, 2H), 7.43-7.38 (m, $1 \mathrm{H}), 6.77-6.72(\mathrm{~m}, 1 \mathrm{H}), 6.67-6.63(\mathrm{~m}, 1 \mathrm{H})$, 3.40-3.29 (m, 1H), $2.94(\mathrm{t}, J=7.2 \mathrm{~Hz}, 2 \mathrm{H}), 2.56-2.42(\mathrm{~m}, 2 \mathrm{H})$, 2.38-2.28 (m, 1H), 2.02-1.90 (m, 1H); $\left.{ }^{13} \mathrm{C} \mathrm{NMR} \mathrm{(100} \mathrm{MHz,} \mathrm{CDCl}_{3}\right) \delta 198.5,145.9,144.7,139.8$, $135.3,129.0,128.6,128.4,128.3,127.3,126.2\left(\mathrm{q}, J_{\mathrm{C}-\mathrm{F}}=263.1 \mathrm{~Hz}\right), 125.8,124.6,41.7\left(\mathrm{q}, J_{\mathrm{C}-\mathrm{F}}=\right.$ $27.3 \mathrm{~Hz}), 35.6,35.4\left(\mathrm{q}, J_{\mathrm{C}-\mathrm{F}}=2.5 \mathrm{~Hz}\right), 31.4 ;{ }^{19} \mathrm{~F}$ NMR $\left(376 \mathrm{MHz}, \mathrm{CDCl}_{3}\right) \delta-63.8$ (s). FT-IR: $v$ $\left(\mathrm{cm}^{-1}\right)$ 2988, 2928, 2894, 2360, 2341, 1675, 1606, 1488, 1409, 1382, 1267, 1221. HRMS [ESI] $\mathrm{m} / \mathrm{z}$ : $[\mathrm{M}+\mathrm{Na}]^{+}$calcd for $\mathrm{C}_{22} \mathrm{H}_{18} \mathrm{ClF}_{3} \mathrm{NaOS} 445.0611$; found 445.0623 .

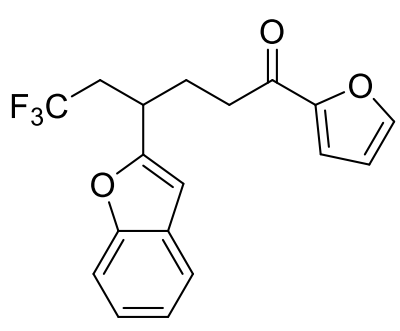

3ab-B: $30.4 \mathrm{mg}, 46 \%$ (ratio >19:1), yellow oil. Purification by flash column chromatography (eluent: EtOAc/Petroleum ether $=1 / 30)$. Major isomer: ${ }^{1} \mathrm{H}$ NMR $\left(400 \mathrm{MHz}, \mathrm{CDCl}_{3}\right) \delta 7.54-7.46(\mathrm{~m}, 2 \mathrm{H})$, 7.45-7.40 (m, 1H), 7.28-7.18 (m, 2H), $7.08(\mathrm{dd}, J=3.6,0.4 \mathrm{~Hz}, 1 \mathrm{H})$, $6.51(\mathrm{~d}, J=0.8 \mathrm{~Hz}, 1 \mathrm{H}), 6.48(\mathrm{dd}, J=3.6,2.0 \mathrm{~Hz}, 1 \mathrm{H}), 3.38-3.28$ (m, $1 \mathrm{H}), 2.78(\mathrm{t}, J=7.2 \mathrm{~Hz}, 2 \mathrm{H}), 2.75-2.65(\mathrm{~m}, 1 \mathrm{H}), 2.58-2.42(\mathrm{~m}, 1 \mathrm{H})$, 
2.32-2.22 (m, 1H), 2.22-2.12 (m, 1H); ${ }^{13} \mathrm{C}$ NMR $\left(100 \mathrm{MHz}, \mathrm{CDCl}_{3}\right) \delta$ 188.2, 157.2, 154.8, 152.5, $146.3,128.2,126.2\left(\mathrm{q}, J_{\mathrm{C}-\mathrm{F}}=275.5 \mathrm{~Hz}\right), 123.9,122.8,120.7,116.9,112.2,111.0,104.0,38.0(\mathrm{q}$, $\left.J_{\mathrm{C}-\mathrm{F}}=27.9 \mathrm{~Hz}\right), 35.4,33.2\left(\mathrm{q}, J_{\mathrm{C}-\mathrm{F}}=2.7 \mathrm{~Hz}\right), 27.6 ;{ }^{19} \mathrm{~F} \mathrm{NMR}\left(376 \mathrm{MHz}, \mathrm{CDCl}_{3}\right) \delta-64.4(\mathrm{~s})$. FT-IR: $v\left(\mathrm{~cm}^{-1}\right)$ 2987, 2902, 1677, 1569, 1469, 1456, 1384, 1251. HRMS [ESI] m/z: $[\mathrm{M}+\mathrm{Na}]^{+}$calcd for $\mathrm{C}_{18} \mathrm{H}_{15} \mathrm{~F}_{3} \mathrm{NaO}_{3} 359.0866$; found 359.0869 .

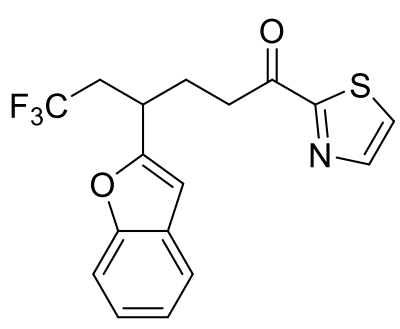

3ac-B: $56.2 \mathrm{mg}$, 80\% (ratio 10:1), yellow oil. Purification by flash column chromatography (eluent: EtOAc/Petroleum ether $=1 / 30$ ). Major isomer: ${ }^{1} \mathrm{H} \mathrm{NMR}\left(400 \mathrm{MHz}, \mathrm{CDCl}_{3}\right) \delta 7.93(\mathrm{~d}, J=2.8 \mathrm{~Hz}$, $1 \mathrm{H}), 7.63(\mathrm{~d}, J=3.2 \mathrm{~Hz}, 1 \mathrm{H}), 7.50-7.45(\mathrm{~m}, 1 \mathrm{H}), 7.43-7.38(\mathrm{~m}, 1 \mathrm{H})$, 7.26-7.16 (m, 2H), $6.52(\mathrm{~s}, 1 \mathrm{H}), 3.42-3.31(\mathrm{~m}, 1 \mathrm{H}), 3.22-3.09(\mathrm{~m}$, $2 \mathrm{H}), 2.81-2.67(\mathrm{~m}, 1 \mathrm{H}), 2.60-2.46(\mathrm{~m}, 1 \mathrm{H}), 2.35-2.22(\mathrm{~m}, 2 \mathrm{H}) ;{ }^{13} \mathrm{C}$ NMR $\left(100 \mathrm{MHz}, \mathrm{CDCl}_{3}\right) \delta 192.7,166.7,157.0,154.8,144.7,128.2$, $126.3,126.2\left(\mathrm{q}, J_{\mathrm{C}-\mathrm{F}}=275.5 \mathrm{~Hz}\right), 123.9,122.7,120.7,111.0,104.1,37.9\left(\mathrm{q}, J_{\mathrm{C}-\mathrm{F}}=27.9 \mathrm{~Hz}\right), 35.8$, $33.2\left(\mathrm{q}, J_{\mathrm{C}-\mathrm{F}}=2.7 \mathrm{~Hz}\right), 27.6 ;{ }^{19} \mathrm{~F} \mathrm{NMR}\left(376 \mathrm{MHz}, \mathrm{CDCl}_{3}\right) \delta-64.3(\mathrm{~s})$. FT-IR: $v\left(\mathrm{~cm}^{-1}\right) 3088,2935$, 1687, 1602, 1586, 1455, 1392, 1322, 1251. HRMS [ESI] m/z: $[\mathrm{M}+\mathrm{H}]^{+}$calcd for $\mathrm{C}_{17} \mathrm{H}_{15} \mathrm{~F}_{3} \mathrm{NO}_{2} \mathrm{~S}$ 354.0770; found 354.0760.<smiles>O=C(CCC(CC(F)(F)F)c1cc2ccccc2o1)c1cccs1</smiles>

3ad-B: $45.4 \mathrm{mg}$, 65\% (ratio >19:1), red solid, m.p. 64-65 ${ }^{\circ} \mathrm{C}$. Purification by flash column chromatography (eluent: EtOAc/Petroleum ether $=1 / 30)$. Major isomer: ${ }^{1} \mathrm{H}$ NMR $(400 \mathrm{MHz}$, $\left.\mathrm{CDCl}_{3}\right) \delta 7.60(\mathrm{dd}, J=4.8,0.8 \mathrm{~Hz}, 1 \mathrm{H}), 7.56(\mathrm{dd}, J=4.0,1.2 \mathrm{~Hz}$, $1 \mathrm{H}), 7.53-7.49(\mathrm{~m}, 1 \mathrm{H}), 7.46-7.41(\mathrm{~m}, 1 \mathrm{H}), 7.27-7.19(\mathrm{~m}, 2 \mathrm{H}), 7.06$ (dd, $J=5.2,4.0 \mathrm{~Hz}, 1 \mathrm{H}), 6.51(\mathrm{~d}, J=0.4 \mathrm{~Hz}, 1 \mathrm{H}), 3.42-3.31(\mathrm{~m}, 1 \mathrm{H})$, $2.86(\mathrm{t}, J=7.6 \mathrm{~Hz}, 2 \mathrm{H}), 2.78-2.68(\mathrm{~m}, 1 \mathrm{H}), 2.56-2.46(\mathrm{~m}, 1 \mathrm{H})$, 2.36-2.27 (m, 1H), 2.25-2.17 (m, 1H); ${ }^{13} \mathrm{C}$ NMR (100 MHz, $\left.\mathrm{CDCl}_{3}\right) \delta$ 191.9, 157.2, 154.8, 143.9, 133.7, 131.8, 128.2, 128.1, $126.2\left(\mathrm{q}, J_{\mathrm{C}-\mathrm{F}}=275.8 \mathrm{~Hz}\right), 123.9,122.8,120.8,111.1,104.1,38.1(\mathrm{q}$, $\left.J_{\mathrm{C}-\mathrm{F}}=27.8 \mathrm{~Hz}\right), 36.3,33.2\left(\mathrm{q}, J_{\mathrm{C}-\mathrm{F}}=2.7 \mathrm{~Hz}\right), 28.1 ;{ }^{19} \mathrm{~F} \mathrm{NMR}\left(376 \mathrm{MHz}, \mathrm{CDCl}_{3}\right) \delta-64.3(\mathrm{~s}) . \mathrm{FT}-\mathrm{IR}$ : $v\left(\mathrm{~cm}^{-1}\right)$ 3083, 2924, 2854, 1666, 1519, 1417, 1354, 1316, 1260, 1205. HRMS [ESI] m/z: $[\mathrm{M}+\mathrm{Na}]^{+}$calcd for $\mathrm{C}_{18} \mathrm{H}_{15} \mathrm{~F}_{3} \mathrm{NaO}_{2} \mathrm{~S} 375.0637$; found 375.0633 .

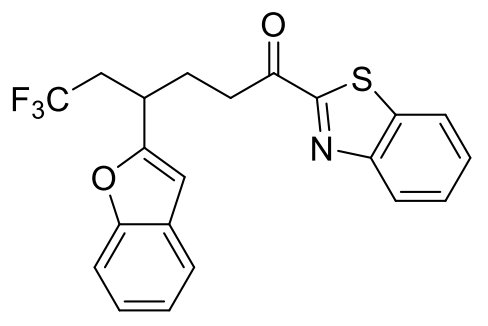

3ae-B: $63.5 \mathrm{mg}, 87 \%$ (ratio 10:1), white solid, m.p. $88-89^{\circ} \mathrm{C}$. Purification by flash column chromatography (eluent: EtOAc/Petroleum ether = 1/30). Major isomer: ${ }^{1} \mathrm{H}$ NMR $(400$ $\left.\mathrm{MHz}, \mathrm{CDCl}_{3}\right) \delta$ 8.06-7.96 (m, 1H), 7.88-7.80 (m, 1H), 7.49-7.35 (m, 3H), 7.34-7.28 (m, 1H), 7.16-7.06 (m, 2H), 6.45 (s, $1 \mathrm{H}), 3.36-3.26(\mathrm{~m}, 1 \mathrm{H}), 3.21-3.12(\mathrm{~m}, 2 \mathrm{H}), 2.75-2.58(\mathrm{~m}$, $1 \mathrm{H}), 2.53-2.38(\mathrm{~m}, 1 \mathrm{H}), 2.32-2.15(\mathrm{~m}, 2 \mathrm{H}) ;{ }^{13} \mathrm{C}$ NMR $(100$ $\left.\mathrm{MHz}, \mathrm{CDCl}_{3}\right) \delta 194.2,165.9,157.0,154.9,153.5,137.2,128.3,127.7,127.0,126.2\left(\mathrm{q}, J_{\mathrm{C}-\mathrm{F}}=\right.$ $275.7 \mathrm{~Hz}), 125.4,123.9,122.8,122.4,120.8,111.1,104.2,38.0\left(\mathrm{q}, J_{\mathrm{C}-\mathrm{F}}=28.0 \mathrm{~Hz}\right), 35.9,33.3(\mathrm{q}$, $\left.J_{\mathrm{C}-\mathrm{F}}=2.7 \mathrm{~Hz}\right), 27.6 ;{ }^{19} \mathrm{~F}$ NMR $\left(376 \mathrm{MHz}, \mathrm{CDCl}_{3}\right) \delta-64.3(\mathrm{~s})$. FT-IR: $v\left(\mathrm{~cm}^{-1}\right) 3750,3649,2989$, 2928, 2359, 1748, 1682, 1541, 1473, 1387, 1261, 1250. HRMS [ESI] m/z: $[\mathrm{M}+\mathrm{Na}]^{+}$calcd for $\mathrm{C} 21 \mathrm{H} 16 \mathrm{~F} 3 \mathrm{NNaO} 2 \mathrm{~S} 426.0746$; found 426.0735 . 


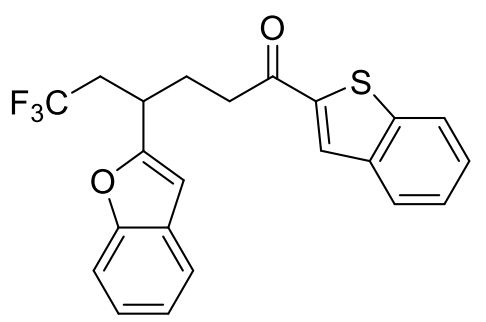

3af-B: $44.7 \mathrm{mg}, 54 \%$ (ratio 10:1), white solid, m.p. $98-99{ }^{\circ} \mathrm{C}$. Purification by flash column chromatography (eluent: EtOAc/Petroleum ether $=1 / 30)$. Major isomer: ${ }^{1} \mathrm{H}$ NMR $(400$ $\left.\mathrm{MHz}, \mathrm{CDCl}_{3}\right) \delta 7.84(\mathrm{~d}, J=8.0 \mathrm{~Hz}, 1 \mathrm{H}), 7.79(\mathrm{~d}, J=8.0 \mathrm{~Hz}$, $1 \mathrm{H}), 7.76(\mathrm{~s}, 1 \mathrm{H}), 7.54-7.50(\mathrm{~m}, 1 \mathrm{H}), 7.48-7.42(\mathrm{~m}, 2 \mathrm{H})$, 7.41-7.36 (m, 1H), 7.30-7.21 (m, 2H), $6.54(\mathrm{~s}, 1 \mathrm{H}), 3.45-3.33$ $(\mathrm{m}, 1 \mathrm{H}), 2.96(\mathrm{t}, J=7.6 \mathrm{~Hz}, 2 \mathrm{H}), 2.82-2.68(\mathrm{~m}, 1 \mathrm{H}), 2.59-2.47$ (m, 1H), 2.40-2.31 (m, 1H), 2.29-2.19 (m, 1H) ; $\left.{ }^{13} \mathrm{C} \mathrm{NMR} \mathrm{(100} \mathrm{MHz,} \mathrm{CDCl}_{3}\right) \delta$ 193.4, 157.1, $154.9,143.2,142.4,139.0,129.0,128.2,127.5,126.4\left(\mathrm{q}, J_{\mathrm{C}-\mathrm{F}}=275.6 \mathrm{~Hz}\right), 125.9,125.0,124.0$, $123.0,122.9,122.9,120.8,111.1,104.2,38.1\left(\mathrm{q}, J_{\mathrm{C}-\mathrm{F}}=28.0 \mathrm{~Hz}\right), 36.2,33.2\left(\mathrm{q}, J_{\mathrm{C}-\mathrm{F}}=2.7 \mathrm{~Hz}\right)$, 28.1; ${ }^{19} \mathrm{~F}$ NMR $\left(376 \mathrm{MHz}, \mathrm{CDCl}_{3}\right) \delta-64.3$ (s). FT-IR: $v\left(\mathrm{~cm}^{-1}\right)$ 2987, 2968, 2894, 1772, 1699 , 1636, 1558, 1521, 1489, 1396. HRMS [ESI] m/z: $[\mathrm{M}+\mathrm{Na}]^{+}$calcd for $\mathrm{C}_{22} \mathrm{H}_{17} \mathrm{~F}_{3} \mathrm{NaO}_{2} \mathrm{~S}$ 425.0794; found 425.0787 .

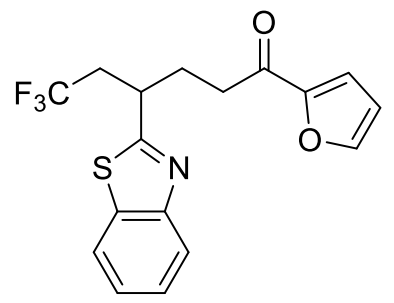

3ag-B: $13.5 \mathrm{mg}, 38 \%$ (ratio 10:1), yellow oil. Purification by flash column chromatography (eluent: EtOAc/Petroleum ether $=1 / 30$ ). Major isomer: ${ }^{1} \mathrm{H} \mathrm{NMR}\left(400 \mathrm{MHz}, \mathrm{CDCl}_{3}\right) \delta 8.00(\mathrm{~d}, J=8.0 \mathrm{~Hz}$, $1 \mathrm{H}), 7.87(\mathrm{~d}, J=8.0 \mathrm{~Hz}, 1 \mathrm{H}), 7.53-7.46(\mathrm{~m}, 2 \mathrm{H}), 7.42-7.36(\mathrm{~m}, 1 \mathrm{H})$, $7.11(\mathrm{dd}, J=3.6,0.4 \mathrm{~Hz}, 1 \mathrm{H}), 6.48(\mathrm{dd}, J=3.6,1.6 \mathrm{~Hz}, 1 \mathrm{H})$, 3.70-3.61 (m, 1H), 3.03-2.88 (m, 1H), 2.88-2.80 (m, 2H), 2.70-2.55 $(\mathrm{m}, 1 \mathrm{H}), 2.43-2.25(\mathrm{~m}, 2 \mathrm{H}) ;{ }^{13} \mathrm{C}$ NMR $\left(100 \mathrm{MHz}, \mathrm{CDCl}_{3}\right) \delta 187.3$, $171.6,152.2,151.9,145.9,134.0,125.8,125.6\left(\mathrm{q}, J_{\mathrm{C}-\mathrm{F}}=275.7 \mathrm{~Hz}\right), 124.9,122.8,121.3,116.6$, $111.8,38.7\left(\mathrm{q}, J_{\mathrm{C}-\mathrm{F}}=28.4 \mathrm{~Hz}\right), 37.4\left(\mathrm{q}, J_{\mathrm{C}-\mathrm{F}}=2.6 \mathrm{~Hz}\right), 34.6,29.2 ;{ }^{19} \mathrm{~F} \mathrm{NMR}\left(376 \mathrm{MHz}, \mathrm{CDCl}_{3}\right) \delta$ -64.1 (s). FT-IR: $v\left(\mathrm{~cm}^{-1}\right)$ 2955, 2921, 2851, 1780, 1674, 1569, 1468, 1439, 1395, 1255, 1144. HRMS [ESI] m/z: $[\mathrm{M}+\mathrm{H}]^{+}$calcd for $\mathrm{C}_{17} \mathrm{H}_{15} \mathrm{~F}_{3} \mathrm{NO}_{2} \mathrm{~S}$ 354.0776; found 354.0777.

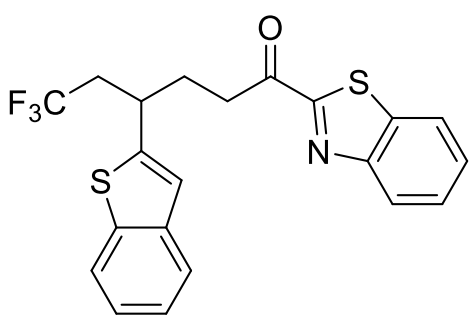

3ah-A: $20.0 \mathrm{mg}$, 24\%, yellow solid, m.p. $114-115{ }^{\circ} \mathrm{C}$. Purification by flash column chromatography (eluent: EtOAc/Petroleum ether $=1 / 30) .{ }^{1} \mathrm{H}$ NMR $\left(400 \mathrm{MHz}, \mathrm{CDCl}_{3}\right) \delta$ 8.14-8.07 $(\mathrm{m}, 1 \mathrm{H}), 7.97-7.92(\mathrm{~m}, 1 \mathrm{H}), 7.80-7.76(\mathrm{~m}, 1 \mathrm{H})$, 7.68-7.64 (m, 1H), 7.57-7.48 (m, 2H), 7.34-7.26 (m, 2H), 7.14 (s, $1 \mathrm{H}), 3.62-3.51(\mathrm{~m}, 1 \mathrm{H}), 3.36-3.20(\mathrm{~m}, 2 \mathrm{H}), 2.69-2.54(\mathrm{~m}$, $2 \mathrm{H}), 2.46-2.37(\mathrm{~m}, 1 \mathrm{H}), 2.24-2.14(\mathrm{~m}, 1 \mathrm{H}) ;{ }^{13} \mathrm{C}$ NMR $(100$ $\left.\mathrm{MHz}, \mathrm{CDCl}_{3}\right) \delta 194.4,165.9,153.4,146.4,139.5,139.2,137.2,127.7,127.0,126.1\left(\mathrm{q}, J_{\mathrm{C}-\mathrm{F}}=\right.$ $275.8 \mathrm{~Hz}), 125.4,124.7,124.4,124.2,123.3,122.4,122.2,41.3\left(\mathrm{q}, J_{\mathrm{C}-\mathrm{F}}=27.5 \mathrm{~Hz}\right), 36.00,35.6(\mathrm{q}$, $\left.J_{\mathrm{C}-\mathrm{F}}=2.5 \mathrm{~Hz}\right), 30.7 ;{ }^{19} \mathrm{~F}$ NMR $\left(376 \mathrm{MHz}, \mathrm{CDCl}_{3}\right) \delta-63.8(\mathrm{~s})$. FT-IR: $v\left(\mathrm{~cm}^{-1}\right) 3069,3049,2923$, $1686,1598,1428,1409,1306,1252,1227$. HRMS [ESI] $\mathrm{m} / \mathrm{z}:[\mathrm{M}+\mathrm{Na}]^{+}$calcd for $\mathrm{C}_{21} \mathrm{H}_{16} \mathrm{~F}_{3} \mathrm{NNaOS}{ }_{2}$ 442.0518; found 442.0512 .

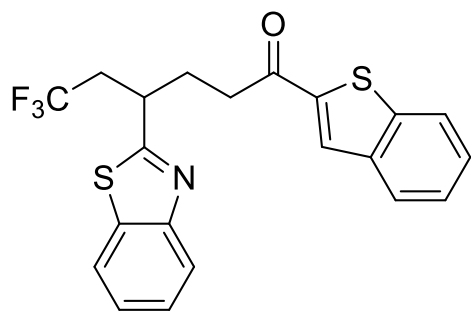

3ah-B: $33.3 \mathrm{mg}$, $40 \%$, yellow solid, m.p. $96-97{ }^{\circ} \mathrm{C}$. Purification by flash column chromatography (eluent: EtOAc/Petroleum ether $=1 / 30) .{ }^{1} \mathrm{H}$ NMR $\left(400 \mathrm{MHz}, \mathrm{CDCl}_{3}\right) \delta 8.00(\mathrm{~d}, J=8.4$ $\mathrm{Hz}, 1 \mathrm{H}), 7.91-7.82(\mathrm{~m}, 2 \mathrm{H}), 7.82-7.78(\mathrm{~m}, 2 \mathrm{H}), 7.52-7.47(\mathrm{~m}$, $1 \mathrm{H}), 7.47-7.42(\mathrm{~m}, 1 \mathrm{H}), 7.42-7.35(\mathrm{~m}, 2 \mathrm{H}), 3.76-3.65(\mathrm{~m}, 1 \mathrm{H})$, 
$3.03(\mathrm{t}, J=7.6 \mathrm{~Hz}, 2 \mathrm{H}), 3.00-2.88(\mathrm{~m}, 1 \mathrm{H}), 2.71-2.58(\mathrm{~m}, 1 \mathrm{H}), 2.51-2.33(\mathrm{~m}, 2 \mathrm{H}) ;{ }^{13} \mathrm{C}$ NMR $(100$ $\left.\mathrm{MHz}, \mathrm{CDCl}_{3}\right) \delta 193.1,171.8,153.1,143.1,142.5,139.0,134.7,129.2,127.5,126.3,126.1\left(\mathrm{q}, J_{\mathrm{C}-\mathrm{F}}\right.$ $=275.7 \mathrm{~Hz}), 125.9,125.3,125.0,123.0,123.0,121.8,39.3\left(\mathrm{q}, J_{\mathrm{C}-\mathrm{F}}=28.3 \mathrm{~Hz}\right), 37.9\left(\mathrm{q}, J_{\mathrm{C}-\mathrm{F}}=2.4\right.$ $\mathrm{Hz}$ ), 35.9, 30.1; ${ }^{19} \mathrm{~F}$ NMR (376 MHz, $\left.\mathrm{CDCl}_{3}\right) \delta$-64.0 (s). FT-IR: $v\left(\mathrm{~cm}^{-1}\right)$ 2955, 2856, 2360, 2342 , $1679,1557,1437,1383,1346,1269,1250$. HRMS [ESI] $\mathrm{m} / \mathrm{z}:[\mathrm{M}+\mathrm{H}]^{+}$calcd for $\mathrm{C}_{21} \mathrm{H}_{17} \mathrm{~F}_{3} \mathrm{NOS}_{2}$ 420.0698; found 420.0700 .

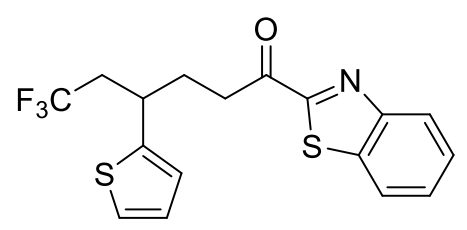

3ai-A: $11.3 \mathrm{mg}, 15 \%$, yellow solid, m.p. $99-100{ }^{\circ} \mathrm{C}$. Purification by flash column chromatography (eluent: EtOAc/Petroleum ether $=1 / 30) .{ }^{1} \mathrm{H}$ NMR $\left(400 \mathrm{MHz}, \mathrm{CDCl}_{3}\right) \delta 8.17-8.11(\mathrm{~m}, 1 \mathrm{H})$, 7.98-7.93 $(\mathrm{m}, 1 \mathrm{H}), 7.59-7.49(\mathrm{~m}, 2 \mathrm{H}), 7.23-7.17(\mathrm{~m}, 1 \mathrm{H})$, 6.96-6.85 (m, 2H), 3.55-3.43 (m, 1H), 3.31-3.12 (m, 2H), 2.60-2.49 (m, 2H), 2.41-2.31 (m, 1H), 2.17-2.07 (m, 1H); ${ }^{13} \mathrm{C}$ NMR (100 MHz, $\left.\mathrm{CDCl}_{3}\right) \delta$ 194.4, 166.0, 153.5, 145.6, 137.2, 127.7, 127.0, 126.8, $126.2\left(\mathrm{q}, J_{\mathrm{C}-\mathrm{F}}=275.9 \mathrm{~Hz}\right), 125.4,125.2,124.1$, $122.4,41.8\left(\mathrm{q}, J_{\mathrm{C}-\mathrm{F}}=27.3 \mathrm{~Hz}\right), 36.1,34.7\left(\mathrm{q}, J_{\mathrm{C}-\mathrm{F}}=3.1 \mathrm{~Hz}\right), 31.2 ;{ }^{19} \mathrm{~F} \mathrm{NMR}\left(376 \mathrm{MHz}, \mathrm{CDCl}_{3}\right) \delta$ -63.8 (s). FT-IR: $v\left(\mathrm{~cm}^{-1}\right)$ 2971, 2920, 1690, 1552, 1484, 1430, 1269, 1251. HRMS [ESI] m/z: $[\mathrm{M}+\mathrm{H}]^{+}$calcd for $\mathrm{C}_{17} \mathrm{H}_{15} \mathrm{~F}_{3} \mathrm{NO}_{2} \mathrm{~S} 370.0542$; found 370.0538 .<smiles>O=C(CCC(CC(F)(F)F)c1nc2ccccc2s1)c1cccs1</smiles>

3ai-B: $45.1 \mathrm{mg}, 61 \%$, yellow solid. m.p. $53-54{ }^{\circ} \mathrm{C}$. Purification by flash column chromatography (eluent: EtOAc/Petroleum ether = 1/30). ${ }^{1} \mathrm{H}$ NMR (400 MHz, $\left.\mathrm{CDCl}_{3}\right) \delta 7.99(\mathrm{~d}, J=8.0 \mathrm{~Hz}, 1 \mathrm{H}), 7.87$ (d, $J=8.0 \mathrm{~Hz}, 1 \mathrm{H}), 7.61-7.57(\mathrm{~m}, 2 \mathrm{H}), 7.49$ (ddd, $J=8.4,7.2,1.2 \mathrm{~Hz}$, $1 \mathrm{H}), 7.42-7.36(\mathrm{~m}, 1 \mathrm{H}), 7.06(\mathrm{dd}, J=4.8,3.6 \mathrm{~Hz}, 1 \mathrm{H}), 3.72-3.63(\mathrm{~m}$, $1 \mathrm{H}), 3.02-2.85(\mathrm{~m}, 3 \mathrm{H}), 2.70-2.55(\mathrm{~m}, 1 \mathrm{H}), 2.47-2.27(\mathrm{~m}, 2 \mathrm{H}) ;{ }^{13} \mathrm{C}$ NMR $\left(100 \mathrm{MHz}, \mathrm{CDCl}_{3}\right) \delta 191.5,171.9,153.0,143.8,134.6,133.8$, $131.9,128.1,126.3,126.1\left(\mathrm{q}, J_{\mathrm{C}-\mathrm{F}}=275.7 \mathrm{~Hz}\right), 125.3,123.0,121.7,39.3\left(\mathrm{q}, J_{\mathrm{C}-\mathrm{F}}=28.3 \mathrm{~Hz}\right), 37.9$ $\left(\mathrm{q}, J_{\mathrm{C}-\mathrm{F}}=2.6 \mathrm{~Hz}\right), 35.9,30.1 ;{ }^{19} \mathrm{~F} \mathrm{NMR}\left(376 \mathrm{MHz}, \mathrm{CDCl}_{3}\right) \delta-64.1(\mathrm{~s})$. FT-IR: $v\left(\mathrm{~cm}^{-1}\right) 3068,2922$, 2852, 1662, 1517, 1437, 1415, 1355, 1251. HRMS [ESI] m/z: $[\mathrm{M}+\mathrm{H}]^{+}$calcd for $\mathrm{C}_{17} \mathrm{H}_{15} \mathrm{~F}_{3} \mathrm{NOS}_{2}$ 370.0542 ; found 370.0540 .<smiles>O=C(CCC(CC(F)(F)F)c1nccs1)c1ccco1</smiles>

3aj-A: $9.9 \mathrm{mg}$, 17\%, red oil. Purification by flash column chromatography (eluent: EtOAc/Petroleum ether $=1 / 30$ ). ${ }^{1} \mathrm{H}$ NMR $\left(400 \mathrm{MHz}, \mathrm{CDCl}_{3}\right) \delta 7.74(\mathrm{~d}, J=3.2 \mathrm{~Hz}, 1 \mathrm{H}), 7.56-7.51(\mathrm{~m}, 1 \mathrm{H})$, $7.25(\mathrm{~d}, J=3.2 \mathrm{~Hz}, 1 \mathrm{H}), 7.11(\mathrm{~d}, J=3.2 \mathrm{~Hz}, 1 \mathrm{H}), 6.50(\mathrm{dd}, J=3.2$, $1.6 \mathrm{~Hz}, 1 \mathrm{H}), 3.63-3.55(\mathrm{~m}, 1 \mathrm{H}), 2.90-2.74(\mathrm{~m}, 3 \mathrm{H}), 2.61-2.49(\mathrm{~m}$, 1H), 2.34-2.17 (m, 2H); ${ }^{13} \mathrm{C}$ NMR (100 MHz, $\left.\mathrm{CDCl}_{3}\right) \delta 187.9,171.0,152.4,146.4,142.7,126.1$ $\left(\mathrm{q}, J_{\mathrm{C}-\mathrm{F}}=275.7 \mathrm{~Hz}\right), 118.6,117.1,112.2,39.5\left(\mathrm{q}, J_{\mathrm{C}-\mathrm{F}}=28.1 \mathrm{~Hz}\right), 36.9\left(\mathrm{q}, J_{\mathrm{C}-\mathrm{F}}=2.5 \mathrm{~Hz}\right), 35.1$, 30.0; ${ }^{19} \mathrm{~F}$ NMR (376 MHz, $\left.\mathrm{CDCl}_{3}\right) \delta-64.1$ (s). FT-IR: $v\left(\mathrm{~cm}^{-1}\right) 2932,2858,1794,1673,1569$, 1469, 1383, 1253. HRMS [ESI] $\mathrm{m} / \mathrm{z}:[\mathrm{M}+\mathrm{Na}]^{+}$calcd for $\mathrm{C}_{13} \mathrm{H}_{12} \mathrm{~F}_{3} \mathrm{NNaO}_{2} \mathrm{~S} 326.0433$; found 326.0440 .<smiles>O=C(CCC(CC(F)(F)F)c1ccco1)c1nccs1</smiles>

3aj-B: $10 \mathrm{mg}$, 17\%, red oil. Purification by flash column chromatography (eluent: EtOAc/Petroleum ether $=1 / 30$ ). ${ }^{1} \mathrm{H}$ NMR $\left(400 \mathrm{MHz}, \mathrm{CDCl}_{3}\right) \delta 7.97(\mathrm{~d}, J=3.2 \mathrm{~Hz}, 1 \mathrm{H}), 7.65(\mathrm{~d}, J=3.2 \mathrm{~Hz}$, 
$1 \mathrm{H}), 7.32(\mathrm{~d}, J=1.2 \mathrm{~Hz}, 1 \mathrm{H}), 6.25(\mathrm{dd}, J=3.2,2.0 \mathrm{~Hz}, 1 \mathrm{H}), 6.09(\mathrm{~d}, J=3.2 \mathrm{~Hz}, 1 \mathrm{H}), 3.27-3.18$ (m, 1H), 3.14-3.02 (m, 2H), 2.67-2.52 (m, 1H), 2.50-2.35 (m, 1H), 2.25-2.10 (m, 2H); ${ }^{13} \mathrm{C}$ NMR $\left(100 \mathrm{MHz}, \mathrm{CDCl}_{3}\right) \delta 192.8,166.8,154.1,144.7,141.8,126.3\left(\mathrm{q}, J_{\mathrm{C}-\mathrm{F}}=275.8 \mathrm{~Hz}\right), 126.2,110.1$, $106.8,38.1\left(\mathrm{q}, J_{\mathrm{C}-\mathrm{F}}=27.8 \mathrm{~Hz}\right), 35.7,32.7\left(\mathrm{q}, J_{\mathrm{C}-\mathrm{F}}=2.7 \mathrm{~Hz}\right), 27.9 ;{ }^{19} \mathrm{~F} \mathrm{NMR}\left(376 \mathrm{MHz}, \mathrm{CDCl}_{3}\right) \delta$ -64.4 (s). FT-IR: $v\left(\mathrm{~cm}^{-1}\right) 3084,2926,2857,1684,1521,1436,1399,1222$. HRMS [ESI] m/z: $[\mathrm{M}+\mathrm{Na}]^{+}$calcd for $\mathrm{C}_{13} \mathrm{H}_{12} \mathrm{~F}_{3} \mathrm{NNaO}_{2} \mathrm{~S} 326.0433$; found 326.0428 .

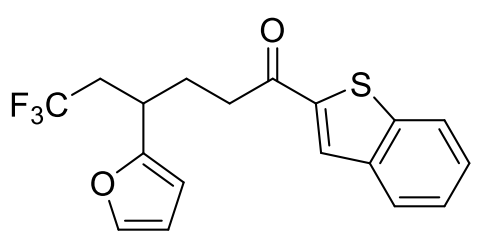

3ak-B: 29 mg, 41\% (ratio 10:1), yellow solid, m.p. 83-84 ${ }^{\circ} \mathrm{C}$. Purification by flash column chromatography (eluent: EtOAc/Petroleum ether $=1 / 30)$. Major isomer: ${ }^{1} \mathrm{H}$ NMR $(400$ $\left.\mathrm{MHz}, \mathrm{CDCl}_{3}\right) \delta$ 7.89-7.83 (m, 2H), $7.81(\mathrm{~s}, 1 \mathrm{H}), 7.50-7.35(\mathrm{~m}$, $3 \mathrm{H}), 6.30(\mathrm{dd}, J=3.2,2.0 \mathrm{~Hz}, 1 \mathrm{H}), 6.12(\mathrm{~d}, J=3.2 \mathrm{~Hz}, 1 \mathrm{H})$, 3.29-3.19 (m, 1H), $2.90(\mathrm{t}, J=7.4 \mathrm{~Hz}, 2 \mathrm{H}), 2.66-2.54(\mathrm{~m}, 1 \mathrm{H}), 2.49-2.38(\mathrm{~m}, 1 \mathrm{H}), 2.29-2.20(\mathrm{~m}$, 1H), 2.16-2.07 (m, 1H) ; $\left.{ }^{13} \mathrm{C} \mathrm{NMR} \mathrm{(100} \mathrm{MHz,} \mathrm{CDCl}_{3}\right) \delta$ 193.6, 154.2, 143.4, 142.5, 141.9, 139.1, $128.9,127.5,126.2\left(\mathrm{q}, J_{\mathrm{C}-\mathrm{F}}=275.7 \mathrm{~Hz}\right), 125.9,125.0,123.0,110.2,106.9,38.3\left(\mathrm{q}, J_{\mathrm{C}-\mathrm{F}}=27.7 \mathrm{~Hz}\right)$, $36.3,32.7\left(\mathrm{q}, J_{\mathrm{C}-\mathrm{F}}=2.5 \mathrm{~Hz}\right), 28.5 ;{ }^{19} \mathrm{~F}$ NMR $\left(376 \mathrm{MHz}, \mathrm{CDCl}_{3}\right) \delta-64.4(\mathrm{~s})$. FT-IR: $v\left(\mathrm{~cm}^{-1}\right) 3058$, 2954, 2856, 1793, 1518, 1457, 1376, 1272, 1251. HRMS [ESI] m/z: $[\mathrm{M}+\mathrm{Na}]^{+}$calcd for $\mathrm{C}_{18} \mathrm{H}_{15} \mathrm{~F}_{3} \mathrm{NaO}_{2} \mathrm{~S}$ 375.0637; found 375.0641.<smiles>O=C(CCC(CC(F)(F)F)c1cccs1)c1nccs1</smiles>

3al-A: $11.2 \mathrm{mg}, 18 \%$, yellow oil. Purification by flash column chromatography (eluent: EtOAc/Petroleum ether $=1 / 30$ ). ${ }^{1} \mathrm{H}$ NMR $\left(400 \mathrm{MHz}, \mathrm{CDCl}_{3}\right) \delta 7.96(\mathrm{~d}, J=2.8 \mathrm{~Hz}, 1 \mathrm{H}), 7.65(\mathrm{~d}, J=2.8 \mathrm{~Hz}$, $1 \mathrm{H}), 7.19(\mathrm{~d}, J=5.2 \mathrm{~Hz}, 1 \mathrm{H}), 6.93-6.89(\mathrm{~m}, 1 \mathrm{H}), 6.89-6.84(\mathrm{~m}, 1 \mathrm{H})$, $6.09(\mathrm{~d}, J=3.2 \mathrm{~Hz}, 1 \mathrm{H}), 3.50-3.39(\mathrm{~m}, 1 \mathrm{H}), 3.19-3.02(\mathrm{~m}, 2 \mathrm{H})$, 2.58-2.46 (m, 2H), 2.36-2.27 (m, 1H), 2.12-2.02 (m, 1H); ${ }^{13} \mathrm{C}$ NMR (100 MHz, $\left.\mathrm{CDCl}_{3}\right) \delta 192.9$, $166.8,145.6,144.7,126.8,126.2,126.1\left(\mathrm{q}, J_{\mathrm{C}-\mathrm{F}}=275.9 \mathrm{~Hz}\right), 125.1,124.1,41.7\left(\mathrm{q}, J_{\mathrm{C}-\mathrm{F}}=27.2 \mathrm{~Hz}\right)$, 35.9, $34.7\left(\mathrm{q}, J_{\mathrm{C}-\mathrm{F}}=2.8 \mathrm{~Hz}\right), 31.2 ;{ }^{19} \mathrm{~F}$ NMR $\left(376 \mathrm{MHz}, \mathrm{CDCl}_{3}\right) \delta-63.9$ (s). FT-IR: $v\left(\mathrm{~cm}^{-1}\right) 3112$, 2930, 2872, 2359, 1718, 1686, 1482, 1392, 1327, 1279, 1253. HRMS [ESI] m/z: [M+Na] ${ }^{+}$calcd for $\mathrm{C}_{13} \mathrm{H}_{12} \mathrm{~F}_{3} \mathrm{NNaOS}_{2} 342.0205$; found 342.0198 .<smiles>O=C(CCC(CC(F)(F)F)c1nccs1)c1cccs1</smiles>

3al-B: $46.6 \mathrm{mg}, 73 \%$, red solid, m.p. $83-84{ }^{\circ} \mathrm{C}$. Purification by flash column chromatography (eluent: EtOAc/Petroleum ether $=1 / 30) .{ }^{1} \mathrm{H}$ NMR (400 MHz, $\left.\mathrm{CDCl}_{3}\right) \delta 7.75(\mathrm{~d}, J=3.2 \mathrm{~Hz}, 1 \mathrm{H}), 7.63-7.56(\mathrm{~m}$, $2 \mathrm{H}), 7.25(\mathrm{~d}, J=3.2 \mathrm{~Hz}, 1 \mathrm{H}), 7.08(\mathrm{dd}, J=4.8,3.6 \mathrm{~Hz}, 1 \mathrm{H})$, 3.67-3.58 (m, 1H), 3.90-2.76 (m, 3H), 2.63-2.52 (m, 1H), 2.38-2.21 $(\mathrm{m}, 2 \mathrm{H}) ;{ }^{13} \mathrm{C}$ NMR $\left(100 \mathrm{MHz}, \mathrm{CDCl}_{3}\right) \delta 191.7,171.0,143.8,142.7,133.7,131.9,128.1,126.1(\mathrm{q}$, $\left.J_{\mathrm{C}-\mathrm{F}}=275.7 \mathrm{~Hz}\right), 118.7,39.6\left(\mathrm{q}, J_{\mathrm{C}-\mathrm{F}}=28.1 \mathrm{~Hz}\right), 36.9\left(\mathrm{q}, J_{\mathrm{C}-\mathrm{F}}=2.6 \mathrm{~Hz}\right), 35.9,30.5 ;{ }^{19} \mathrm{~F}$ NMR $(376$ MHz, $\left.\mathrm{CDCl}_{3}\right) \delta$-64.1 (s). FT-IR: $v\left(\mathrm{~cm}^{-1}\right)$ 3069, 2919, 1688, 1590, 1428, 1364, 1304, 1257, 1212. HRMS [ESI] m/z: [M+Na] $]^{+}$calcd for $\mathrm{C}_{13} \mathrm{H}_{12} \mathrm{~F}_{3} \mathrm{NNaOS}_{2} 342.0205$; found 342.0206.

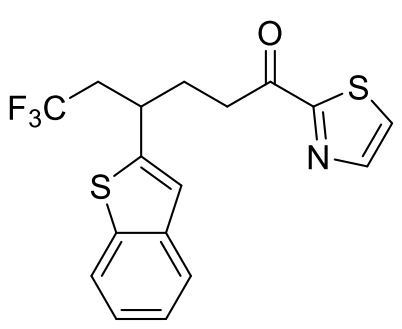

3am-B: $31.1 \mathrm{mg}, 42 \%$, white solid, m.p. $77-78^{\circ} \mathrm{C}$. Purification by flash column chromatography (eluent: EtOAc/Petroleum ether = 1/30). ${ }^{1} \mathrm{H} \mathrm{NMR}\left(400 \mathrm{MHz}, \mathrm{CDCl}_{3}\right) \delta 7.92(\mathrm{~d}, J=2.8 \mathrm{~Hz}, 1 \mathrm{H})$, 7.79-7.75 (m, 1H), 7.69-7.66 (m, 1H), $7.63(\mathrm{~d}, J=2.8 \mathrm{~Hz}, 1 \mathrm{H})$, 
7.35-7.26 (m, 2H), $7.12(\mathrm{~s}, 1 \mathrm{H}), 3.56-3.47(\mathrm{~m}, 1 \mathrm{H}), 3.24-3.10(\mathrm{~m}, 2 \mathrm{H}), 2.66-2.52(\mathrm{~m}, 2 \mathrm{H})$, 2.41-2.32 (m, 1H), 2.20-2.10 (m, 1H); ${ }^{13} \mathrm{C}$ NMR (100 MHz, $\left.\mathrm{CDCl}_{3}\right) \delta 192.8,166.6,146.4,144.7$, $139.5,139.1,126.3,126.1\left(\mathrm{q}, J_{\mathrm{C}-\mathrm{F}}=275.9 \mathrm{~Hz}\right), 124.4,124.1,123.3,122.4,122.1,41.3\left(\mathrm{q}, J_{\mathrm{C}-\mathrm{F}}=\right.$ $27.5 \mathrm{~Hz}), 35.9,35.6\left(\mathrm{q}, J_{\mathrm{C}-\mathrm{F}}=2.7 \mathrm{~Hz}\right), 30.7 ;{ }^{19} \mathrm{~F}$ NMR $\left(376 \mathrm{MHz}, \mathrm{CDCl}_{3}\right) \delta-63.9$ (s). FT-IR: $v$ $\left(\mathrm{cm}^{-1}\right)$ 2988, 2901, 1683, 1541, 1404, 1394, 1294, 1273, 1244. HRMS [ESI] m/z: [M+Na] $]^{+}$calcd for $\mathrm{C}_{17} \mathrm{H}_{14} \mathrm{~F}_{3} \mathrm{NNaOS}_{2} 392.0361$; found 392.0352 .

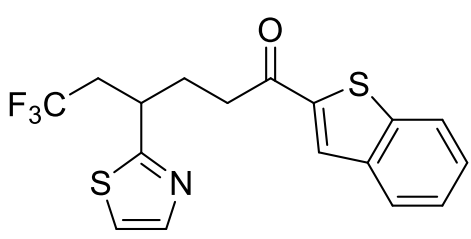

3am-A: $22.7 \mathrm{mg}, 31 \%$, white solid, m.p. 115-116 ${ }^{\circ} \mathrm{C}$. Purification by flash column chromatography (eluent: EtOAc/Petroleum ether $=1 / 30) .{ }^{1} \mathrm{H}$ NMR $\left(400 \mathrm{MHz}, \mathrm{CDCl}_{3}\right) \delta$ 791-7.81 (m, 3H), $7.78(\mathrm{~d}, J=3.2 \mathrm{~Hz}, 1 \mathrm{H}), 7.49-7.43(\mathrm{~m}, 1 \mathrm{H})$, 7.42-7.37 (m, 1H), $7.29(\mathrm{~d}, J=3.6 \mathrm{~Hz}, 1 \mathrm{H}), 3.73-3.64(\mathrm{~m}, 1 \mathrm{H})$, $2.97(\mathrm{t}, J=7.2 \mathrm{~Hz}, 2 \mathrm{H}), 2.92-2.81(\mathrm{~m}, 1 \mathrm{H}), 2.67-2.54(\mathrm{~m}, 1 \mathrm{H}), 2.44-2.27(\mathrm{~m}, 2 \mathrm{H}) ;{ }^{13} \mathrm{C}$ NMR $(100$ $\left.\mathrm{MHz}, \mathrm{CDCl}_{3}\right) \delta 193.2,171.1,143.2,142.6,142.5,139.0,129.1,127.5,126.1\left(\mathrm{q}, J_{\mathrm{C}-\mathrm{F}}=275.7 \mathrm{~Hz}\right)$, 126.0, 125.1, 124.7, 123.0, 118.8, $39.6\left(\mathrm{q}, J_{\mathrm{C}-\mathrm{F}}=28.1 \mathrm{~Hz}\right), 36.8\left(\mathrm{q}, J_{\mathrm{C}-\mathrm{F}}=2.6 \mathrm{~Hz}\right), 35.8,30.4 ;{ }^{19} \mathrm{~F}$ NMR $\left(376 \mathrm{MHz}, \mathrm{CDCl}_{3}\right) \delta-64.1$ (s). FT-IR: $v\left(\mathrm{~cm}^{-1}\right) 2988,2901,1662,1558,1435,1385,1270$, 1252. HRMS [ESI] m/z: [M+Na] ${ }^{+}$calcd for $\mathrm{C}_{17} \mathrm{H}_{14} \mathrm{~F}_{3} \mathrm{NNaOS}_{2}$ 392.0361; found 392.0360 .<smiles>O=C(CCC(CC(F)(F)F)c1cccs1)c1cc2ccccc2s1</smiles>

3an-A: $14.4 \mathrm{mg}, 20 \%$, white solid, m.p. $69-70{ }^{\circ} \mathrm{C}$. Purification by flash column chromatography (eluent: EtOAc/Petroleum ether $=1 / 30) .{ }^{1} \mathrm{H}$ NMR $\left(400 \mathrm{MHz}, \mathrm{CDCl}_{3}\right) \delta$ 7.89-7.82 (m, 2H), $7.89(\mathrm{~s}, 1 \mathrm{H}), 7.49-7.42(\mathrm{~m}, 1 \mathrm{H}), 7.42-7.36(\mathrm{~m}, 1 \mathrm{H}), 7.23(\mathrm{dd}, J$ $=5.2,0.8 \mathrm{~Hz}, 1 \mathrm{H}), 6.95(\mathrm{dd}, J=5.2,3.6 \mathrm{~Hz}, 1 \mathrm{H}), 6.88(\mathrm{dd}, J=$ 3.2, $0.4 \mathrm{~Hz}, 1 \mathrm{H}), 3.50-3.41(\mathrm{~m}, 1 \mathrm{H}), 2.96-2.88(\mathrm{~m}, 2 \mathrm{H}), 2.60-2.45(\mathrm{~m}, 2 \mathrm{H}), 2.43-2.34(\mathrm{~m}, 1 \mathrm{H})$, 2.08-1.99 (m, 1H); ${ }^{13} \mathrm{C}$ NMR (100 MHz, CDCl3) $\delta 193.6,145.7,143.4,142.5,139.0,129.0,127.5$, $126.9,126.1\left(\mathrm{q}, J_{\mathrm{C}-\mathrm{F}}=275.9 \mathrm{~Hz}\right), 125.9,125.2,125.0,124.1,123.0,41.9\left(\mathrm{q}, J_{\mathrm{C}-\mathrm{F}}=27.1 \mathrm{~Hz}\right), 36.4$, $34.7\left(\mathrm{q}, J_{\mathrm{C}-\mathrm{F}}=2.7 \mathrm{~Hz}\right), 31.7 ;{ }^{19} \mathrm{~F} \mathrm{NMR}(376 \mathrm{MHz}, \mathrm{CDCl} 3) \delta-63.9$ (s). FT-IR: $v\left(\mathrm{~cm}^{-1}\right) 3069,3050$, 1688, 1602, 1590, 1428, 1257, 1212. HRMS [ESI] $\mathrm{m} / \mathrm{z}:[\mathrm{M}+\mathrm{Na}]^{+}$calcd for $\mathrm{C}_{18} \mathrm{H}_{15} \mathrm{~F}_{3} \mathrm{NaOS}_{2}$ 391.0409; found 391.0414 .

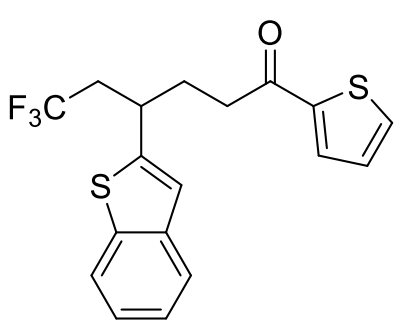

3an-B: $47.8 \mathrm{mg}, 65 \%$, yellow oil. Purification by flash column chromatography (eluent: EtOAc/Petroleum ether $=1 / 30) .{ }^{1} \mathrm{H}$ NMR $\left(400 \mathrm{MHz}, \mathrm{CDCl}_{3}\right) \delta$ 7.83-7.77 (m, 1H), 7.72-7.66 (m, 1H), 7.59 (dd, $J=4.8,0.8 \mathrm{~Hz}, 1 \mathrm{H}), 7.54(\mathrm{dd}, J=3.6,0.8 \mathrm{~Hz}, 1 \mathrm{H}), 7.37-7.28(\mathrm{~m}$, $2 \mathrm{H}), 7.11(\mathrm{~s}, 1 \mathrm{H}), 7.05(\mathrm{dd}, J=4.8,3.6 \mathrm{~Hz}, 1 \mathrm{H}), 3.56-3.46(\mathrm{~m}, 1 \mathrm{H})$, $2.87(\mathrm{t}, J=7.2 \mathrm{~Hz}, 2 \mathrm{H}), 2.65-2.50(\mathrm{~m}, 2 \mathrm{H}), 2.44-2.35(\mathrm{~m}, 1 \mathrm{H})$, 2.12-2.01 (m, 1H) ; ${ }^{13} \mathrm{C}$ NMR $\left(100 \mathrm{MHz}, \mathrm{CDCl}_{3}\right) \delta 192.0,146.6$, $143.9,139.5,139.1,133.7,131.9,128.1,126.1\left(\mathrm{q}, J_{\mathrm{C}-\mathrm{F}}=275.9 \mathrm{~Hz}\right), 124.5,124.2,123.3,122.5$, $122.1,41.5\left(\mathrm{q}, J_{\mathrm{C}-\mathrm{F}}=27.5 \mathrm{~Hz}\right), 36.4,35.6\left(\mathrm{q}, J_{\mathrm{C}-\mathrm{F}}=2.6 \mathrm{~Hz}\right), 31.3 ;{ }^{19} \mathrm{~F} \mathrm{NMR}\left(376 \mathrm{MHz}, \mathrm{CDCl}_{3}\right) \delta$ -63.8 (s). FT-IR: $v\left(\mathrm{~cm}^{-1}\right)$ 3064, 2942, 1772, 1541, 1457, 1380, 1356, 1270, 1214. HRMS [ESI] $\mathrm{m} / \mathrm{z}:[\mathrm{M}+\mathrm{Na}]^{+}$calcd for $\mathrm{C}_{18} \mathrm{H}_{15} \mathrm{~F}_{3} \mathrm{NaOS}_{2}$ 391.0409; found 391.0416 . 


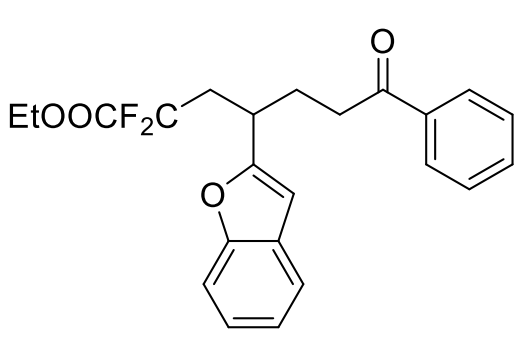

4a: $47.0 \mathrm{mg}, 59 \%$, white solid, m.p. $73-74{ }^{\circ} \mathrm{C}$. Purification by flash column chromatography (eluent: EtOAc/Petroleum ether $=1 / 30) .{ }^{1} \mathrm{H}$ NMR $\left(400 \mathrm{MHz}, \mathrm{CDCl}_{3}\right) \delta$ 7.89-7.78 (m, $2 \mathrm{H})$, 7.56-7.45 (m, 2H), 7.44-7.37 (m, 3H), 7.26-7.17 (m, $2 \mathrm{H}), 6.47(\mathrm{~d}, J=0.4 \mathrm{~Hz}, 1 \mathrm{H}), 4.06-3.96(\mathrm{~m}, 1 \mathrm{H}), 3.96-3.87$ $(\mathrm{m}, 1 \mathrm{H}), 3.37-3.27(\mathrm{~m}, 1 \mathrm{H}), 2.97-2.85(\mathrm{~m}, 2 \mathrm{H}), 2.82-2.72(\mathrm{~m}$, $1 \mathrm{H}), 2.54-2.41(\mathrm{~m}, 1 \mathrm{H}), 2.30-2.19(\mathrm{~m}, 2 \mathrm{H}), 1.15(\mathrm{t}, J=7.0$ $\mathrm{Hz}, 3 \mathrm{H}) ;{ }^{13} \mathrm{C}$ NMR $\left(100 \mathrm{MHz}, \mathrm{CDCl}_{3}\right) \delta 199.0,163.7\left(\mathrm{t}, J_{\mathrm{C}-\mathrm{F}}=31.9 \mathrm{~Hz}\right), 157.6,154.8,136.7$, $133.1,128.5,128.2,127.9,123.9,122.8,120.7,115.4\left(\mathrm{dd}, J_{\mathrm{C}-\mathrm{F}}=250.6,248.0 \mathrm{~Hz}\right), 111.0,104.3$, $62.9,38.7\left(\mathrm{t}, J_{\mathrm{C}-\mathrm{F}}=23.0 \mathrm{~Hz}\right), 35.7,32.9\left(\mathrm{dd}, J_{\mathrm{C}-\mathrm{F}}=5.3,3.2 \mathrm{~Hz}\right), 28.5,13.7 ;{ }^{19} \mathrm{~F} \mathrm{NMR}(376 \mathrm{MHz}$, $\left.\mathrm{CDCl}_{3}\right) \delta-101.8(\mathrm{~d}, J=263.2 \mathrm{~Hz}),-106.4(\mathrm{~d}, J=262.8 \mathrm{~Hz})$. FT-IR: $v\left(\mathrm{~cm}^{-1}\right) 2988,2901,1705$, 1519, 1457, 1395, 1252, 1230. HRMS [ESI] m/z: $[\mathrm{M}+\mathrm{Na}]^{+}$calcd for $\mathrm{C}_{23} \mathrm{H}_{22} \mathrm{~F}_{2} \mathrm{NaO}_{4} 423.1378$; found 423.1386

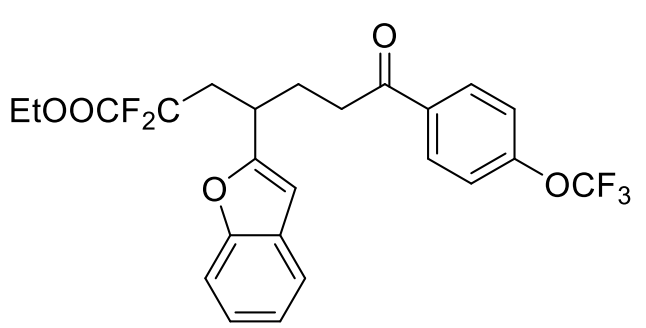

4b: $92.1 \mathrm{mg}, 95 \%$, yellow solid, m.p. Purification by flash column chromatography (eluent: EtOAc/Petroleum ether $=1 / 30) .41-42{ }^{\circ} \mathrm{C} .{ }^{1} \mathrm{H}$ NMR $\left(400 \mathrm{MHz}, \mathrm{CDCl}_{3}\right) \delta 7.78(\mathrm{~d}, J=8.4 \mathrm{~Hz}, 2 \mathrm{H}), 7.38$ $(\mathrm{d}, J=7.2 \mathrm{~Hz}, 1 \mathrm{H}), 7.32(\mathrm{~d}, J=8.0 \mathrm{~Hz}, 1 \mathrm{H})$, 7.17-7.08 (m, 4H), $6.37(\mathrm{~s}, 1 \mathrm{H}), 3.97-3.79(\mathrm{~m}, 2 \mathrm{H})$, 3.28-3.16 (m, 1H), $2.79(\mathrm{t}, J=7.0 \mathrm{~Hz}, 2 \mathrm{H})$, 2.75-2.61 (m, 1H), 2.43-2.32 (m, 1H), 2.21-2.08 (m, 2H), $1.06(\mathrm{t}, J=7.2 \mathrm{~Hz}, 3 \mathrm{H}) ;{ }^{13} \mathrm{C}$ NMR $(100$ $\left.\mathrm{MHz}, \mathrm{CDCl}_{3}\right) \delta 197.4,163.7\left(\mathrm{t}, J_{\mathrm{C}-\mathrm{F}}=32.4 \mathrm{~Hz}\right), 157.5,154.8,152.6,134.9,130.0,128.2,124.0$, $122.9,120.7,120.3\left(\mathrm{q}, J_{\mathrm{C}-\mathrm{F}}=256.8 \mathrm{~Hz}\right), 120.3,115.4\left(\mathrm{dd}, J_{\mathrm{C}-\mathrm{F}}=250.9,248.3 \mathrm{~Hz}\right), 111.0,104.3$, $62.9,38.7\left(\mathrm{t}, J_{\mathrm{C}-\mathrm{F}}=23.1 \mathrm{~Hz}\right), 35.8,32.9\left(\mathrm{dd}, J_{\mathrm{C}-\mathrm{F}}=4.9,3.2 \mathrm{~Hz}\right), 28.4,13.6 ;{ }^{19} \mathrm{~F}$ NMR $(376 \mathrm{MHz}$, $\left.\mathrm{CDCl}_{3}\right) \delta-57.6(\mathrm{~s}),-101.8(\mathrm{~d}, J=262.8 \mathrm{~Hz}),-106.4(\mathrm{~d}, J=262.8 \mathrm{~Hz})$. FT-IR: $v\left(\mathrm{~cm}^{-1}\right) 2968,2922$, $2855,1692,1508,1423,1385,1314,1276,1206$. HRMS [ESI] $\mathrm{m} / \mathrm{z}:[\mathrm{M}+\mathrm{Na}]^{+}$calcd for $\mathrm{C}_{24} \mathrm{H}_{21} \mathrm{~F}_{5} \mathrm{NaO}_{5}$ 507.1201; found 507.1095.

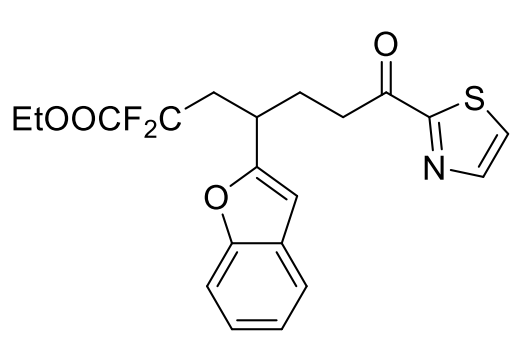

4c: $51.7 \mathrm{mg}, 64 \%$, white solid, m.p. $53-54{ }^{\circ} \mathrm{C}$. Purification by flash column chromatography (eluent: EtOAc/Petroleum ether $=1 / 30) .{ }^{1} \mathrm{H}$ NMR $\left(400 \mathrm{MHz}, \mathrm{CDCl}_{3}\right) \delta 7.92(\mathrm{~d}, J=3.2$ $\mathrm{Hz}, 1 \mathrm{H}), 7.61(\mathrm{~d}, J=3.2 \mathrm{~Hz}, 1 \mathrm{H}), 7.48-7.43(\mathrm{~m}, 1 \mathrm{H})$, 7.42-7.37 (m, 1H), 7.24-7.14 (m, 2H), $6.48(\mathrm{~d}, J=0.4 \mathrm{~Hz}$, $1 \mathrm{H}), 4.05-3.96(\mathrm{~m}, 1 \mathrm{H}), 3.96-3.87(\mathrm{~m}, 1 \mathrm{H}), 3.36-3.28(\mathrm{~m}$, $1 \mathrm{H}), 3.21-3.07(\mathrm{~m}, 2 \mathrm{H}), 2.85-2.70(\mathrm{~m}, 1 \mathrm{H}), 2.54-2.41(\mathrm{~m}$, 1H), 2.32-2.22 (m, 2H), $1.15(\mathrm{t}, J=7.2 \mathrm{~Hz}, 3 \mathrm{H}) ;{ }^{13} \mathrm{C} \mathrm{NMR}\left(100 \mathrm{MHz}, \mathrm{CDCl}_{3}\right) \delta$ 192.7, 166.7, $163.6\left(\mathrm{t}, J_{\mathrm{C}-\mathrm{F}}=32.5 \mathrm{~Hz}\right), 157.2,154.8,144.7,128.2,126.2,123.9,122.7,120.7,115.4\left(\mathrm{dd}, J_{\mathrm{C}-\mathrm{F}}=\right.$ $250.5,248.1 \mathrm{~Hz}), 111.0,104.4,62.9,38.5\left(\mathrm{t}, J_{\mathrm{C}-\mathrm{F}}=23.1 \mathrm{~Hz}\right), 35.9,32.9\left(\mathrm{dd}, J_{\mathrm{C}-\mathrm{F}}=5.3,3.1 \mathrm{~Hz}\right)$, 28.2, 13.7; ${ }^{19} \mathrm{~F}$ NMR $\left(376 \mathrm{MHz}, \mathrm{CDCl}_{3}\right) \delta-101.7(\mathrm{~d}, J=262.4 \mathrm{~Hz}),-106.4(\mathrm{~d}, J=262.4 \mathrm{~Hz})$. FT-IR: $v\left(\mathrm{~cm}^{-1}\right)$ 2988, 2901, 1765, 1653, 1522, 1437, 1394, 1319, 1252, 1221. HRMS [ESI] m/z: $[\mathrm{M}+\mathrm{Na}]^{+}$calcd for $\mathrm{C}_{20} \mathrm{H}_{19} \mathrm{~F}_{2} \mathrm{NNaO}_{4} \mathrm{~S} 430.0895$; found 430.0893 . 


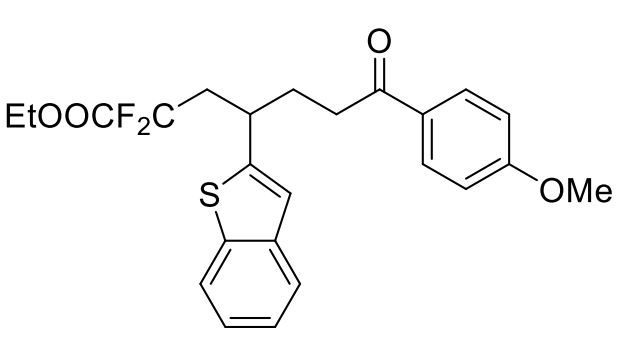

4d: $56.0 \mathrm{mg}, 63 \%$, white solid, m.p. $76-77{ }^{\circ} \mathrm{C}$. Purification by flash column chromatography (eluent: EtOAc/Petroleum ether $=1 / 30) .{ }^{1} \mathrm{H}$ NMR $(400 \mathrm{MHz}$, $\left.\mathrm{CDCl}_{3}\right) \delta 7.86-7.80(\mathrm{~m}, 2 \mathrm{H}), 7.78(\mathrm{~d}, J=7.6 \mathrm{~Hz}, 1 \mathrm{H})$, 7.70-7.64 (m, 1H), 7.36-7.25 (m, 2H), $7.06(\mathrm{~s}, 1 \mathrm{H})$, 6.89-6.83 (m, 2H), 4.05-3.90 (m, 2H), 3.52-3.40 (m, $1 \mathrm{H}), 3.83(\mathrm{~s}, 3 \mathrm{H}), 2.87(\mathrm{t}, J=7.4 \mathrm{~Hz}, 3 \mathrm{H}), 2.74-2.61$ (m, 1H), 2.59-2.48 (m, 1H), 2.38-2.28 (m, 1H), 2.12-2.02 (m, $1 \mathrm{H}), 1.16(\mathrm{t}, J=7.2 \mathrm{~Hz}, 3 \mathrm{H}) ;{ }^{13} \mathrm{C}$ NMR $\left(100 \mathrm{MHz}, \mathrm{CDCl}_{3}\right) \delta 197.7,163.8\left(\mathrm{t}, J_{\mathrm{C}-\mathrm{F}}=32.4 \mathrm{~Hz}\right), 163.5,147.2,139.5,139.2,130.3$, $129.8,124.4,124.1,123.2,122.4,122.3,115.4\left(\mathrm{dd}, J_{\mathrm{C}-\mathrm{F}}=251.0,248.1 \mathrm{~Hz}\right), 113.7,62.9,55.5$, $42.1\left(\mathrm{t}, J_{\mathrm{C}-\mathrm{F}}=22.9 \mathrm{~Hz}\right), 35.5,35.5\left(\mathrm{dd}, J_{\mathrm{C}-\mathrm{F}}=5.4,3.1 \mathrm{~Hz}\right), 32.0,13.7 ;{ }^{19} \mathrm{~F} \mathrm{NMR}\left(376 \mathrm{MHz}, \mathrm{CDCl}_{3}\right)$ $\delta-101.2(\mathrm{~d}, J=262.4 \mathrm{~Hz}),-106.4(\mathrm{~d}, J=262.1 \mathrm{~Hz})$. FT-IR: $v\left(\mathrm{~cm}^{-1}\right) 2987,2924,2360,2342,1774$, $1683,1603,1519,1439,1372,1308,1265,1219$. HRMS $[\mathrm{ESI}] \mathrm{m} / \mathrm{z}:[\mathrm{M}+\mathrm{Na}]^{+}$calcd for $\mathrm{C}_{24} \mathrm{H}_{24} \mathrm{~F}_{2} \mathrm{NaO}_{4} \mathrm{~S}$ 469.1256; found 469.1249 .

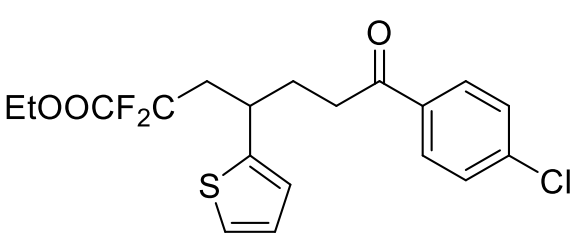

4e: $52.0 \mathrm{mg}, 65 \%$, white solid, m.p. $61-62{ }^{\circ} \mathrm{C}$. Purification by flash column chromatography (eluent: EtOAc/Petroleum ether $=1 / 30) .{ }^{1} \mathrm{H}$ NMR $(400 \mathrm{MHz}$, $\left.\mathrm{CDCl}_{3}\right) \delta$ 7.82-7.72 (m, 2H), 7.41-7.34 (m, 2H), 7.22-7.16 (m, 1H), $6.91(\mathrm{dd}, J=5.2,3.6 \mathrm{~Hz}, 1 \mathrm{H})$, 6.85-6.78 (m, 1H), 4.14-4.02 (m, 2H), 3.42-3.32 (m, 1H), 2.88-2.75 (m, 2H), 2.65-2.40 (m, 2H), 2.32-2.23 (m, 1H), 2.04-1.94 (m, 1H), $1.26(\mathrm{t}, J=7.0 \mathrm{~Hz}, 3 \mathrm{H}) ;{ }^{13} \mathrm{C} \mathrm{NMR}\left(100 \mathrm{MHz}, \mathrm{CDCl}_{3}\right) \delta$ $198.0,163.8\left(\mathrm{t}, J_{\mathrm{C}-\mathrm{F}}=32.3 \mathrm{~Hz}\right), 146.0,139.5,135.0,129.4,128.9,126.7,125.4,124.1,115.4(\mathrm{dd}$, $\left.J_{\mathrm{C}-\mathrm{F}}=250.8,248.0 \mathrm{~Hz}\right), 62.9,42.5\left(\mathrm{t}, J_{\mathrm{C}-\mathrm{F}}=22.8 \mathrm{~Hz}\right), 35.9,34.5\left(\mathrm{dd}, J_{\mathrm{C}-\mathrm{F}}=5.3,3.0 \mathrm{~Hz}\right), 32.3$, 13.8; ${ }^{19} \mathrm{~F}$ NMR $\left(376 \mathrm{MHz}, \mathrm{CDCl}_{3}\right) \delta-101.1(\mathrm{~d}, J=261.7 \mathrm{~Hz}),-106.6(\mathrm{~d}, J=261.7 \mathrm{~Hz})$. FT-IR: $v$ $\left(\mathrm{cm}^{-1}\right)$ 2982, 2926, 2853, 1767, 1685, 1589, 1225. HRMS [ESI] $\mathrm{m} / \mathrm{z}:[\mathrm{M}+\mathrm{Na}]^{+}$calcd for $\mathrm{C}_{19} \mathrm{H}_{19} \mathrm{ClF}_{2} \mathrm{NaO}_{3} \mathrm{~S}$ 423.0604; found 423.0604 .

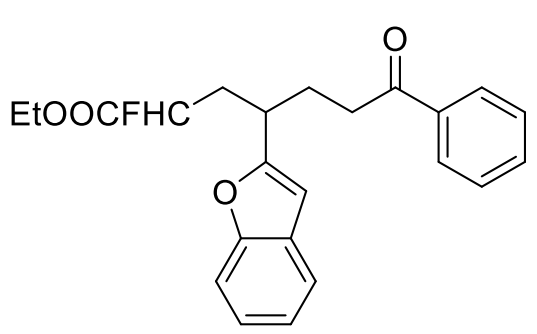

5a $($ d.r. $=1: 1): 70.9 \mathrm{mg}, 93 \%$, yellow oil. Purification by flash column chromatography (eluent: EtOAc/Petroleum ether $=1 / 30) .{ }^{1} \mathrm{H}$ NMR $\left(400 \mathrm{MHz}, \mathrm{CDCl}_{3}\right) \delta 7.91-7.80(\mathrm{~m}$, $4 \mathrm{H}$, two isomers), 7.55-7.47 ( $\mathrm{m}, 4 \mathrm{H}$, two isomers), 7.46-7.38 (m, 6H, two isomers), 7.26-7.17 (m, 4H, two isomers), 6.56 (s, $1 \mathrm{H}$, one isomer), $6.49(\mathrm{~s}, 1 \mathrm{H}$, one isomer), 5.09-4.93 $(\mathrm{m}, 1 \mathrm{H}$, one isomer $)$, 4.81-4.63 $(\mathrm{m}, 1 \mathrm{H}$, one isomer), 4.25-4.19 (m, $2 \mathrm{H}$, two isomers), 4.13-3.96 (m, 2H, two isomers), 3.33-3.22 (m, $2 \mathrm{H}$, two isomers), 3.01-2.88 ( $\mathrm{m}, 4 \mathrm{H}$, two isomers), 2.53-2.37 ( $\mathrm{m}, 2 \mathrm{H}$, two isomers), 2.37-2.18 (m, $6 \mathrm{H}$, two isomers), 1.28 (t, $J=7.2 \mathrm{~Hz}, 3 \mathrm{H}$, one isomer), 1.19 (t, $J=7.2 \mathrm{~Hz}, 3 \mathrm{H}$, one isomer); ${ }^{13} \mathrm{C}$ NMR (100 $\left.\mathrm{MHz} \mathrm{CDCl}_{3}\right) \delta 199.3 \& 199.3$ (two isomers), $169.7\left(\mathrm{~d}, J_{\mathrm{C}-\mathrm{F}}=23.1 \mathrm{~Hz}\right) \& 169.5\left(\mathrm{~d}, J_{\mathrm{C}-\mathrm{F}}=23.7 \mathrm{~Hz}\right)$ (two isomers), $158.7 \& 158.0$ (two isomers), $154.9 \& 154.8$ (two isomers), $136.8 \& 136.8$ (overlap, two isomers), $133.1 \& 133.1$ (overlap, two isomers), $128.6 \& 128.6$ (overlap, two isomers), 128.3 $\& 128.3$ (overlap, two isomers), $128.0 \& 128.0$ (overlap, two isomers), $123.8 \& 123.8$ (two isomers), $122.8 \& 122.7$ (two isomers), $120.7 \& 120.6$ (two isomers), $111.2 \& 111.0$ (two isomers), $104.7 \& 104.0$ (two isomers), $88.0\left(\mathrm{~d}, J_{\mathrm{C}-\mathrm{F}}=10.1 \mathrm{~Hz}\right) \& 86.1\left(\mathrm{~d}, J_{\mathrm{C}-\mathrm{F}}=11.7 \mathrm{~Hz}\right.$ ) (two isomers), 
$61.7 \& 61.6$ (two isomers), $37.0\left(\mathrm{~d}, J_{\mathrm{C}-\mathrm{F}}=20.9 \mathrm{~Hz}\right) \& 36.6\left(\mathrm{~d}, J_{\mathrm{C}-\mathrm{F}}=20.8 \mathrm{~Hz}\right)$ (two isomers), 36.1 \& 35.9 (two isomers), 35.1 (d, $\left.J_{\mathrm{C}-\mathrm{F}}=1.5 \mathrm{~Hz}\right) \& 34.5$ (d, $J_{\mathrm{C}-\mathrm{F}}=3.6 \mathrm{~Hz}$ ) (two isomers), $28.4 \& 27.4$ (two isomers), $14.1 \& 14.0$ (two isomers); $\left.{ }^{19} \mathrm{~F} \mathrm{NMR} \mathrm{(376} \mathrm{MHz,} \mathrm{CDCl}_{3}\right) \delta-191.4$ (s, one isomer), -193.0 (s, one isomer). FT-IR: $v\left(\mathrm{~cm}^{-1}\right)$ 2987, 2902, 1759, 1685, 1582, 1455, 1373, 1253, 1209. HRMS [ESI] m/z: [M+Na] ${ }^{+}$calcd for $\mathrm{C}_{23} \mathrm{H}_{23} \mathrm{FNaO}_{4} 405.1473$; found 405.1471 .

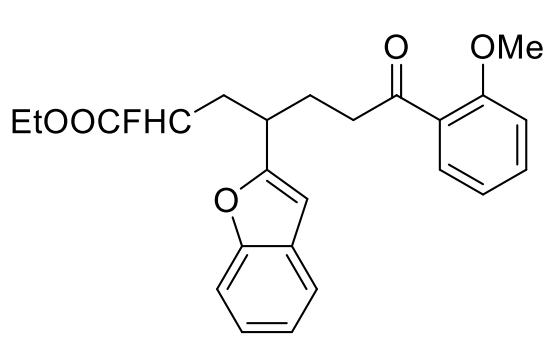

5b $(d . r .=1: 1): 67.9 \mathrm{mg}, 83 \%$, yellow oil. Purification by flash column chromatography (eluent: EtOAc/Petroleum ether $=1 / 30) .{ }^{1} \mathrm{H}$ NMR $\left(400 \mathrm{MHz}, \mathrm{CDCl}_{3}\right) \delta$ 7.65-7.55 (m, $2 \mathrm{H}$, two isomers), 7.53-7.46 ( $\mathrm{m}, 2 \mathrm{H}$, two isomers), 7.46-7.39 (m, $4 \mathrm{H}$, two isomers), 7.25-7.15 (m, 4H, two isomers), 6.96 (t, $J=7.4 \mathrm{~Hz}, 2 \mathrm{H}$, two isomers), 6.89 (d, $J$ $=8.4 \mathrm{~Hz}, 2 \mathrm{H}$, two isomers), $6.53(\mathrm{~s}, 1 \mathrm{H}$, one isomer), 6.46 (s, $1 \mathrm{H}$, one isomer), $5.08-4.90(\mathrm{~m}, 1 \mathrm{H}$, one isomer), 4.82-4.62 $(\mathrm{m}, 1 \mathrm{H}$, one isomer), 4.24-4.18 (m, $2 \mathrm{H}$, two isomers), 4.11-3.96 (m, $2 \mathrm{H}$, two isomers), 3.76-3.70 (m, $6 \mathrm{H}$, two isomers), 3.30-3.18 (m, $2 \mathrm{H}$, two isomers), 3.02-2.86 (m, $4 \mathrm{H}$, two isomers), 2.52-2.35 (m, $2 \mathrm{H}$, two isomers), 2.33-2.09 (m, $6 \mathrm{H}$, two isomers), 1.28 (t, $J=7.2 \mathrm{~Hz}, 3 \mathrm{H}$, one isomer), 1.19 (t, $J=7.0 \mathrm{~Hz}, 3 \mathrm{H}$, one isomer); ${ }^{13} \mathrm{C}$ NMR $\left(100 \mathrm{MHz}, \mathrm{CDCl}_{3}\right) \delta 201.9 \& 201.8$ (two isomers), 169.8 (d, $\left.J_{\mathrm{C}-\mathrm{F}}=23.2 \mathrm{~Hz}\right) \& 169.5$ (d, $J_{\mathrm{C}-\mathrm{F}}=23.7 \mathrm{~Hz}$ ) (two isomers), $159.1 \& 159.1$ (overlap, two isomers), $158.4 \& 158.4$ (two isomers), $154.9 \& 154.8$ (two isomers), $133.4 \& 133.4$ (two isomers), $130.2 \& 130.2$ (overlap, two isomers), $128.4 \& 128.4$ (two isomers), $128.3 \& 128.2$ (two isomers), $123.7 \& 123.6$ (two isomers), $122.7 \&$ 122.6 (two isomers), $120.6 \& 120.6$ (overlap, two isomers), $120.6 \& 120.5$ (two isomers), $110.5 \&$ 110.5 (overlap, two isomers), $111.1 \& 111.0$ (two isomers), $104.4 \& 103.7$ (two isomers), 88.0 (d, $\left.J_{\mathrm{C}-\mathrm{F}}=12.4 \mathrm{~Hz}\right) \& 86.2\left(\mathrm{~d}, J_{\mathrm{C}-\mathrm{F}}=13.0 \mathrm{~Hz}\right)$ (two isomers), $61.6 \& 61.6$ (two isomers), $55.3 \& 55.3$ (two isomers), $41.4 \& 41.2$ (two isomers), $36.9\left(\mathrm{~d}, J_{\mathrm{C}-\mathrm{F}}=20.9 \mathrm{~Hz}\right) \& 36.5\left(\mathrm{~d}, J_{\mathrm{C}-\mathrm{F}}=20.9 \mathrm{~Hz}\right.$ ) (two isomers), $35.2\left(\mathrm{~d}, J_{\mathrm{C}-\mathrm{F}}=1.7 \mathrm{~Hz}\right) \& 34.6\left(\mathrm{~d}, J_{\mathrm{C}-\mathrm{F}}=3.7 \mathrm{~Hz}\right)$ (two isomers), $28.8 \& 27.8$ (two isomers), $14.1 \& 14.0$ (two isomers); $\left.{ }^{19} \mathrm{~F} \mathrm{NMR} \mathrm{(376} \mathrm{MHz,} \mathrm{CDCl}_{3}\right) \delta-191.4$ (s, one isomer), -193.1 (s, one isomer). FT-IR: $v\left(\mathrm{~cm}^{-1}\right)$ 2970, 2924, 1759, 1598, 1437, 1374, 1285, 1250. HRMS [ESI] $\mathrm{m} / \mathrm{z}$ : $[\mathrm{M}+\mathrm{Na}]^{+}$calcd for $\mathrm{C}_{24} \mathrm{H}_{25} \mathrm{FNaO}_{5} 435.1578$; found 435.1579 .

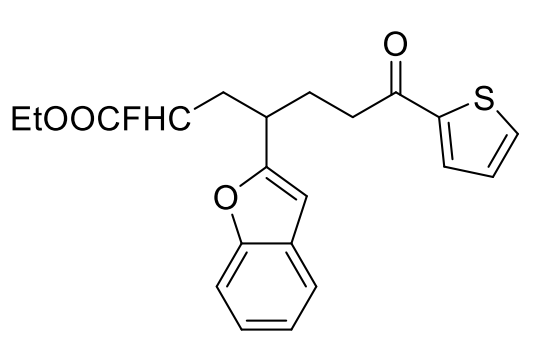

5c $($ d.r. $=1: 1): 51.7 \mathrm{mg}, 67 \%$, yellow oil. Purification by flash column chromatography (eluent: EtOAc/Petroleum ether $=1 / 30) .{ }^{1} \mathrm{H}$ NMR $\left(400 \mathrm{MHz}, \mathrm{CDCl}_{3}\right) \delta$ 7.63-7.54 (m, $4 \mathrm{H}$, two isomers), 7.54-7.40 (m, $4 \mathrm{H}$, two isomers), 7.26-7.17 (m, 4H, two isomers), 7.10-7.02 (m, 2H, two isomers), $6.56(\mathrm{~s}, 1 \mathrm{H}$, one isomer), $6.50(\mathrm{~s}, 1 \mathrm{H}$, one isomer), 5.08-4.91 (m, 1H, one isomer), 4.80-4.62 $(\mathrm{m}, 1 \mathrm{H}$, one isomer), 4.25-4.18 (m, $2 \mathrm{H}$, two isomers), 4.15-3.94 (m, $2 \mathrm{H}$, two isomers), 3.32-3.21 (m, $2 \mathrm{H}$, two isomers), 2.94-2.81 ( $\mathrm{m}, 4 \mathrm{H}$, two isomers), 2.52-2.36 ( $\mathrm{m}, 2 \mathrm{H}$, two isomers), 2.33-2.17 (m, 6H, two isomers), 1.28 (t, $J=7.2 \mathrm{~Hz}, 3 \mathrm{H}$, one isomer), 1.19 (t, $J=7.2 \mathrm{~Hz}, 3 \mathrm{H}$, one isomer); ${ }^{13} \mathrm{C}$ NMR (100 $\left.\mathrm{MHz}, \mathrm{CDCl}_{3}\right) \delta 192.3 \& 192.2$ (two isomers), $169.7\left(\mathrm{~d}, J_{\mathrm{C}-\mathrm{F}}=23.1 \mathrm{~Hz}\right) \& 169.4\left(\mathrm{~d}, J_{\mathrm{C}-\mathrm{F}}=23.4 \mathrm{~Hz}\right.$ ) (two isomers), $158.5 \& 157.8$ (two isomers), $154.9 \& 154.8$ (two isomers), $144.0 \& 144.0$ (overlap, two isomers), $133.6 \& 133.6$ (overlap, two isomers), $131.9 \& 131.8$ (two isomers), $128.3 \& 128.3$ (overlap, two isomers), $128.0 \& 128.0$ (overlap, two isomers), $123.9 \& 123.8$ (two isomers), 122.8 
\& 122.7 (two isomers), $120.7 \& 120.6$ (two isomers), $111.2 \& 111.0$ (two isomers), $104.8 \& 104.1$ (two isomers), $88.0\left(\mathrm{~d}, J_{\mathrm{C}-\mathrm{F}}=10.6 \mathrm{~Hz}\right) \& 86.2\left(\mathrm{~d}, J_{\mathrm{C}-\mathrm{F}}=11.3 \mathrm{~Hz}\right.$ ) (two isomers), $61.7 \& 61.6$ (two isomers), $37.0\left(\mathrm{~d}, J_{\mathrm{C}-\mathrm{F}}=20.9 \mathrm{~Hz}\right) \& 36.5\left(\mathrm{~d}, J_{\mathrm{C}-\mathrm{F}}=20.9 \mathrm{~Hz}\right)$ (two isomers), $36.8 \& 36.6$ (two isomers), $35.1\left(\mathrm{~d}, J_{\mathrm{C}-\mathrm{F}}=1.8 \mathrm{~Hz}\right) \& 34.5\left(\mathrm{~d}, J_{\mathrm{C}-\mathrm{F}}=3.5 \mathrm{~Hz}\right.$ ) (two isomers), $28.6 \& 27.7$ (two isomers), $14.1 \& 14.0$ (two isomers); $\left.{ }^{19} \mathrm{~F} \mathrm{NMR} \mathrm{(376} \mathrm{MHz,} \mathrm{CDCl}_{3}\right) \delta-191.3$ (s, one isomer), -193.0 (s, one isomer). FT-IR: $v\left(\mathrm{~cm}^{-1}\right)$ 2968, 2852, 1757, 1662, 1585, 1455, 1355, 1253, 1237. HRMS [ESI] m/z: [M+Na] $]^{+}$calcd for $\mathrm{C}_{21} \mathrm{H}_{21} \mathrm{FNaO}_{4} \mathrm{~S}$ 411.1037; found 411.1044 .

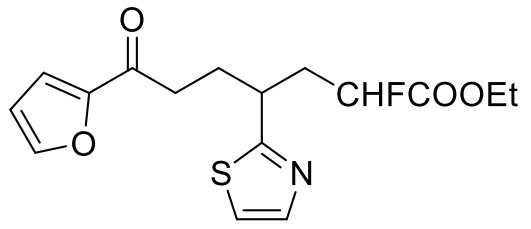

5d-A $($ d.r. $=1: 1): 17.6 \mathrm{mg}, 26 \%$, yellow oil. Purification by flash column chromatography (eluent: EtOAc/Petroleum ether $=1 / 30) .{ }^{1} \mathrm{H}$ NMR $\left(400 \mathrm{MHz}, \mathrm{CDCl}_{3}\right) \delta 7.76(\mathrm{~d}, J=3.2$ $\mathrm{Hz}, 1 \mathrm{H}$, one isomer), $7.72(\mathrm{~d}, J=3.2 \mathrm{~Hz}, 1 \mathrm{H}$, one isomer), 7.53 (s, $2 \mathrm{H}$, two isomers), 7.26-7.22 (m, $2 \mathrm{H}$, two isomers), 7.11 (d, $J=3.6 \mathrm{~Hz}, 2 \mathrm{H}$, two isomers), 6.55-6.44 (m, 2H, two isomers), 5.09-4.93 (m, 1H, one isomer), 4.80-4.63 (m, $1 \mathrm{H}$, one isomer), $4.25-4.15$ (m, $4 \mathrm{H}$, two isomers), 3.57-3.45 (m, $2 \mathrm{H}$, two isomers), 2.87-2.72 ( $\mathrm{m}, 4 \mathrm{H}$, two isomers), 2.52-2.19 (m, $8 \mathrm{H}$, two isomers), 1.29-1.25 (m, $6 \mathrm{H}$, two isomers); ${ }^{13} \mathrm{C}$ NMR $\left(100 \mathrm{MHz}, \mathrm{CDCl}_{3}\right) \delta 188.2 \& 188.2$ (two isomers), $172.4 \& 171.7$ (two isomers), $169.5\left(\mathrm{~d}, J_{\mathrm{C}-\mathrm{F}}=23.2 \mathrm{~Hz}\right) \& 169.4\left(\mathrm{~d}, J_{\mathrm{C}-\mathrm{F}}=23.4 \mathrm{~Hz}\right.$ ) (two isomers), $152.4 \& 152.4$ (overlap, two isomers), $146.3 \& 146.3$ (two isomers), $142.8 \& 142.5$ (two isomers), $118.6 \& 118.5$ (two isomers), $117.1 \& 117.0$ (two isomers), $112.2 \& 112.2$ (two isomers), 87.8 (d, $J_{\mathrm{C}-\mathrm{F}}=2.9 \mathrm{~Hz}$ ) $\& 85.9\left(\mathrm{~d}, J_{\mathrm{C}-\mathrm{F}}=2.2 \mathrm{~Hz}\right)$ (two isomers), 61.7 (overlap, two isomers), $38.8\left(\mathrm{~d}, J_{\mathrm{C}-\mathrm{F}}=1.9 \mathrm{~Hz}\right) \&$ $38.7\left(\mathrm{~d}, J_{\mathrm{C}-\mathrm{F}}=3.2 \mathrm{~Hz}\right)\left(\right.$ two isomers), $38.5\left(\mathrm{~d}, J_{\mathrm{C}-\mathrm{F}}=20.8 \mathrm{~Hz}\right) \& 38.2\left(\mathrm{~d}, J_{\mathrm{C}-\mathrm{F}}=20.7 \mathrm{~Hz}\right)(\mathrm{two}$ isomers), $35.6 \& 35.5$ (two isomers), $30.5 \& 29.2$ (two isomers), $14.1 \& 14.1$ (two isomers); ${ }^{19} \mathrm{~F}$ NMR $\left(376 \mathrm{MHz}, \mathrm{CDCl}_{3}\right) \delta$-190.6--190.8 (m, one isomer), -192.9--193.1 (m, one isomer). FT-IR: $v\left(\mathrm{~cm}^{-1}\right) 2981,2929,2855,1793,1754,1568,1468,1373,1265$. HRMS [ESI] m/z: [M+Na] ${ }^{+}$calcd for $\mathrm{C}_{16} \mathrm{H}_{18} \mathrm{FNNaO}_{4} \mathrm{~S} 362.0833$; found 362.0834 .

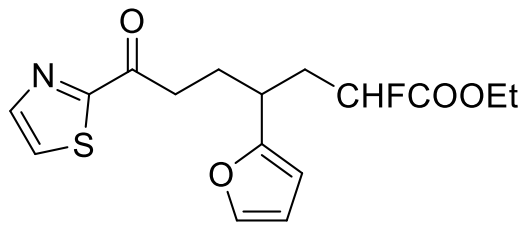

5d-B $(d . r .=1: 1): 14.3 \mathrm{mg}, 21 \%$, yellow oil. Purification by flash column chromatography (eluent: EtOAc/Petroleum ether $=1 / 30) .{ }^{1} \mathrm{H}$ NMR $\left(400 \mathrm{MHz}, \mathrm{CDCl}_{3}\right) \delta 8.00-7.88(\mathrm{~m}$, $2 \mathrm{H}$, two isomers), 7.63 (d, $J=3.2 \mathrm{~Hz}, 2 \mathrm{H}$, two isomers), 7.35-7.28 (m, 2H, two isomers), 6.29-6.19 (m, 2H, two isomers), 6.12 (d, $J=3.2 \mathrm{~Hz}, 1 \mathrm{H}$, two isomers), 6.06 (d, $J=3.2 \mathrm{~Hz}, 1 \mathrm{H}$, two isomers), $5.00-4.83$ (m, $1 \mathrm{H}$, one isomer), 4.71-4.54 (m, $1 \mathrm{H}$, one isomer), 4.22-4.08 (m, 4H, two isomers), 3.17-3.00 (m, $6 \mathrm{H}$, two isomers), 2.39-2.24 (m, $2 \mathrm{H}$, two isomers), 2.23-2.06 (m, $6 \mathrm{H}$, two isomers), 1.27-1.23 (m, $6 \mathrm{H}$, two isomers); ${ }^{13} \mathrm{C}$ NMR $\left(100 \mathrm{MHz}, \mathrm{CDCl}_{3}\right) \delta 193.1 \& 193.0$ (two isomers), 169.9 (d, $J_{\mathrm{C}-\mathrm{F}}=$ $23.2 \mathrm{~Hz}) \& 169.5\left(\mathrm{~d}, J_{\mathrm{C}-\mathrm{F}}=23.5 \mathrm{~Hz}\right.$ ) (two isomers), $166.9 \& 166.9$ (overlap, two isomers), 155.4 $\& 154.6$ (two isomers), $144.6 \& 144.6$ (overlap, two isomers), $141.9 \& 141.6$ (two isomers), 126.1 $\& 126.1$ (overlap, two isomers), $110.0 \& 110.0$ (two isomers), $107.5 \& 106.8$ (two isomers), 88.0 $\left(\mathrm{d}, J_{\mathrm{C}-\mathrm{F}}=11.0 \mathrm{~Hz}\right) \& 86.1\left(\mathrm{~d}, J_{\mathrm{C}-\mathrm{F}}=11.6 \mathrm{~Hz}\right)$ (two isomers), $61.5 \& 61.5$ (two isomers), $37.1(\mathrm{~d}$, $\left.J_{\mathrm{C}-\mathrm{F}}=21.0 \mathrm{~Hz}\right) \& 36.5\left(\mathrm{~d}, J_{\mathrm{C}-\mathrm{F}}=20.8 \mathrm{~Hz}\right.$ ) (two isomers), $36.1 \& 36.0$ (two isomers), 34.6 (d, $J_{\mathrm{C}-\mathrm{F}}$ $=1.8 \mathrm{~Hz}) \& 34.0\left(\mathrm{~d}, J_{\mathrm{C}-\mathrm{F}}=3.6 \mathrm{~Hz}\right.$ ) (two isomers), $28.4 \& 27.4$ (two isomers), $14.1 \& 14.0$ (two isomers); ${ }^{19} \mathrm{~F}$ NMR $\left(376 \mathrm{MHz}, \mathrm{CDCl}_{3}\right) \delta$-191.2--191.4 (m, one isomer), -193.0--193.2 (m, one isomer). FT-IR: $v\left(\mathrm{~cm}^{-1}\right)$ 2980, 2961, 1755, 1684, 1507, 1481, 1391, 1280, 1209. HRMS [ESI] m/z: 
<smiles>CCOC=CCC(CCC(=O)c1ccc(C)cc1)c1cccs1</smiles>

5e $($ d.r. $=1: 1): 38 \mathrm{mg}, 53 \%$, white solid, m.p. $48-49^{\circ} \mathrm{C}$. Purification by flash column chromatography (eluent: EtOAc/Petroleum ether $=1 / 30) .{ }^{1} \mathrm{H}$ NMR $(400 \mathrm{MHz}$, $\left.\mathrm{CDCl}_{3}\right) \delta$ 7.82-7.72 (m, 4H, two isomers), 7.25-7.17 (m, $6 \mathrm{H}$, two isomers), 6.99-6.84 ( $\mathrm{m}, 4 \mathrm{H}$, two isomers),

5.04-4.87 (m, $1 \mathrm{H}$, one isomer), 4.73-4.56 (m, 1H, one isomer), 4.26-4.10 (m, $4 \mathrm{H}$, two isomers), 3.39-3.25 (m, $2 \mathrm{H}$, two isomers), 2.95-2.79 (m, $4 \mathrm{H}$, two isomers), 2.39 (s, 6H, two isomers), 2.36-2.25 (m, $4 \mathrm{H}$, two isomers), 2.24-2.13 ( $\mathrm{m}, 2 \mathrm{H}$, two isomers), 2.08-1.97 ( $\mathrm{m}, 2 \mathrm{H}$, two isomers), 1.30-1.25 (m, 6H, two isomers); ${ }^{13} \mathrm{C}$ NMR (100 MHz, $\left.\mathrm{CDCl}_{3}\right) \delta 199.2 \& 199.1$ (two isomers), $170.0\left(\mathrm{~d}, J_{\mathrm{C}-\mathrm{F}}=23.0 \mathrm{~Hz}\right) \& 169.6\left(\mathrm{~d}, J_{\mathrm{C}-\mathrm{F}}=23.3 \mathrm{~Hz}\right)$ (two isomers), $147.0 \& 145.9$ (two isomers), $143.8 \& 143.8$ (overlap, two isomers), $134.4 \& 134.4$ (overlap, two isomers), $129.2 \& 129.2$ (overlap, two isomers), $128.1 \& 128.1$ (overlap, two isomers), $126.9 \& 126.7$ (two isomers), 125.7 \&125.0 (two isomers), $124.1 \& 123.8$ (two isomers), 87.9 (d, $\left.J_{\mathrm{C}-\mathrm{F}}=2.9 \mathrm{~Hz}\right) \& 86.1$ (d, $J_{\mathrm{C}-\mathrm{F}}=2.4$ $\mathrm{Hz}$ ) (two isomers), $61.6 \& 61.6$ (overlap, two isomers), $40.8\left(\mathrm{~d}, J_{\mathrm{C}-\mathrm{F}}=20.9 \mathrm{~Hz}\right) \& 40.5\left(\mathrm{~d}, J_{\mathrm{C}-\mathrm{F}}=\right.$ $20.8 \mathrm{~Hz}$ ) (two isomers), $36.7\left(\mathrm{~d}, J_{\mathrm{C}-\mathrm{F}}=1.3 \mathrm{~Hz}\right) \& 36.1\left(\mathrm{~d}, J_{\mathrm{C}-\mathrm{F}}=4.2 \mathrm{~Hz}\right.$ ) (two isomers), $36.2 \&$ 36.0 (two isomers), $32.6 \& 31.2$ (two isomers), $21.6 \& 21.6$ (overlap, two isomers), $14.1 \& 14.1$ (two isomers); ${ }^{19} \mathrm{~F}$ NMR (376 MHz, $\left.\mathrm{CDCl}_{3}\right) \delta-191.0$ (s, one isomer), -194.1 (s, one isomer). FT-IR: $v\left(\mathrm{~cm}^{-1}\right)$ 2981, 2901, 1758, 1674, 1507, 1456, 1374, 1252, 1216. HRMS [ESI] m/z: $[\mathrm{M}+\mathrm{Na}]^{+}$calcd for $\mathrm{C}_{20} \mathrm{H}_{23} \mathrm{FNaO}_{3} \mathrm{~S} 385.1244$; found 385.1252.

\section{7. ${ }^{1} \mathrm{H},{ }^{13} \mathrm{C},{ }^{19} \mathrm{~F}$, and HMBC NMR spectra}
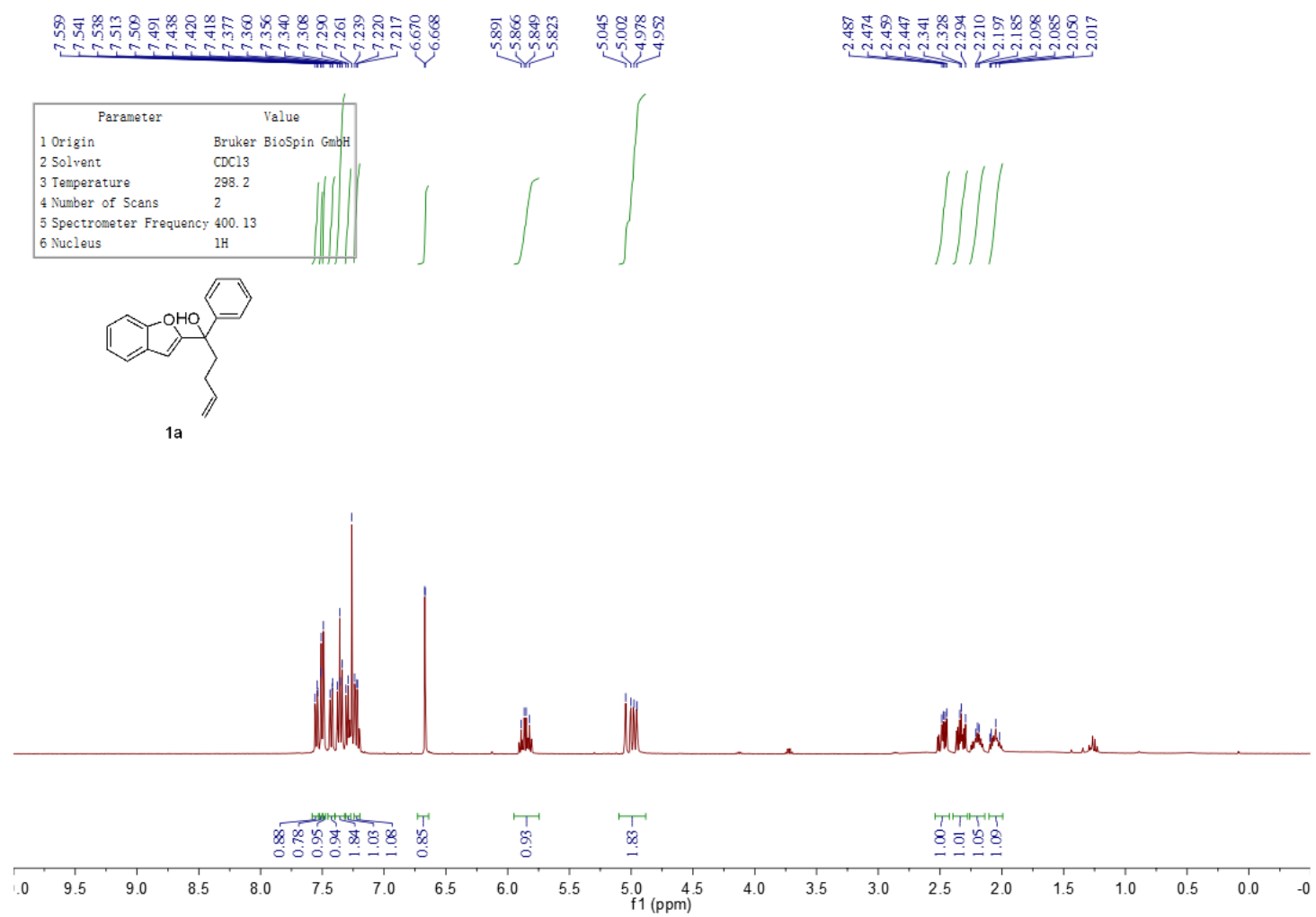


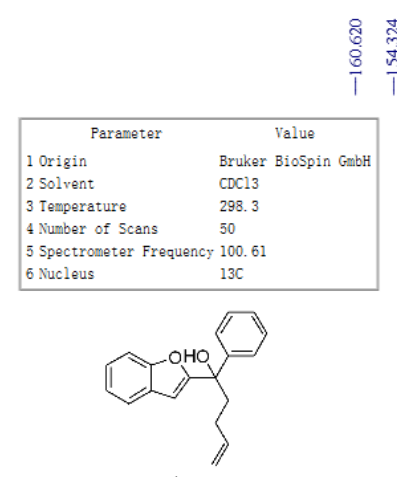

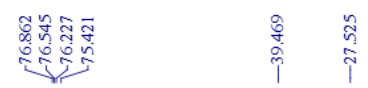
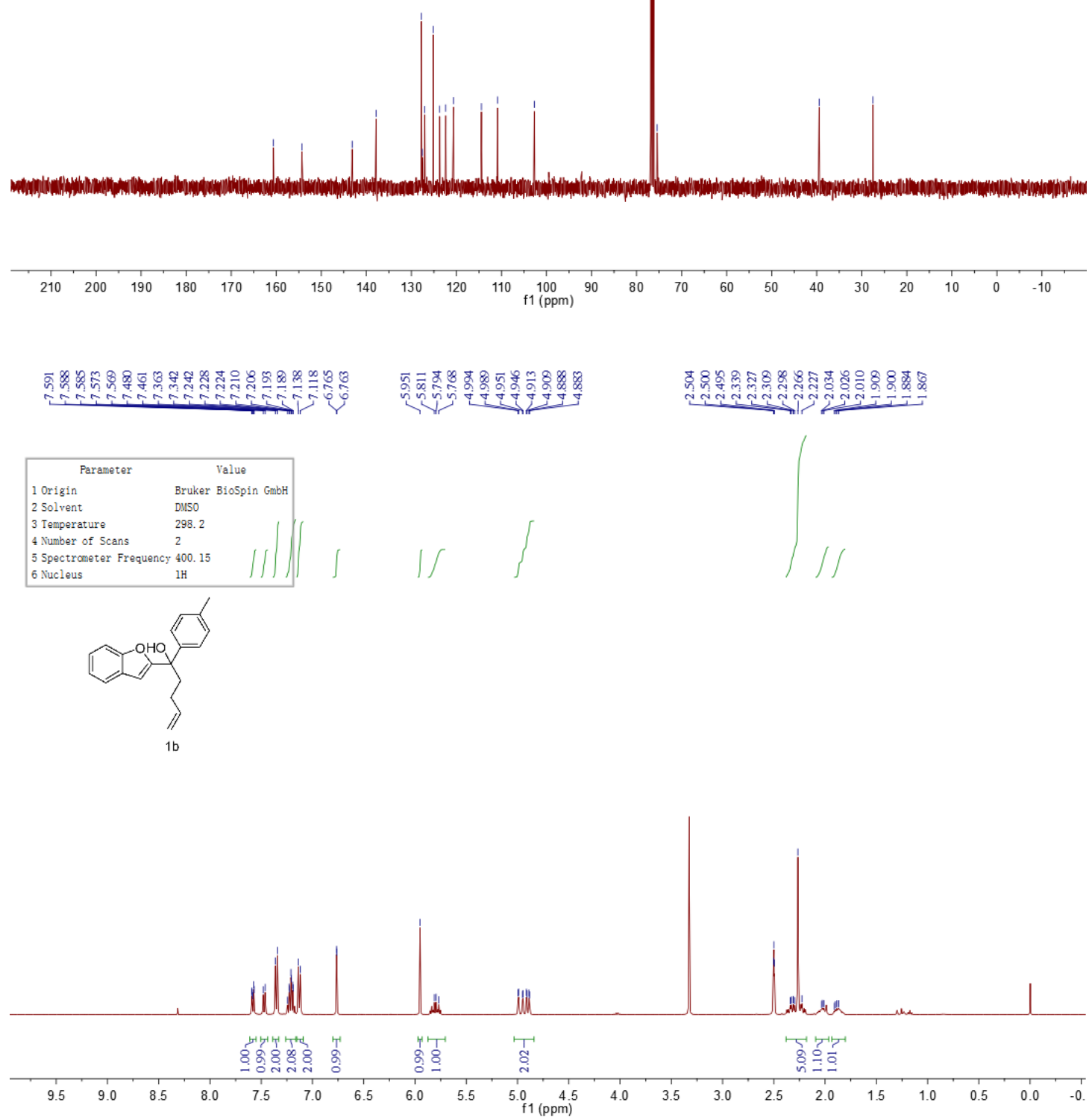


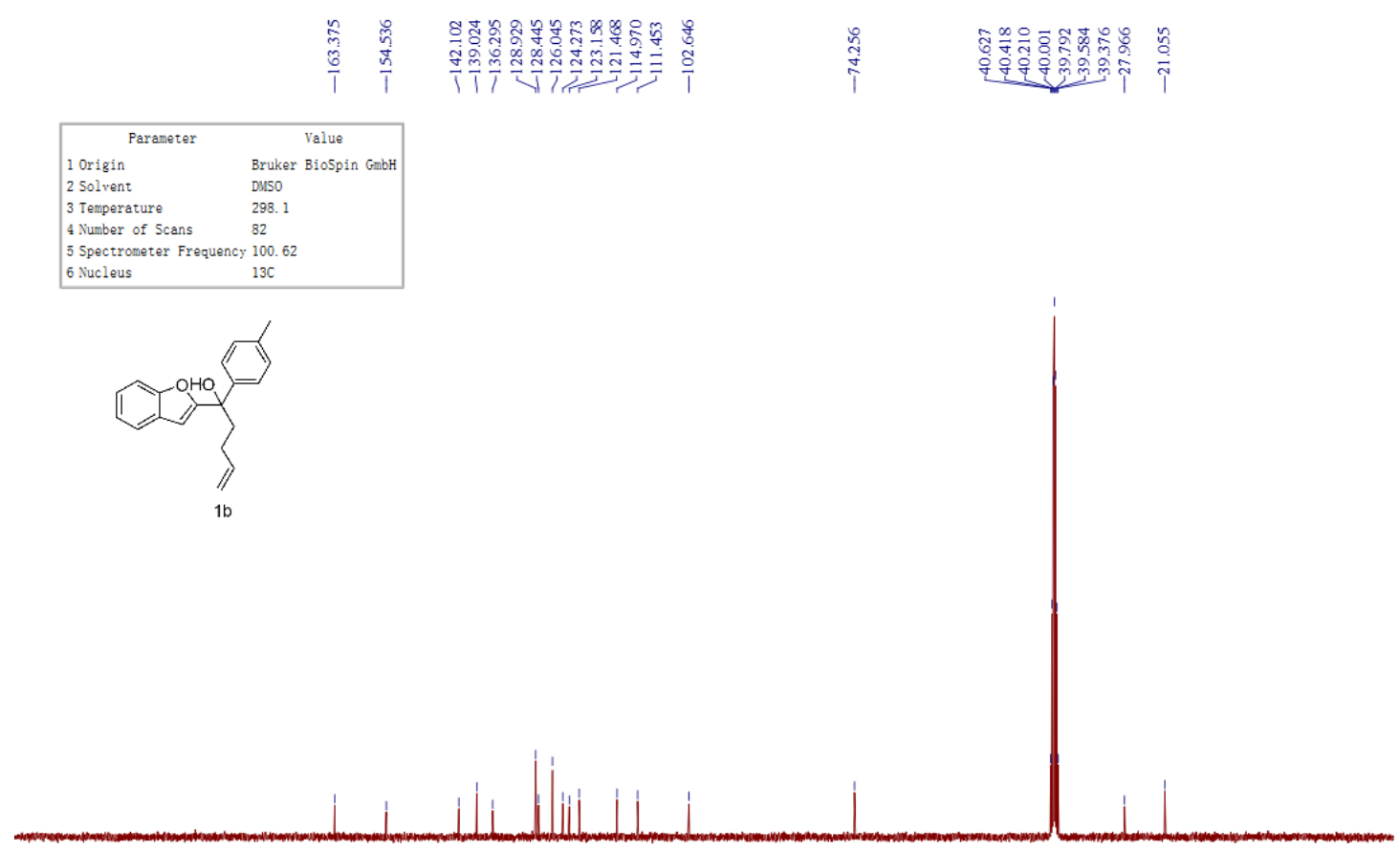

$\begin{array}{llllllllllllllllllllllllllll}210 & 200 & 190 & 180 & 170 & 160 & 150 & 140 & 130 & 120 & 110 & 100 & 90 & 80 & 70 & 60 & 50 & 40 & 30 & 20 & 10 & 0 & -10\end{array}$

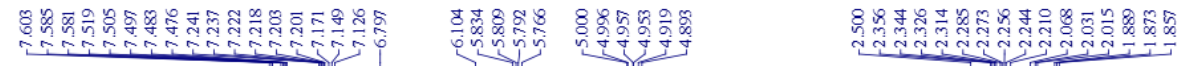
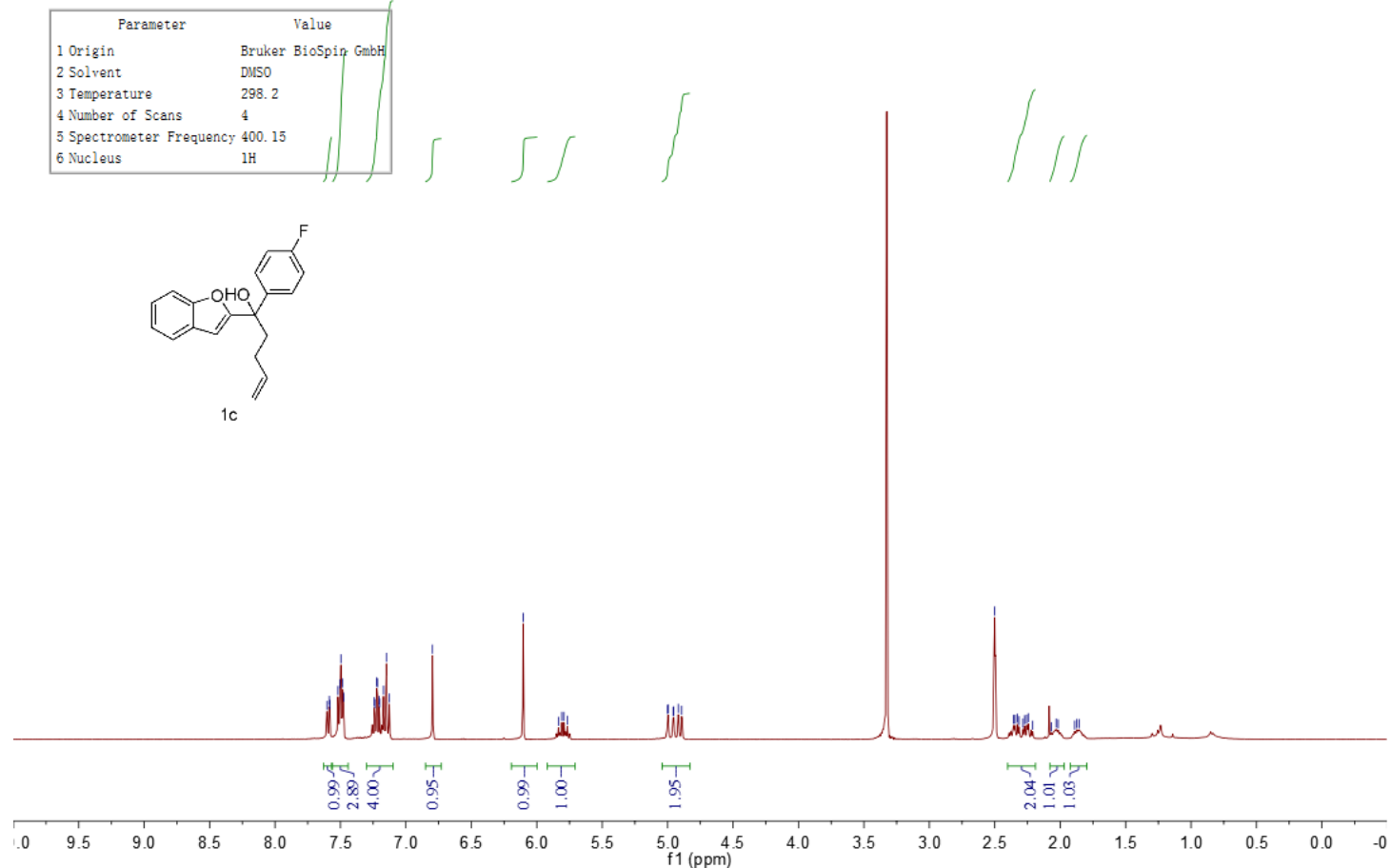

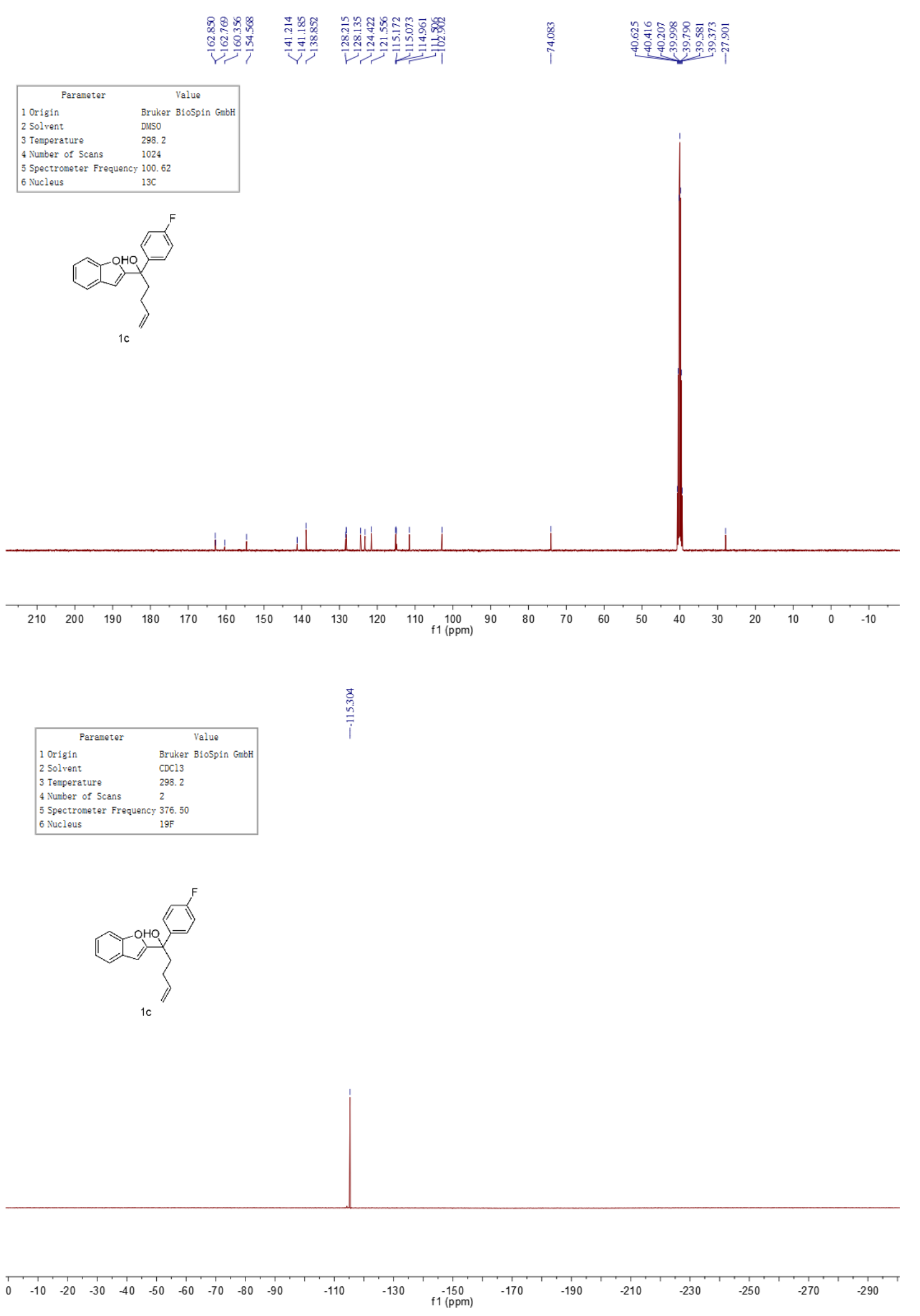

35 


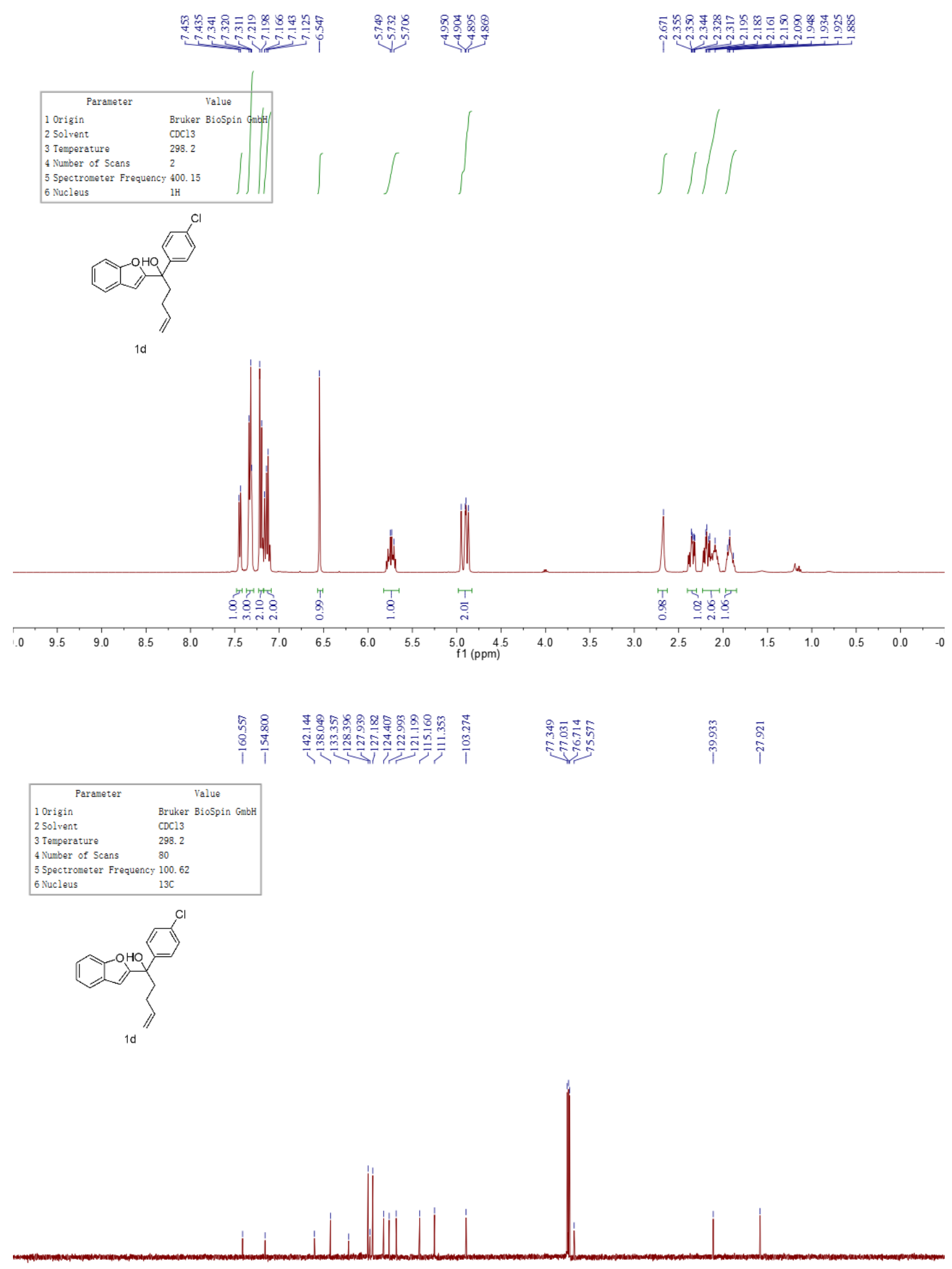

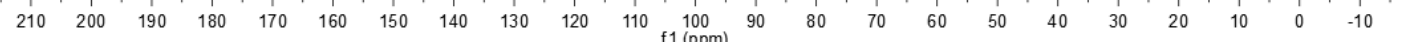




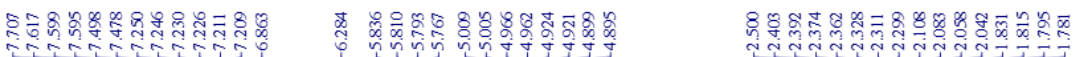

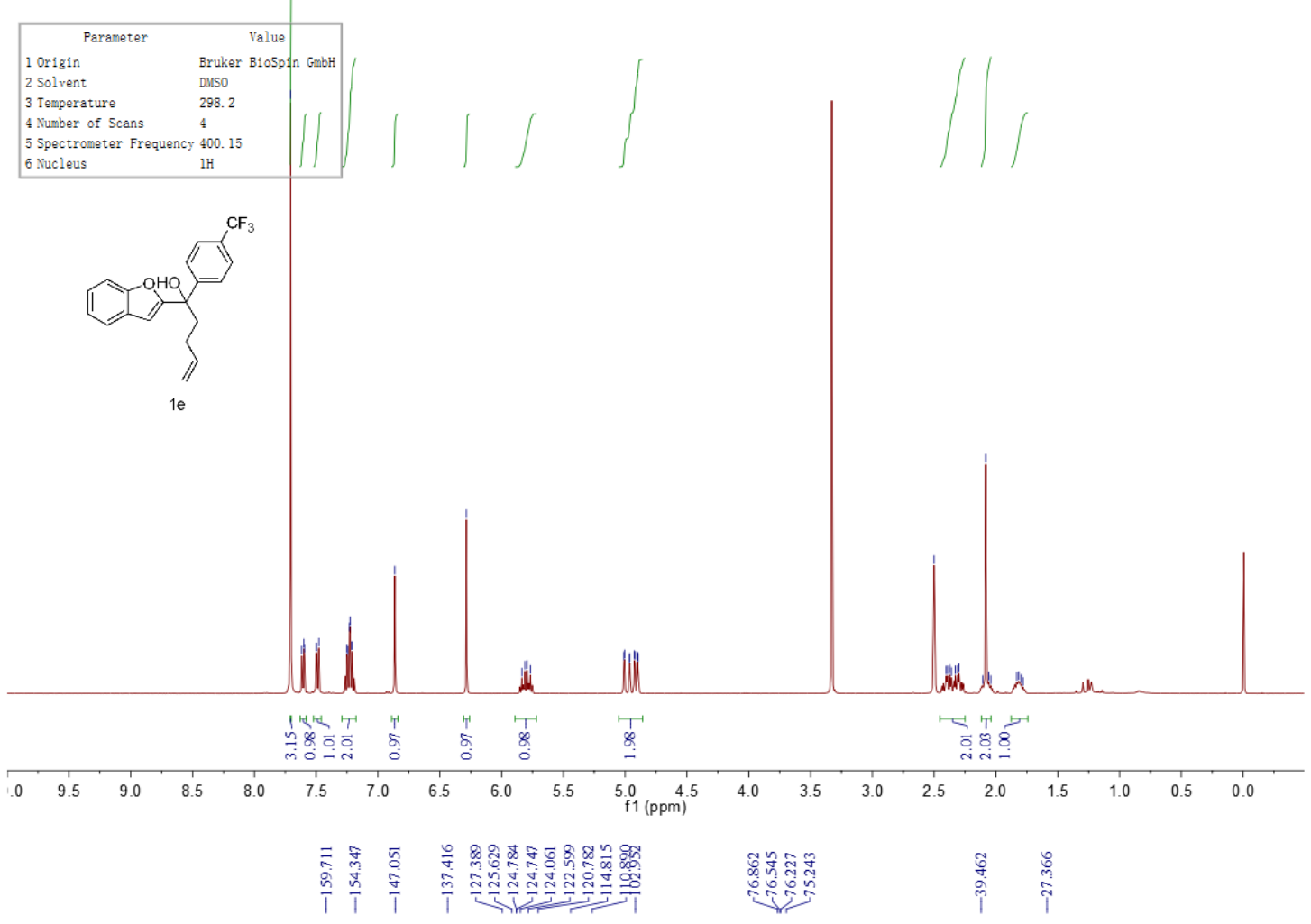

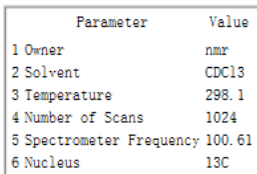
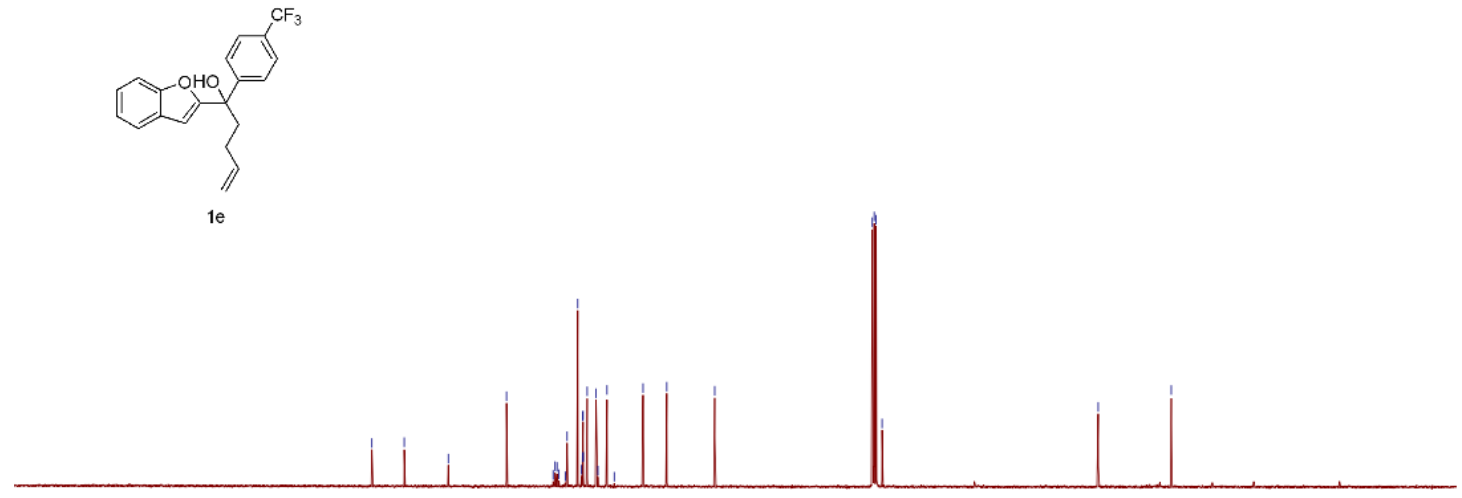

$\begin{array}{lllllllllllllllllllllllllll} & 1 & 210 & 200 & 190 & 180 & 170 & 160 & 150 & 140 & 130 & 120 & 110 & 100 & 90 & 80 & 70 & 60 & 50 & 40 & 30 & 20 & 10 & 0 & -10\end{array}$ 

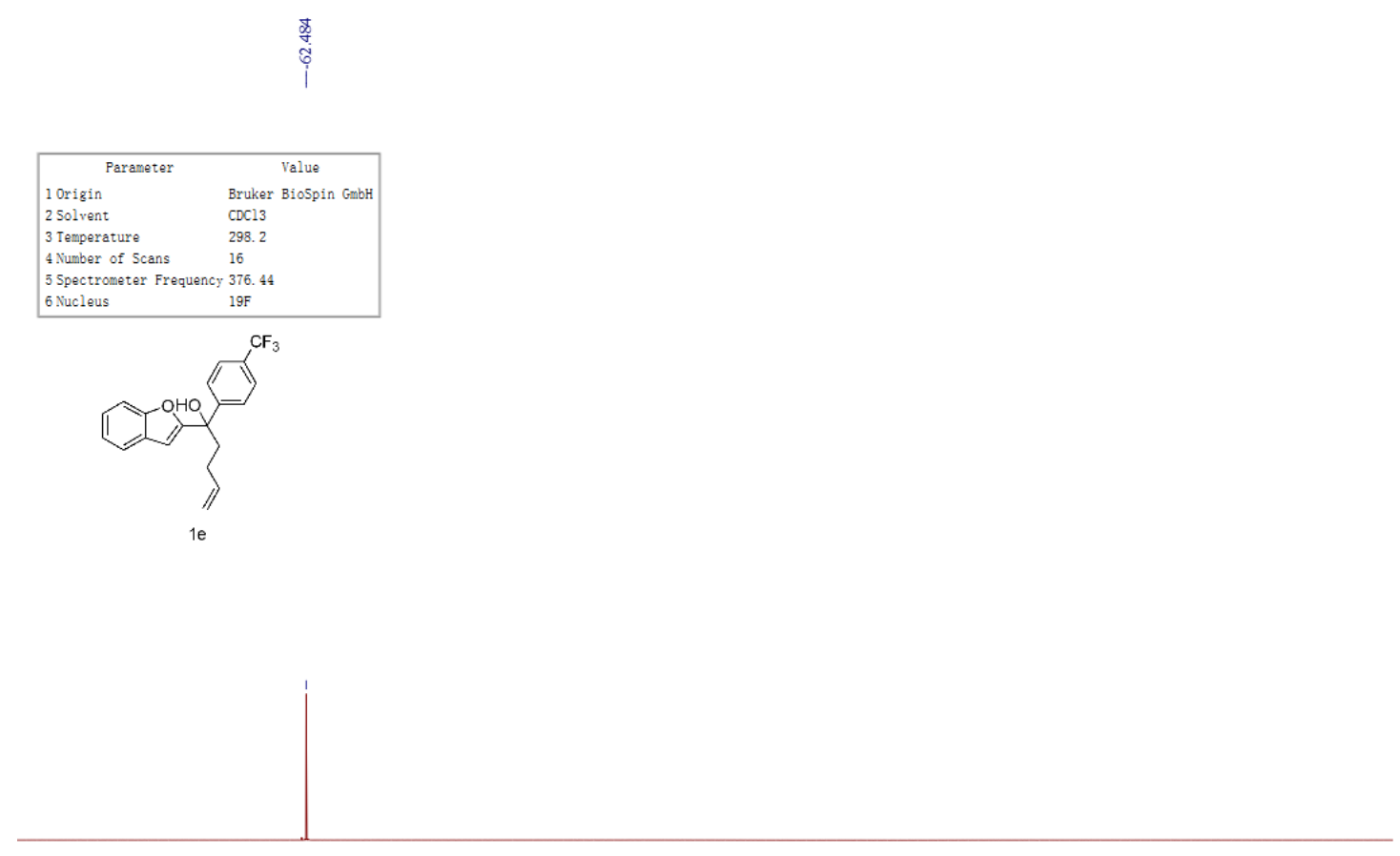

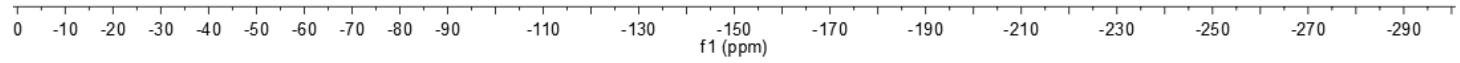

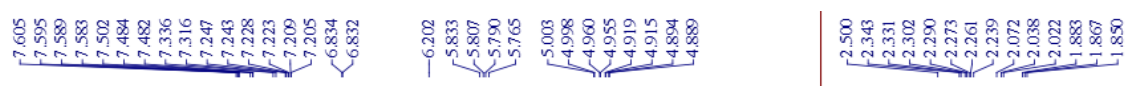

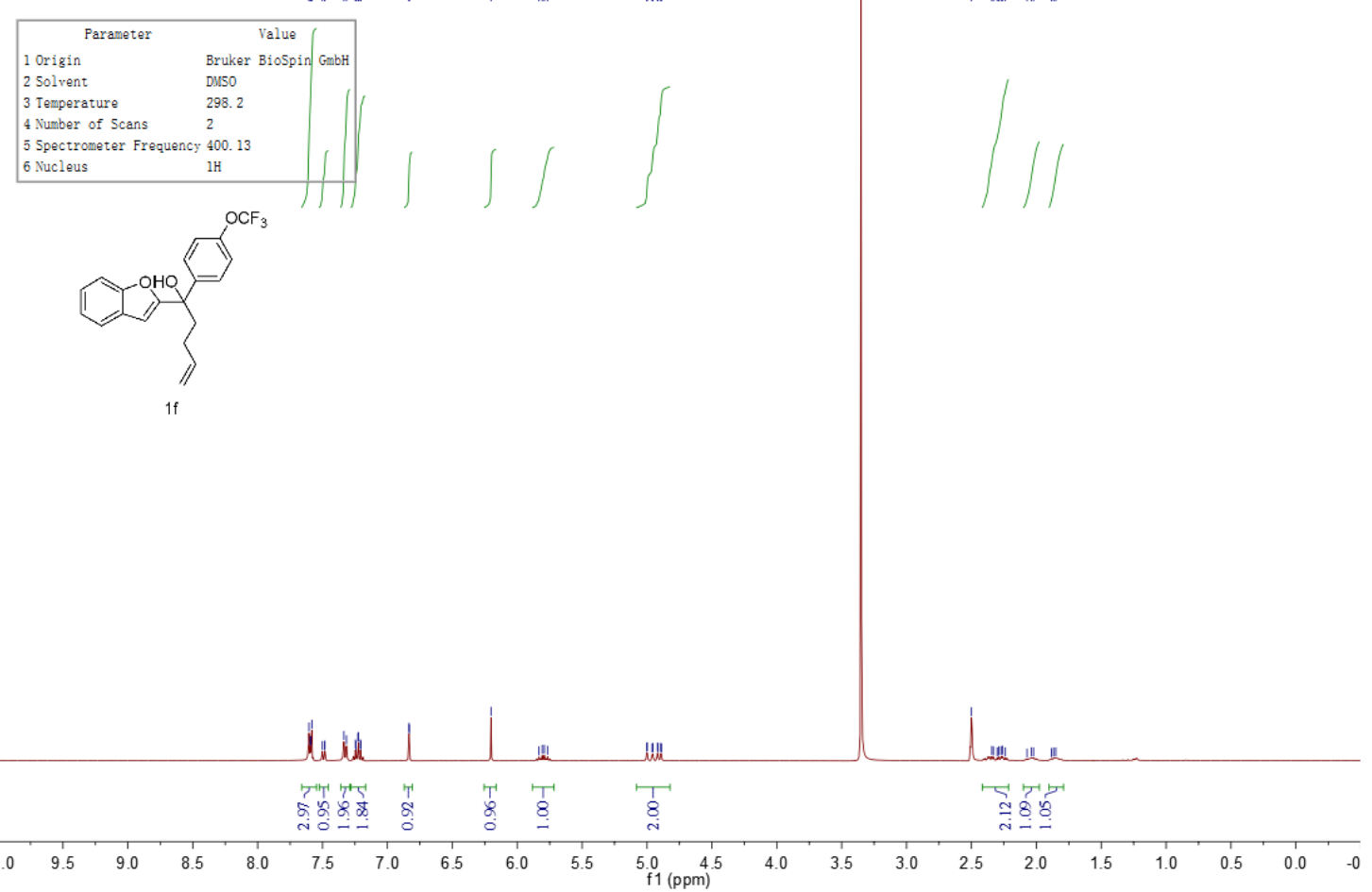



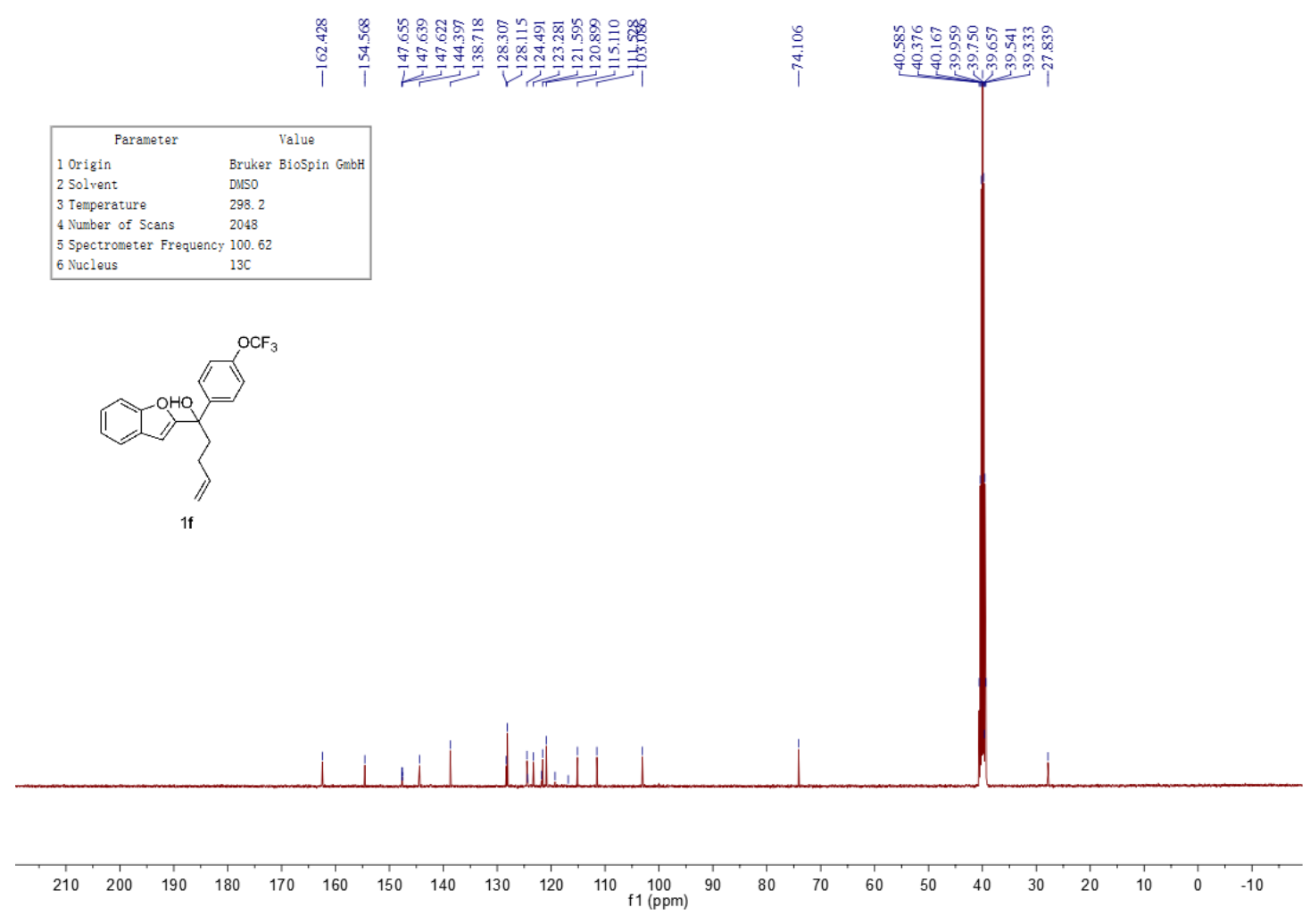

\begin{tabular}{|ll|}
\hline \multicolumn{1}{|c|}{ Parameter } & \multicolumn{1}{c|}{ Value } \\
1 Origin & Bruker Biospin GmbH \\
2 Solvent & DUSO \\
3 Temperature & 298.2 \\
4 Number of Scans & 4 \\
5 Spectrometer Frequency 376.46 \\
6 Nucleus & $19 \mathrm{~F}$ \\
\hline
\end{tabular}

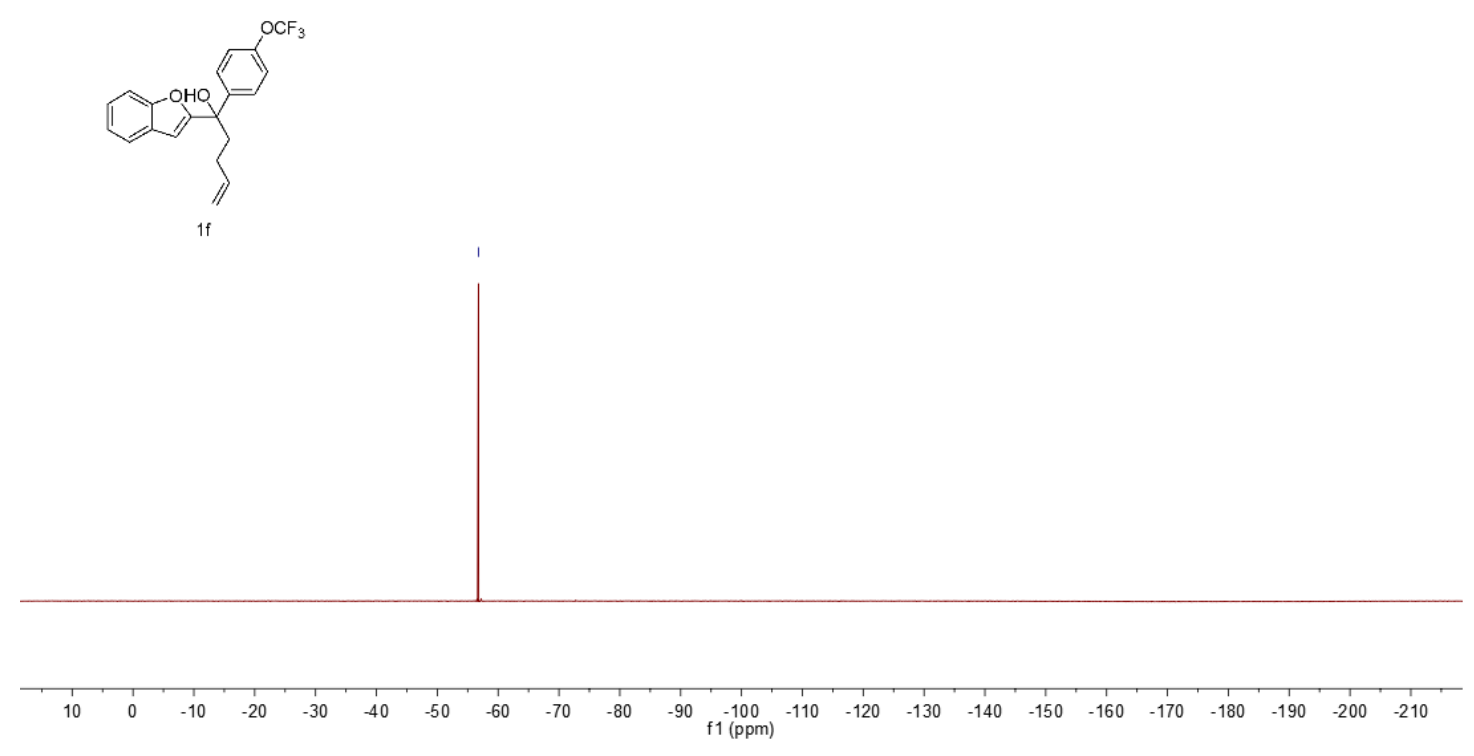




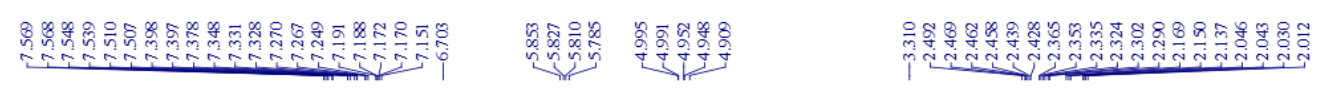
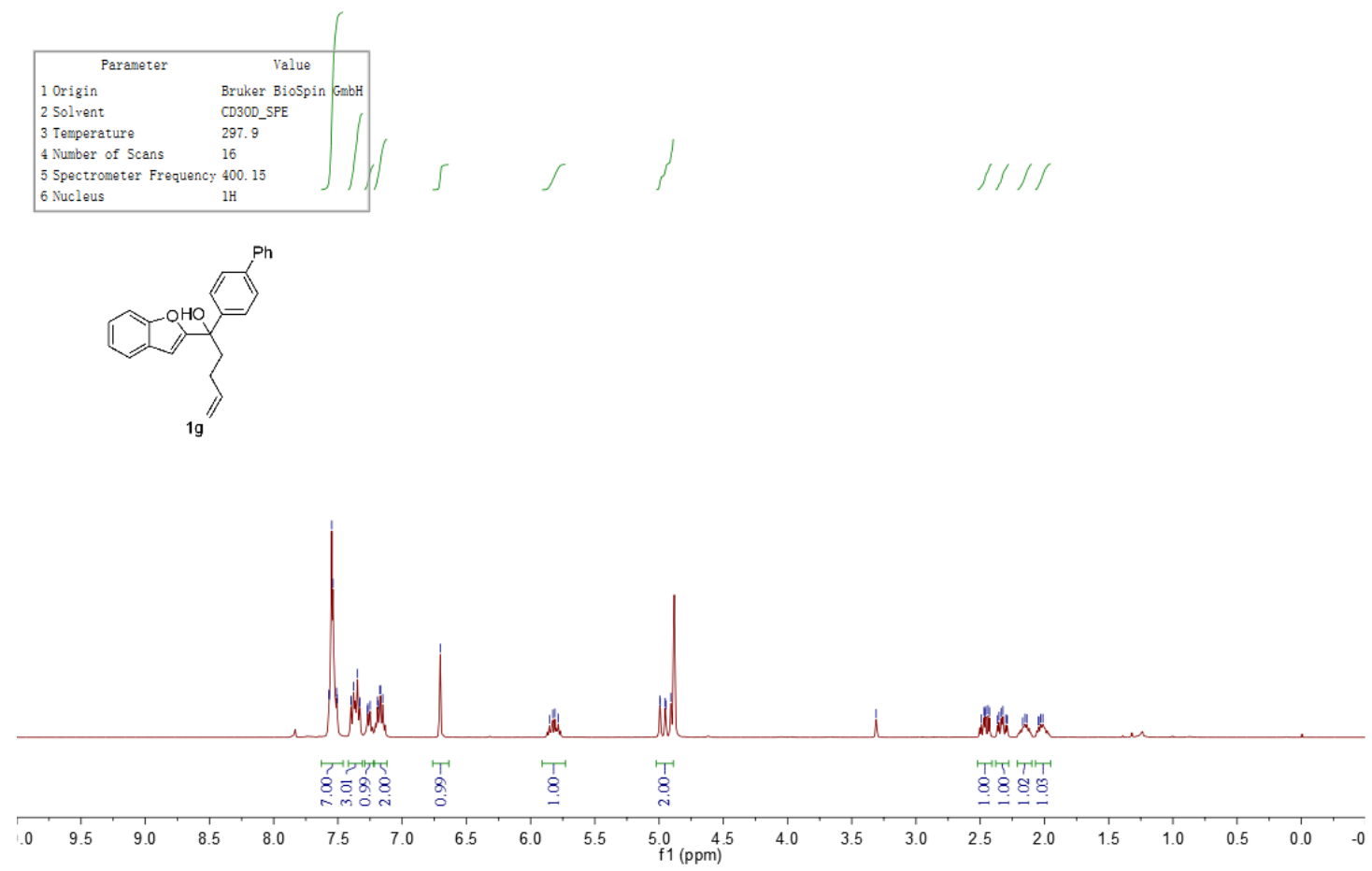

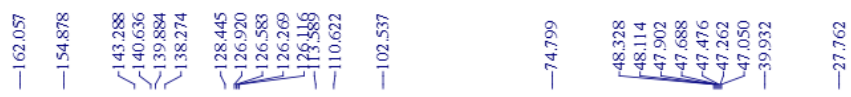

\begin{tabular}{|ll|}
\hline \multicolumn{1}{|c|}{ Parameter } & \multicolumn{1}{c|}{ Value } \\
1 Origin & Bruker Bio5pin GmbH \\
2 Solvent & CD30D_SPE \\
3 Temperature & 298. 2 \\
4 Number of Scans & 1024 \\
5 Spectrometer Frequency & 100.62 \\
6 Nucleus & $13 \mathrm{C}$ \\
\hline
\end{tabular}
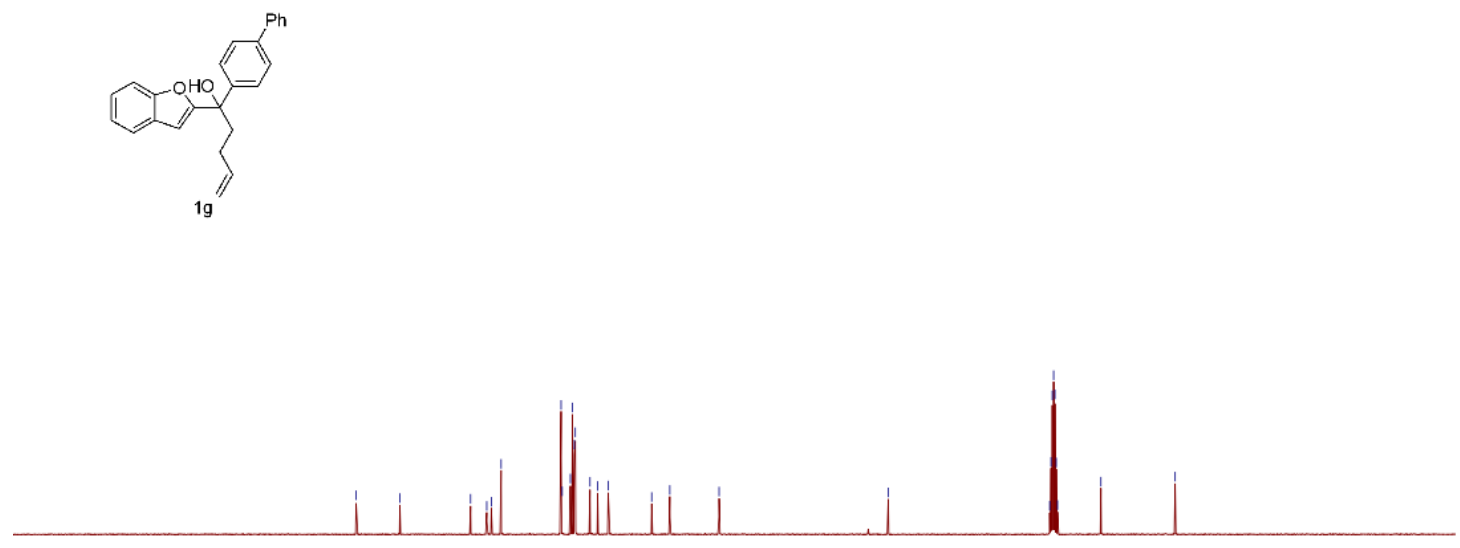

$\begin{array}{lllllllllllllllllllllllllllllll}1 & 110 & 200 & 190 & 180 & 170 & 160 & 150 & 140 & 130 & 120 & 110 & 100 & 90 & 80 & 70 & 60 & 50 & 40 & 30 & 20 & 10 & 0 & -10\end{array}$ 

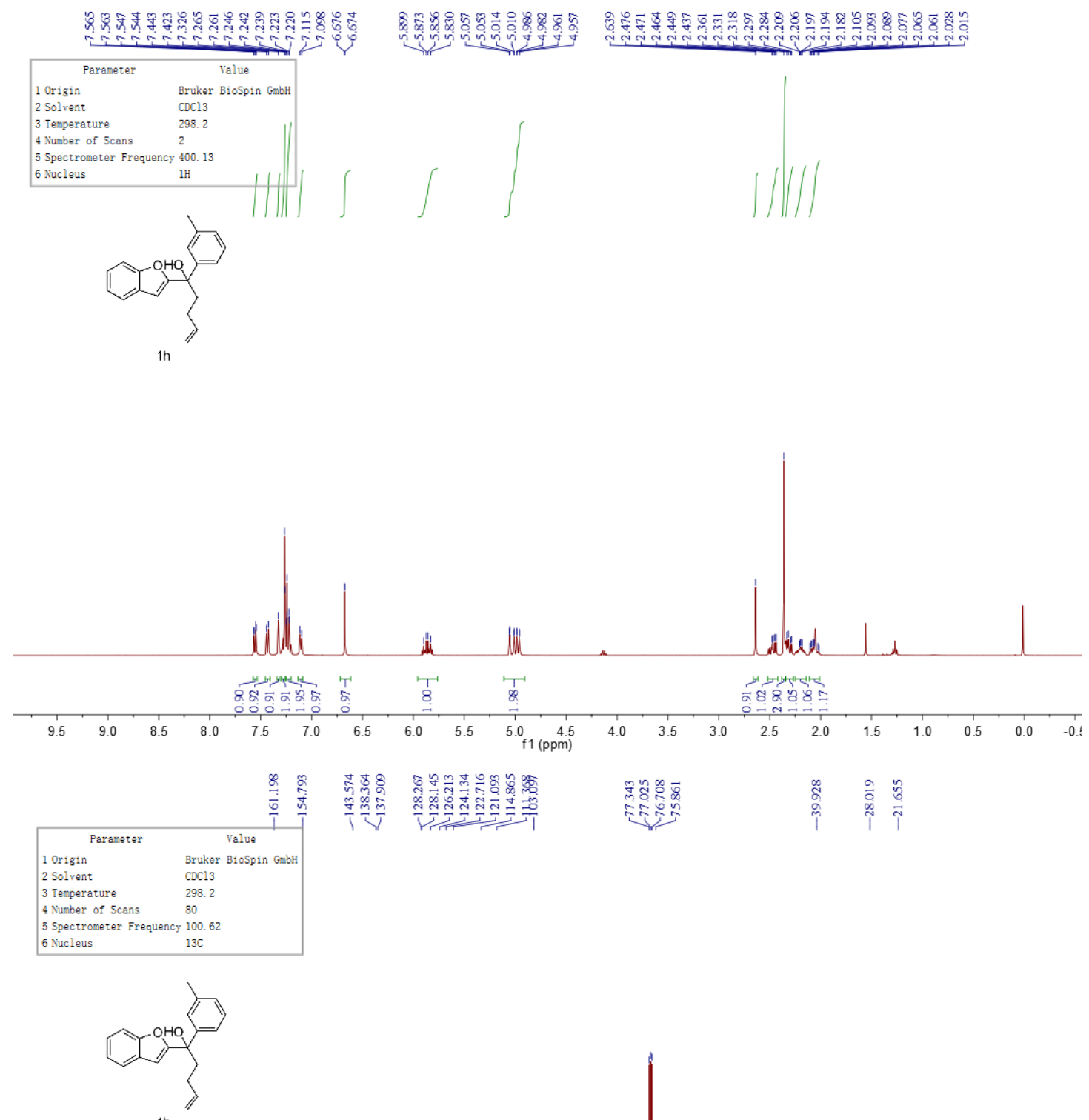

in

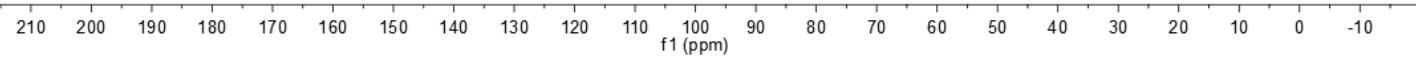




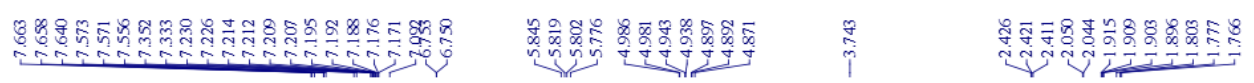

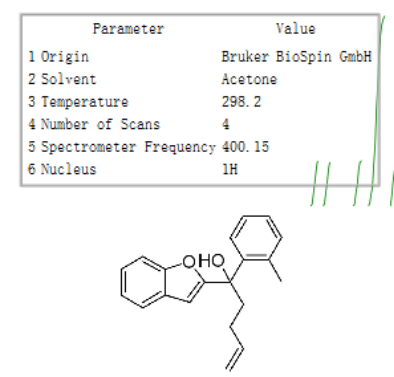

$1 i$

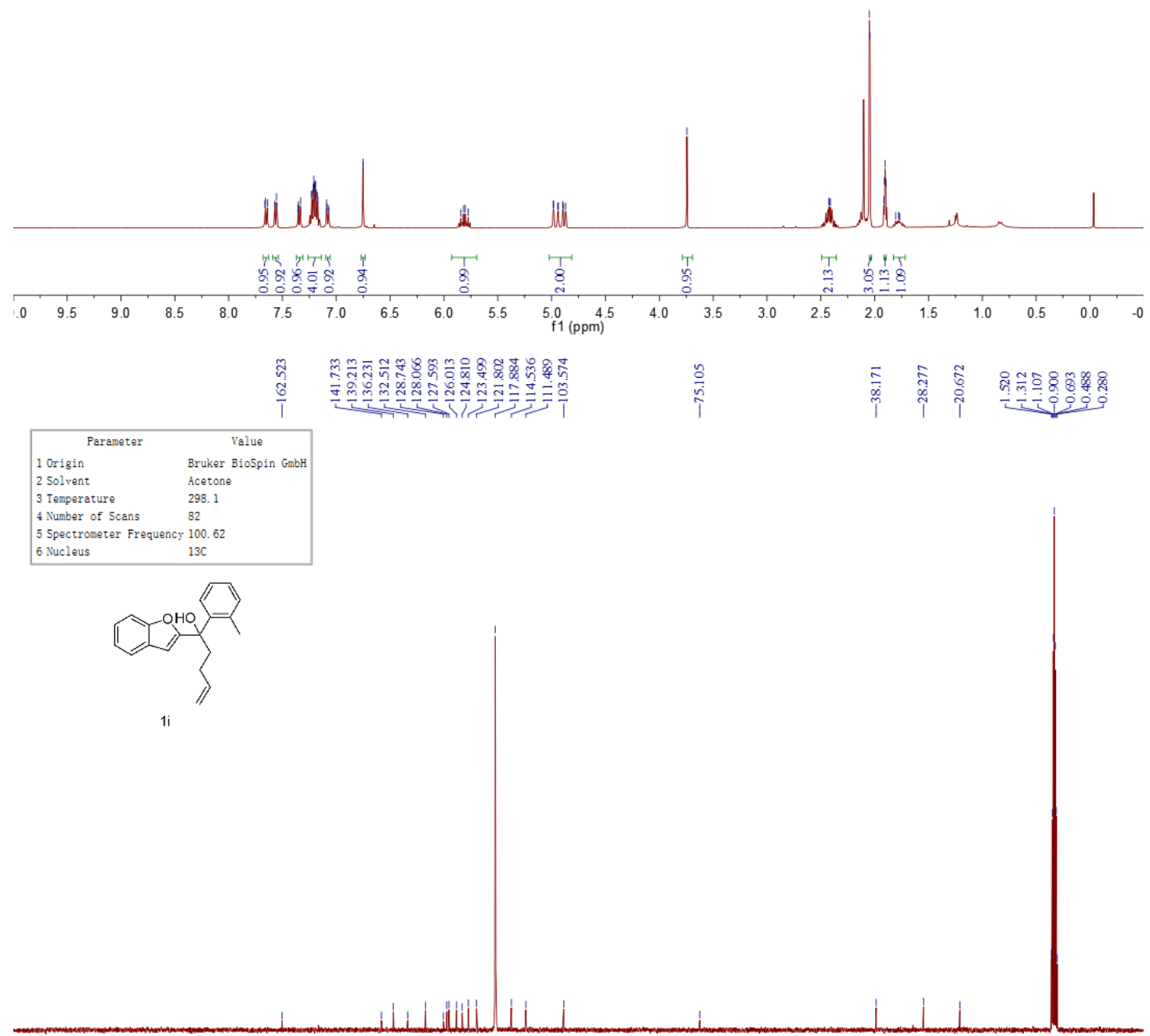

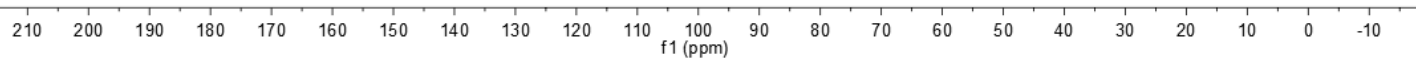




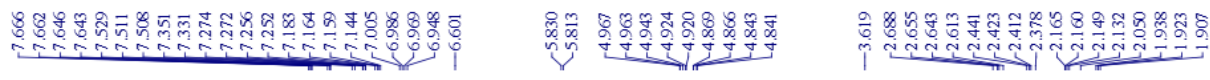
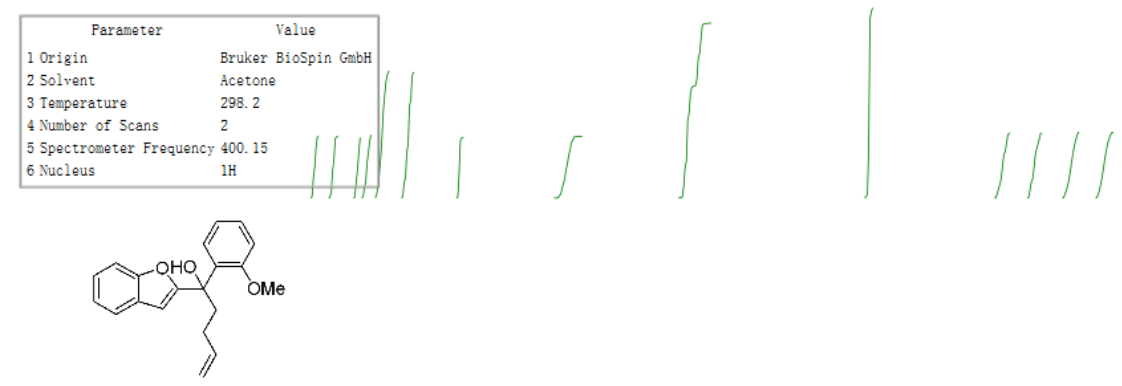

1j
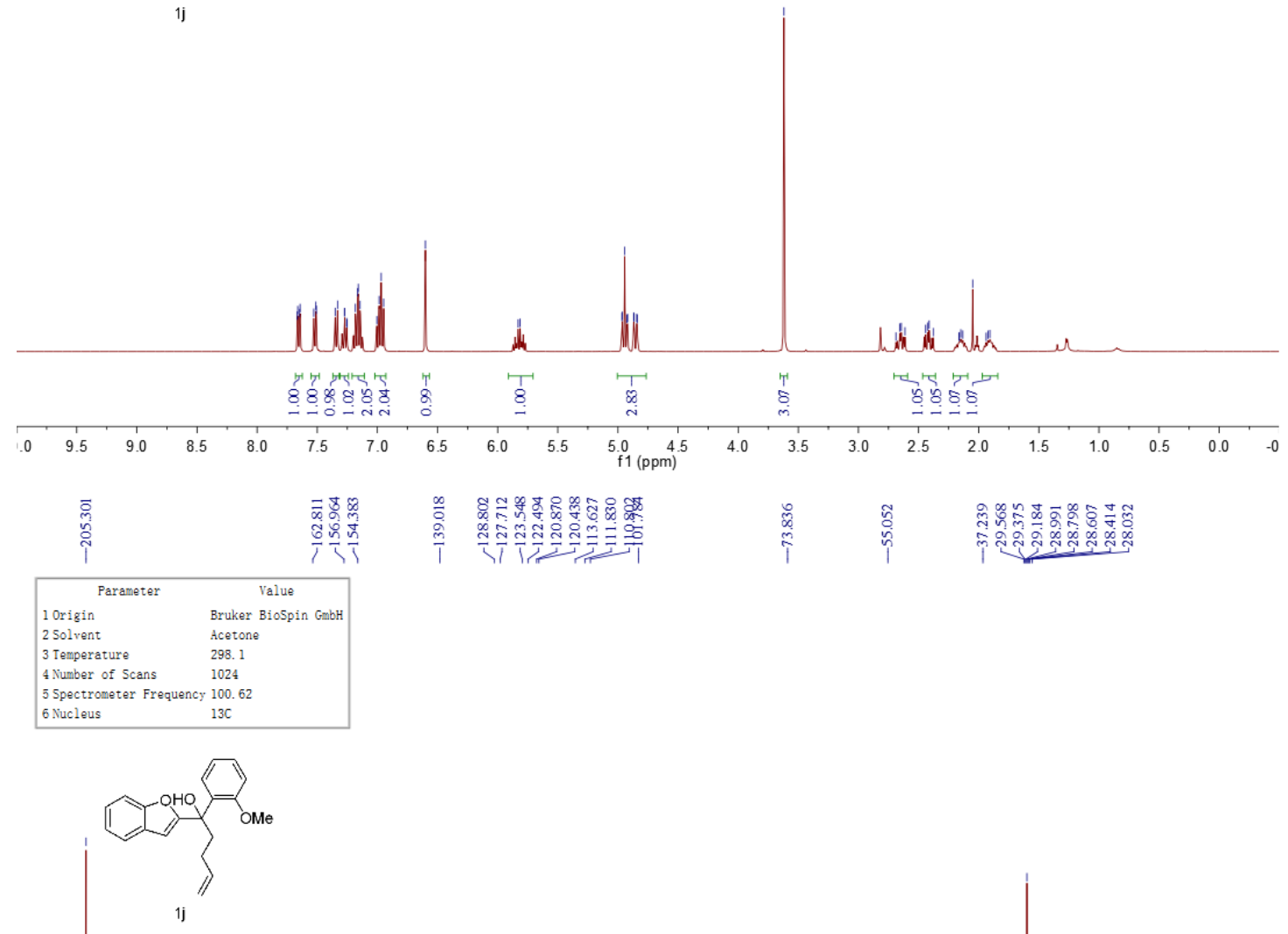

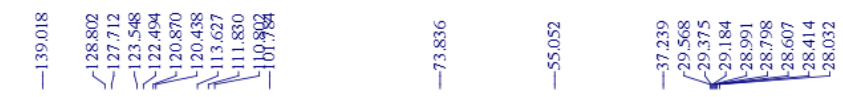

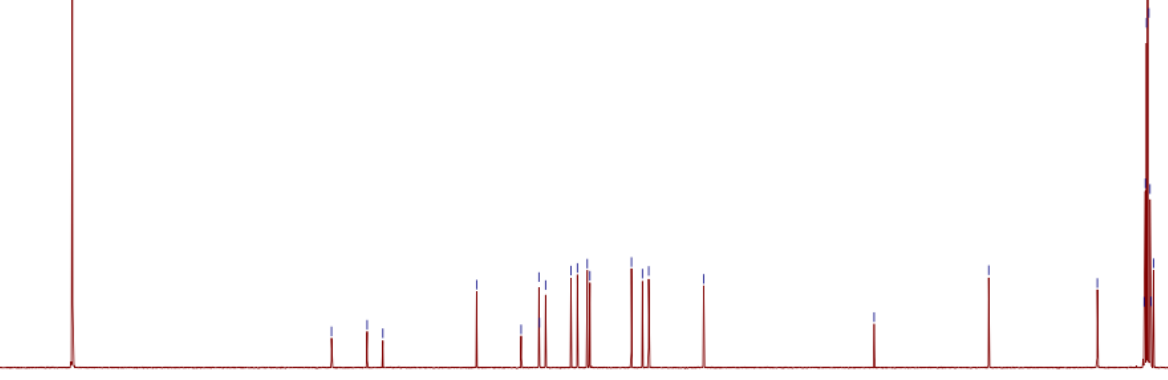

$\begin{array}{llllllllllllllllllllllllll}210 & 200 & 190 & 180 & 170 & 160 & 150 & 140 & 130 & 120 & 110 & 100 & 90 & 80 & 70 & 60 & 50 & 40 & 30 & 20 & 10 & 0 & -10\end{array}$ 


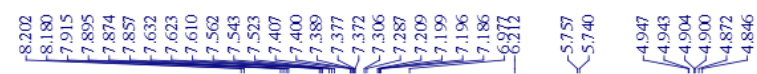

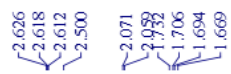

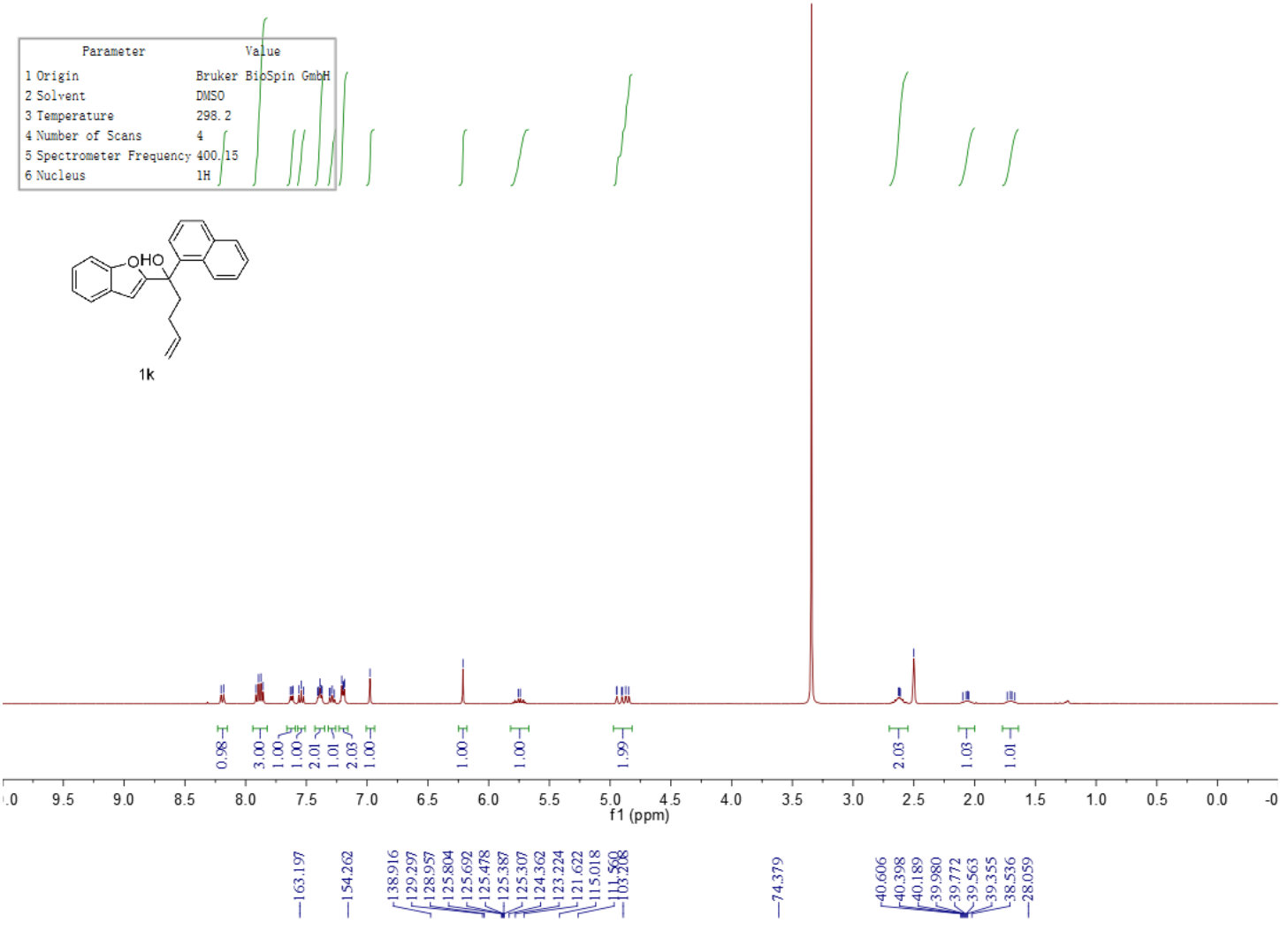

\begin{tabular}{|ll|}
\hline \multicolumn{1}{|c|}{ Parameter } & \multicolumn{1}{c|}{ Value } \\
1 Origin & Bruker BioSpin GmbH \\
2 Solvent & DUSO \\
3 Temperature & 298.1 \\
4 Number of Scans & 1024 \\
5 Spectrometer Frequency & 100.62 \\
6 . Nucleus & $13 \mathrm{C}$ \\
\hline
\end{tabular}

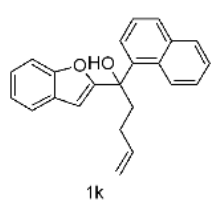

$1 \mathrm{k}$ 


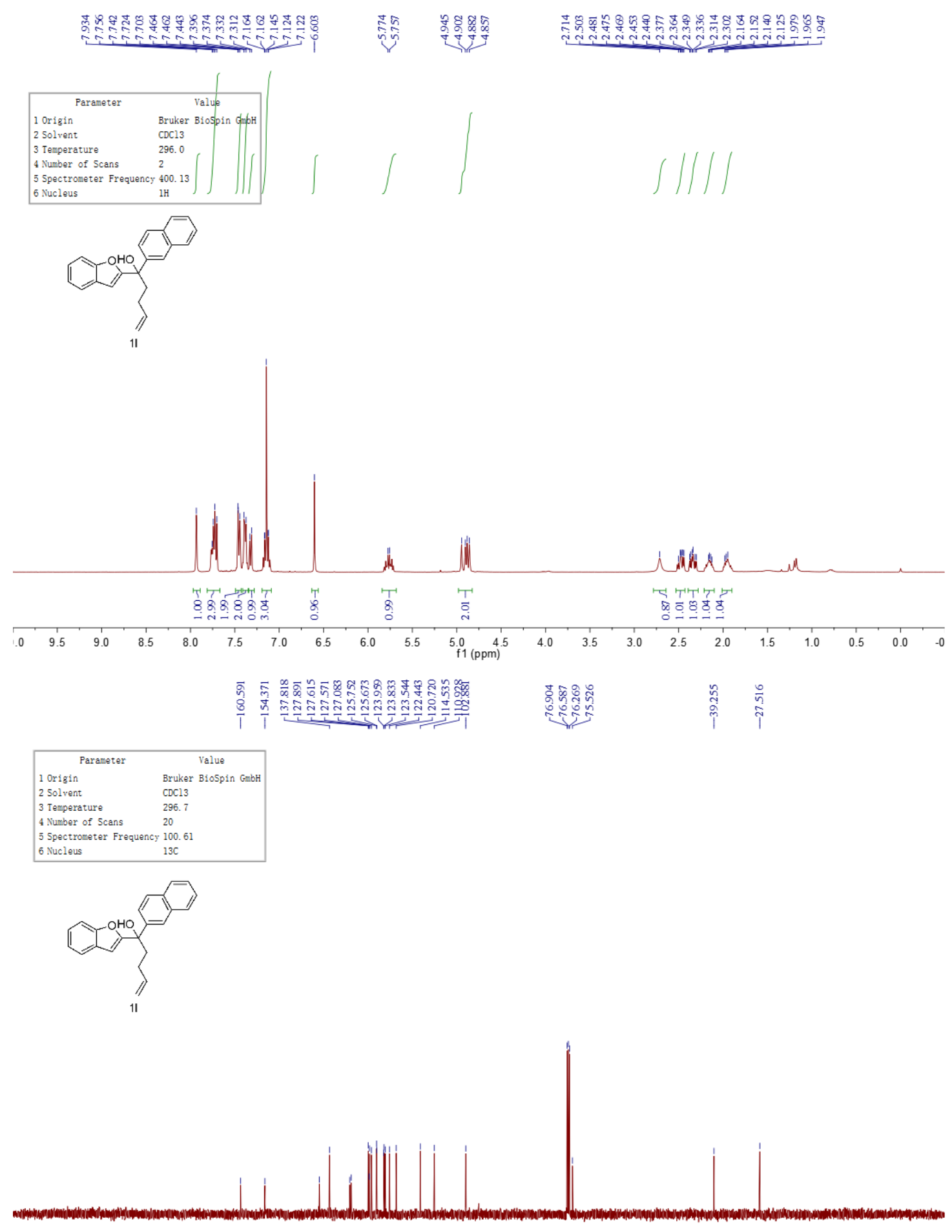

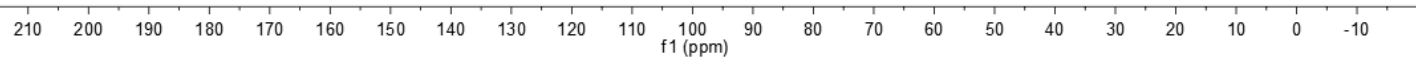




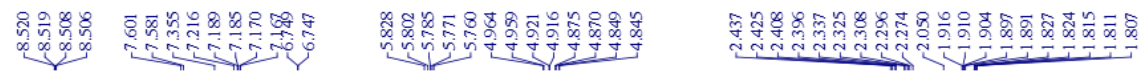

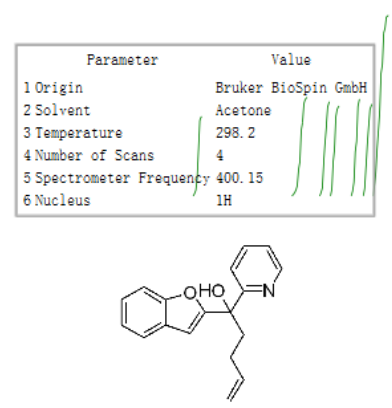

$1 \mathrm{~m}$

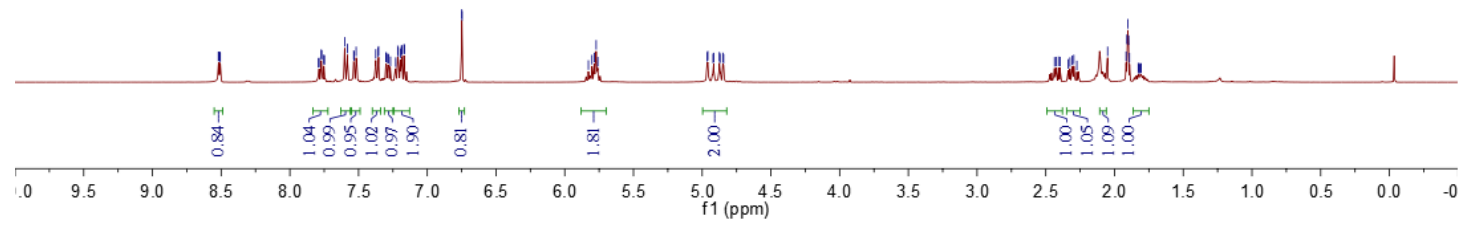

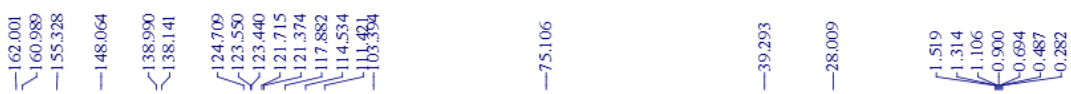

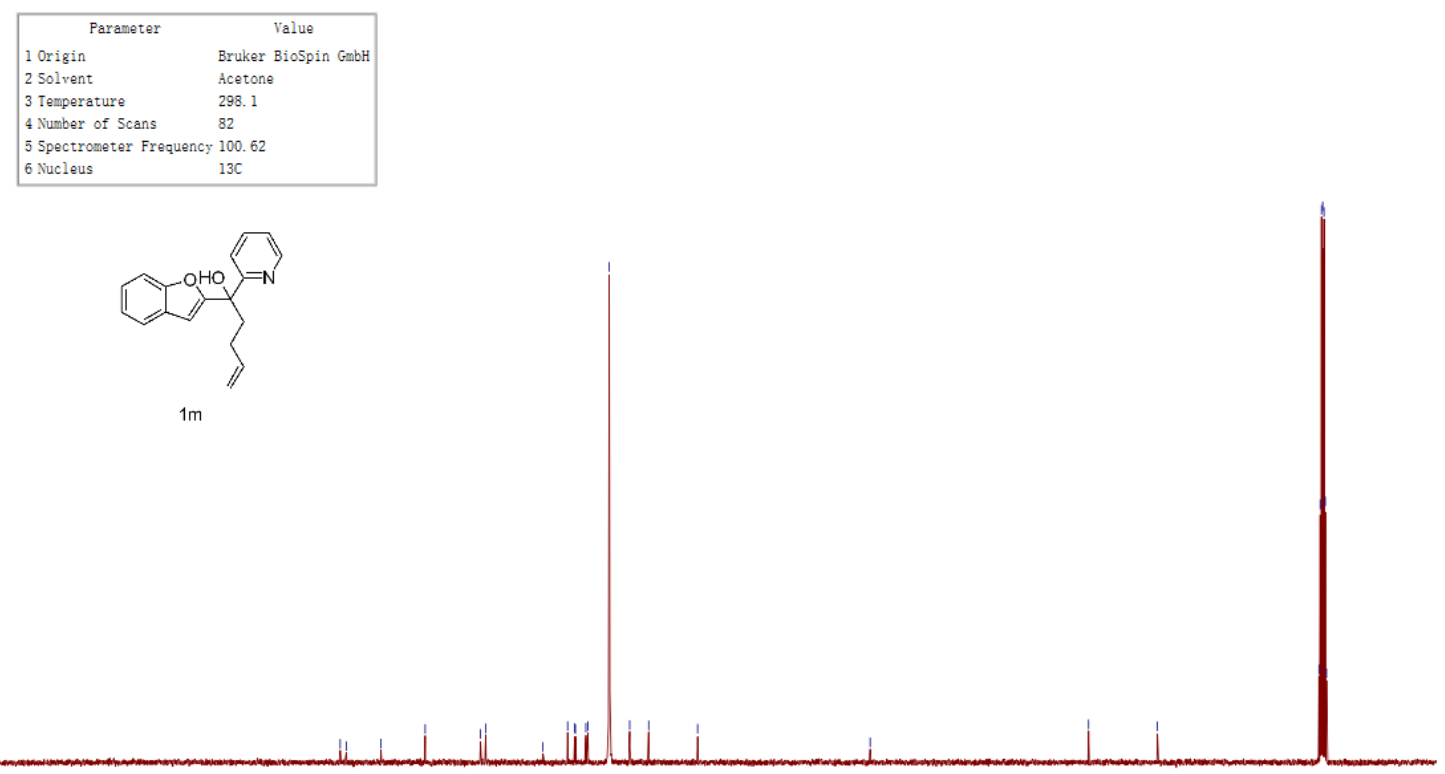

$\begin{array}{llllllllllllllllllllllll} & 110 & 200 & 190 & 180 & 170 & 160 & 150 & 140 & 130 & 120 & 110 & 100 & 10 & 10 & 70 & 60 & 50 & 40 & 30 & 20 & 10 & 0 & -10\end{array}$ 


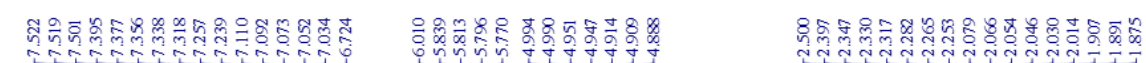
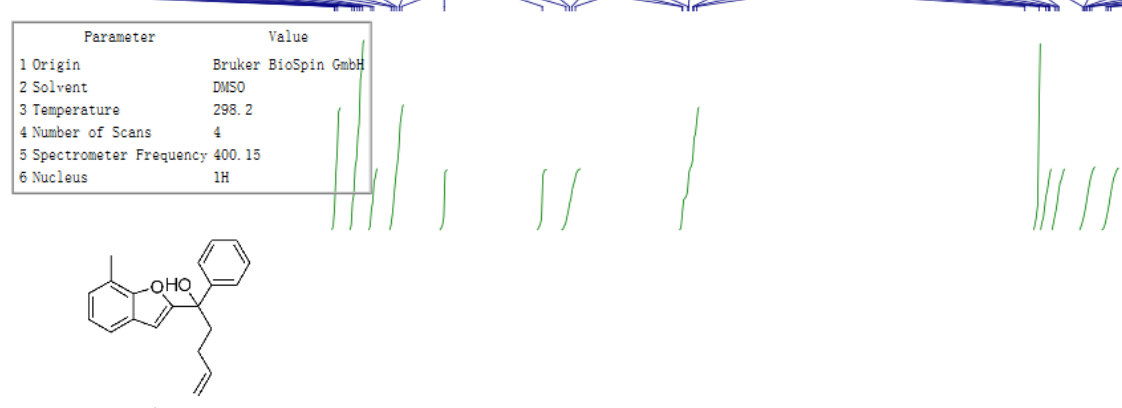

1n

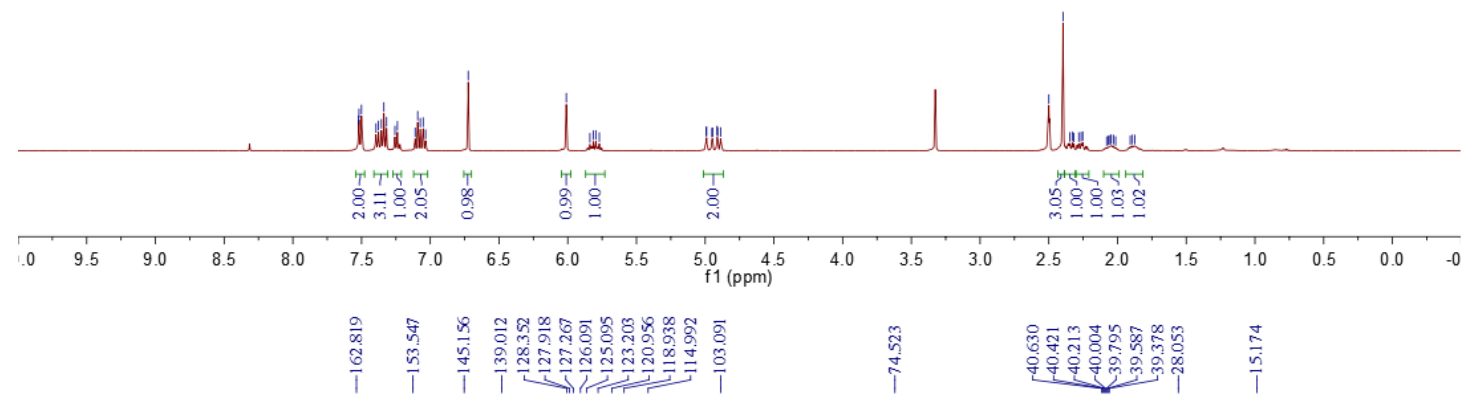

\begin{tabular}{|ll|}
\hline \multicolumn{1}{|c|}{ Parameter } & \multicolumn{1}{c|}{ Value } \\
1 Origin & Bruker BioSpin GmbH \\
2 Solvent & DMSO \\
3 Temperature & 298.2 \\
4 Number of Scans & 339 \\
5 Spectrometer Frequency & 100.62 \\
6 Nucleus & $13 C$ \\
\hline
\end{tabular}

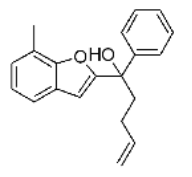

$1 \mathrm{n}$

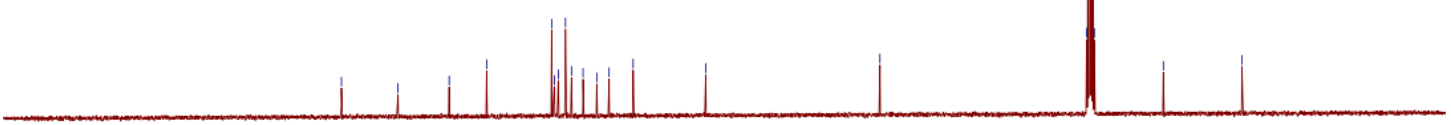

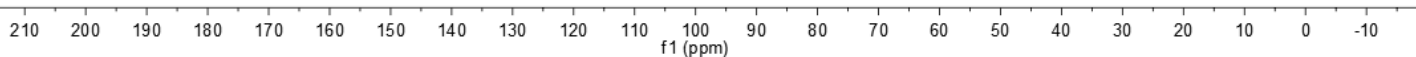




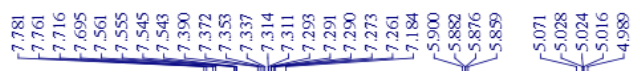

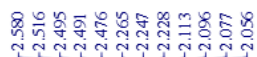
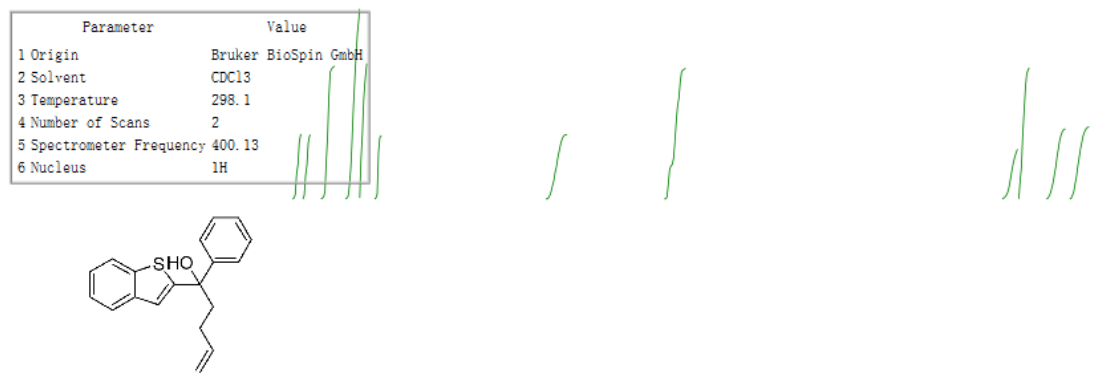

10
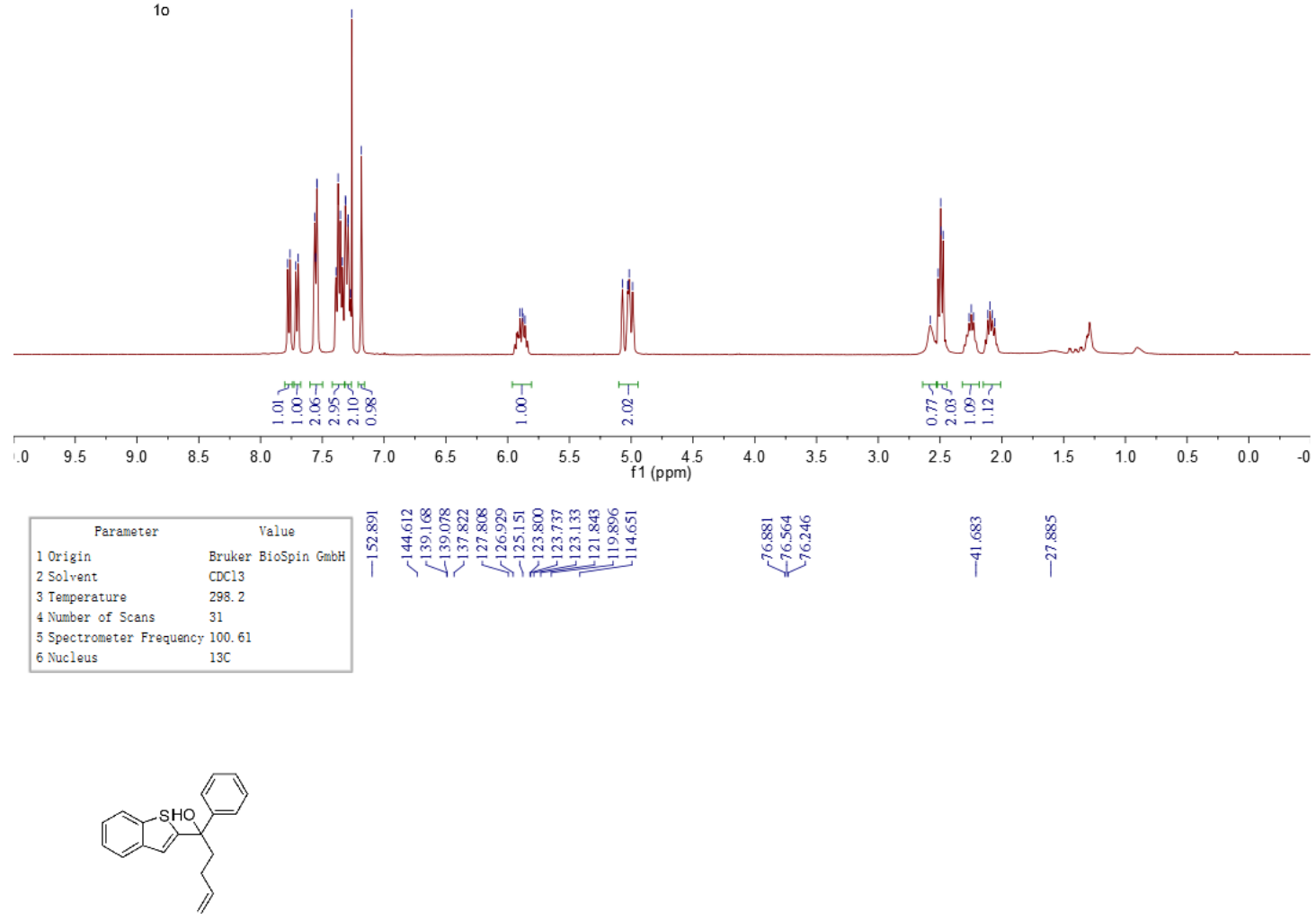

10

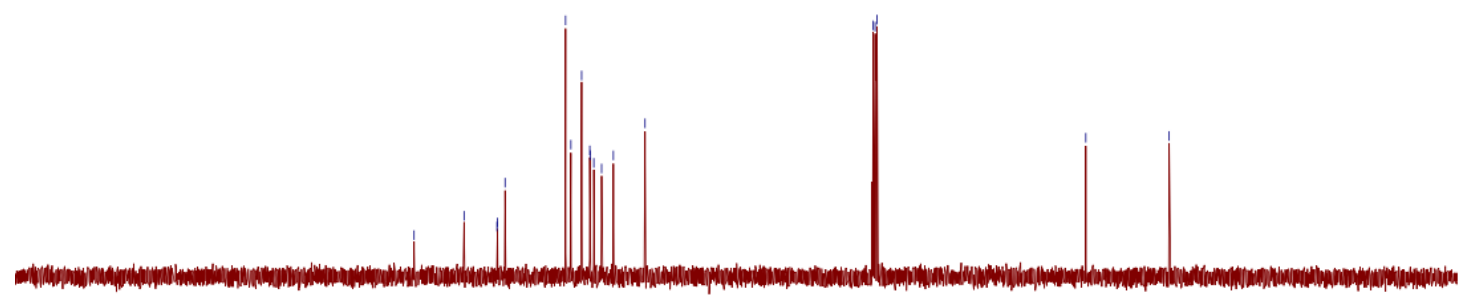

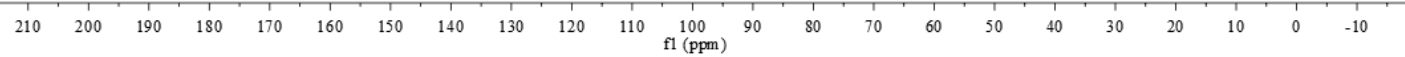




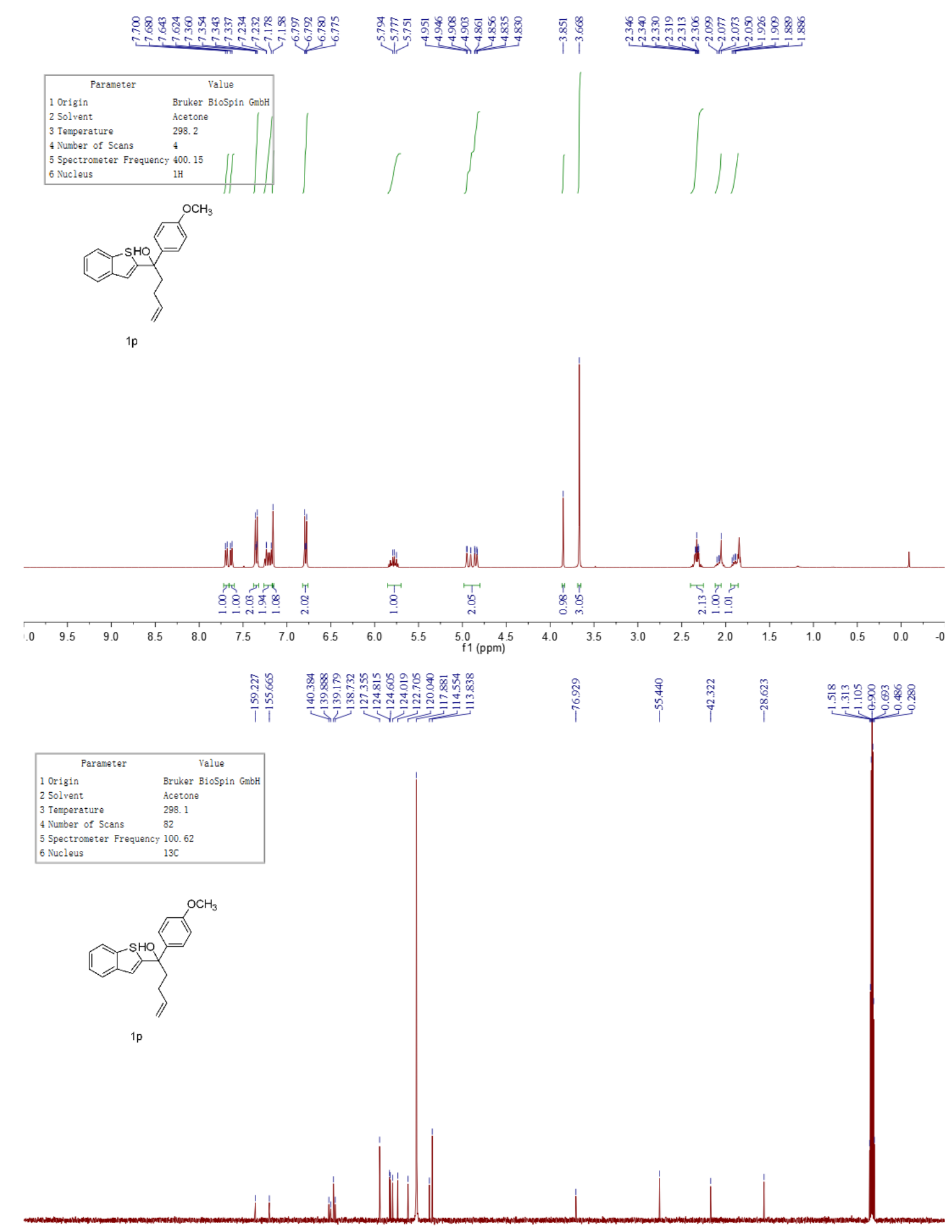

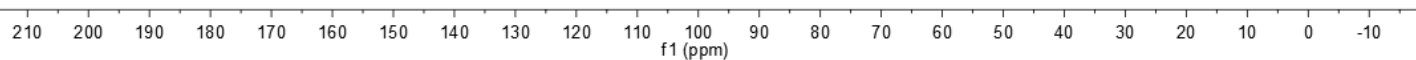



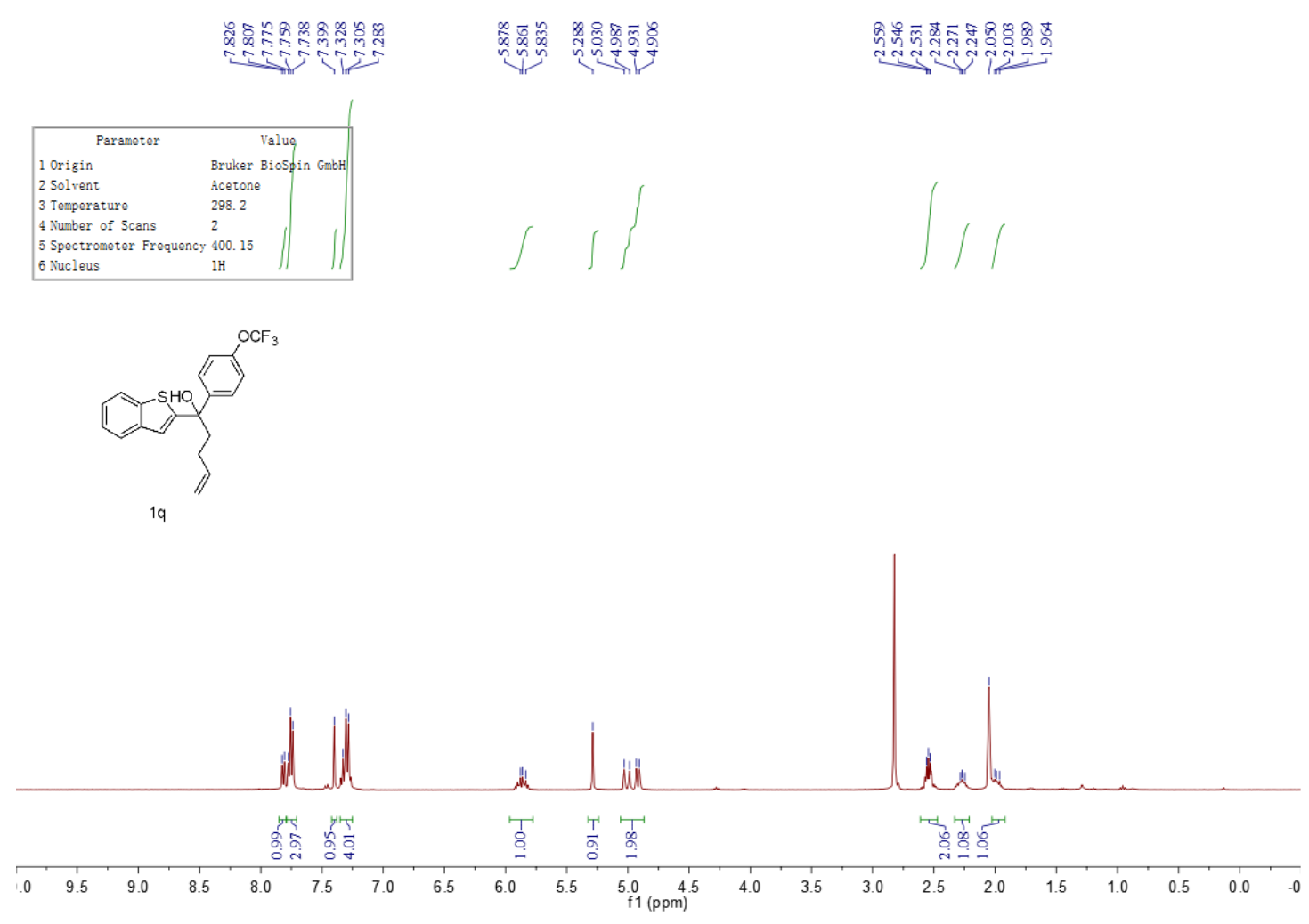

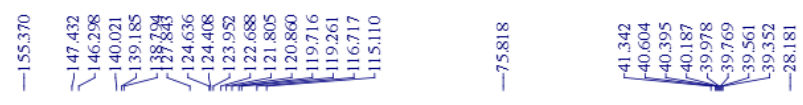

\begin{tabular}{|c|c|}
\hline Parameter & Value \\
\hline 1 0rigin & Bruker BioSpin GmbH \\
\hline 2 Solvent & DMSO \\
\hline 3 Temper ature & 298.2 \\
\hline 4 Humber of Scans & 2048 \\
\hline \multicolumn{2}{|c|}{5 Spectrometer Frequency 100.62} \\
\hline 6 Hucleus & $13 \mathrm{C}$ \\
\hline
\end{tabular}
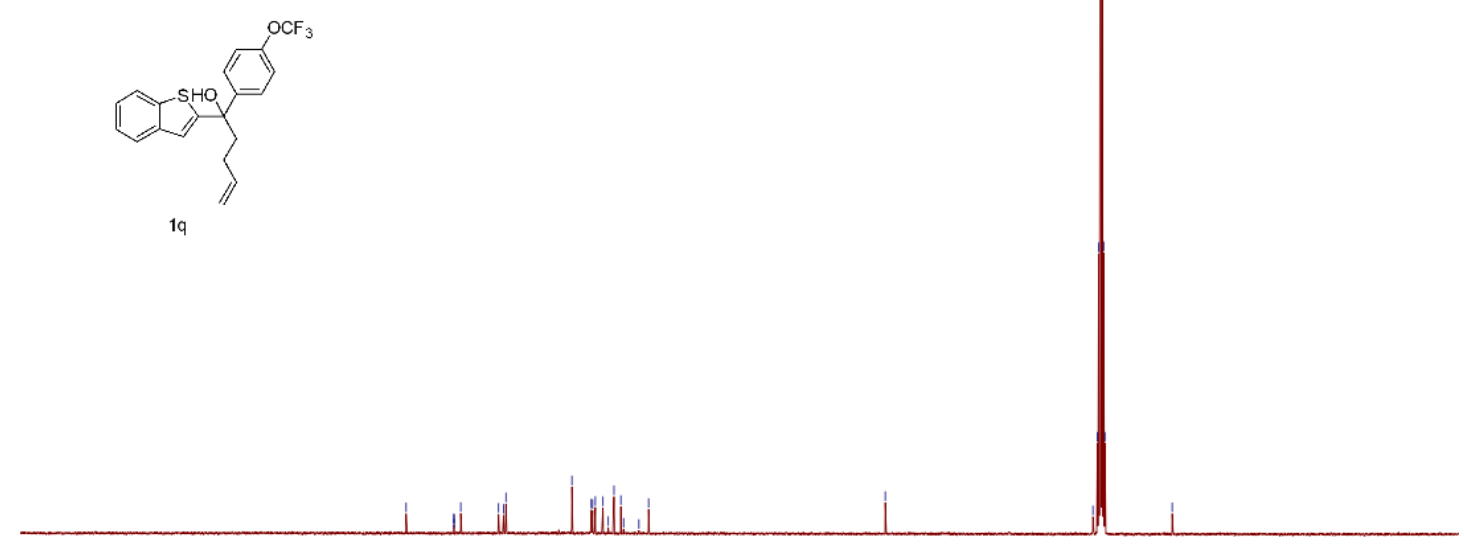

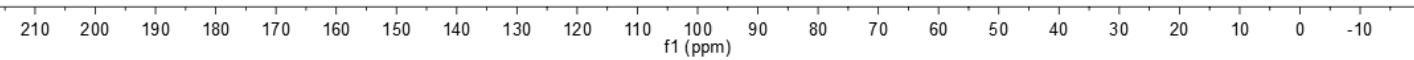




\begin{tabular}{|ll|}
\hline \multicolumn{1}{|c|}{ Parameter } & \multicolumn{1}{c|}{ Value } \\
1 Origin & Bruker Biospin GmbH \\
2 Solvent & Acetone \\
3 Temperature & 298.2 \\
4 Number of Scans & 2 \\
5 Spectrometer Frequency 376.52 \\
6 Nucleus & $19 \mathrm{~F}$ \\
\hline
\end{tabular}

6 Nucleus $\quad 19 \mathrm{~F}$

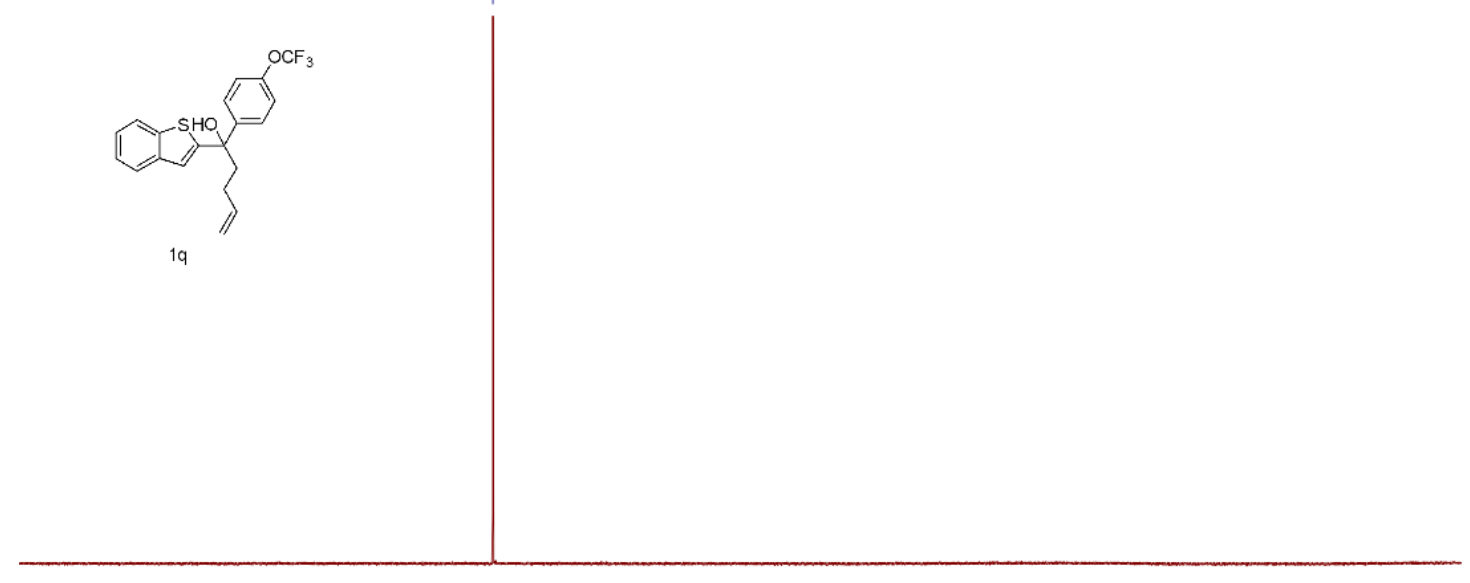

\begin{tabular}{lllllllllllllllllllllllllllll}
\hline 0 & 10 & 0 & -10 & -20 & -30 & -40 & -50 & -60 & -70 & -80 & -90 & -100 & -110 & -120 & -130 & -140 & -150 & -160 & -170 & -180 & -190 & -200 & -210 & $-2 c^{\prime}$
\end{tabular}

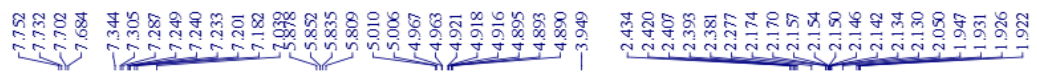
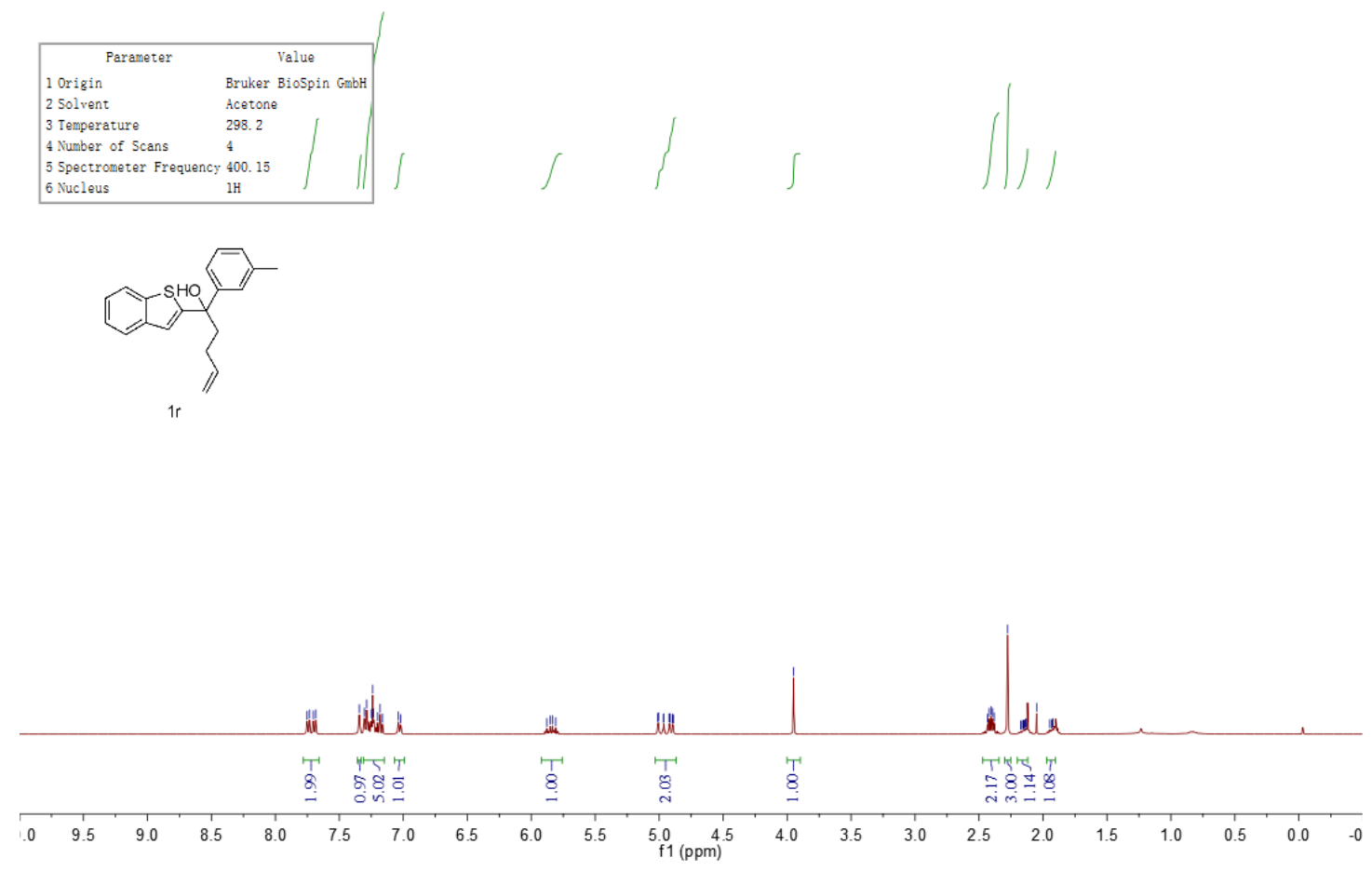


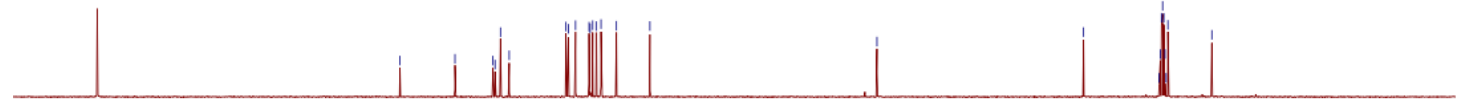

$\begin{array}{llllllllllllllllllllllll}210 & 200 & 190 & 180 & 170 & 160 & 150 & 140 & 130 & 120 & 110 & 100 & 90 & 80 & 70 & 60 & 50 & 40 & 30 & 20 & 10 & 0 & -10\end{array}$

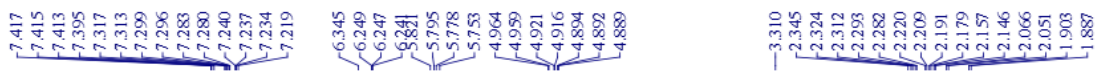

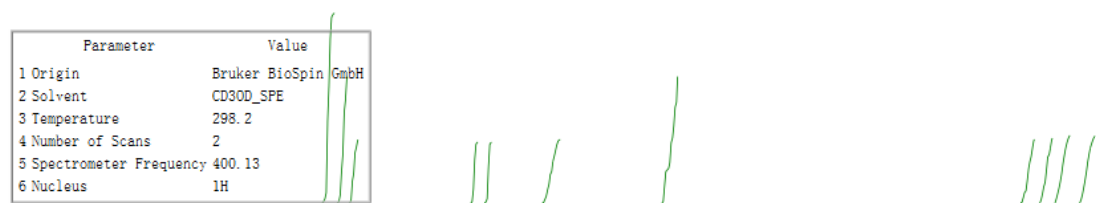

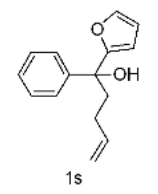

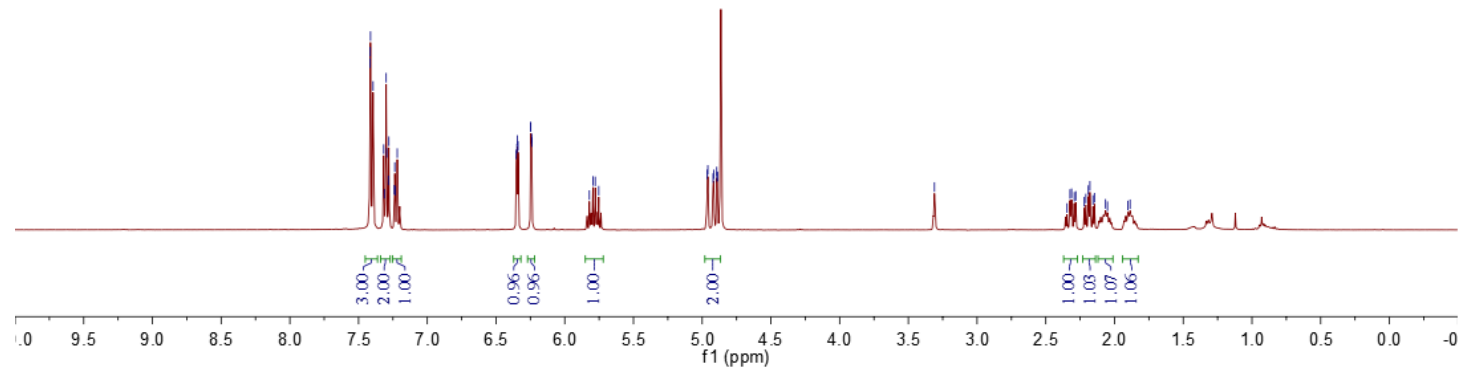




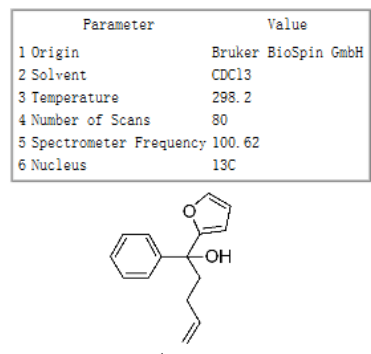

$1 \mathrm{~s}$

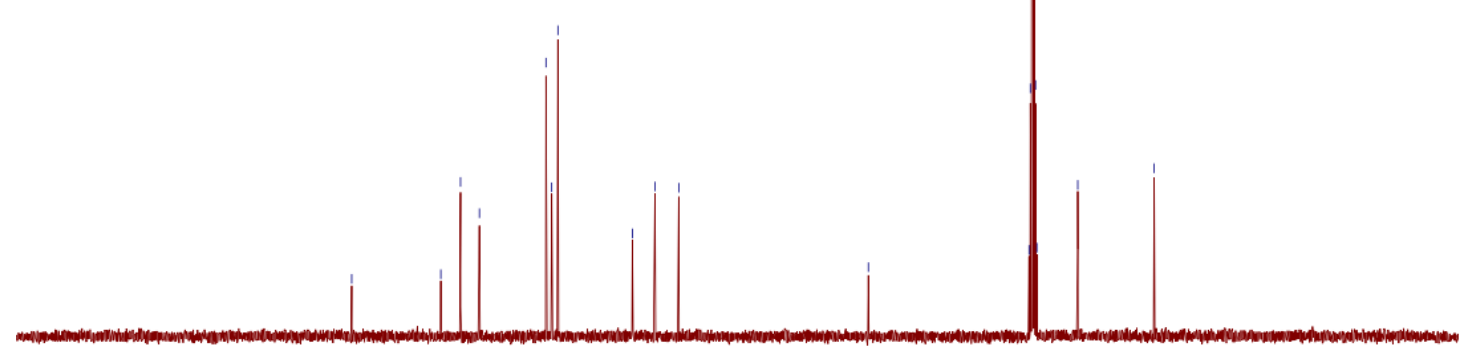

$\begin{array}{llllllllllllllllllllllll}210 & 200 & 190 & 180 & 170 & 160 & 150 & 140 & 130 & 120 & 110 & 100 & 90 & 80 & 70 & 60 & 50 & 40 & 30 & 20 & 10 & 0 & -10\end{array}$

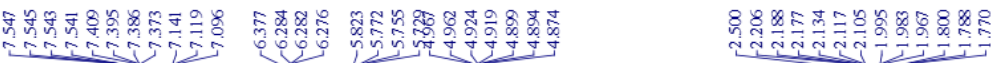

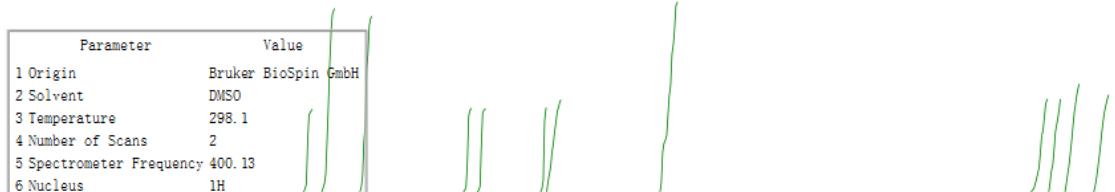

5 Spectrometer Frequency 400.

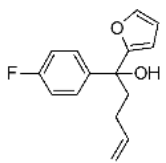

$1 \mathrm{t}$

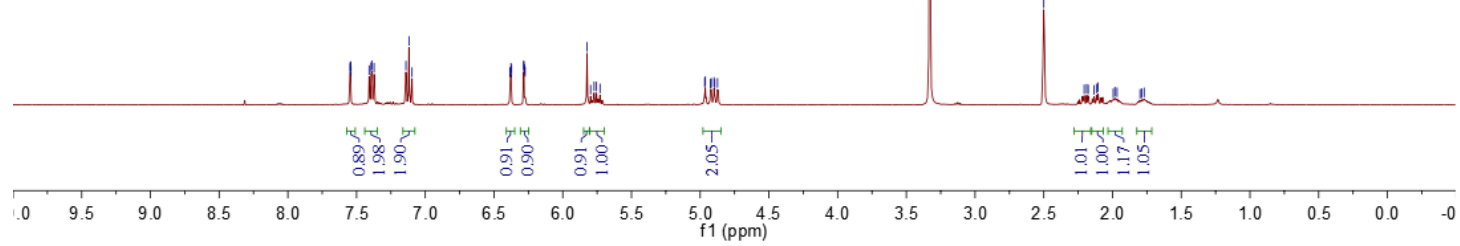




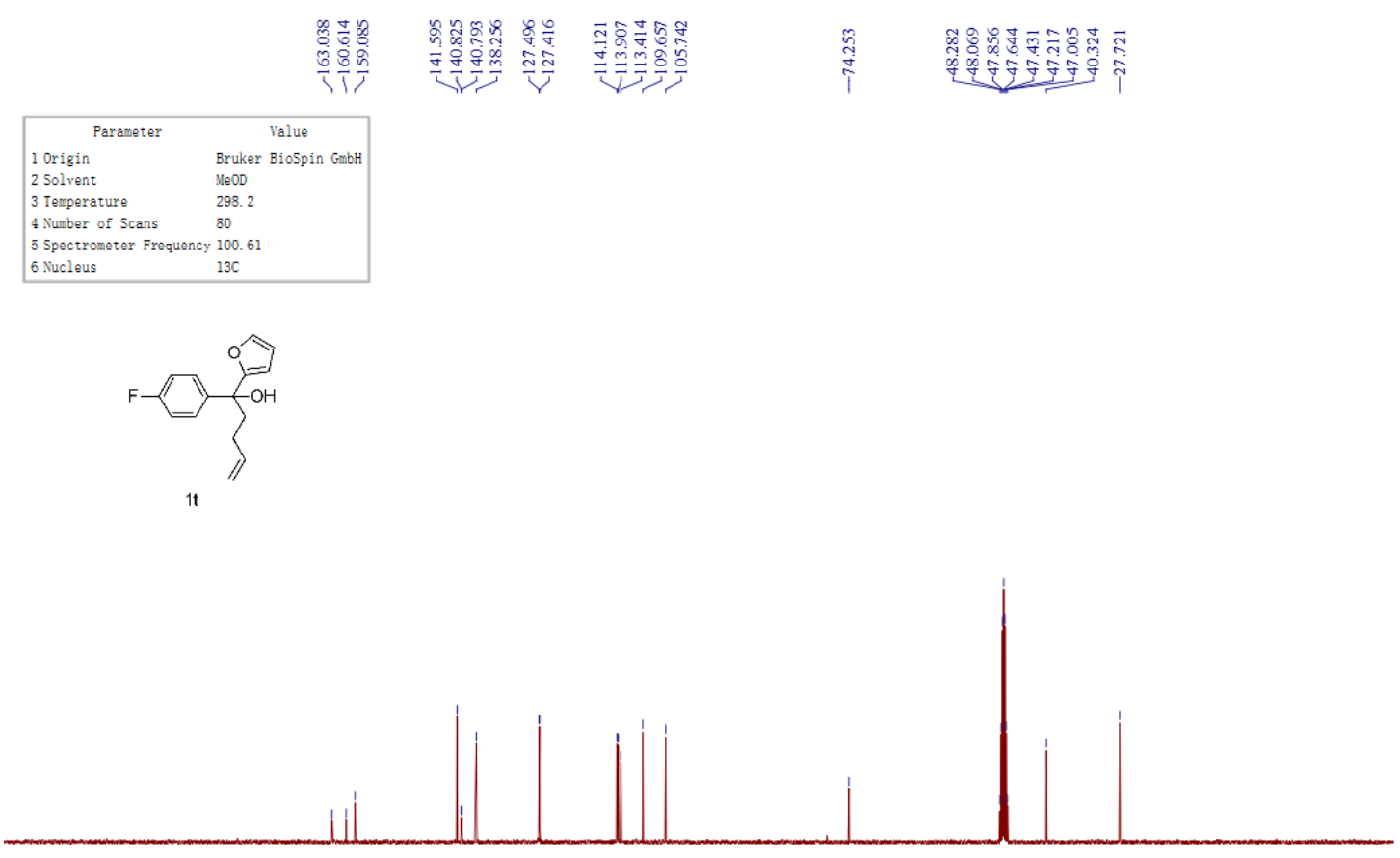

$\begin{array}{lllllllllllllllllllllllllll}1 & 10 & 210 & 190 & 180 & 170 & 160 & 150 & 140 & 130 & 120 & 110 & \underset{f 1}{100} & 90 & 80 & 70 & 60 & 50 & 40 & 30 & 20 & 10 & 0 & -10\end{array}$

$$
\begin{array}{|ll}
\hline \multicolumn{1}{|c}{\text { Parameter }} \\
\text { 1 Origin } & \text { Bruker } \\
\text { 2 S01vent } & \text { DISO } \\
\text { 3 Temperature } & \text { 298. } 2 \\
\text { 4 Number of Scans } & 2 \\
\text { 5 Spectrometer Frequency } 376.50 \\
\text { 6 Nucleus }
\end{array}
$$

$1 \mathbf{t}$
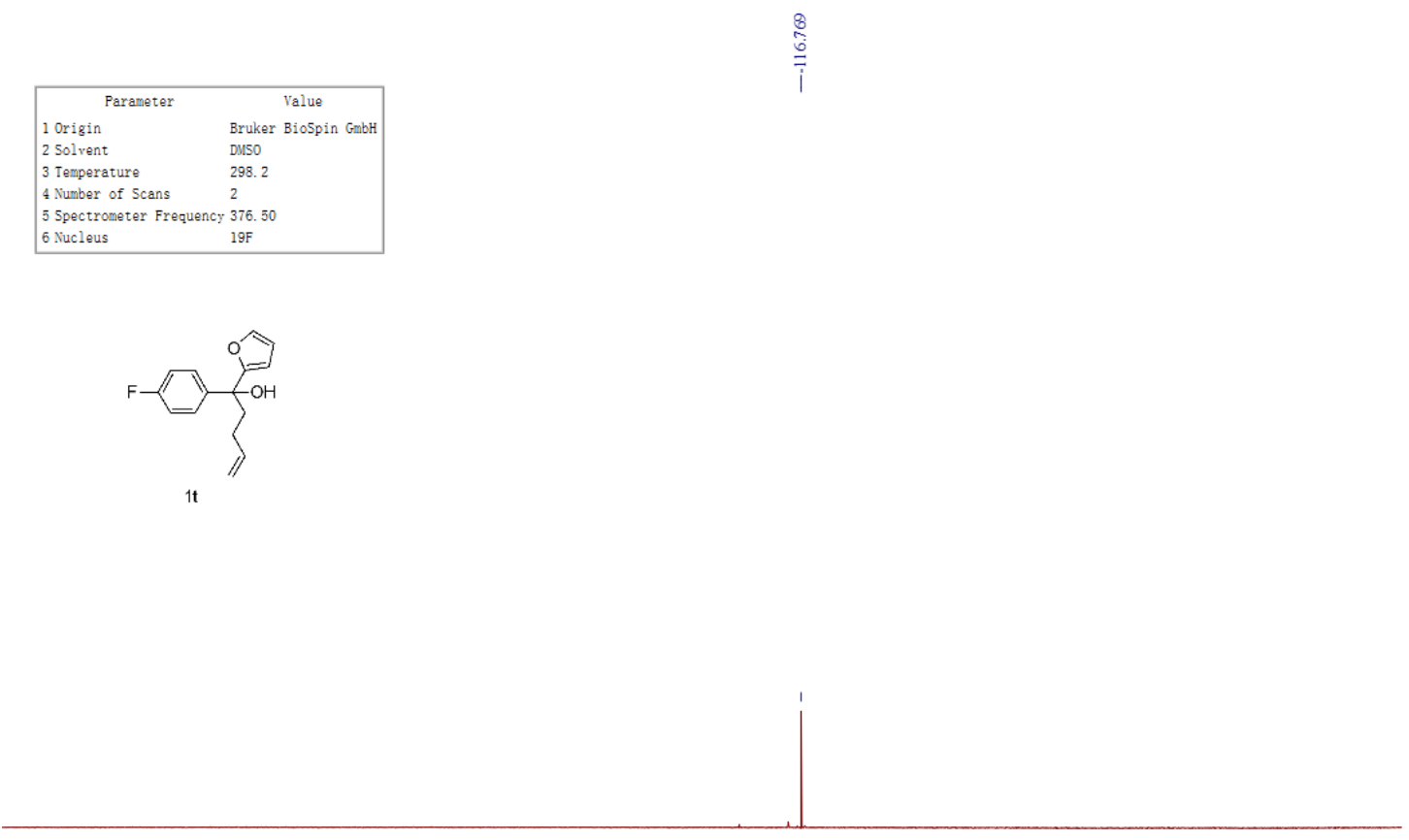

$\begin{array}{llllllllllllllllllllllllllll}10 & 0 & -10 & -20 & -30 & -40 & -50 & -60 & -70 & -80 & -90 & -100 & -110 & -120 & -130 & -140 & -150 & -160 & -170 & -180 & -190 & -200 & -210\end{array}$ 


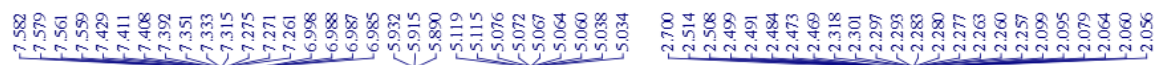
\begin{tabular}{|ll|}
\hline \multicolumn{1}{|c|}{ Parameter } & \multicolumn{1}{c|}{ Value } \\
1 Origin & Bruker BioSpin \\
2 Solvbh & \\
3 Tempert & CDC13 \\
4 Number of Scans & 298.1 \\
5 Spectrometer Frequency & 2 \\
6 Nucleus & 1H \\
\hline
\end{tabular}

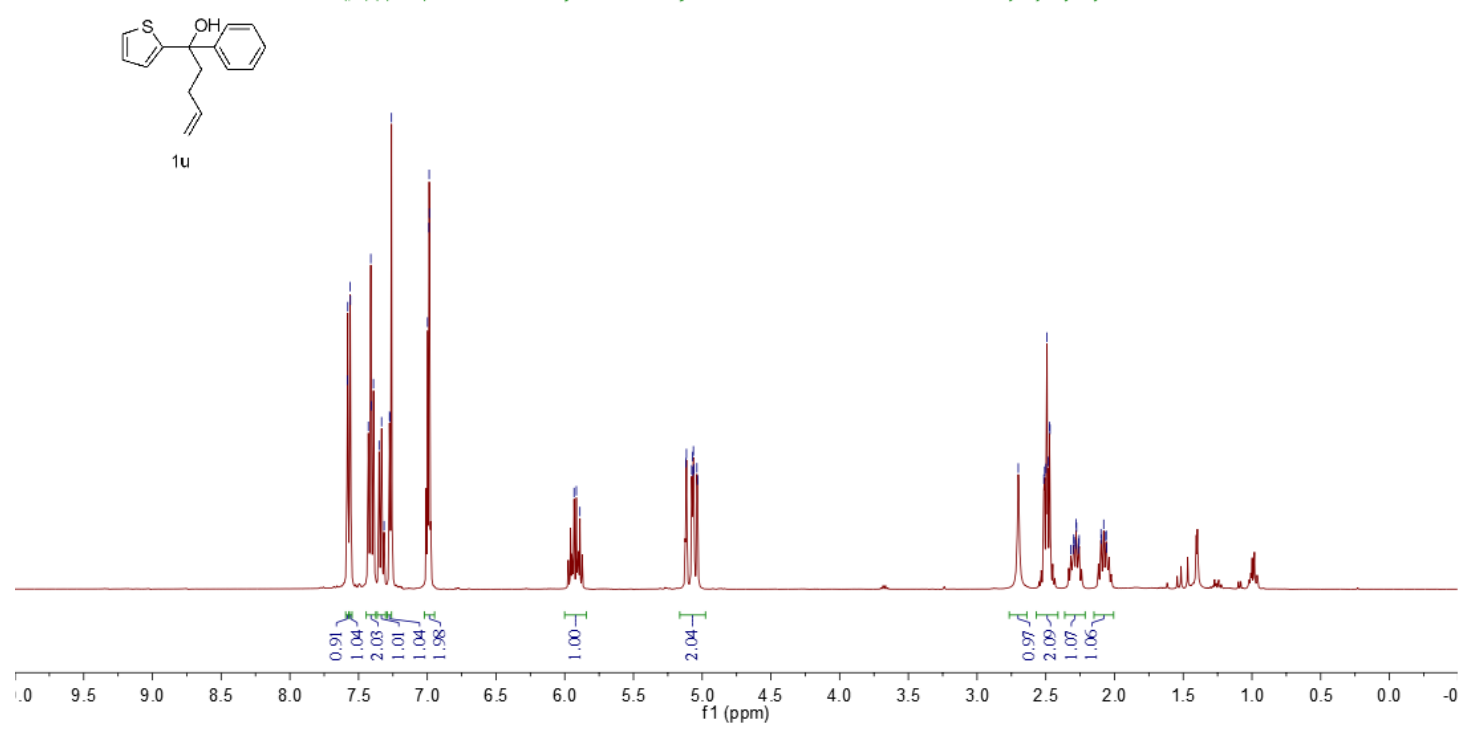

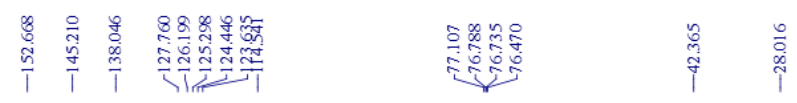

\begin{tabular}{|ll|}
\hline \multicolumn{1}{|c|}{ Parameter } & \multicolumn{1}{c|}{ Value } \\
1 Origin & Bruker BioSpin GmbH \\
2 Solvent & CDC13 \\
3 Temperature & 298.3 \\
4 Number of Scans & 5 \\
5 Spectrometer Frequency & 100.61 \\
6 Nucleus & $13 \mathrm{C}$ \\
\hline
\end{tabular}<smiles>C=CCCC(O)(c1ccccc1)c1cccs1</smiles>

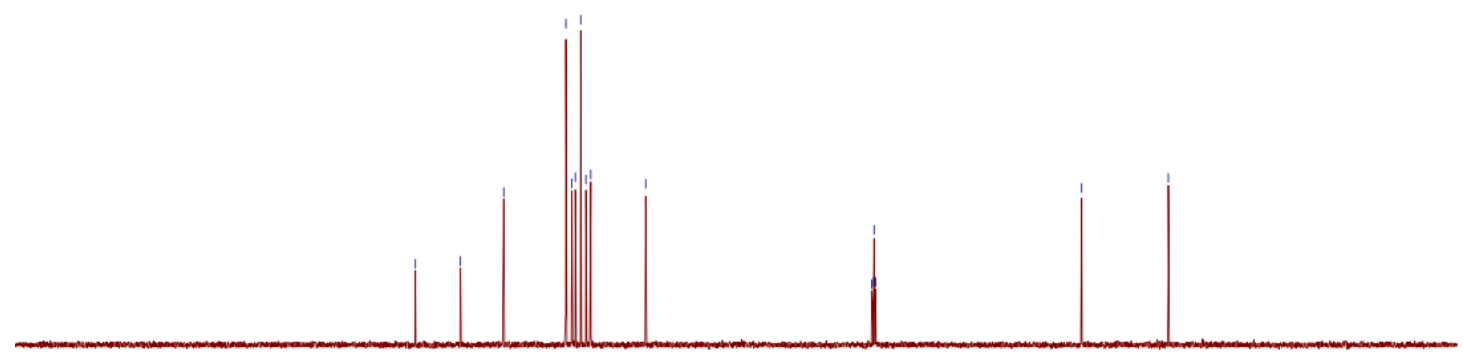

$\begin{array}{llllllllllllllllllllllllll}210 & 200 & 190 & 180 & 170 & 160 & 150 & 140 & 130 & 120 & 110 & 100 & 90 & 80 & 70 & 60 & 50 & 40 & 30 & 20 & 10 & 0 & -10\end{array}$ 


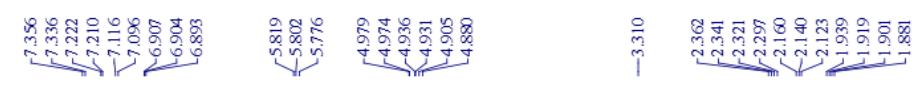
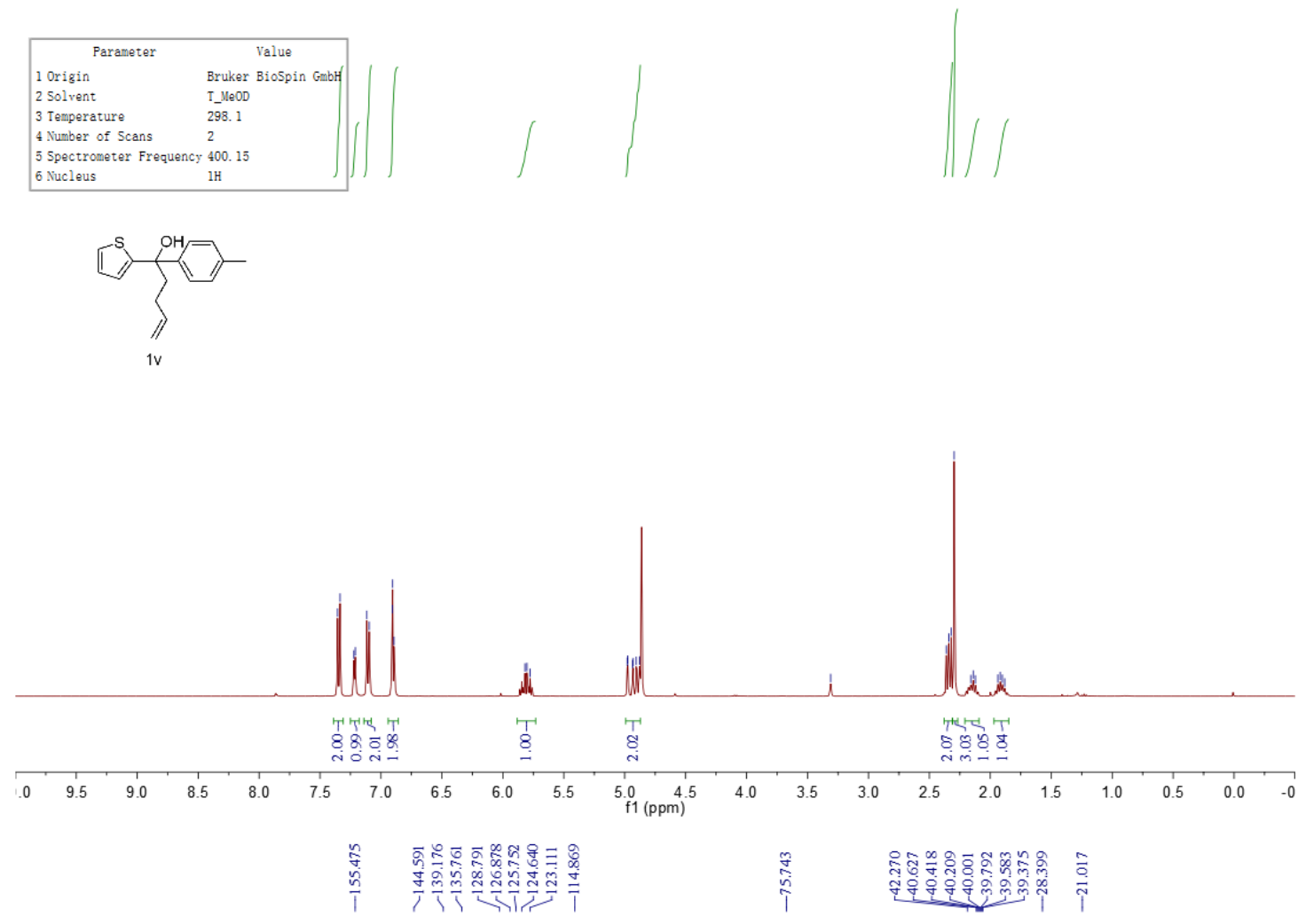

\begin{tabular}{|ll|}
\hline \multicolumn{1}{|c|}{ Parameter } & \multicolumn{1}{c|}{ Value } \\
1 Origin & Bruker BioSpin GmbH \\
2 Solvent & DMSO \\
3 Temperature & 298.1 \\
4 Number of Scans & 1024 \\
5 Spectrometer Frequency & 100.62 \\
6 Nucleus & $13 \mathrm{C}$ \\
\hline
\end{tabular}
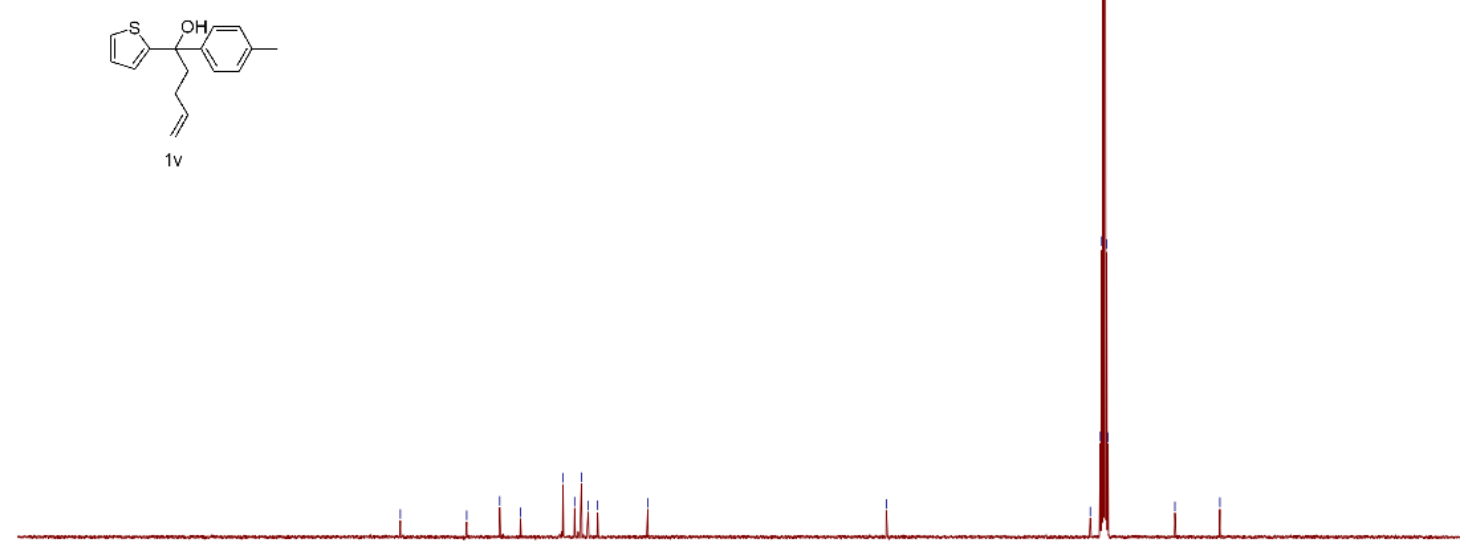

$\begin{array}{llllllllllllllllllllllllllllll}1 & 210 & 200 & 190 & 180 & 170 & 160 & 150 & 140 & 130 & 120 & 110 & 100 & 90 & 80 & 70 & 60 & 50 & 40 & 30 & 20 & 10 & 0 & -10\end{array}$ 


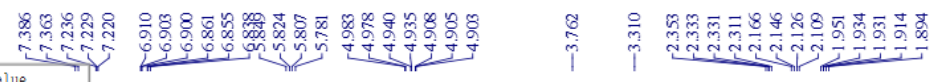
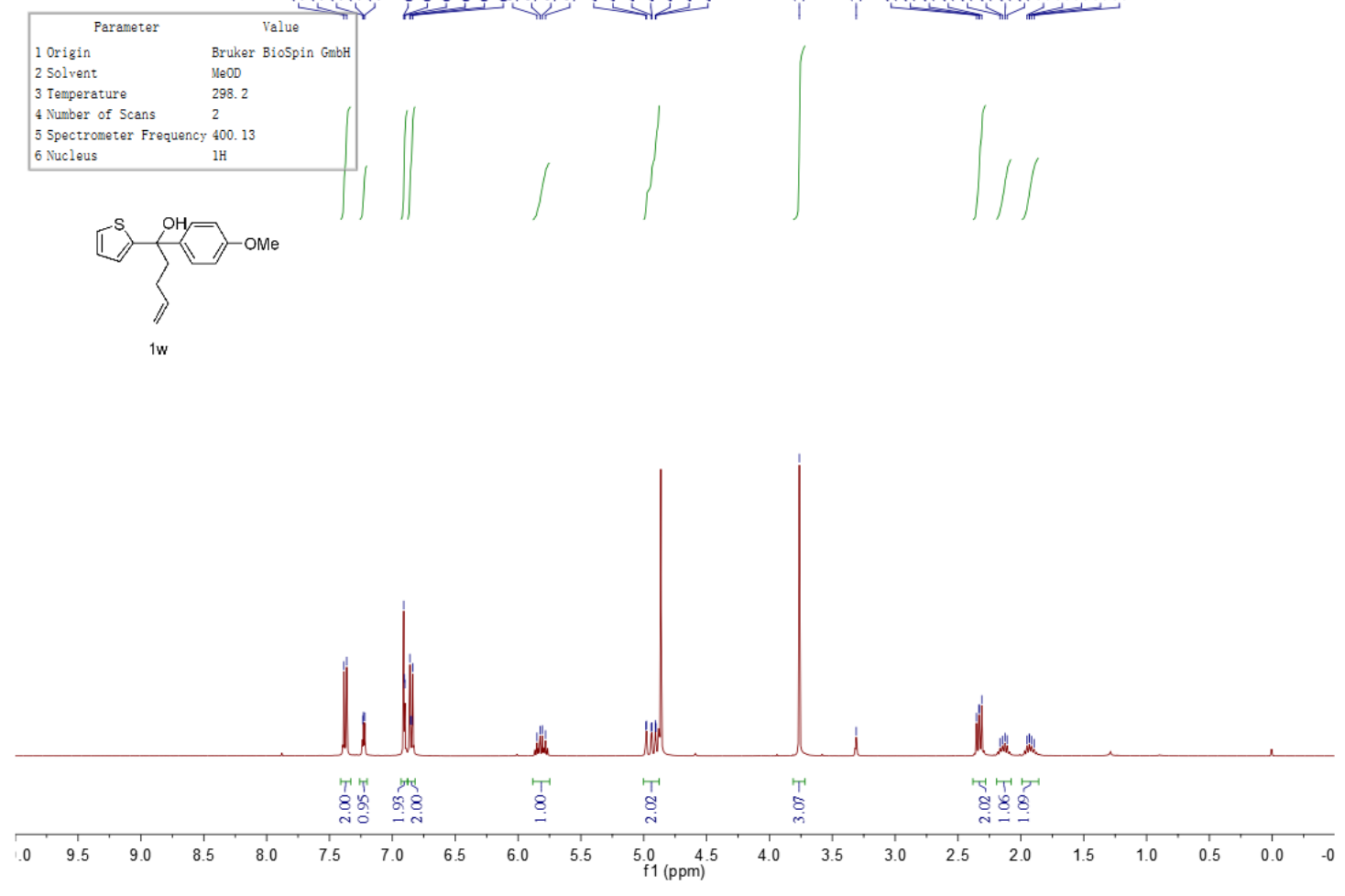

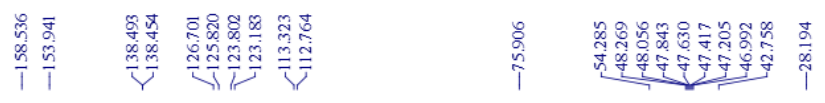

\begin{tabular}{|ll|}
\hline \multicolumn{1}{|c|}{ Parameter } & \multicolumn{1}{c|}{ Value } \\
1 Origin & Bruker BioSpin GmbH \\
2 Solvent & MeOD \\
3 Temperature & 298.1 \\
4 Number of Scans & 1024 \\
5 Spectrometer Frequency & 100.62 \\
6 Nucleus & $13 \mathrm{C}$ \\
\hline
\end{tabular}

[1 $\rangle\langle\langle-\mathrm{OMe}$

$1 w$

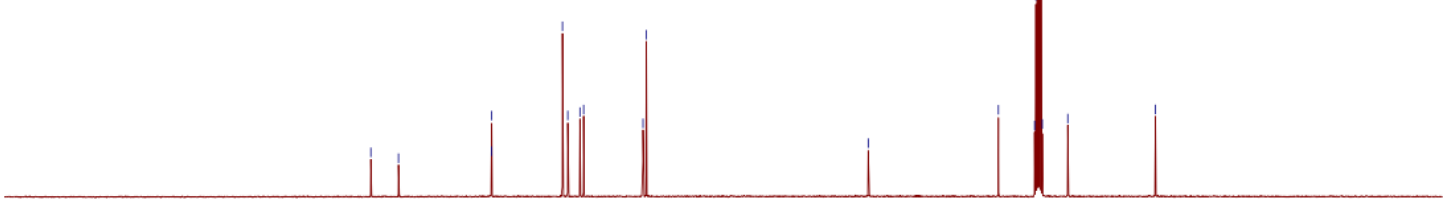

$\begin{array}{lllllllllllllllllllllllllll}210 & 200 & 190 & 180 & 170 & 160 & 150 & 140 & 130 & 120 & 110 & \begin{array}{c}100 \\ \mathrm{f} 1(\mathrm{ppm})\end{array} & 90 & 80 & 70 & 60 & 50 & 40 & 30 & 20 & 10 & 0 & -10\end{array}$ 


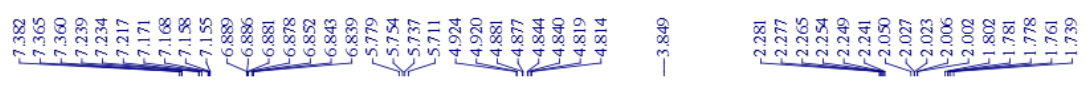
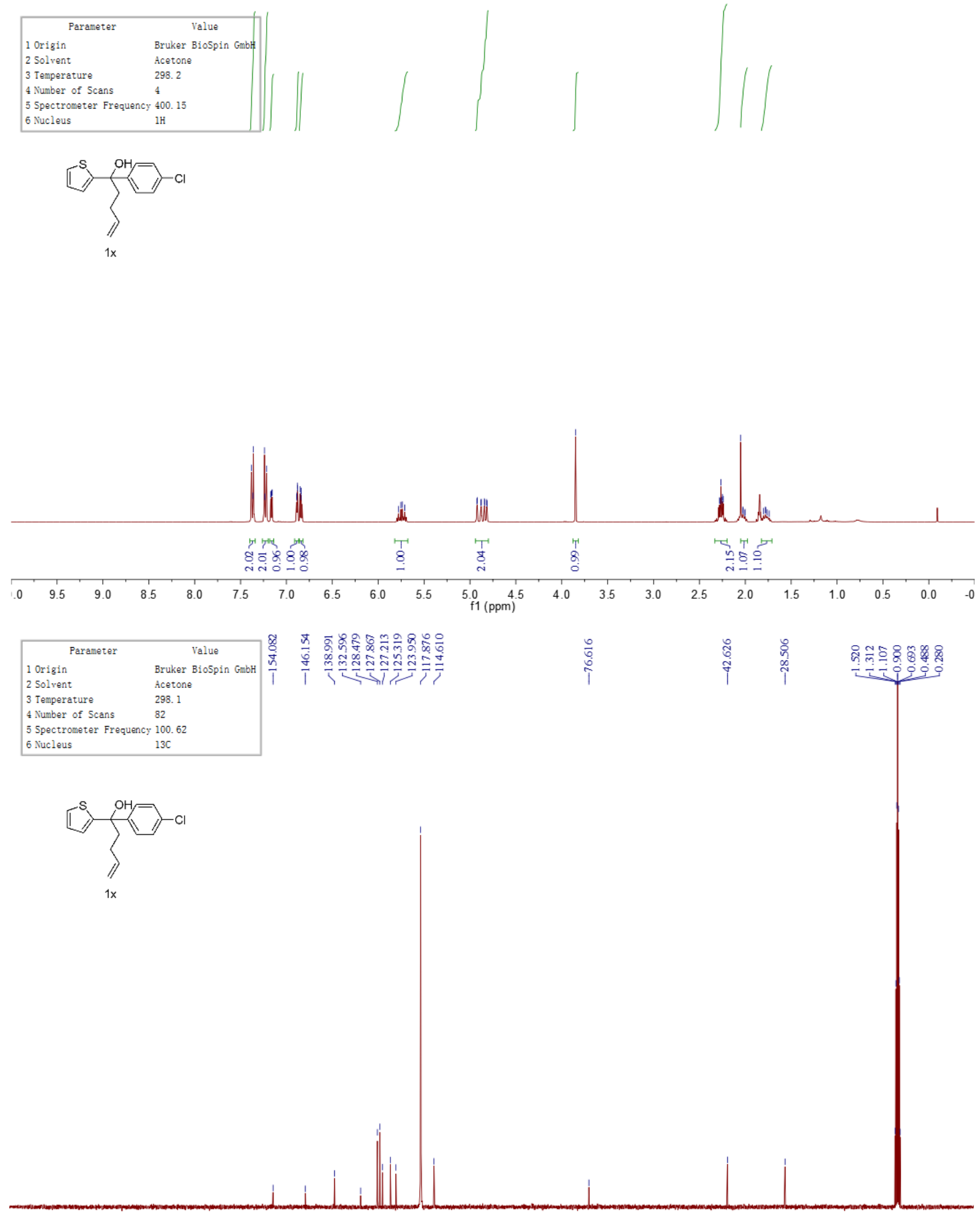

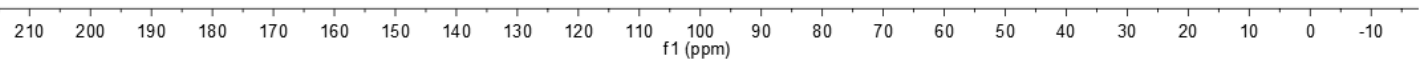




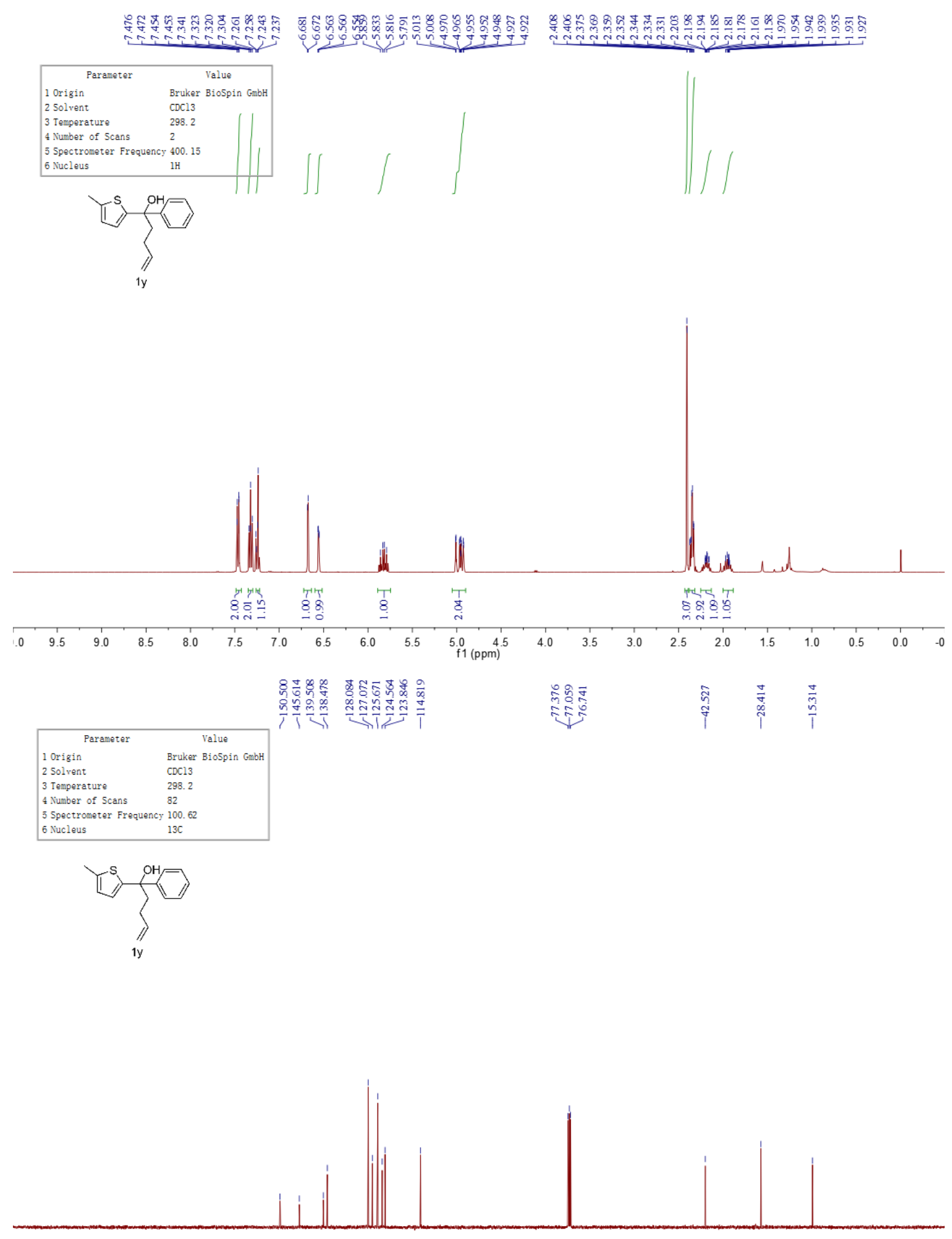

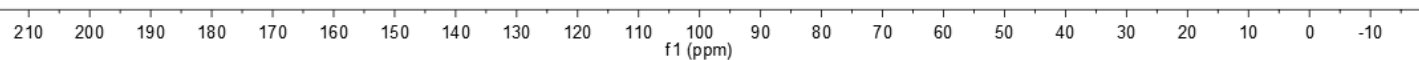




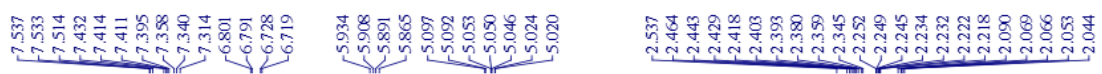
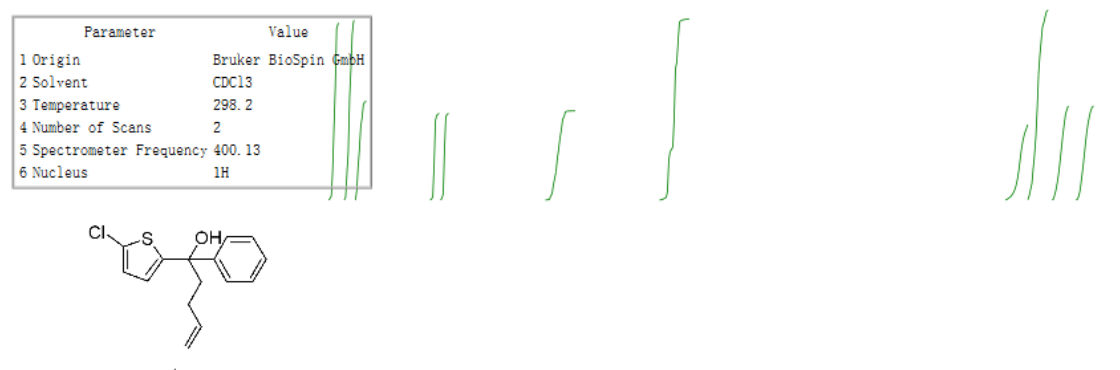

$1 z$
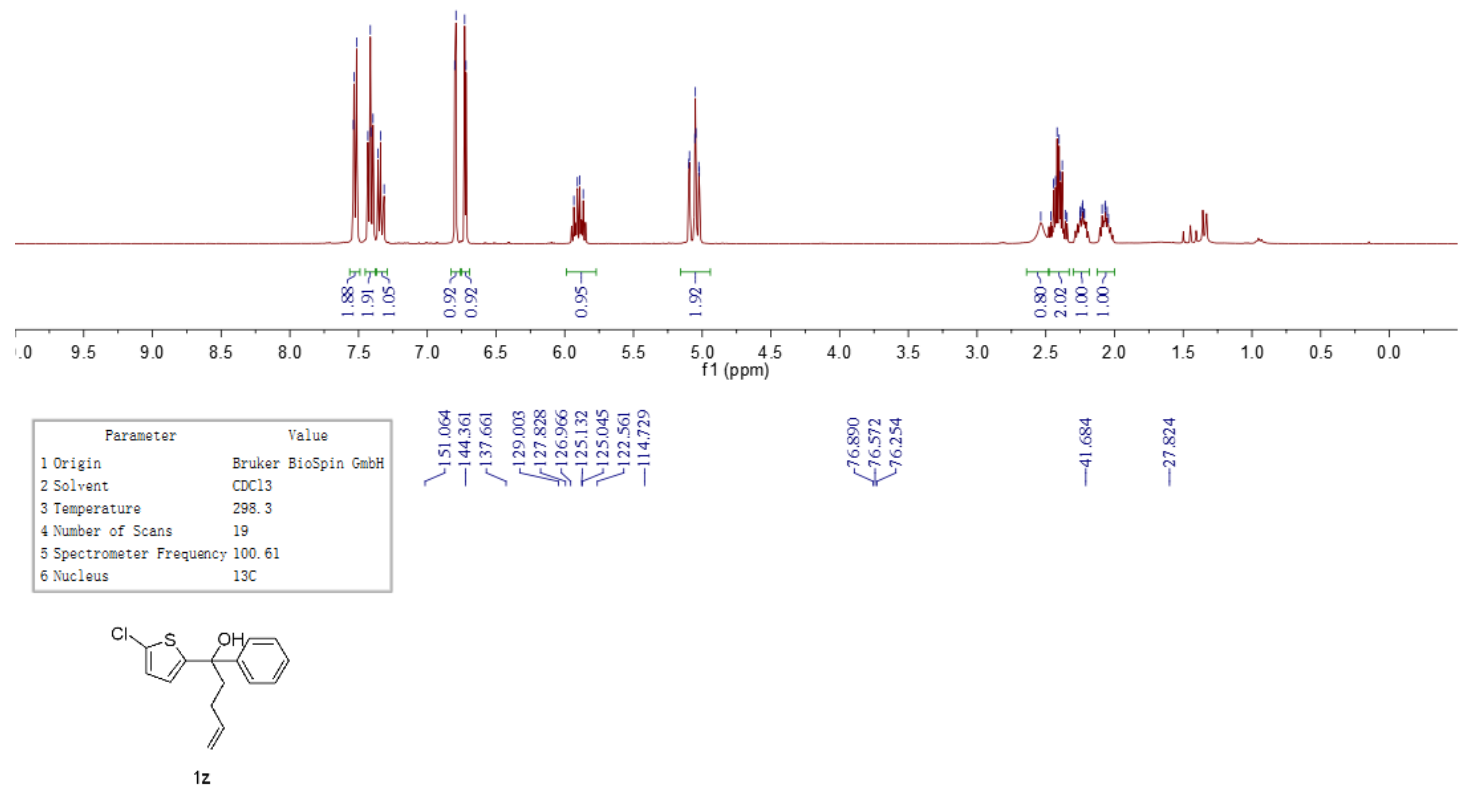

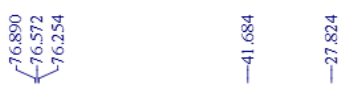

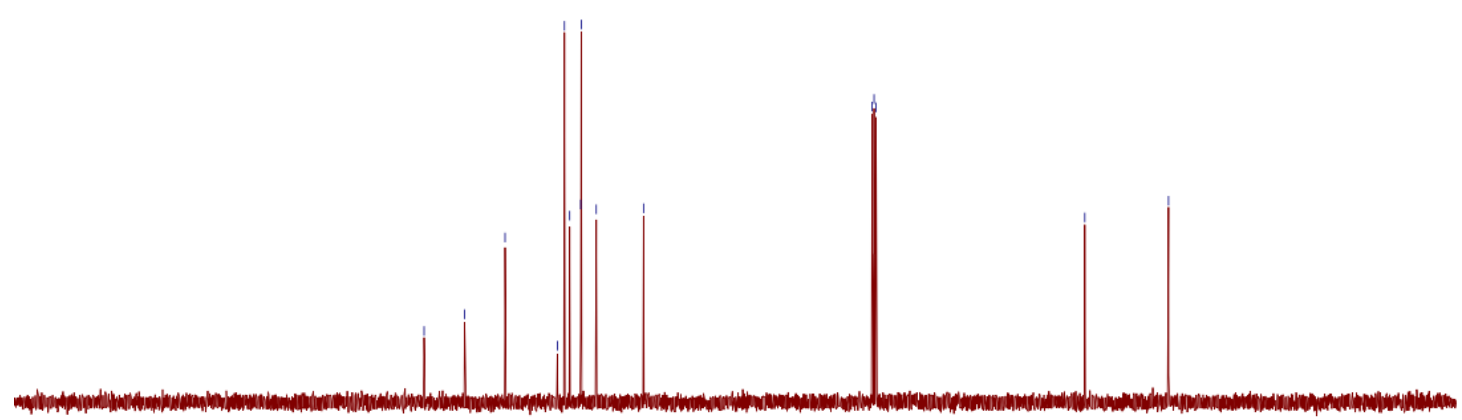

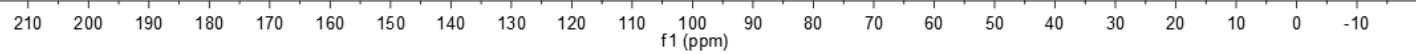




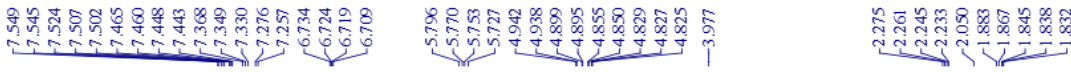
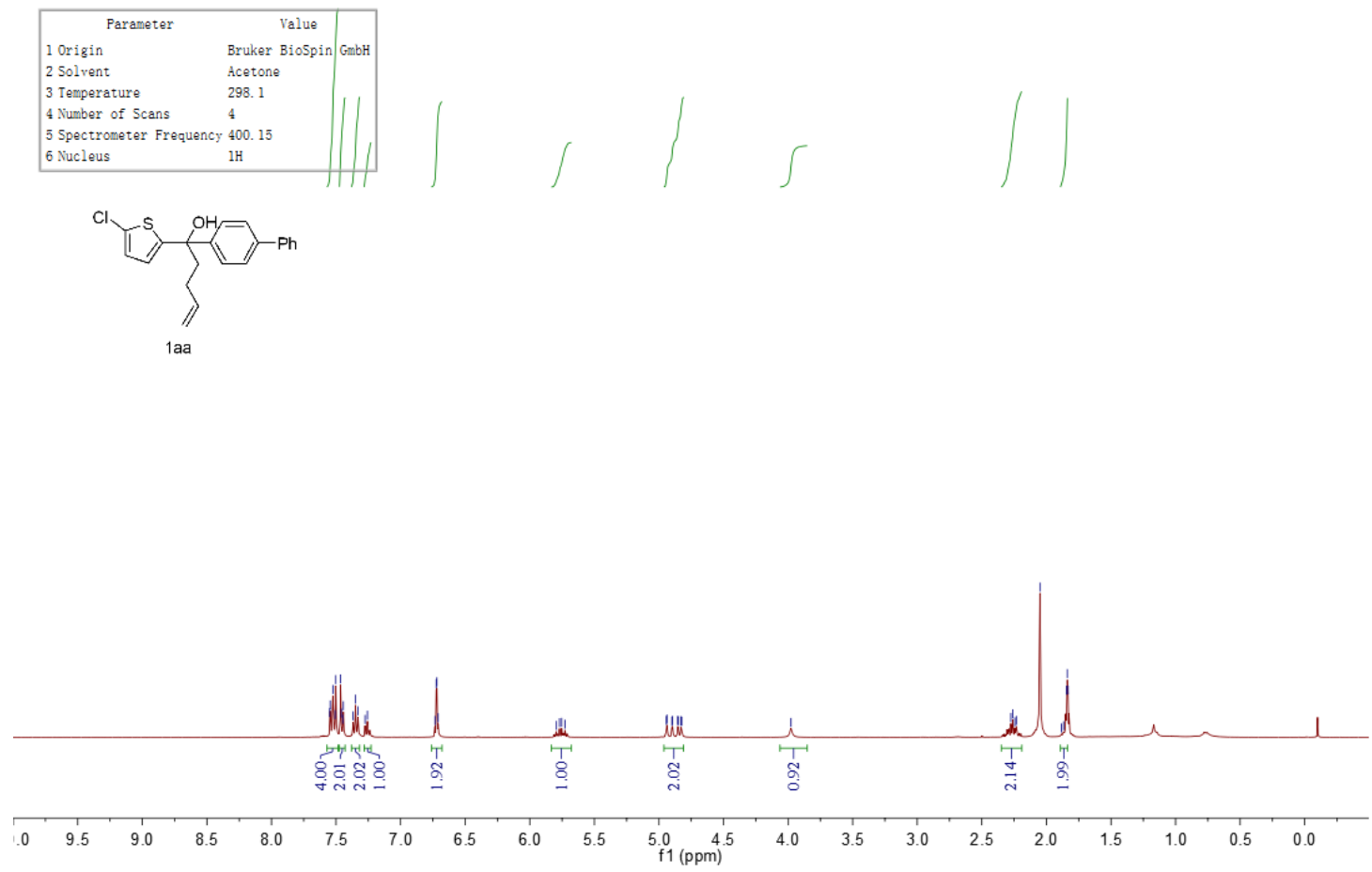

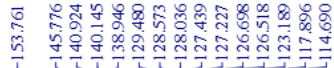

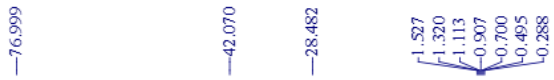

\begin{tabular}{|ll|}
\hline \multicolumn{1}{|c|}{ Parameter } & \multicolumn{1}{c|}{ Value } \\
1 Origin & Bruker BioSpin GmbH \\
2 Solvent & Acetone \\
3 Temperature & 298.1 \\
4 Number of Scans & 512 \\
5 Spectrometer Frequency 100.62 \\
6 Nucleus & $13 \mathrm{C}$ \\
\hline
\end{tabular}

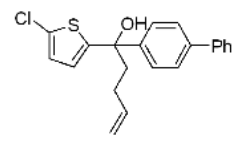

1 aa

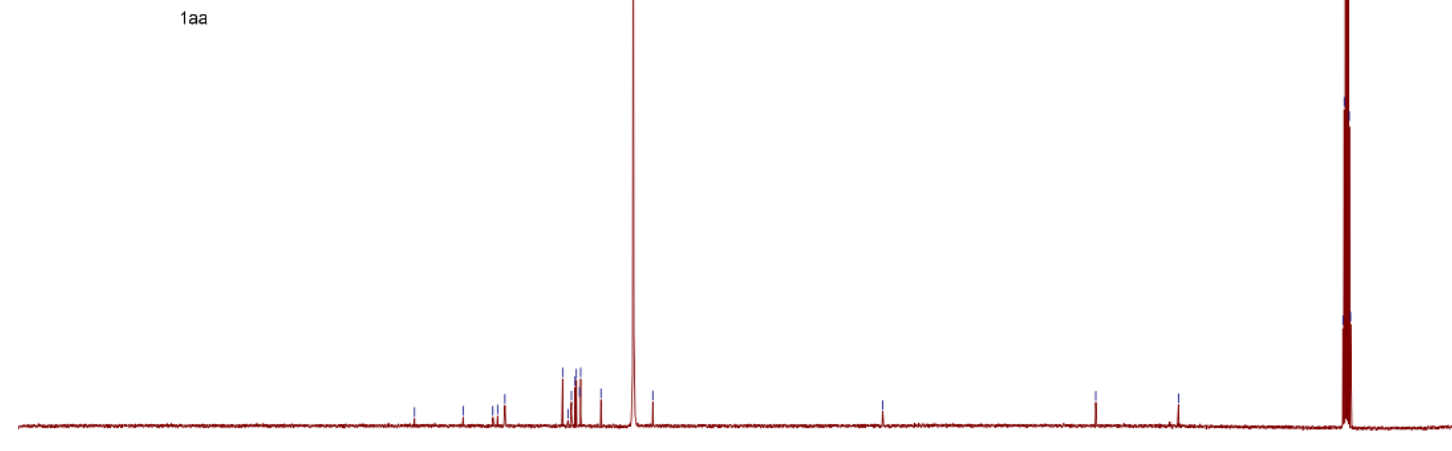

$\begin{array}{lllllllllllllllllllllllll}210 & 200 & 190 & 180 & 170 & 160 & 150 & 140 & 130 & 120 & 110 & 100 & 90 & 80 & 70 & 60 & 50 & 40 & 30 & 20 & 10 & 0 & -10\end{array}$ 


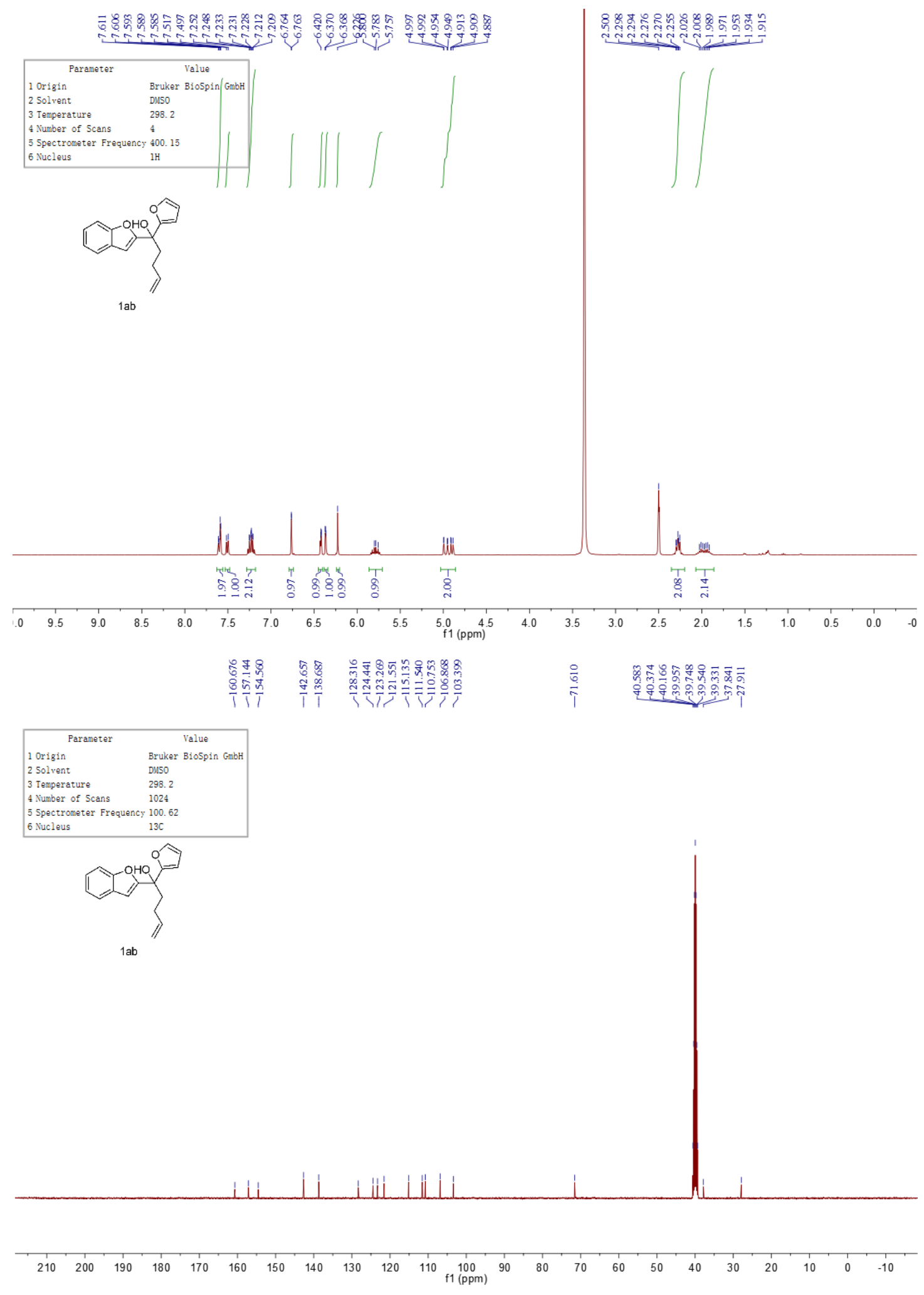




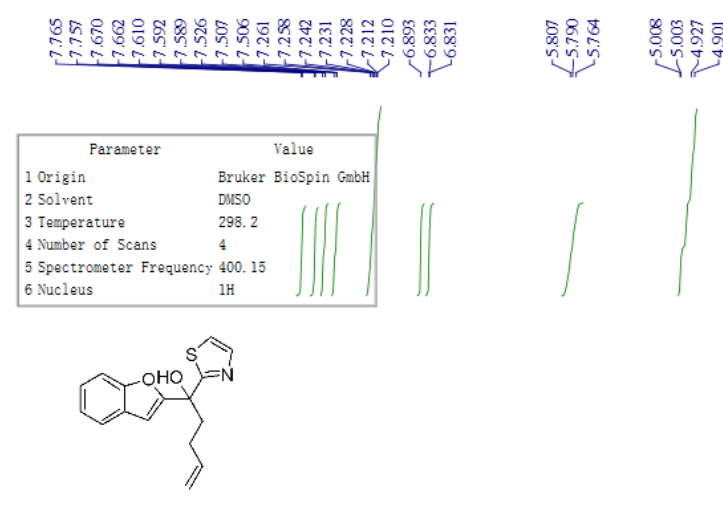

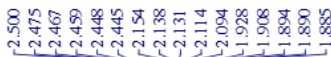

1 ac

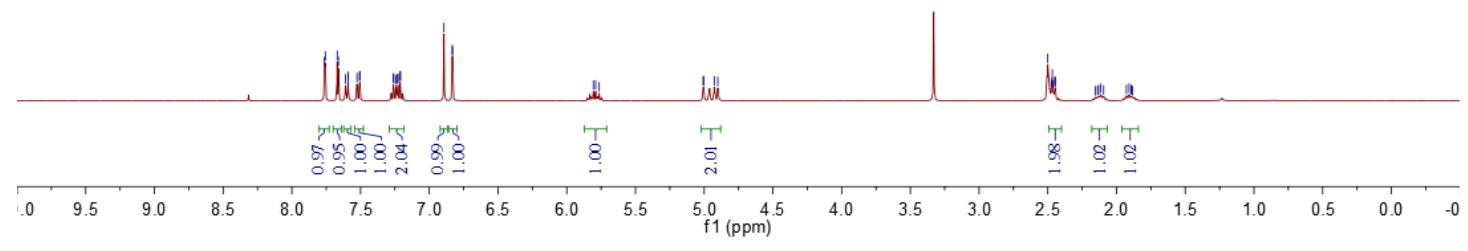

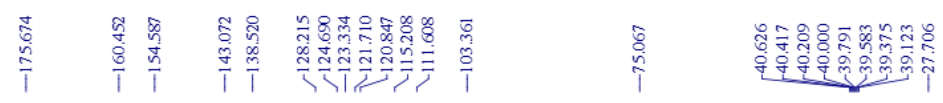

\begin{tabular}{|ll|}
\hline \multicolumn{1}{|c|}{ Parameter } & \multicolumn{1}{c|}{ Value } \\
1 Origin & Bruker Biospin GmbH \\
2 Solvent & DMSO \\
3 Temperature & 298.2 \\
4 Number of Scans & 82 \\
5 Spectrometer Frequency & 100.62 \\
6 Nucleus & $13 \mathrm{C}$ \\
\hline
\end{tabular}

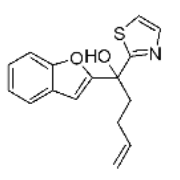

1 ac

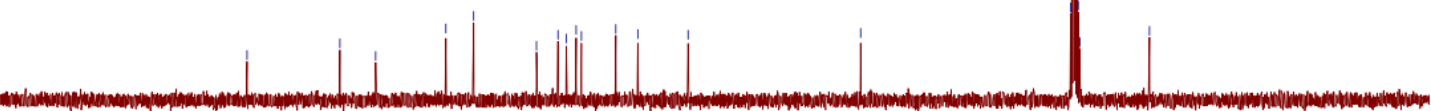

$\begin{array}{llllllllllllllllllllllllll}210 & 200 & 190 & 180 & 170 & 160 & 150 & 140 & 130 & 120 & 110 & 100 & 90 & 80 & 70 & 60 & 50 & 40 & 30 & 20 & 10 & 0 & -10\end{array}$ 


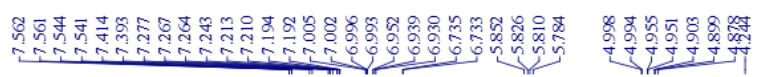

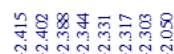

\begin{tabular}{|ll|}
\hline \multicolumn{1}{|c|}{ Parameter } & \multicolumn{1}{c|}{ Value } \\
1 Origin & Bruker BioSpin GmbH \\
2 Solvent & Acetone \\
3 Temperature & 298.2 \\
4 Number of Scans & 4 \\
5 5pectrometer Frequency & 400.15 \\
6 Nucleus & 1H
\end{tabular}||||||

10.

\|\|

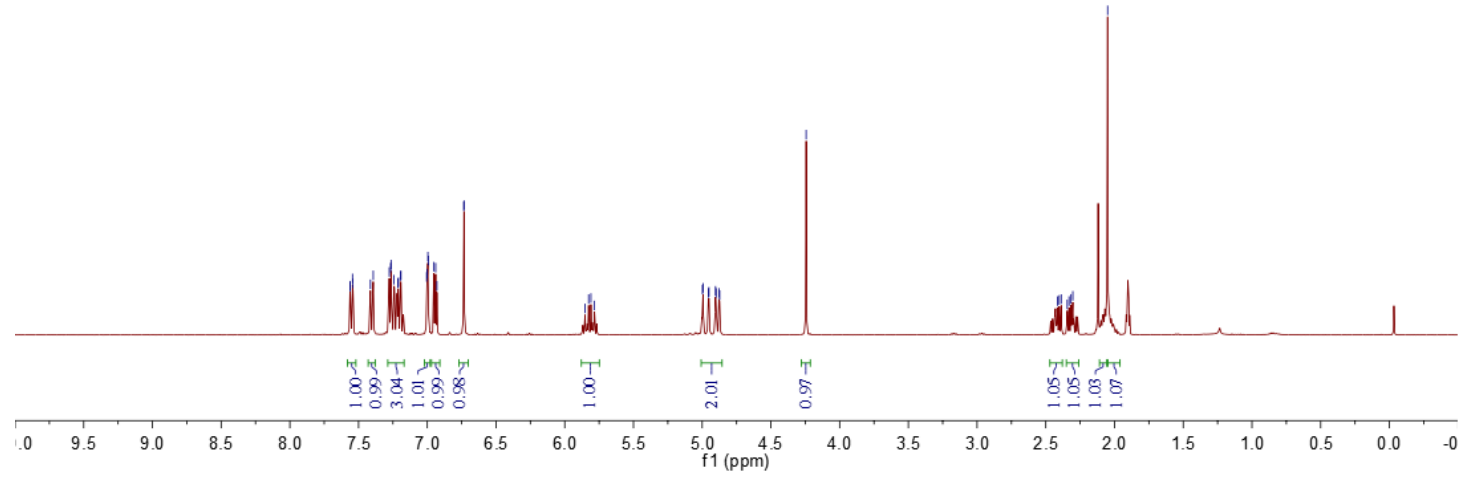

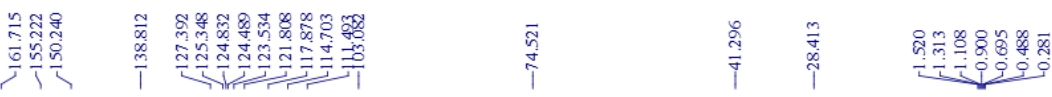

\begin{tabular}{|c|c|}
\hline Parameter & Value \\
\hline 1 Origin & Bruker Biospin GmbH \\
\hline 2 Solvent & Acetone \\
\hline 3 Temperature & 298.1 \\
\hline 4 Number of Scans & 82 \\
\hline 5 Spectrometer Frequency & 100.62 \\
\hline 6 Nucleus & $13 \mathrm{C}$ \\
\hline
\end{tabular}

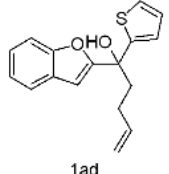

$1 \mathrm{ad}$

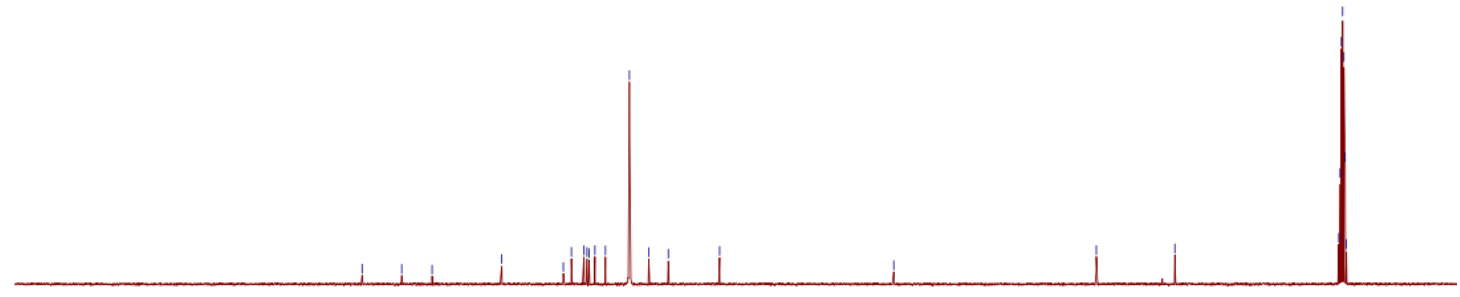

$\begin{array}{lllllllllllllllllllllll}210 & 200 & 190 & 180 & 170 & 160 & 150 & 140 & 130 & 120 & 110 & 100 & 90 & 80 & 70 & 60 & 50 & 40 & 30 & 20 & 10 & 0 & -10\end{array}$ 


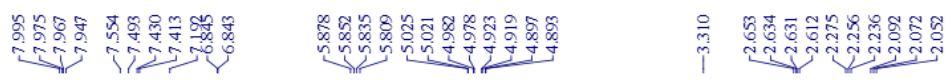

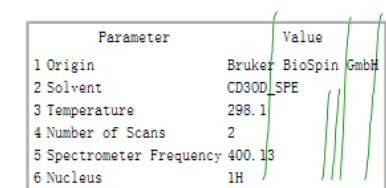

6 Nuclemeter Frequency 400.

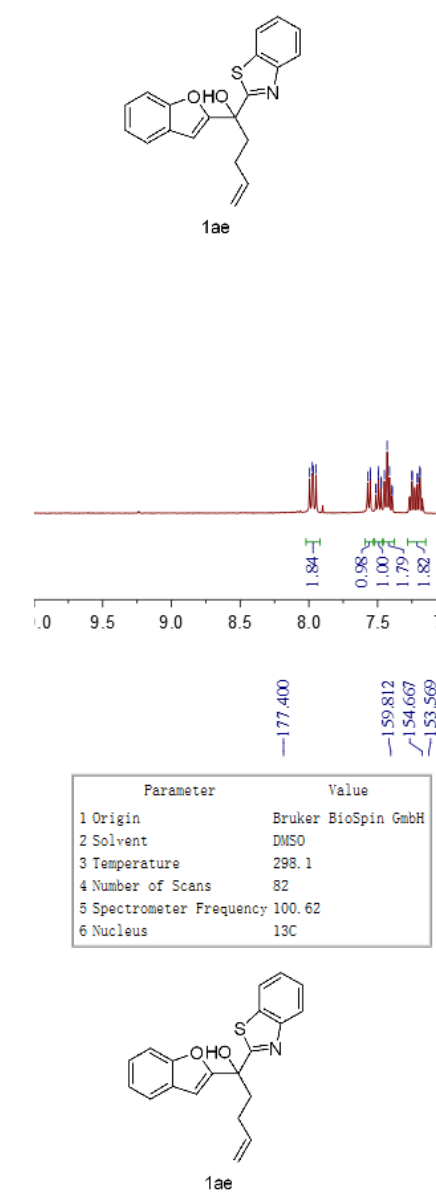

1ae
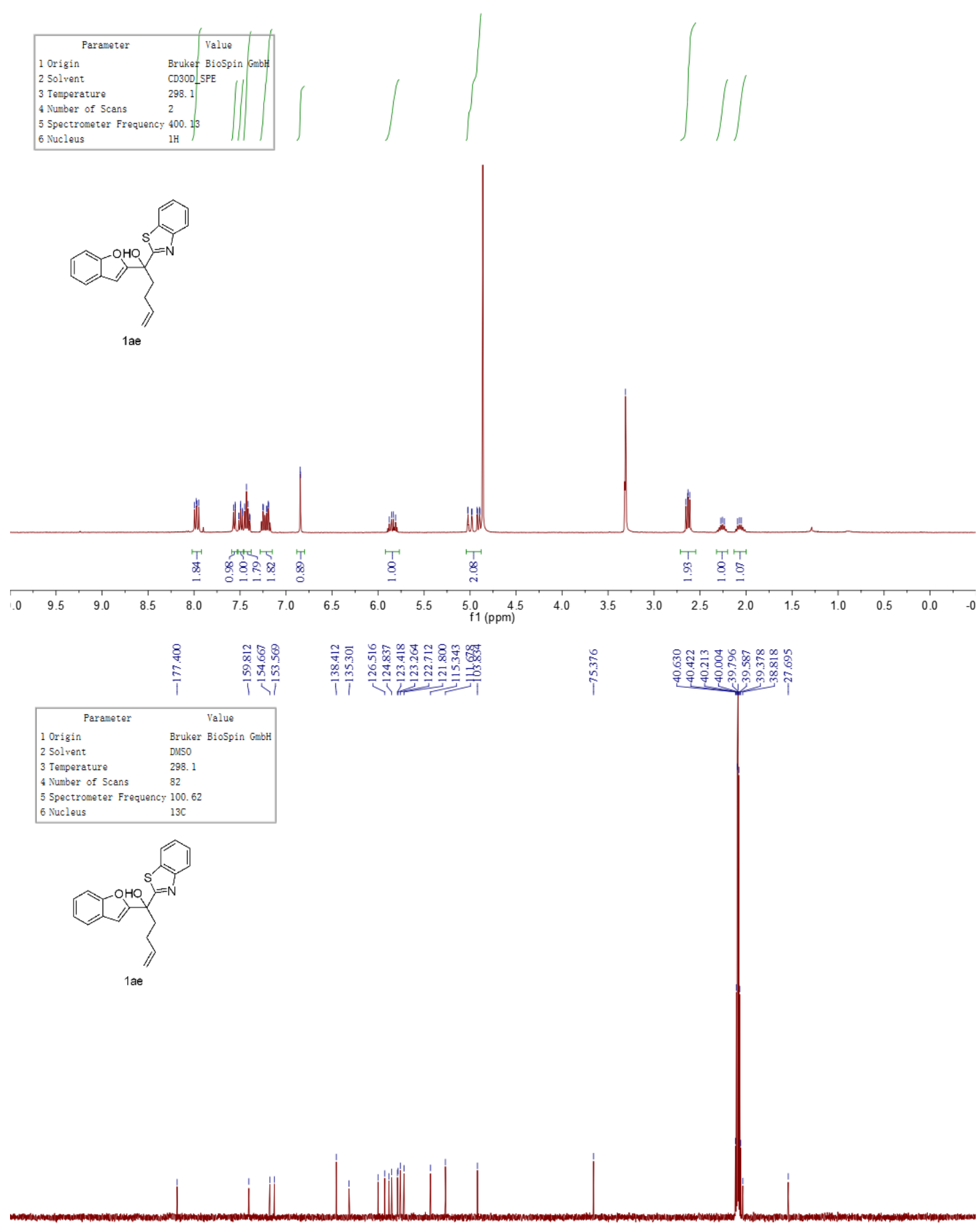

$\begin{array}{lllllllllllllllllllllllll}210 & 200 & 190 & 180 & 170 & 160 & 150 & 140 & 130 & 120 & 110 & 100 & 90 & 80 & 70 & 60 & 50 & 40 & 30 & 20 & 10 & 0 & -10\end{array}$ 


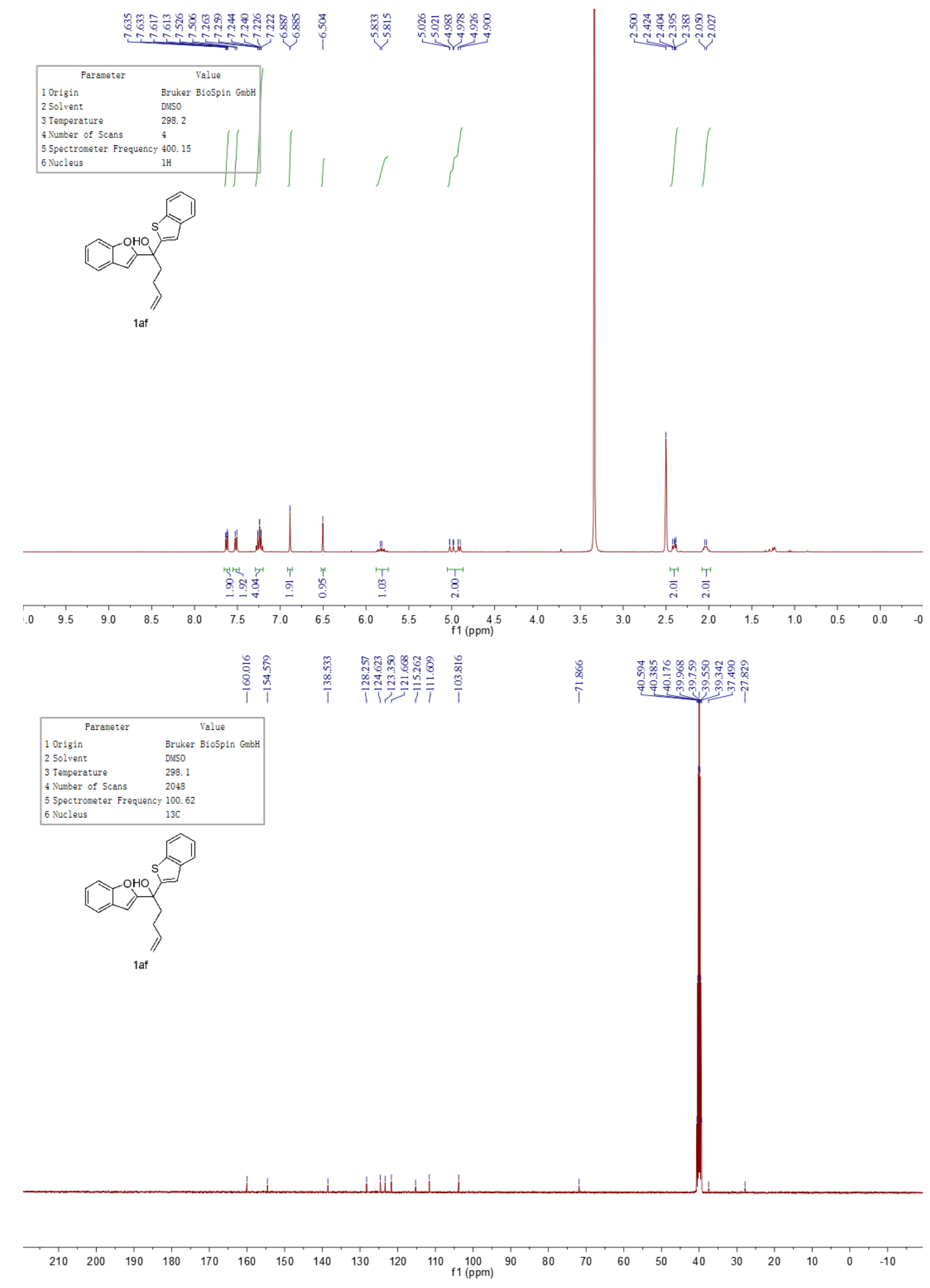




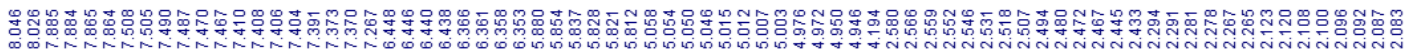
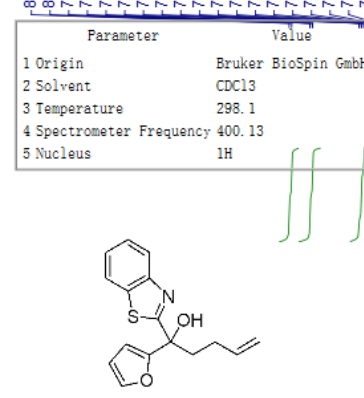

1ag

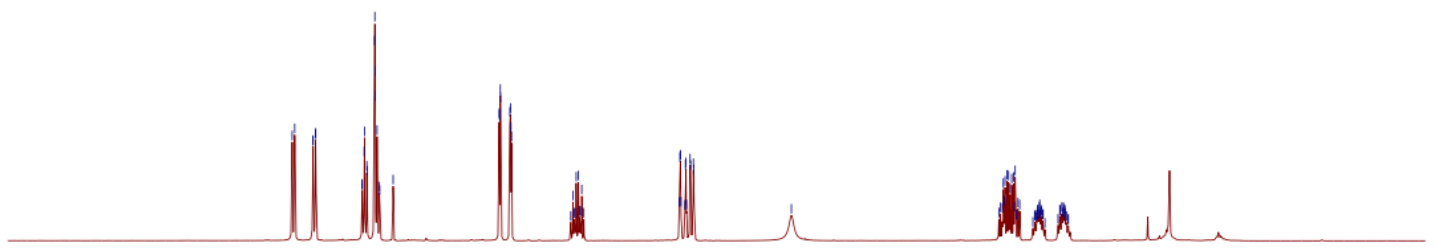

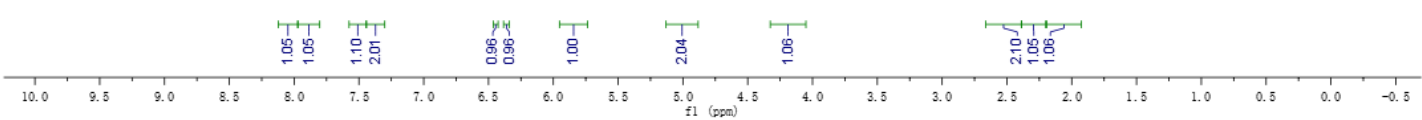

\begin{tabular}{|c|c|}
\hline $\begin{array}{l}\text { Parameter } \\
1 \text { Origin } \\
2 \text { Solvent } \\
3 \text { Temperature } \\
4 \text { Number of Scans } \\
5 \text { Spepectroneter Freque } \\
6 \text { Nucleus }\end{array}$ & 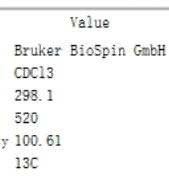 \\
\hline
\end{tabular}

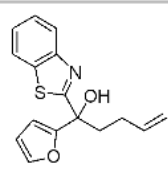

1ag
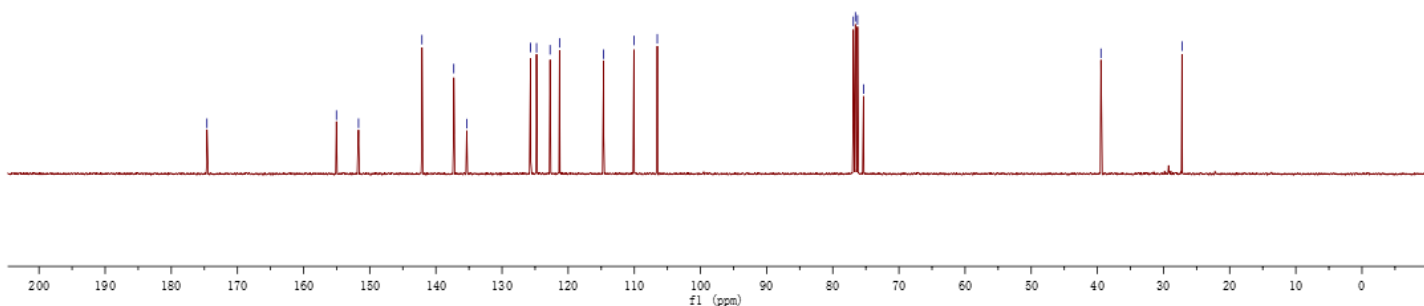


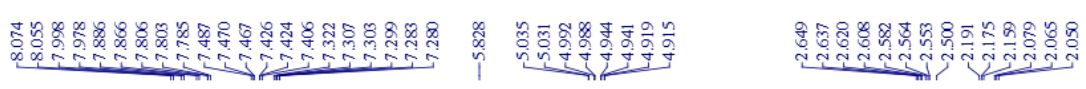
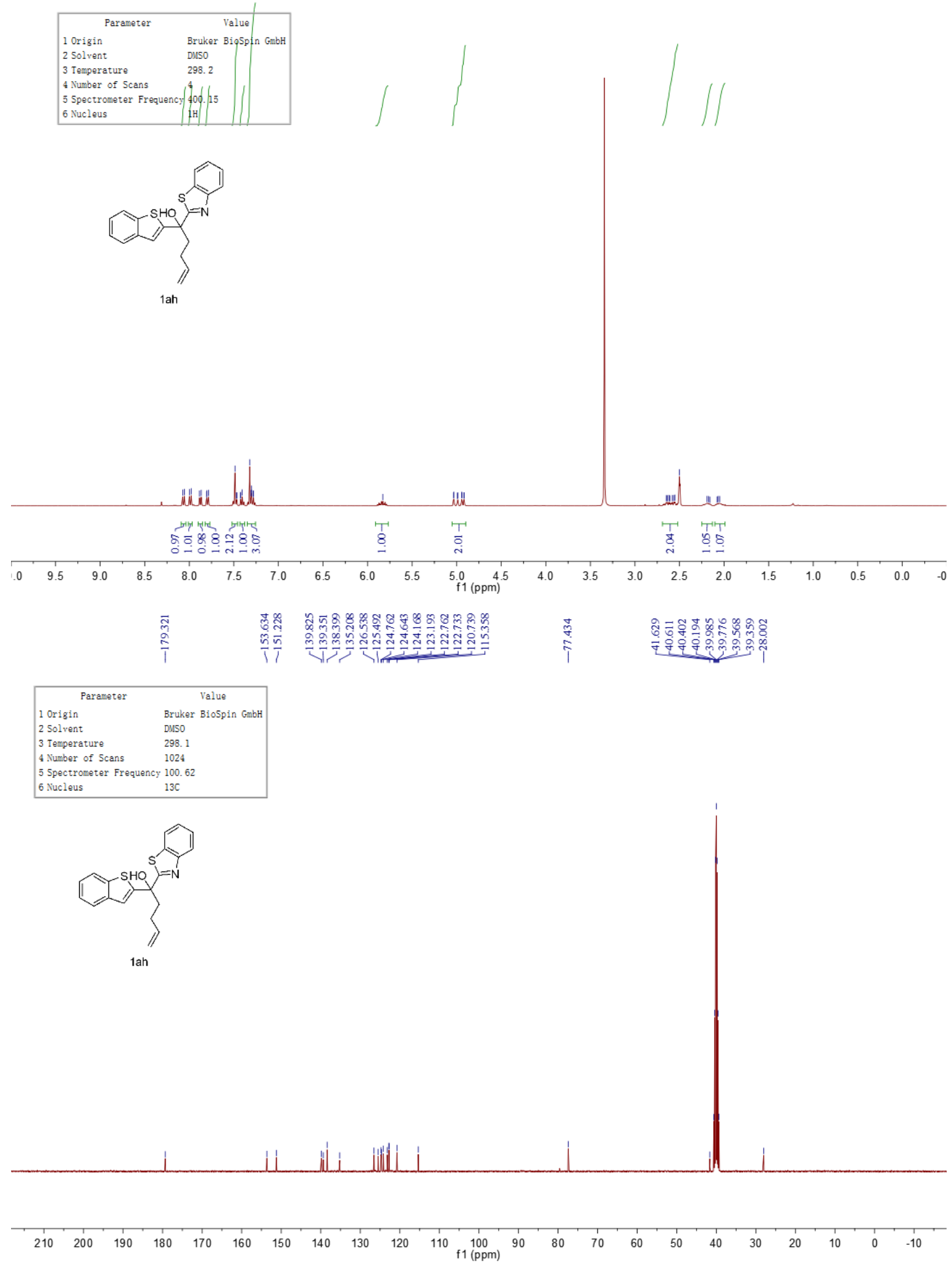


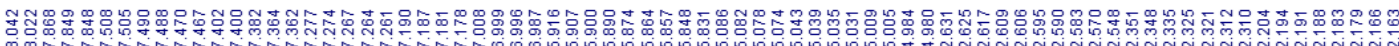

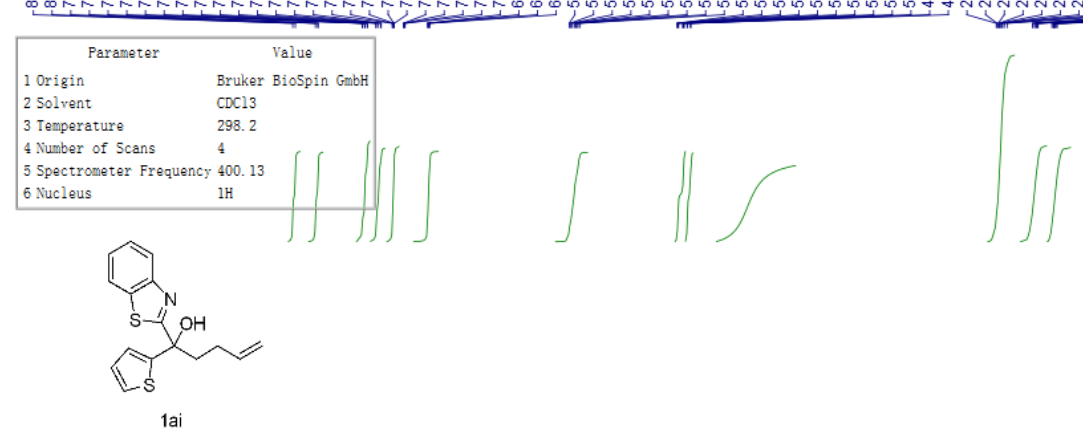

1ai
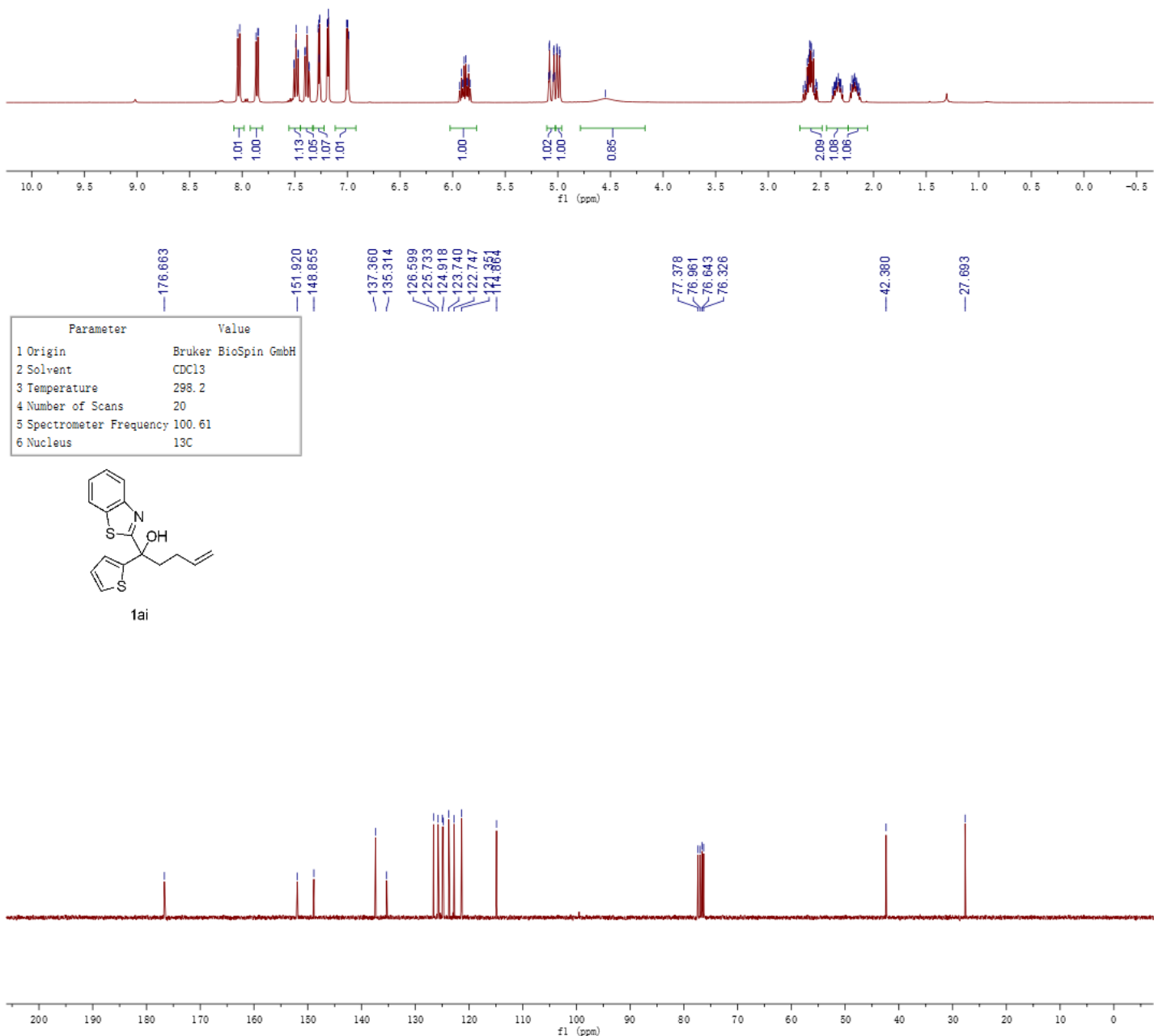


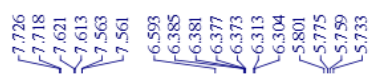

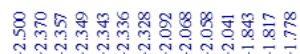

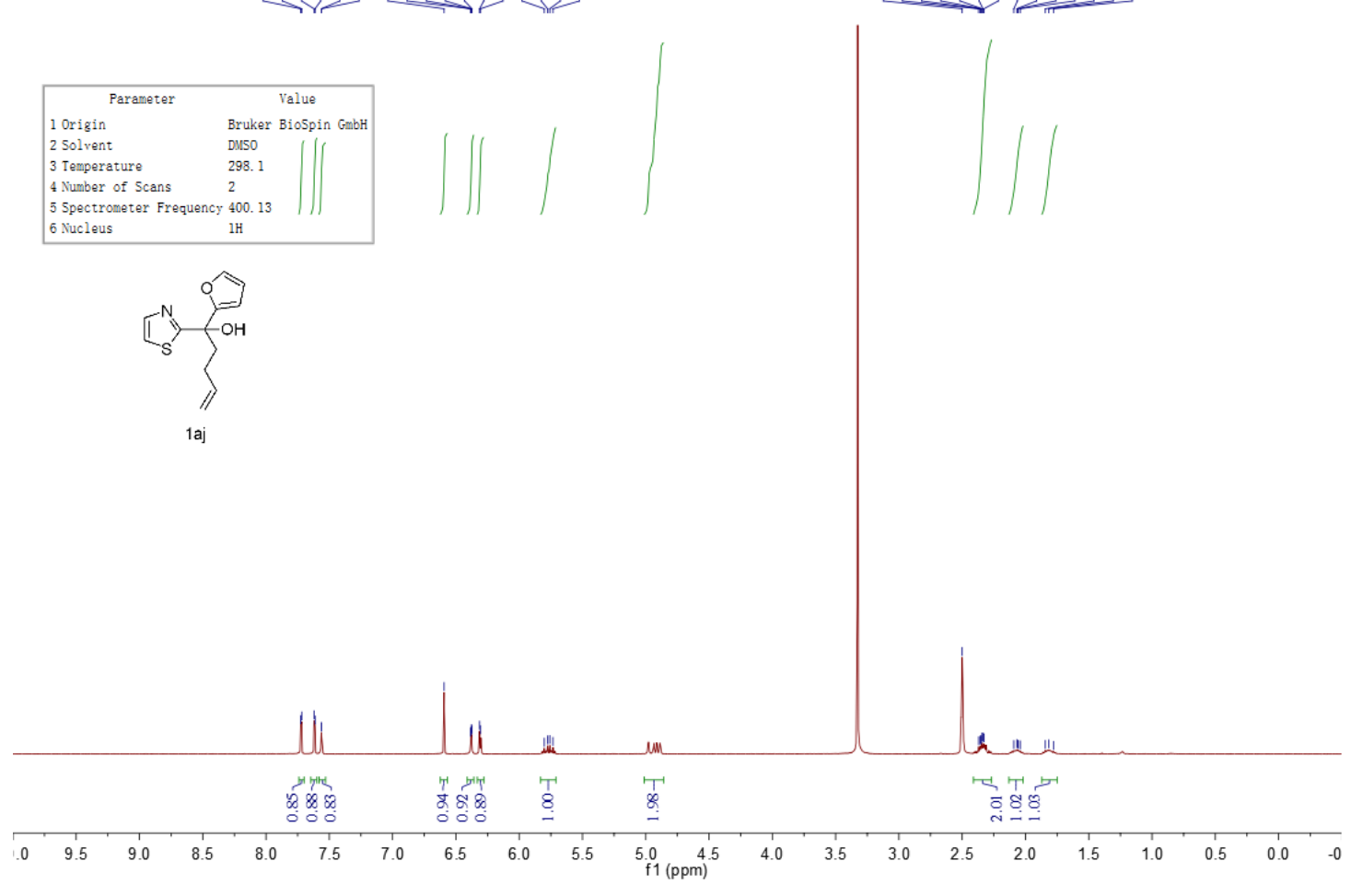

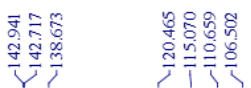

$\underset{⿱ 亠 䒑}{\stackrel{7}{*}}$

\begin{tabular}{|ll}
\multicolumn{1}{c}{ Parameter } & \multicolumn{1}{c}{ Value } \\
1 Origin & Bruker BioSpin GmbH \\
2 Solvent & DMSO
\end{tabular}

298.2

4 Humber of Scans 1024
5 Spectrometer Frequency 100.61

6 Hucleus $13 \mathrm{C}$

$\sum_{\text {1aj }}$

$\begin{array}{llllllllllllllllllllllllll}210 & 200 & 190 & 180 & 170 & 160 & 150 & 140 & 130 & 120 & 110 & 100 & 90 & 80 & 70 & 60 & 50 & 40 & 30 & 20 & 10 & 0 & -10\end{array}$ 


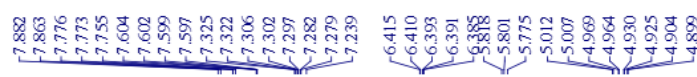

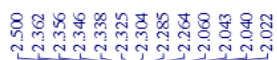

\begin{tabular}{|ll|}
\hline \multicolumn{1}{|c|}{ Parameter } & \multicolumn{1}{c|}{ Value } \\
1 Origin & Bruker Biospin GmbH \\
2 Solvent & DMSO \\
3 Temperature & 298.2 \\
4 Number of 5cans & 16 \\
5 Spectrometer Frequency & 400.15 \\
6 Nucleus & 1H
\end{tabular}||||||||
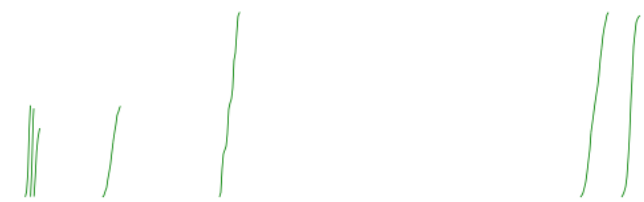

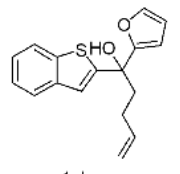

$1 \mathrm{ak}$

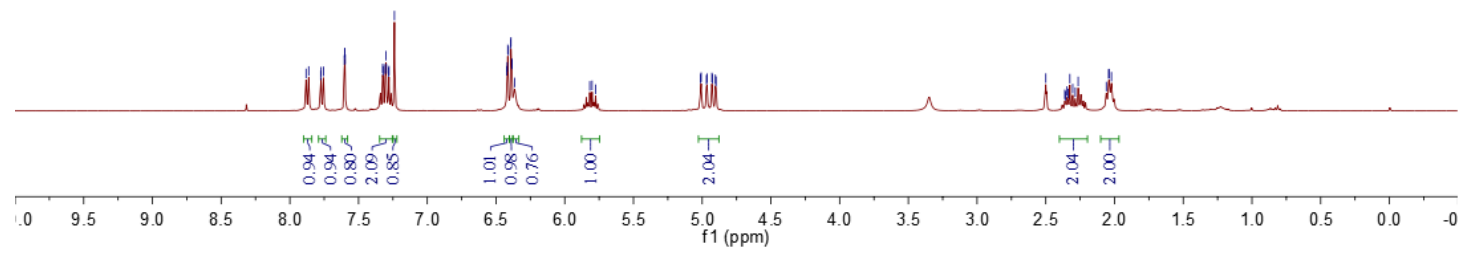

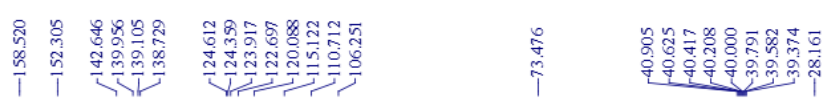

\begin{tabular}{|ll|}
\hline \multicolumn{1}{|c|}{ Parameter } & \multicolumn{1}{c|}{ Value } \\
1 Origin & Bruker Biospin GmbH \\
2 Solvent & DuSO \\
3 Temperature & 298.1 \\
4 Number of Scans & 75 \\
5 Spectrometer Frequency 100.62 \\
6 Nucleus & $13 \mathrm{C}$ \\
\hline
\end{tabular}

5 Spectrometer Frequency 100.62

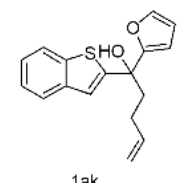

1ak

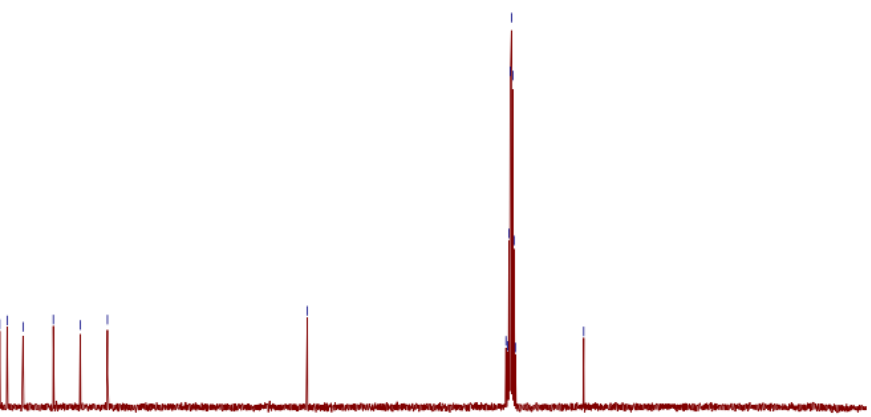

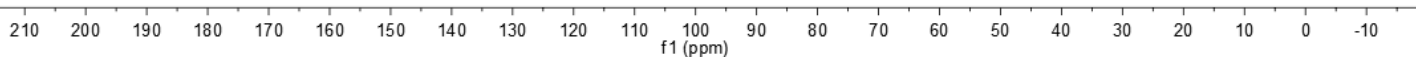




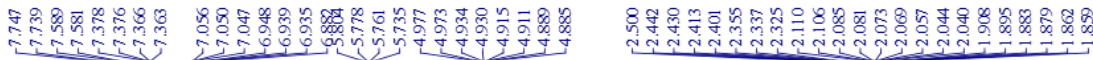
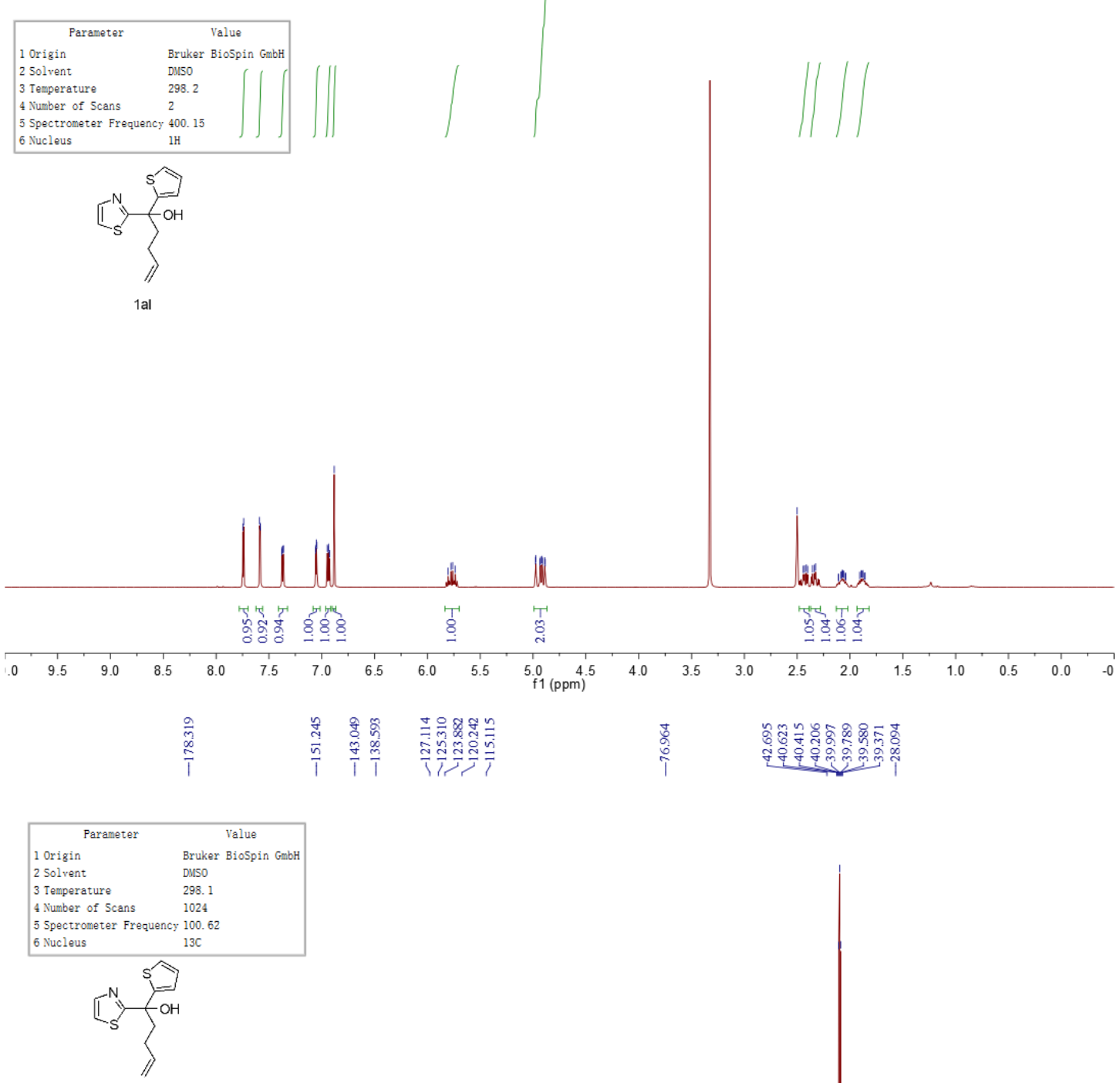

1al

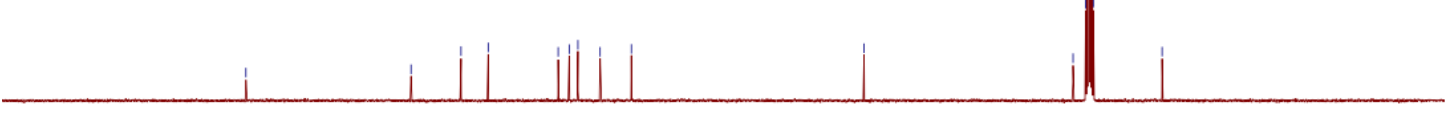

$\begin{array}{lllllllllllllllllllllllllllllll}1 & 110 & 200 & 190 & 180 & 170 & 160 & 150 & 140 & 130 & 120 & 110 & 100 & 90 & 80 & 70 & 60 & 50 & 40 & 30 & 20 & 10 & 0 & -10\end{array}$ 


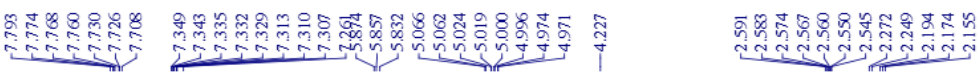

\begin{tabular}{|ll|}
\hline \multicolumn{1}{|c|}{ Parameter } & \multicolumn{1}{c|}{ Value } \\
1 Origin & Bruker Biospin GmbH \\
2 Solvent & CDC13 \\
3 Temperature & 298.2 \\
4 Number of Scans & 2 \\
5 Spectrometer Frequency & 400.15 \\
6 Nucleus & 1H
\end{tabular} \mid

5 Spectrometer Frequency 400. 15

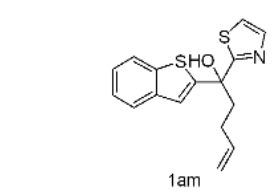

$1 \mathrm{am}$
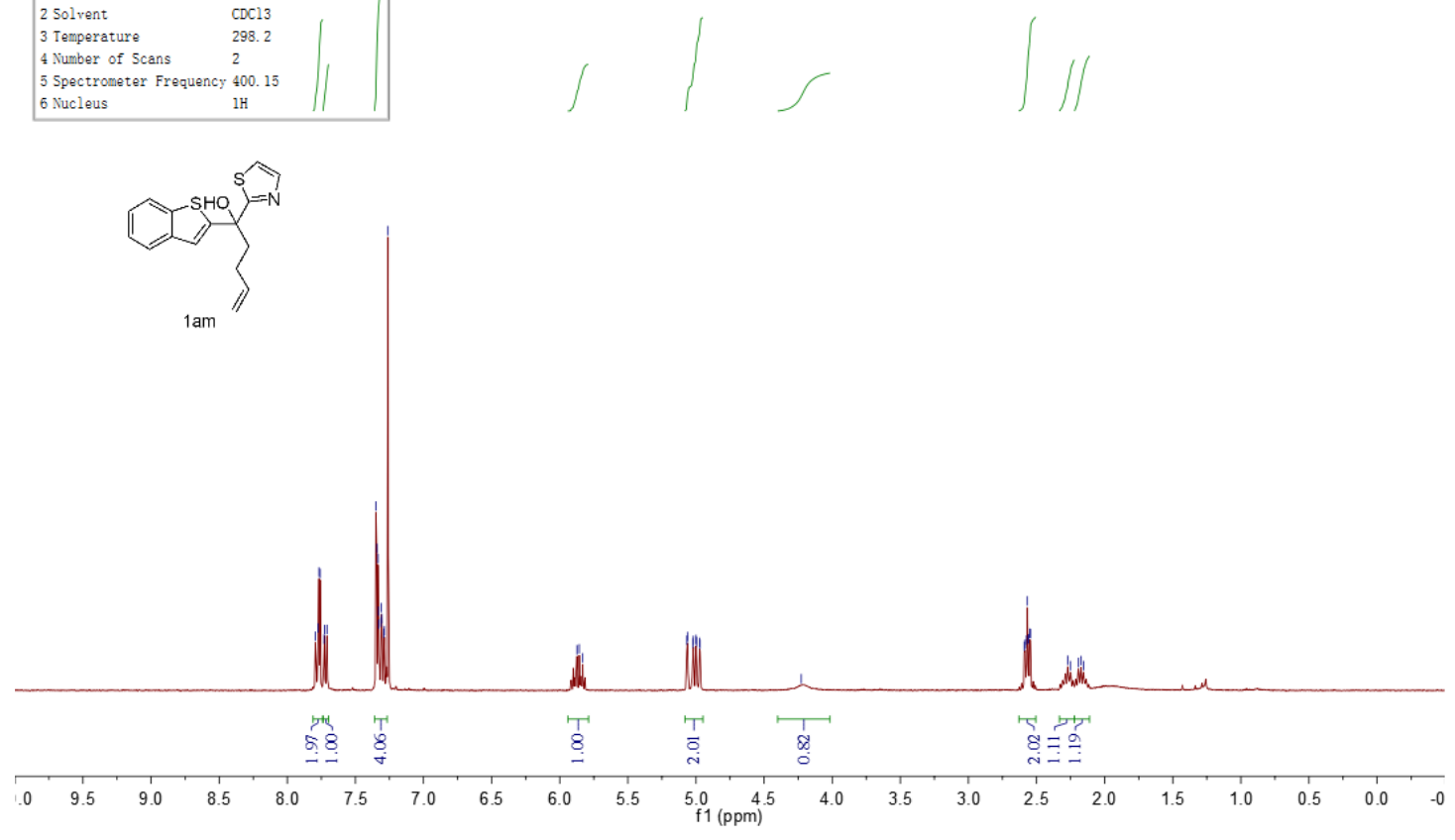

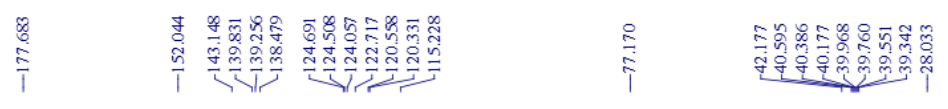

\begin{tabular}{|ll|}
\hline \multicolumn{1}{|c|}{ Parameter } & \multicolumn{1}{c|}{ Value } \\
1 Origin & Bruker Bio5pin GmbH \\
2 Solvent & DMSO \\
3 Temperature & 298.2 \\
4 Number of Scans & 512 \\
5 Spectrometer Frequency 100.61 \\
6 Nucleus & $13 \mathrm{C}$ \\
\hline
\end{tabular}

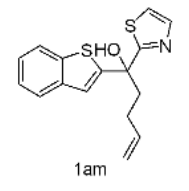

$1 \mathrm{am}$

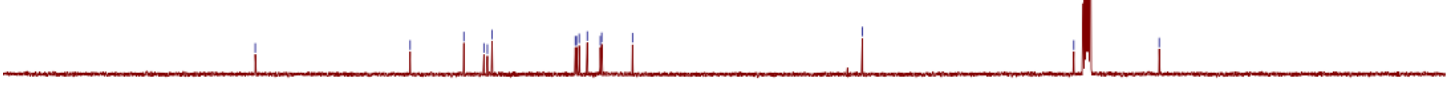

\begin{tabular}{lllllllllllllllllllllllll}
\hline & 110 & 200 & 190 & 180 & 170 & 160 & 150 & 140 & 130 & 120 & 110 & $\begin{array}{c}100 \\
\mathrm{f} 1(\mathrm{ppm})\end{array}$ & 90 & 80 & 70 & 60 & 50 & 40 & 30 & 20 & 10 & 0 & -10
\end{tabular} 


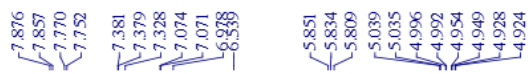

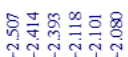

\begin{tabular}{|ll|}
\hline \multicolumn{1}{|c|}{ Parameter } & \multicolumn{1}{c|}{ Value } \\
1 Origin & Bruker BioSpin GmbH \\
2 Solvent & DMSO \\
3 Temperature & 298.2 \\
4 Number of Scans & 2 \\
5 Spectrometer Frequency 400.13 \\
6 Nucleus & 1H
\end{tabular}
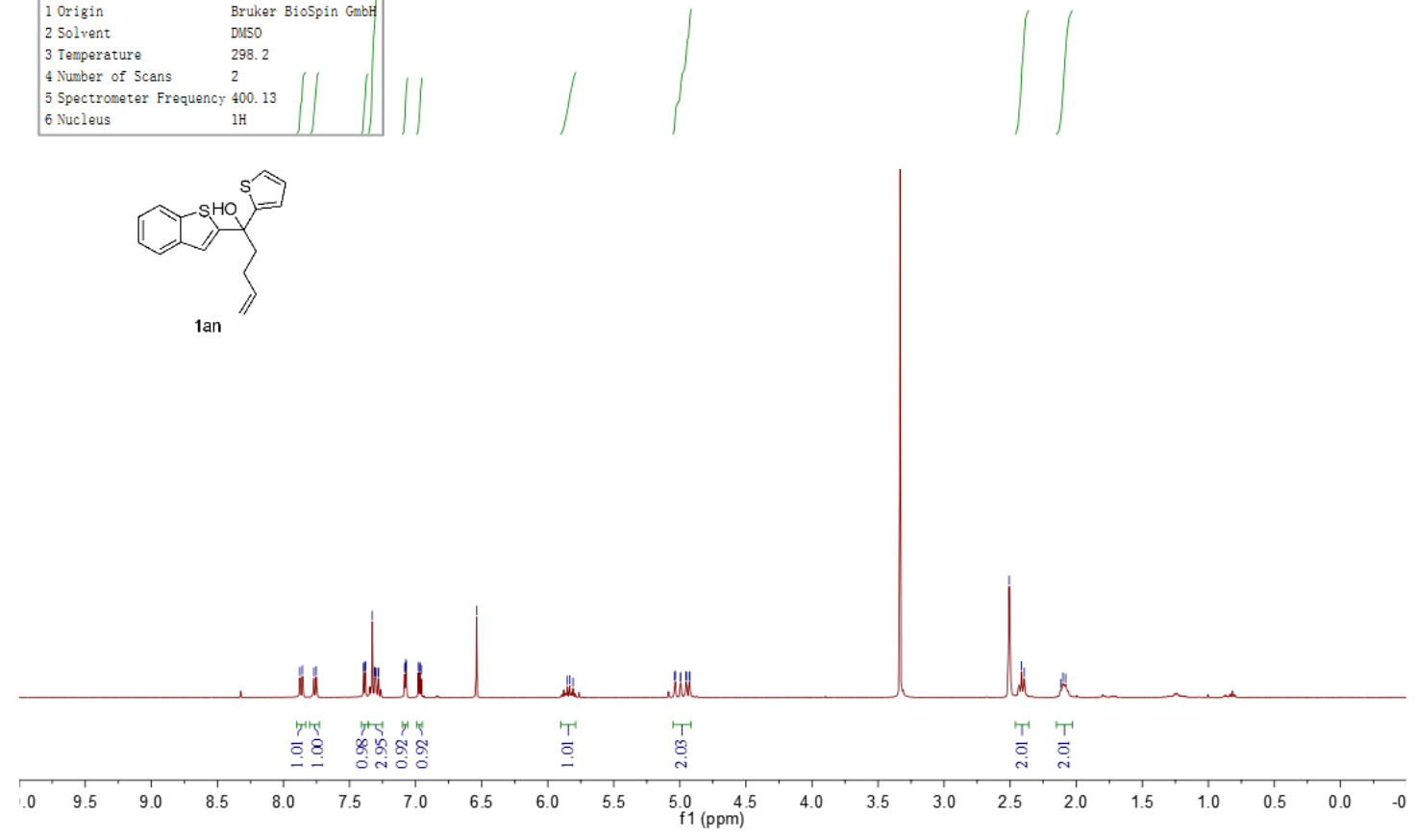

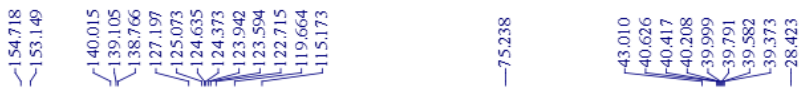

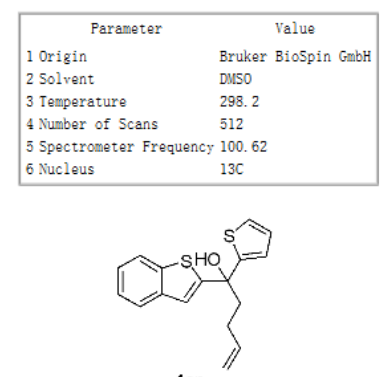

1an

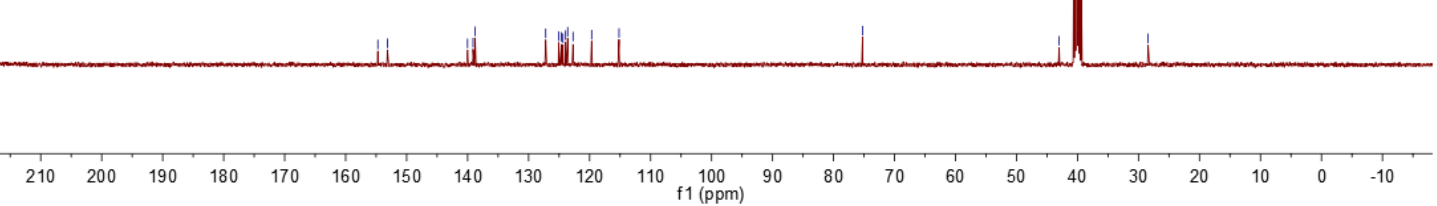




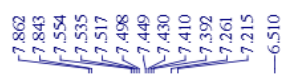

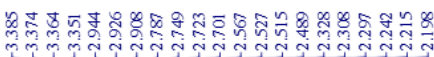

\begin{tabular}{|l|l|}
\hline \multicolumn{1}{|c|}{ Parameter } \\
1 Origin & Balu \\
2 Solvent & CDC13 Bio5 \\
3 Temperature & 298.2 \\
4 Number of Scans & 2 \\
5 Spectrometer Frequency & 400.13 \\
6 Nucleus & 1H
\end{tabular}

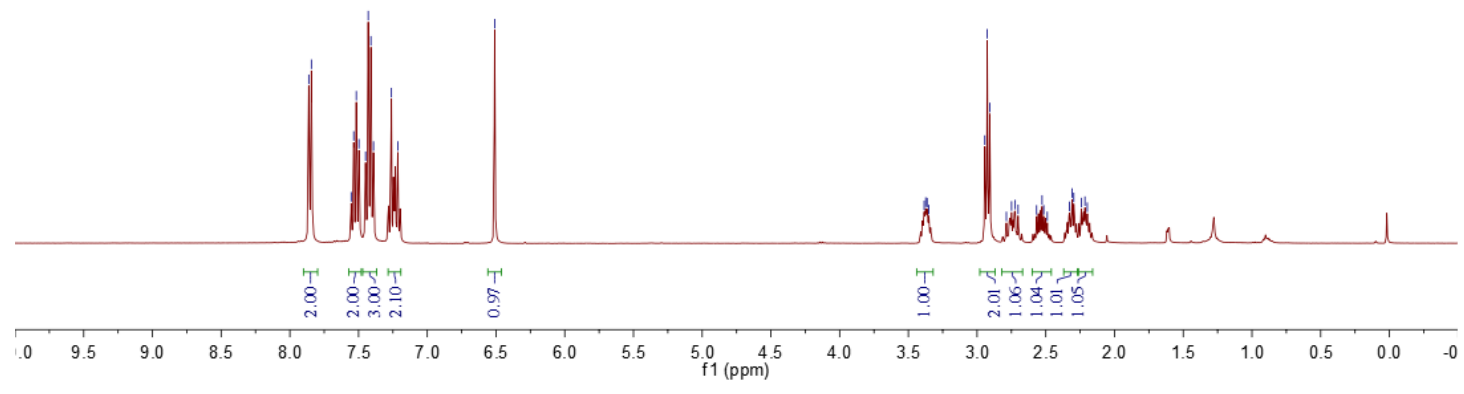

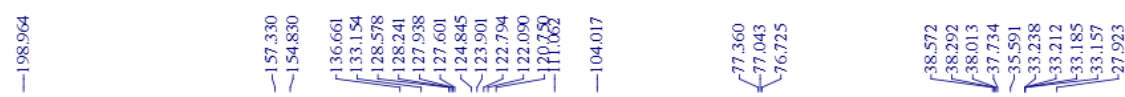

\begin{tabular}{|ll|}
\hline \multicolumn{1}{|c|}{ Parameter } & \multicolumn{1}{c|}{ Value } \\
1 Origin & Bruker BioSpin GmbH \\
2 Solvent & CDC13 \\
3 Temperature & 298.1 \\
4 Number of Scans & 82 \\
5 Spectrometer Frequency 100.62 \\
6 Nucleus & $13 \mathrm{C}$ \\
\hline
\end{tabular}

6 Nucleus $\quad 13 \mathrm{C}$<smiles>O=C(CCC(CC(F)(F)F)c1cc2ccccc2o1)c1ccccc1</smiles>

$2 a$

$\begin{array}{lllllllllllllllllllllll}210 & 200 & 190 & 180 & 170 & 160 & 150 & 140 & 130 & 120 & 110 & \begin{array}{c}100 \\ \mathrm{f} 1(\mathrm{ppm})\end{array} & 90 & 80 & 70 & 60 & 50 & 40 & 30 & 20 & 10 & 0 & -10\end{array}$ 


\section{$\frac{\text { m }}{\text { के }}$}
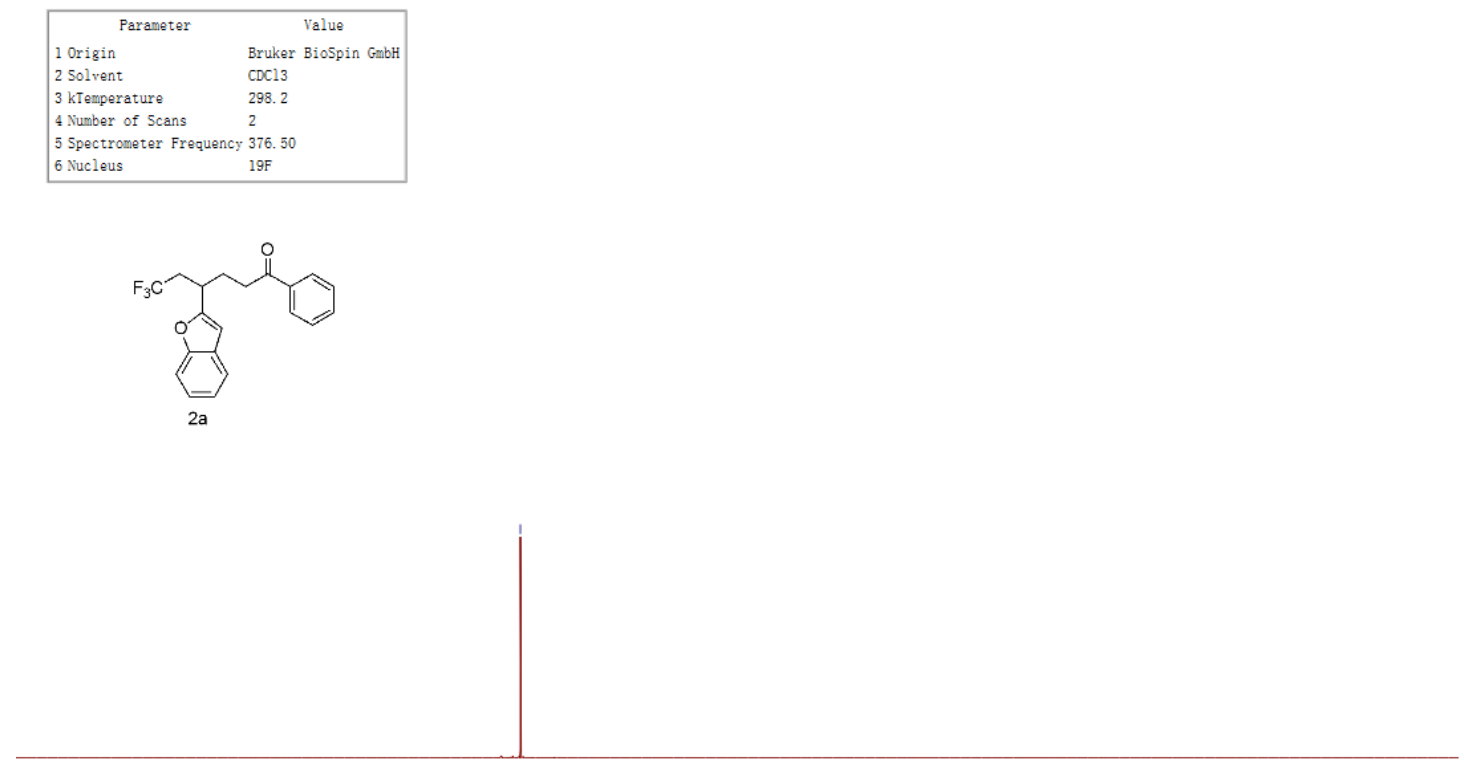

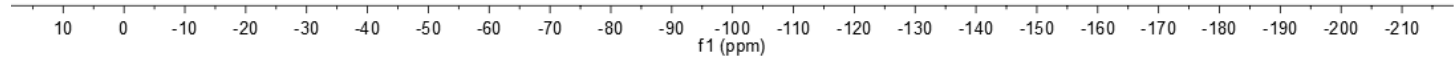

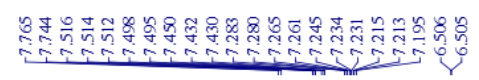

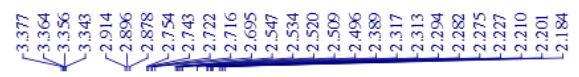

\begin{tabular}{|ll|}
\hline \multicolumn{1}{|c|}{ Parameter } & \multicolumn{1}{c|}{ Value } \\
1 Origin & Bruker BioSpin GmbH \\
2 Solvent & CDC13 \\
3 Temperature & 298.2 \\
4 N Number of Scans & 2 \\
5 Spectrometer Frequency 400.13 \\
6 Nucleus & 1H \\
\hline
\end{tabular}

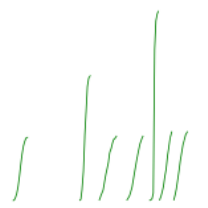

(2b

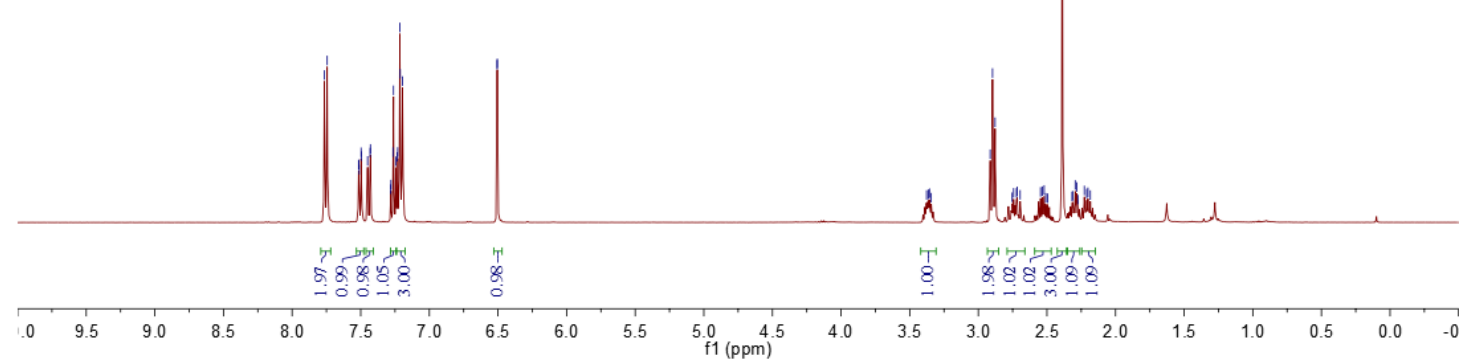




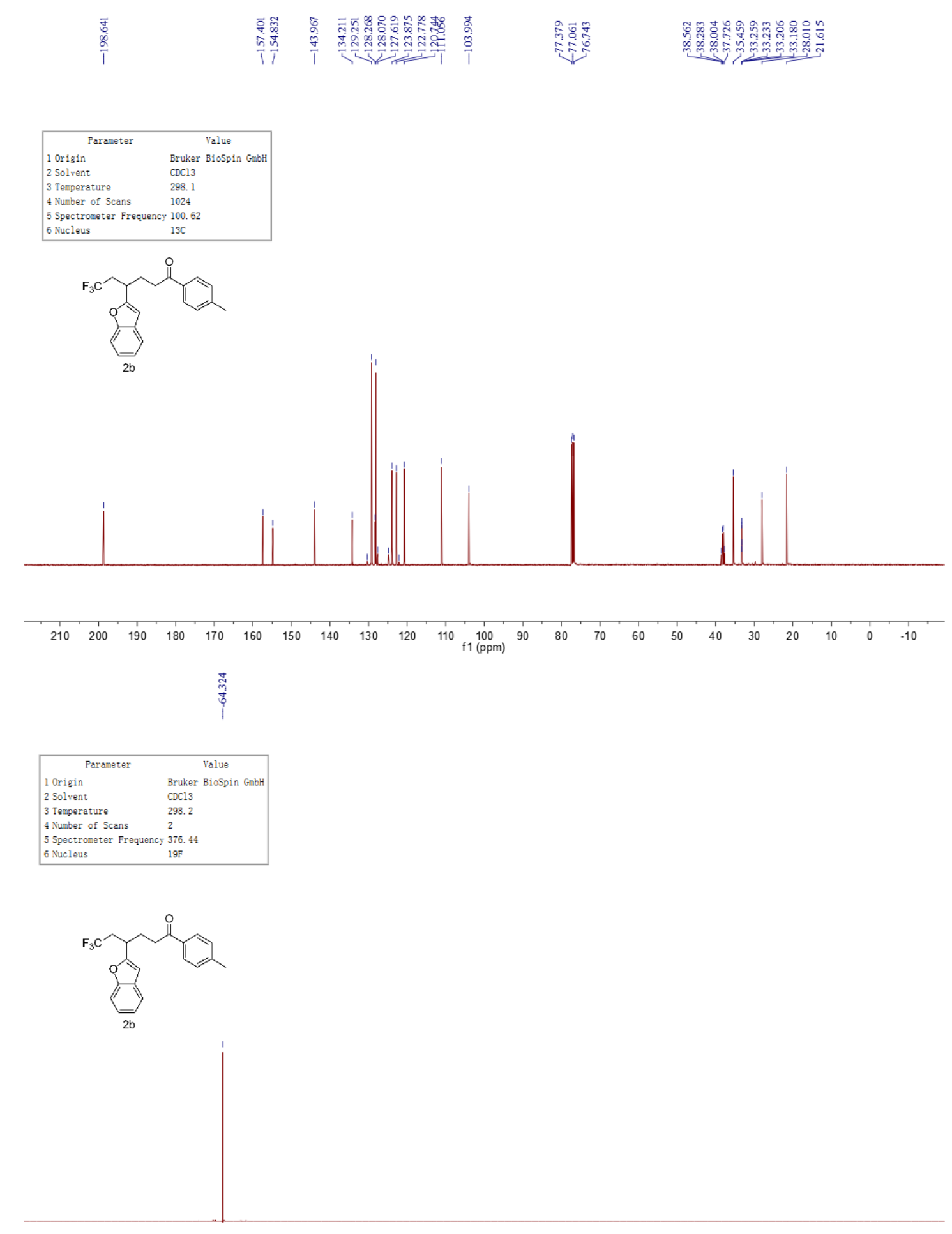

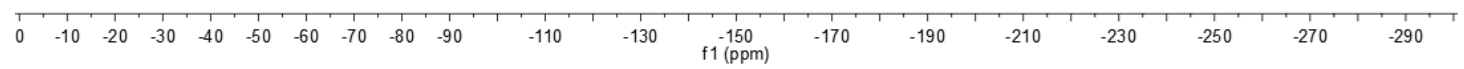




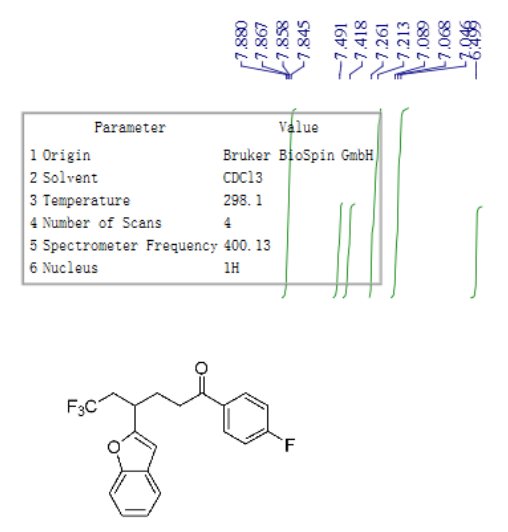

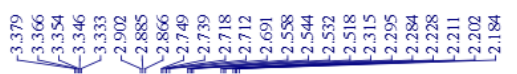

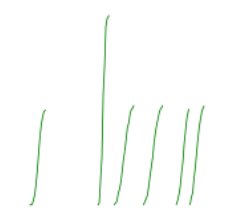

$2 \mathrm{c}$

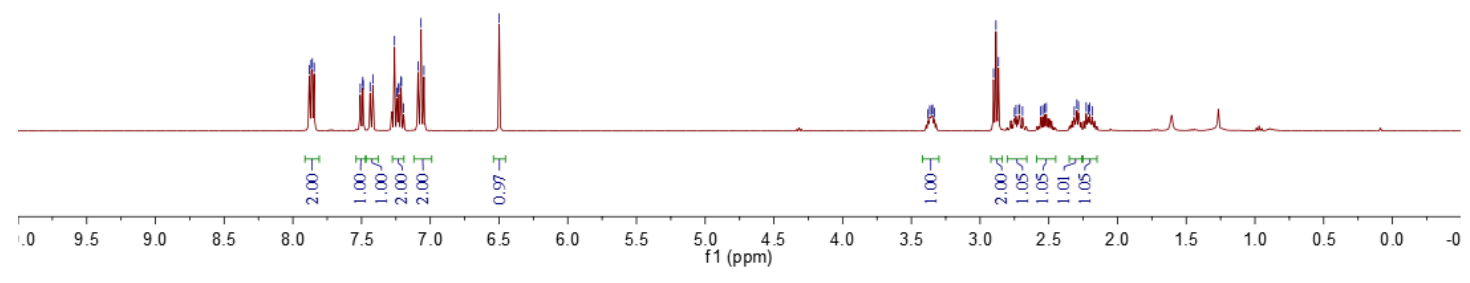

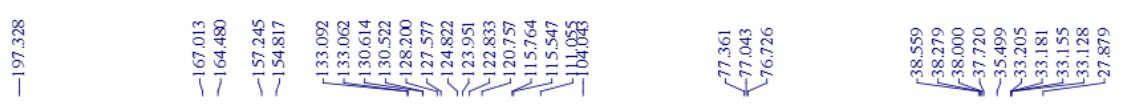

\begin{tabular}{|ll|}
\hline \multicolumn{1}{|c|}{ Parameter } & \multicolumn{1}{c|}{ Value } \\
1 Origin & Bruker Bio5pin GmbH \\
2 Solvent & CDC13 \\
3 Temperature & 298.1 \\
4 Number of Scans & 1024 \\
5 Spectrometer Frequency & 100.62 \\
6 Nucleus & $13 \mathrm{C}$ \\
\hline
\end{tabular}

is

$2 c$

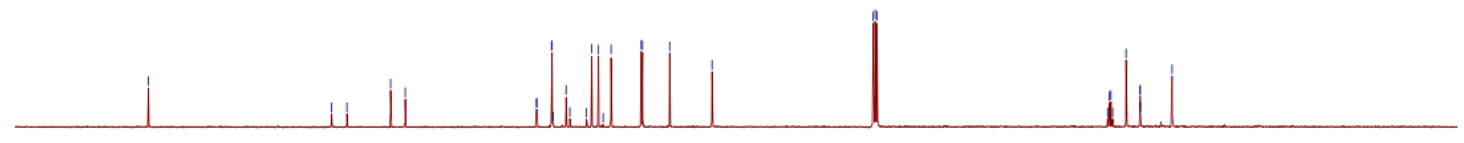

$\begin{array}{lllllllllllllllllllllllllll}1 & 1 & 200 & 190 & 180 & 170 & 160 & 150 & 140 & 130 & 120 & 110 & 100 & 90 & 80 & 70 & 60 & 50 & 40 & 30 & 20 & 10 & 0 & -10\end{array}$ 

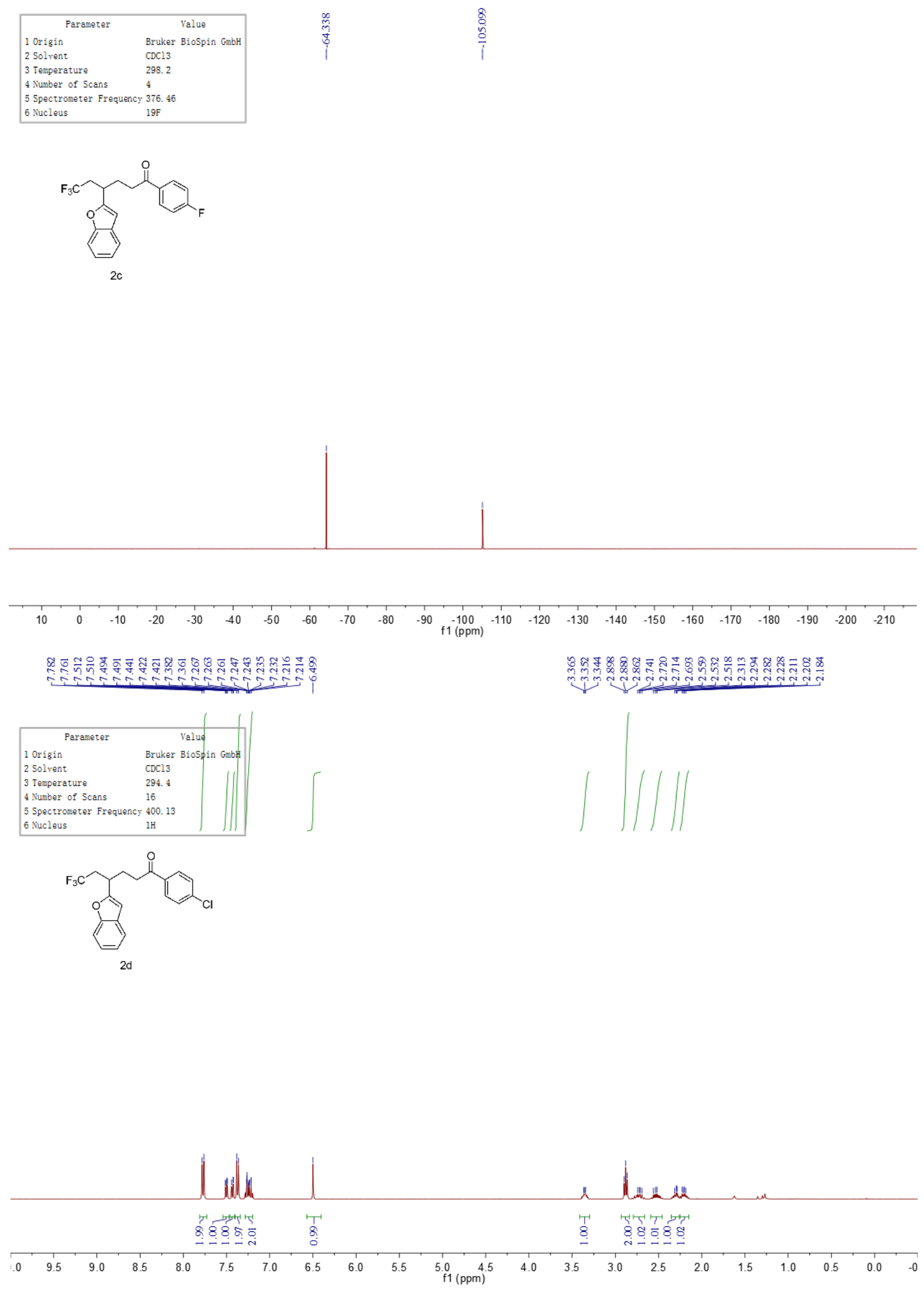


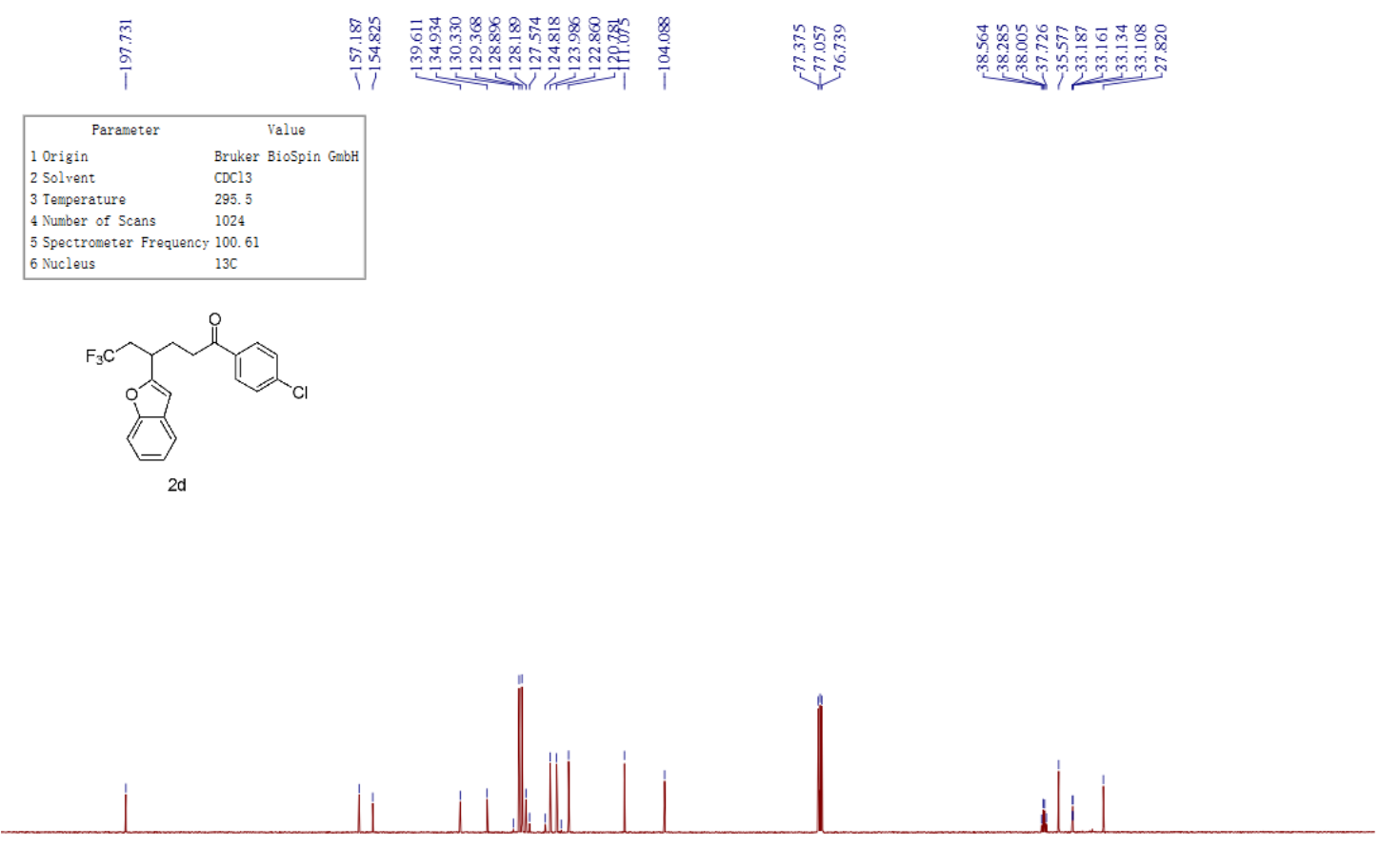

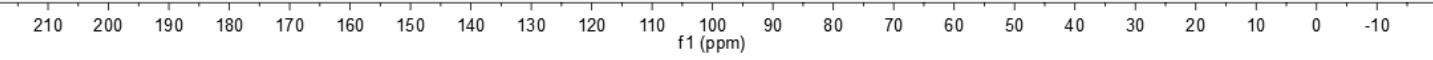

\begin{tabular}{|ll|}
\hline \multicolumn{1}{|c|}{ Parameter } & \multicolumn{1}{c|}{ Value } \\
1 Origin & Bruker BioSpin GmbH \\
2 Solvent & CDC13 \\
3 Temperature & 294.5 \\
4 Number of Scans & 16 \\
5 Spectrometer Frequency & 376.46 \\
6 Nucleus & $19 \mathrm{~F}$ \\
\hline
\end{tabular}

$\stackrel{m}{\frac{m}{0}}$<smiles>O=C(CCC(CCF)c1cc2ccccc2o1)c1ccc(Cl)cc1</smiles>

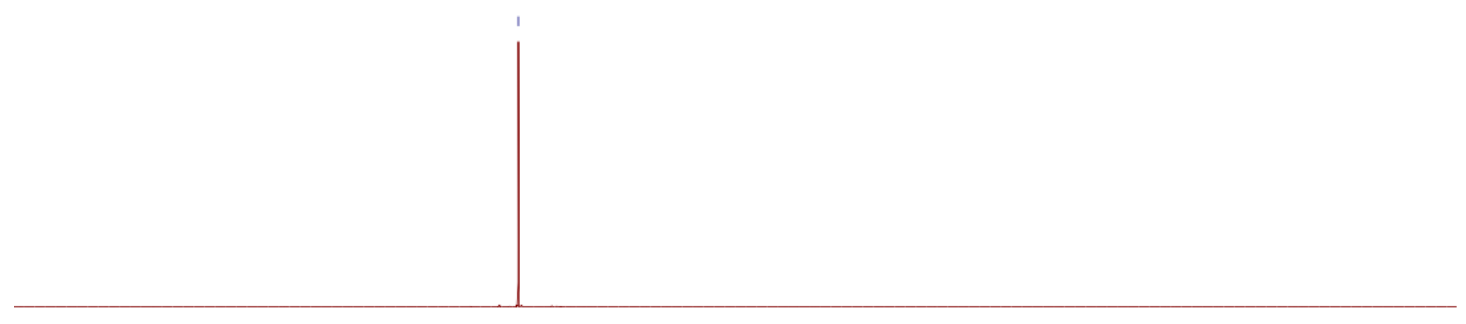

\begin{tabular}{rlllllllllllllllllllllll}
\hline 10 & 0 & -10 & -20 & -30 & -40 & -50 & -60 & -70 & -80 & -90 & -100 & -110 & -120 & -130 & -140 & -150 & -160 & -170 & -180 & -190 & -200 & -210
\end{tabular} 


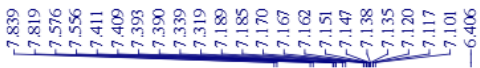
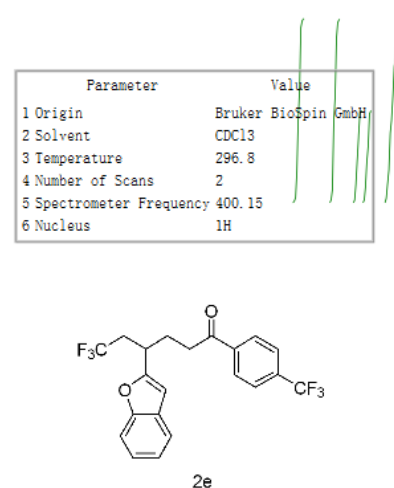

$2 e$

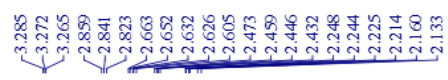

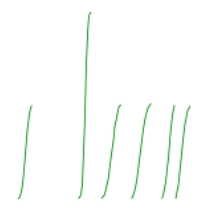

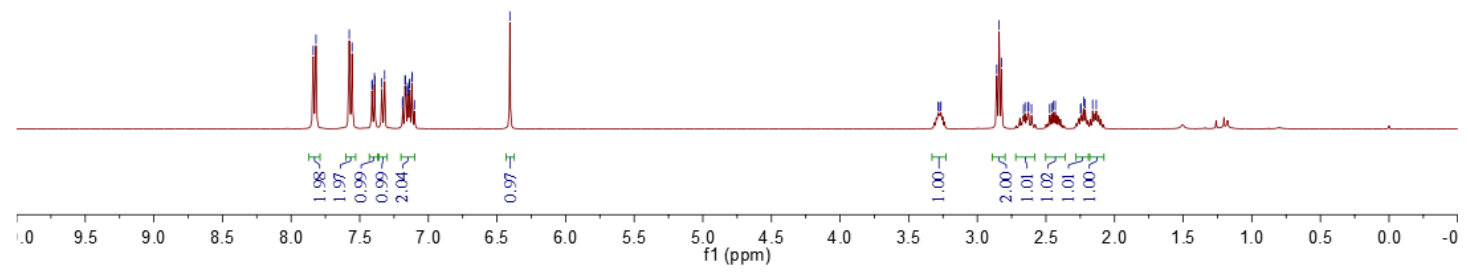

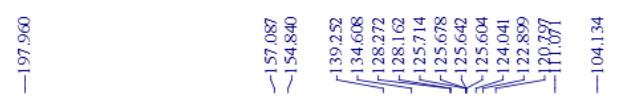

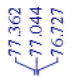

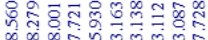

क्लm ले लेल

\begin{tabular}{|ll|}
\hline \multicolumn{1}{|c|}{ Parameter } & \multicolumn{1}{c|}{ Value } \\
1 Origin & Bruker Biospin GmbH \\
2 Solvent & CDC13 \\
3 Temperature & 297.5 \\
4 Number of Scans & 1024 \\
5 Spectrometer Frequency & 100.62 \\
6 Nucleus & $13 \mathrm{C}$ \\
\hline
\end{tabular}

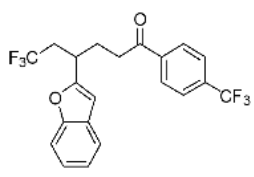

28

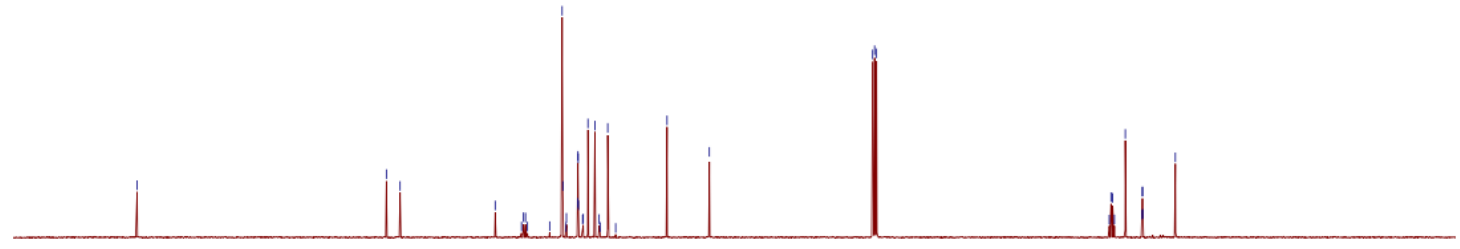

$\begin{array}{llllllllllllllllllllllll}210 & 200 & 190 & 180 & 170 & 160 & 150 & 140 & 130 & 120 & 110 & \begin{array}{c}100 \\ \mathrm{f} 1(\mathrm{ppm})\end{array} & 90 & 80 & 70 & 60 & 50 & 40 & 30 & 20 & 10 & 0 & -10\end{array}$ 

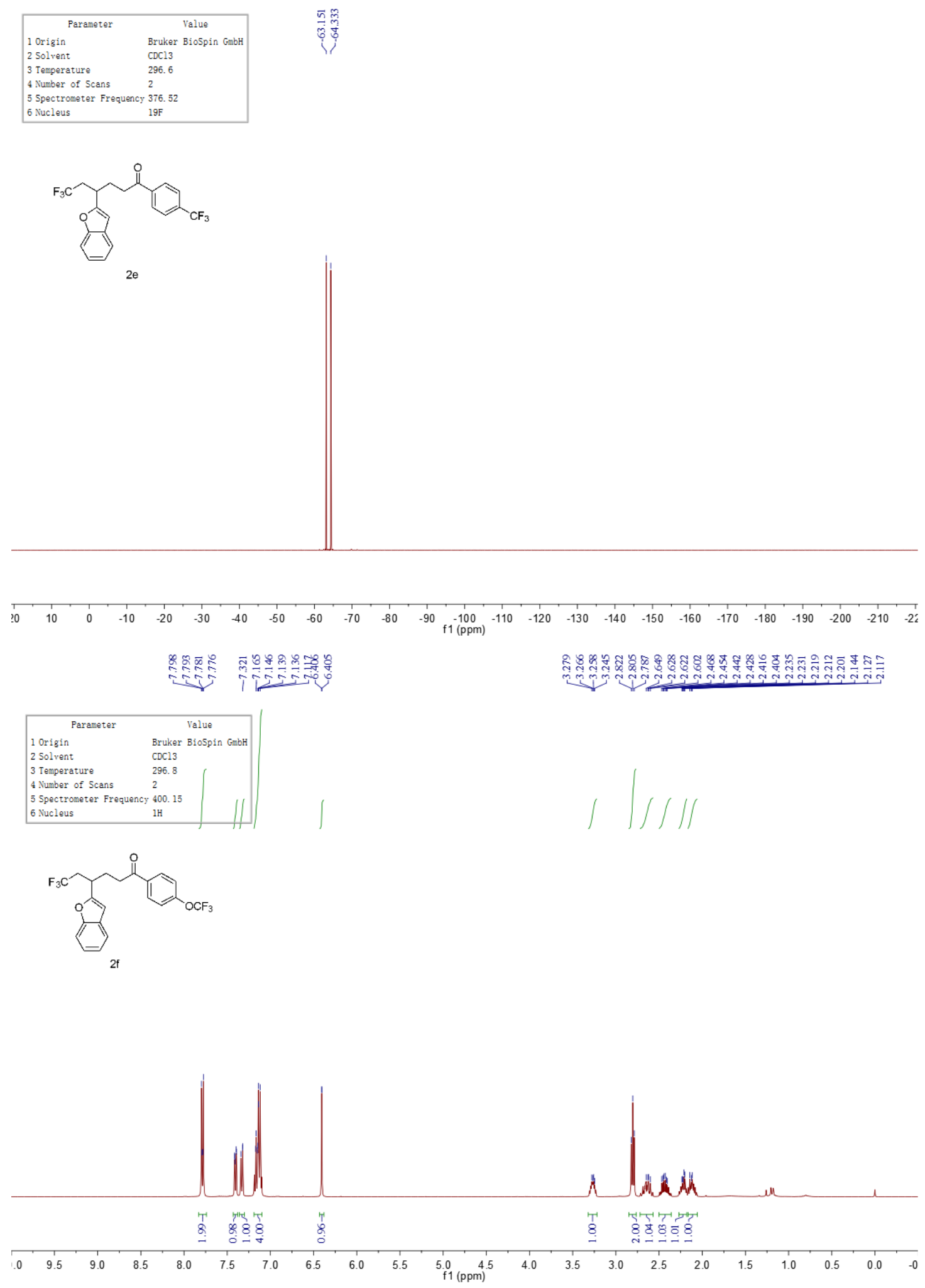


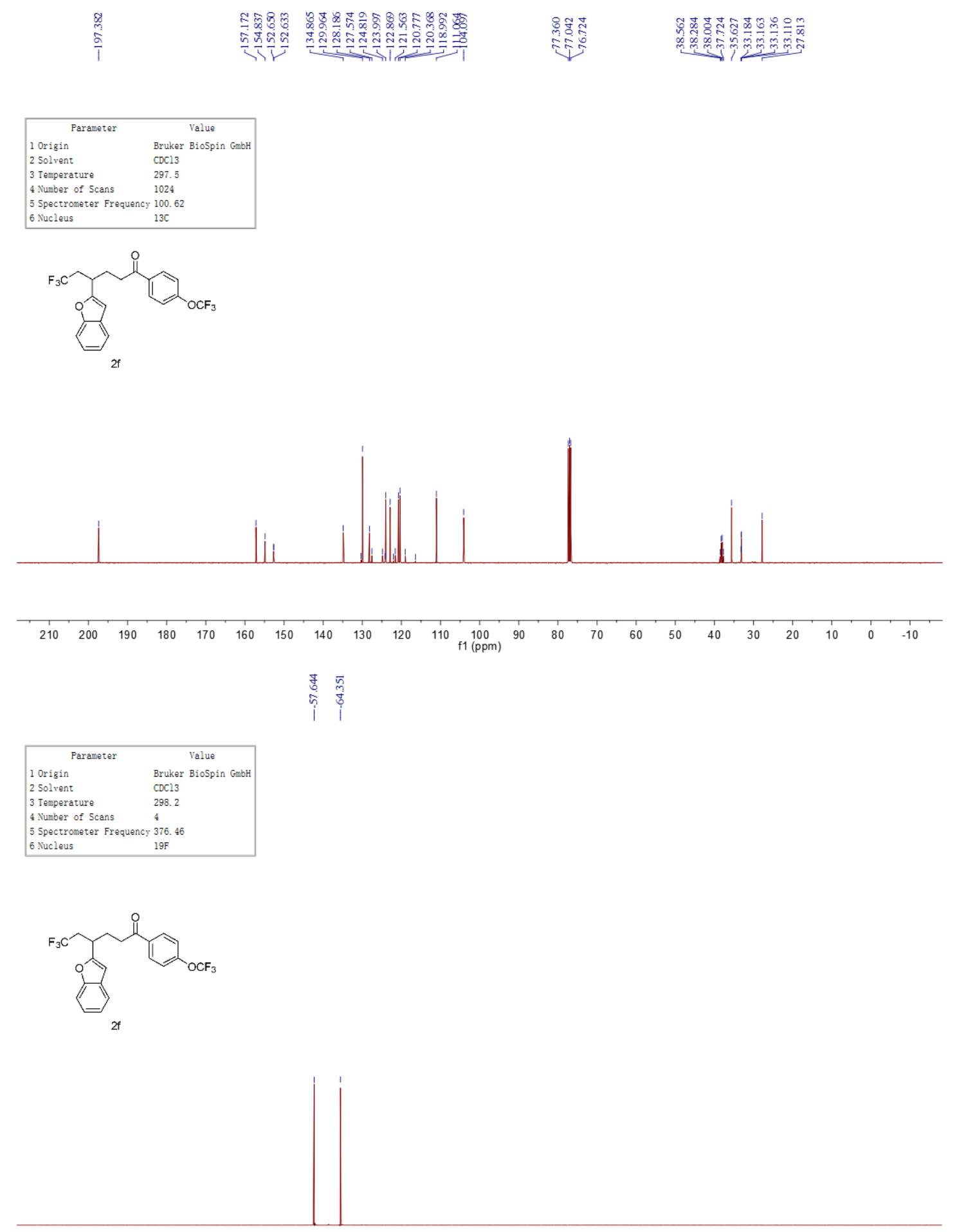

$\begin{array}{lllllllllllllllllllllllllllllll}10 & 0 & -10 & -20 & -30 & -40 & -50 & -60 & -70 & -80 & -90 & -100 & -110 & -120 & -130 & -140 & -150 & -160 & -170 & -180 & -190 & -200 & -210 & 1\end{array}$ 


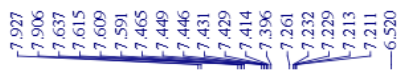
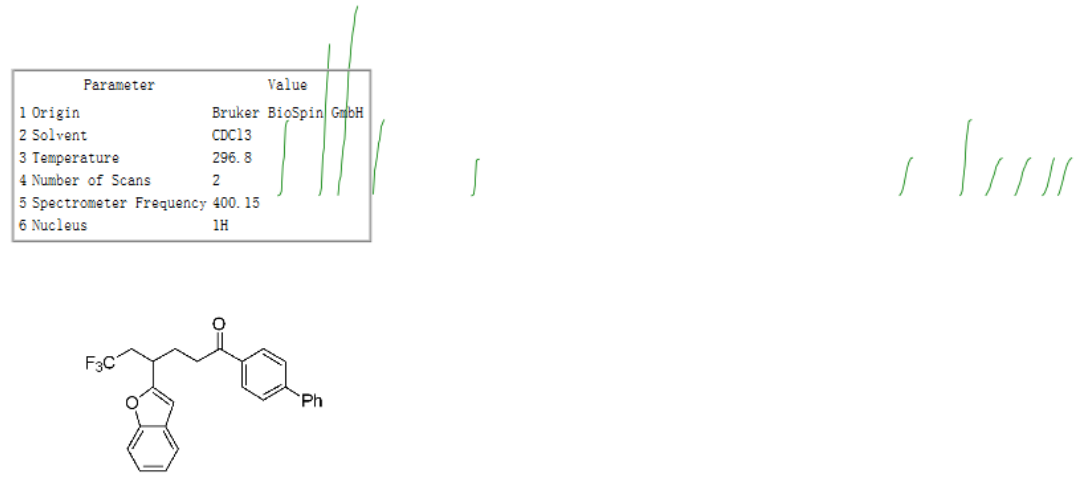

$2 \mathrm{~g}$
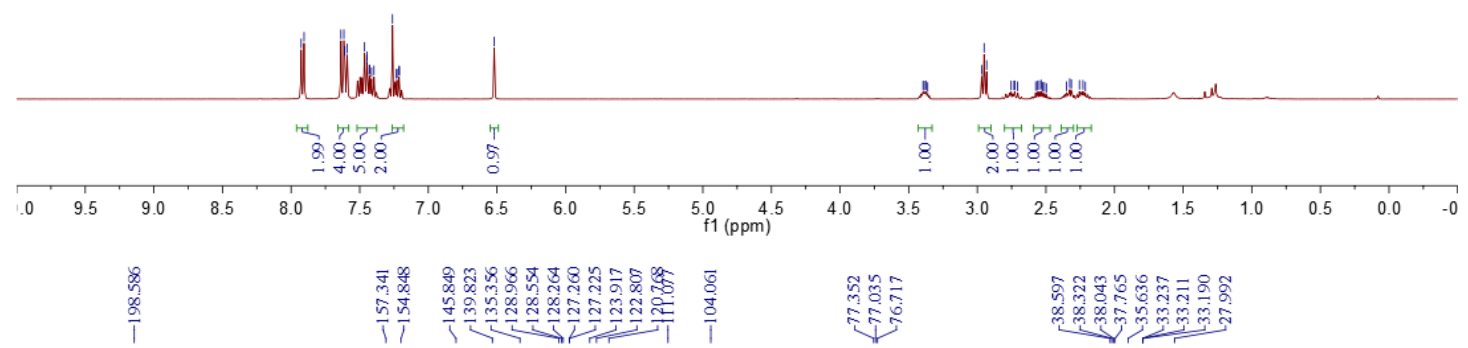

ํํ을

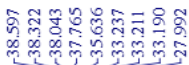

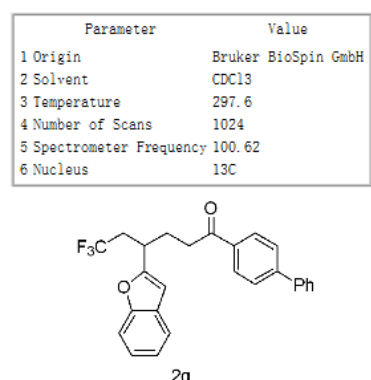

$2 \mathrm{~g}$

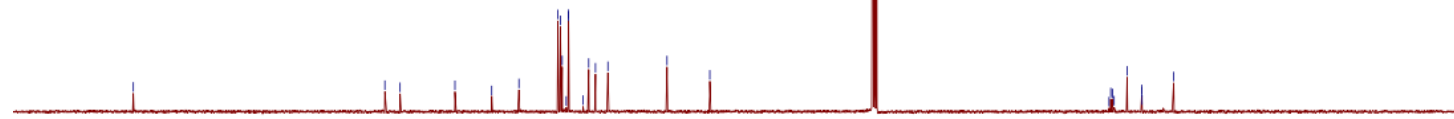

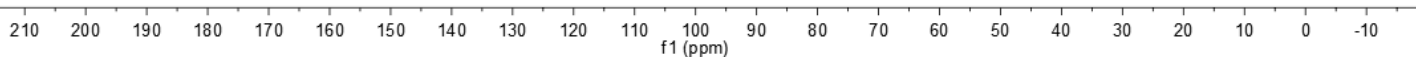




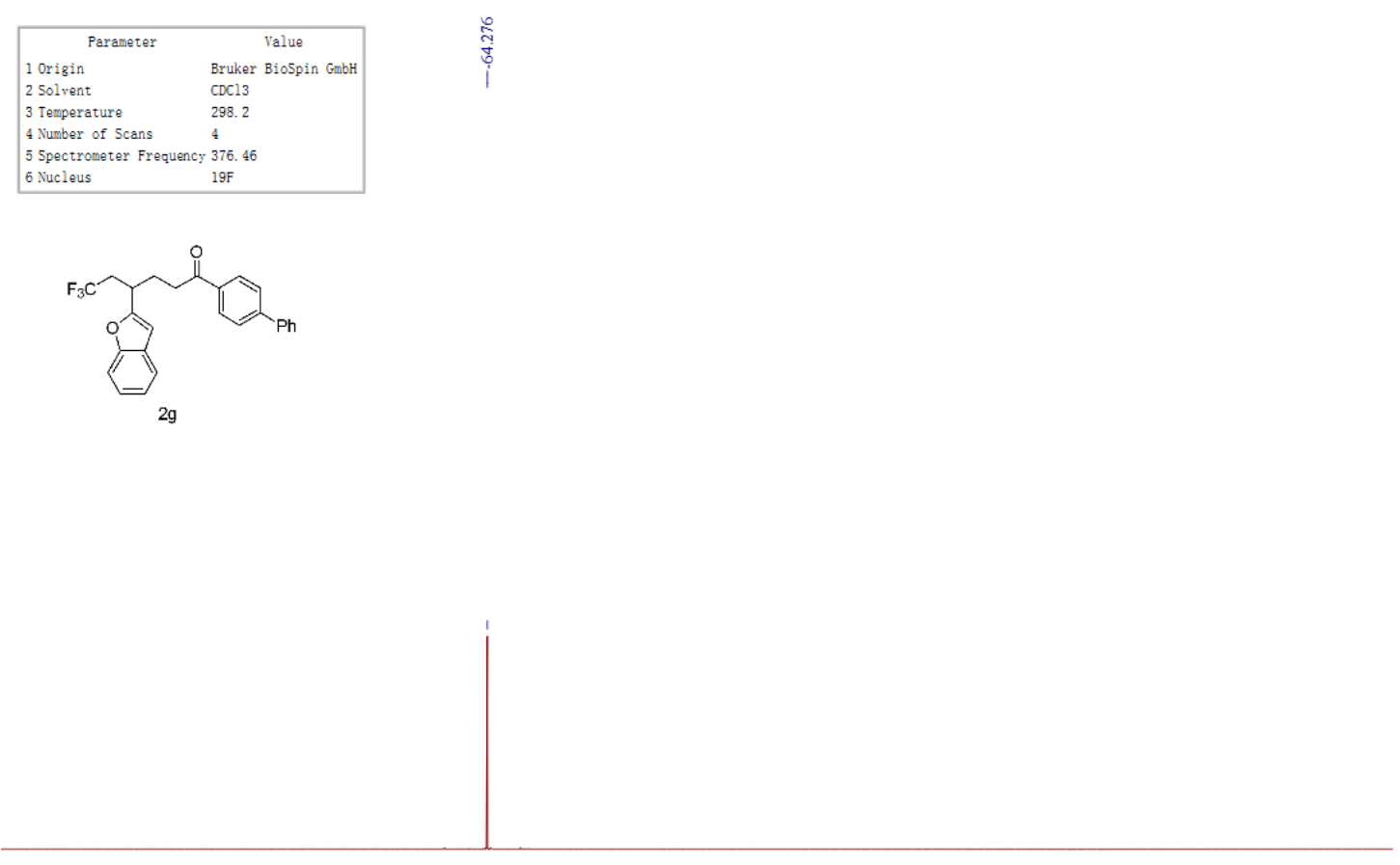

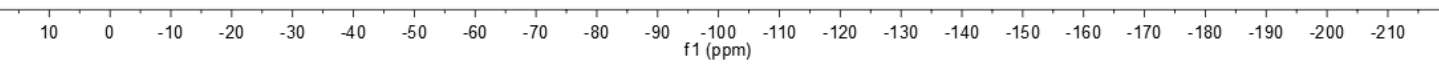

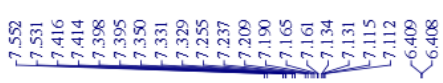

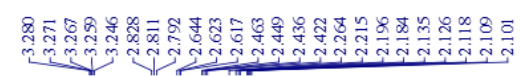
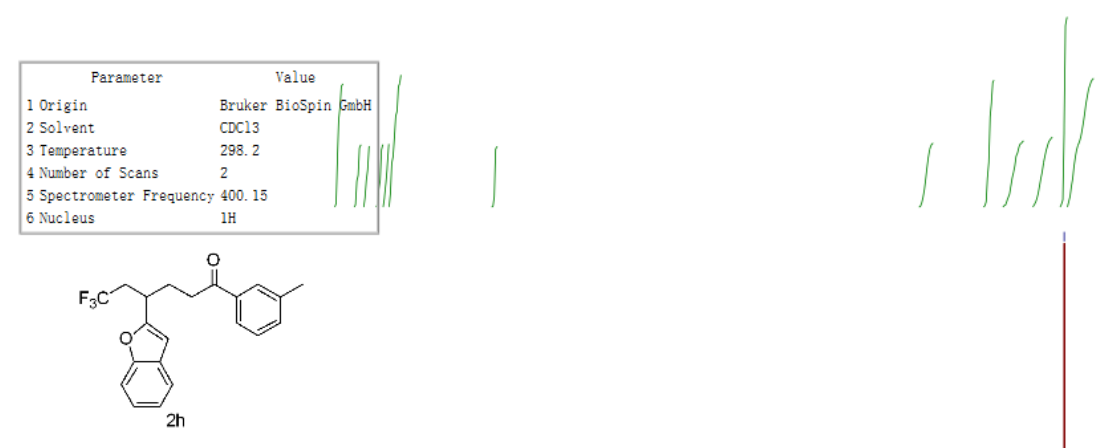

4 Number of Scans
5 Spectrometer Frequency 400.15
6 Nucleus

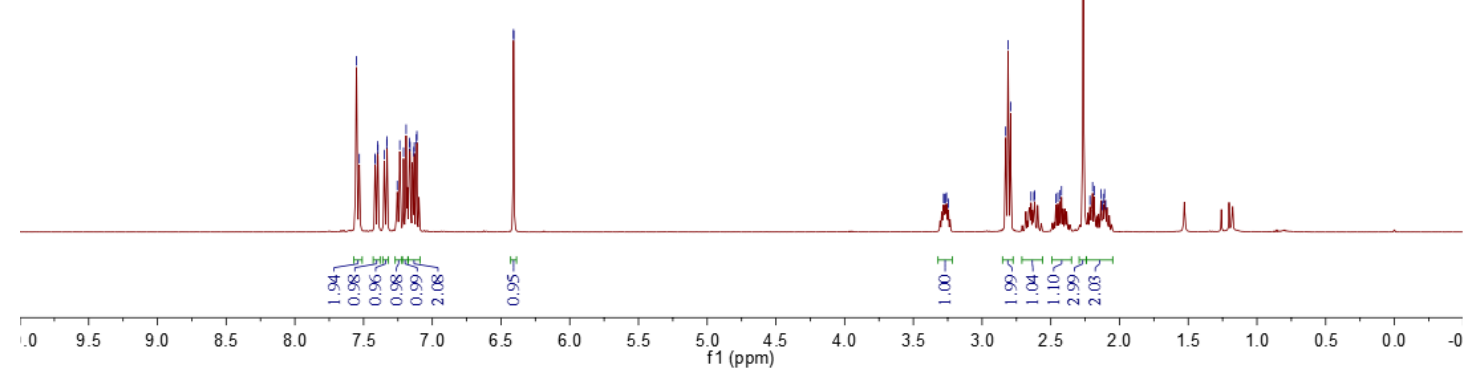




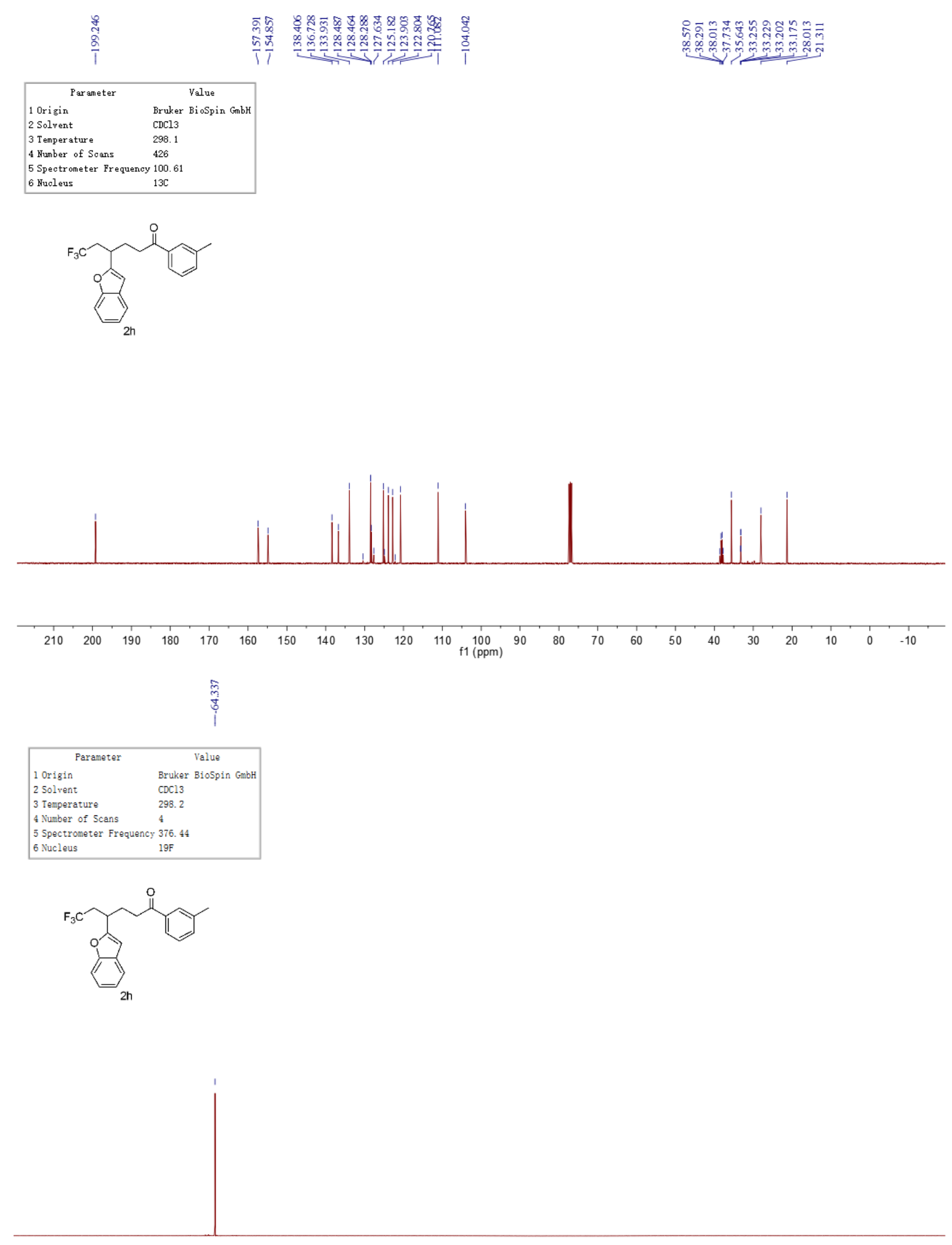

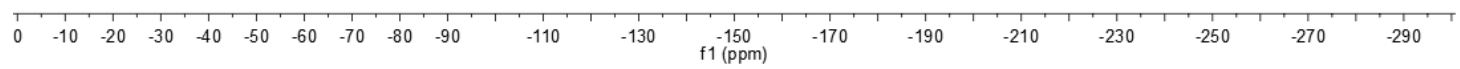



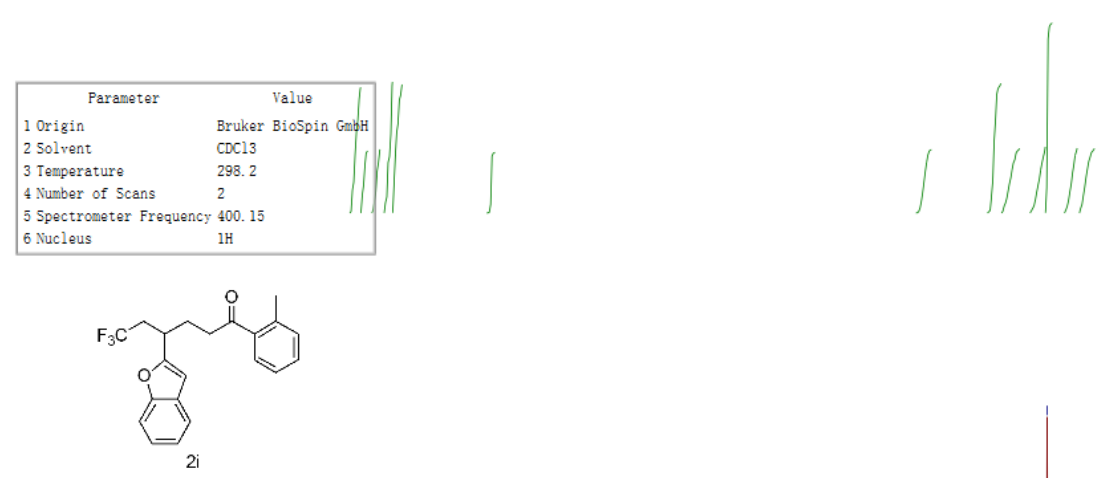

$$
\begin{array}{lll}
5 \text { Spectrometer Frequency } 400.15 \\
6 \text { Nucleus } & 1 \mathrm{H}
\end{array}
$$

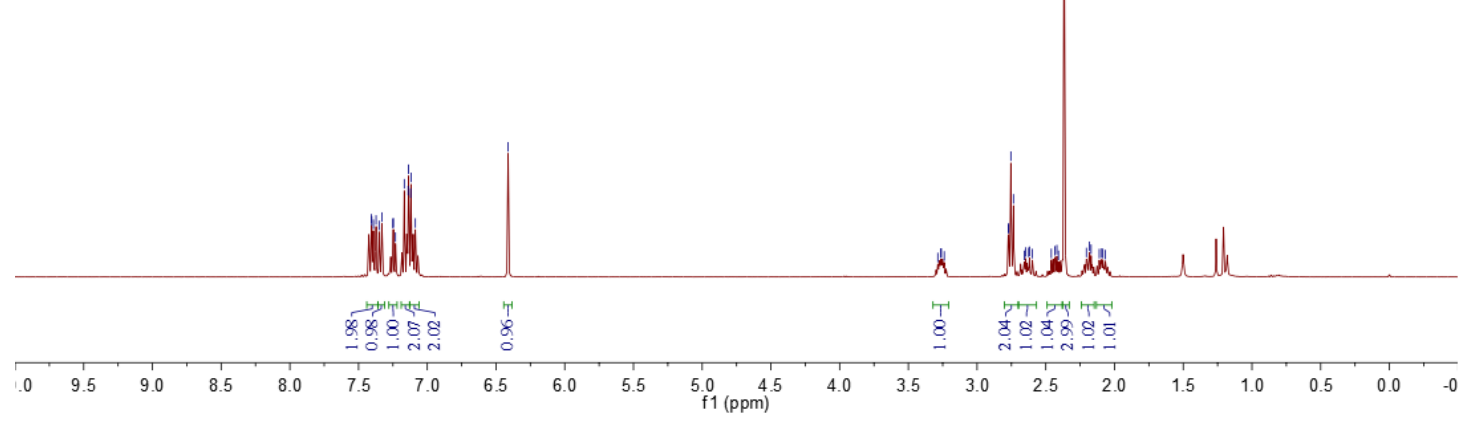

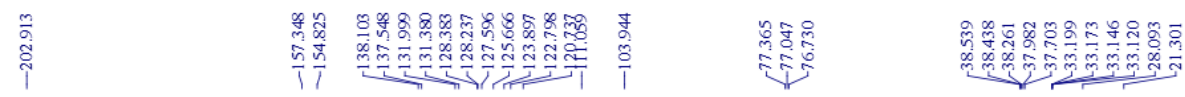

\begin{tabular}{|ll|}
\hline \multicolumn{1}{|c|}{ Parameter } & \multicolumn{1}{c|}{ Value } \\
1 Origin & Bruker BioSpin GmbH \\
2 Solvent & CDC13 \\
3 Temperature & 298.2 \\
4 Number of Scans & 1024 \\
5 Spectrometer Frequency 100.62 \\
6 Nucleus & $13 C$ \\
\hline
\end{tabular}
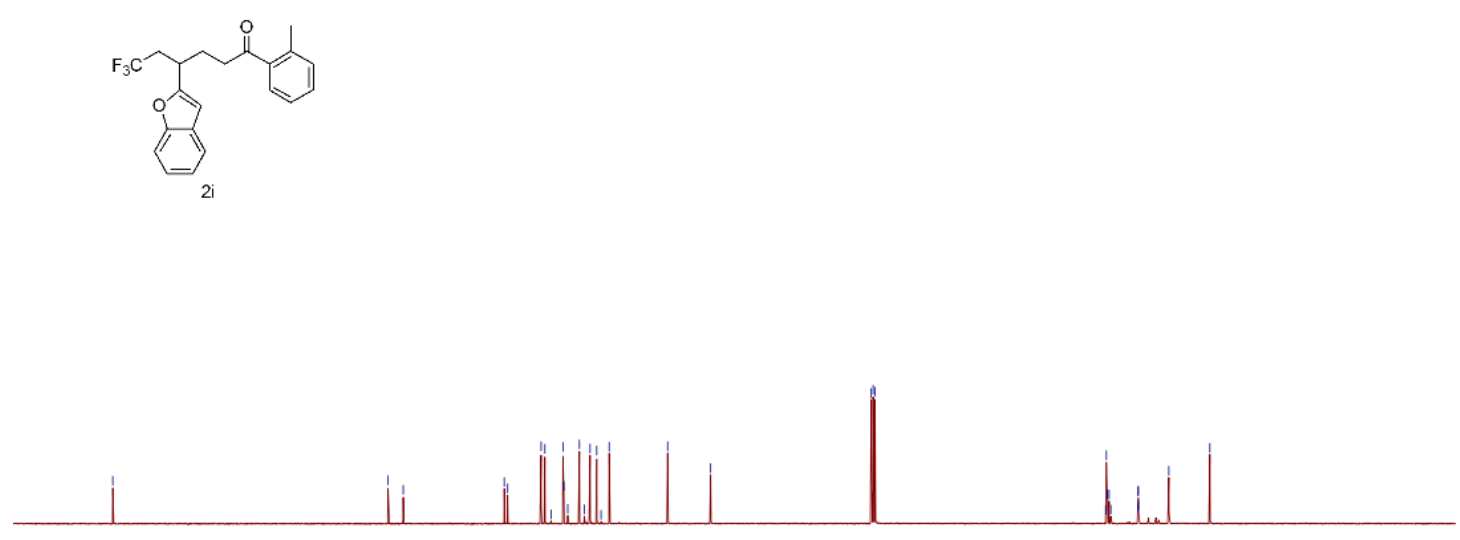

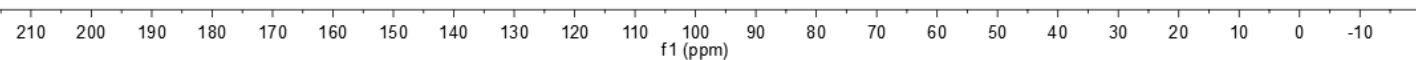




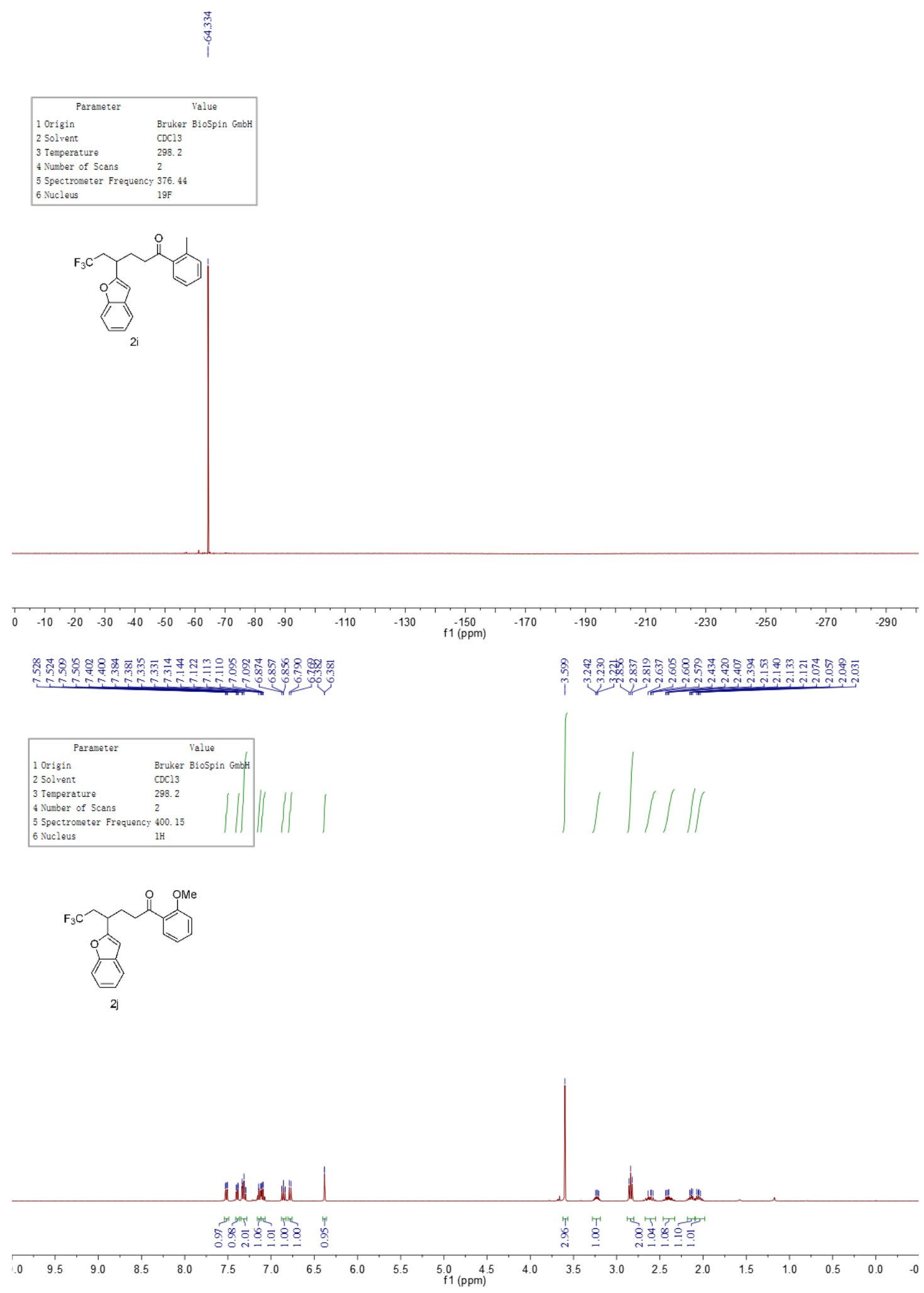



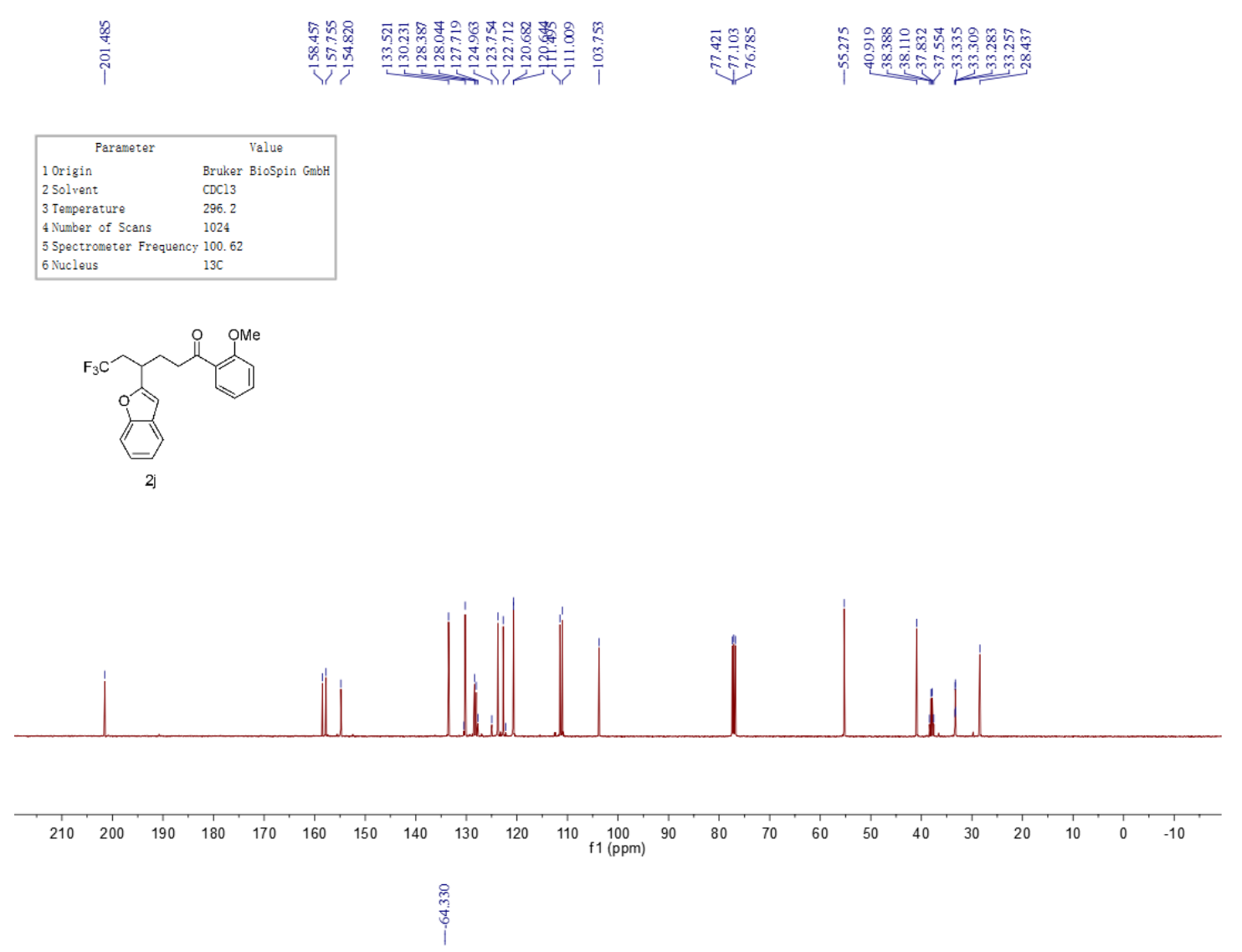

\begin{tabular}{|ll|}
\hline \multicolumn{1}{|c|}{ Parameter } & \multicolumn{1}{c|}{ Value } \\
1 Origin & Bruker BioSpin GmbH \\
2 Solvent & CDC13 \\
3 Temperature & 298.2 \\
4 Number of Scans & 2 \\
5 Spectrometer Frequency 376.52 \\
6 Nucleus & 19F \\
\hline
\end{tabular}

5 Spectrometer Frequency 376.52
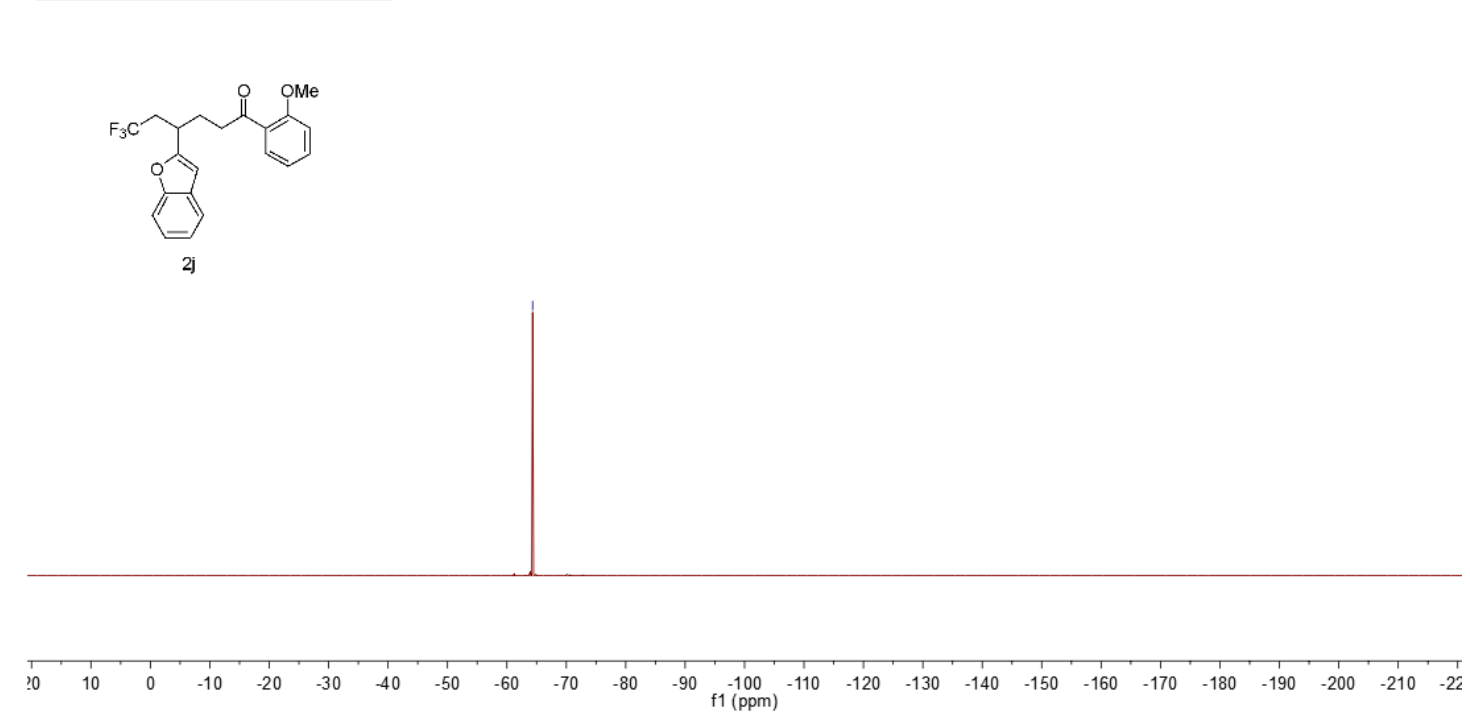


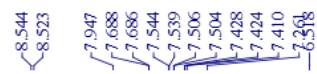
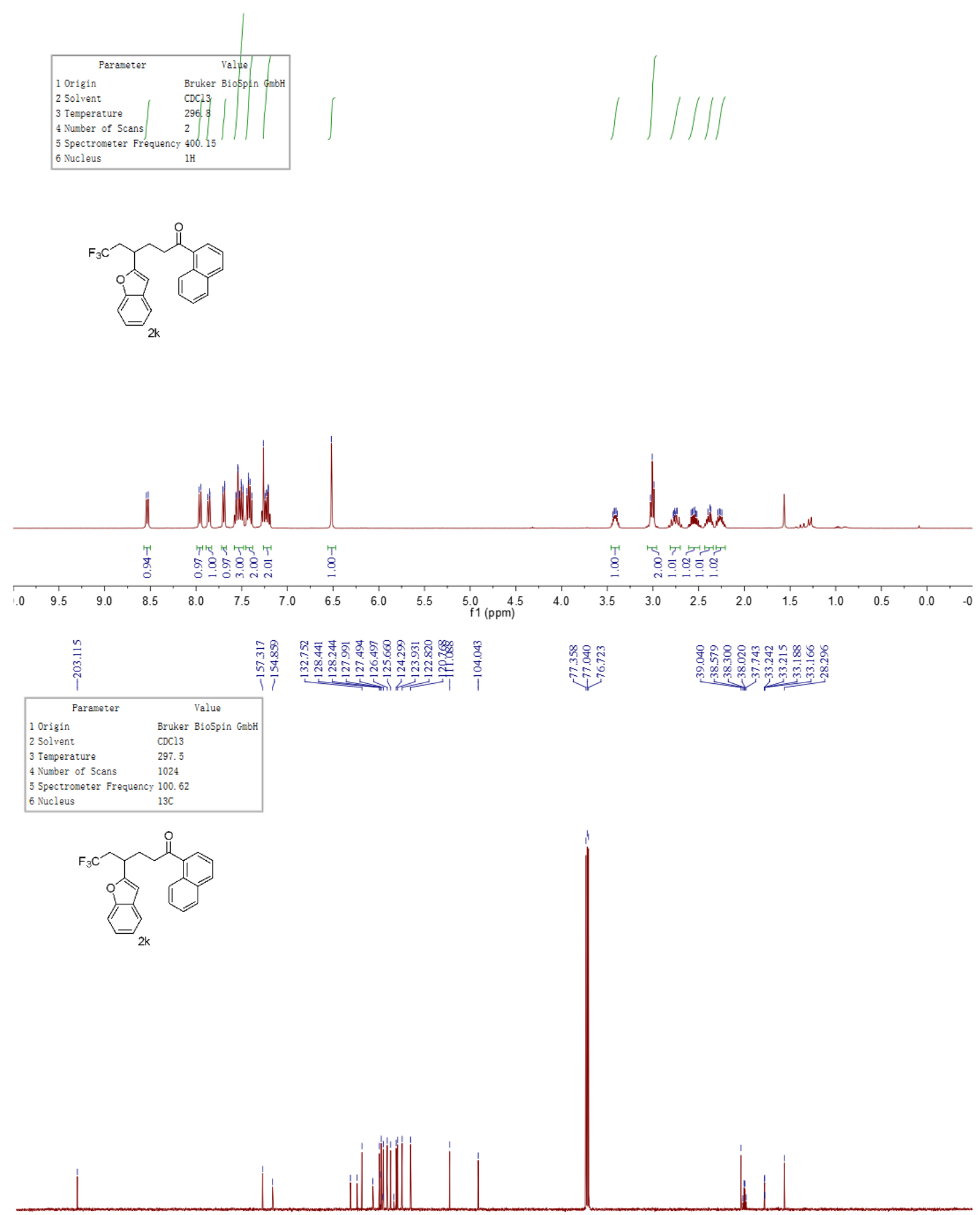

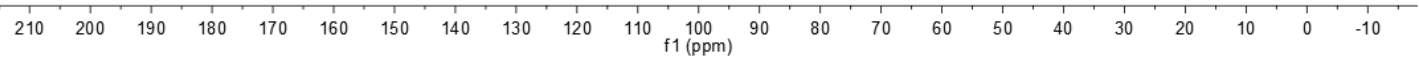




\begin{tabular}{|ll|}
\hline \multicolumn{1}{|c|}{ Parameter } & \multicolumn{1}{c|}{ Value } \\
1 Origin & Bruker BioSpin GmbH \\
2 Solvent & CDC13 \\
3 Temperature & 298.2 \\
4 Number of Scans & 4 \\
5 Spectrometer Frequency & 376.46 \\
6 Nucleus & $19 \mathrm{~F}$ \\
\hline
\end{tabular}<smiles>O=C(CCC(Cc1cccc2ccccc12)CC(F)(F)F)c1cccc2ccccc12</smiles>

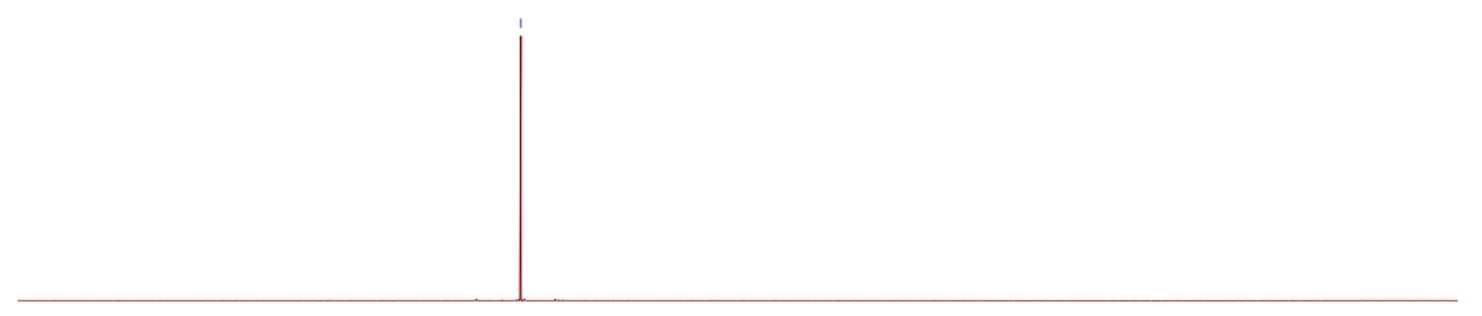
\begin{tabular}{rlllllllllllllllllllllllllll}
\hline 10 & 0 & -10 & -20 & -30 & -40 & -50 & -60 & -70 & -80 & -90 & -100 & -110 & -120 & -130 & -140 & -150 & -160 & -170 & -180 & -190 & -200 & -210 \\
\hline
\end{tabular}

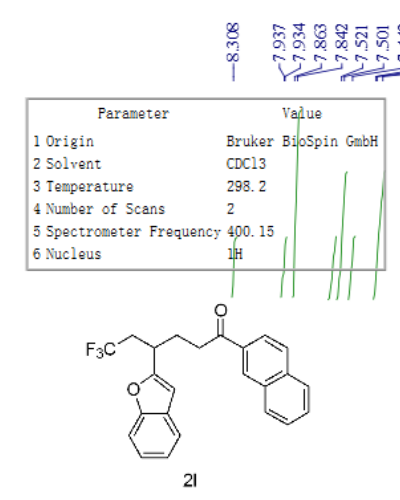

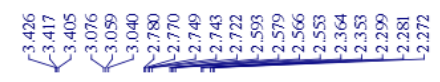

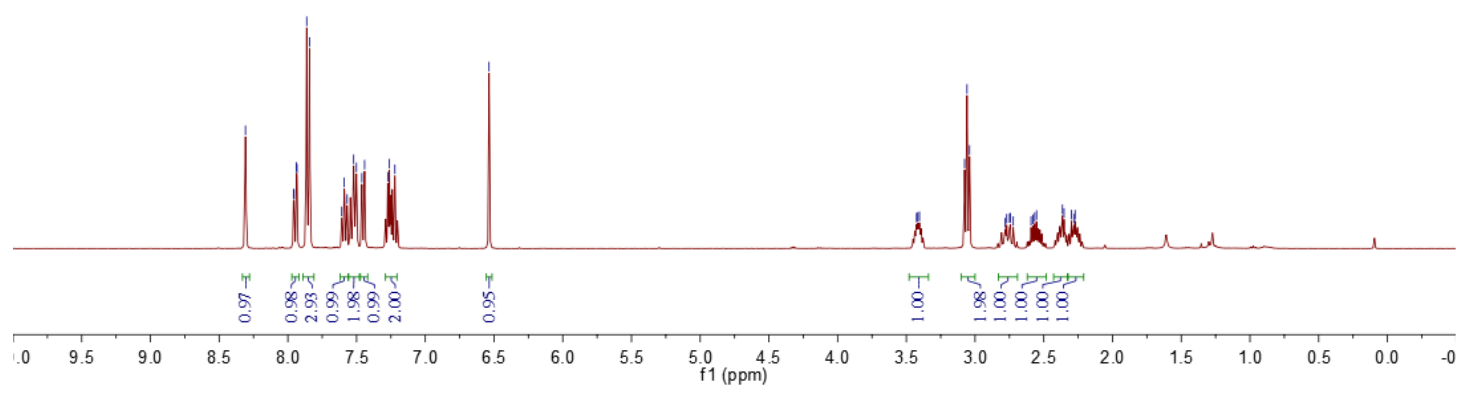




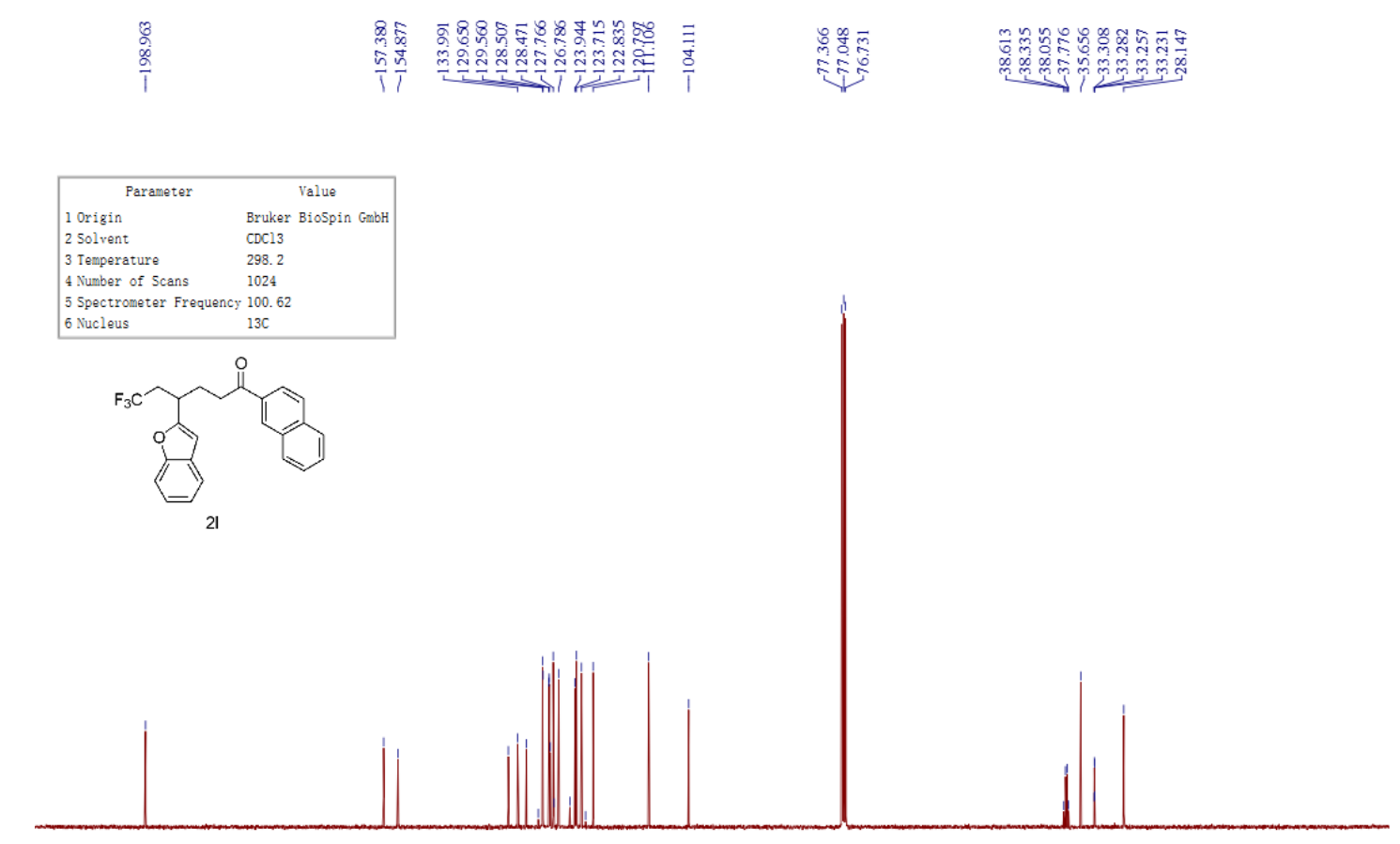

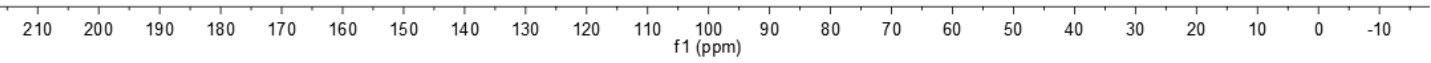
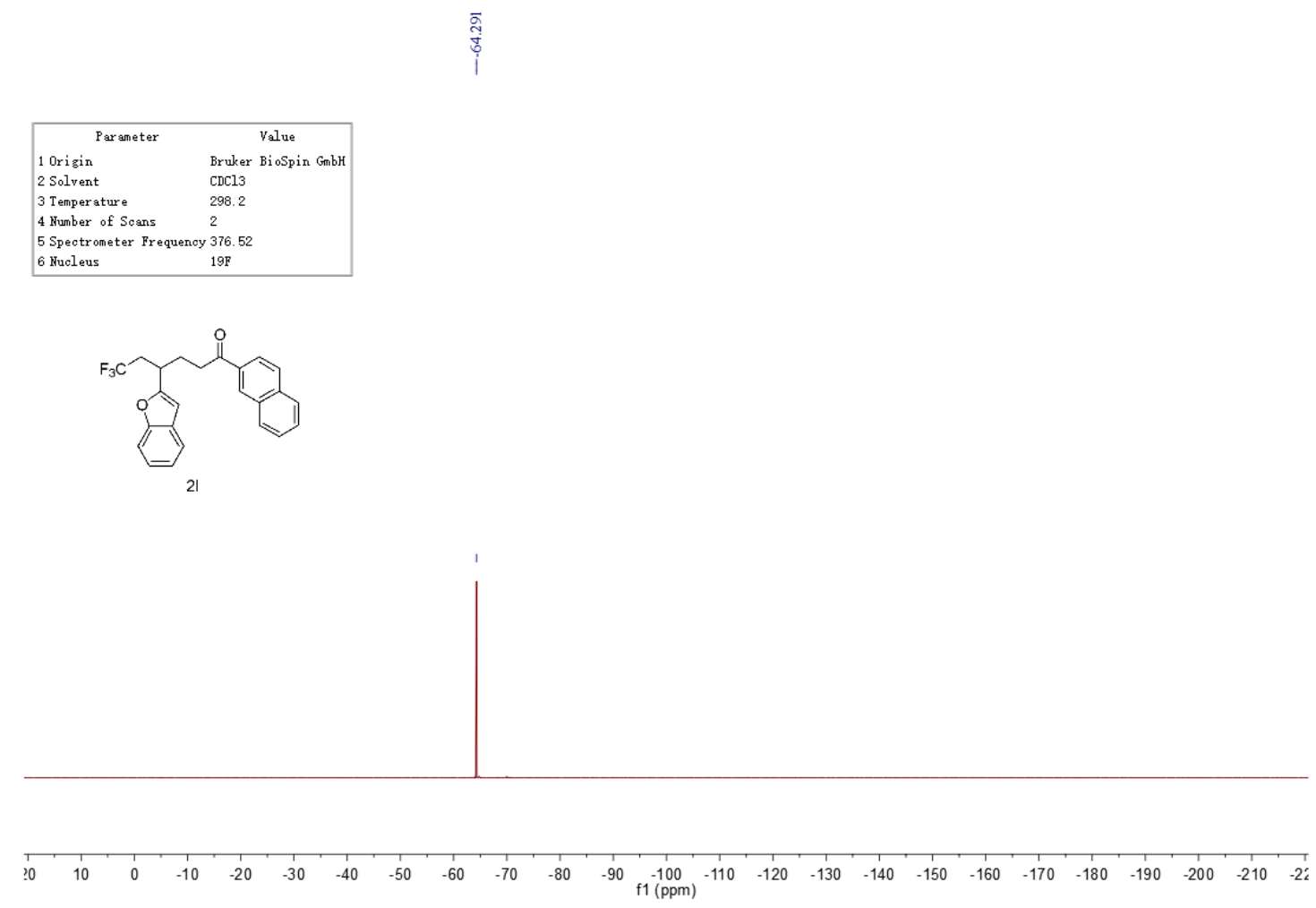


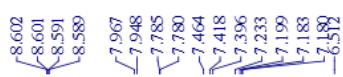

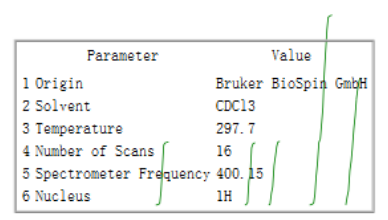

(m)

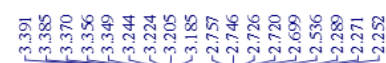

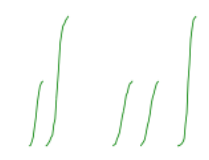

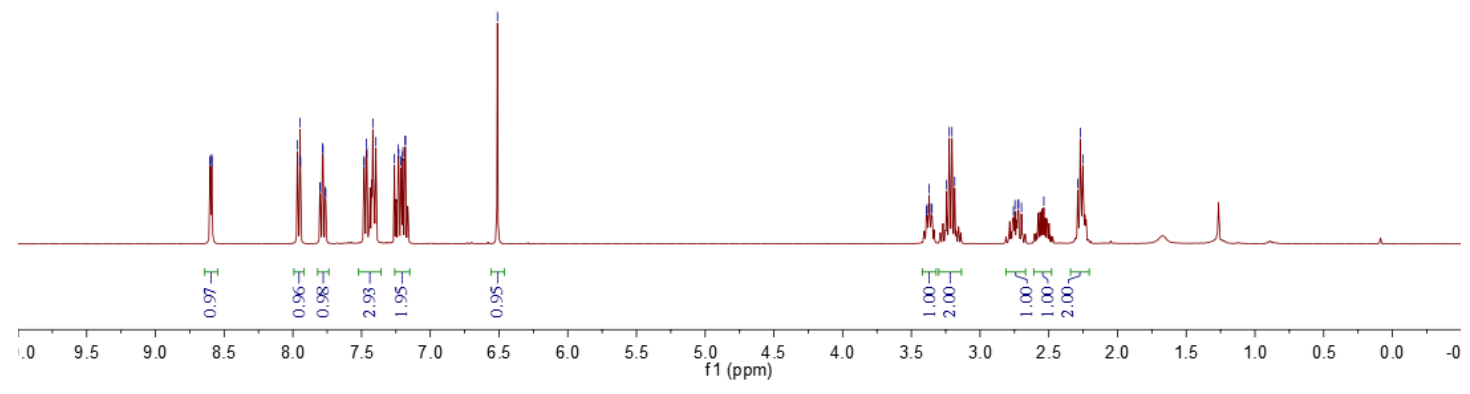
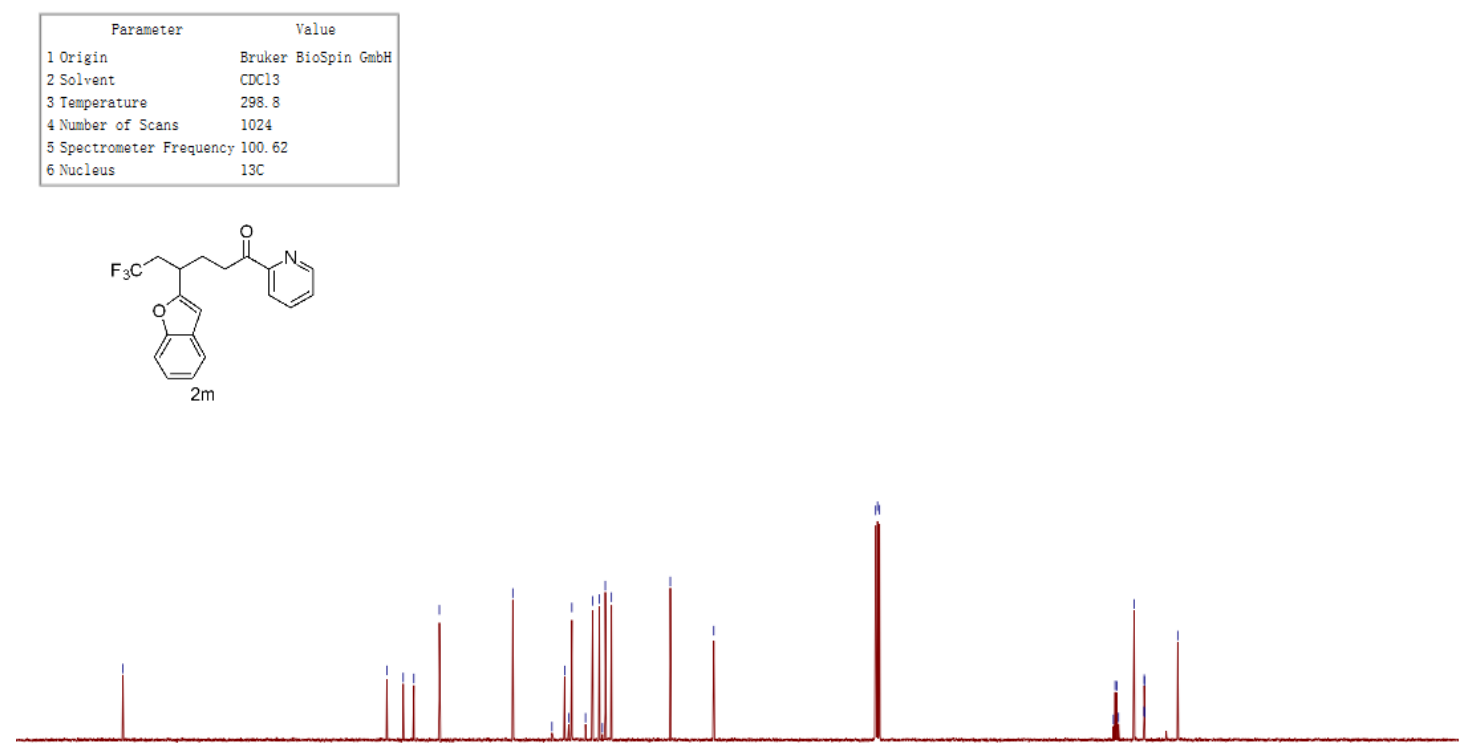

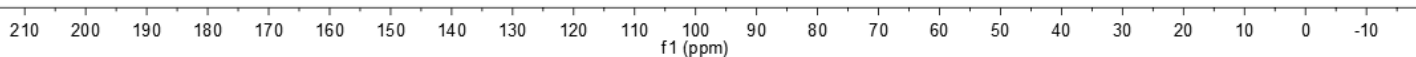



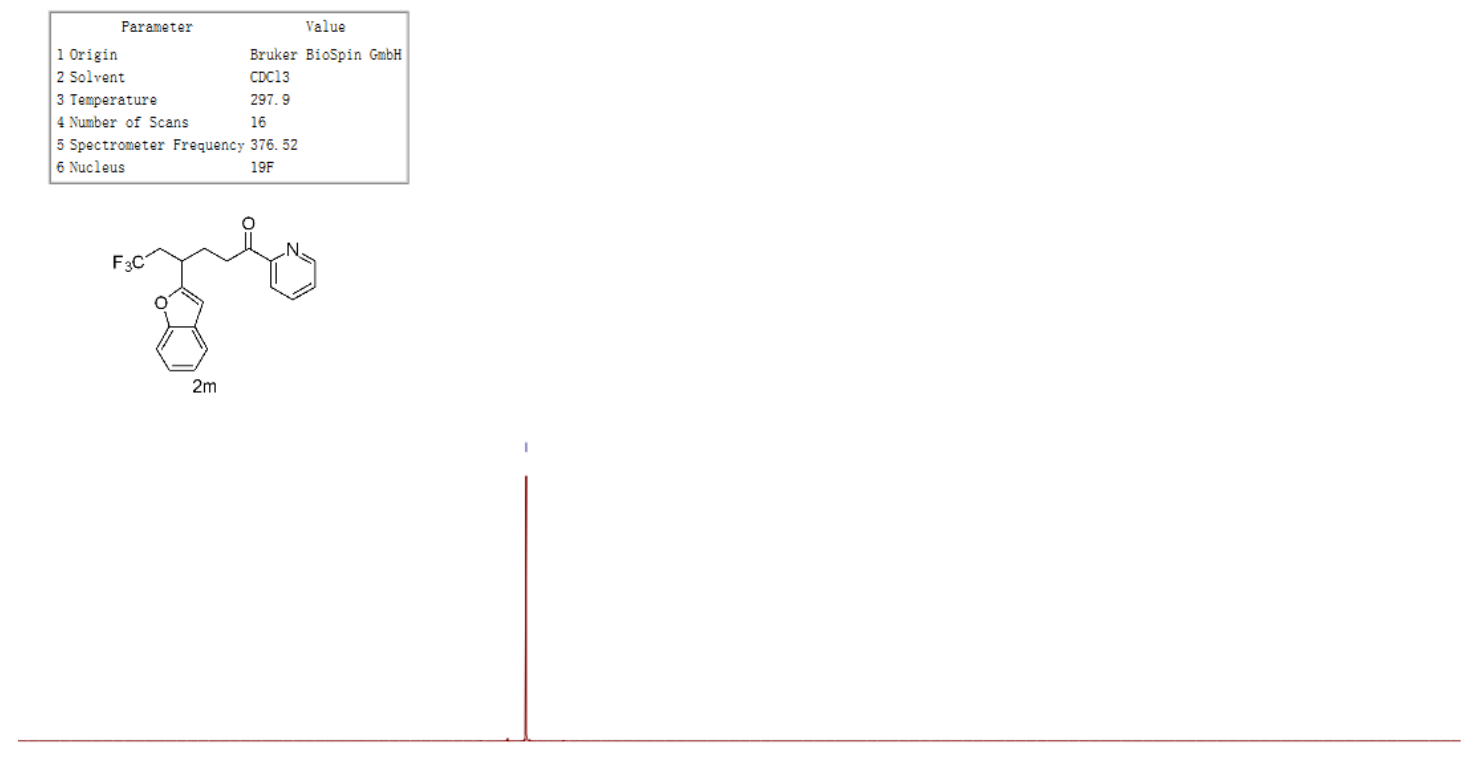

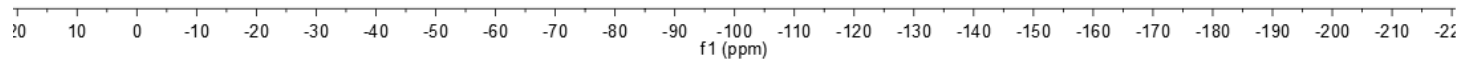

\section{HMBC}

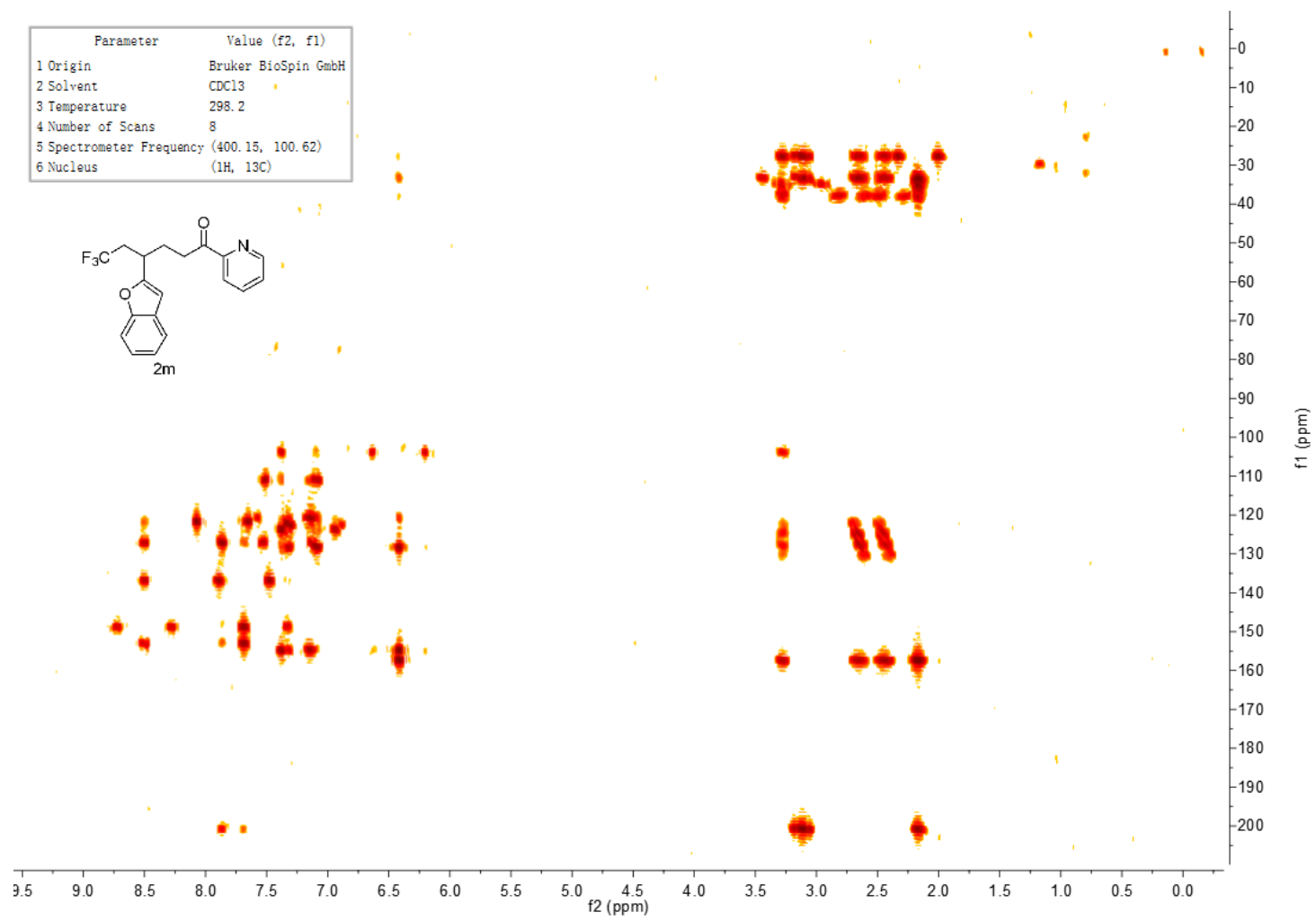



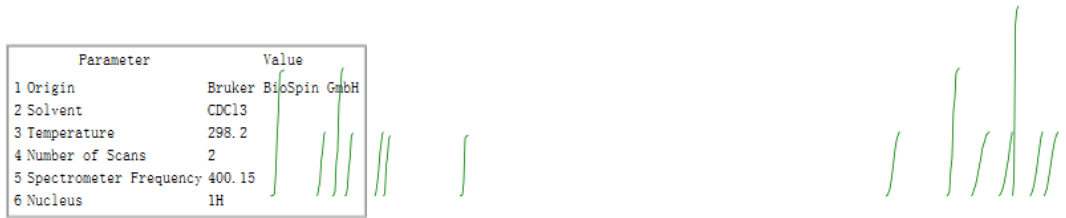

6 Nucleus<smiles>Cc1cccc2cc(CCC(=O)c3ccccc3)oc12</smiles>

2 n

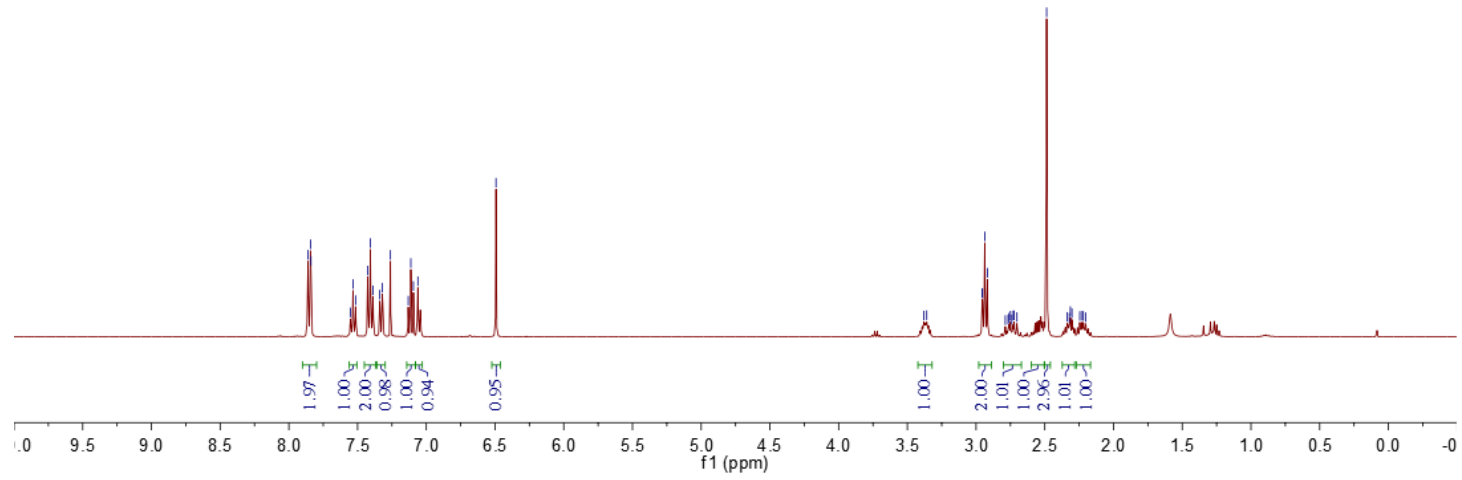

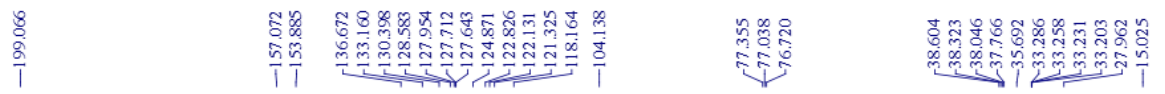

\begin{tabular}{|ll|}
\hline \multicolumn{1}{|c|}{ Parameter } & \multicolumn{1}{c|}{ Value } \\
1 Origin & Bruker BioSpin GmbH \\
2 Solvent & CDC13 \\
3 Temperature & 298.2 \\
4 Number of Scans & 1024 \\
5 Spectrometer Frequency & 100.62 \\
6 Nucleus & $13 \mathrm{C}$ \\
\hline
\end{tabular}<smiles>Cc1cccc2cc(C(CCC(=O)c3ccccc3)CC(F)(F)F)oc12</smiles>

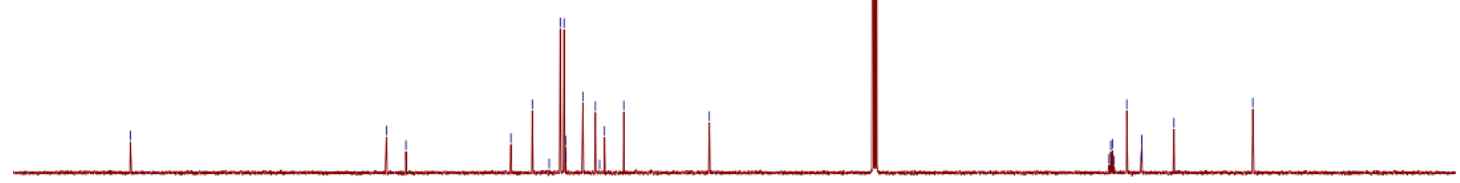

$\begin{array}{lllllllllllllllllllllll}210 & 200 & 190 & 180 & 170 & 160 & 150 & 140 & 130 & 120 & 110 & 100 & 90 & 80 & 70 & 60 & 50 & 40 & 30 & 20 & 10 & 0 & -10\end{array}$ 

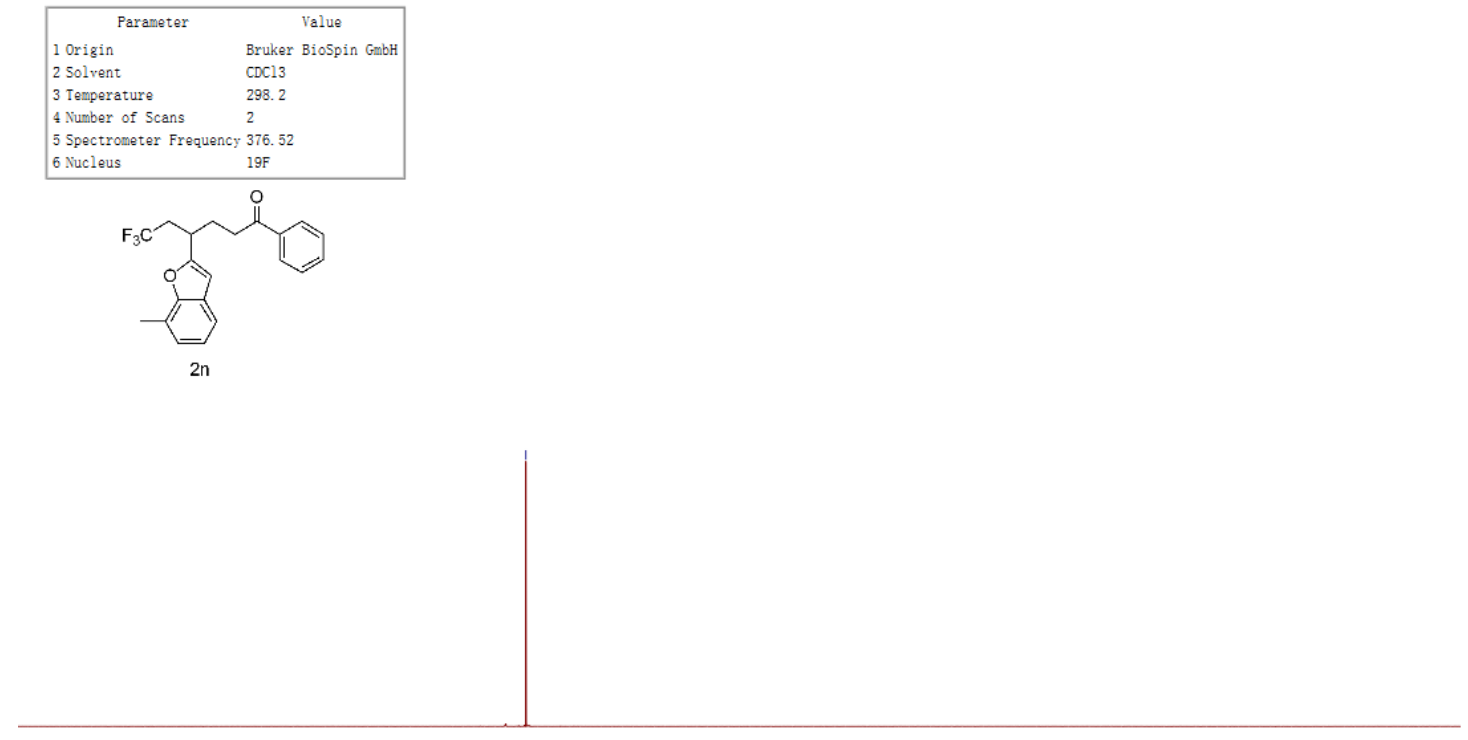

$\begin{array}{lllllllllllllllllllllllllllllll}20 & 10 & 0 & -10 & -20 & -30 & -40 & -50 & -60 & -70 & -80 & -90 & -100 & -110 & -120 & -130 & -140 & -150 & -160 & -170 & -180 & -190 & -200 & -210 & -2.2\end{array}$

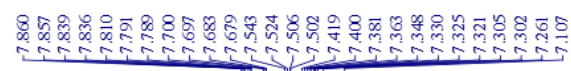

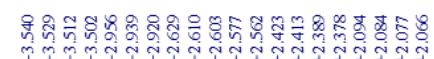

\begin{tabular}{|ll|}
\hline \multicolumn{1}{|c|}{ Parameter } & \multicolumn{2}{c|}{ Value } \\
1 Origin & Bruker Biospin Gmbb \\
2 Solment & CDC13 \\
3 Temperature & 298.2 \\
4 Number of Scans & 2 \\
5 Spectrometer Frequency 400.15 \\
6 Nucleus & $1 \mathrm{H}$
\end{tabular}

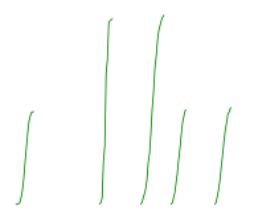

(1)

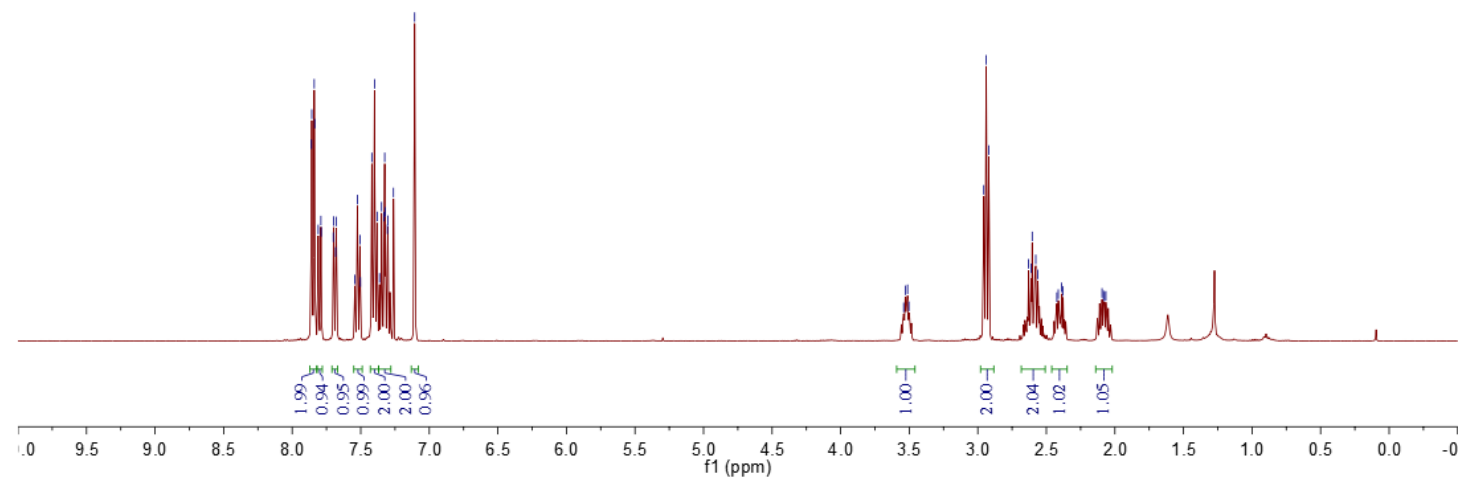



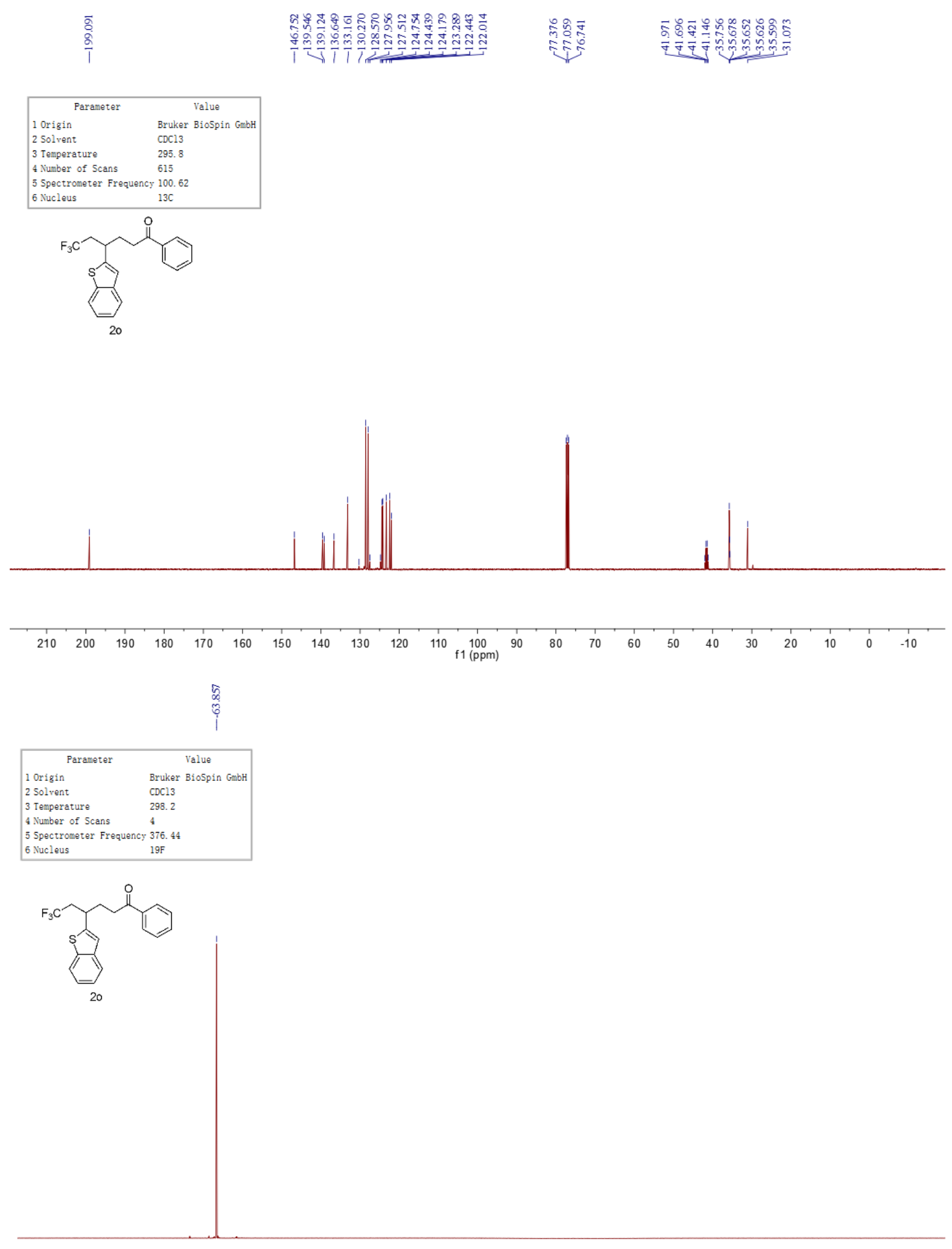

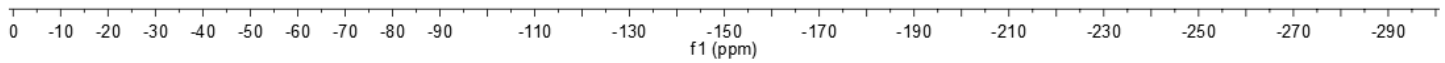




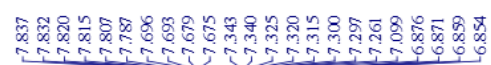
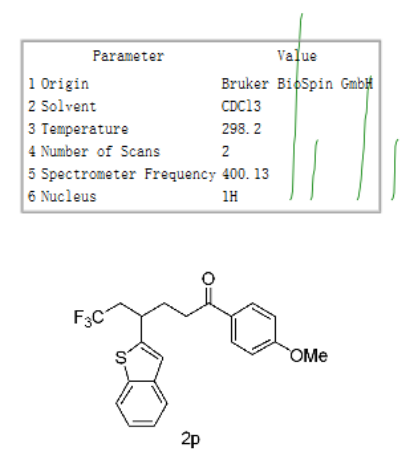

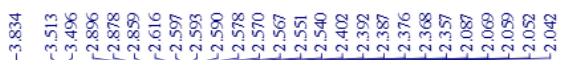

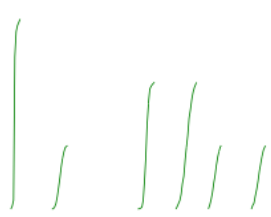

Nhat

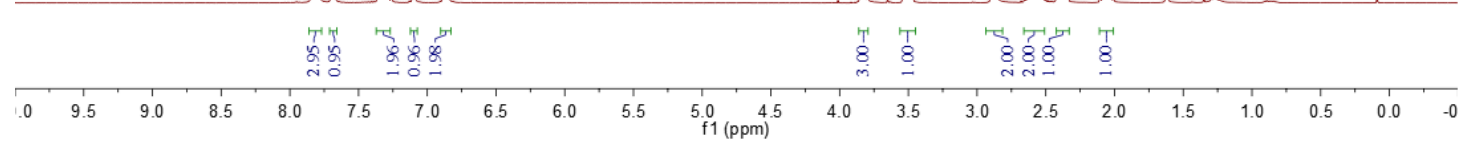

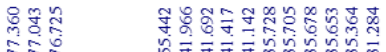

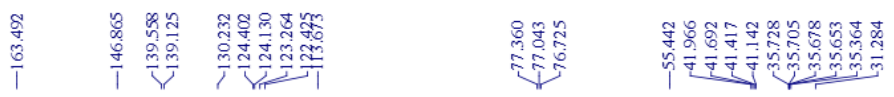

\begin{tabular}{|ll|}
\hline \multicolumn{1}{|c|}{ Parameter } & \multicolumn{1}{c|}{ Value } \\
1 Origin & Bruker Biospin GmbH \\
2 Solvent & CDC13 \\
3 Temperature & 298.2 \\
4 Number of Scans & 1024 \\
5 Spectrometer Frequency 100.62 \\
6 Nucleus & $13 \mathrm{C}$ \\
\hline
\end{tabular}<smiles>COc1ccc(C(=O)CCC(CC(F)(F)F)c2cc3ccccc3s2)cc1</smiles>

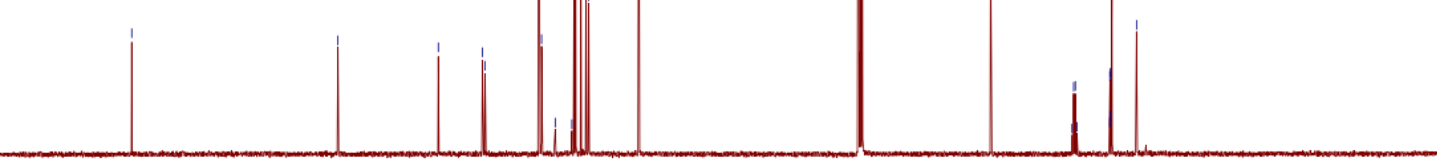

$\begin{array}{lllllllllllllllllllllllllll} & 210 & 200 & 190 & 180 & 170 & 160 & 150 & 140 & 130 & 120 & 110 & 100 & 90 & 80 & 70 & 60 & 50 & 40 & 30 & 20 & 10 & 0 & -10\end{array}$ 

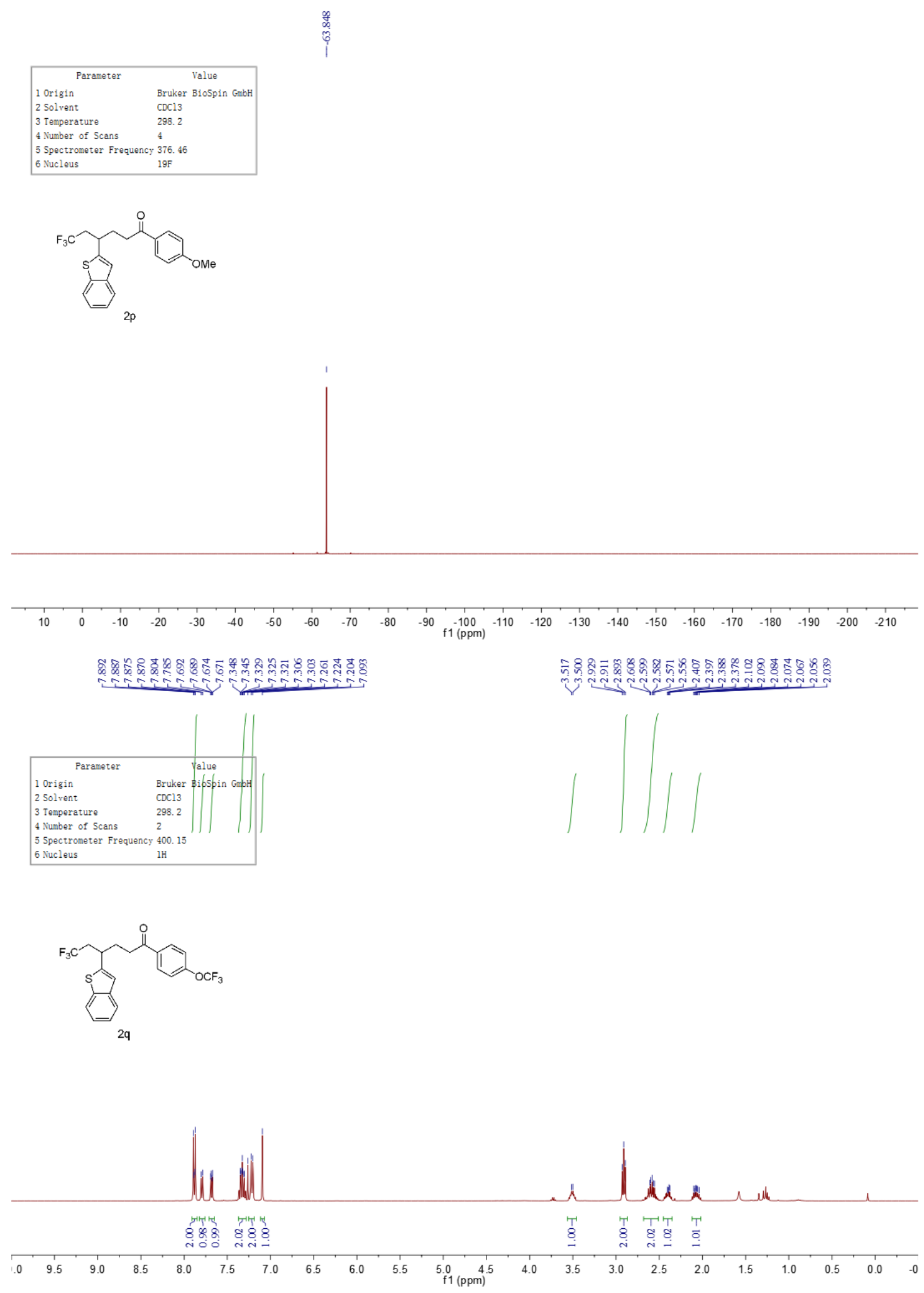

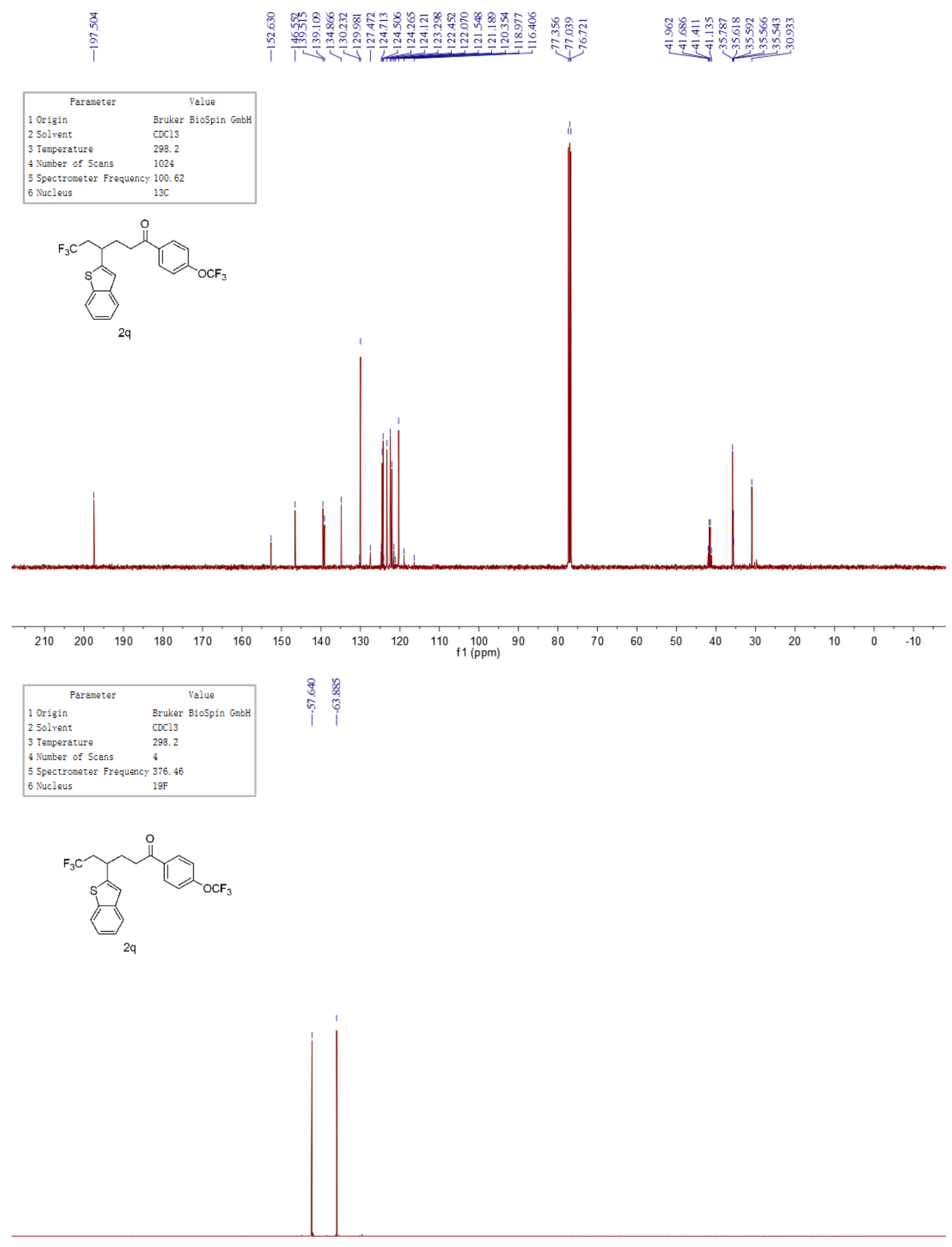

$\begin{array}{lllllllllllllllllllllllllll}1 & 10 & 0 & -10 & -20 & -30 & -40 & -50 & -60 & -70 & -80 & -90 & -100 & -110 & -120 & -130 & -140 & -150 & -160 & -170 & -180 & -190 & -200 & -210\end{array}$ 


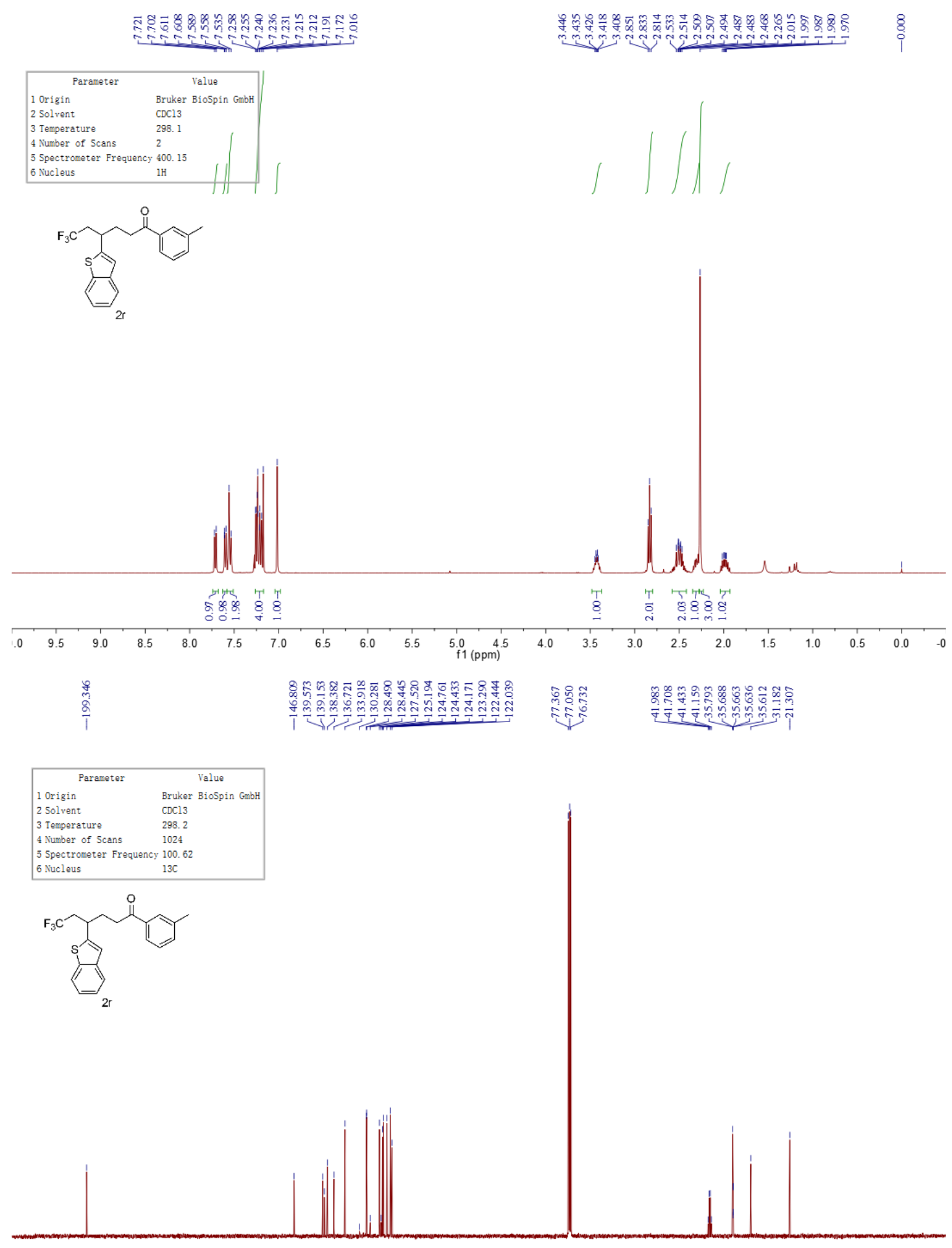

$\begin{array}{lllllllllllllllllllllllll}1 & 1 \\ 210 & 200 & 190 & 180 & 170 & 160 & 150 & 140 & 130 & 120 & 110 & 100 & 90 & 80 & 70 & 60 & 50 & 40 & 30 & 20 & 10 & 0 & -10\end{array}$ 

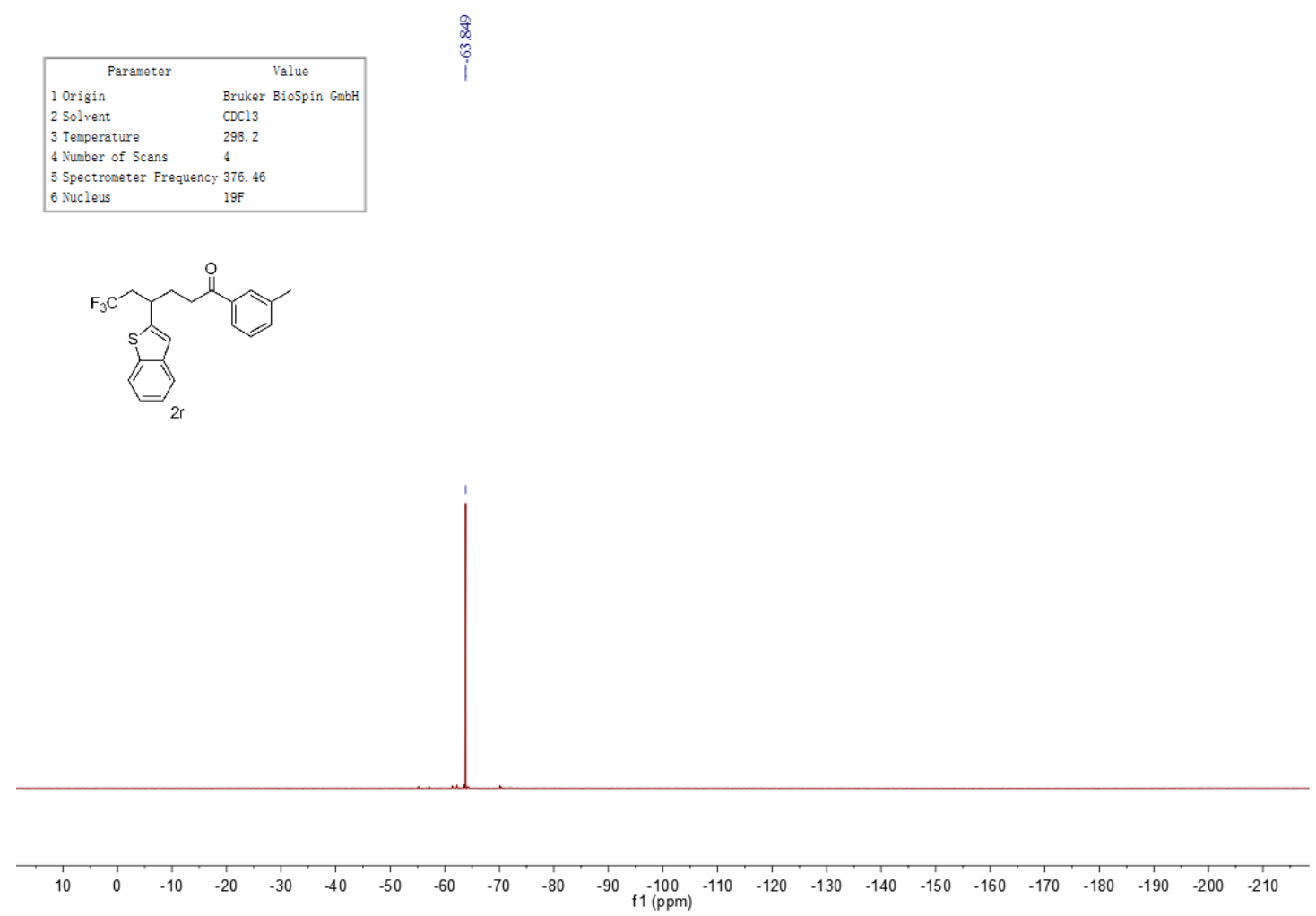

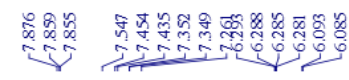

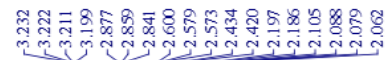
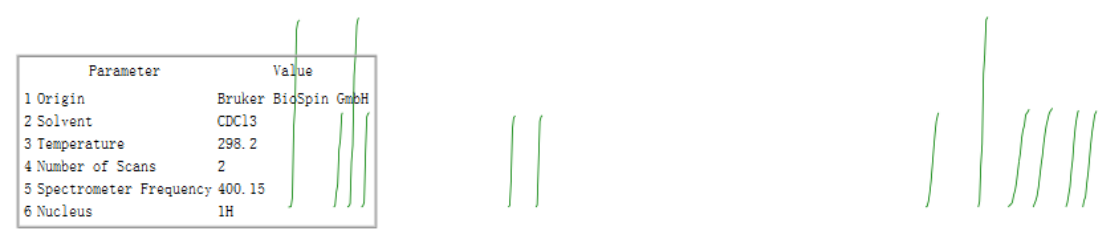<smiles>CCC(CCC(=O)C1CCCC1)C1CCCC1</smiles>

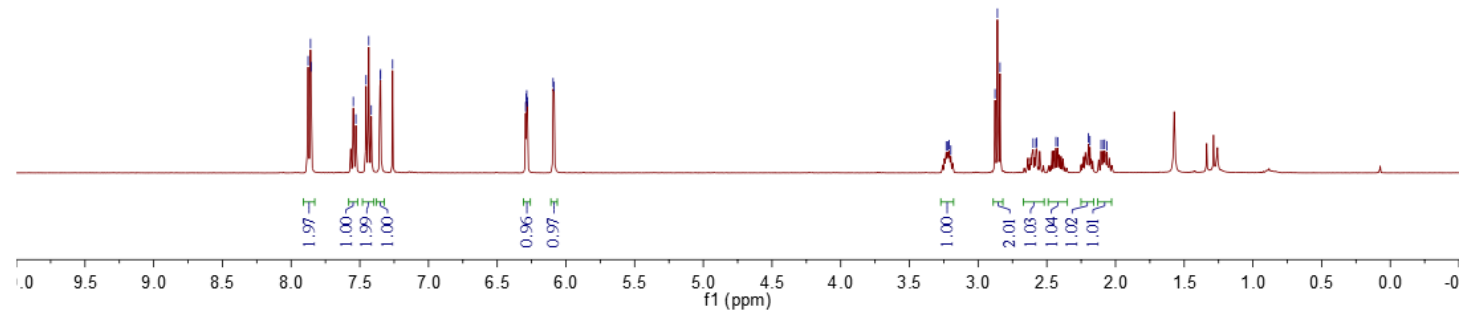



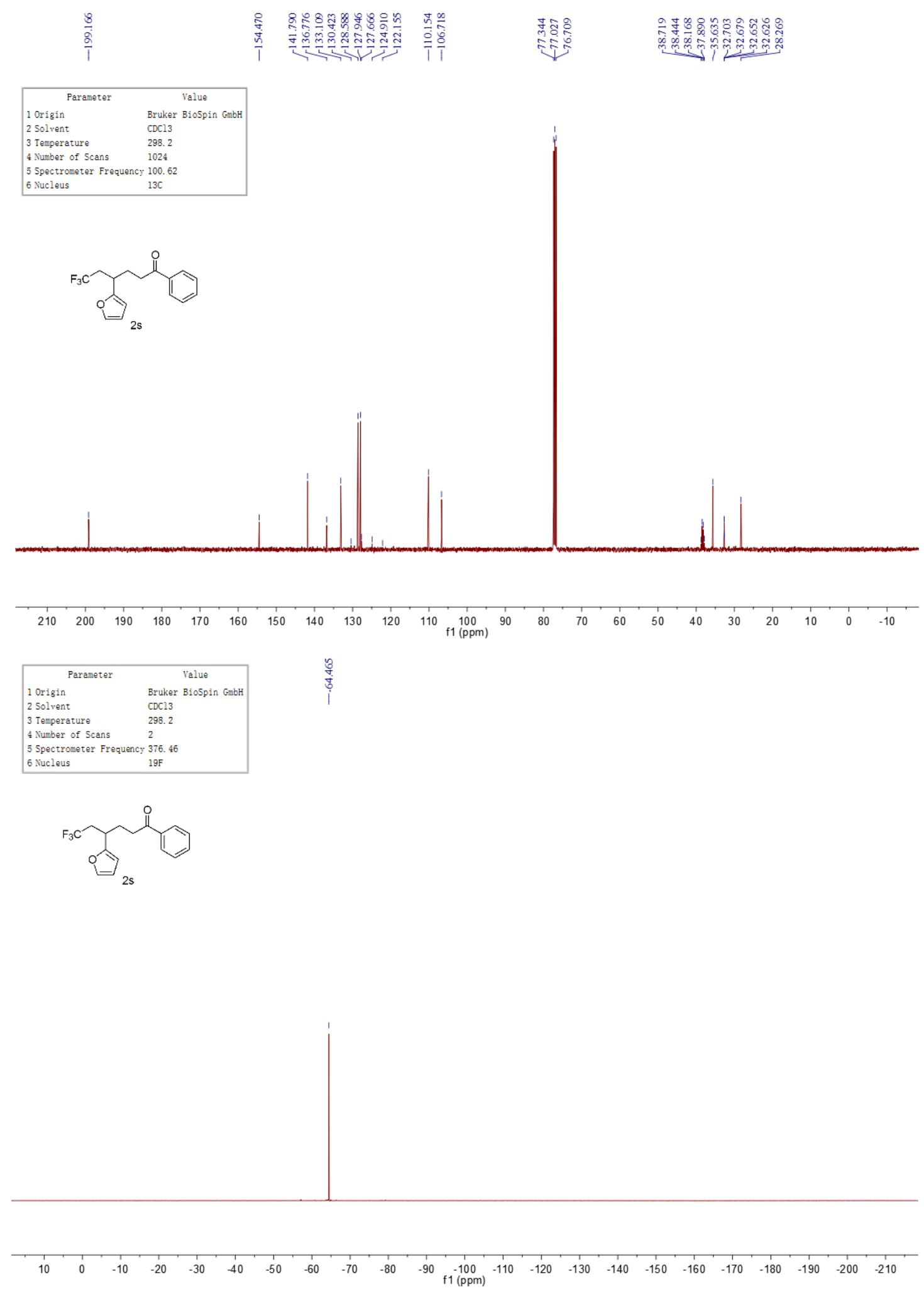

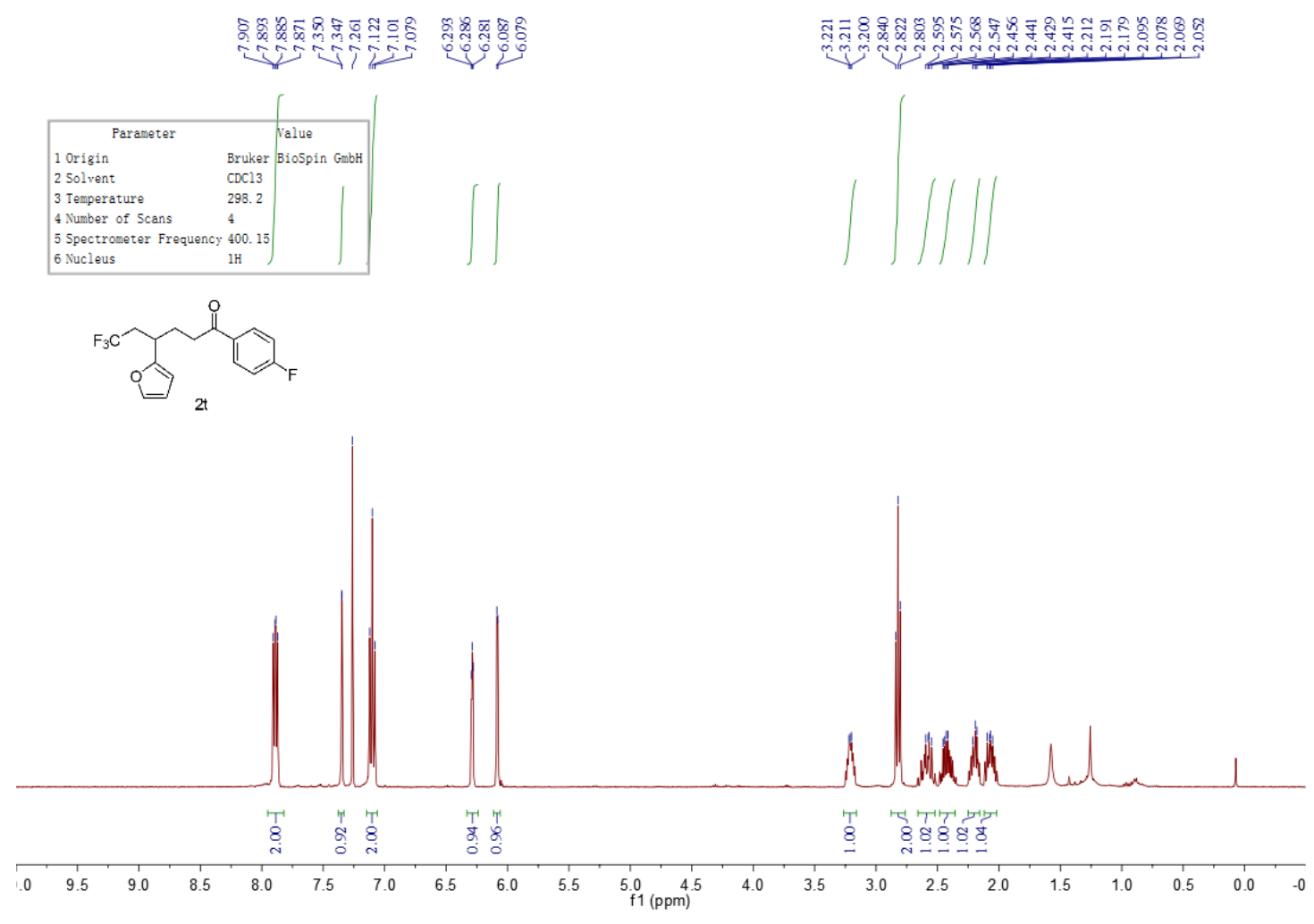

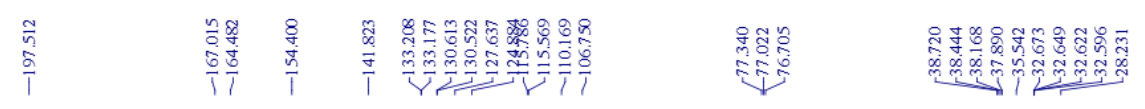

\begin{tabular}{|ll|}
\hline \multicolumn{1}{|c|}{ Parameter } & \multicolumn{1}{c|}{ Value } \\
1 Origin & Bruker \\
2 Solvospin GmbH & CDC13 \\
3 Temperature & 298.1 \\
4 Number of Scans & 1024 \\
5 Spectrometer Frequency & 100.62 \\
6 Nucleus & $13 \mathrm{C}$ \\
\hline
\end{tabular}<smiles>CCC(CCC(=O)c1ccc(C)cc1)C(=O)O</smiles>

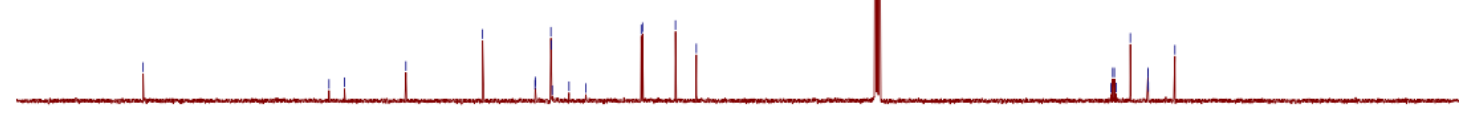

$\begin{array}{llllllllllllllllllllllll}210 & 200 & 190 & 180 & 170 & 160 & 150 & 140 & 130 & 120 & 110 & 100 & 90 & 80 & 70 & 60 & 50 & 40 & 30 & 20 & 10 & 0 & -10\end{array}$ 

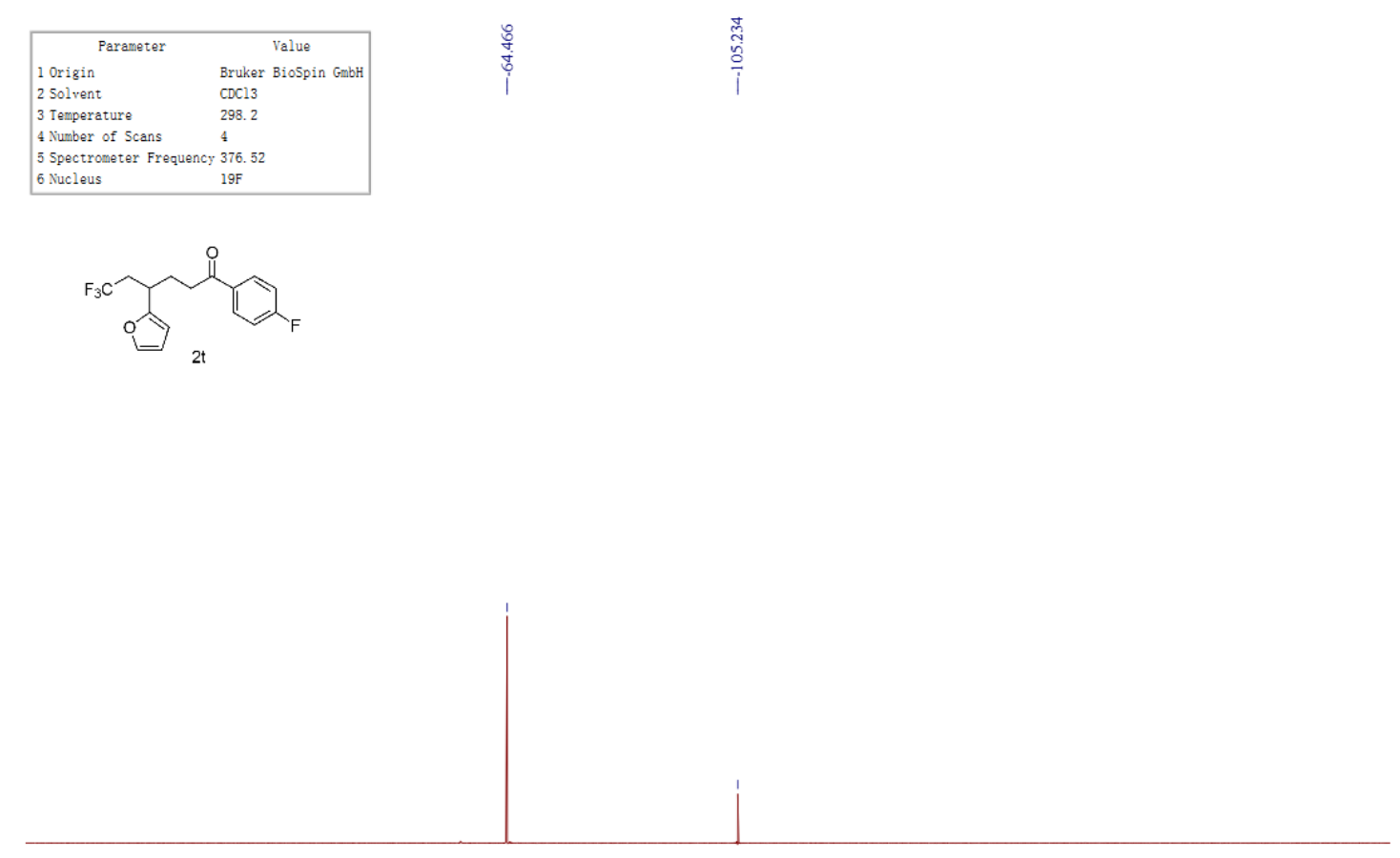

\begin{tabular}{lllllllllllllllllllllllllllll}
\hline 0 & 10 & 0 & -10 & -20 & -30 & -40 & -50 & -60 & -70 & -80 & -90 & -100 & -110 & -120 & -130 & -140 & -150 & -160 & -170 & -180 & -190 & -200 & -210 & $-2 \varepsilon$
\end{tabular}

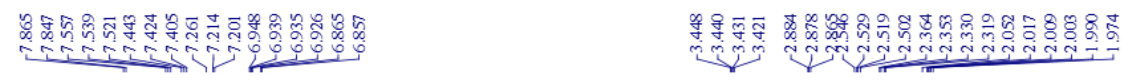

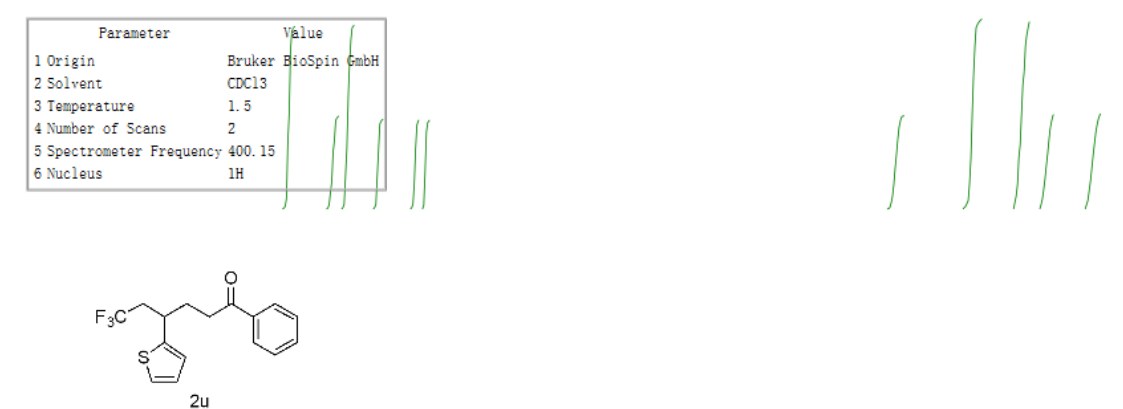

$2 \mathrm{u}$

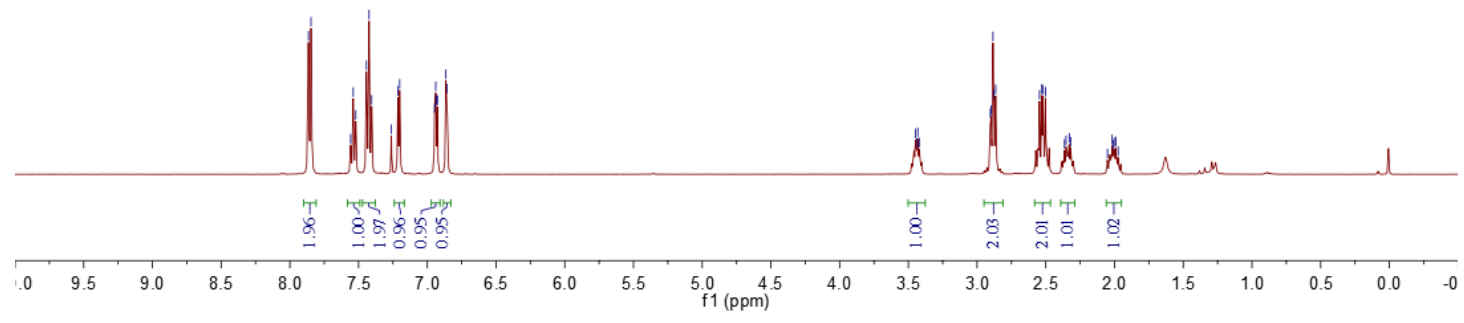



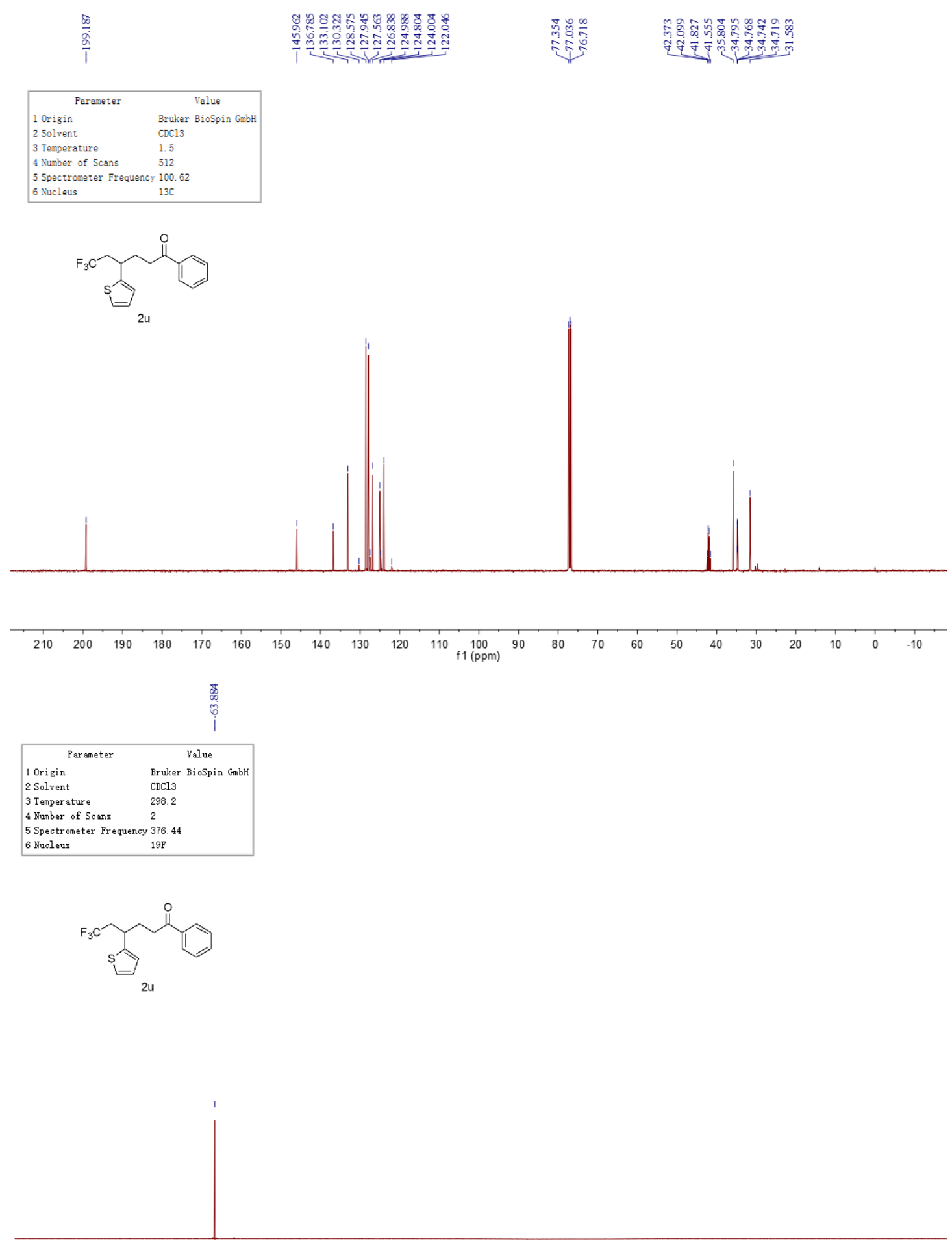

\begin{tabular}{llllllllllllllllllllllll}
\hline & 0 & -10 & -20 & -30 & -40 & -50 & -60 & -70 & -80 & -90 & -110 & -130 & -150 & -170 & -190 & -210 & -230 & -250 & -270 & -290
\end{tabular} 


\begin{tabular}{|ll|}
\hline \multicolumn{1}{|c|}{ Parameter } & \multicolumn{2}{c|}{ Value } \\
1 Origin & Bruker BioSpin GmbH \\
2 Solvent & CDC13 \\
3 Temperature & 298.2 \\
4 Number of Scans & 2 \\
5 Spectrometer Frequency & 400.13 \\
6 Nucleus & 1H \\
\hline
\end{tabular}

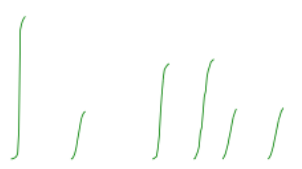<smiles>CCCC(CC)CCC(=O)c1ccc(C)cc1</smiles>

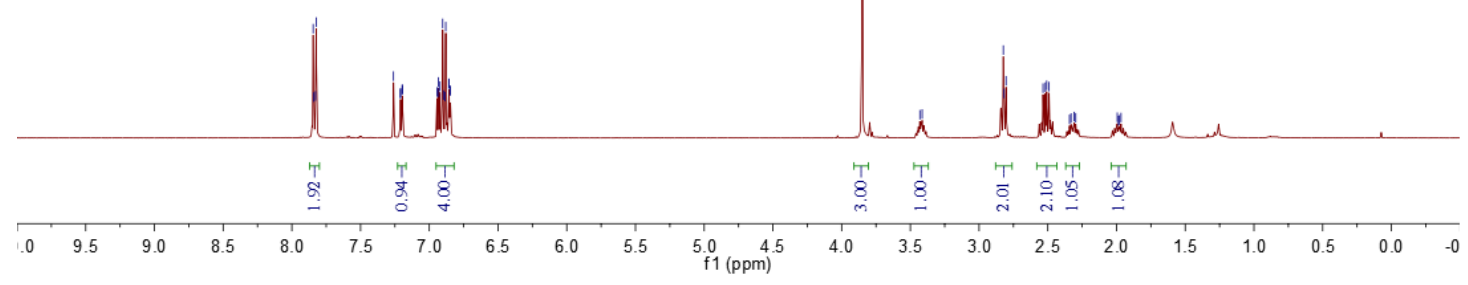

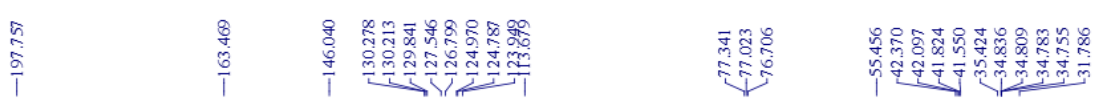
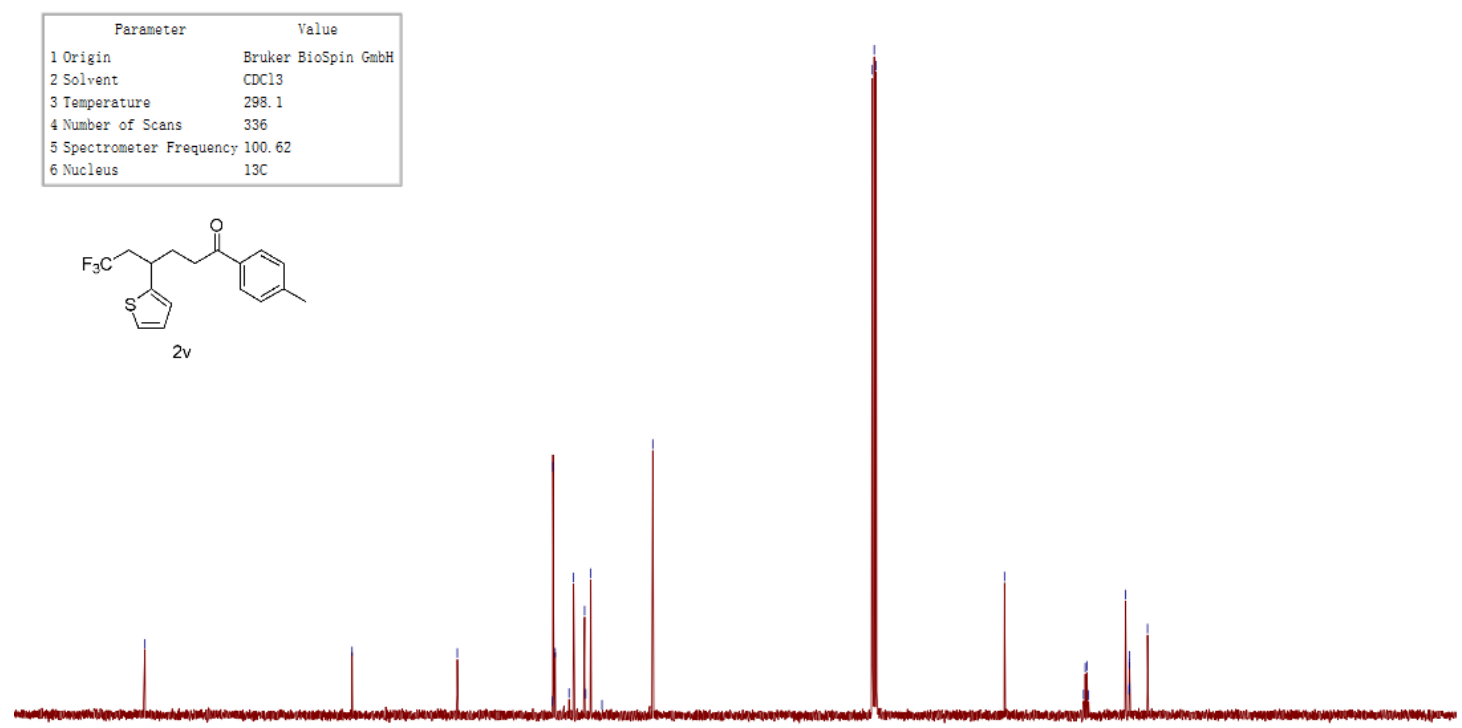

$\begin{array}{llllllllllllllllllllllllll}210 & 200 & 190 & 180 & 170 & 160 & 150 & 140 & 130 & 120 & 110 & 100 & 90 & 80 & 70 & 60 & 50 & 40 & 30 & 20 & 10 & 0 & -10\end{array}$ 

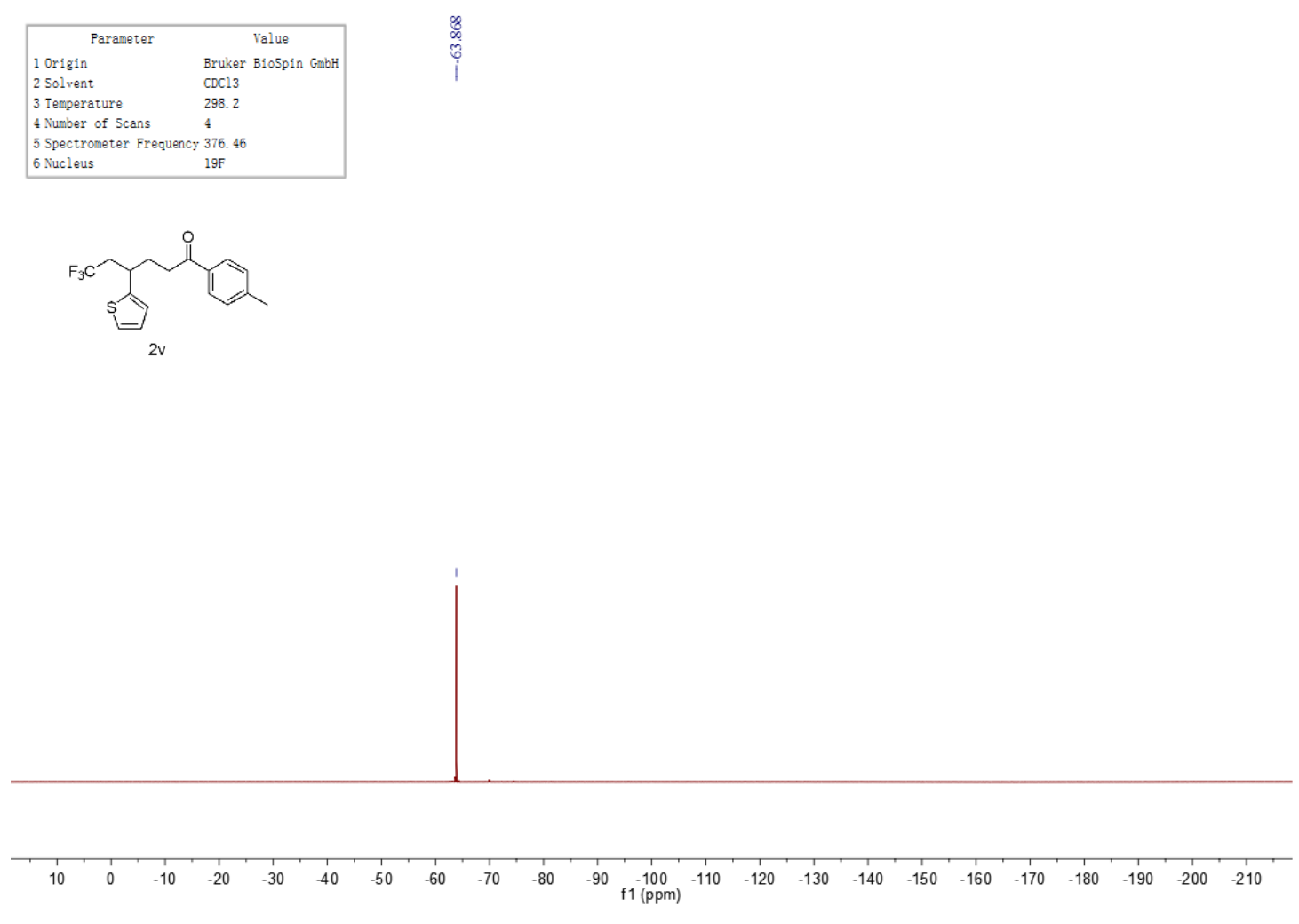

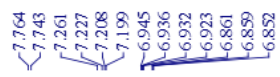

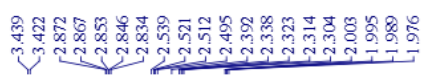
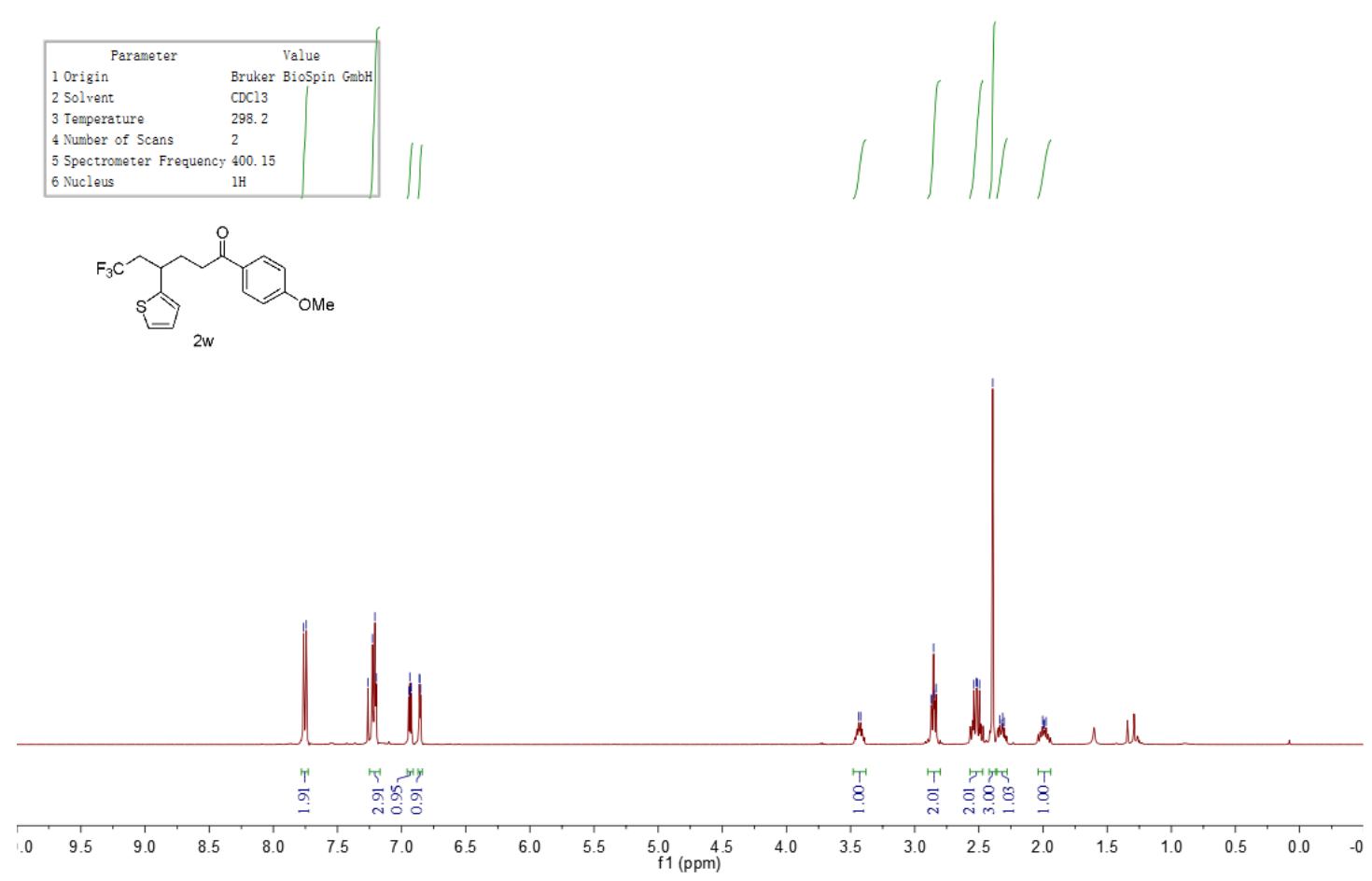


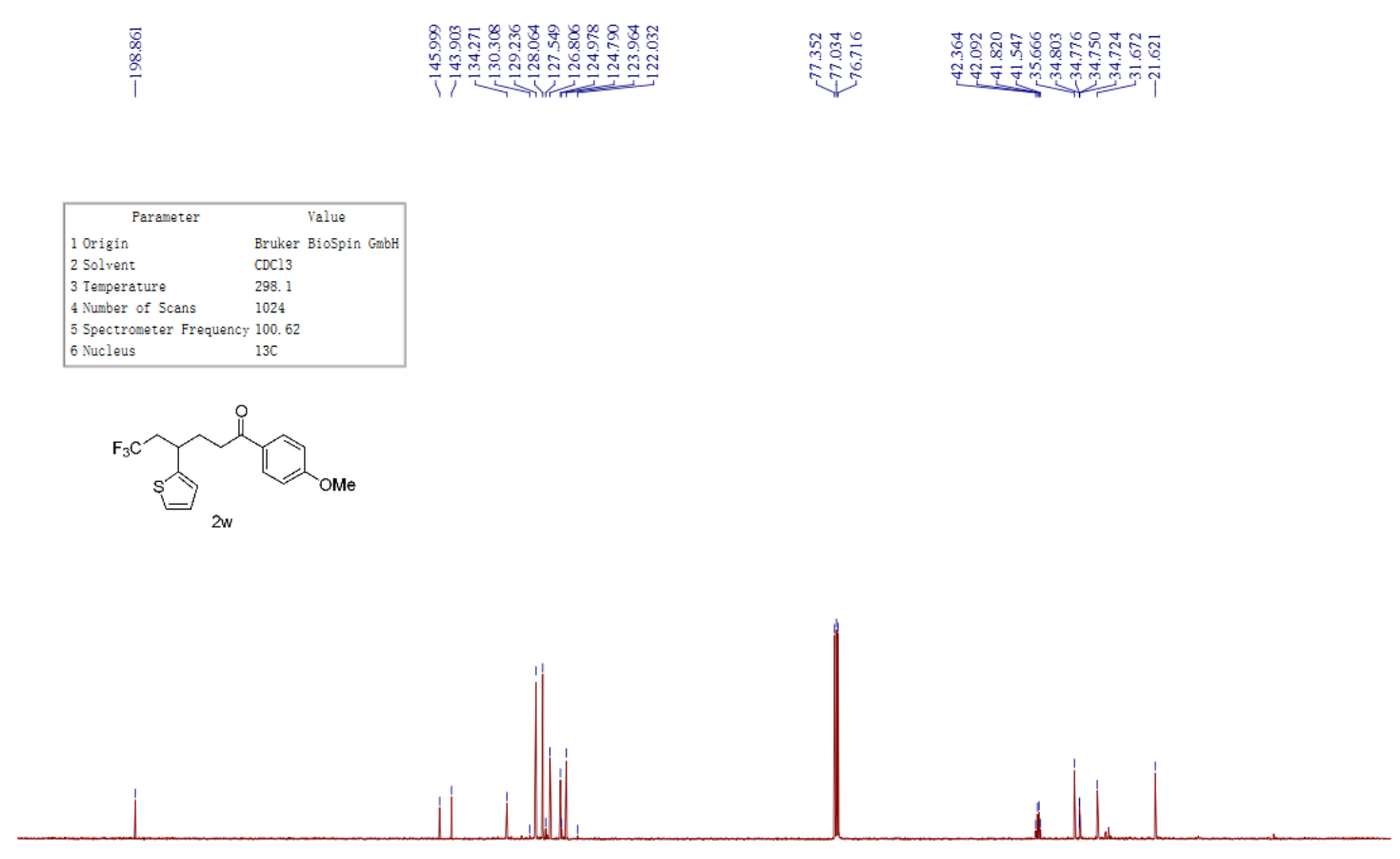

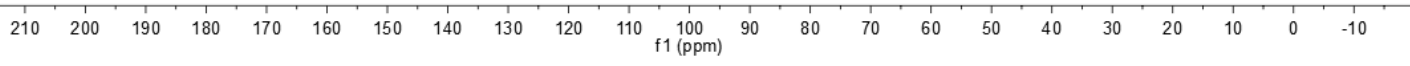
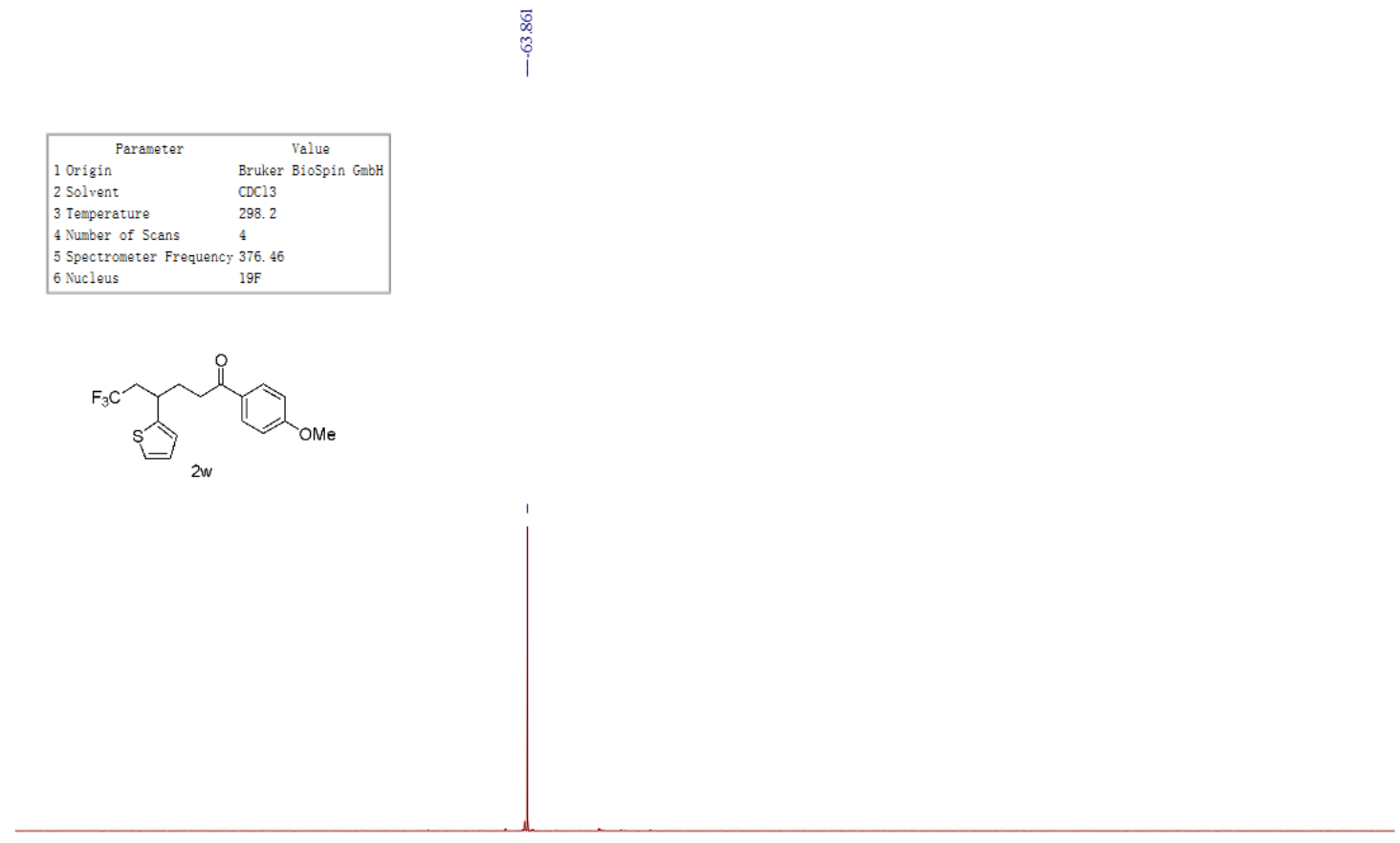

\begin{tabular}{llllllllllllllllllllllllllll}
\hline 0 & -25 & -30 & -35 & -40 & -45 & -50 & -55 & -60 & -65 & -70 & -75 & -80 & -85 & -90 & -95 & -100 & -105 & -110 & -115 & -120 & -125 & -130 & -135
\end{tabular} 


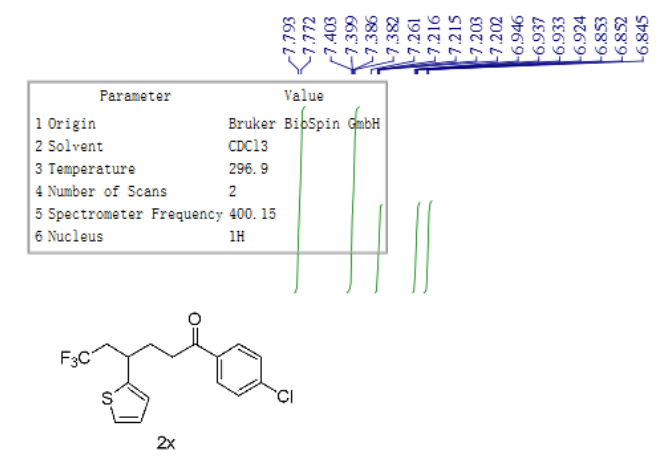

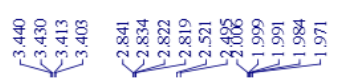
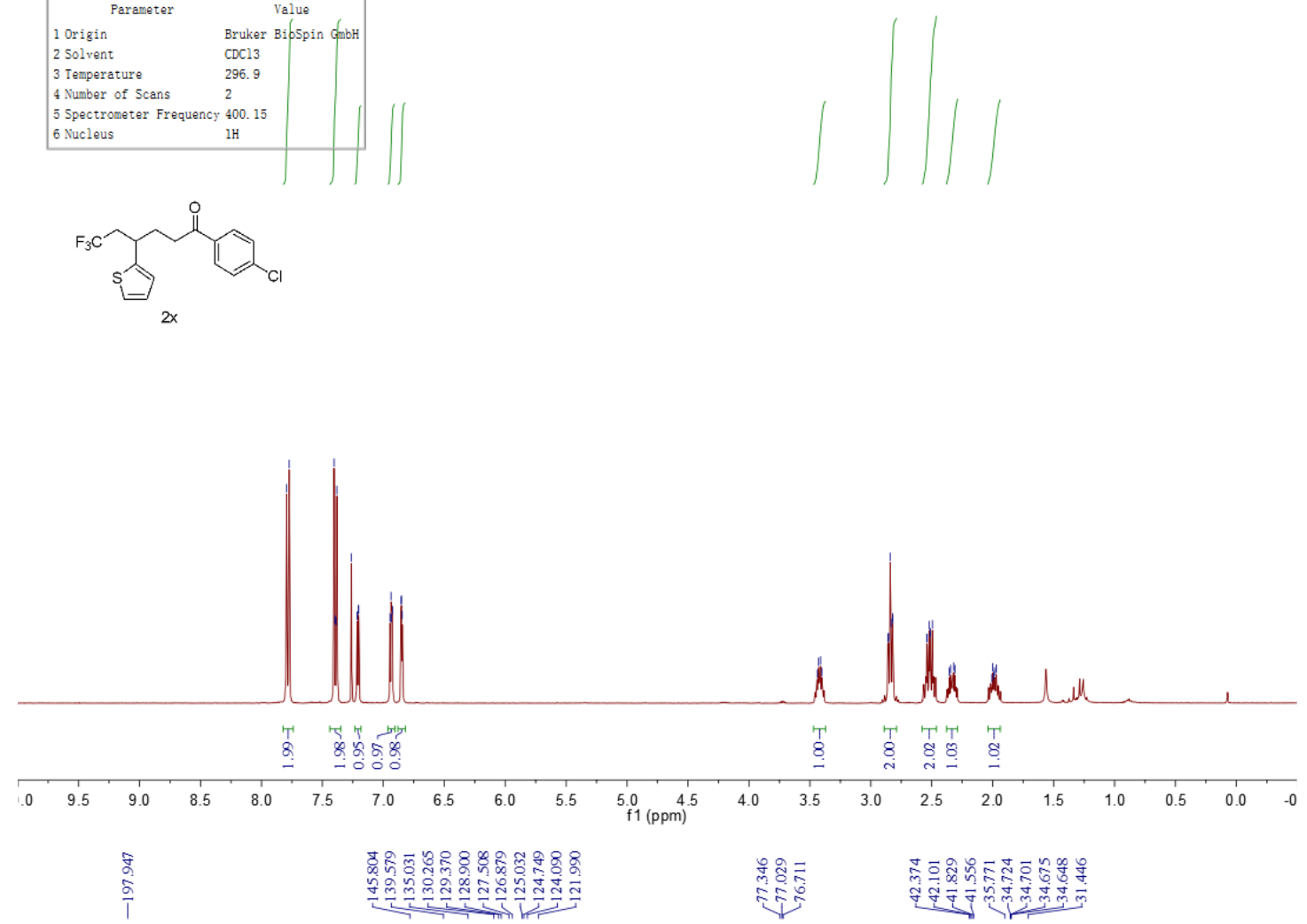

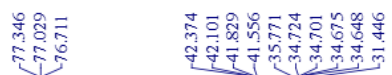

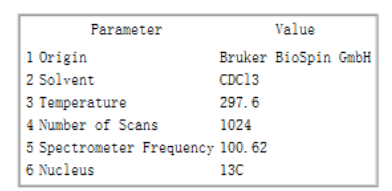

$\overbrace{2 x}^{1}$

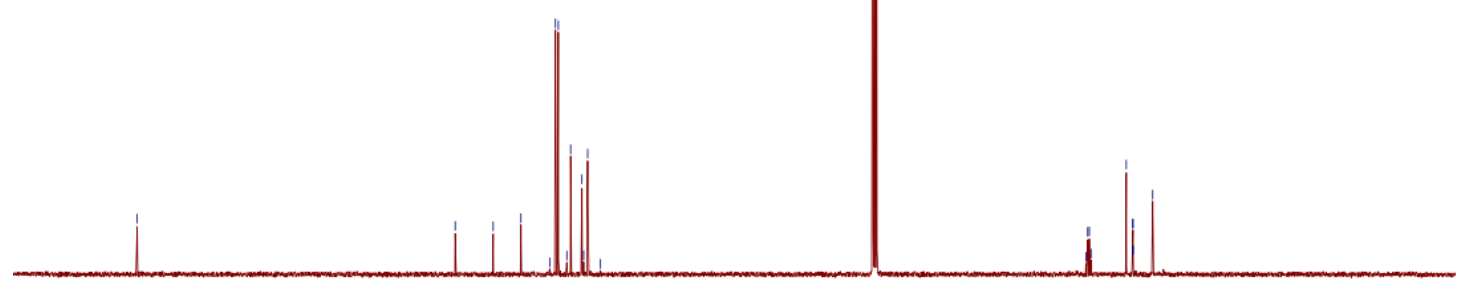

$\begin{array}{llllllllllllllllllllllllll}210 & 200 & 190 & 180 & 170 & 160 & 150 & 140 & 130 & 120 & 110 & 100 & 90 & 80 & 70 & 60 & 50 & 40 & 30 & 20 & 10 & 0 & -10\end{array}$ 


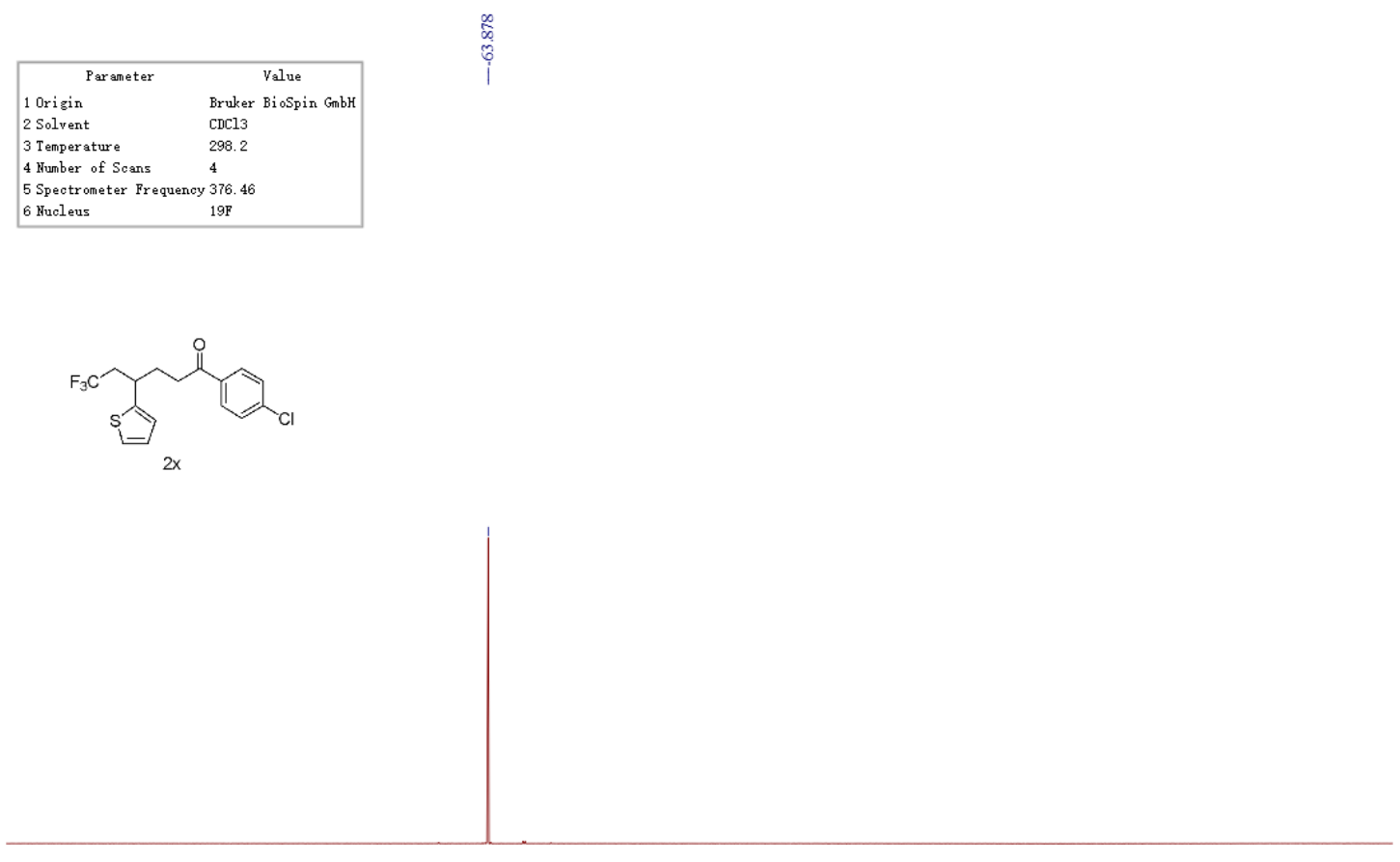

\begin{tabular}{rlllllllllllllllllllllllll}
\hline 10 & 0 & -10 & -20 & -30 & -40 & -50 & -60 & -70 & -80 & -90 & -100 & -110 & -120 & -130 & -140 & -150 & -160 & -170 & -180 & -190 & -200 & -210 & 1
\end{tabular}

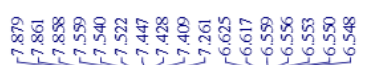

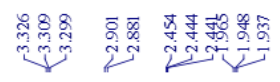
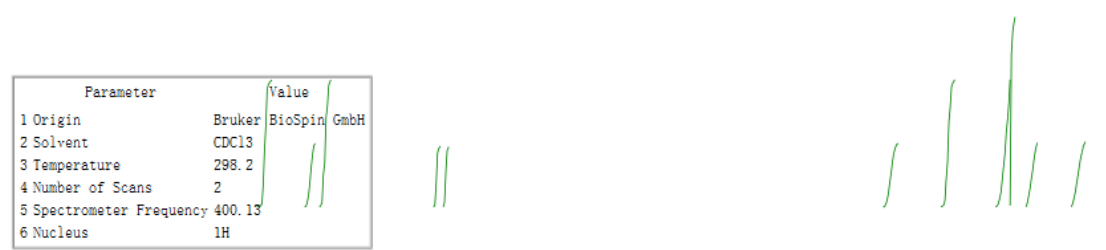

5 Spectromet
6 Nucleus
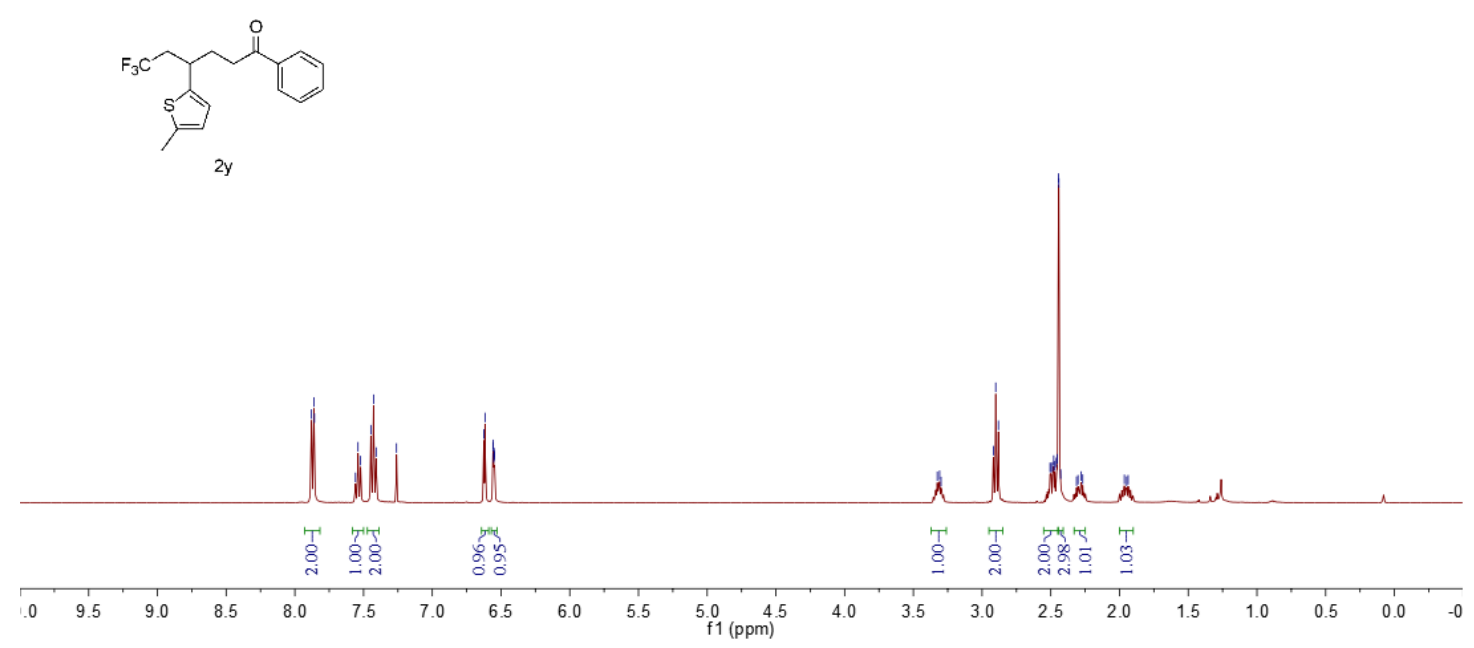


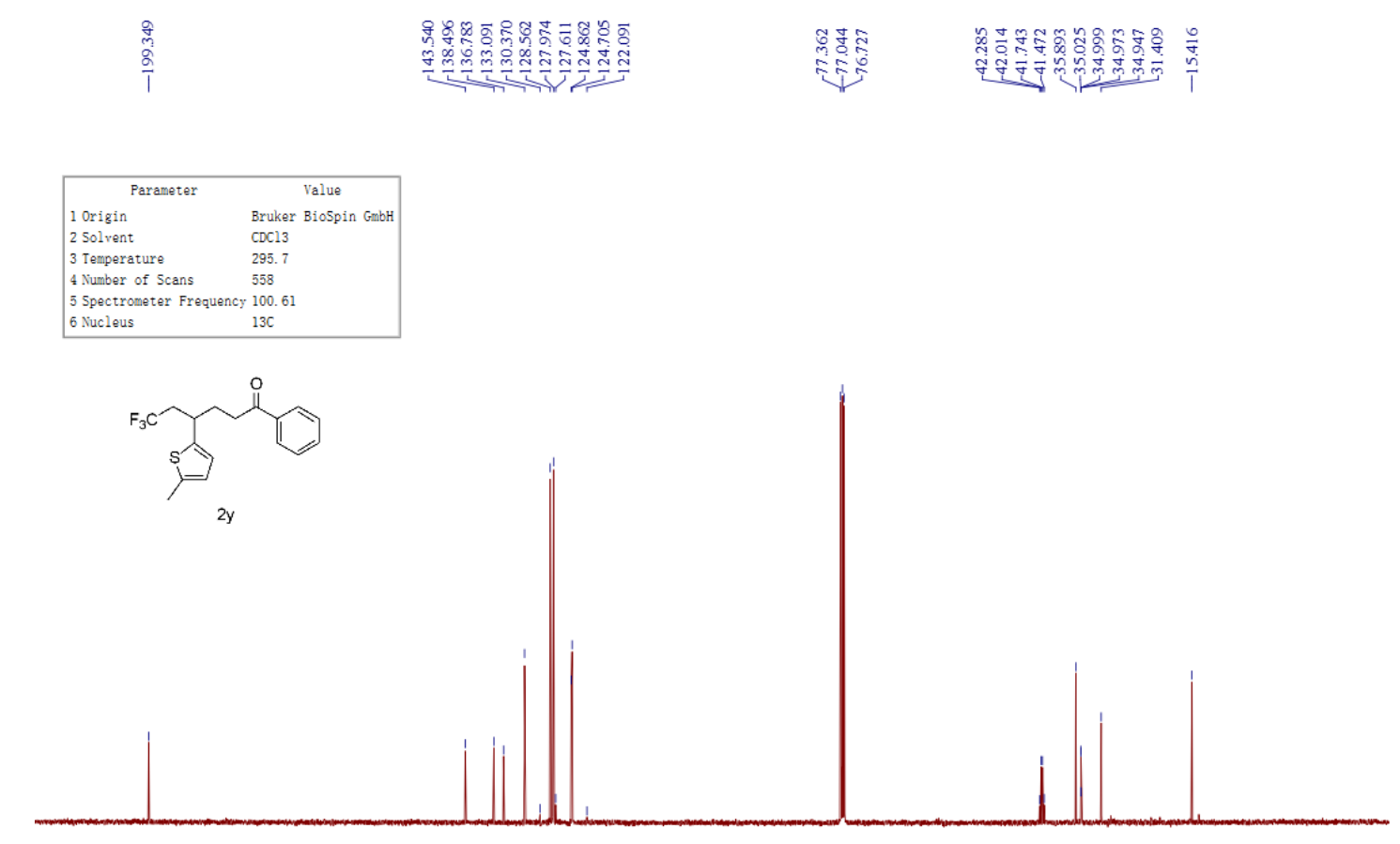

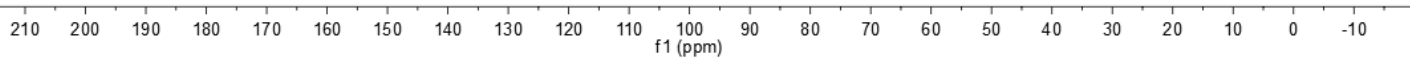
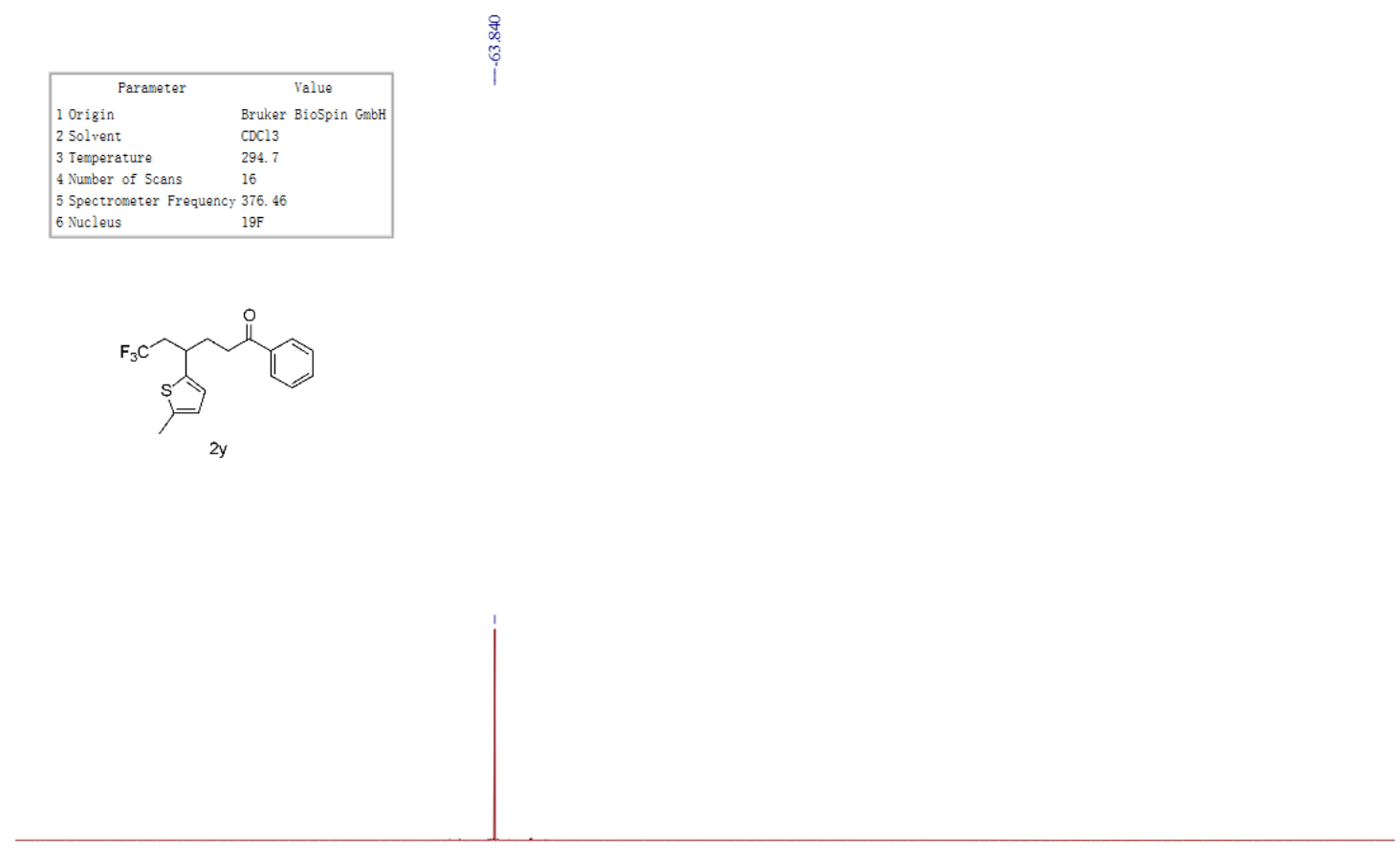

$\begin{array}{llllllllllllllllllllllllllll}10 & 0 & -10 & -20 & -30 & -40 & -50 & -60 & -70 & -80 & -90 & -100 & -110 & -120 & -130 & -140 & -150 & -160 & -170 & -180 & -190 & -200 & -210\end{array}$ 


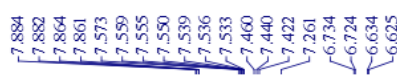

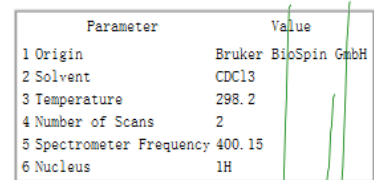

(1)

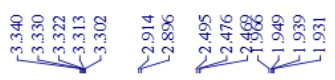

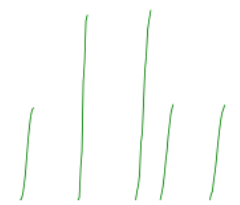

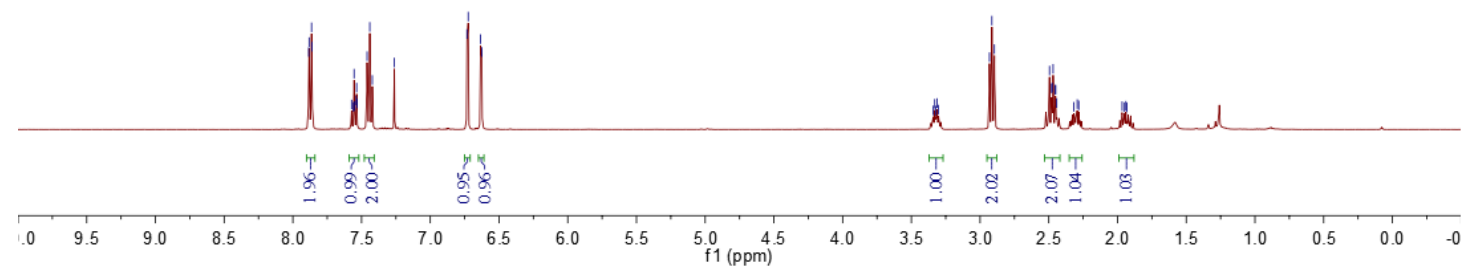
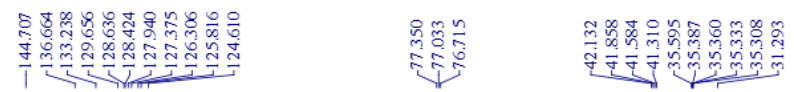

$\begin{array}{cc}\text { Parameter } & \text { Value } \\ 1 \text { Origin } & \text { Bruker Biospin Gmbt }\end{array}$

CDCla

4 Number of Scans $\quad 80$

6 Nucleus
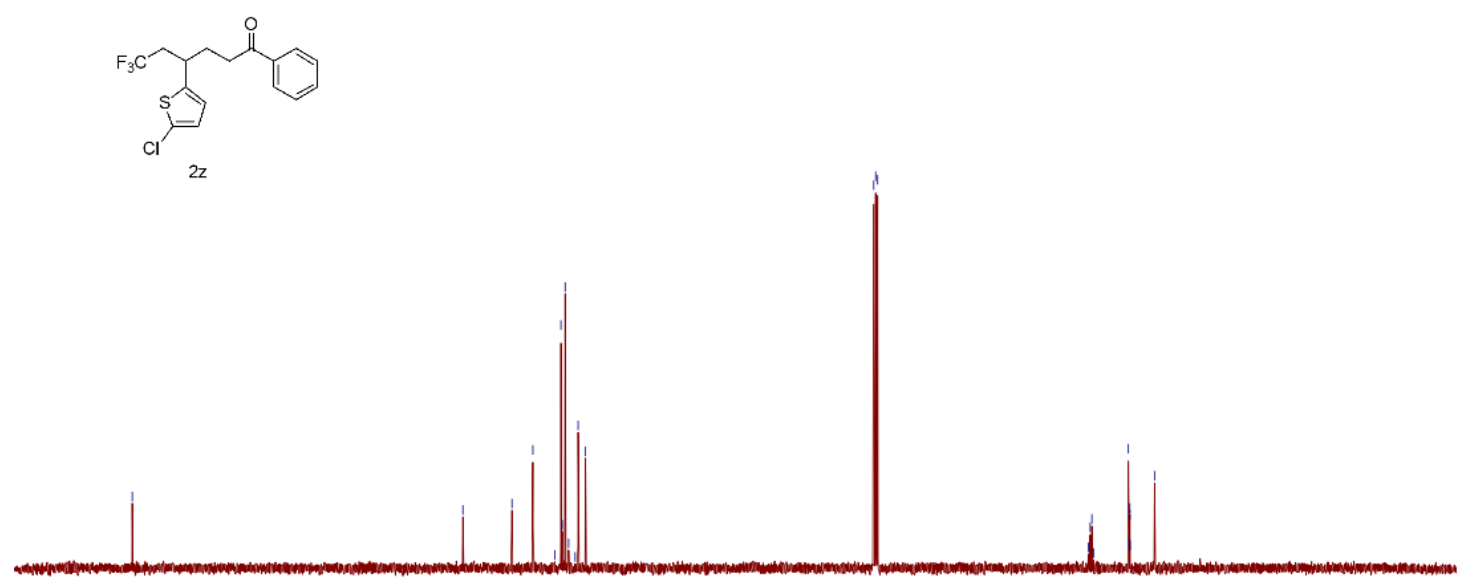

$\begin{array}{llllllllllllllllllllllllll}210 & 200 & 190 & 180 & 170 & 160 & 150 & 140 & 130 & 120 & 110 & 100 & 90 & 80 & 70 & 60 & 50 & 40 & 30 & 20 & 10 & 0 & -10\end{array}$ 


$$
\begin{array}{|ll|}
\hline \multicolumn{1}{|c|}{\text { Parameter }} & \multicolumn{1}{c|}{\text { Value }} \\
\text { 1 Origin } & \text { Bruker Bio5pin GmbH } \\
\text { 2 Solvent } & \text { CDC13 } \\
\text { 3 Temperature } & 298.2 \\
\text { 4 Number of Scans } & 2 \\
\text { 5 Spectrometer Frequency } 376.52 \\
\text { 6 Nucleus } & 19 \mathrm{~F} \\
\hline
\end{array}
$$<smiles>CC=CC(CC)CCC(=O)c1ccccc1</smiles>

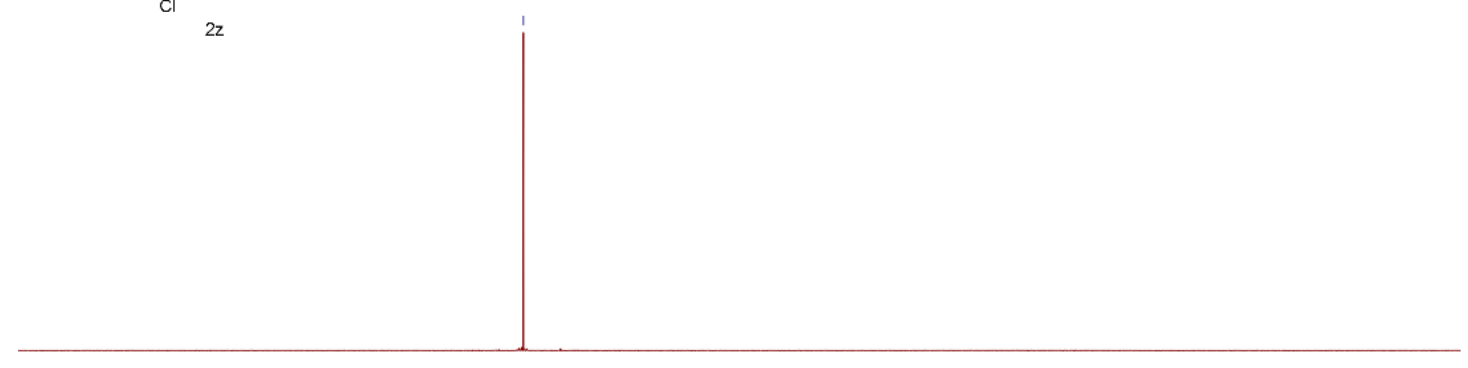
\begin{tabular}{lllllllllllllllllllllllll}
\hline 0 & 10 & 0 & -10 & -20 & -30 & -40 & -50 & -60 & -70 & -80 & -90 & -100 & -110 & -120 & -130 & -140 & -150 & -160 & -170 & -180 & -190 & -200 & -210 & -2.
\end{tabular}

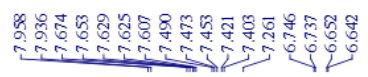

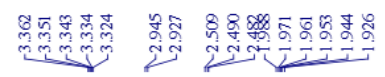
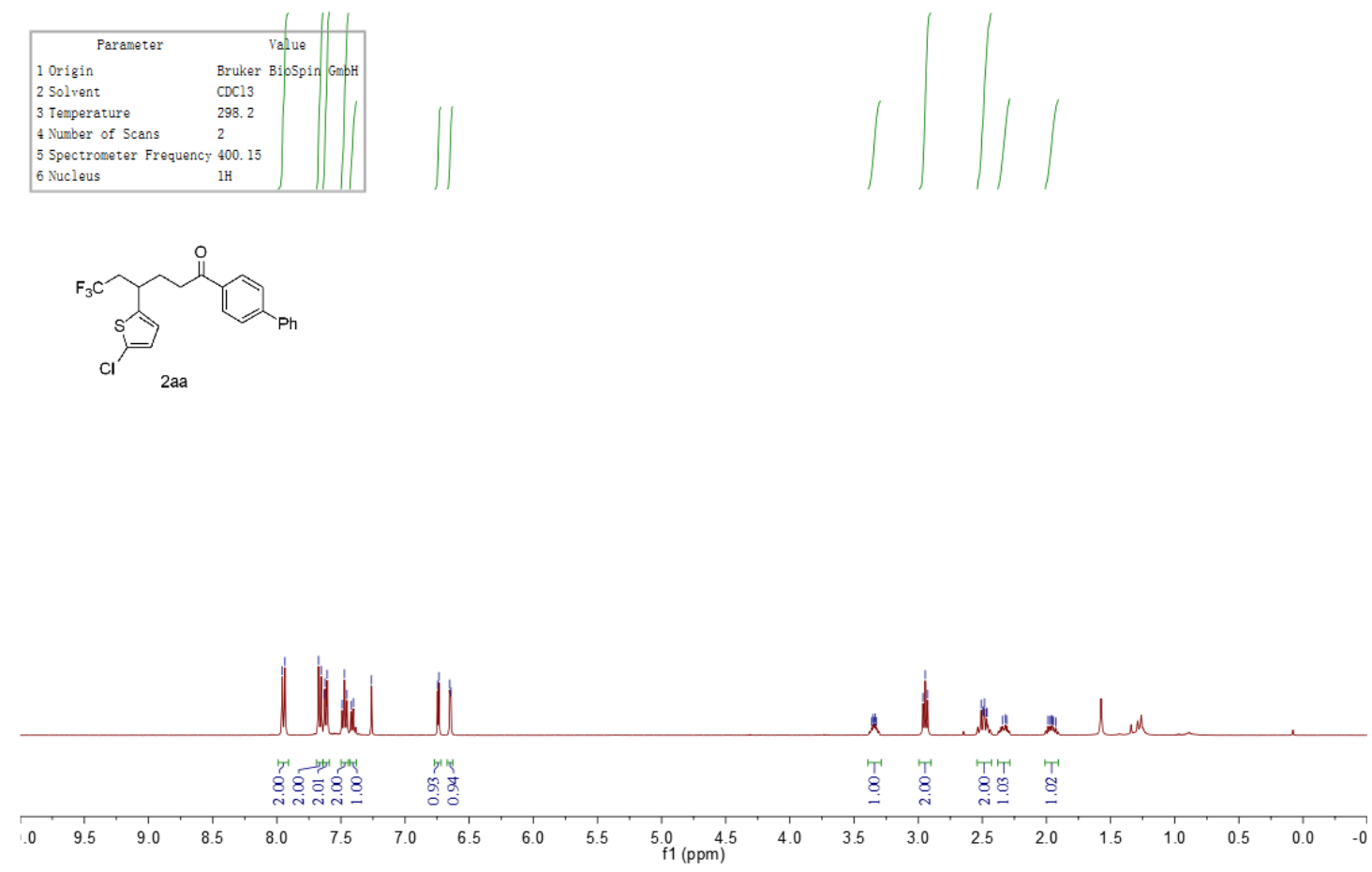

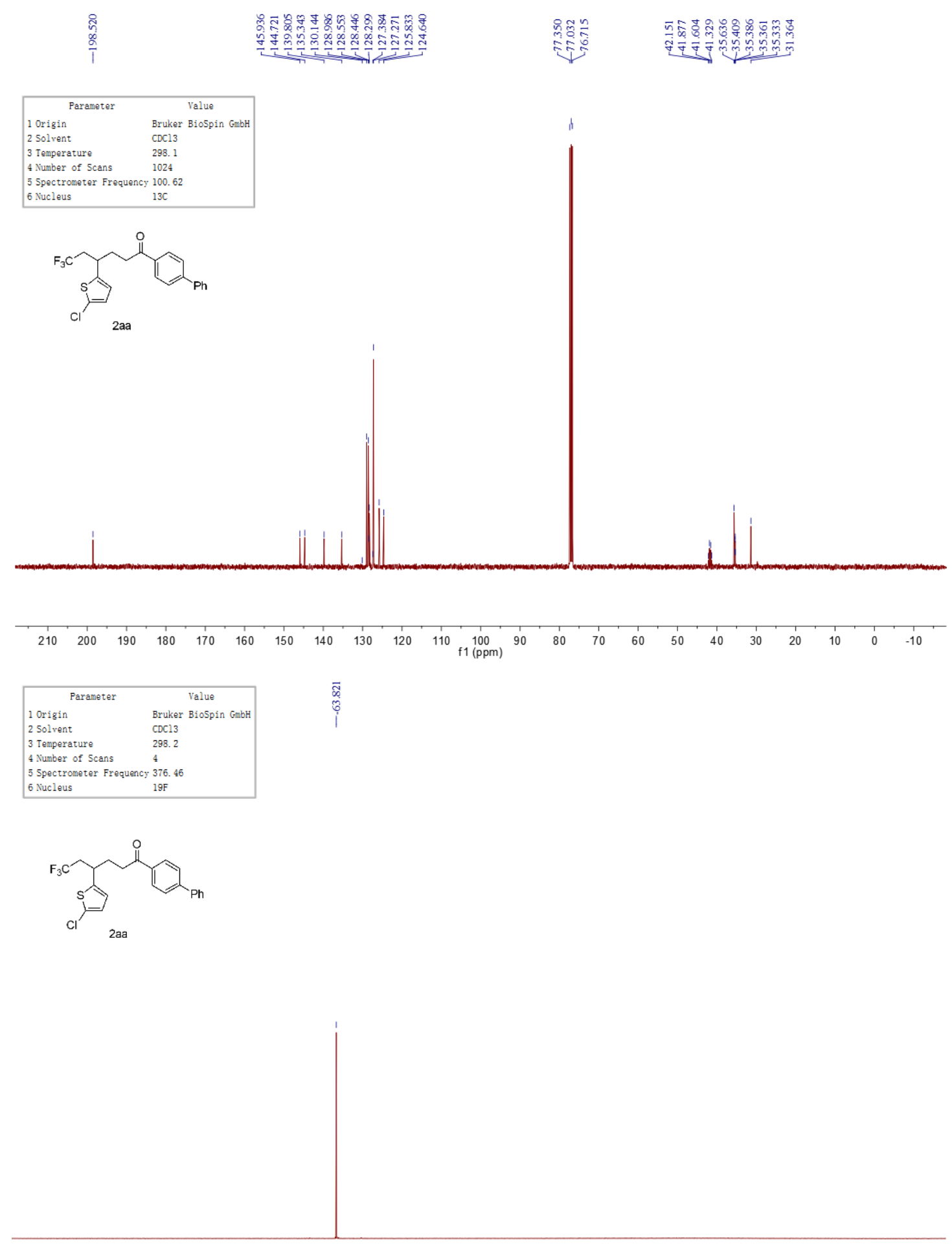

$\begin{array}{lllllllllllllllllllllllllll}1 & 10 & 0 & -10 & -20 & -30 & -40 & -50 & -60 & -70 & -80 & -90 & -100 & -110 & -120 & -130 & -140 & -150 & -160 & -170 & -180 & -190 & -200 & -210\end{array}$ 

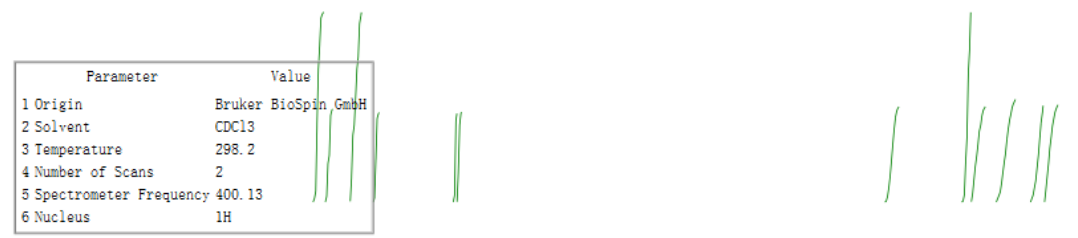

$$
\text { 3ab-B }
$$
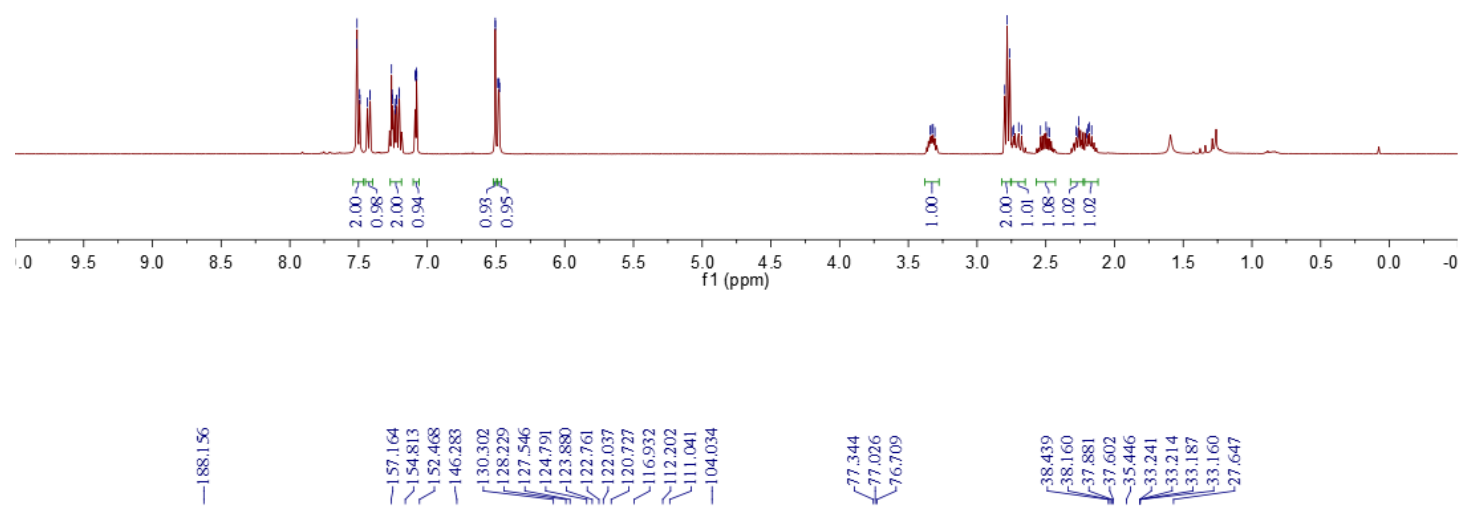

\begin{tabular}{|ll|}
\hline \multicolumn{1}{|c|}{ Parameter } & \multicolumn{1}{c|}{ Value } \\
1 Origin & Bruker Biospin GmbH \\
2 Solvent & CDC13 \\
3 Temperature & 298.1 \\
4 Number of Scans & 1024 \\
5 Spectrometer Frequency 100.62 \\
6 Nucleus & $13 \mathrm{C}$ \\
\hline
\end{tabular}
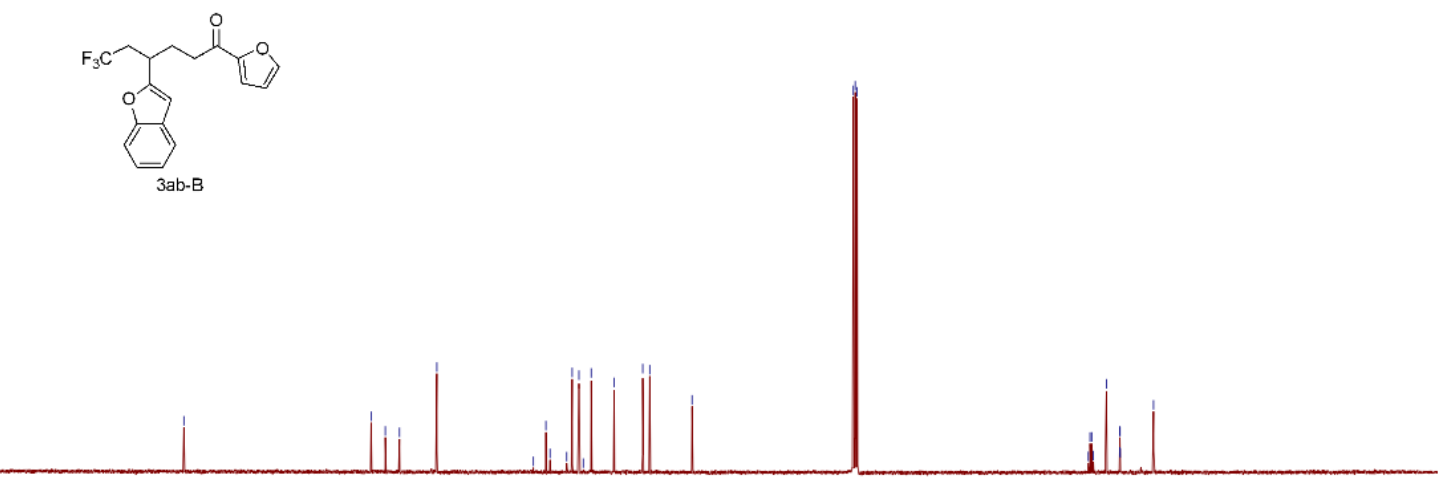

$\begin{array}{lllllllllllllllllllllllllll}210 & 200 & 190 & 180 & 170 & 160 & 150 & 140 & 130 & 120 & 110 & 100 & 90 & 80 & 70 & 60 & 50 & 40 & 30 & 20 & 10 & 0 & -10\end{array}$ 

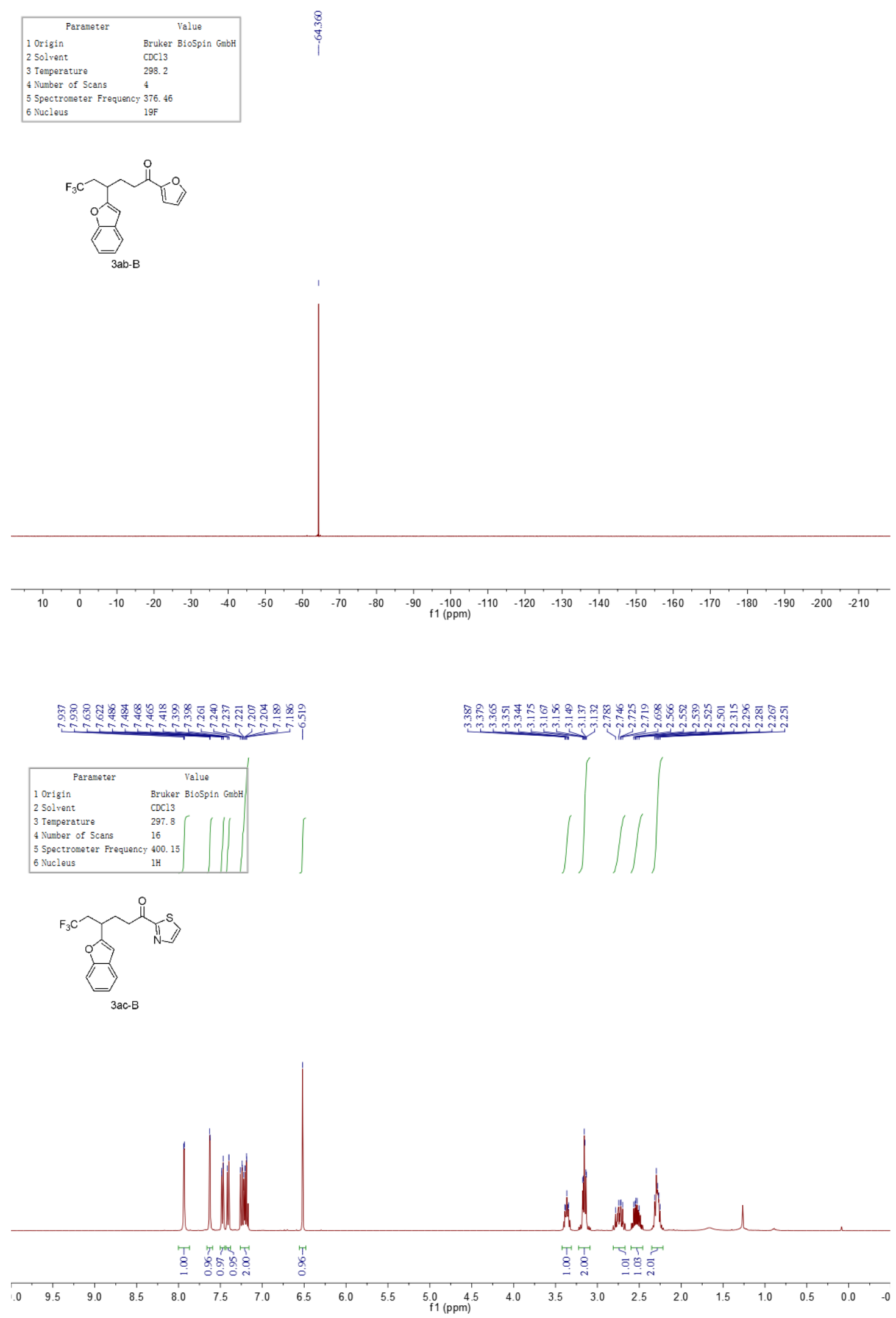

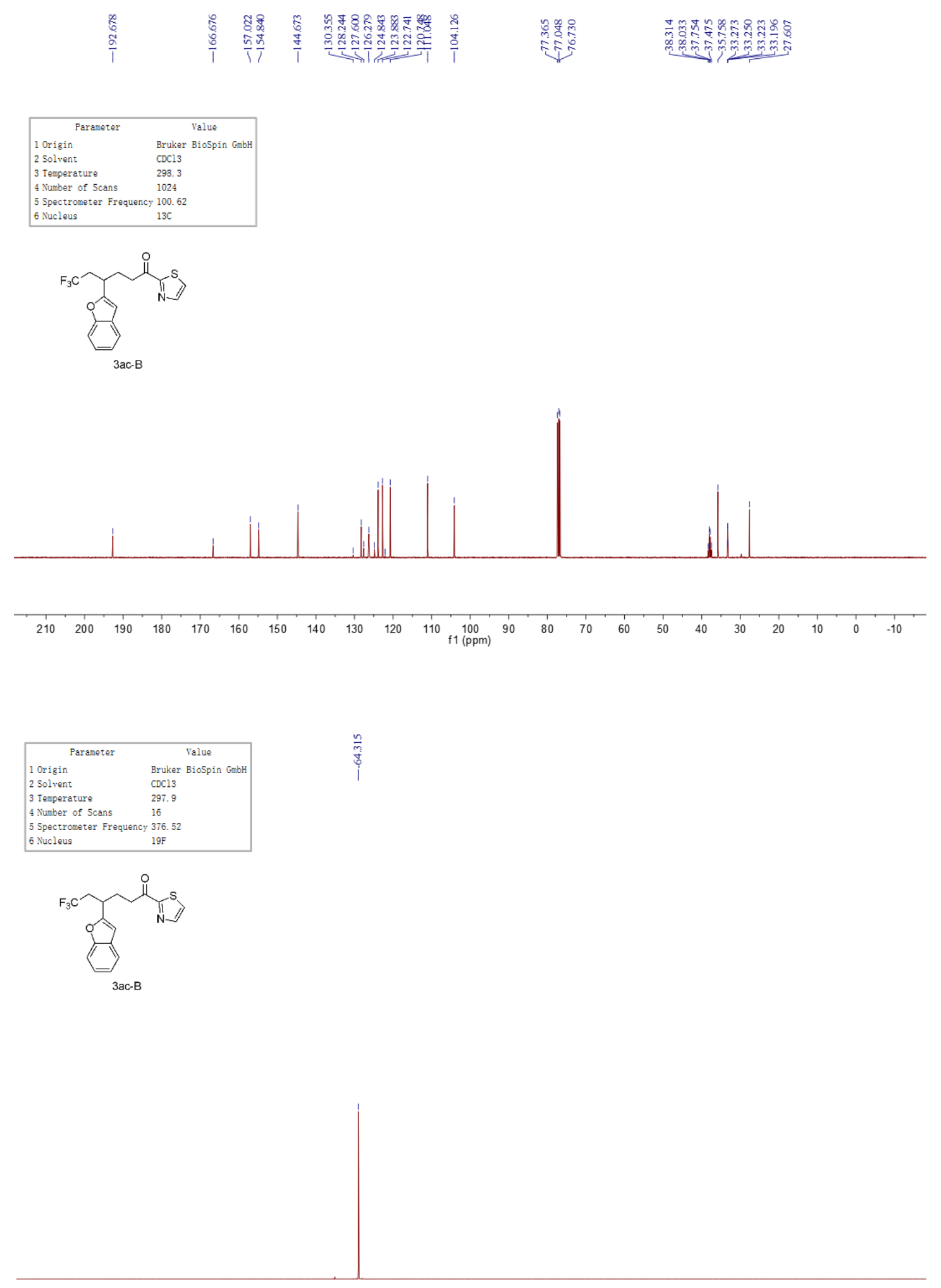

$\begin{array}{lllllllllllllllllllllllllllllllll}-20 & -25 & -30 & -35 & -40 & -45 & -50 & -55 & -60 & -65 & -70 & -75 & -80 & -85 & -90 & -95 & -100 & -105 & -110 & -115 & -120 & -125 & -130 & -135\end{array}$ 


\section{HMBC}

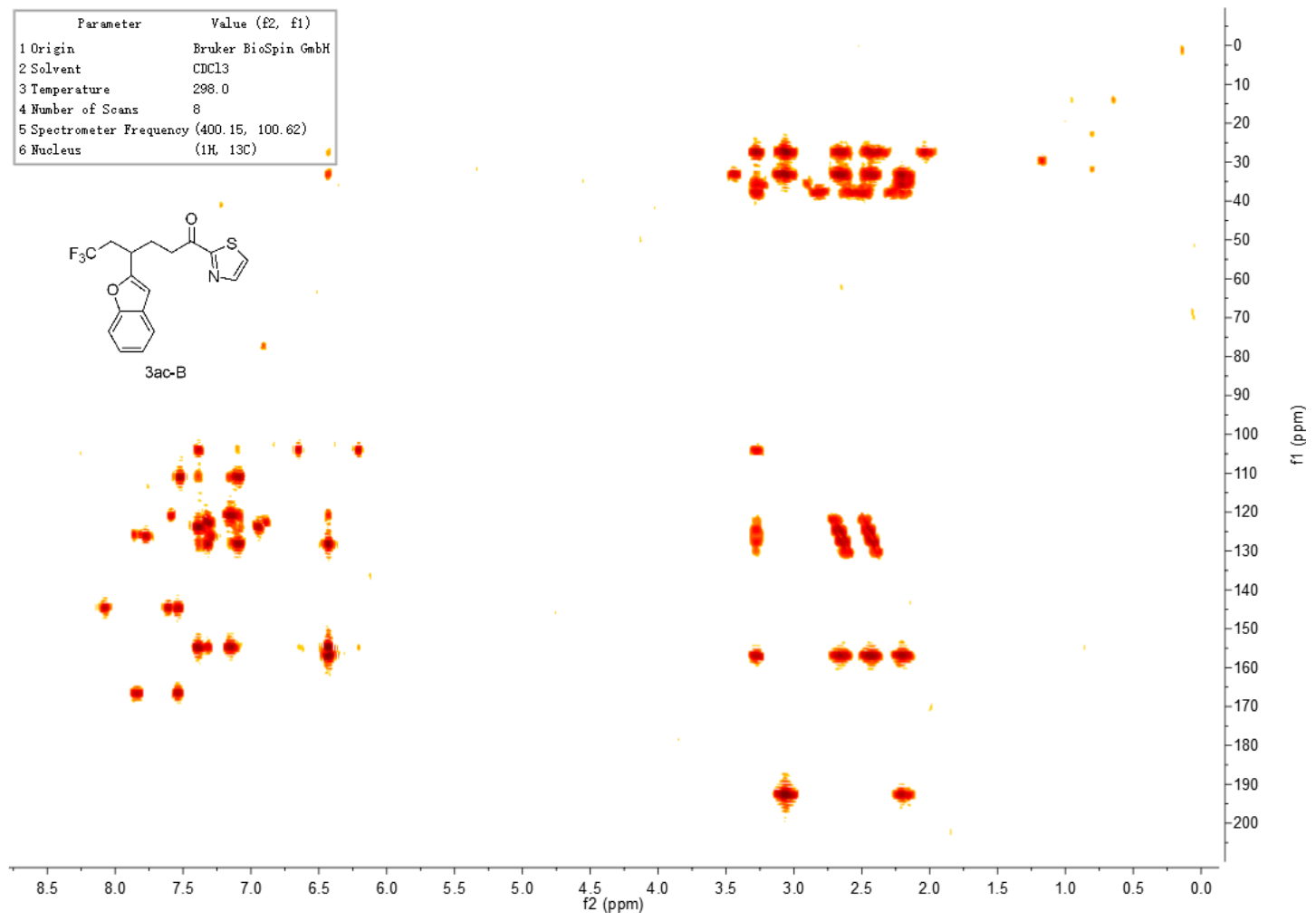

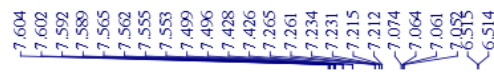

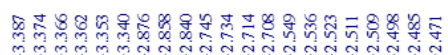

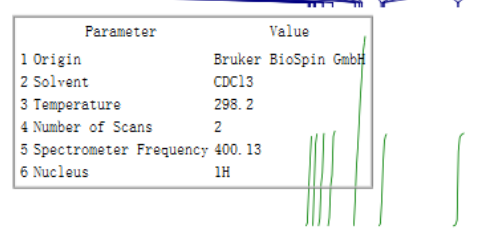

[ad-B
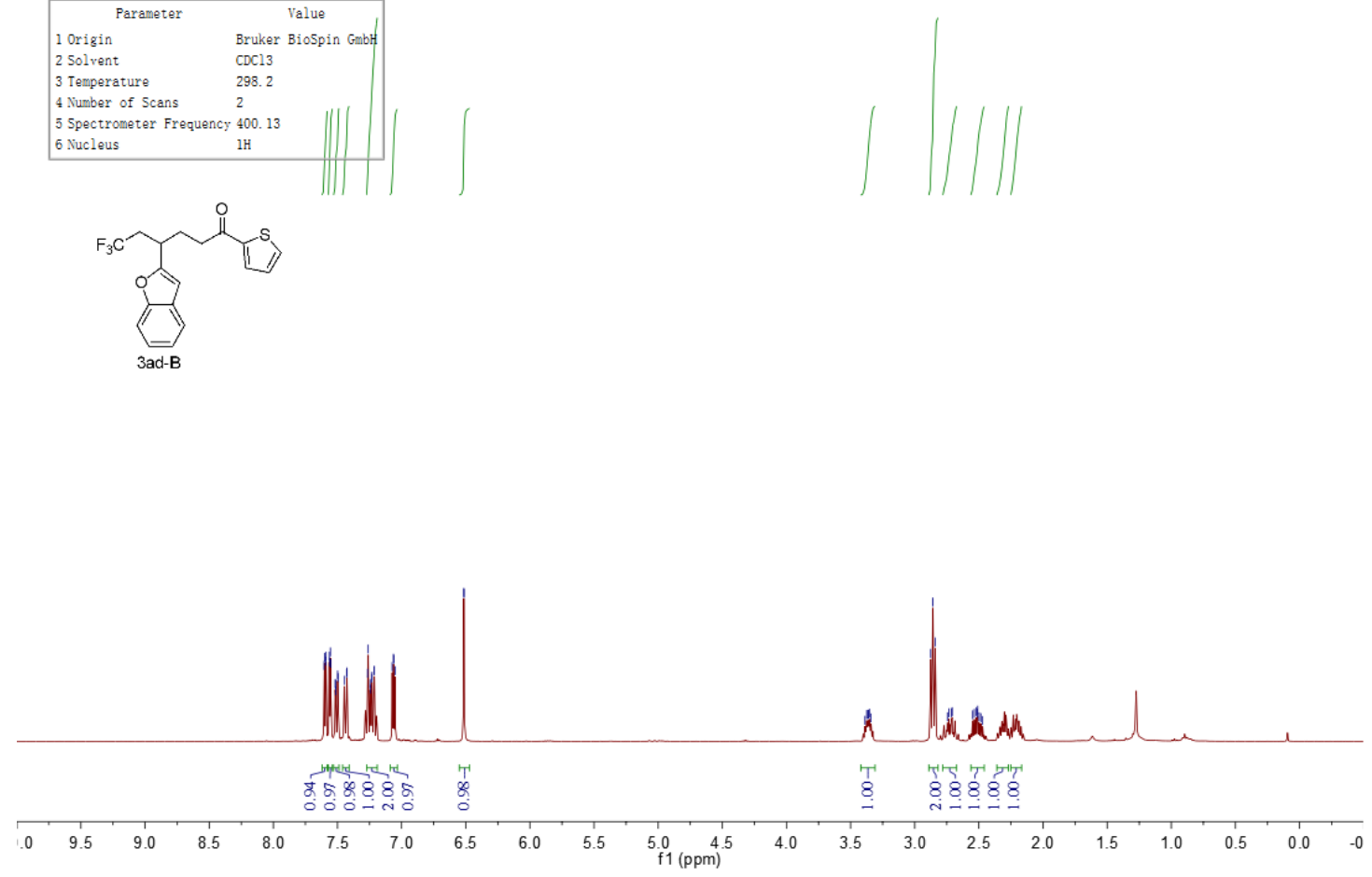


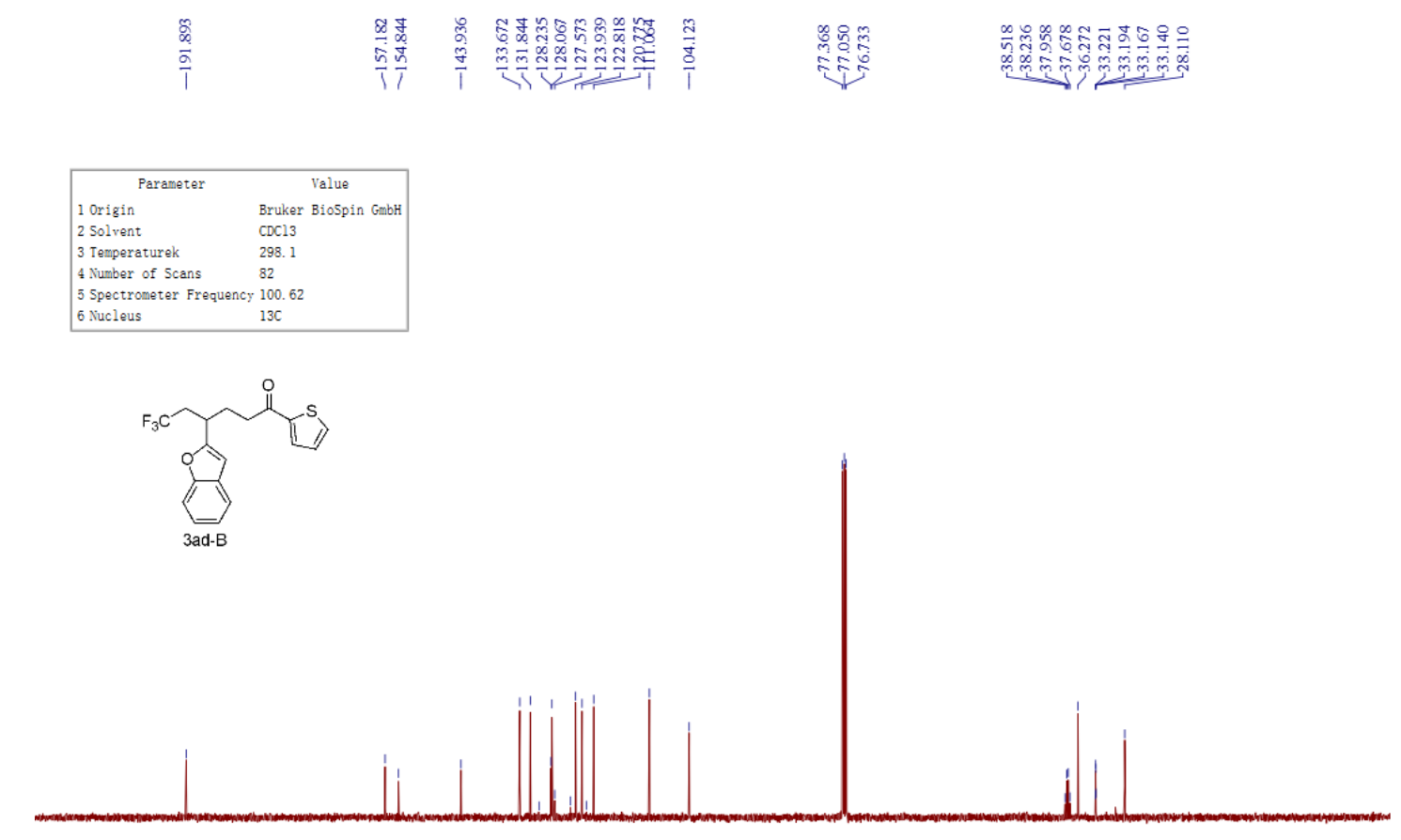

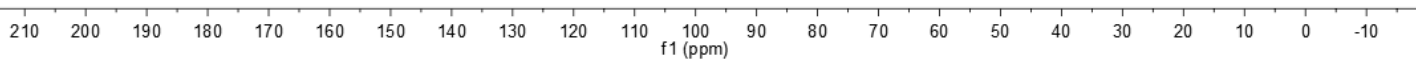

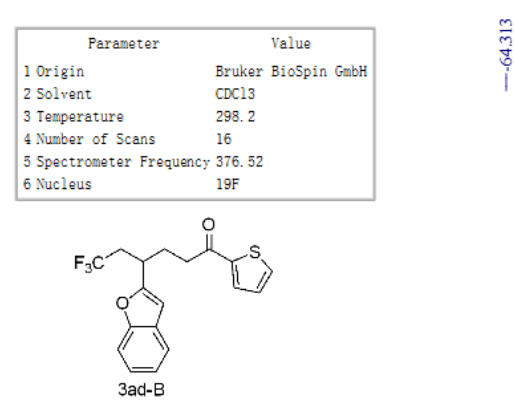

\begin{tabular}{llllllllllllllllllllllllllll}
\hline 0 & 10 & 0 & -10 & -20 & -30 & -40 & -50 & -60 & -70 & -80 & -90 & -100 & -110 & -120 & -130 & -140 & -150 & -160 & -170 & -180 & -190 & -200 & -210 & $-2 c^{2}$
\end{tabular} 


\section{HMBC}

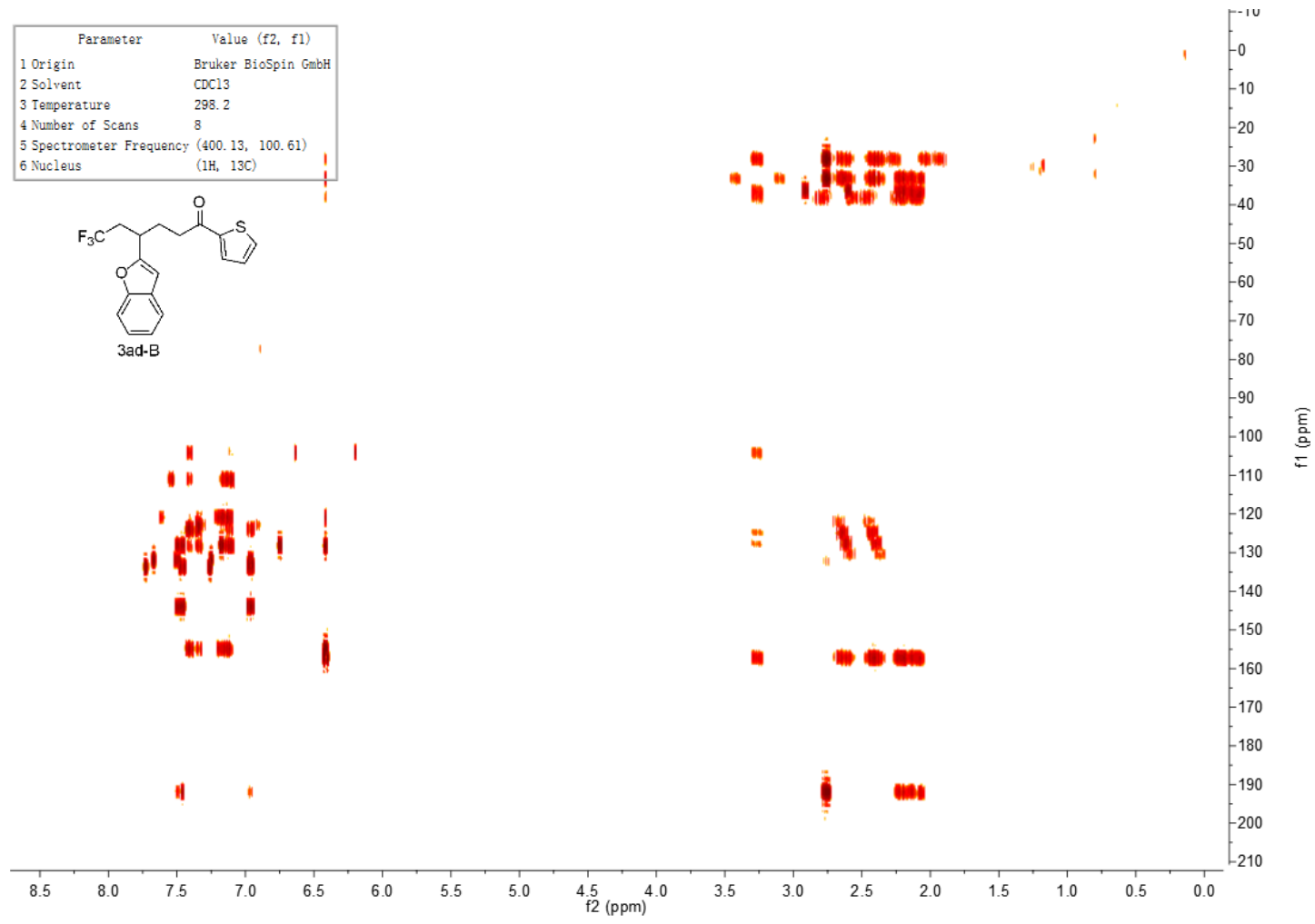

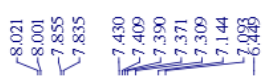

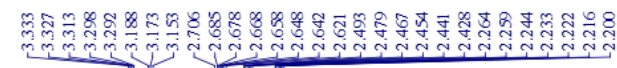
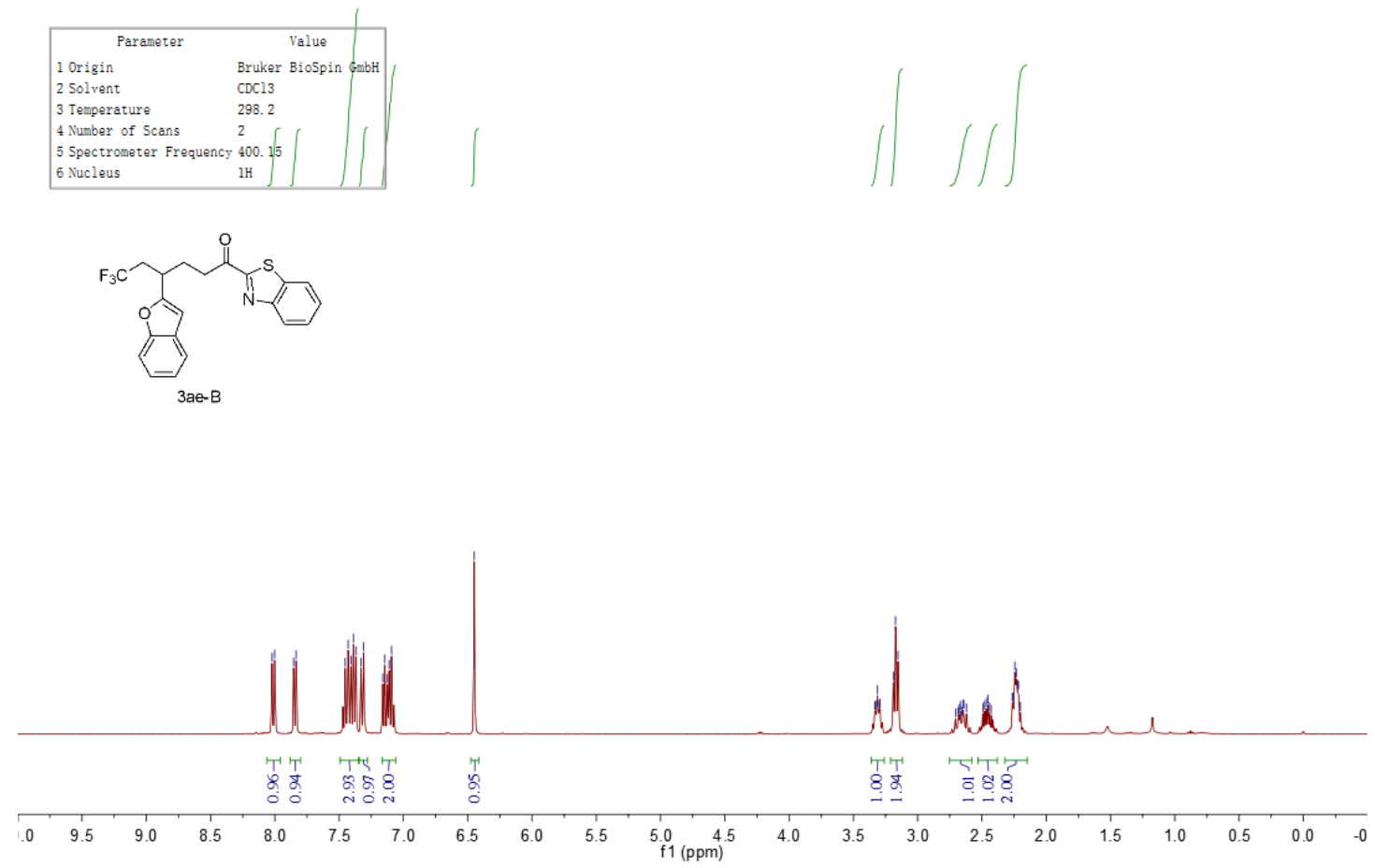


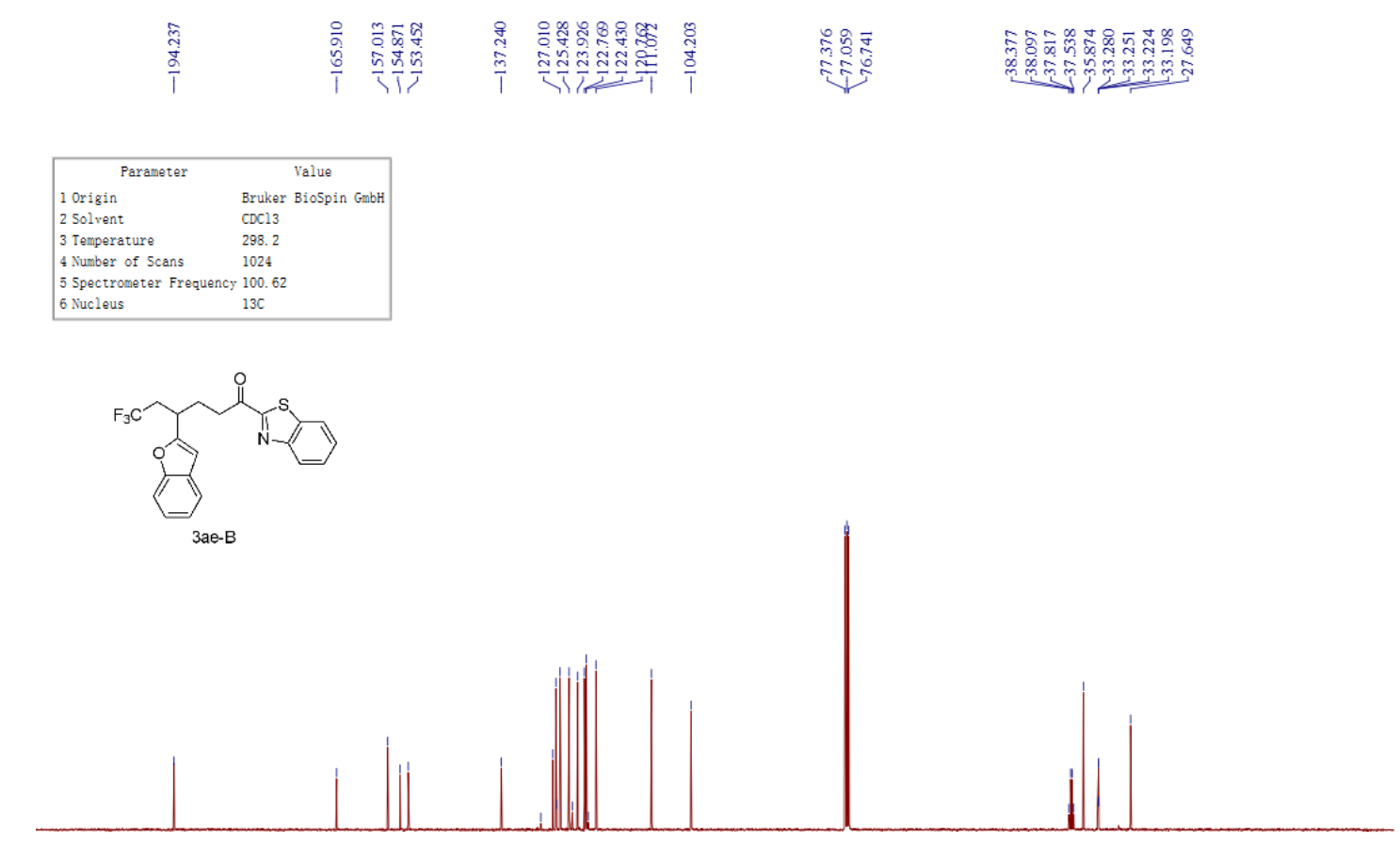

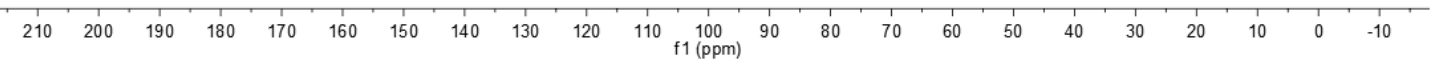
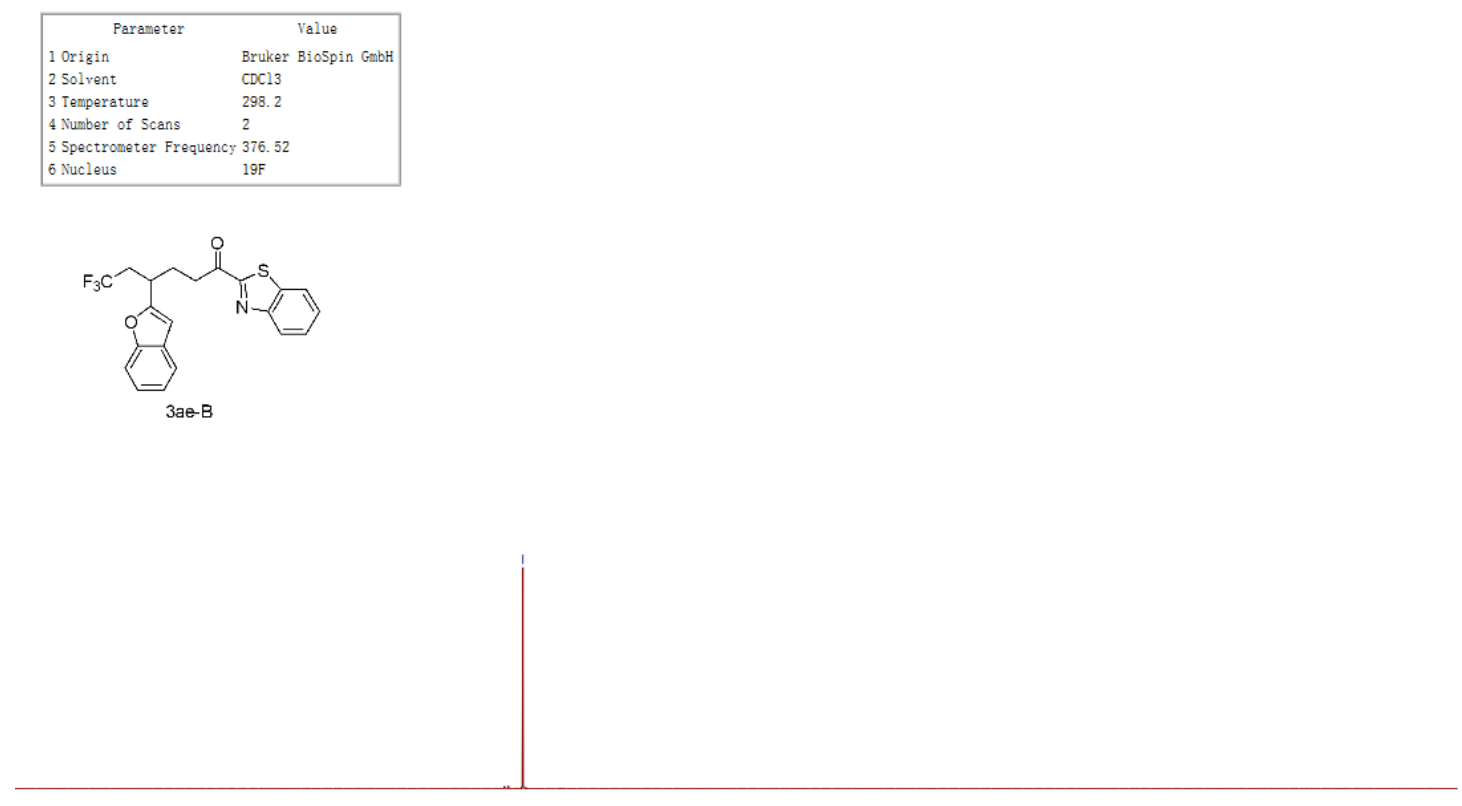

\begin{tabular}{llllllllllllllllllllllllllllll}
\hline 0 & 10 & 0 & -10 & -20 & -30 & -40 & -50 & -60 & -70 & -80 & -90 & -100 & -110 & -120 & -130 & -140 & -150 & -160 & -170 & -180 & -190 & -200 & -210 & $-2 c^{\prime}$
\end{tabular} 
HMBC

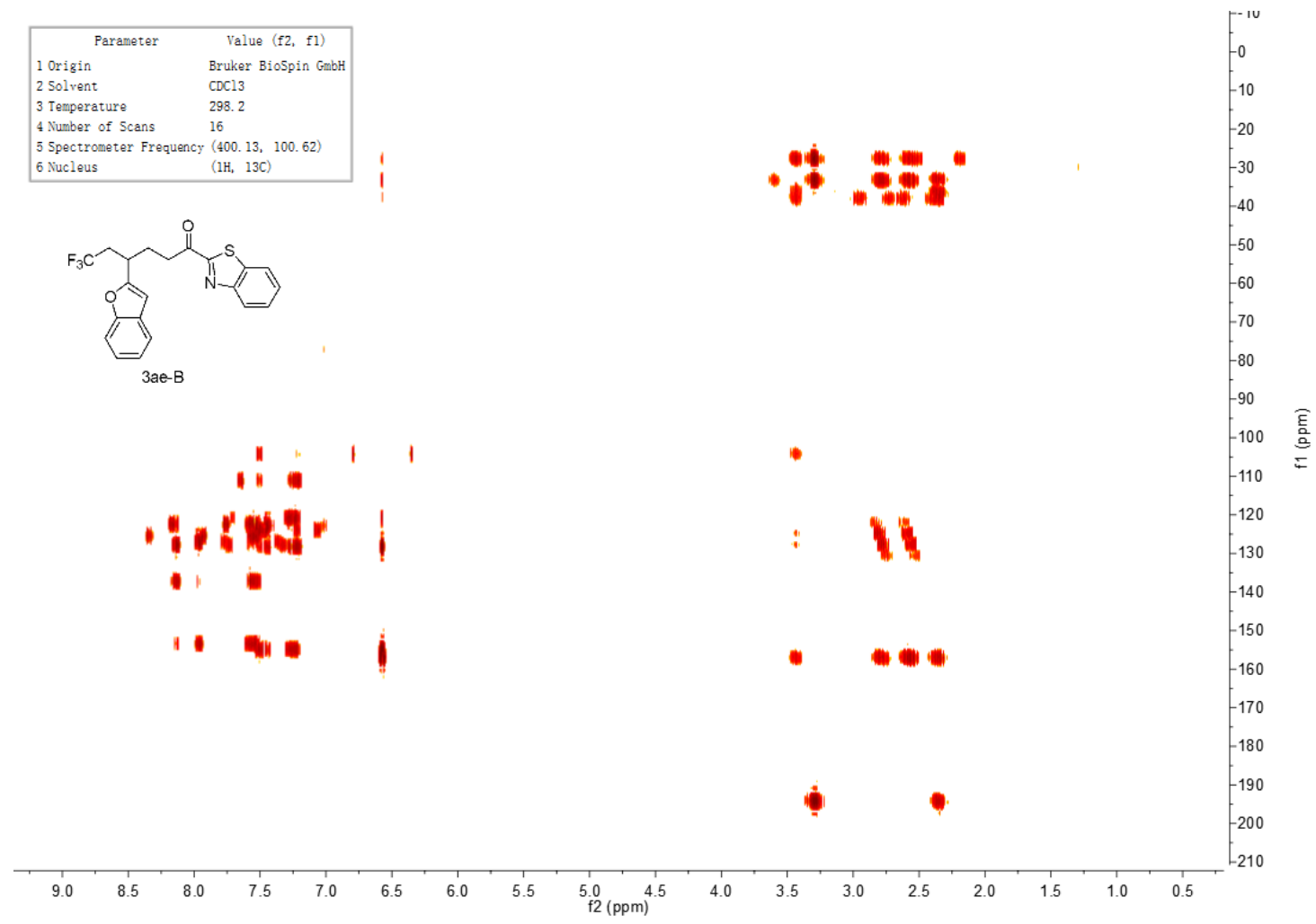

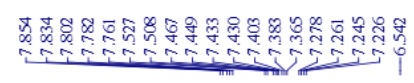

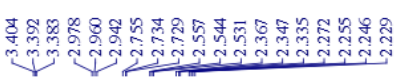
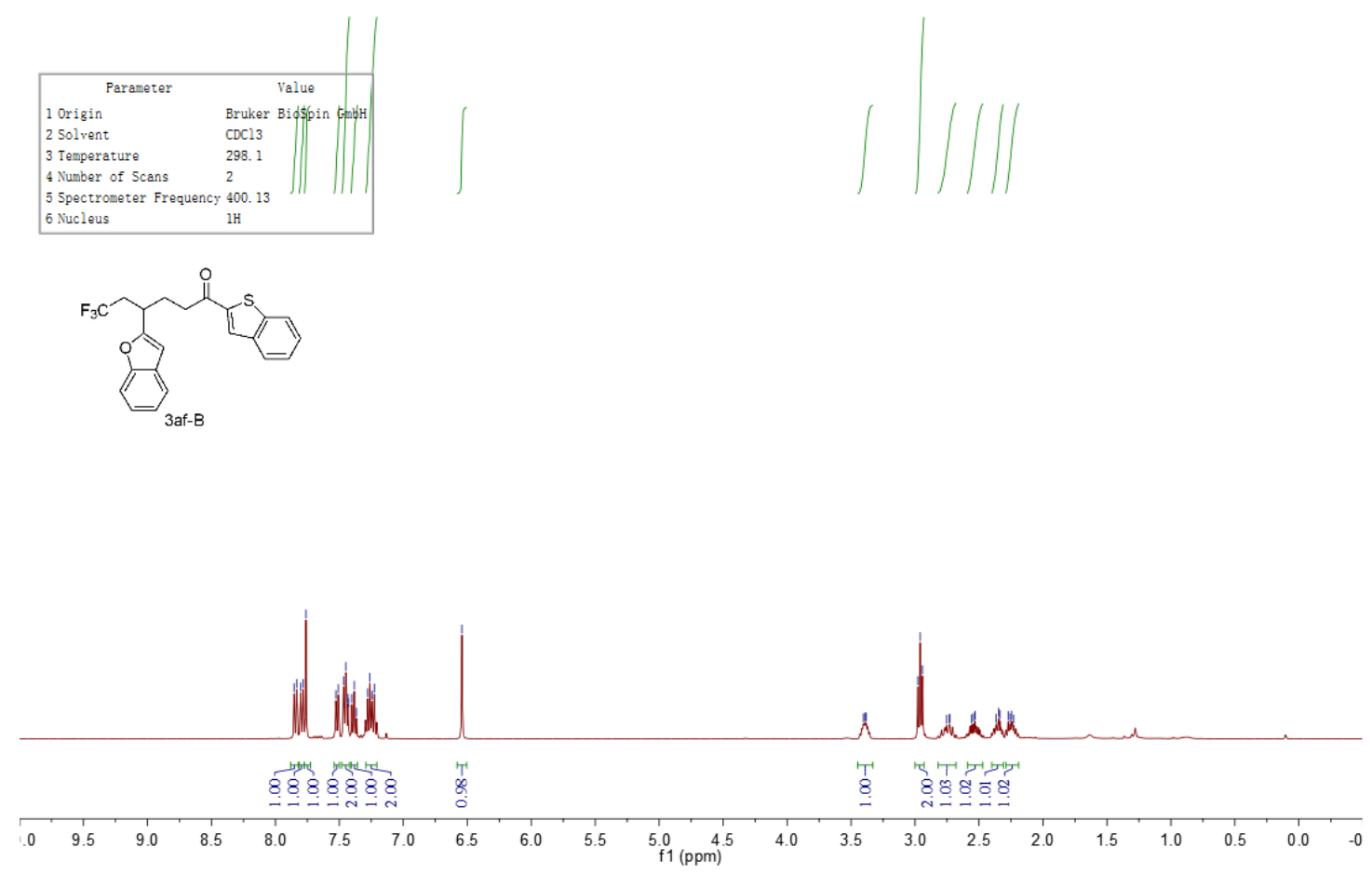


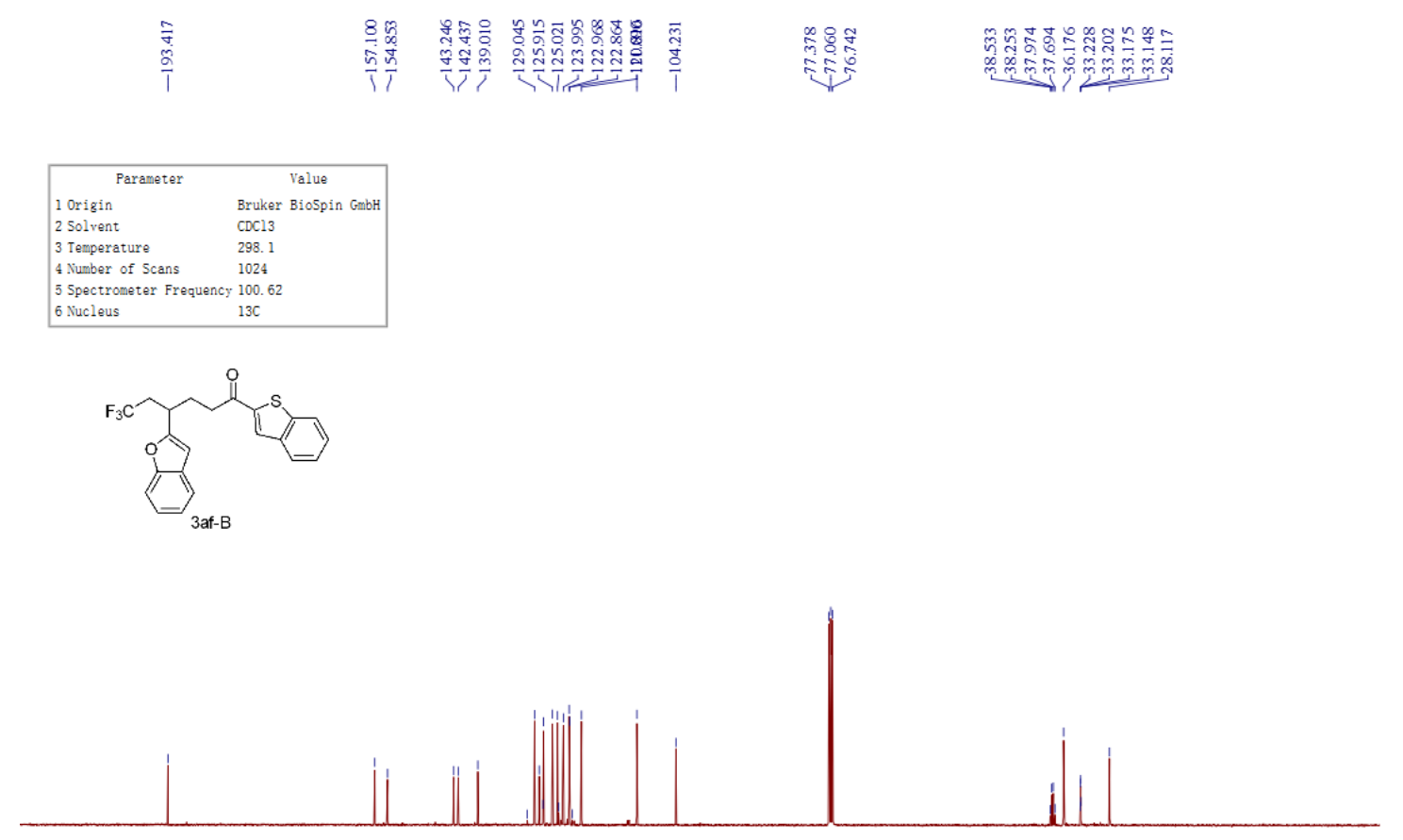

$\begin{array}{llllllllllllllllllllllllll}1 & 210 & 200 & 190 & 180 & 170 & 160 & 150 & 140 & 130 & 120 & 110 & 100 & 90 & 80 & 70 & 60 & 50 & 40 & 30 & 20 & 10 & 0 & -10\end{array}$
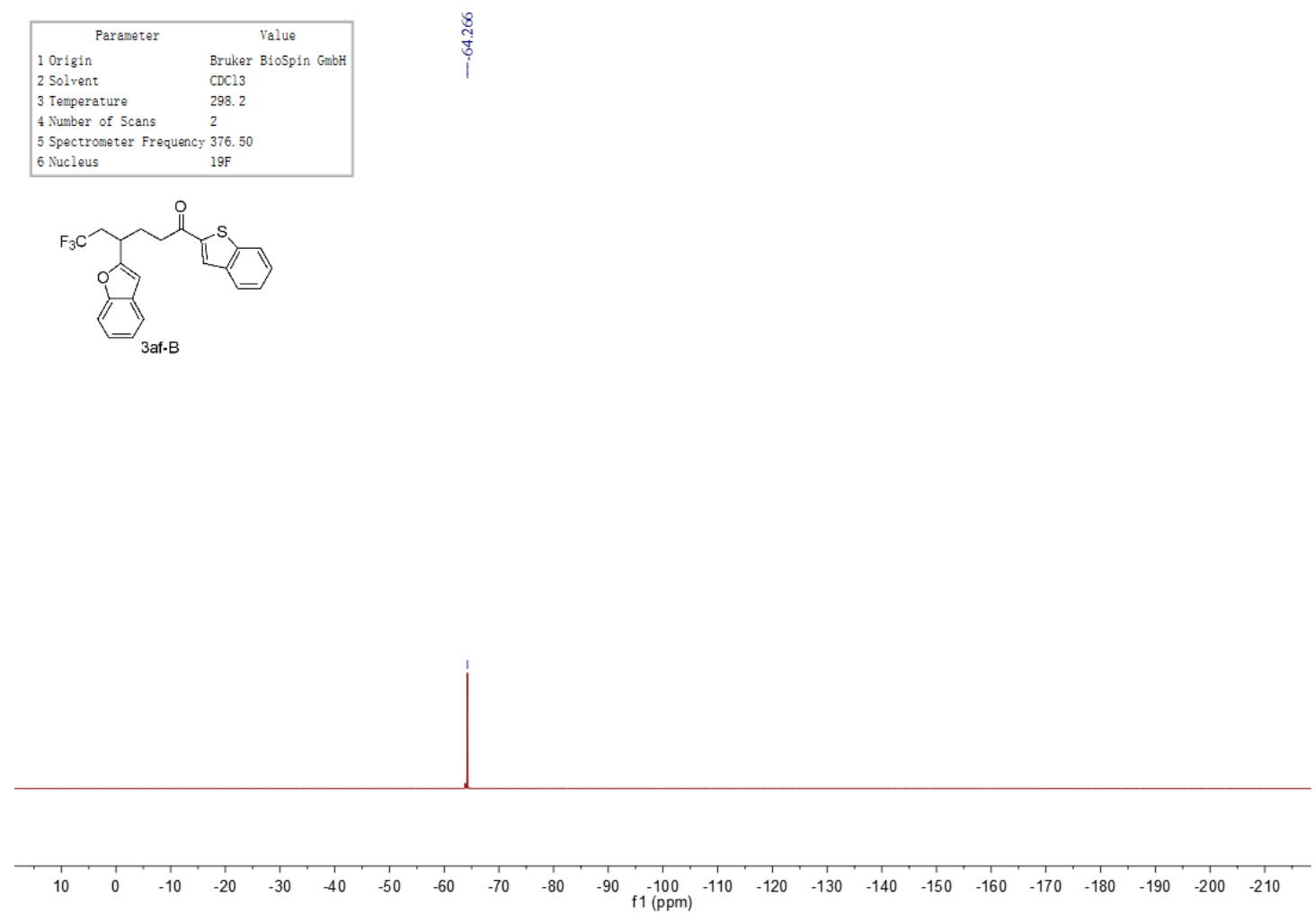


\section{HMBC}

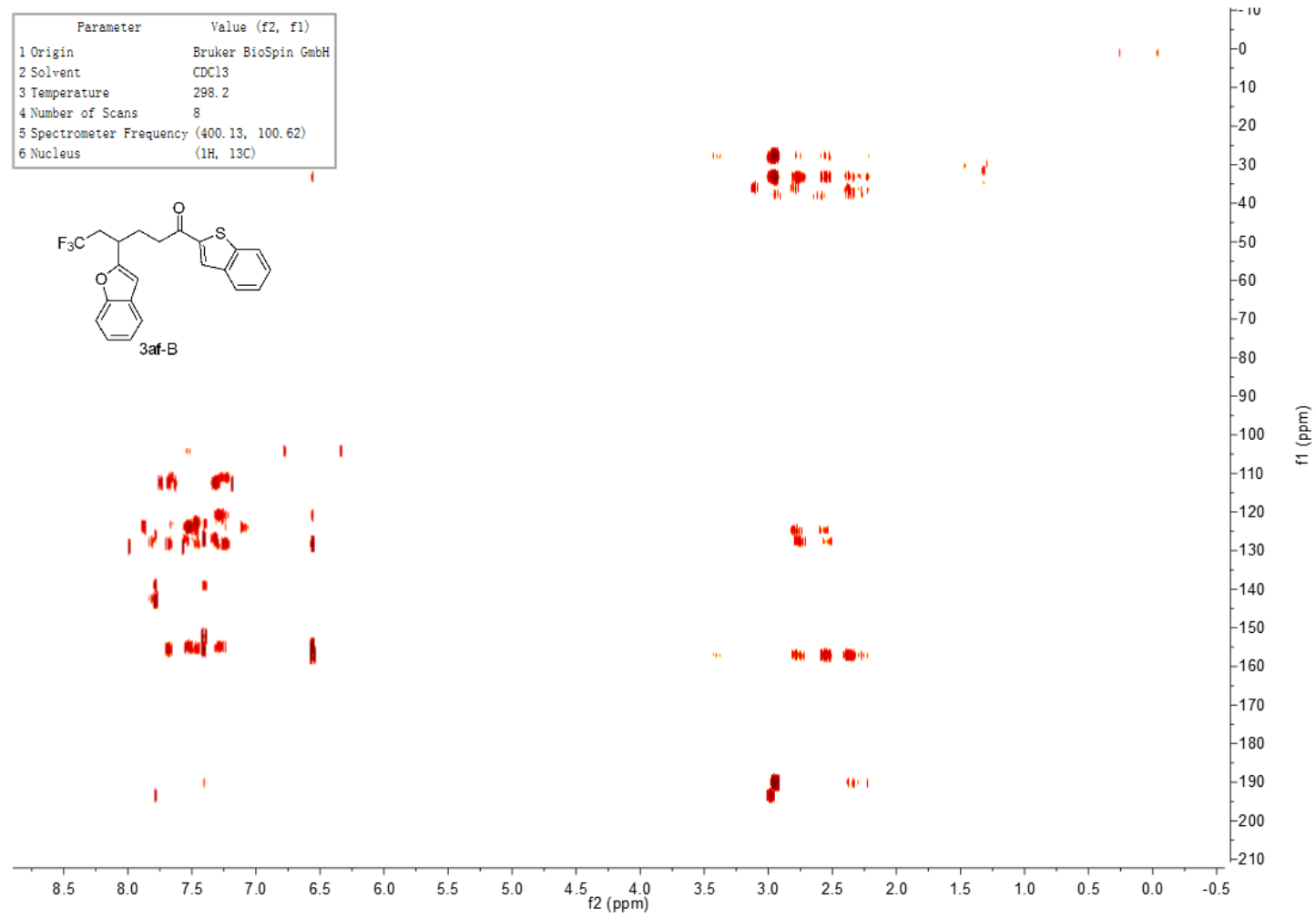

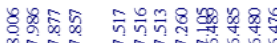
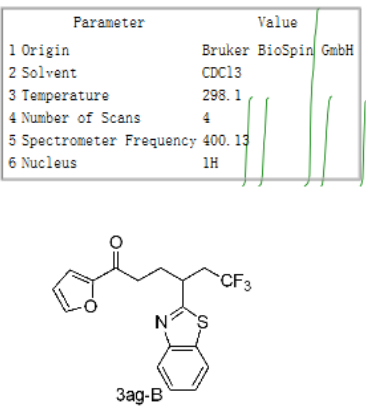

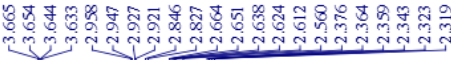

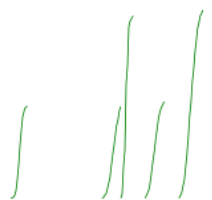

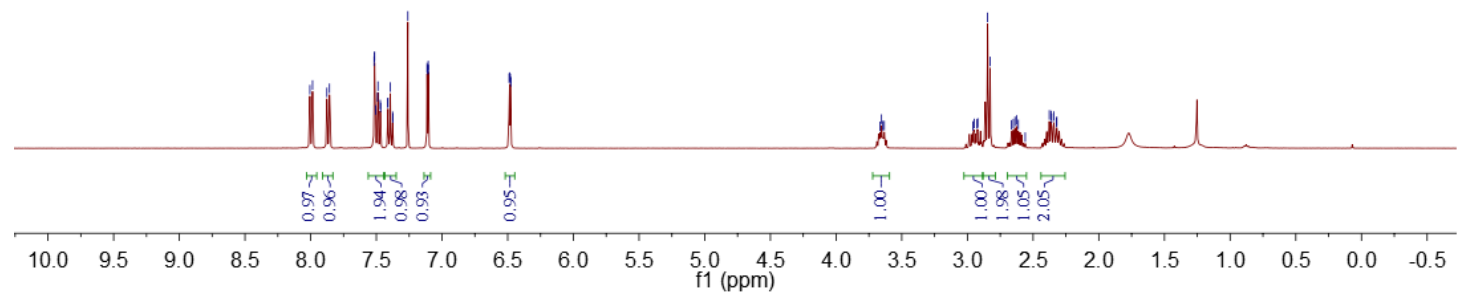




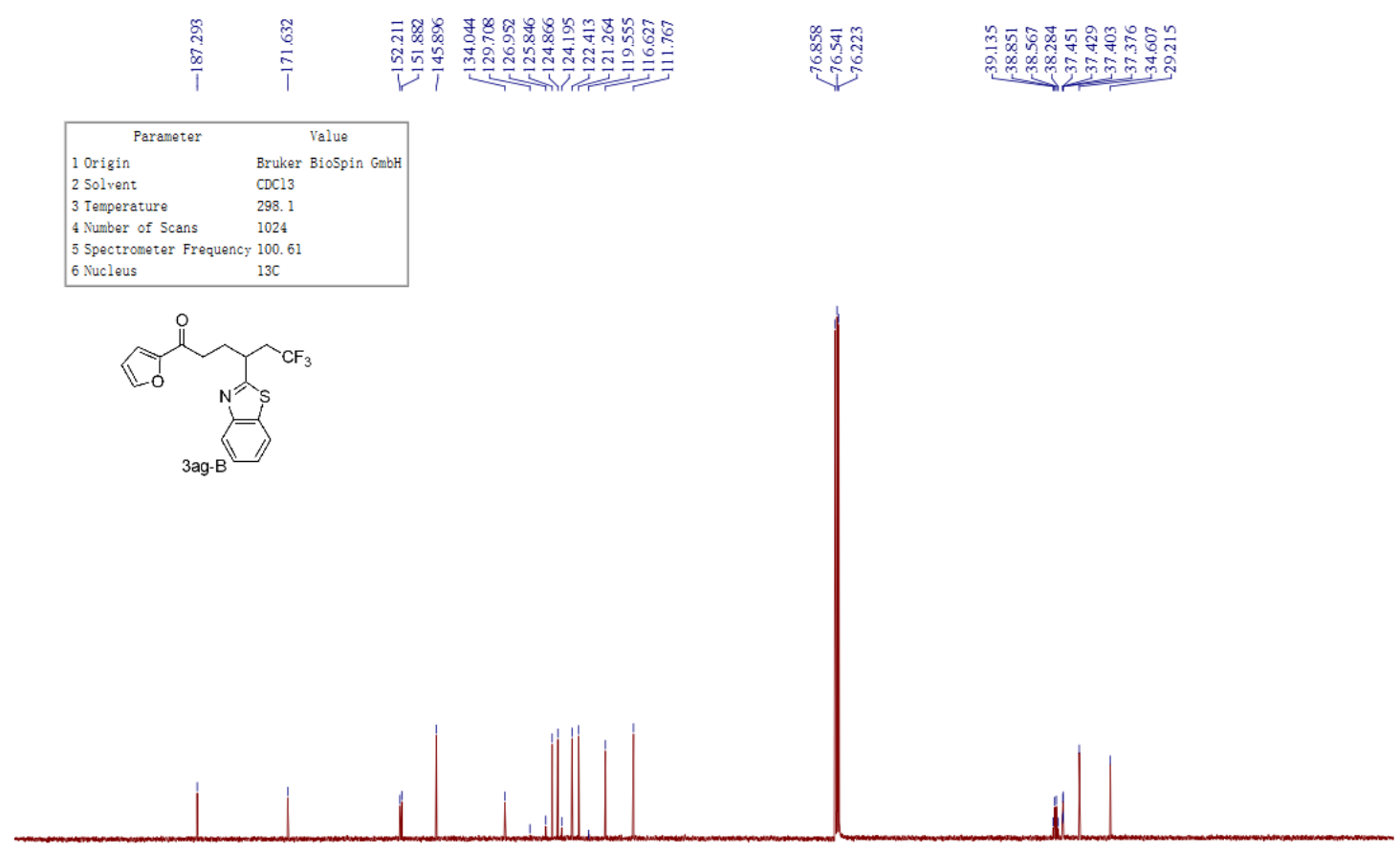

$\begin{array}{lllllllllllllllllllllll}210 & 200 & 190 & 180 & 170 & 160 & 150 & 140 & 130 & 120 & 110 & \begin{array}{c}100 \\ \mathrm{f} 1(\mathrm{ppm})\end{array} & 90 & 80 & 70 & 60 & 50 & 40 & 30 & 20 & 10 & 0 & -10\end{array}$

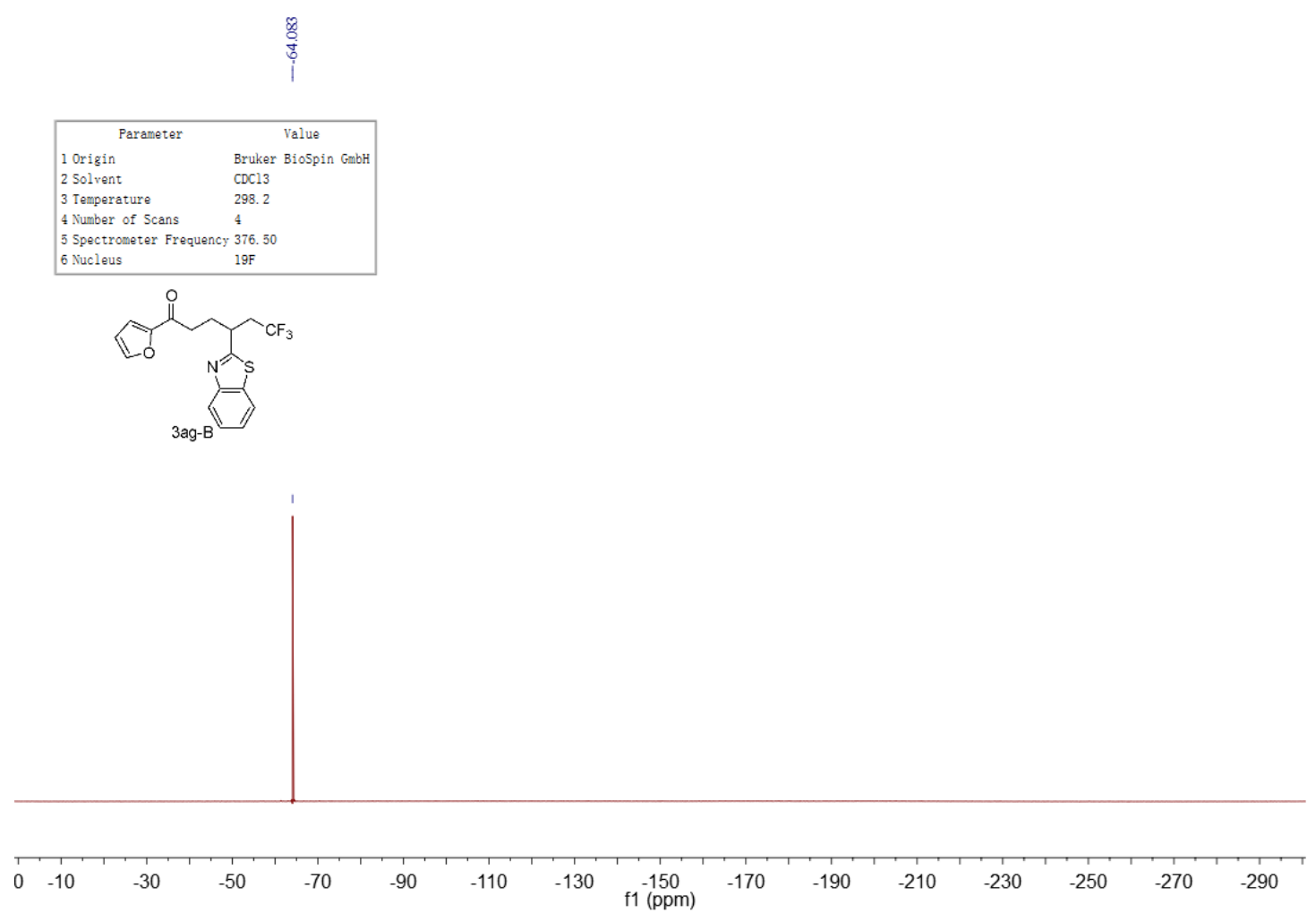




\section{HMBC}

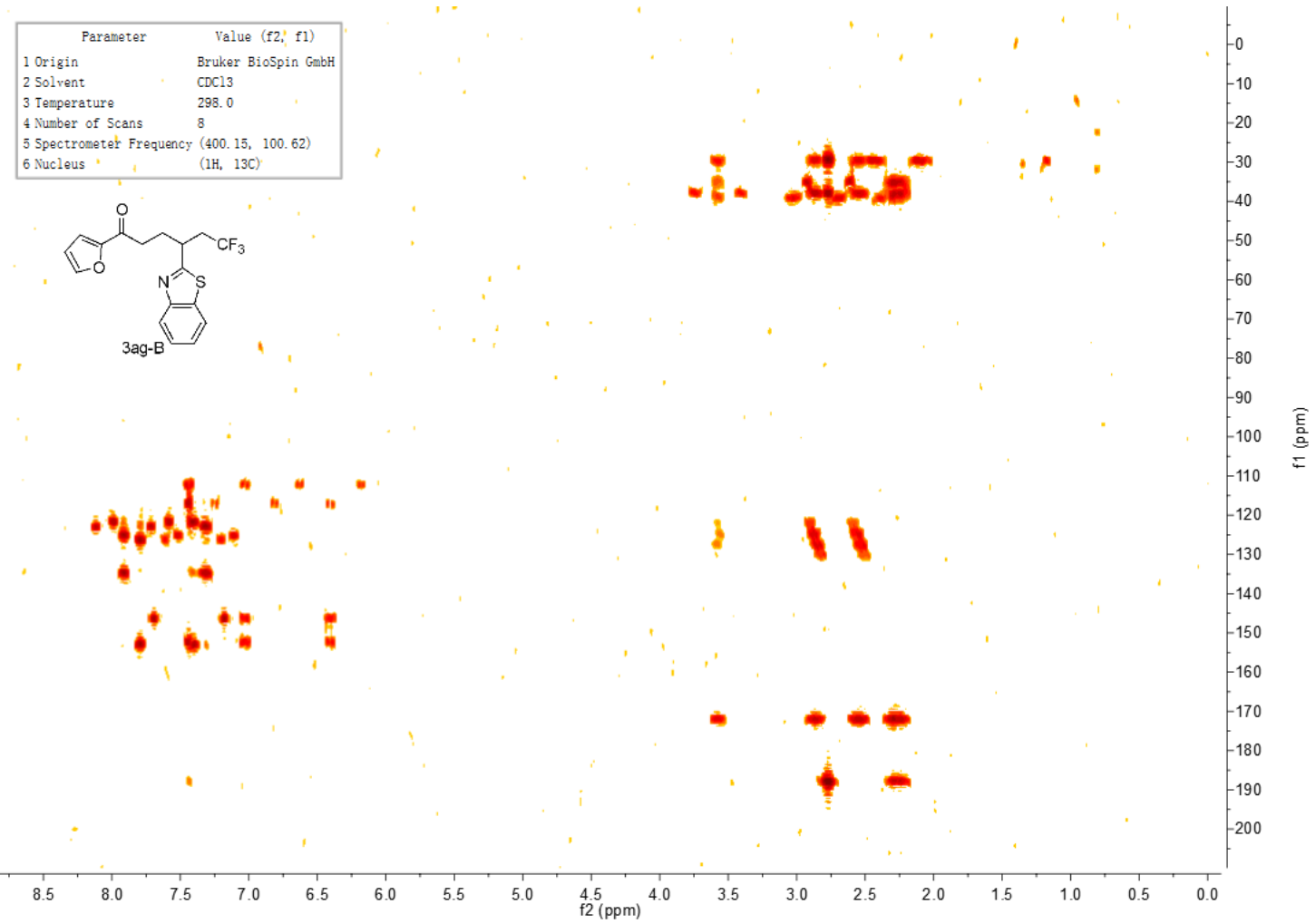

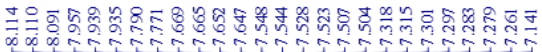

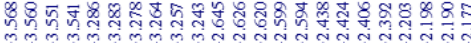
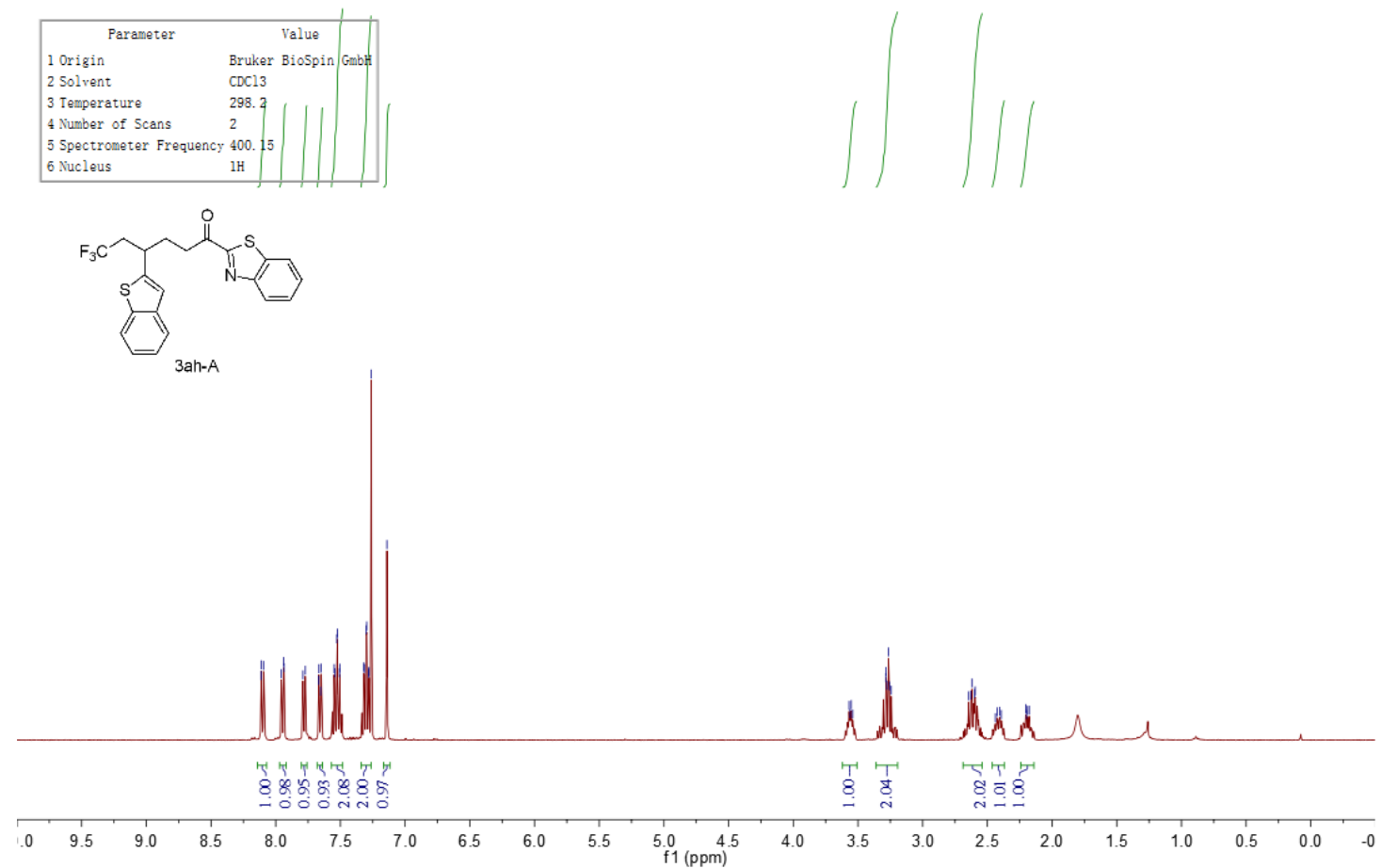


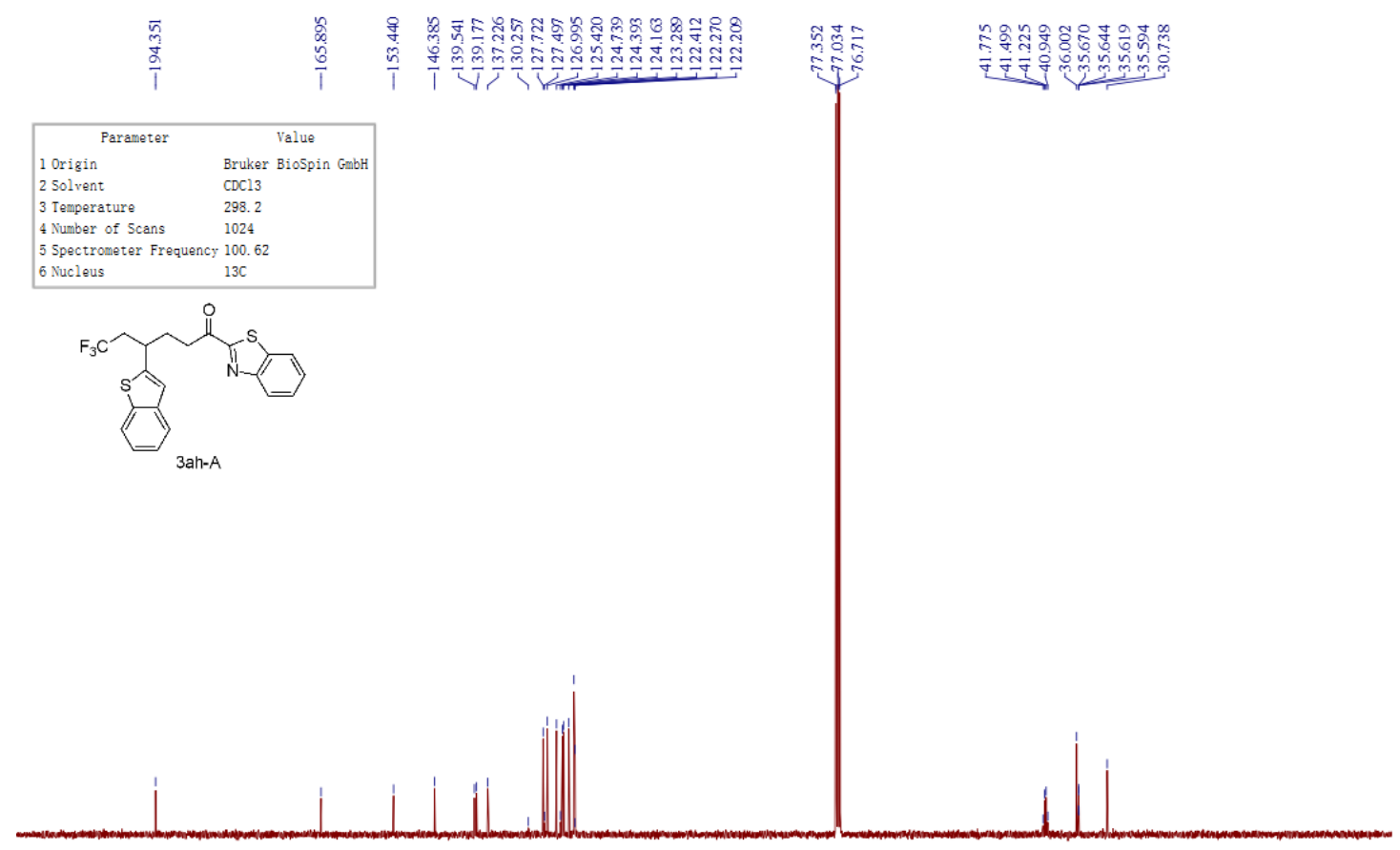

$\begin{array}{llllllllllllllllllllllllllll}210 & 200 & 190 & 180 & 170 & 160 & 150 & 140 & 130 & 120 & 110 & 100 & 90 & 80 & 70 & 60 & 50 & 40 & 30 & 20 & 10 & 0 & -10\end{array}$

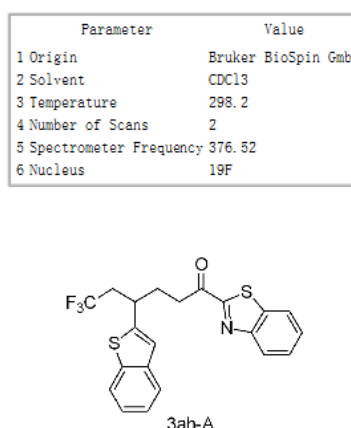

3ah-A

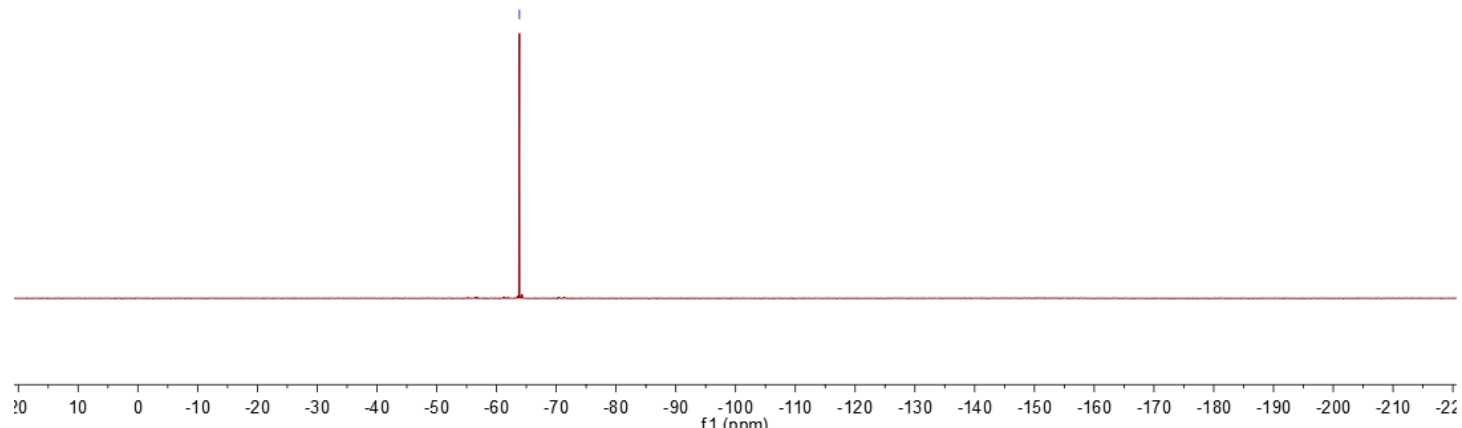




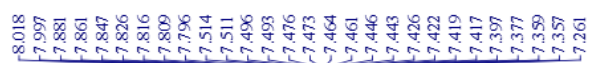

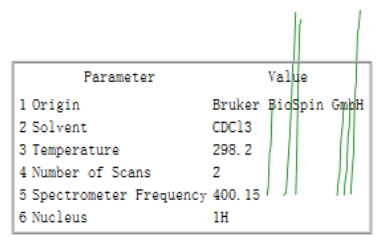

5 Spectrometer Frequency 400
6 Nucleus
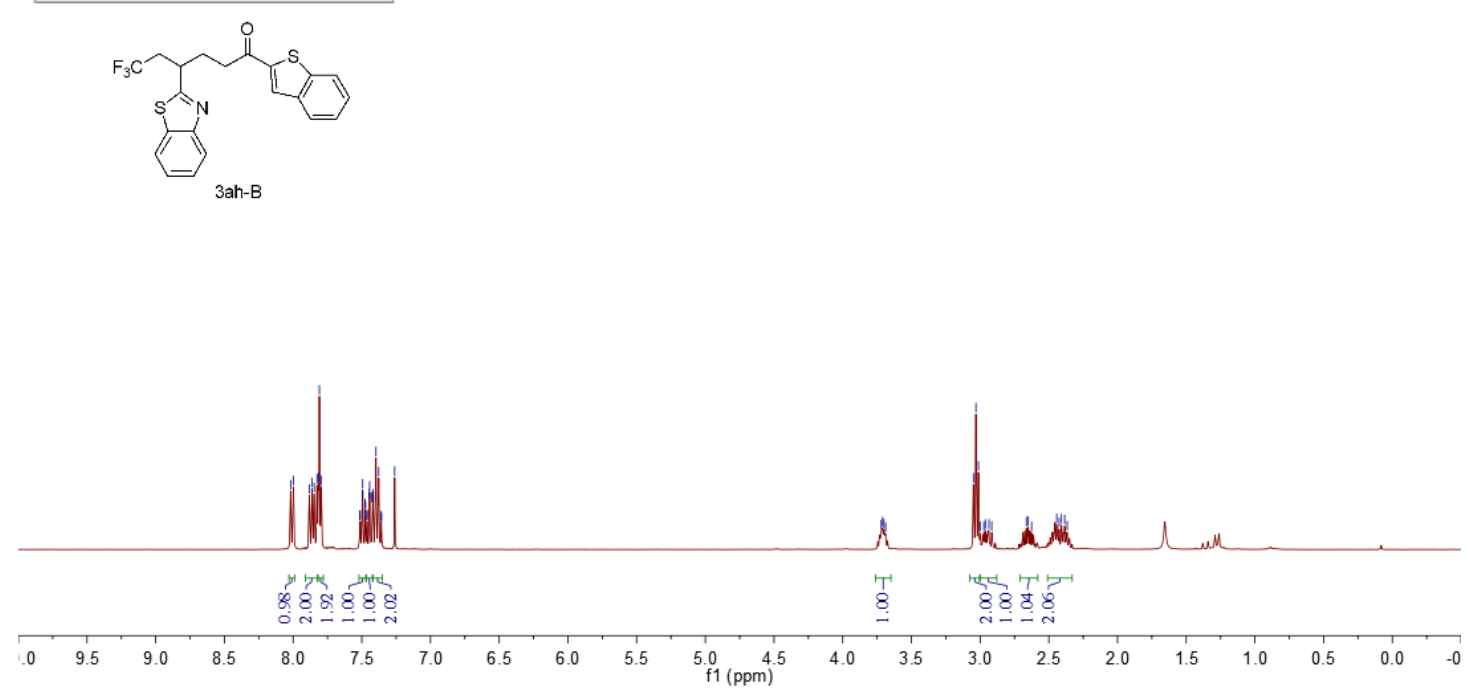

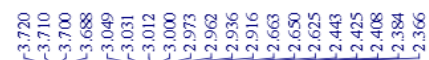

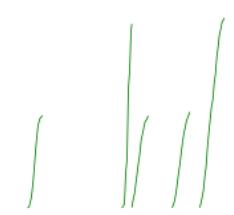

人

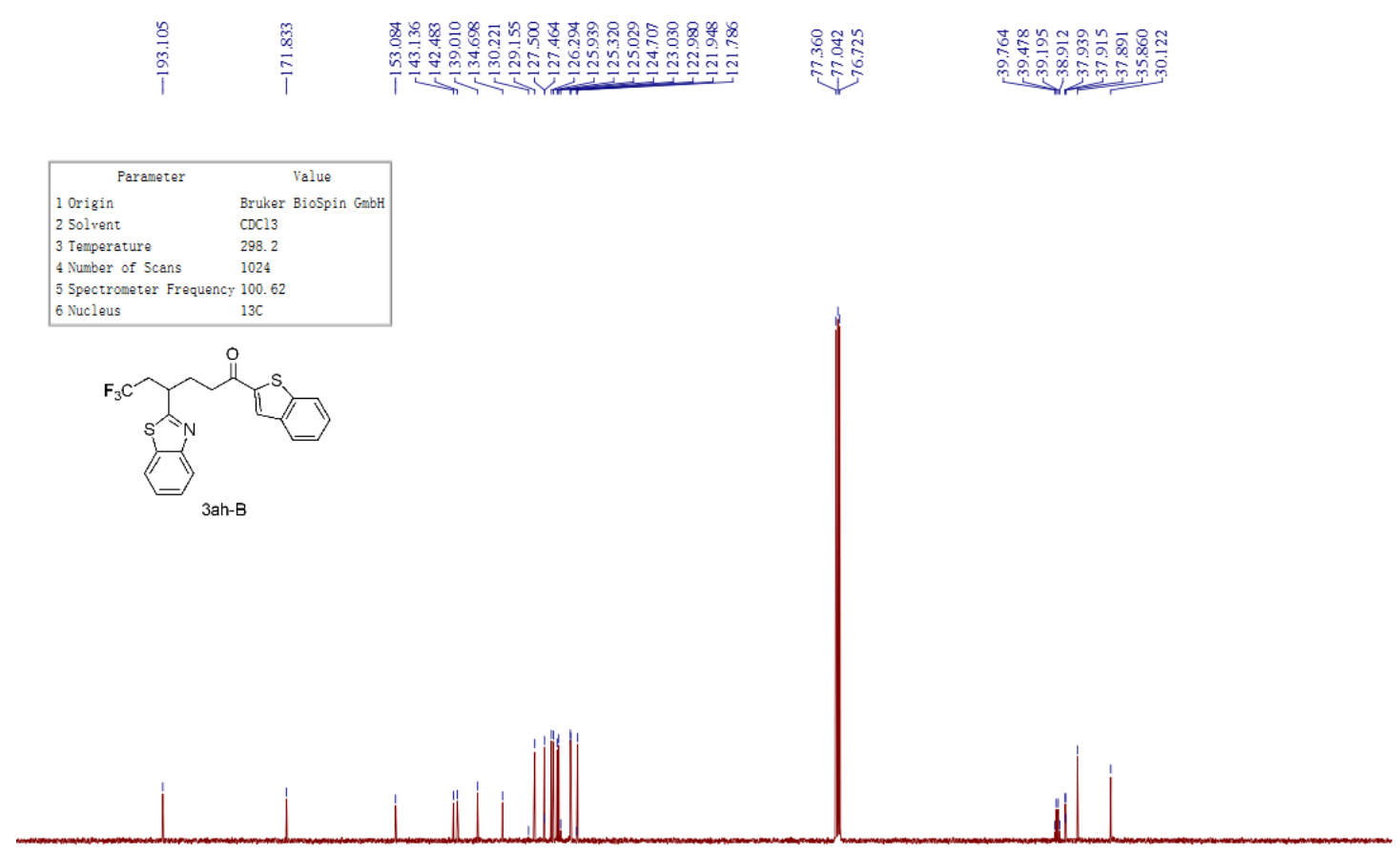

$\begin{array}{lllllllllllllllllllllll}210 & 200 & 190 & 180 & 170 & 160 & 150 & 140 & 130 & 120 & 110 & 100 & 90 & 80 & 70 & 60 & 50 & 40 & 30 & 20 & 10 & 0 & -10\end{array}$ 


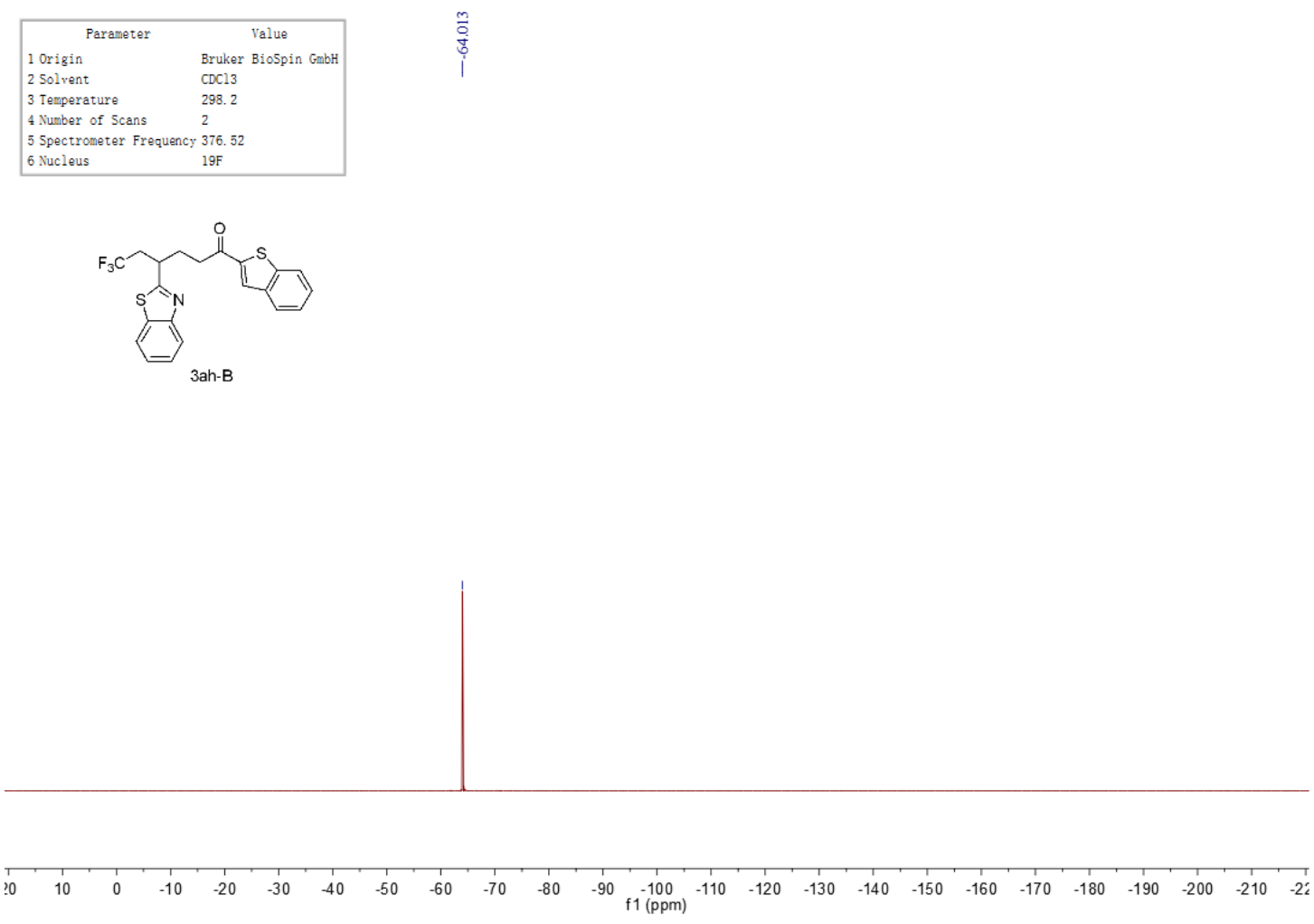

HMBC
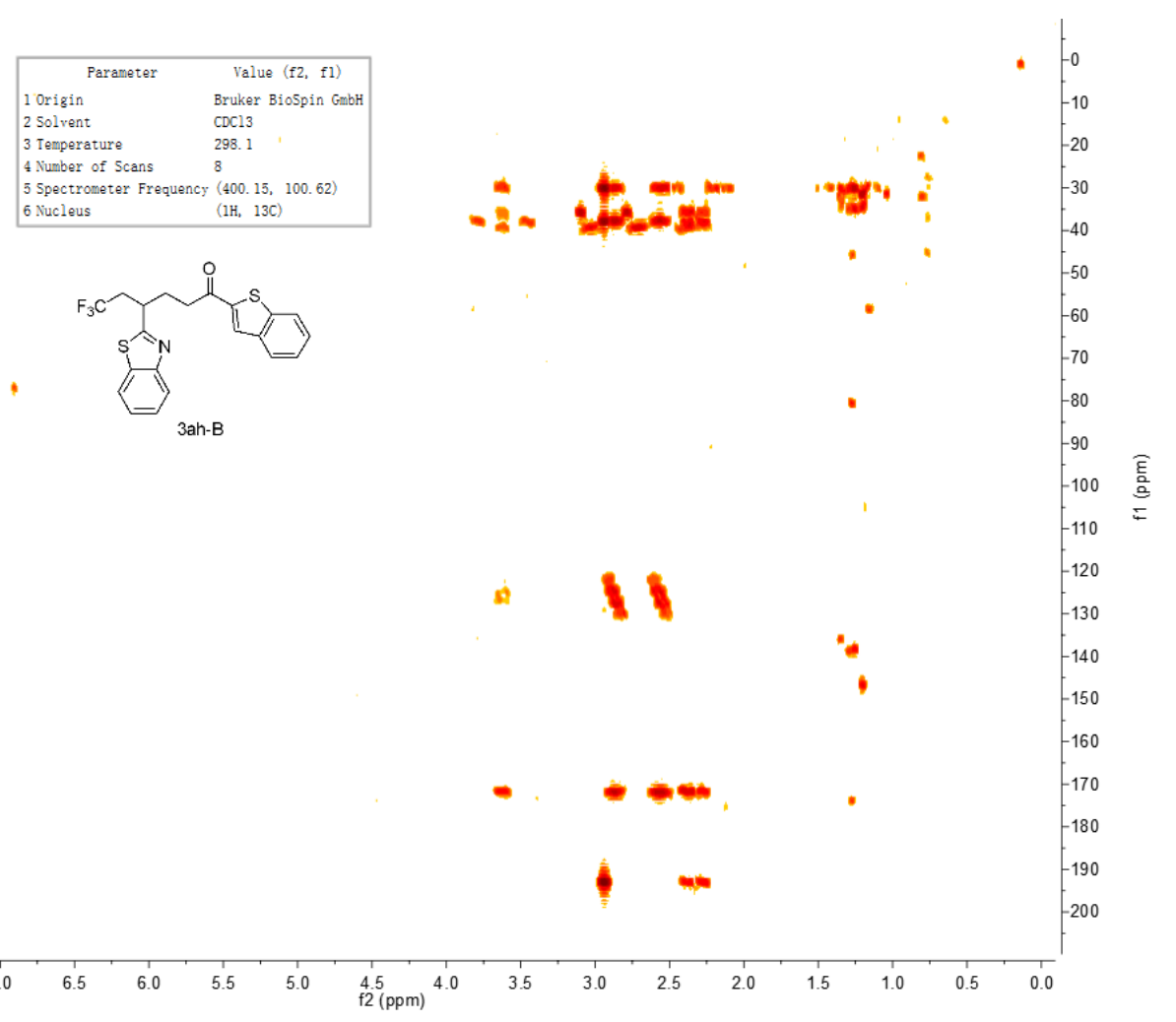

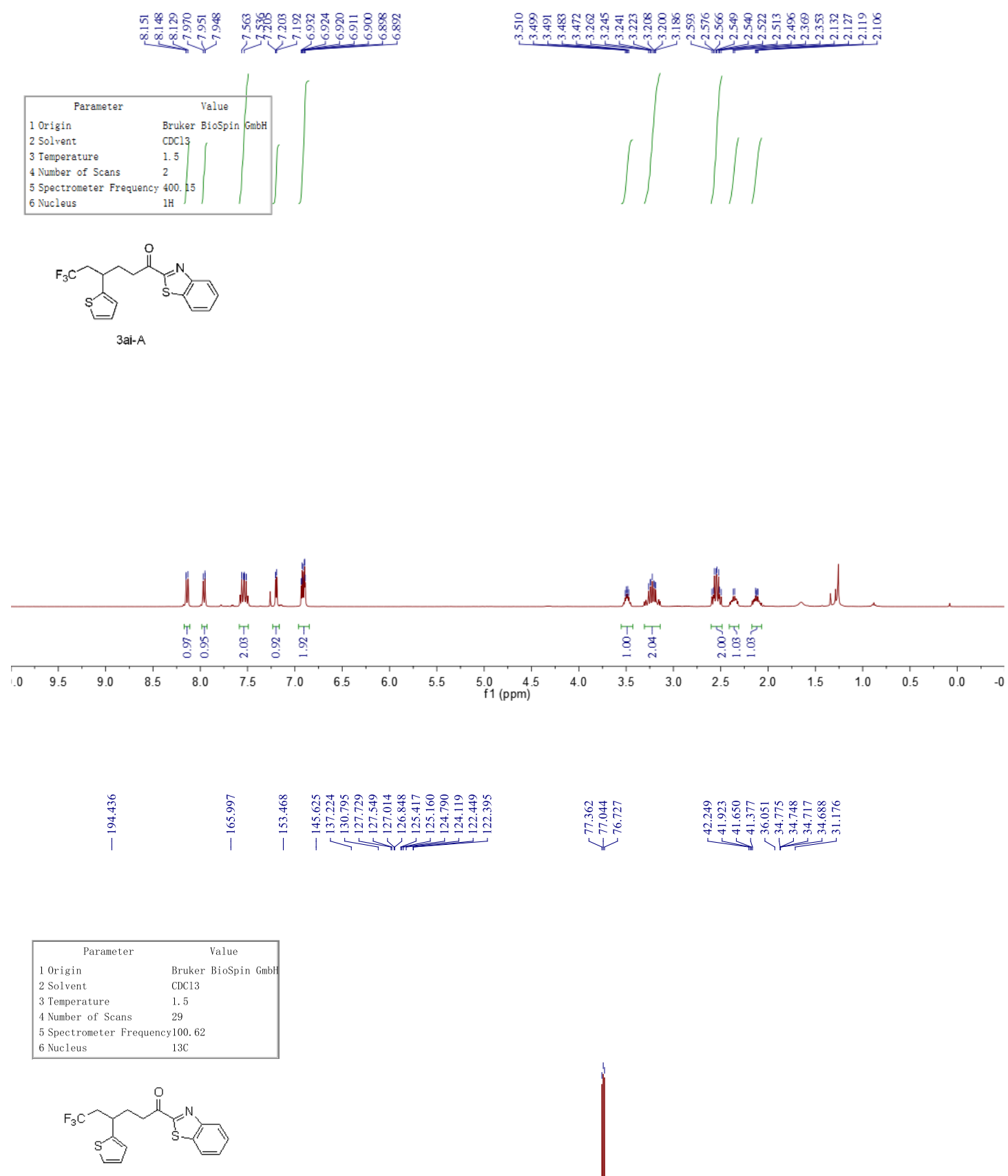

3ai-A
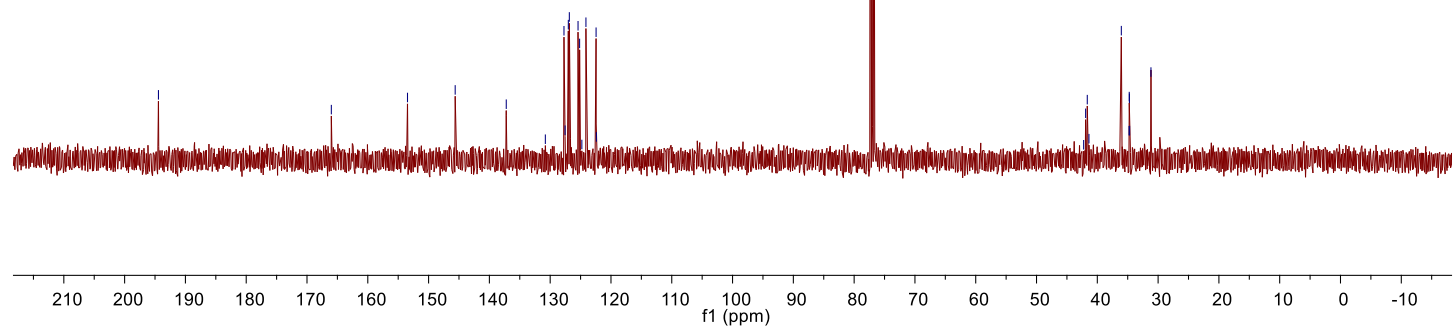

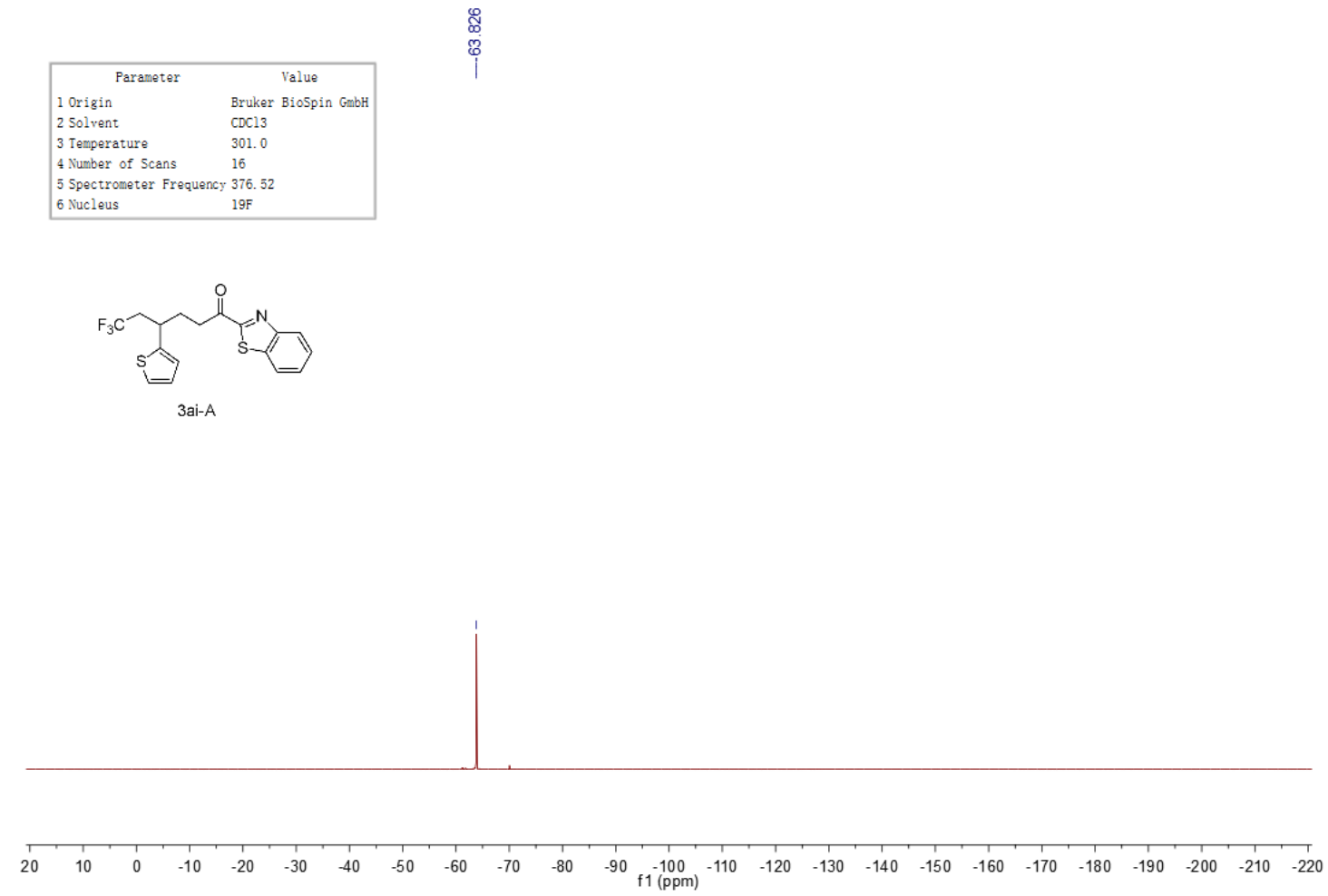

\section{8 .}

\begin{tabular}{|c|c|c|}
\hline Parameter & & Value \\
\hline 1 Origin & Bruker & Biospin GmbH \\
\hline 2 Solvent & CDC13 & \\
\hline 3 Temperature & 298.2 & \\
\hline 4 Number of Scans & 4 & \\
\hline 5 Spectrometer Frequency & 400.13 & \\
\hline 6 Nucleus & $1 \mathrm{H}$ & \\
\hline
\end{tabular}

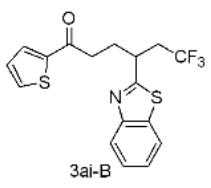

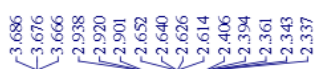

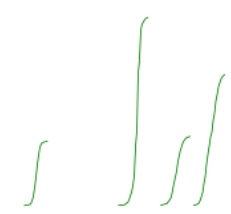

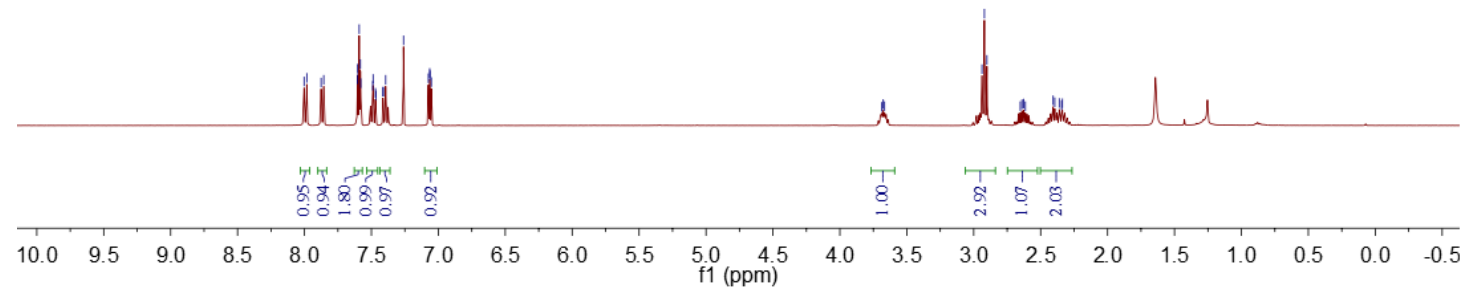




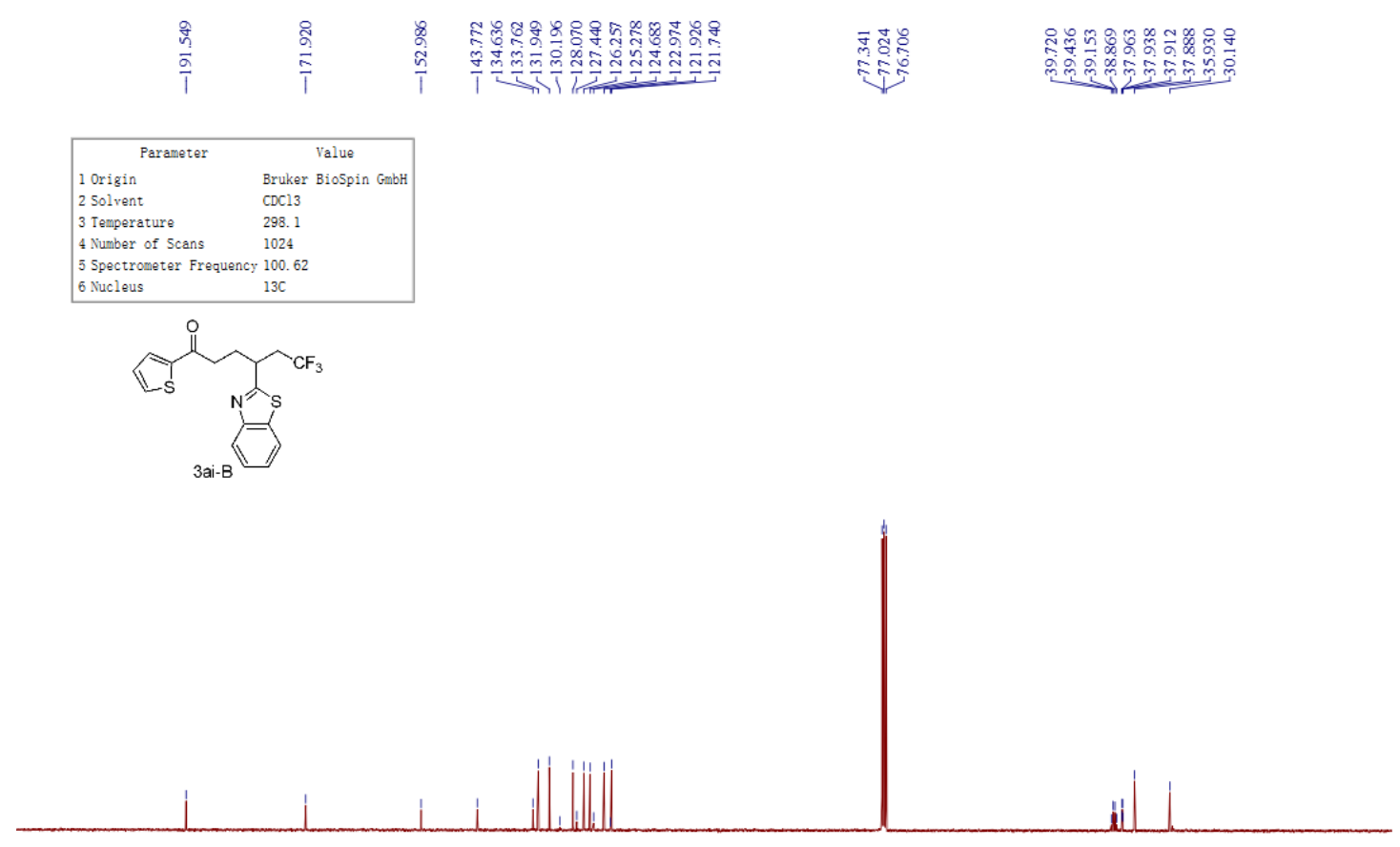

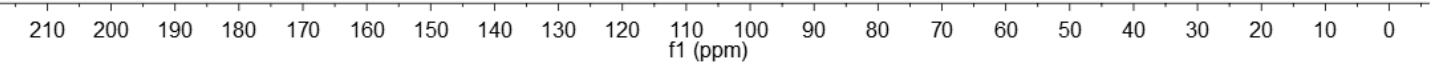
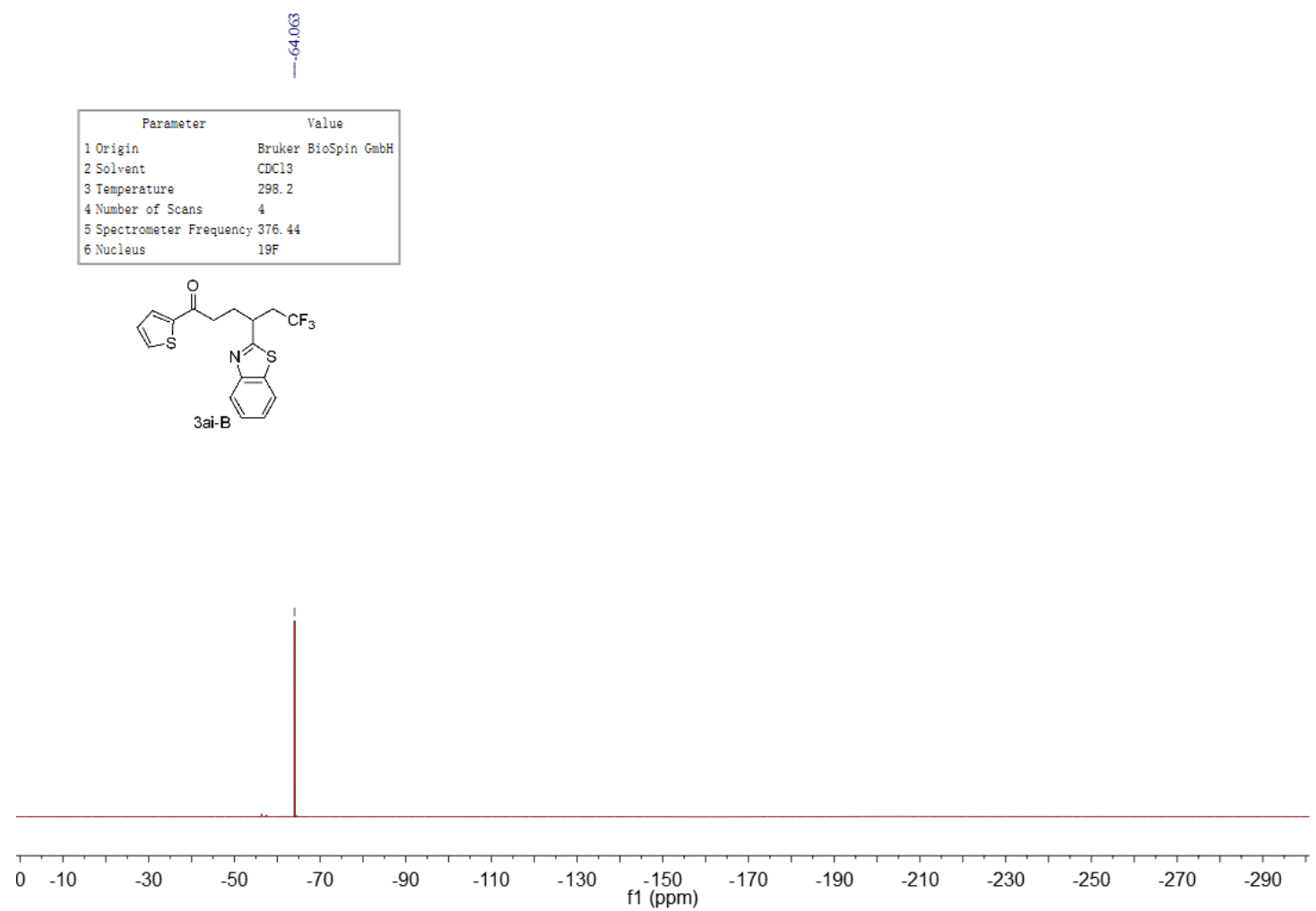

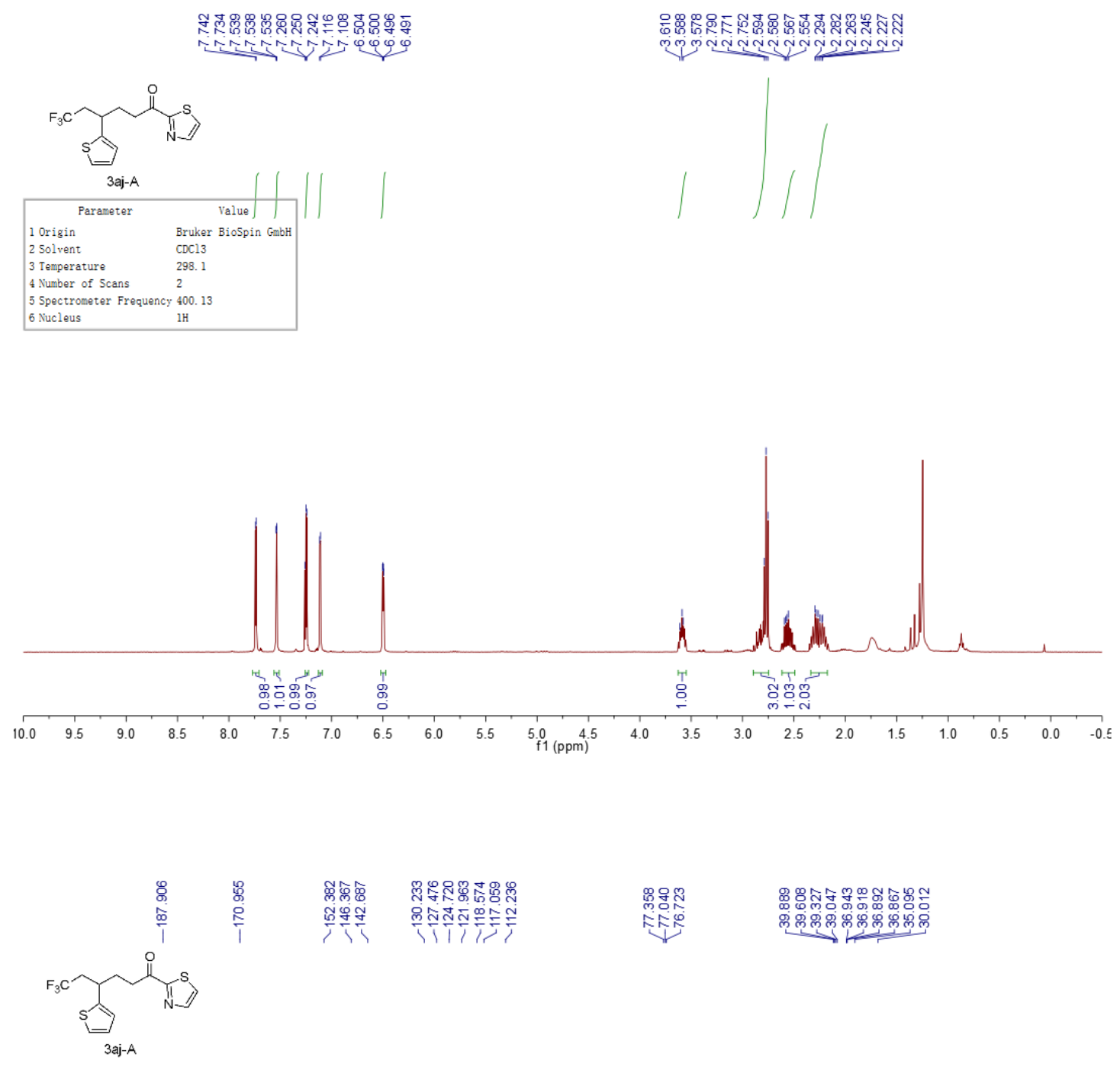

\begin{tabular}{|ll|}
\hline \multicolumn{1}{|c|}{ Parameter } & \multicolumn{1}{c|}{ Value } \\
1 Origin & Bruker \\
2 Solospin Gment & CDC13 \\
3 Temperature & 298.1 \\
4 Number of Scans & 512 \\
5 Spectrometer Frequency & 100.62 \\
6 Nucleus & $13 \mathrm{C}$ \\
\hline
\end{tabular}

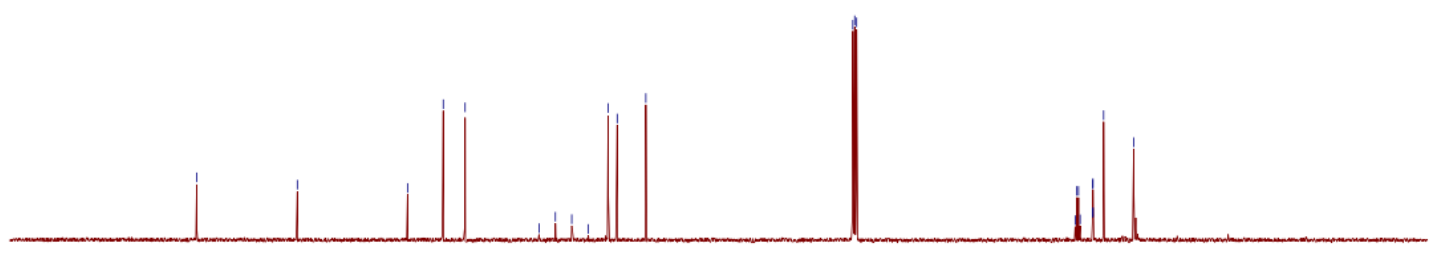

$\begin{array}{lllllllllllllllllllllllllll}210 & 200 & 190 & 180 & 170 & 160 & 150 & 140 & 130 & 120 & 110 & 100 & 90 & 80 & 70 & 60 & 50 & 40 & 30 & 20 & 10 & 0 & -10\end{array}$ 


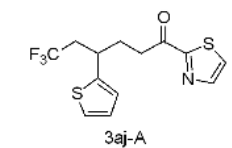

\begin{tabular}{|ll|}
\hline \multicolumn{1}{|c|}{ Parameter } & \multicolumn{1}{c|}{ Value } \\
1 Origin & Bruker BioSpin GmbH \\
2 Solvent & CDC13 \\
3 Temperature & 0.0 \\
4 Number of Scans & 2 \\
5 Spectrometer Frequency & 376.52 \\
6 Nucleus & $19 \mathrm{~F}$ \\
\hline
\end{tabular}

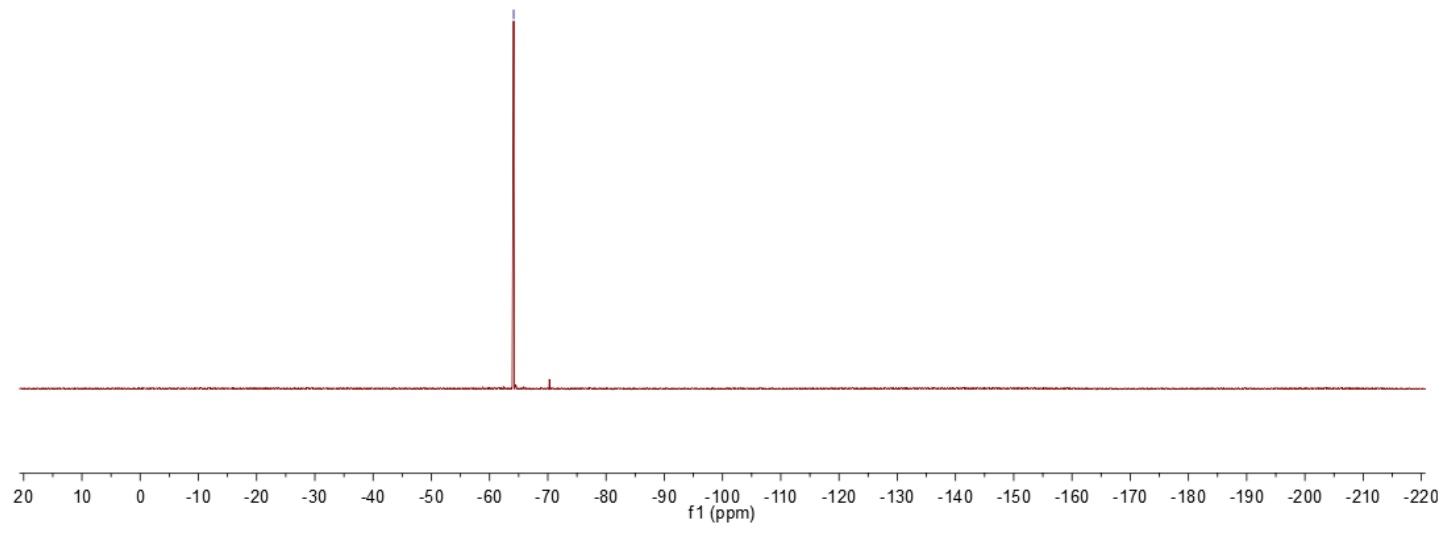

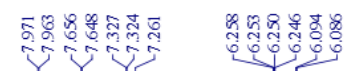

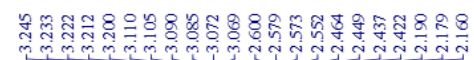
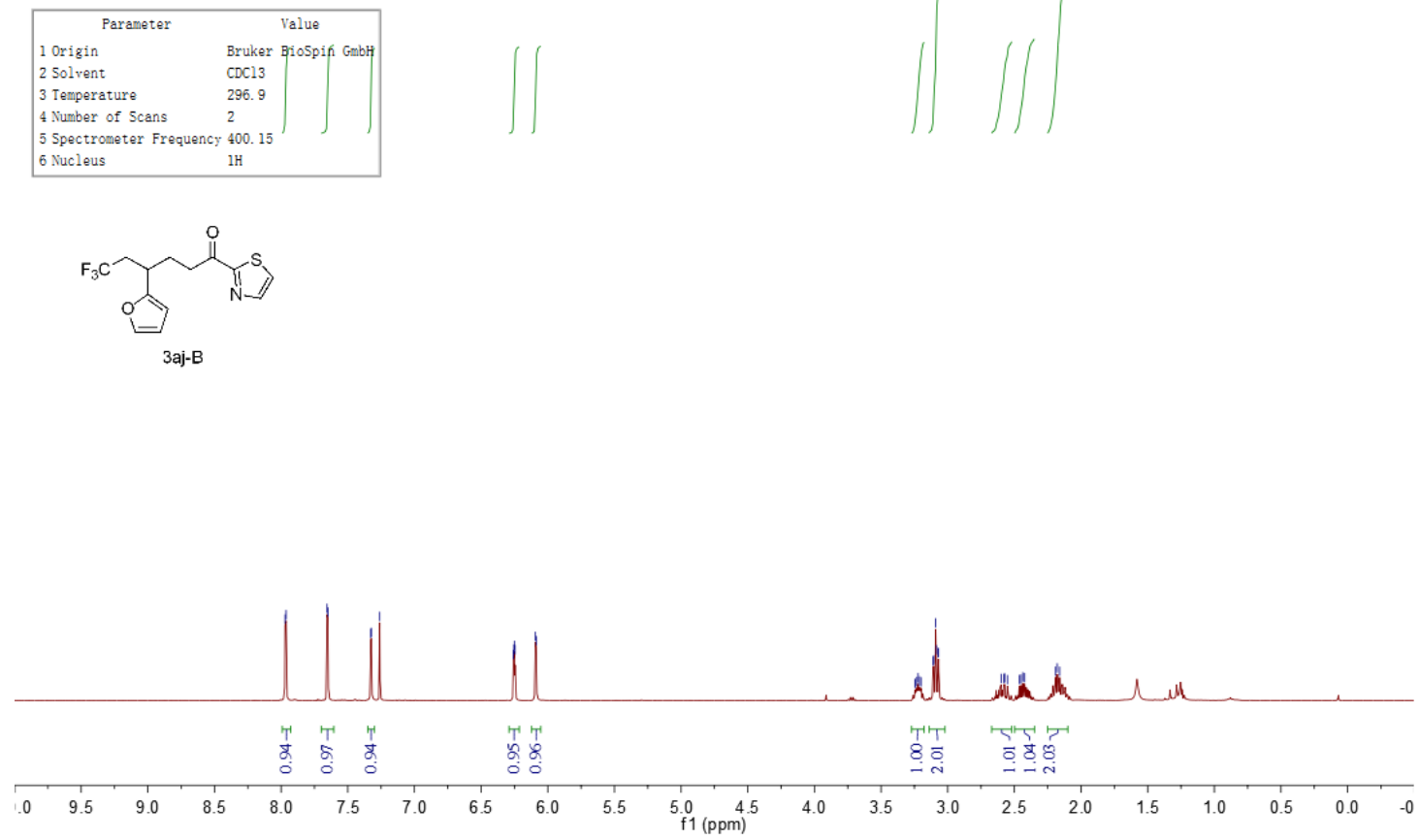


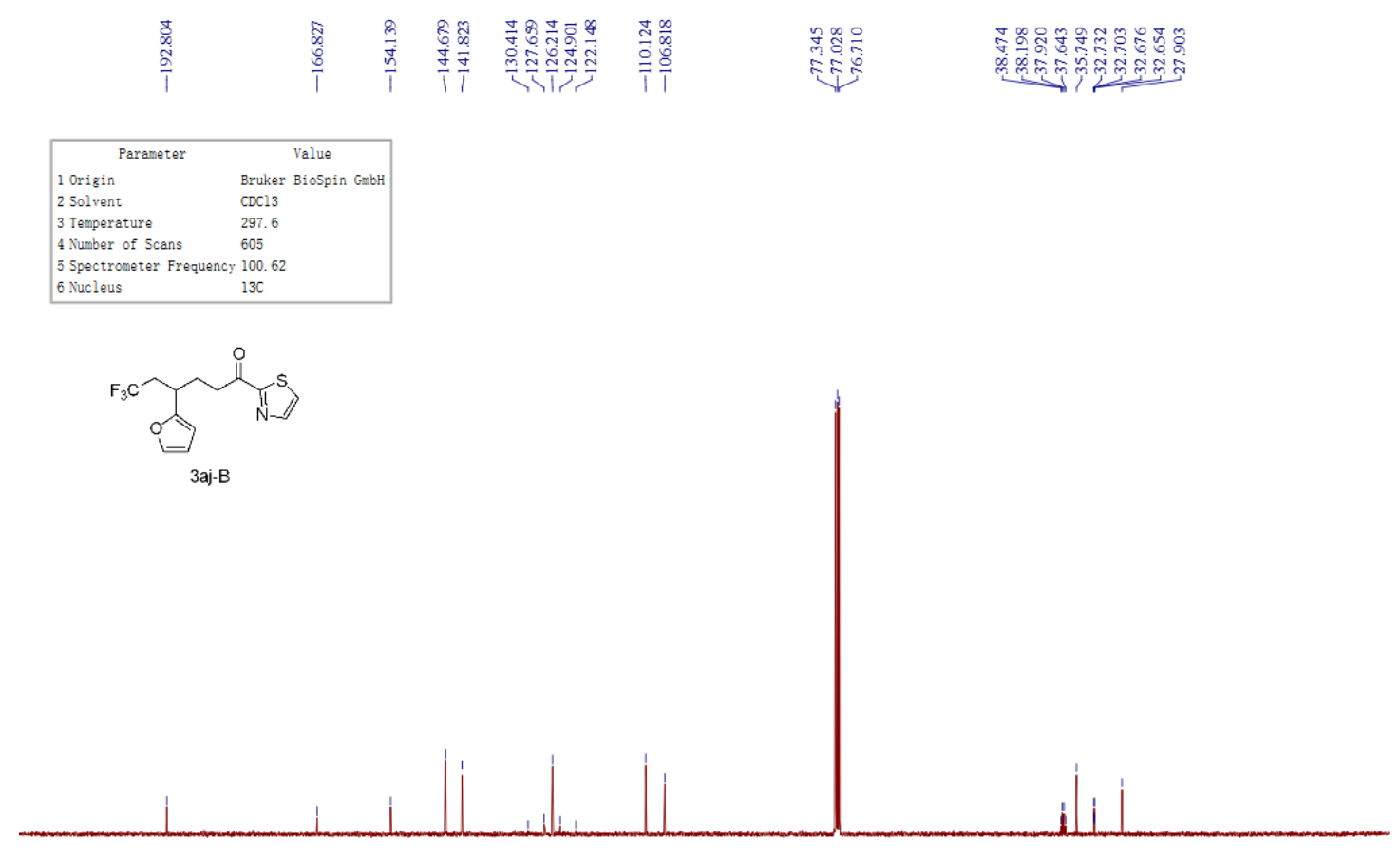

$\begin{array}{lllllllllllllllllllllllll}210 & 200 & 190 & 180 & 170 & 160 & 150 & 140 & 130 & 120 & 110 & 100 & 90 & 80 & 70 & 60 & 50 & 40 & 30 & 20 & 10 & 0 & -10\end{array}$
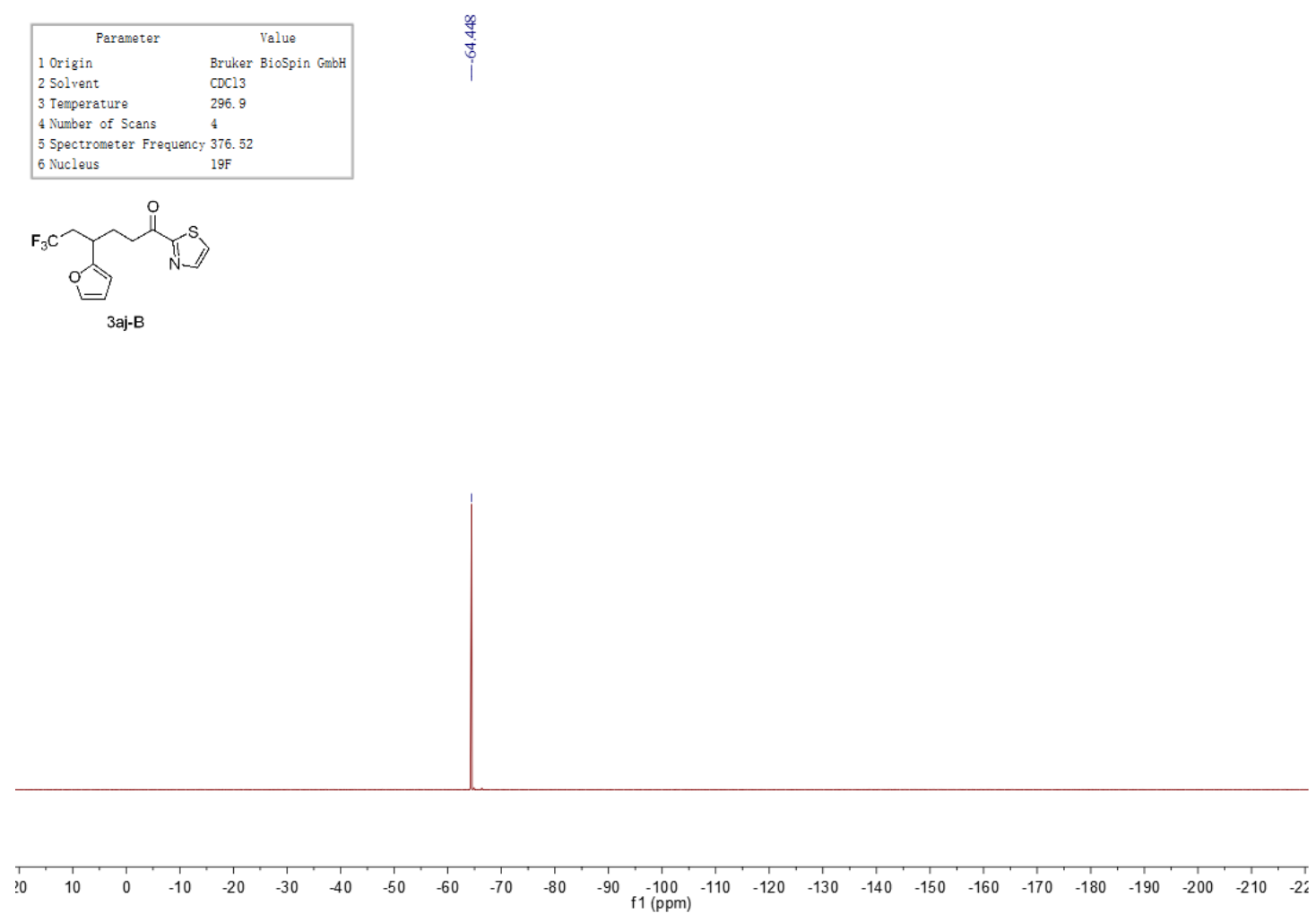

HMBC 


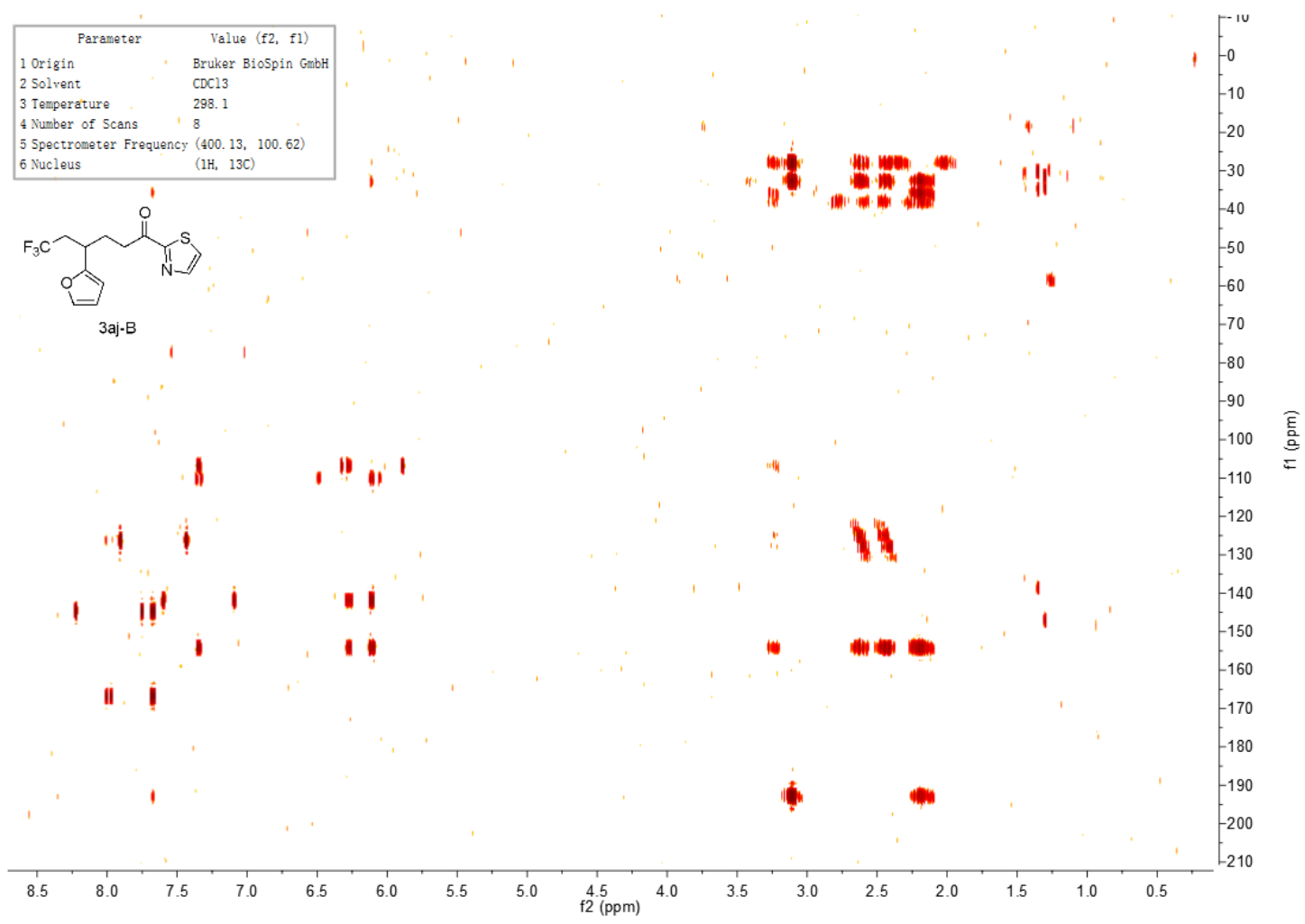

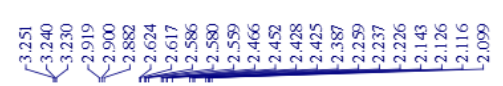
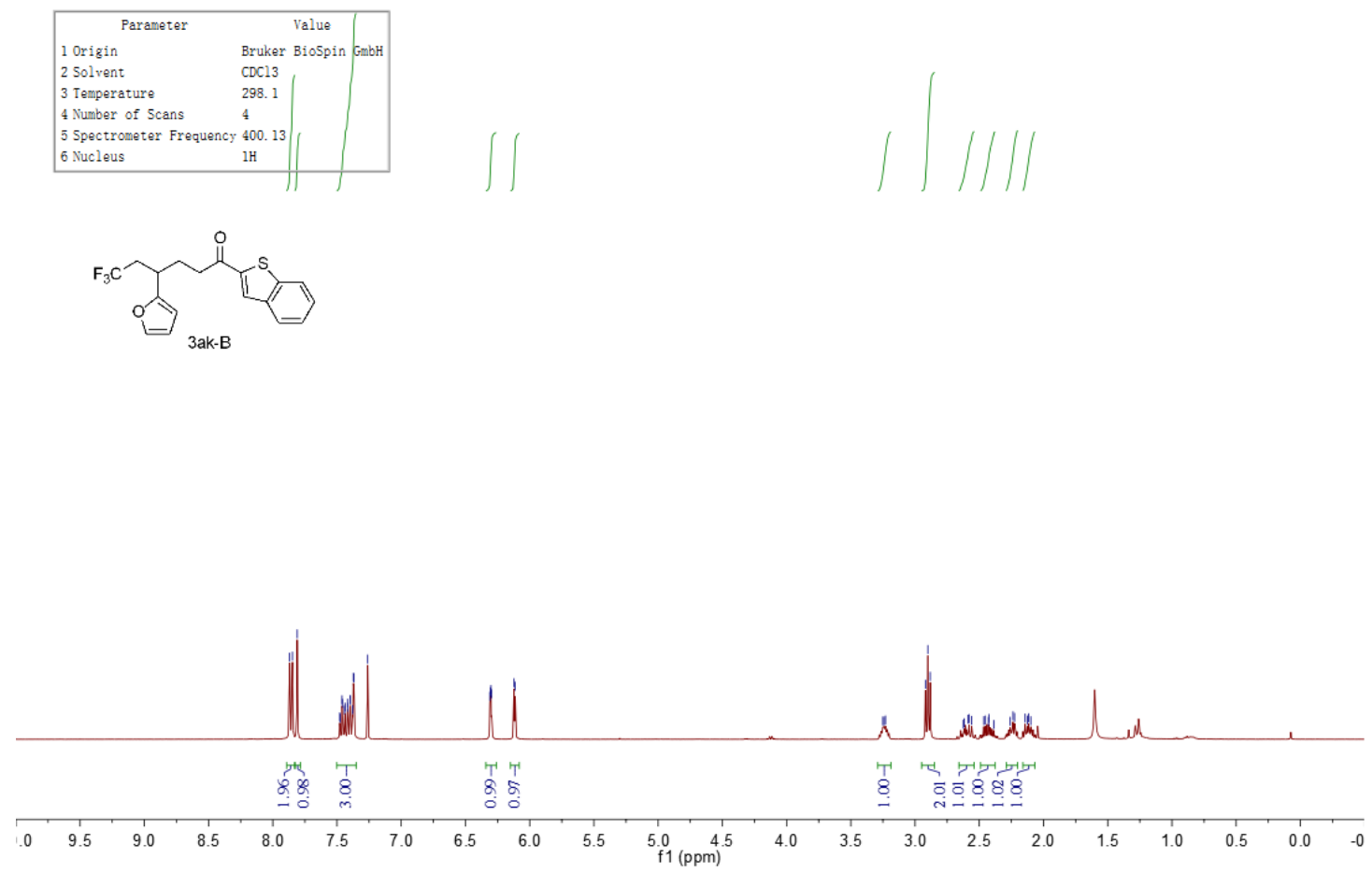


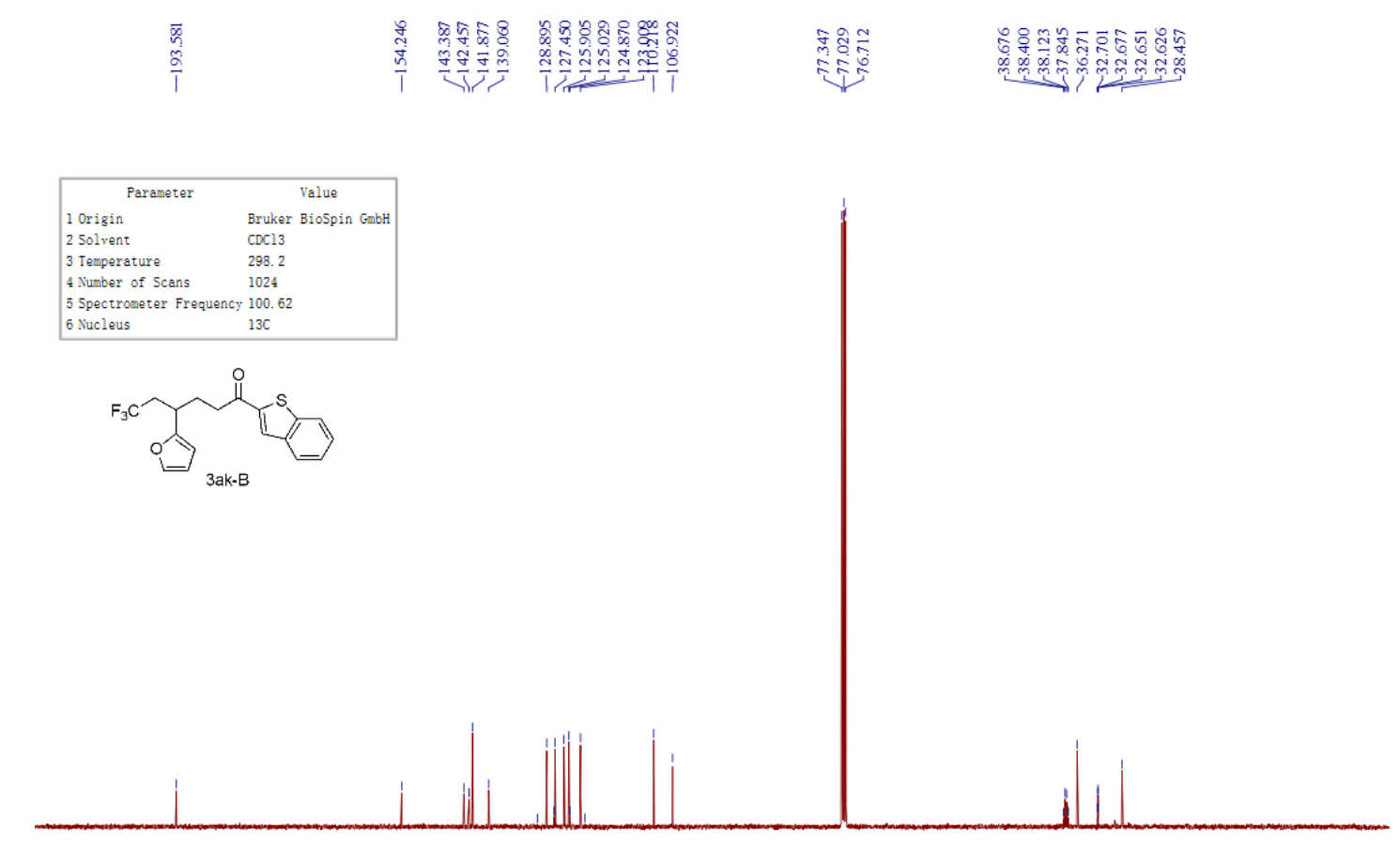

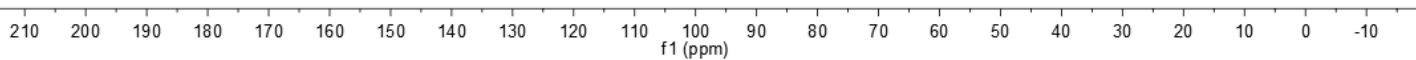
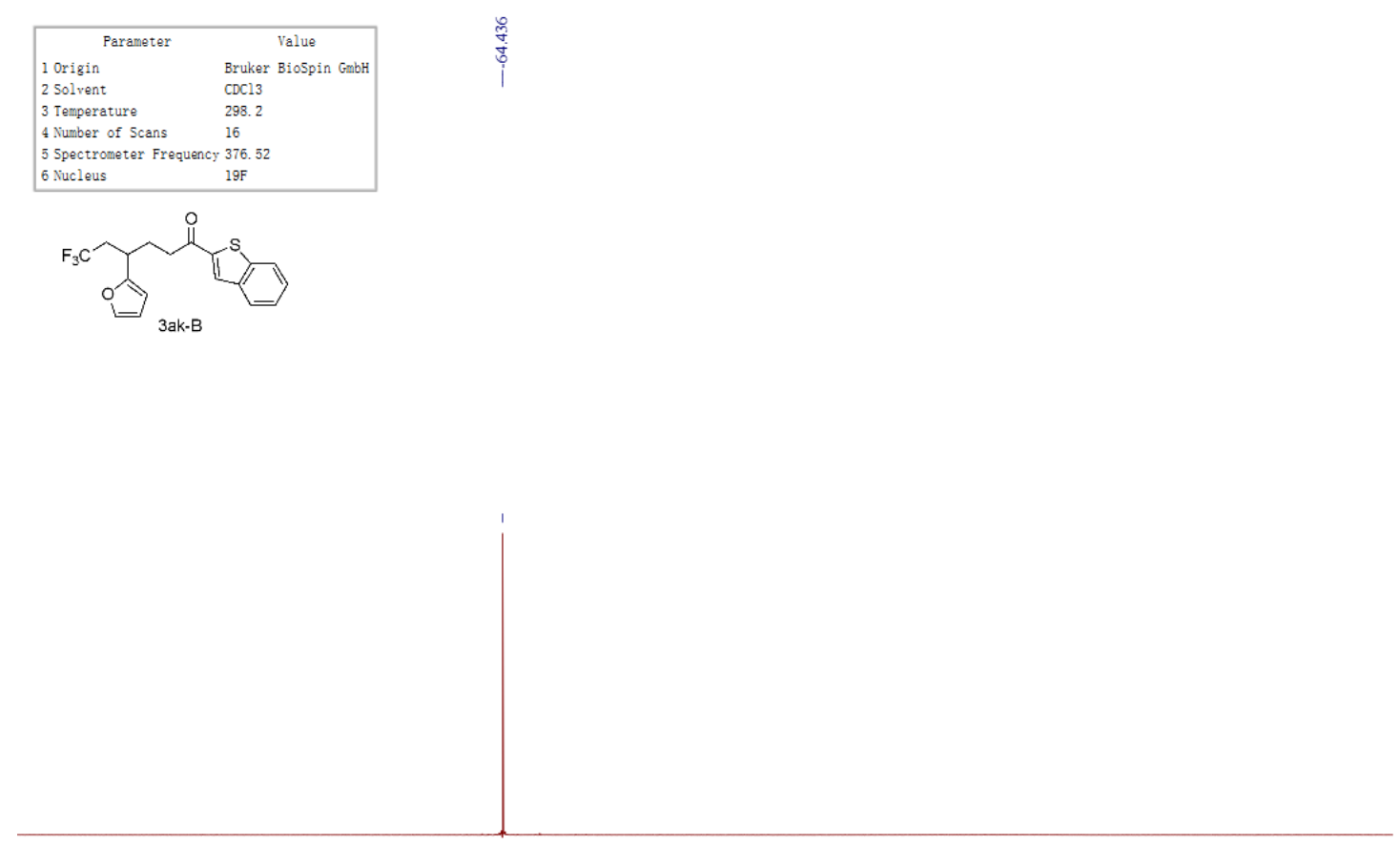

\begin{tabular}{llllllllllllllllllllllllllllllll}
\hline 0 & 10 & 0 & -10 & -20 & -30 & -40 & -50 & -60 & -70 & -80 & -90 & -100 & -110 & -120 & -130 & -140 & -150 & -160 & -170 & -180 & -190 & -200 & -210 & -2 \\
\hline
\end{tabular} 


\section{HMBC}

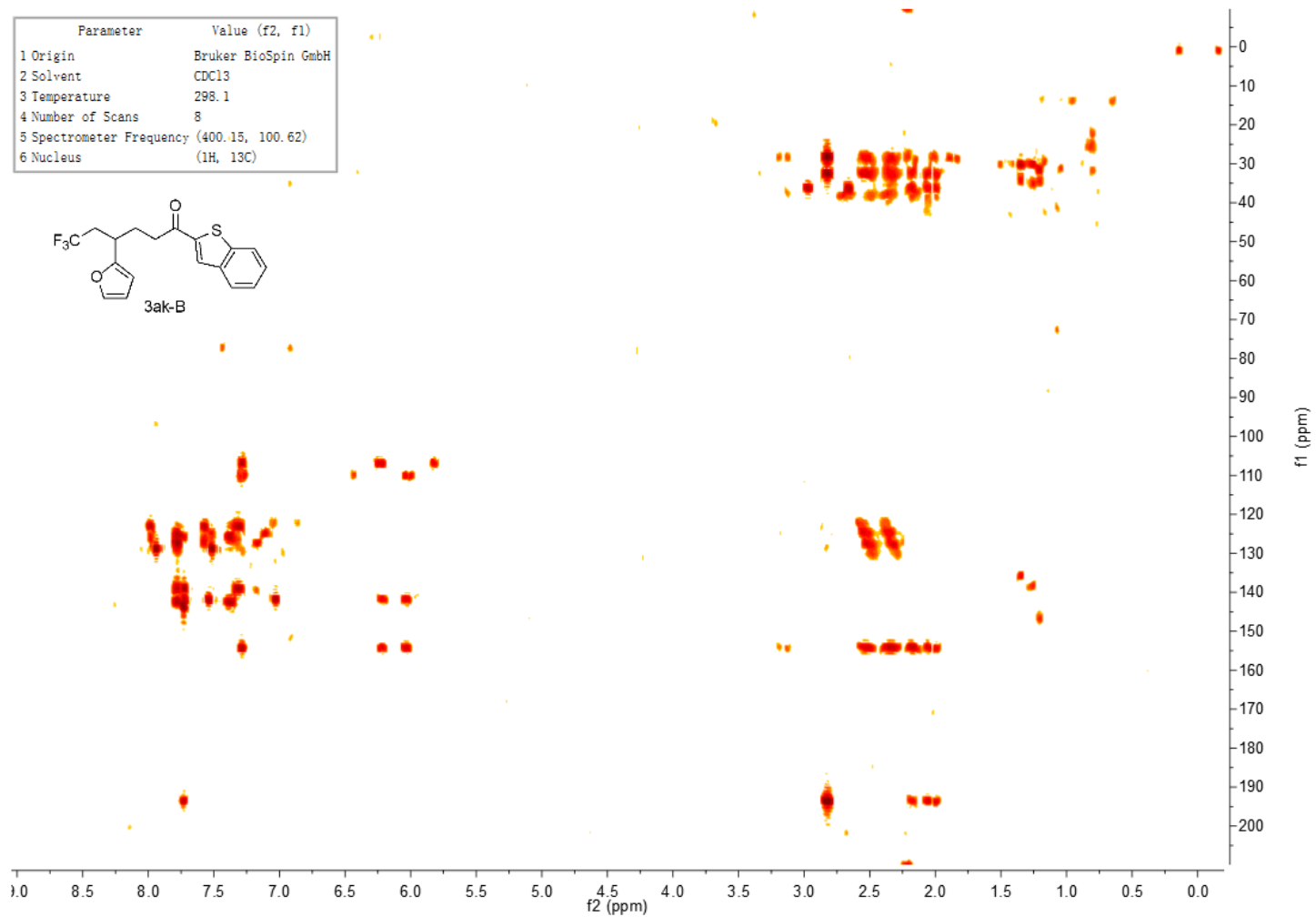

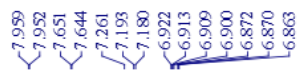

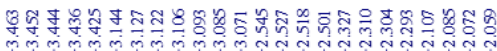
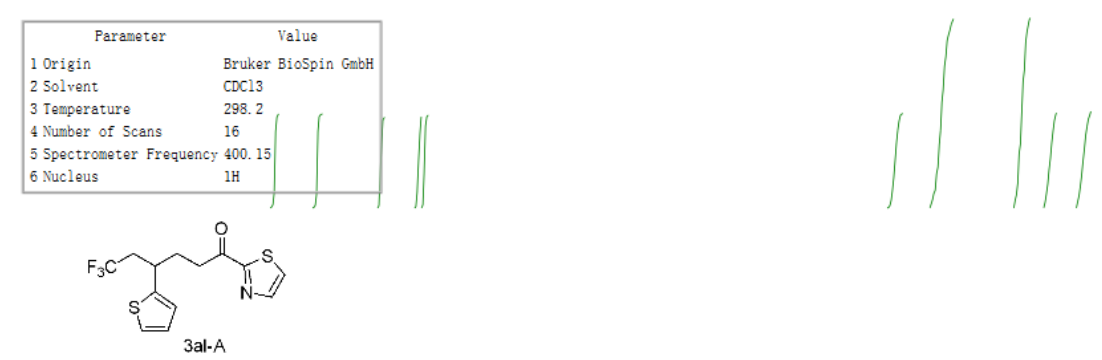

3al-A

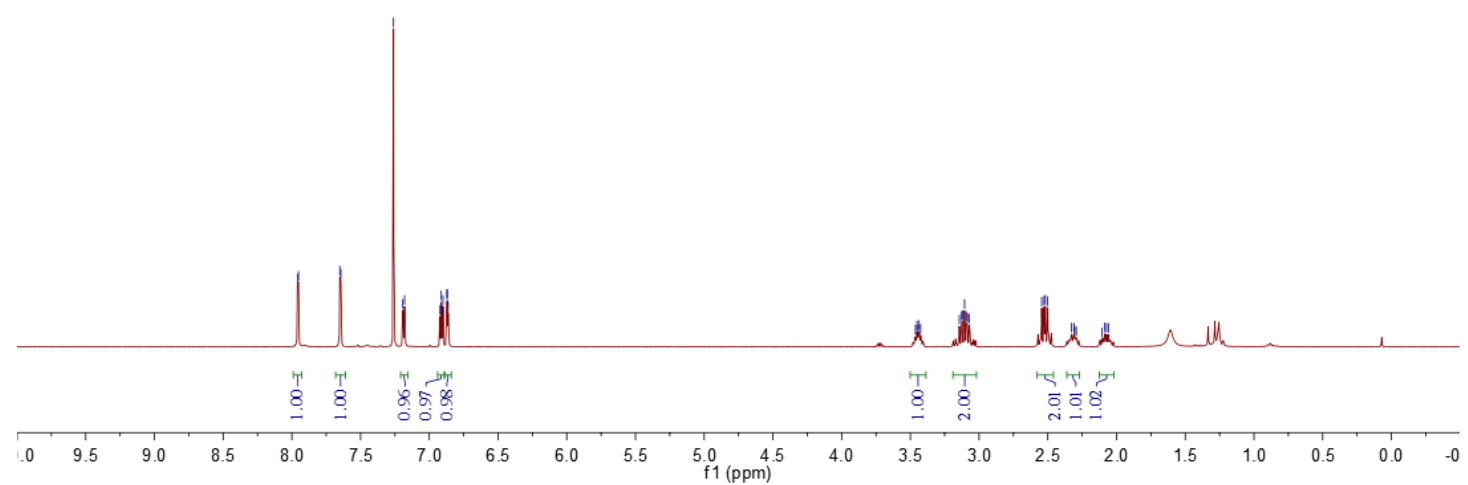




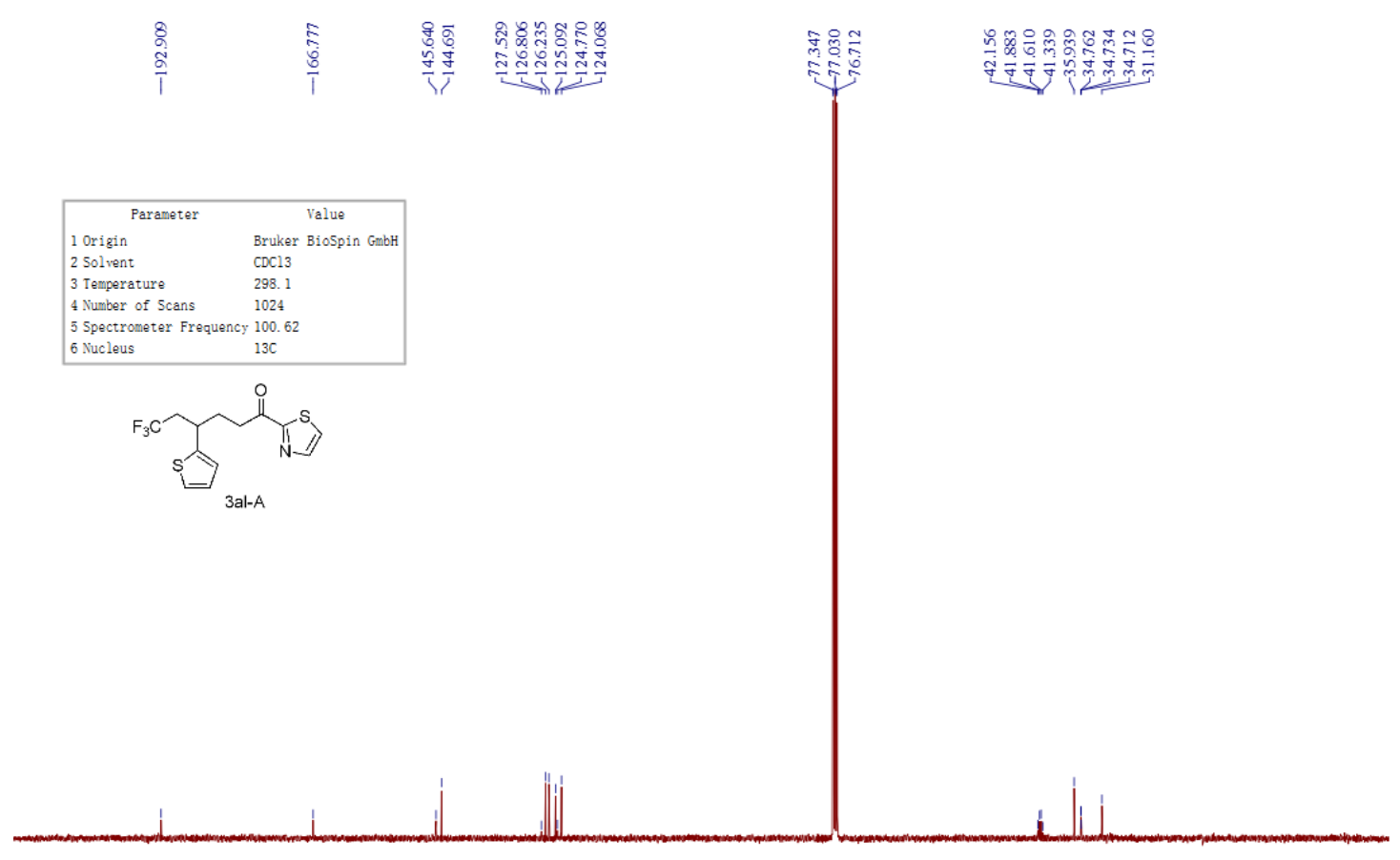

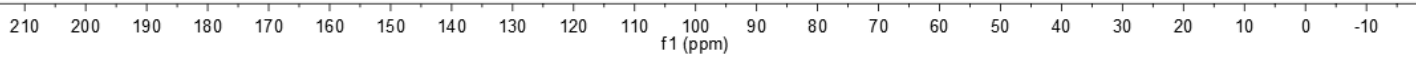
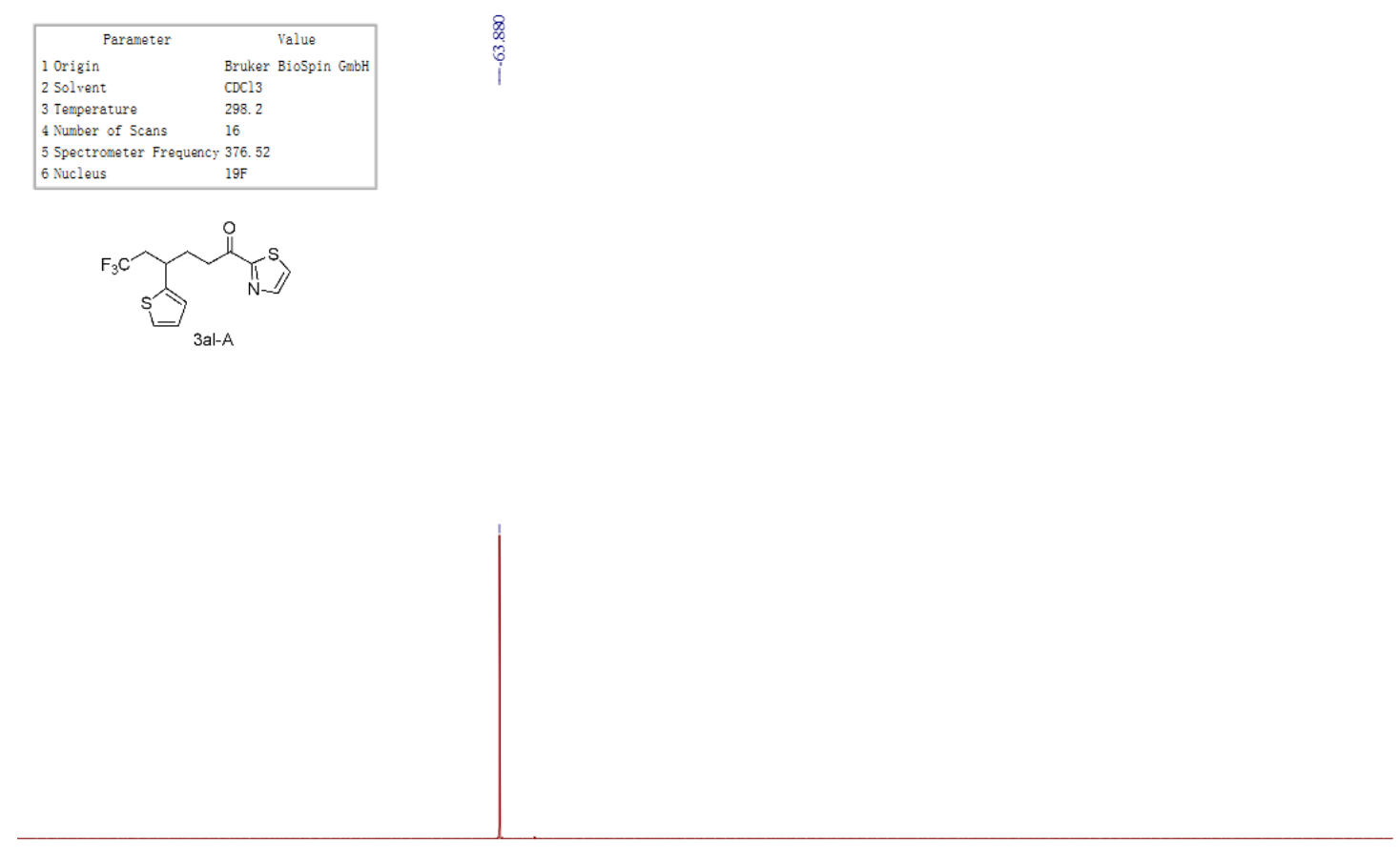

\begin{tabular}{llllllllllllllllllllllllllllll}
\hline 0 & 10 & 0 & -10 & -20 & -30 & -40 & -50 & -60 & -70 & -80 & -90 & -100 & -110 & -120 & -130 & -140 & -150 & -160 & -170 & -180 & -190 & -200 & -210 & $-2 c^{\prime}$
\end{tabular} 


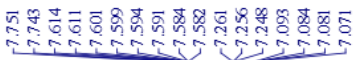

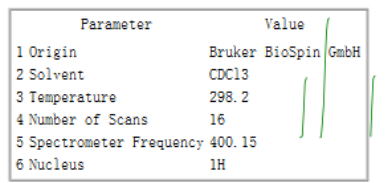

$$
\underbrace{S_{1}^{N}}_{3 a l-B}
$$

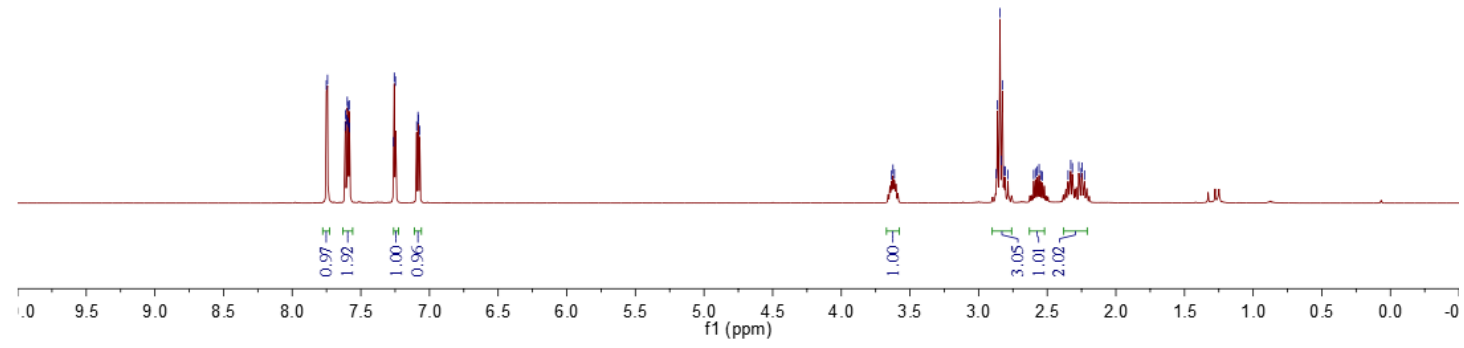

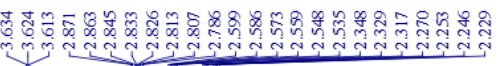

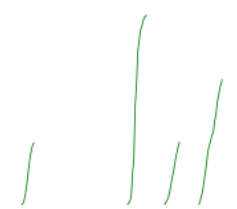

5 Spectrometer Frequency 400 .

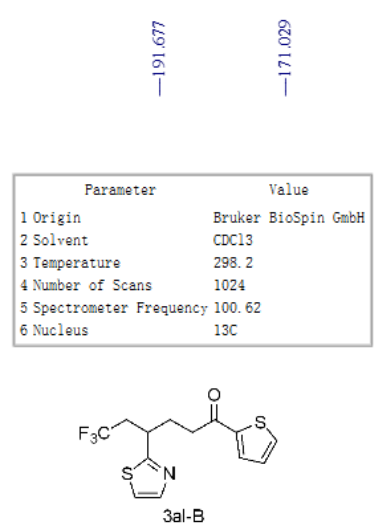

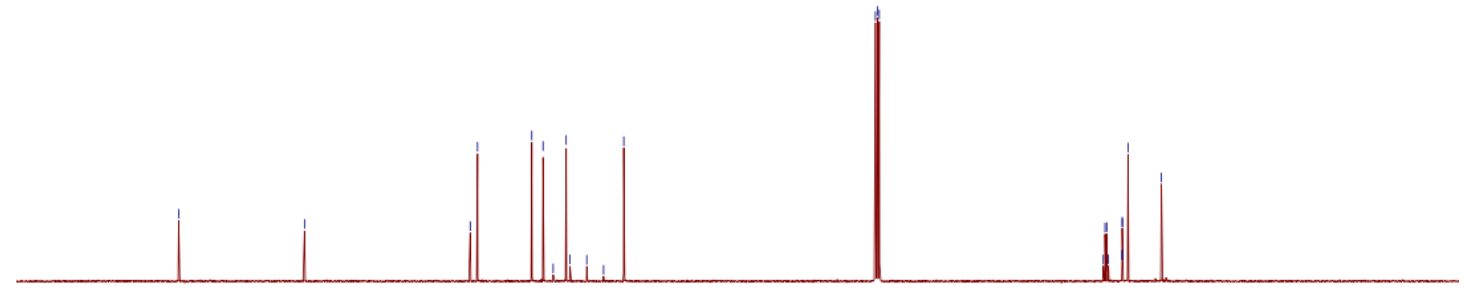

$\begin{array}{lllllllllllllllllllllll}210 & 200 & 190 & 180 & 170 & 160 & 150 & 140 & 130 & 120 & 110 & 100 & 90 & 80 & 70 & 60 & 50 & 40 & 30 & 20 & 10 & 0 & -10\end{array}$ 


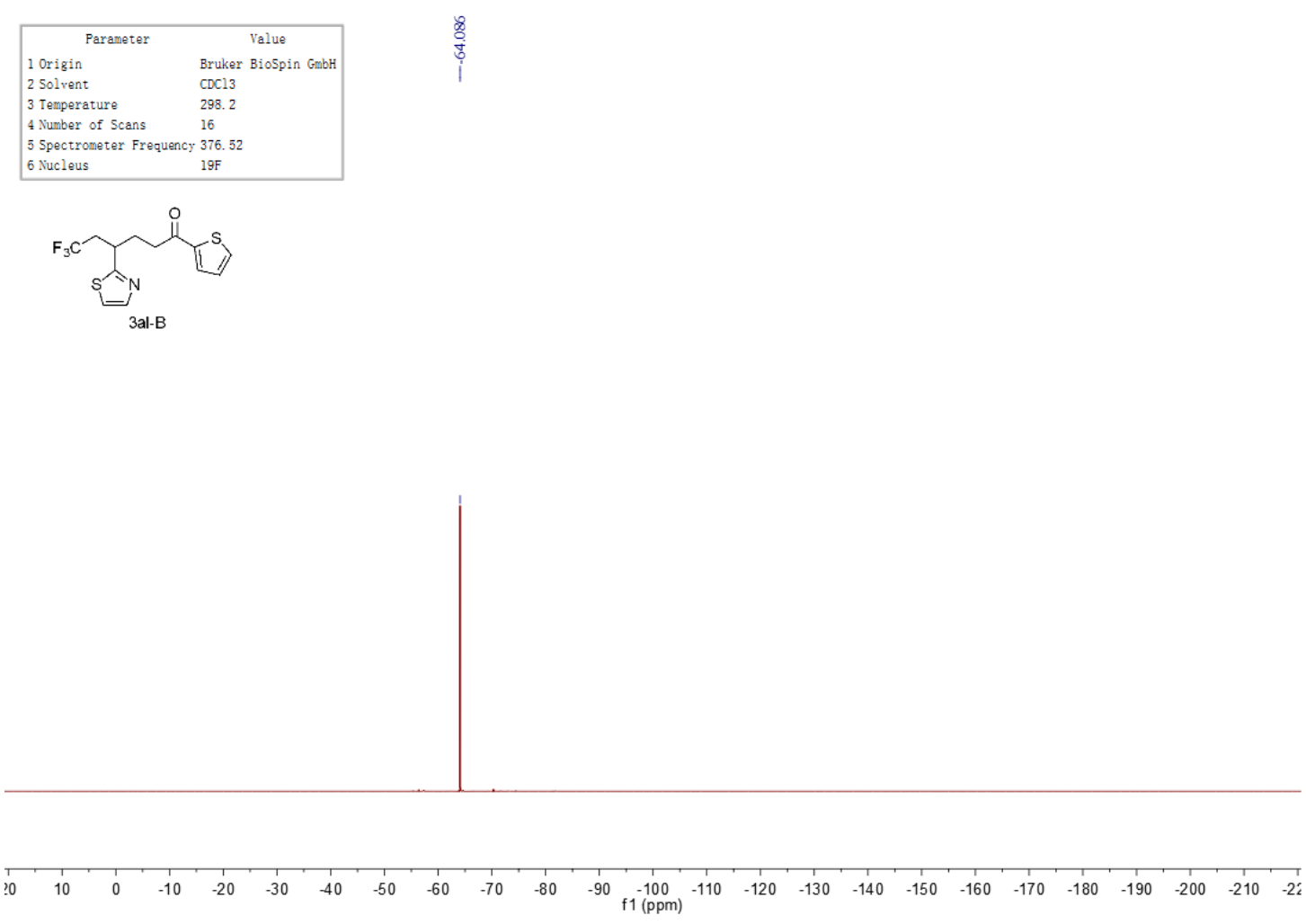

\section{HMBC}

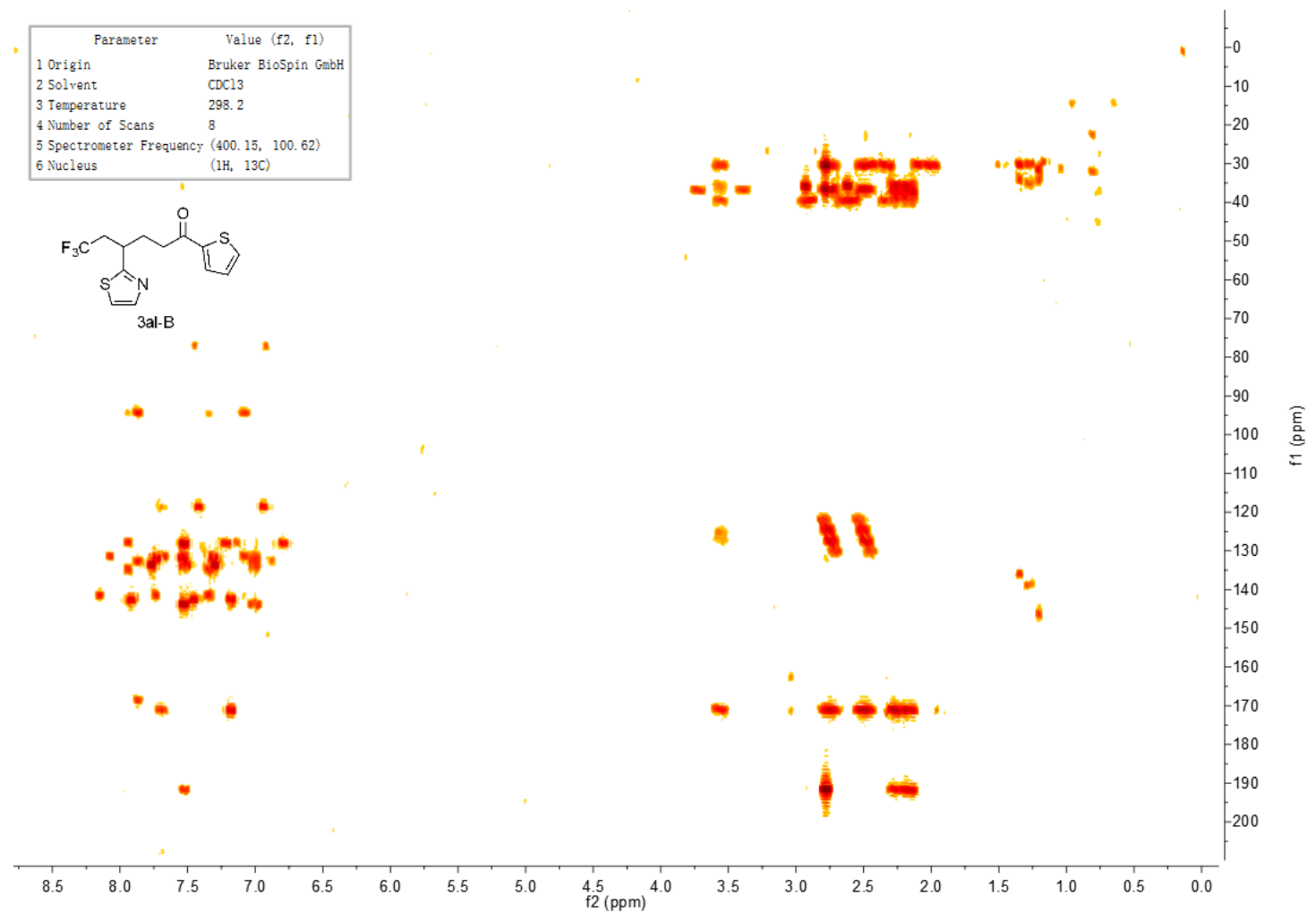




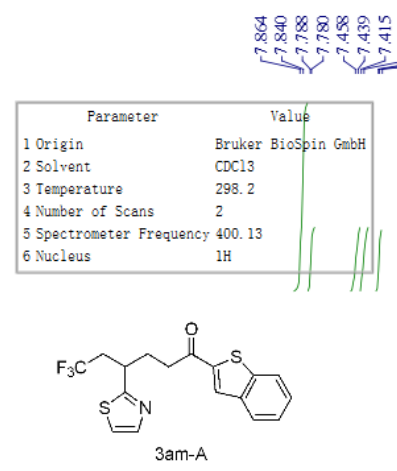

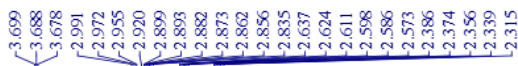
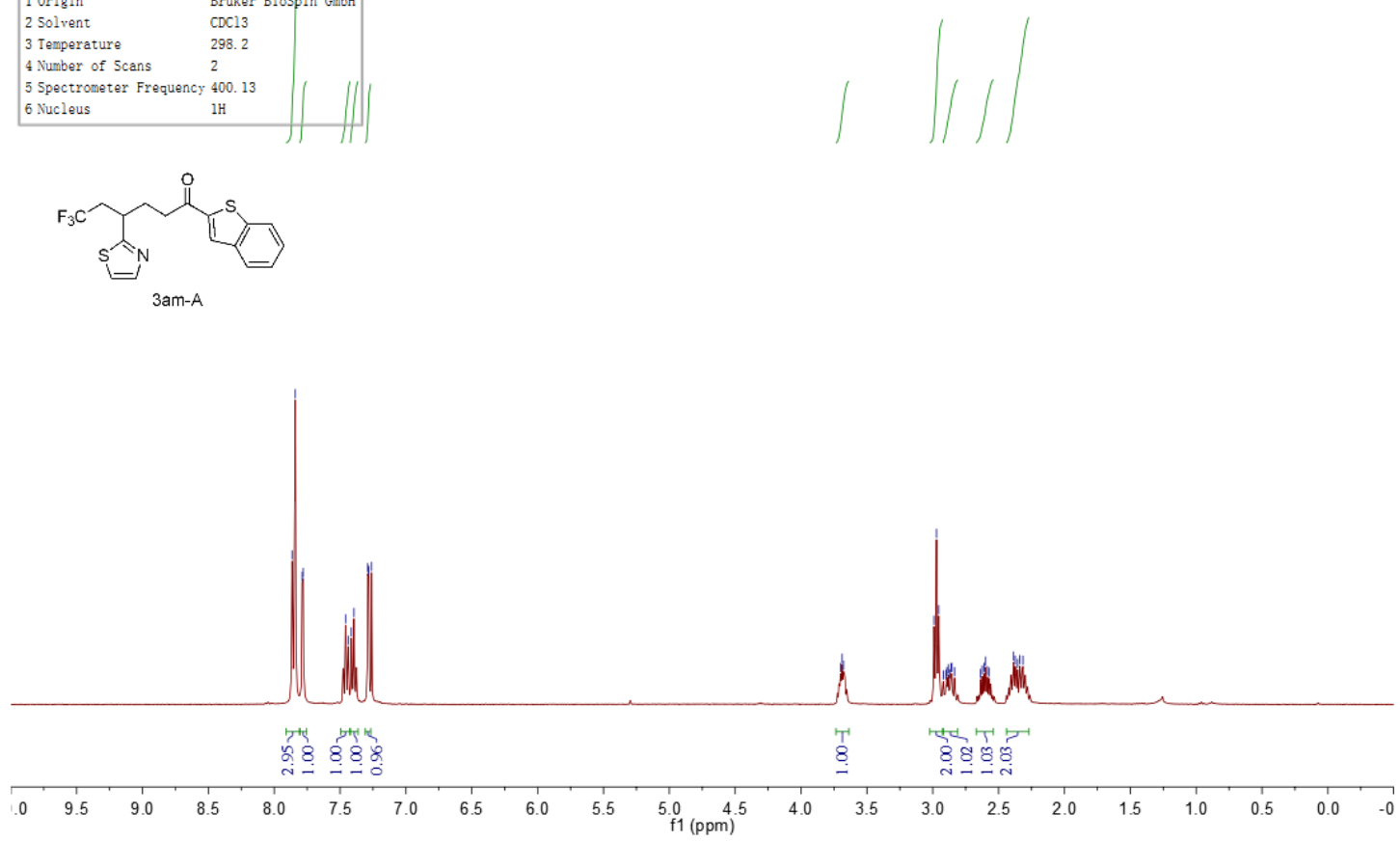

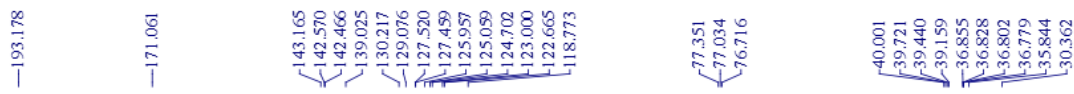

\begin{tabular}{|ll|}
\hline \multicolumn{1}{|c|}{ Parameter } & \multicolumn{1}{c|}{ Value } \\
1 Origin & Bruker BioSpin GmbH \\
2 Solvent & CDC13 \\
3 Temperature & 298.2 \\
4 N Number of Scans & 368 \\
5 Spectrometer Frequency & 100.61 \\
6 Nucleus & $13 \mathrm{C}$ \\
\hline
\end{tabular}

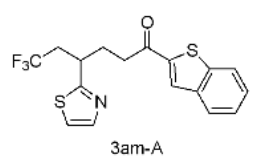

3am-A

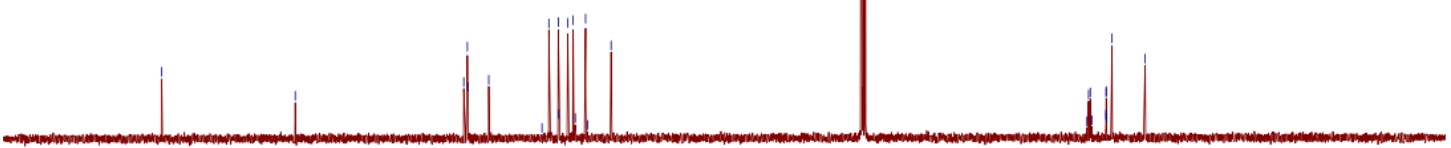

$\begin{array}{llllllllllllllllllllllllllllll}1 & 1 & 200 & 190 & 180 & 170 & 160 & 150 & 140 & 130 & 120 & 110 & 100 & 90 & 80 & 70 & 60 & 50 & 40 & 30 & 20 & 10 & 0 & -10\end{array}$ 

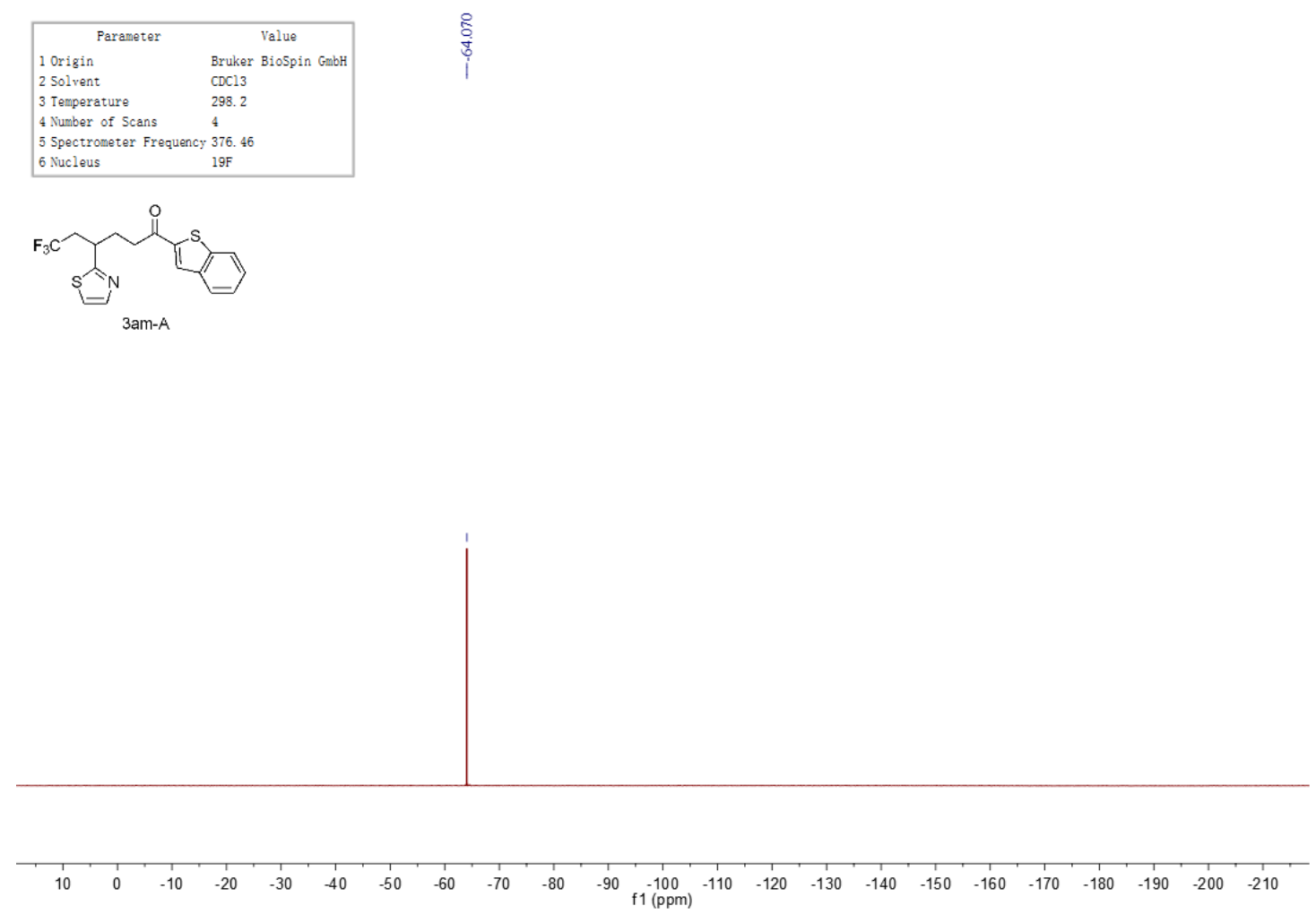

\section{HMBC}

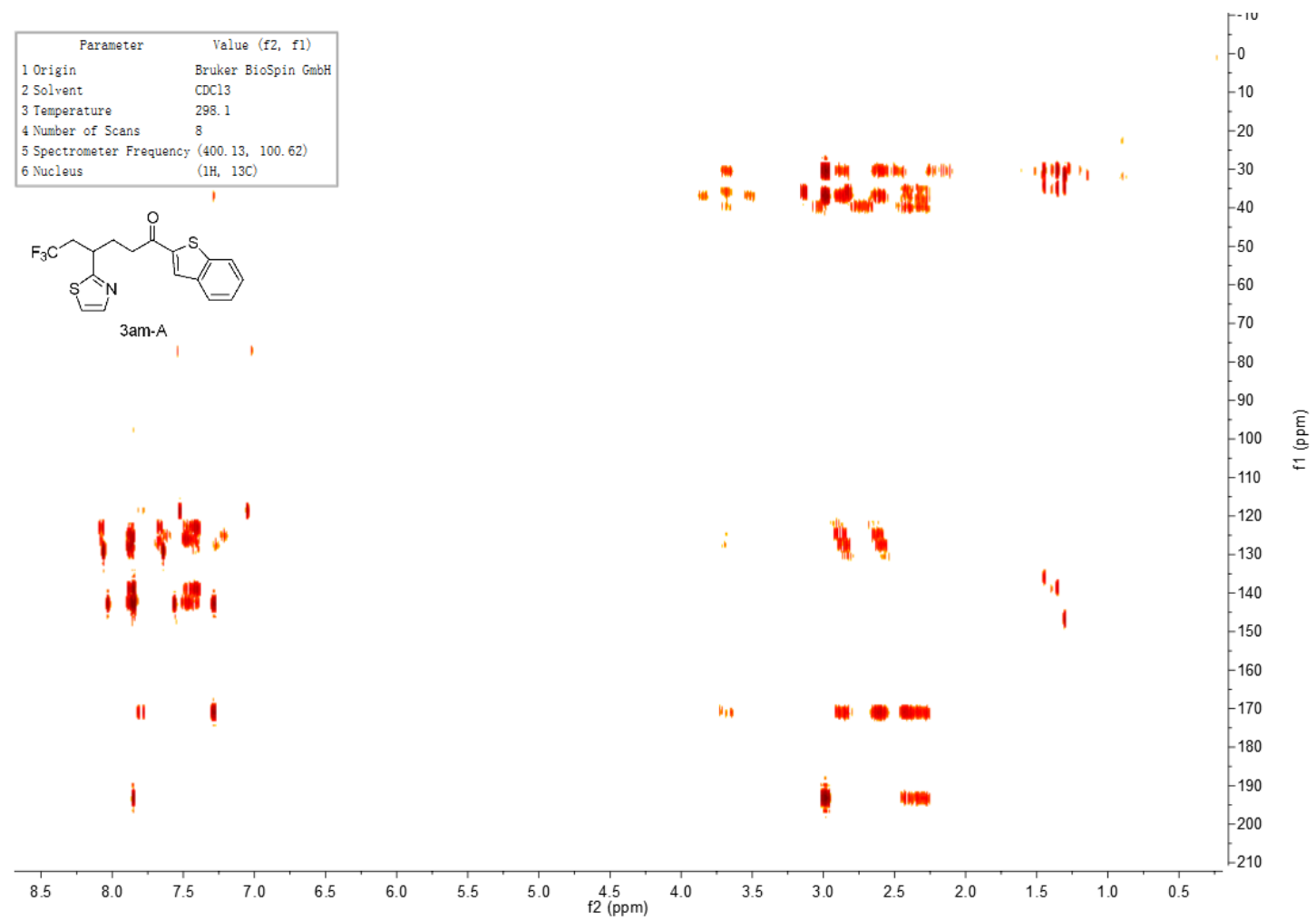


参

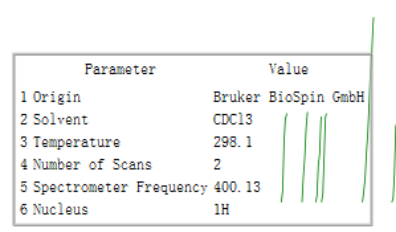

5 Spectrometer Frequency 400.

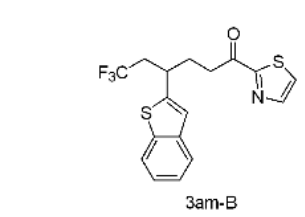

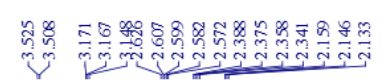

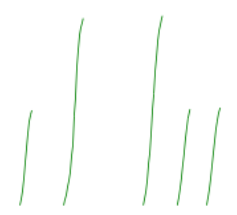

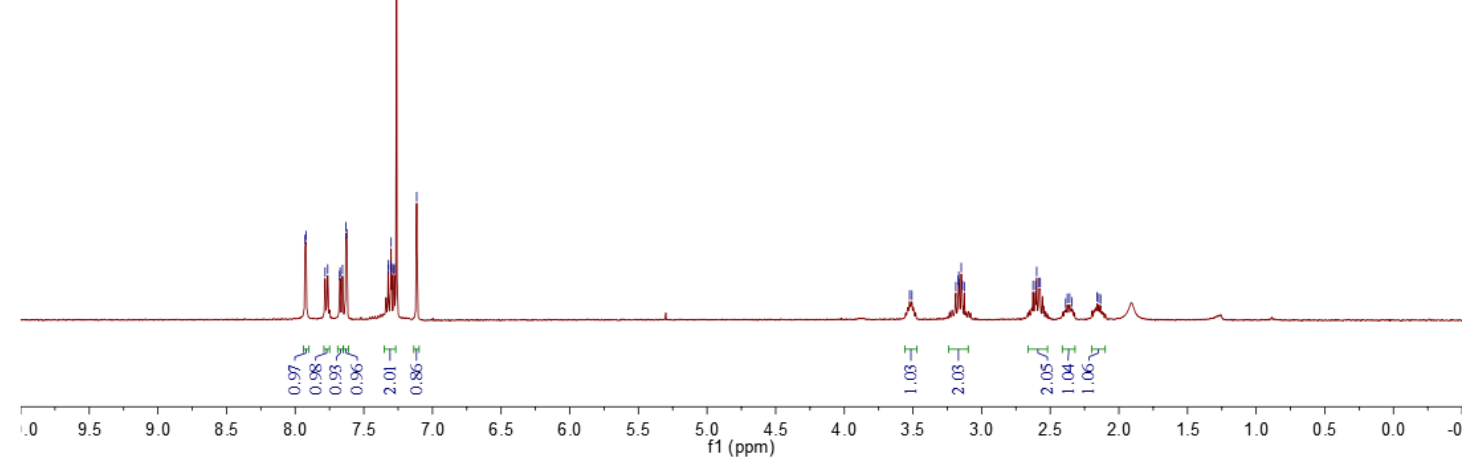
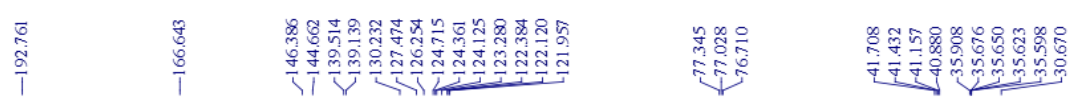

\begin{tabular}{|ll|}
\hline \multicolumn{1}{|c|}{ Parameter } & \multicolumn{1}{c|}{ Value } \\
1 Origin & Bruker BioSpin GmbH \\
2 Solvent & CDC13 \\
3 Temperature & 298.1 \\
4 Number of Scans & 1024 \\
5 Spectrometer Frequency 100.62 \\
6 Nucleus & $13 \mathrm{C}$ \\
\hline
\end{tabular}

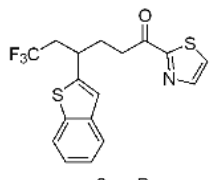

3am-B
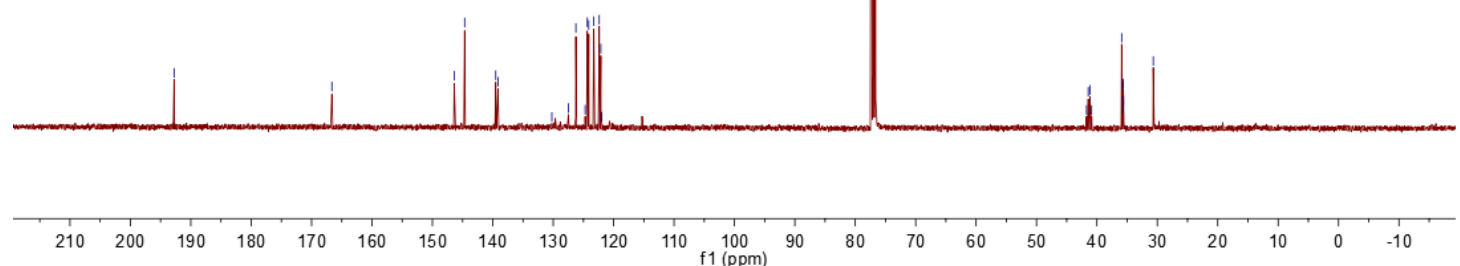

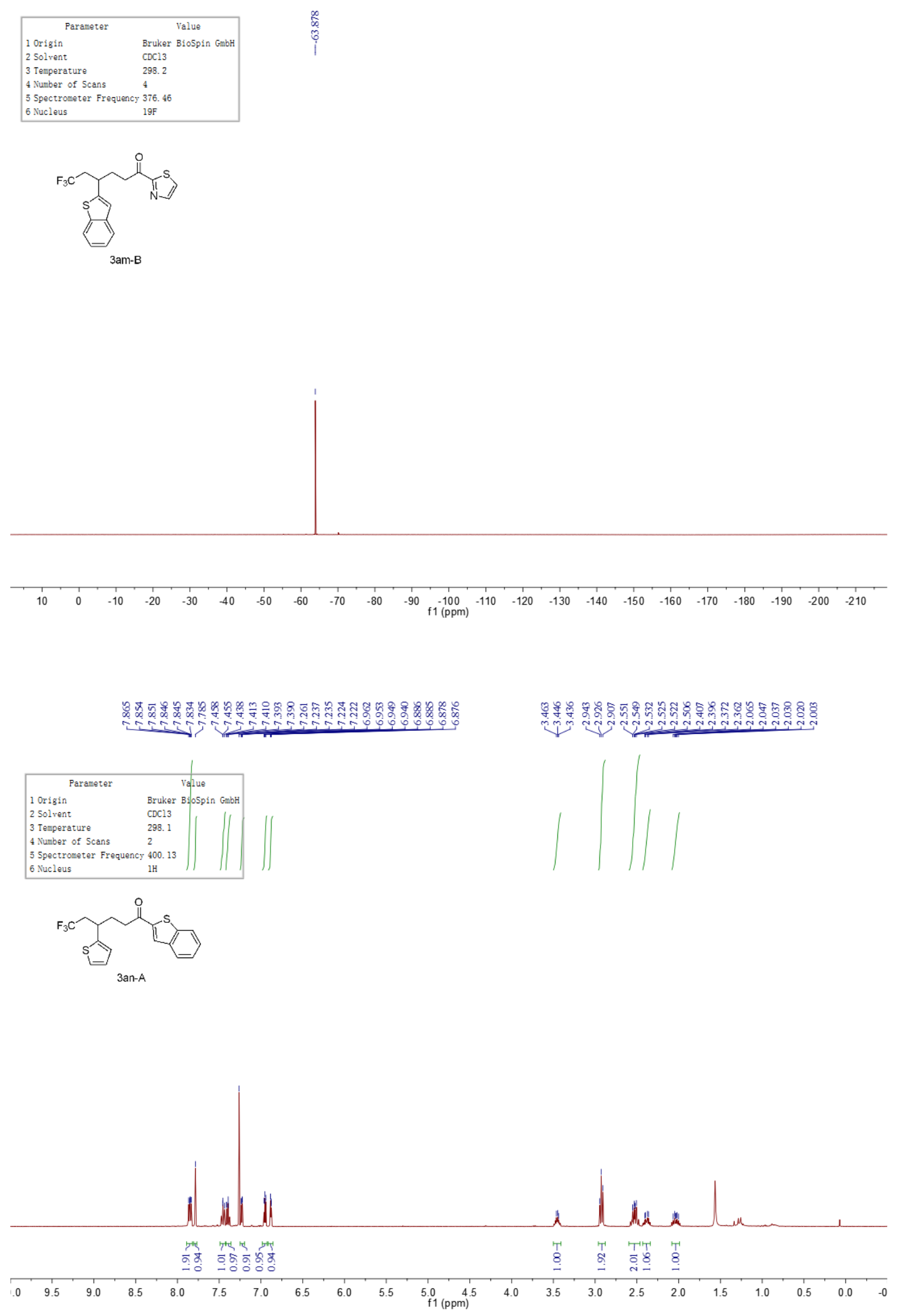


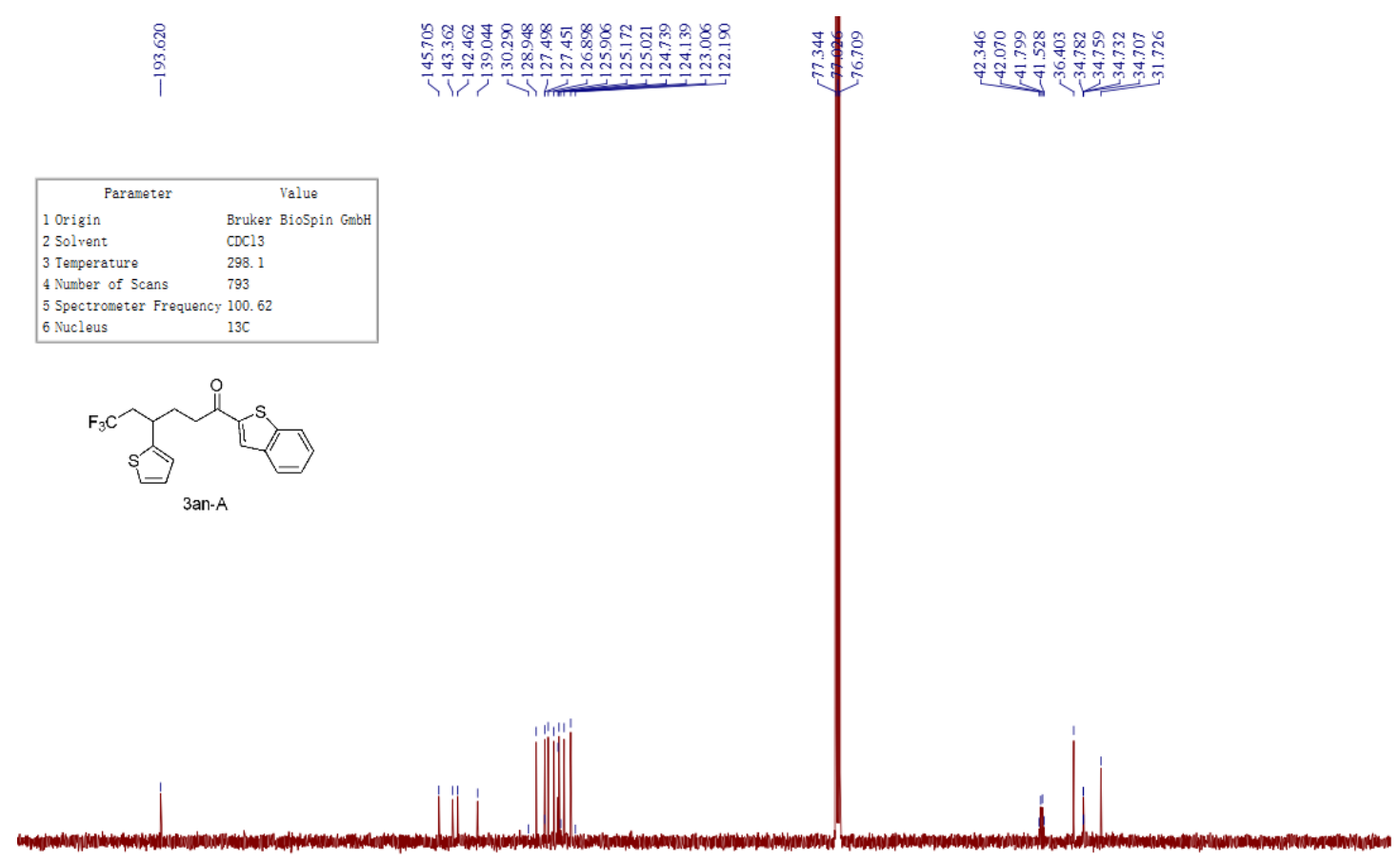

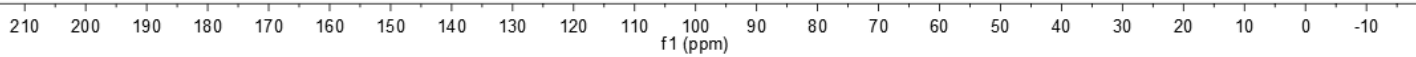

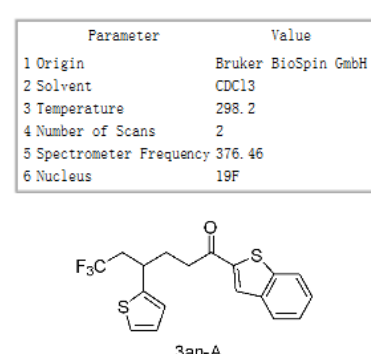

3an-A

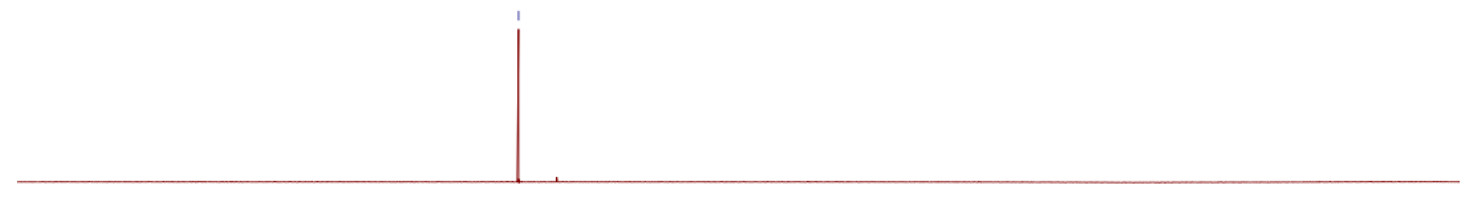

$\begin{array}{llllllllllllllllllllllllllllll}10 & 0 & -10 & -20 & -30 & -40 & -50 & -60 & -70 & -80 & -90 & -100 & -110 & -120 & -130 & -140 & -150 & -160 & -170 & -180 & -190 & -200 & -210\end{array}$ 


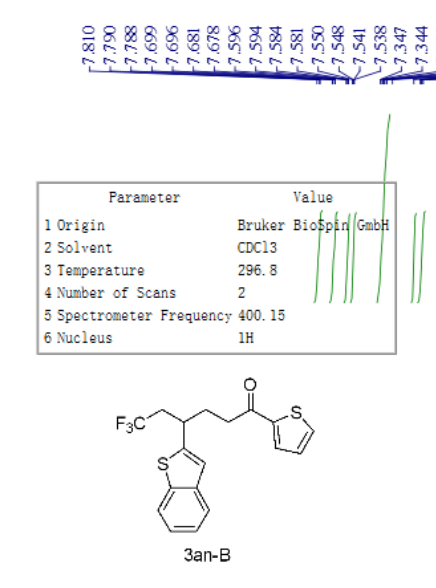

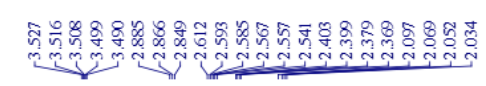

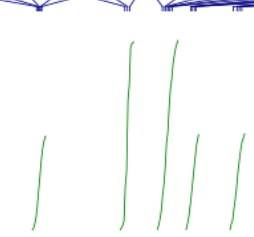

3 Temperat
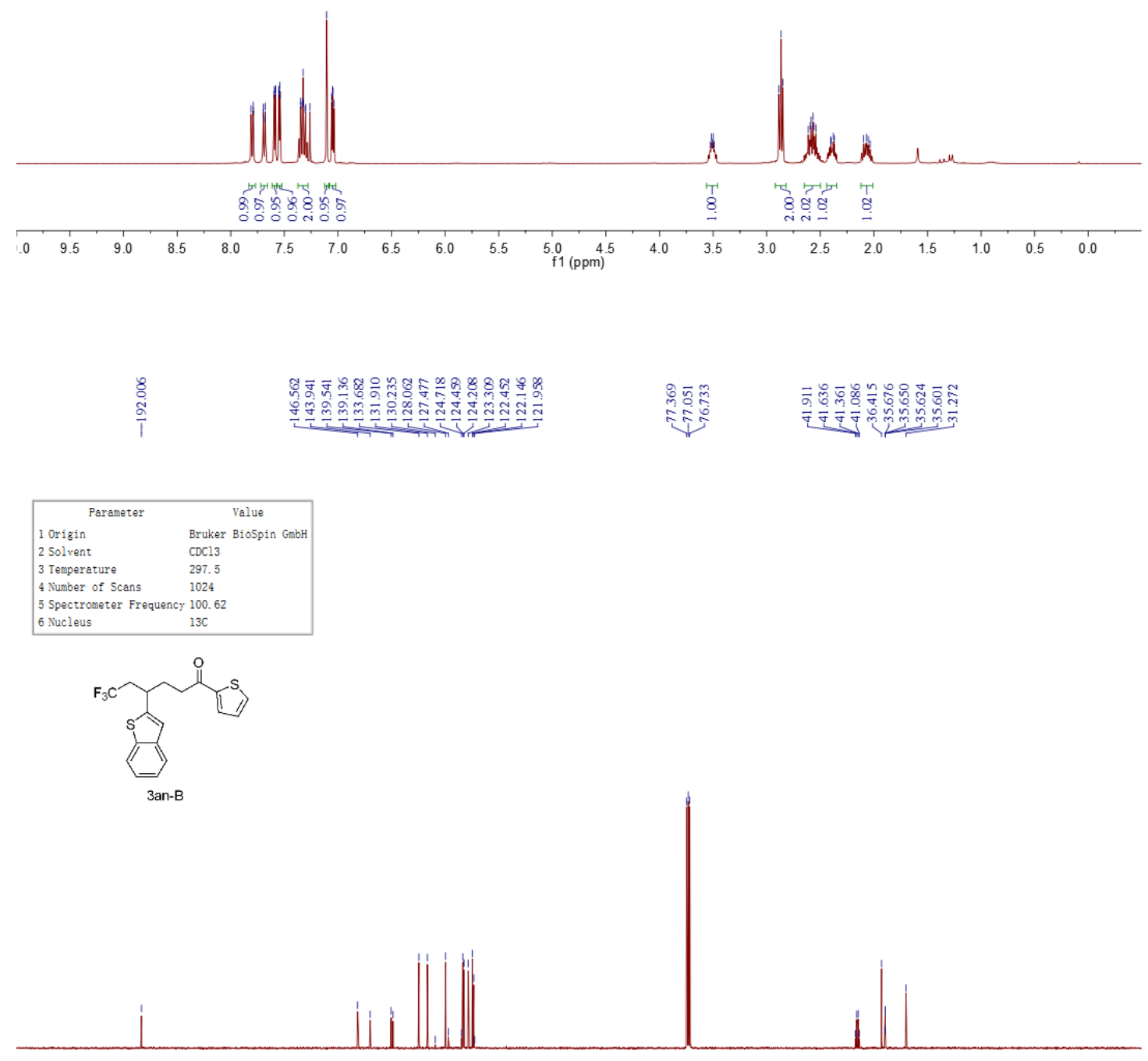

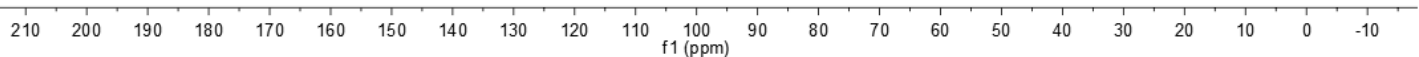




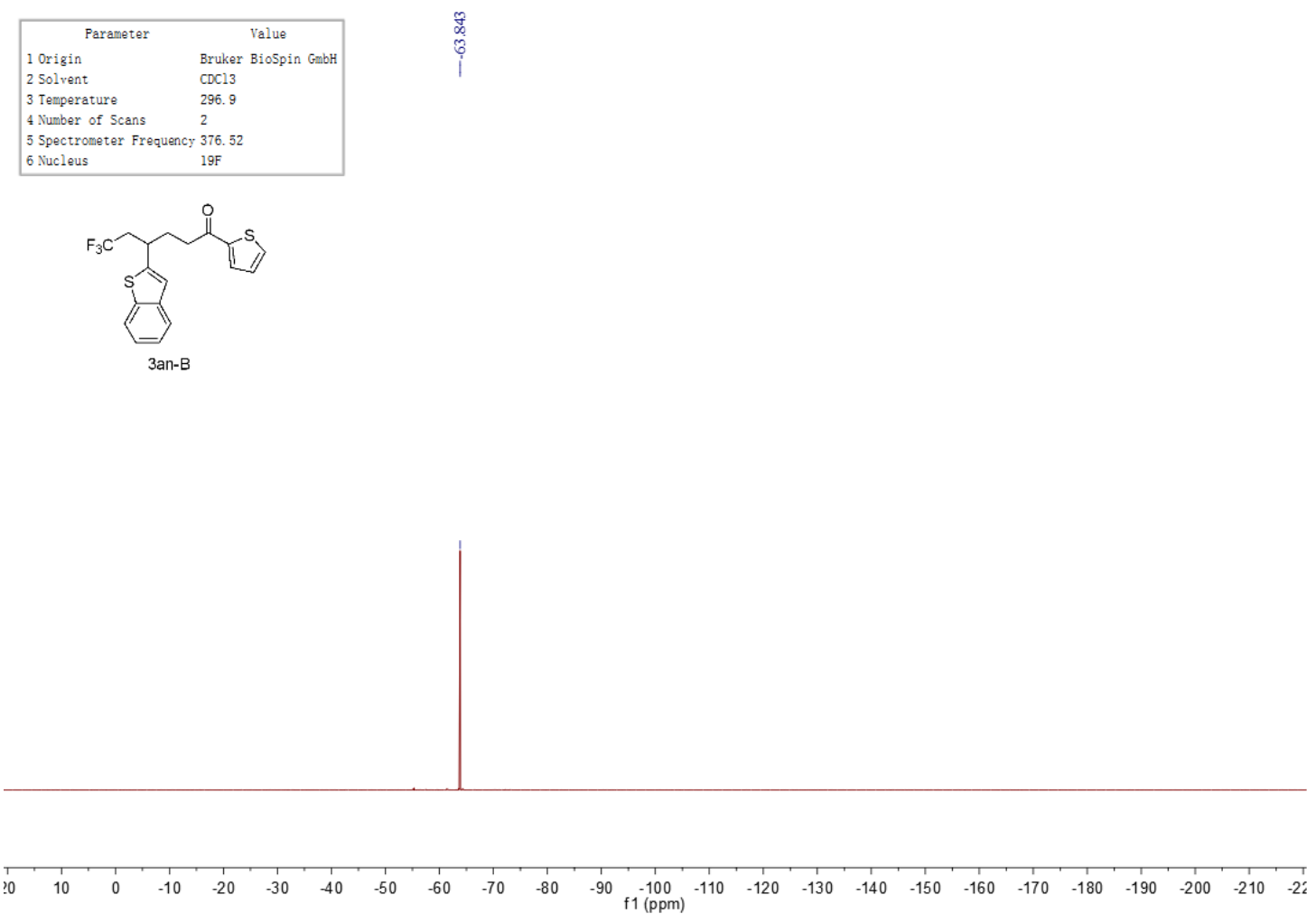

HMBC

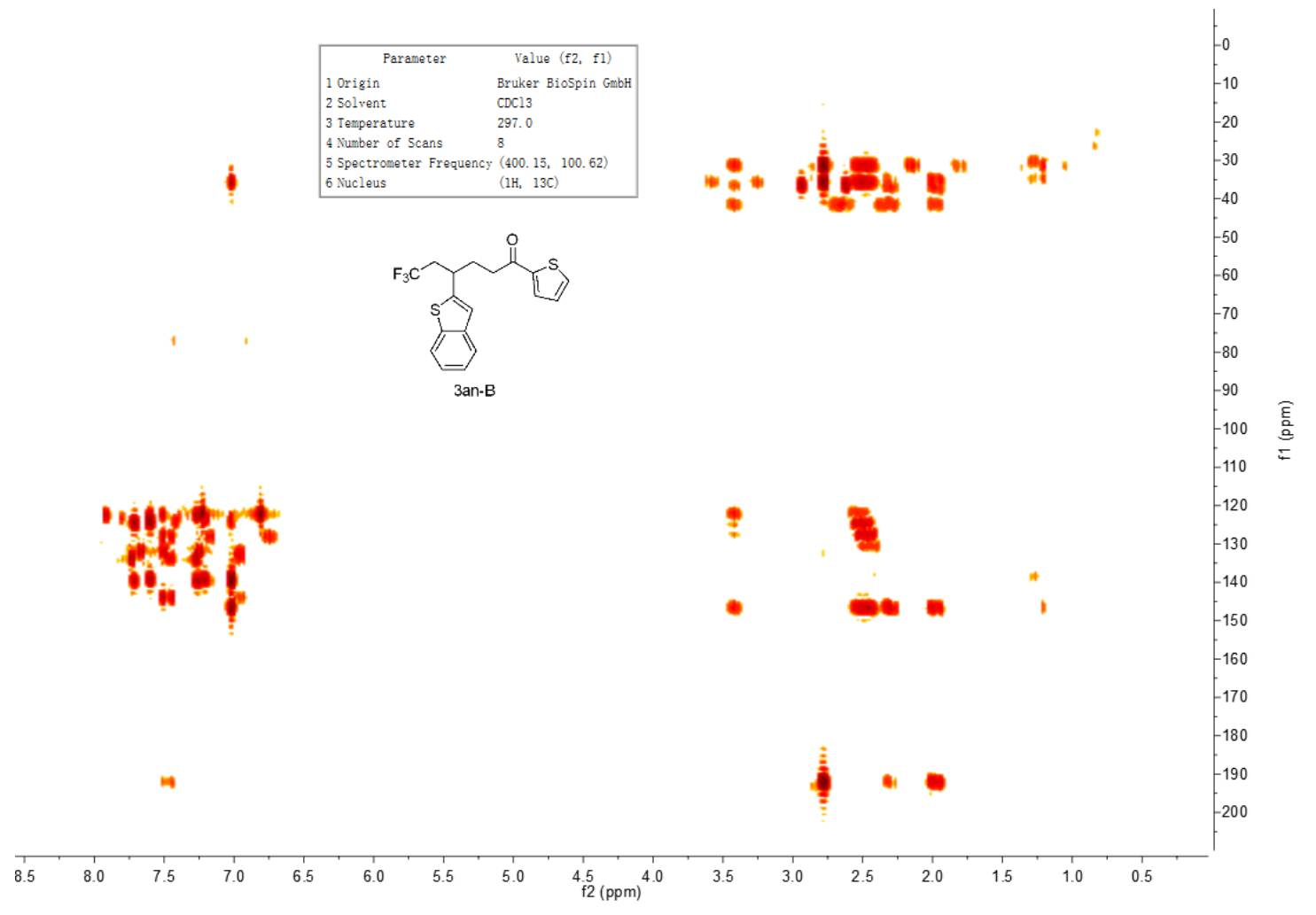



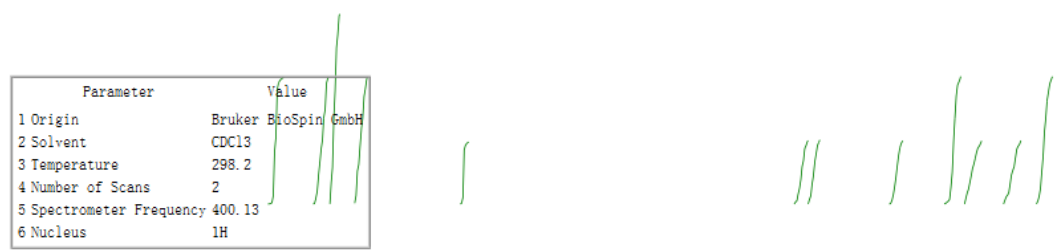

6 Nucleus 1H
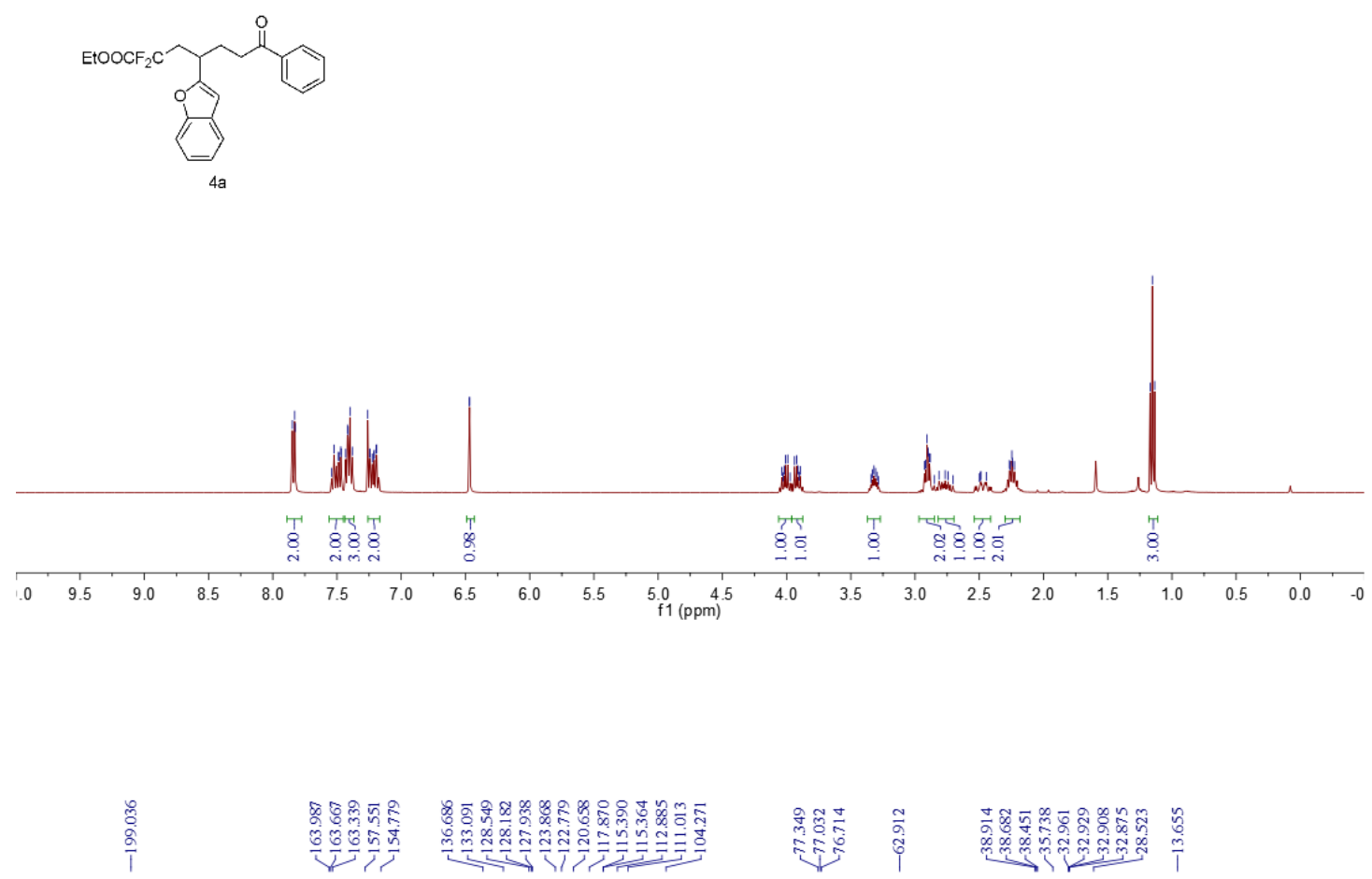

\begin{tabular}{|ll|}
\hline \multicolumn{1}{|c|}{ Parameter } & \multicolumn{1}{c|}{ Value } \\
1 Origin & Bruker BioSpin GmbH \\
2 Solvent & CDC13 \\
3 Temperature & 298.1 \\
4 Number of Scans & 1024 \\
5 Spectrometer Frequency & 100.62 \\
6 N Nucleus & $13 \mathrm{C}$ \\
\hline
\end{tabular}

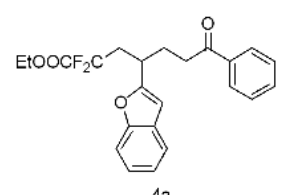

$4 a$

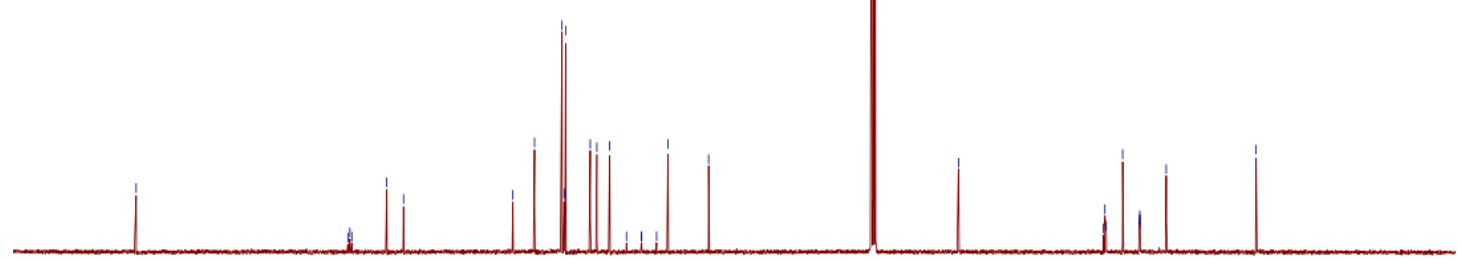

$\begin{array}{llllllllllllllllllllllllllllllll}210 & 200 & 190 & 180 & 170 & 160 & 150 & 140 & 130 & 120 & 110 & 100 & 90 & 80 & 70 & 60 & 50 & 40 & 30 & 20 & 10 & 0 & -10\end{array}$ 

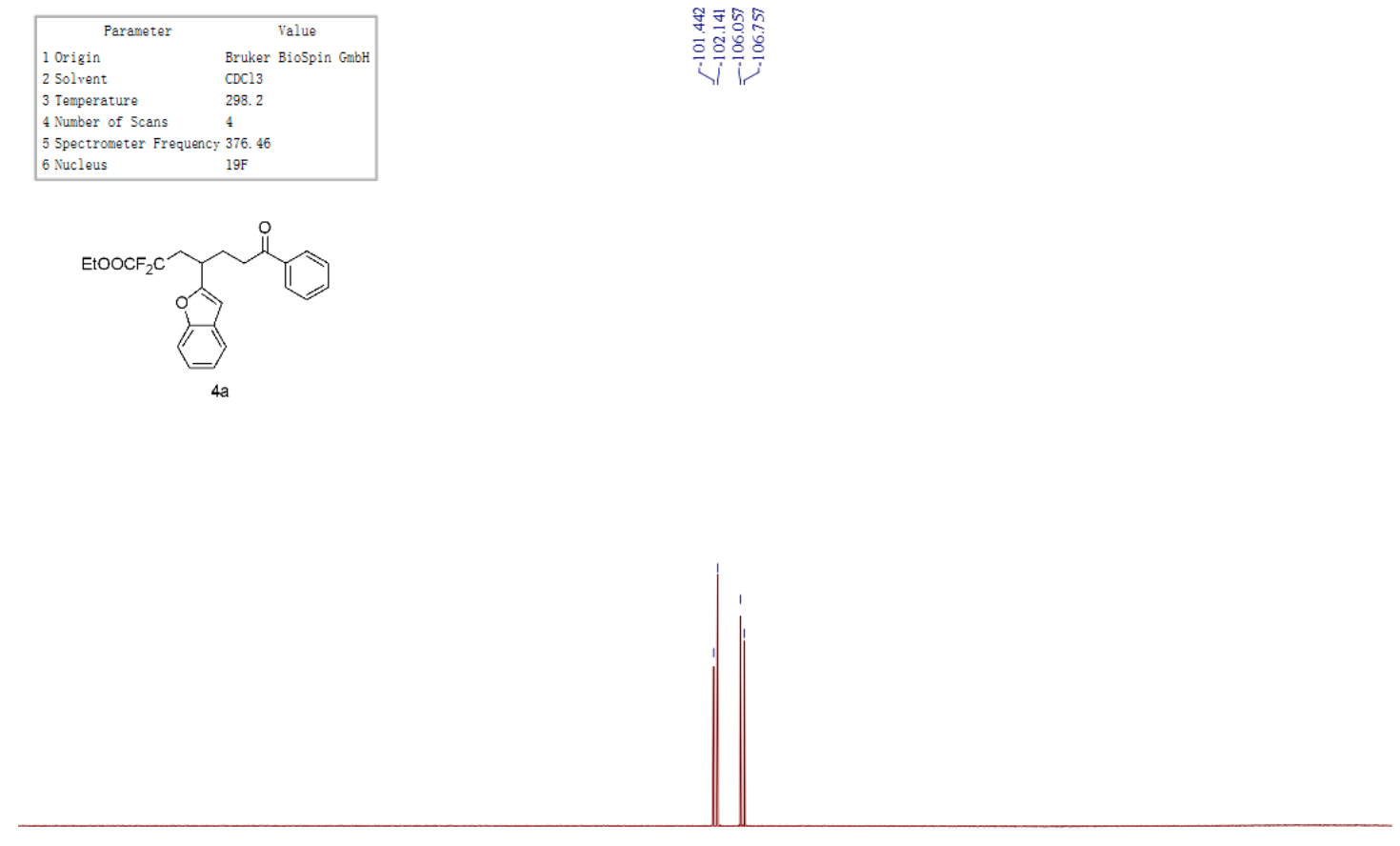

$\begin{array}{llllllllllllllllllllllllllll}10 & 0 & -10 & -20 & -30 & -40 & -50 & -60 & -70 & -80 & -90 & -100 & -110 & -120 & -130 & -140 & -150 & -160 & -170 & -180 & -190 & -200 & -210\end{array}$

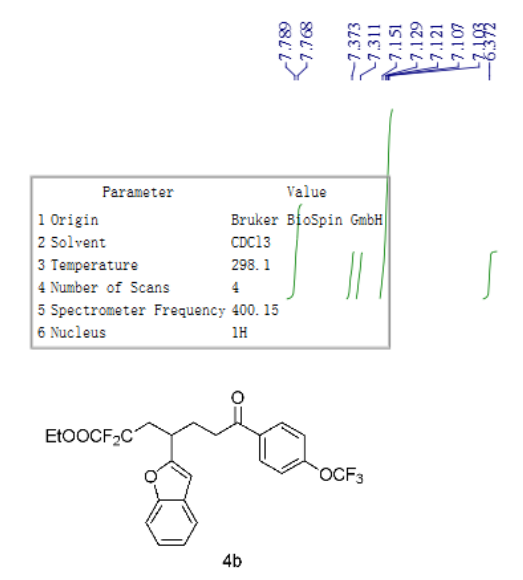

:

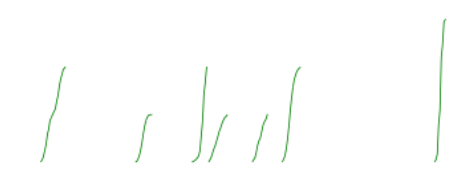

6 . Nucleus $\quad$ it

$\mathrm{EtOOCF}_{2}$

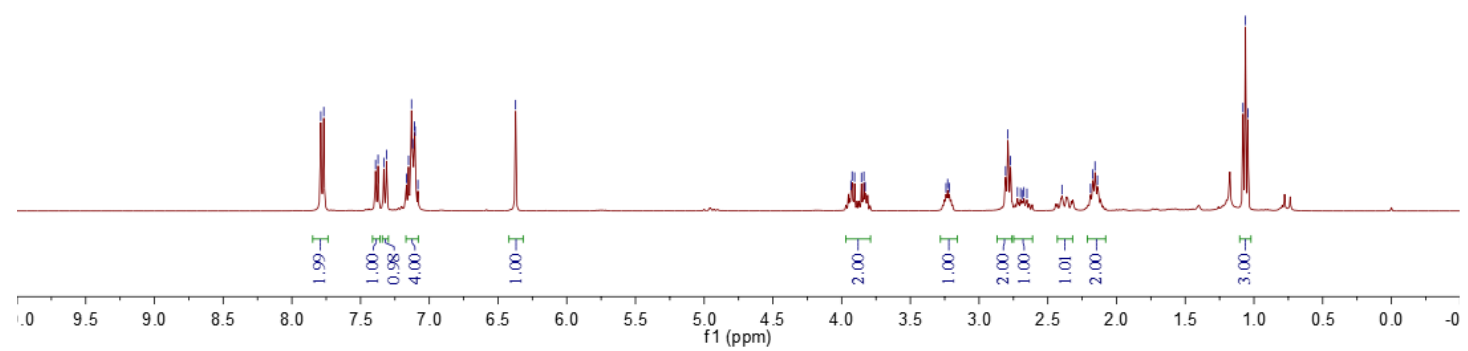




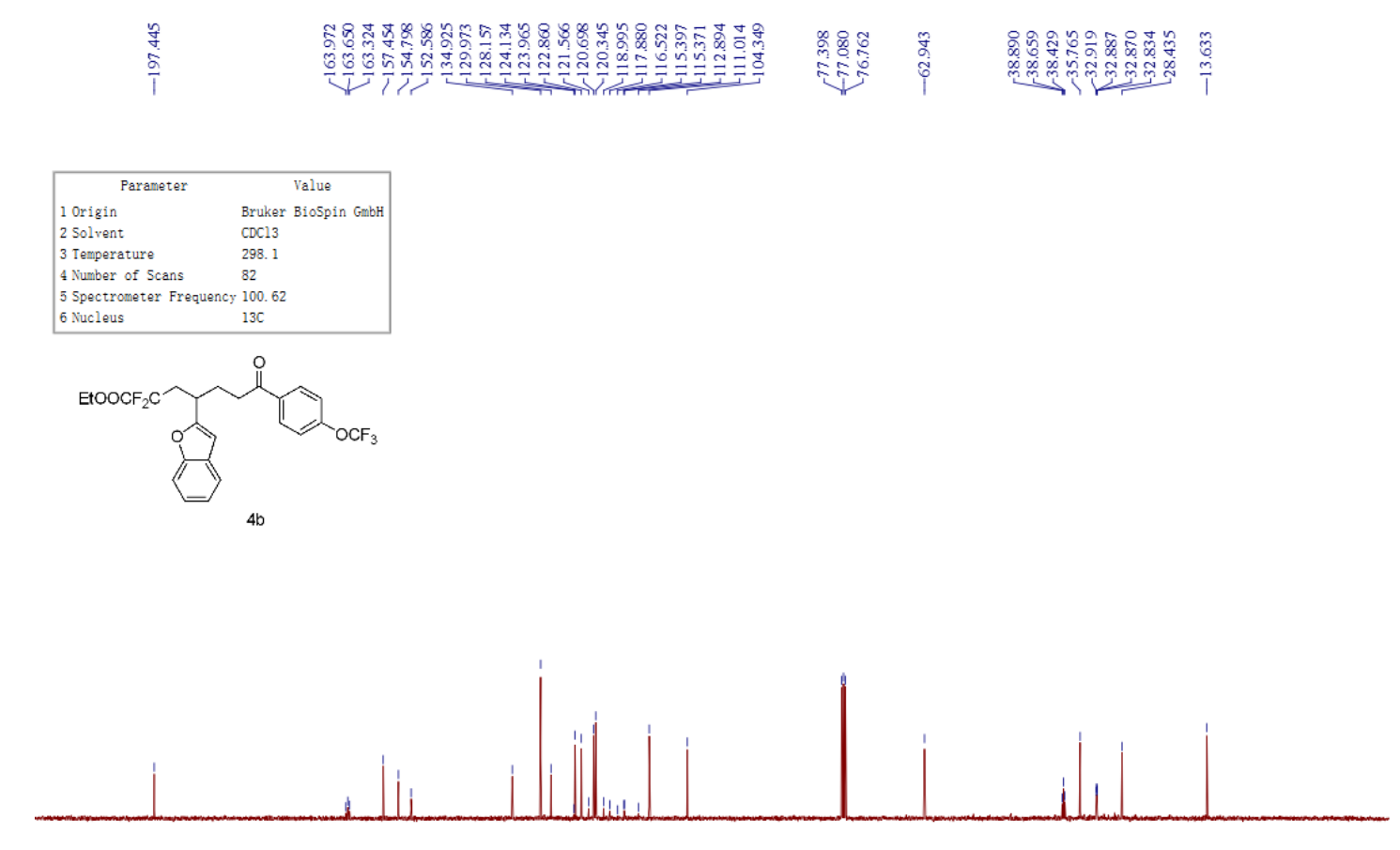

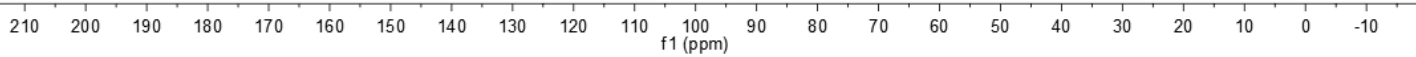

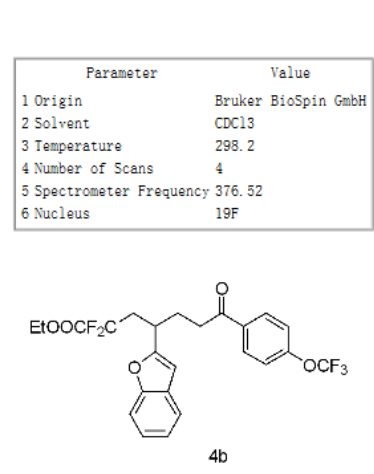

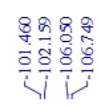

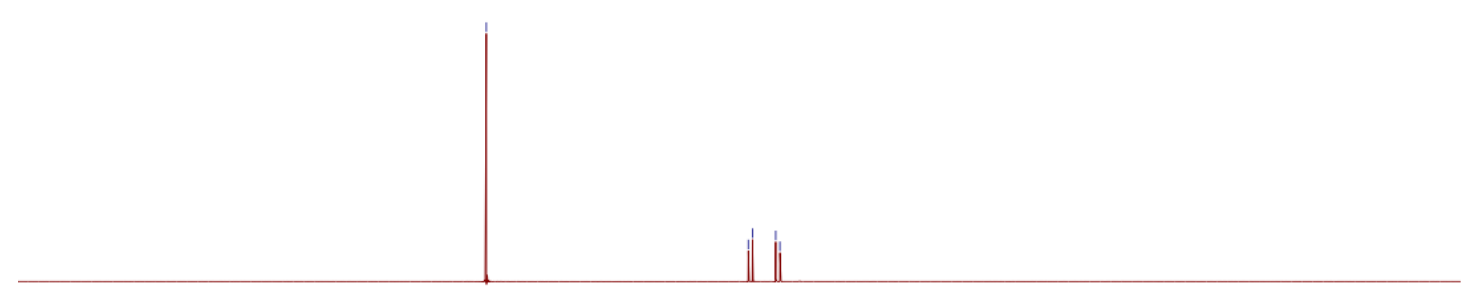

$\begin{array}{llllllllllllllllllllllllllllll}10 & 10 & 0 & -10 & -20 & -30 & -40 & -50 & -60 & -70 & -80 & -90 & -100 & -110 & -120 & -130 & -140 & -150 & -160 & -170 & -180 & -190 & -200 & -210 & -2 .\end{array}$ 


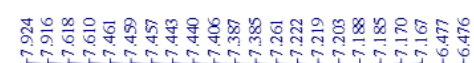

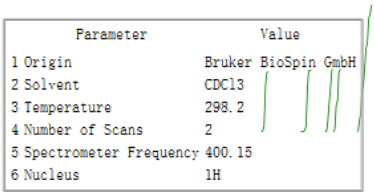

5 5pectrometer Frequency 400.15

EtOOCF 2

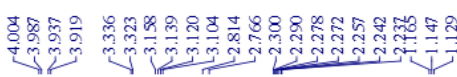

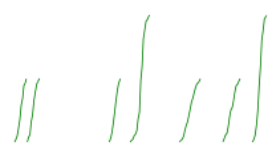

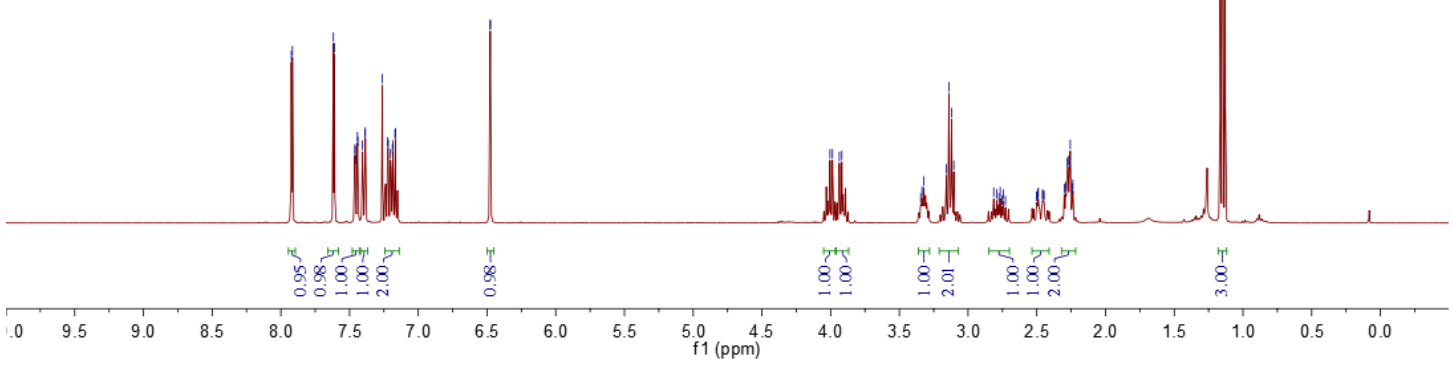

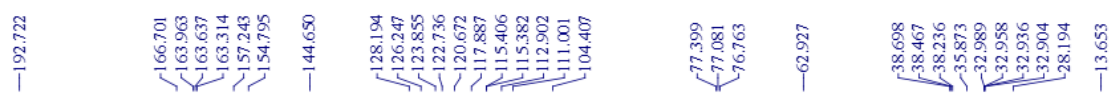

\begin{tabular}{|ll|}
\hline \multicolumn{1}{|c|}{ Parameter } & \multicolumn{1}{c|}{ Value } \\
1 Origin & Bruker BioSpin GmbH \\
2 Solvent & CDC13 \\
3 Temperature & 298.1 \\
4 Number of Scans & 1024 \\
5 Spectrometer Frequency & 100.61 \\
6 Nucleus & 13C \\
\hline
\end{tabular}

EtoocF 2

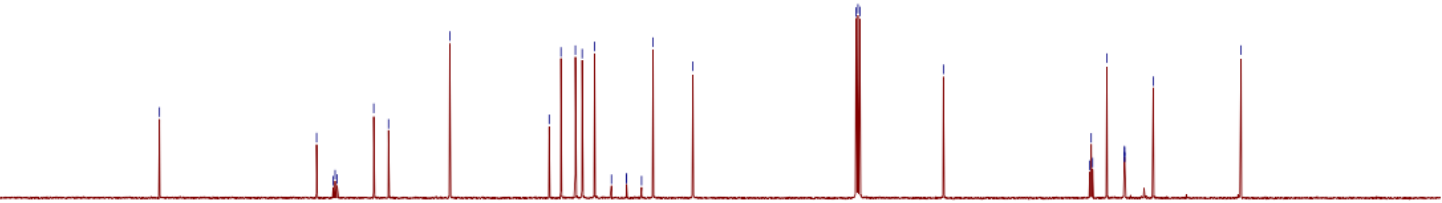

$\begin{array}{llllllllllllllllllllllllll}210 & 200 & 190 & 180 & 170 & 160 & 150 & 140 & 130 & 120 & 110 & 100 & 90 & 80 & 70 & 60 & 50 & 40 & 30 & 20 & 10 & 0 & -10\end{array}$ 


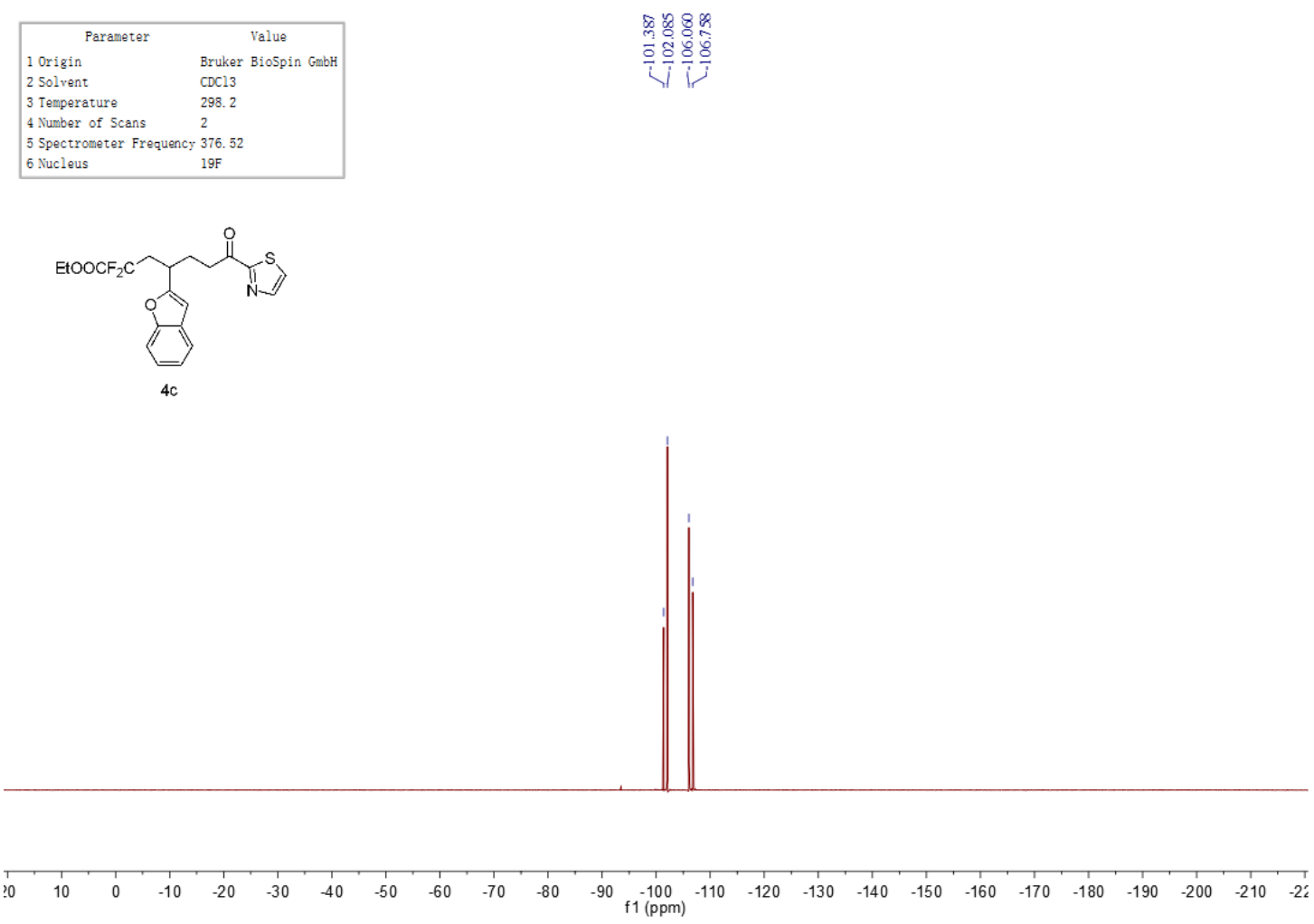

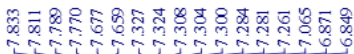 4 บ}
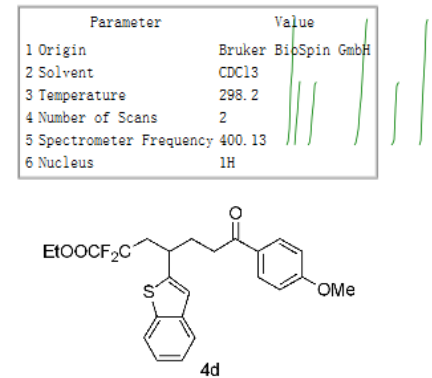

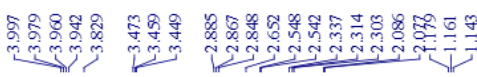
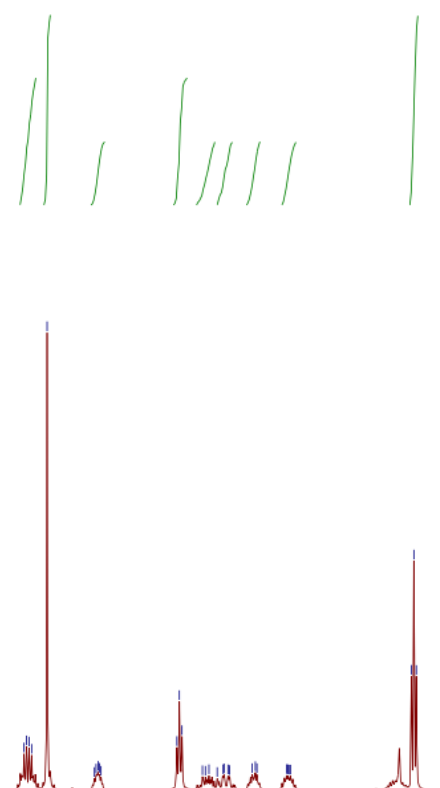

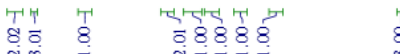

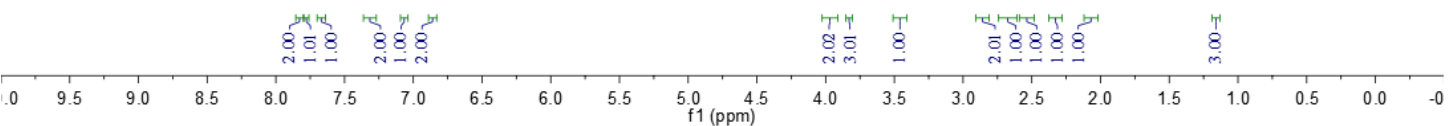




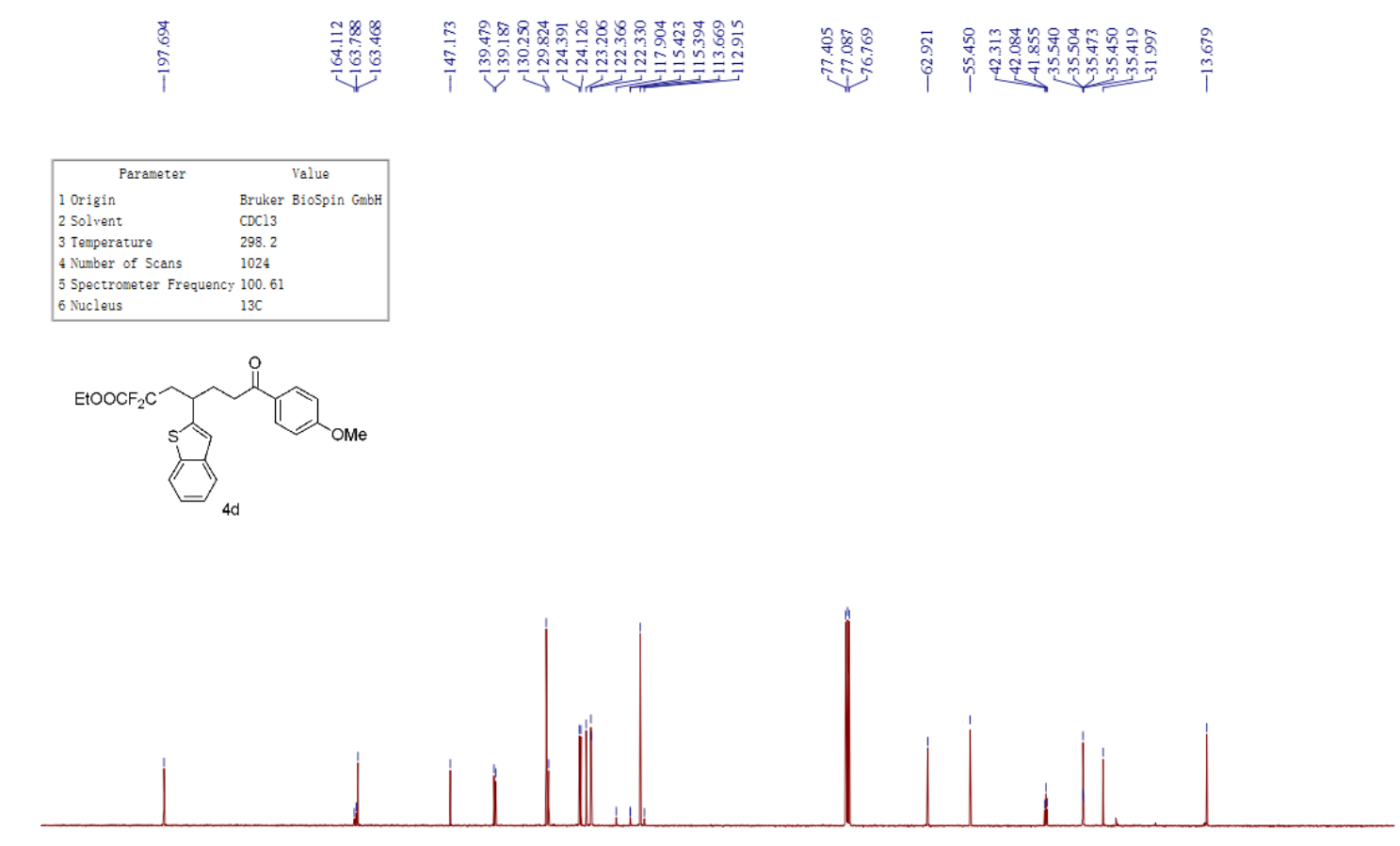

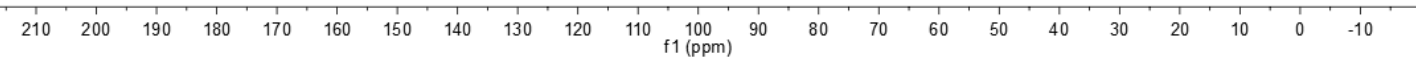

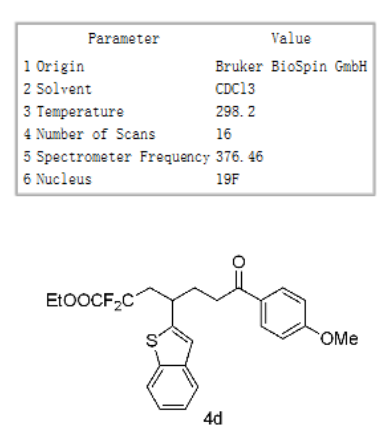

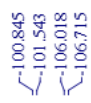

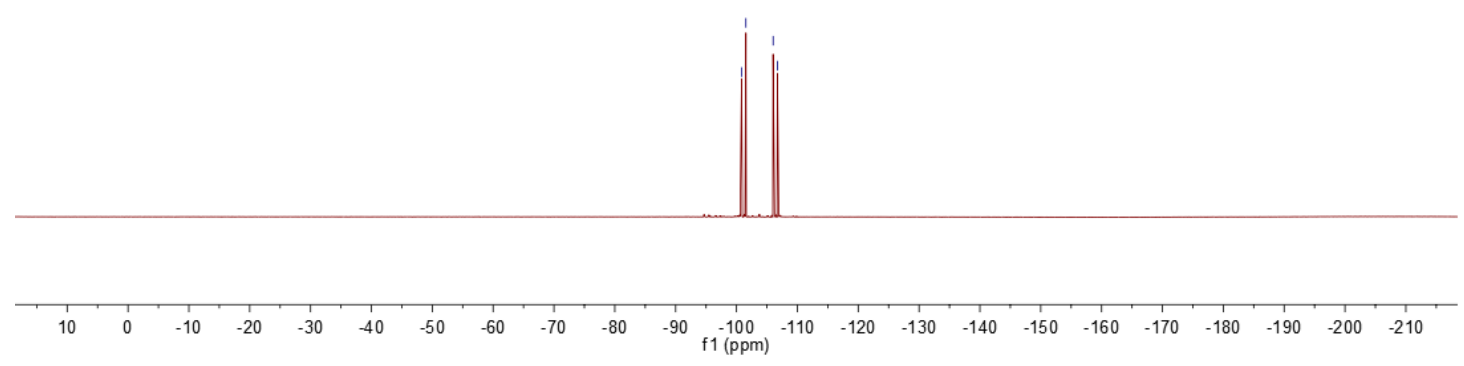




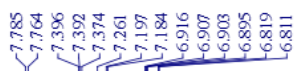

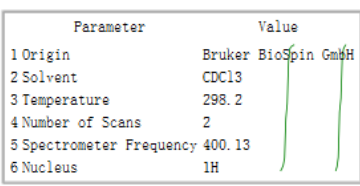

5 Spectrometer Frequency 400. 13

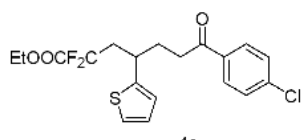

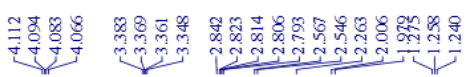

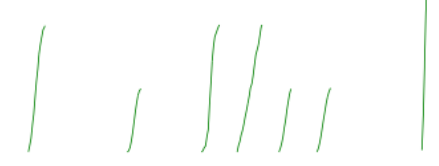

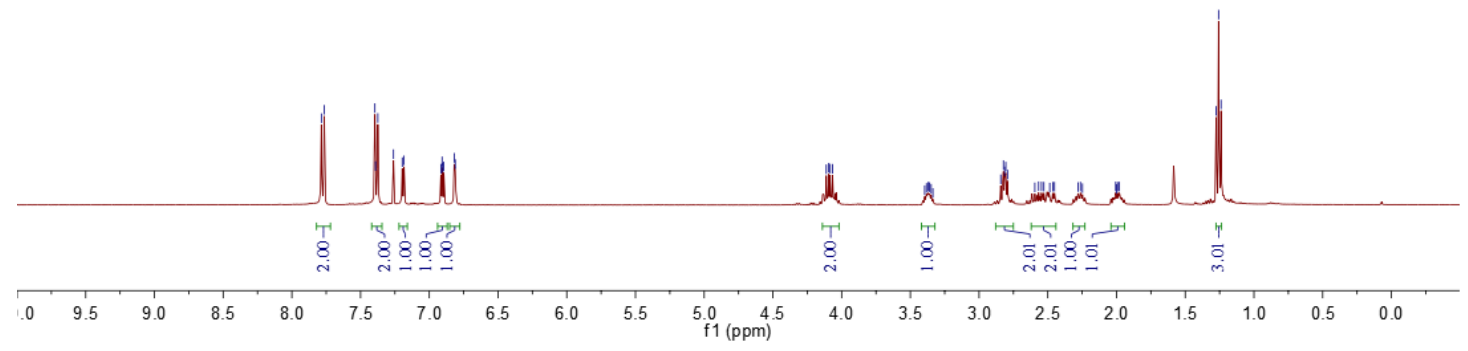

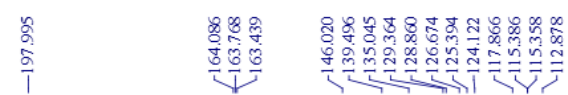

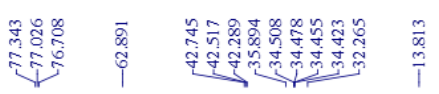

\begin{tabular}{|ll|}
\hline \multicolumn{1}{|c|}{ Parameter } & \multicolumn{1}{c|}{ Value } \\
1 Origin & Bruker \\
2 Solospin Gmbent & CDC13 \\
3 Temperature & 298.1 \\
4 Number of Scans & 1024 \\
5 Spectrometer Frequency & 100.62 \\
6 Nucleus & $13 \mathrm{C}$ \\
\hline
\end{tabular}

$\mathrm{EtoOCF}_{2} \mathrm{C}$

$4 \mathrm{e}$

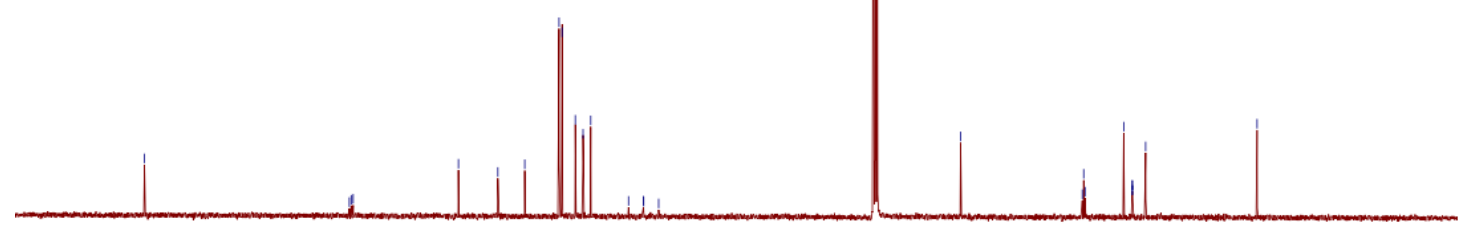

$\begin{array}{lllllllllllllllllllllllllllll}1 & 1 & 200 & 190 & 180 & 170 & 160 & 150 & 140 & 130 & 120 & 110 & 100 & 90 & 80 & 70 & 60 & 50 & 40 & 30 & 20 & 10 & 0 & -10\end{array}$ 

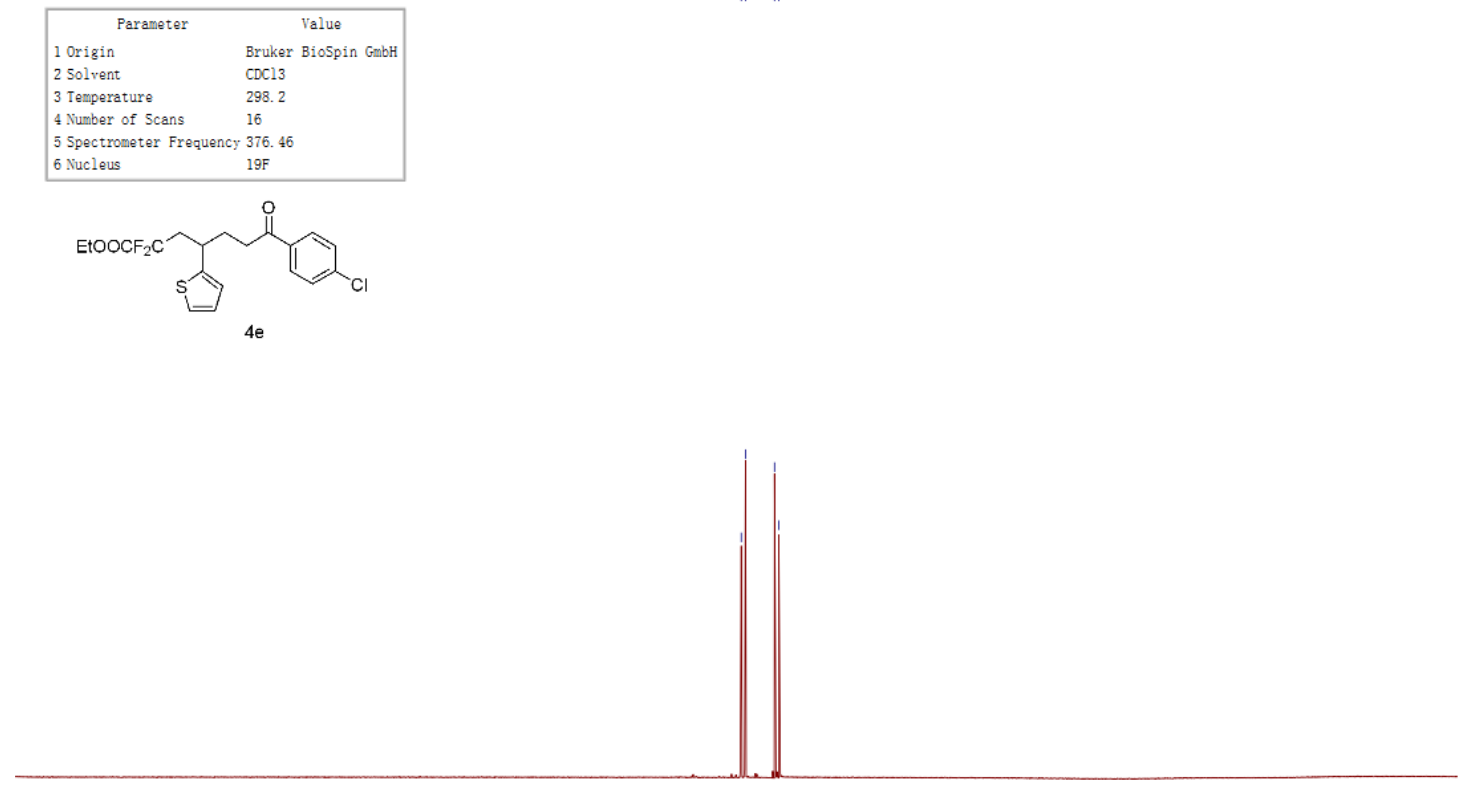

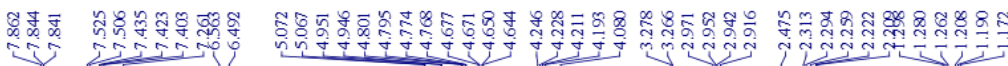
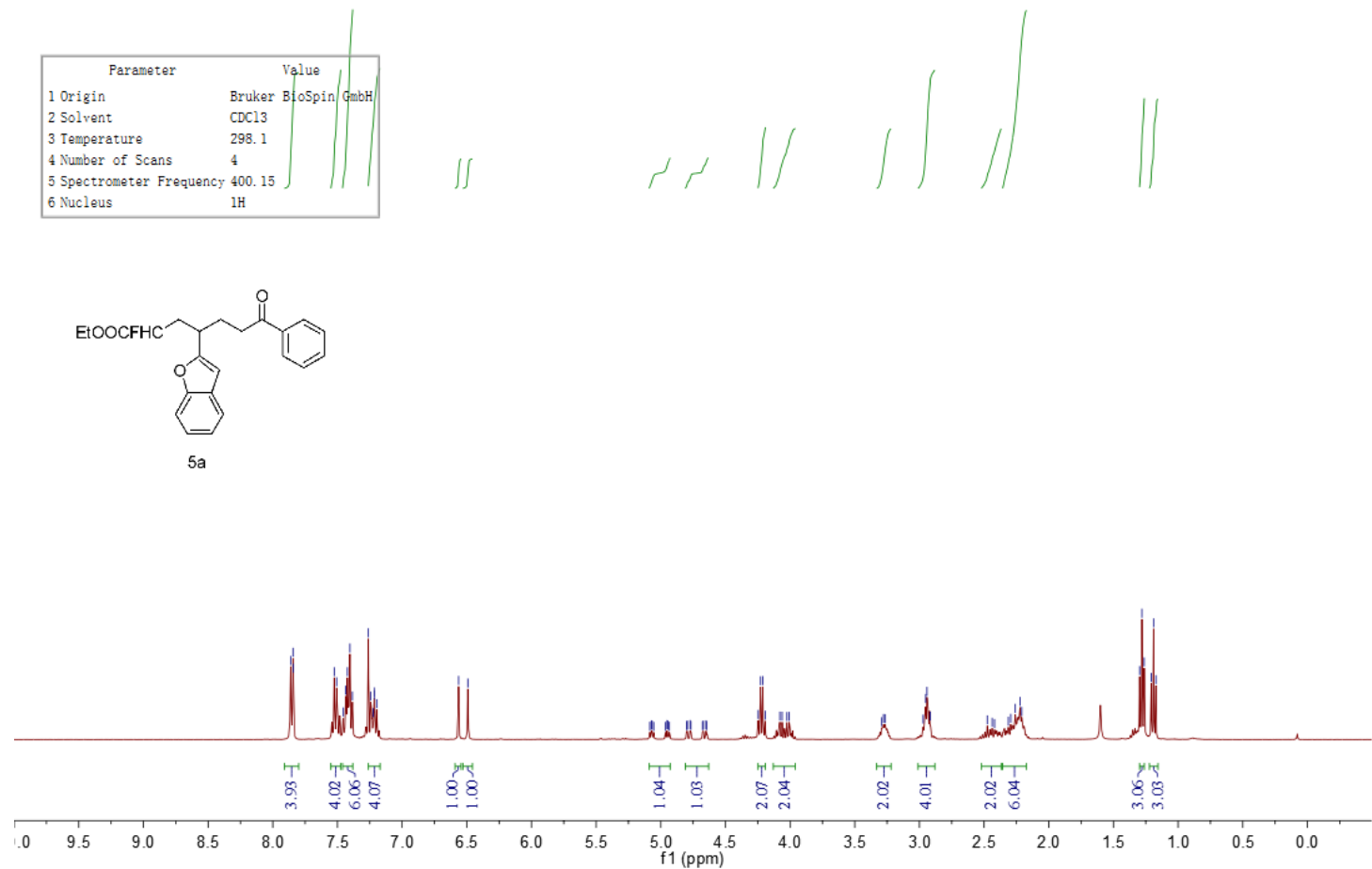


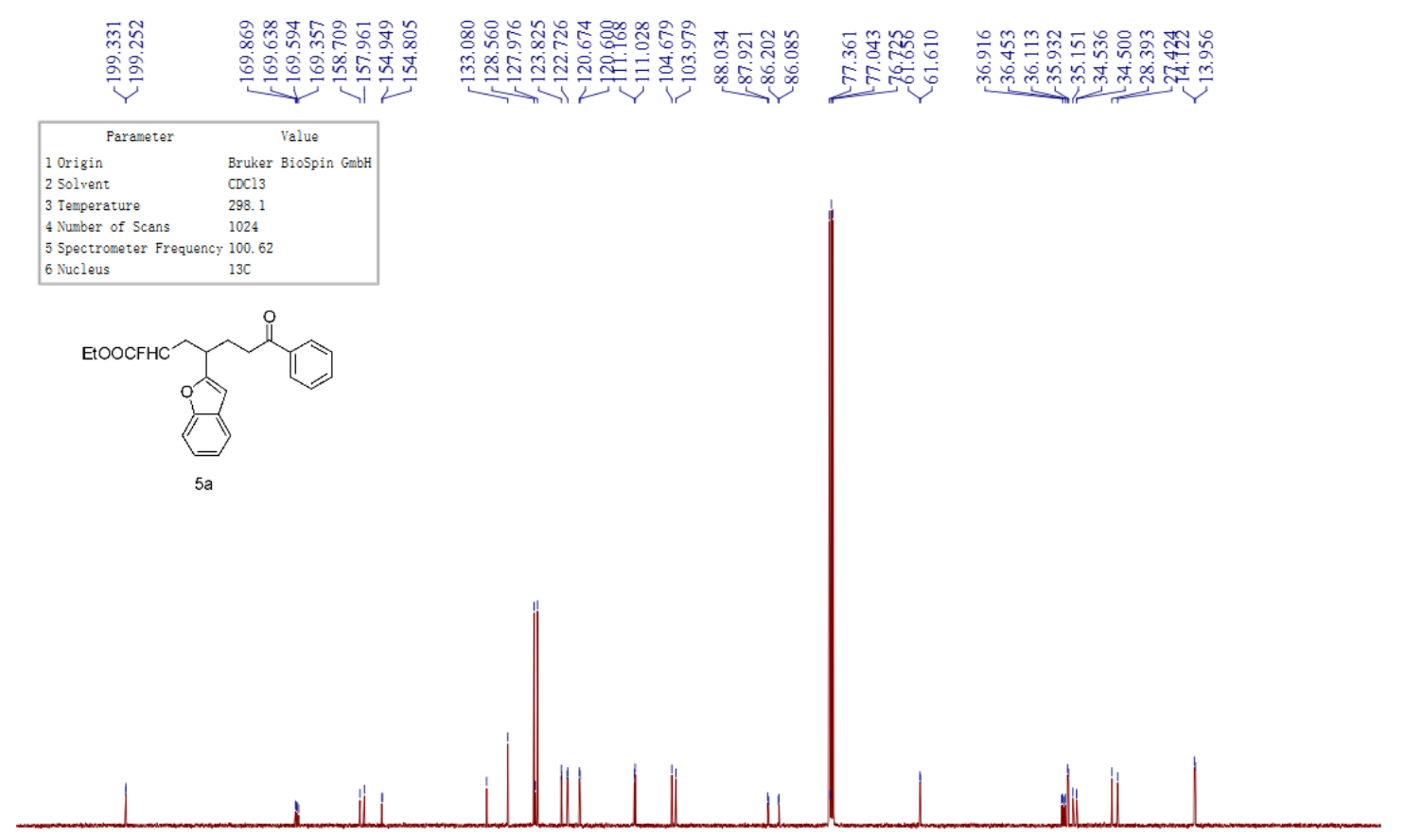

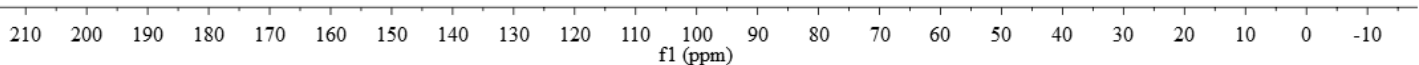

\begin{tabular}{|ll|}
\hline \multicolumn{1}{|c|}{ Parameter } & \multicolumn{1}{c|}{ Value } \\
1 Origin & Bruker \\
2 Solvespin GmbH & CDC13 \\
3 Temperature & 298.2 \\
4 Number of Scans & 4 \\
5 Spectrometer Frequency & 376.52 \\
6 Nucleus & $19 \mathrm{~F}$ \\
\hline
\end{tabular}<smiles>CCOC=CCC(CCC(=O)c1ccccc1)c1cc2ccccc2o1</smiles>

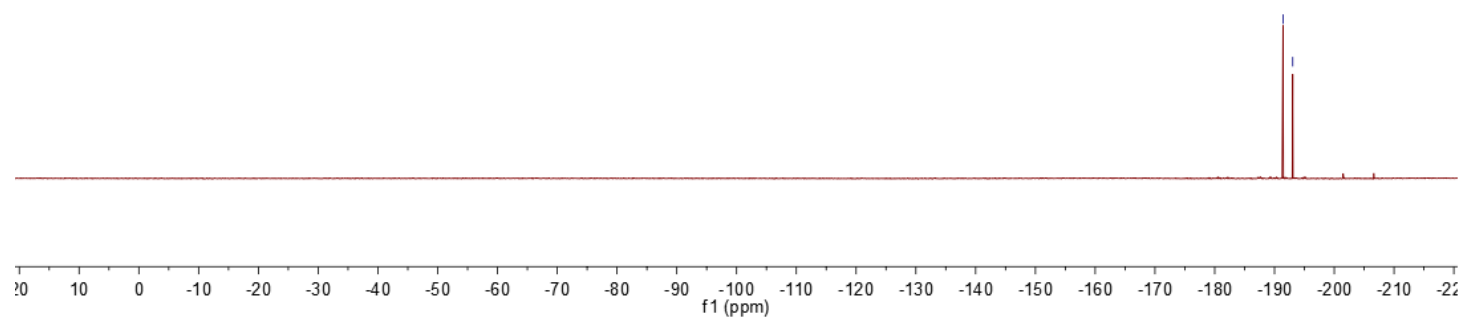




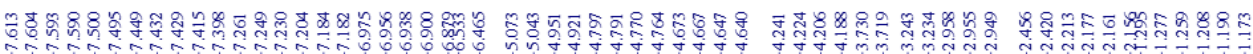
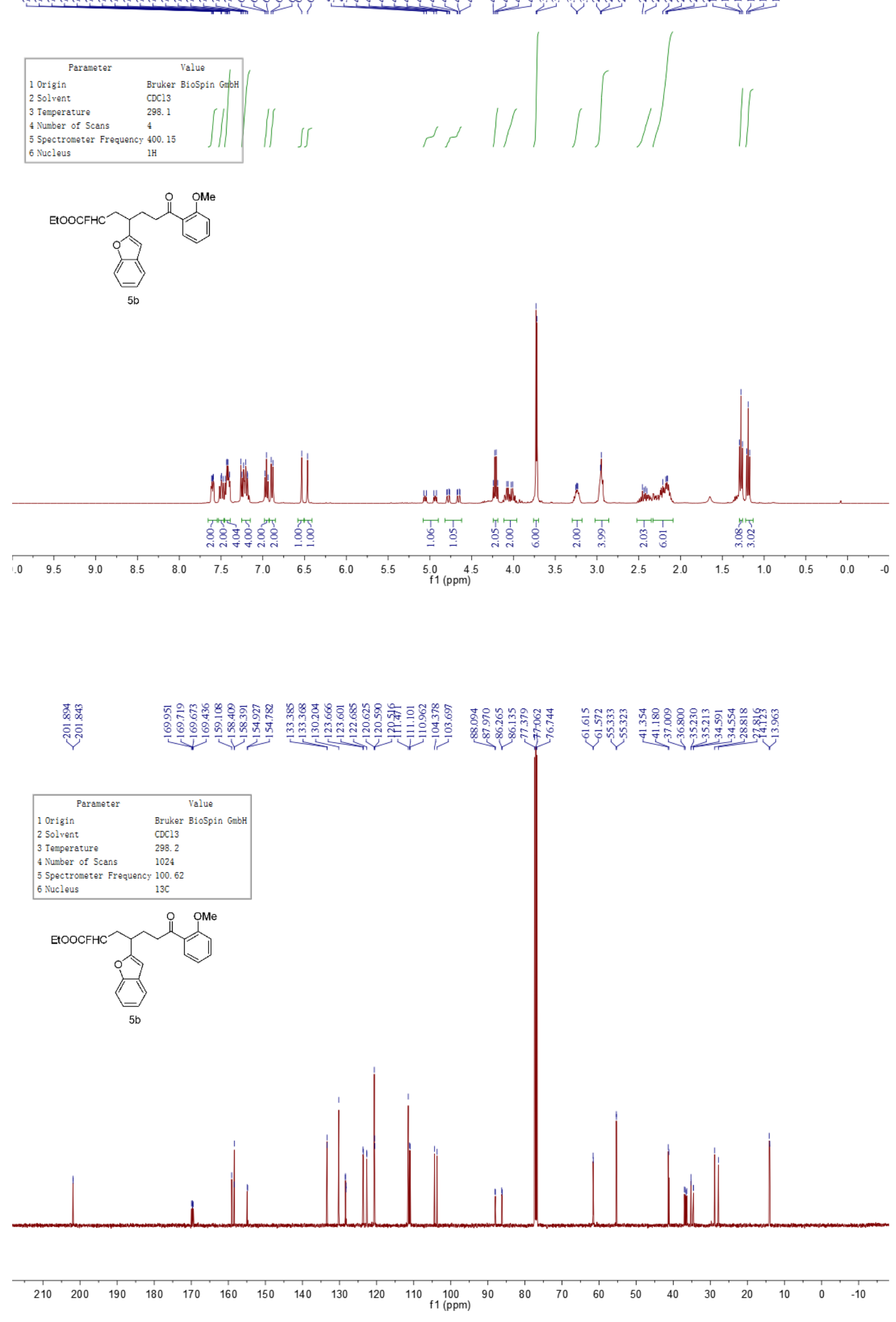


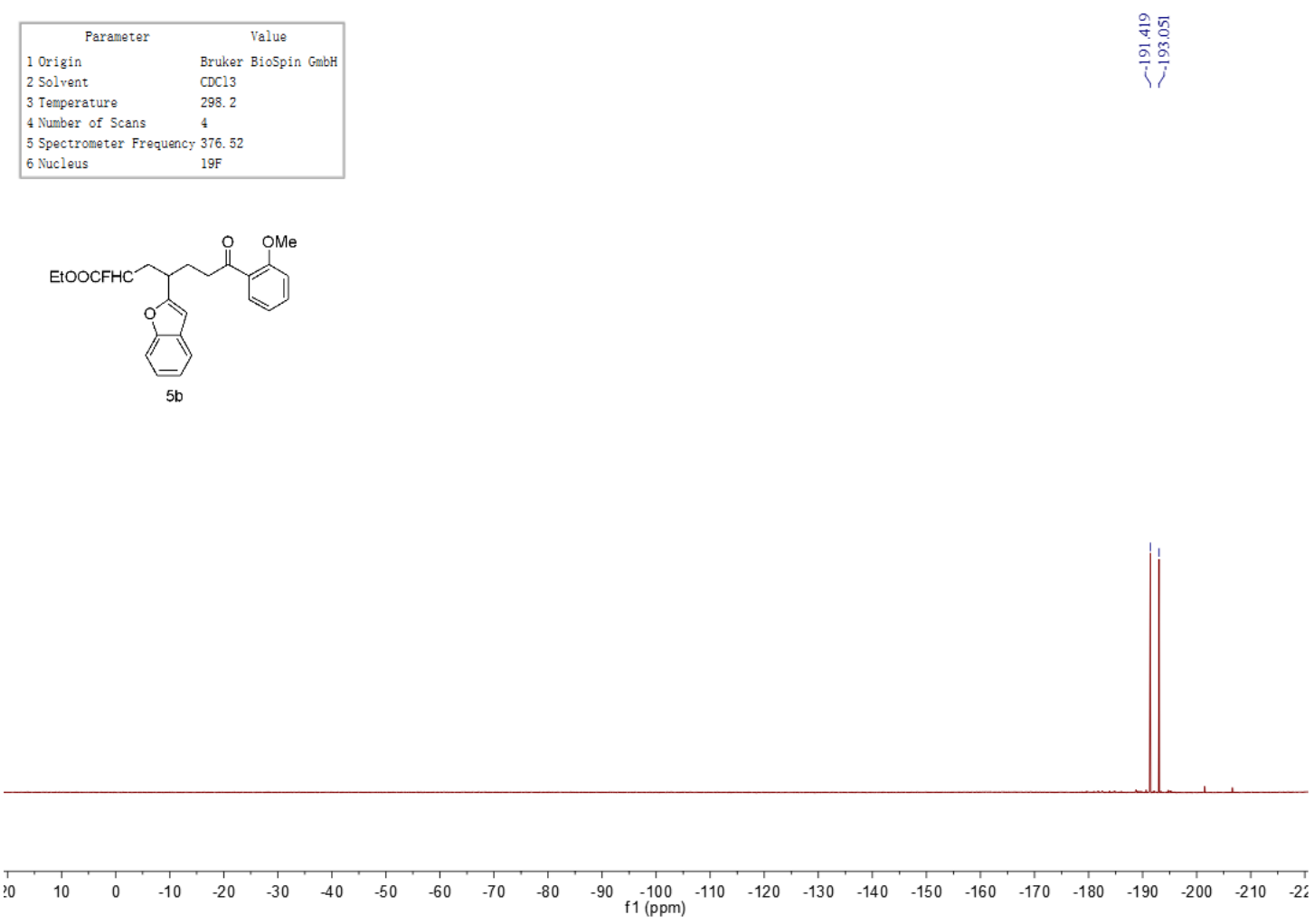

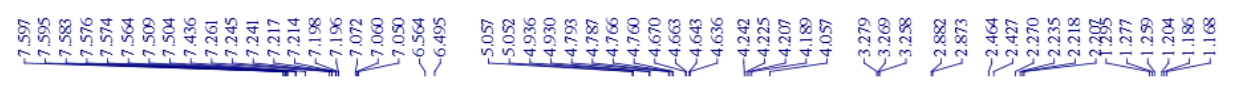
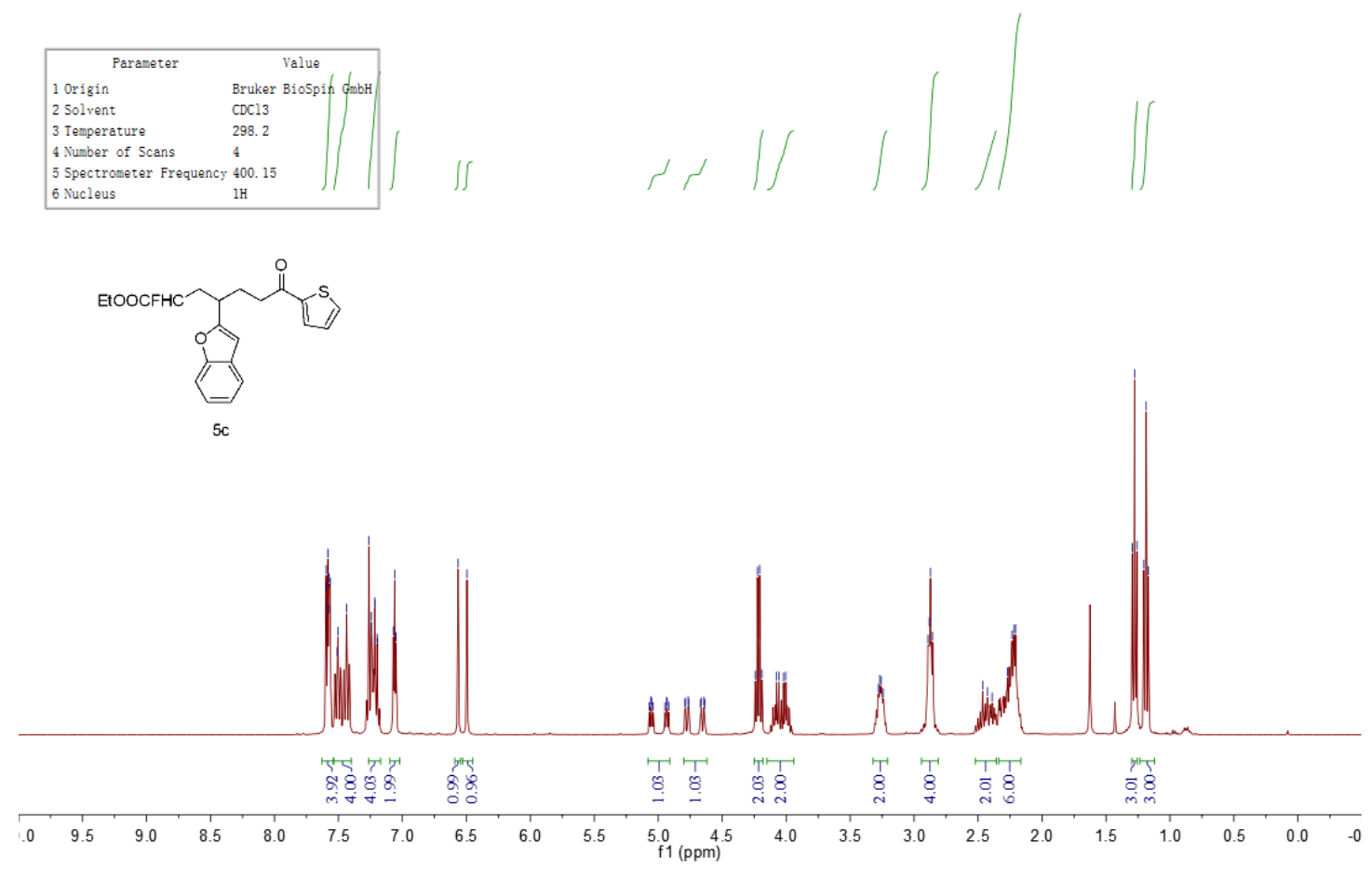


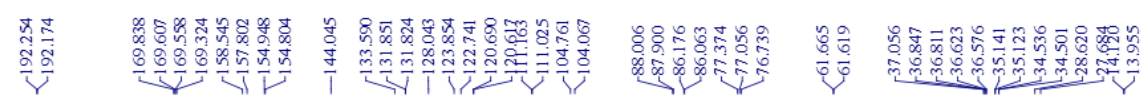

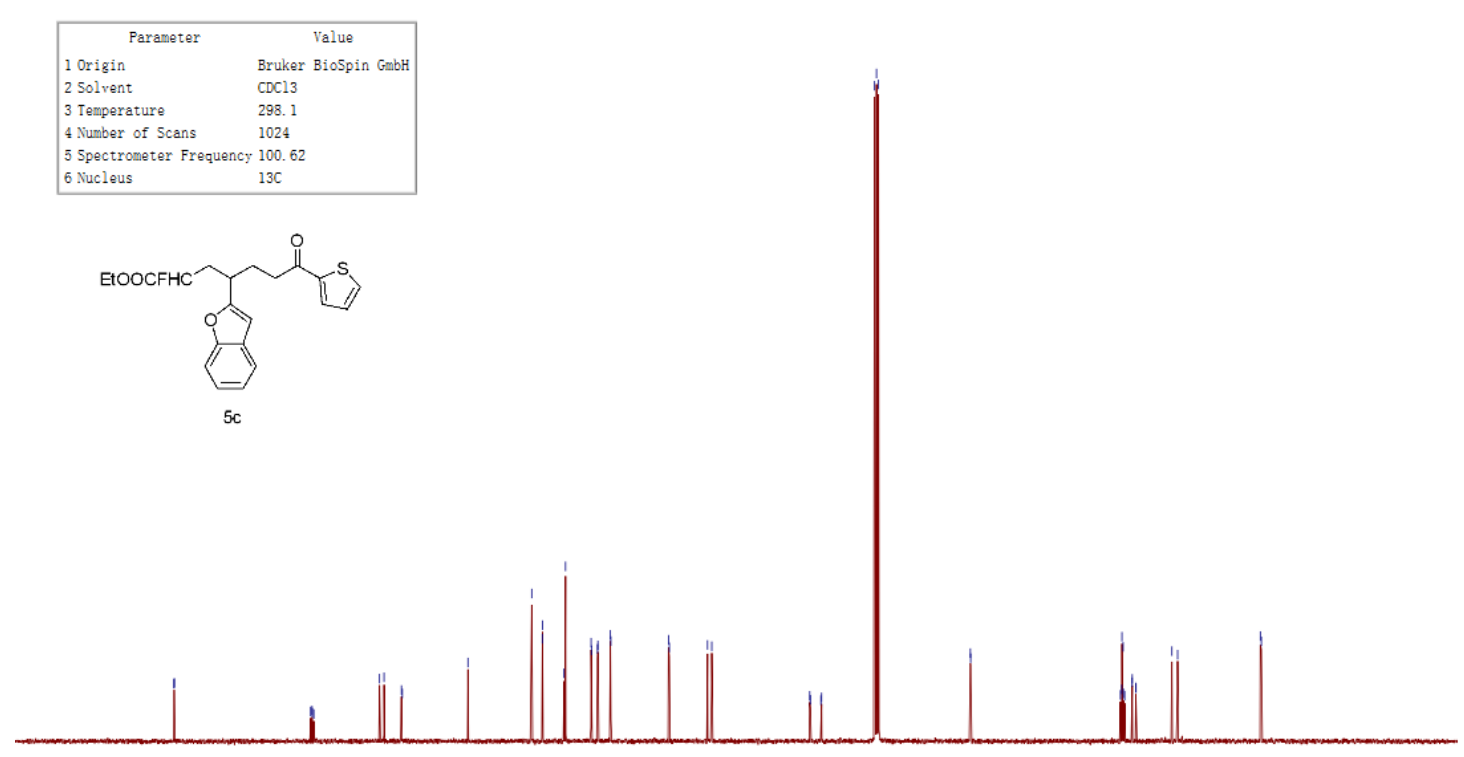

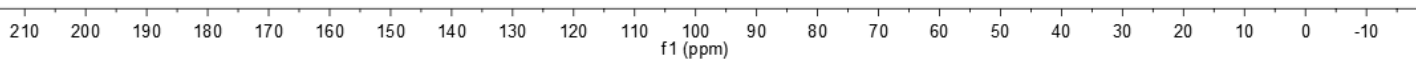

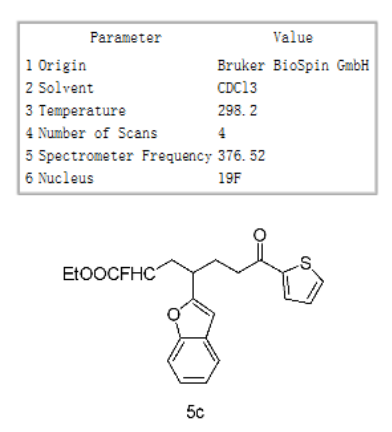

Nucleus $\quad 19 \mathrm{~F}$

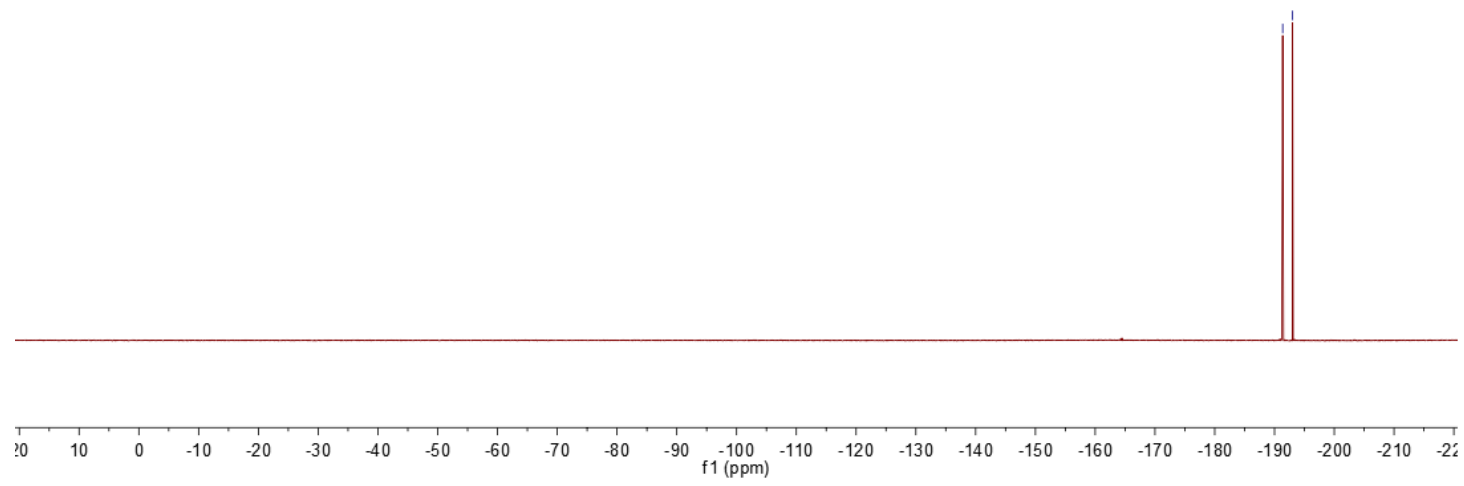



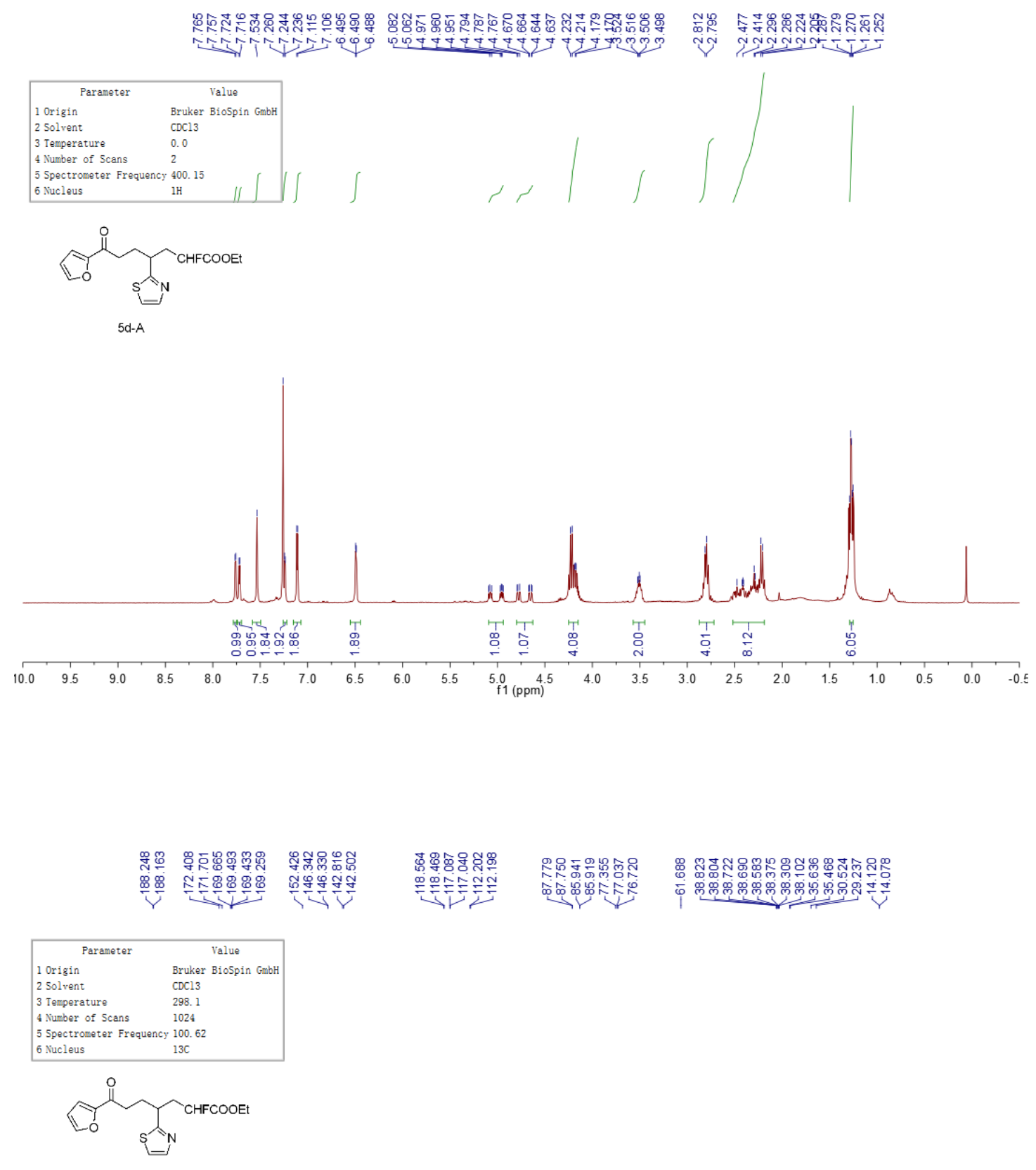

$5 \mathrm{~d}-\mathrm{A}$

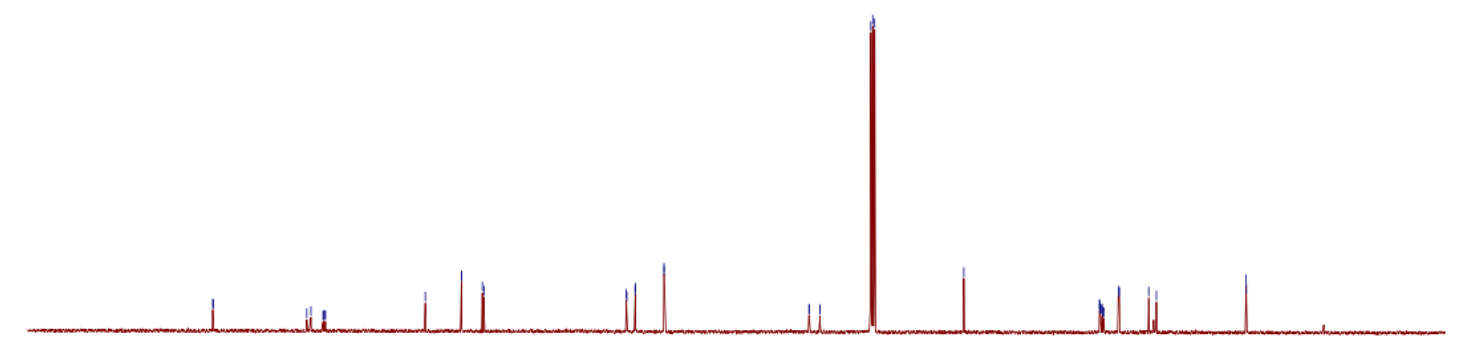

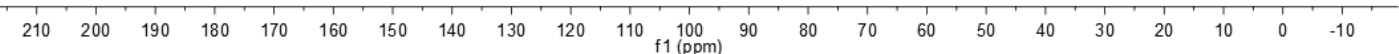




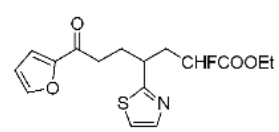

$5 \mathrm{~d}-\mathrm{A}$

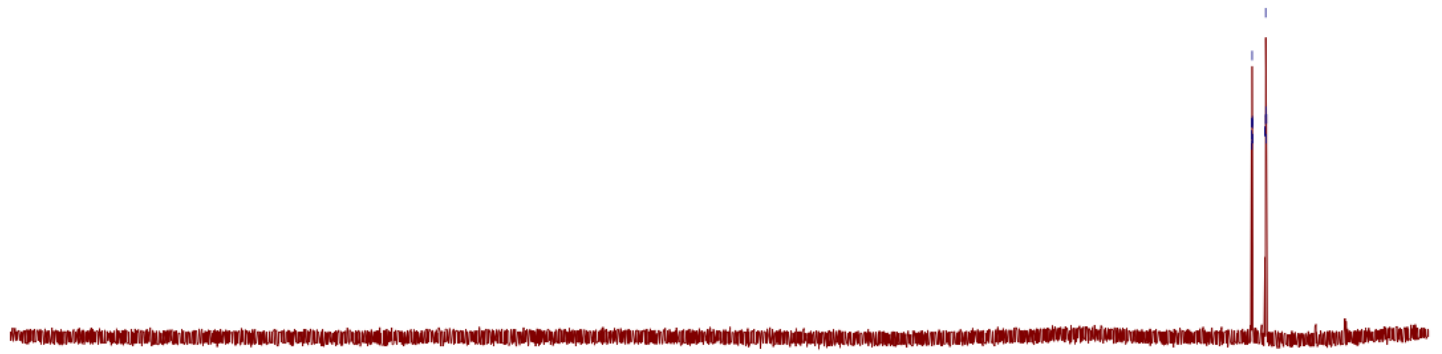

$\begin{array}{llllllllllllllllllllllllllllllll}20 & 10 & 0 & -10 & -20 & -30 & -40 & -50 & -60 & -70 & -80 & -90 & -100 & -110 & -120 & -130 & -140 & -150 & -160 & -170 & -180 & -190 & -200 & -210 & -220\end{array}$

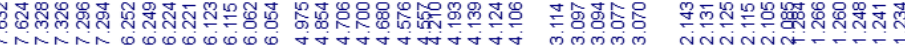

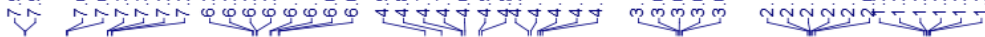

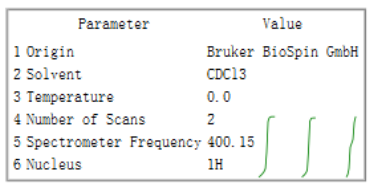

$\underbrace{\text { CHFCOOEt }}_{\text {S }}$

5d-B

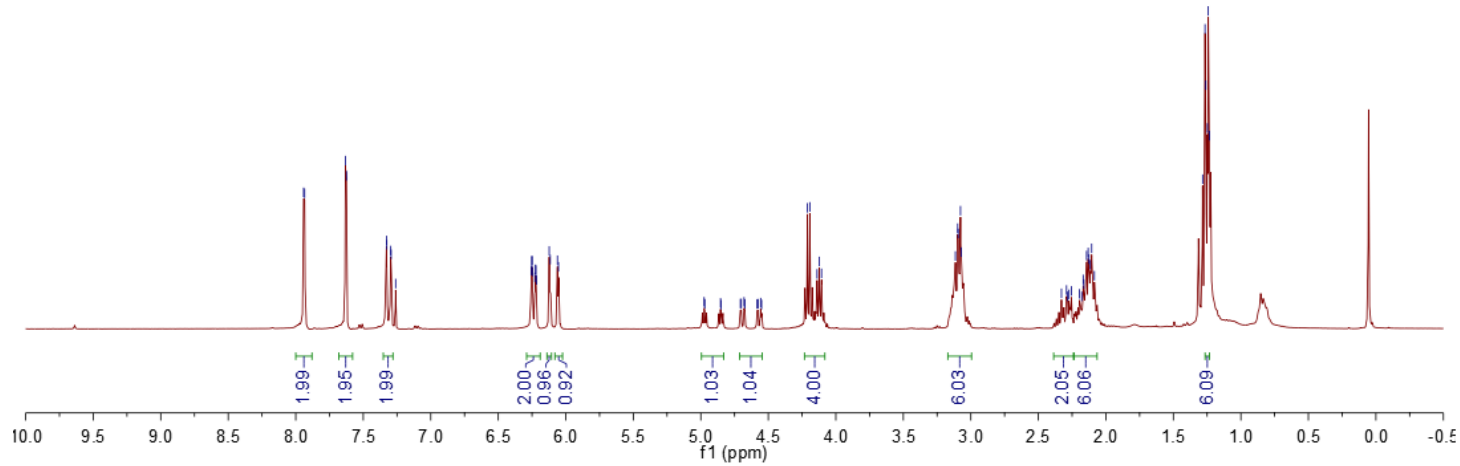




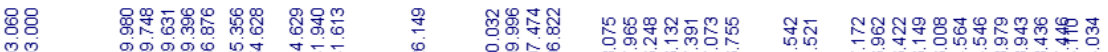

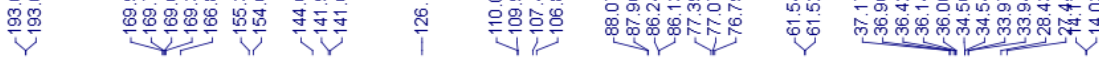

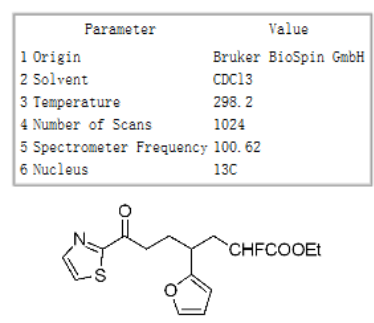

5d-B

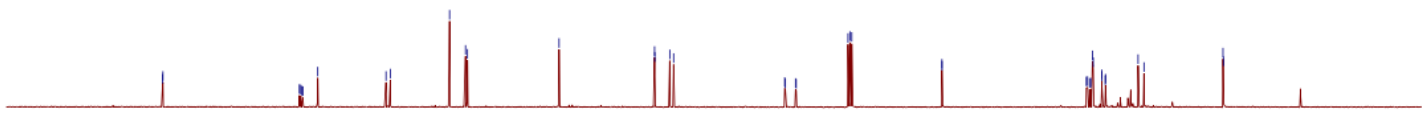

$\begin{array}{lllllllllllllllllllllll}210 & 200 & 190 & 180 & 170 & 160 & 150 & 140 & 130 & 120 & 110 & \begin{array}{c}\mathrm{f} 1(\mathrm{pom}) \\ 90\end{array} & 80 & 70 & 60 & 50 & 40 & 30 & 20 & 10 & 0 & -10\end{array}$

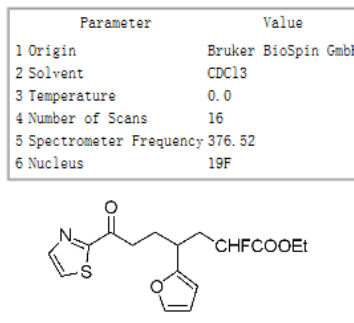

$5 d-B$

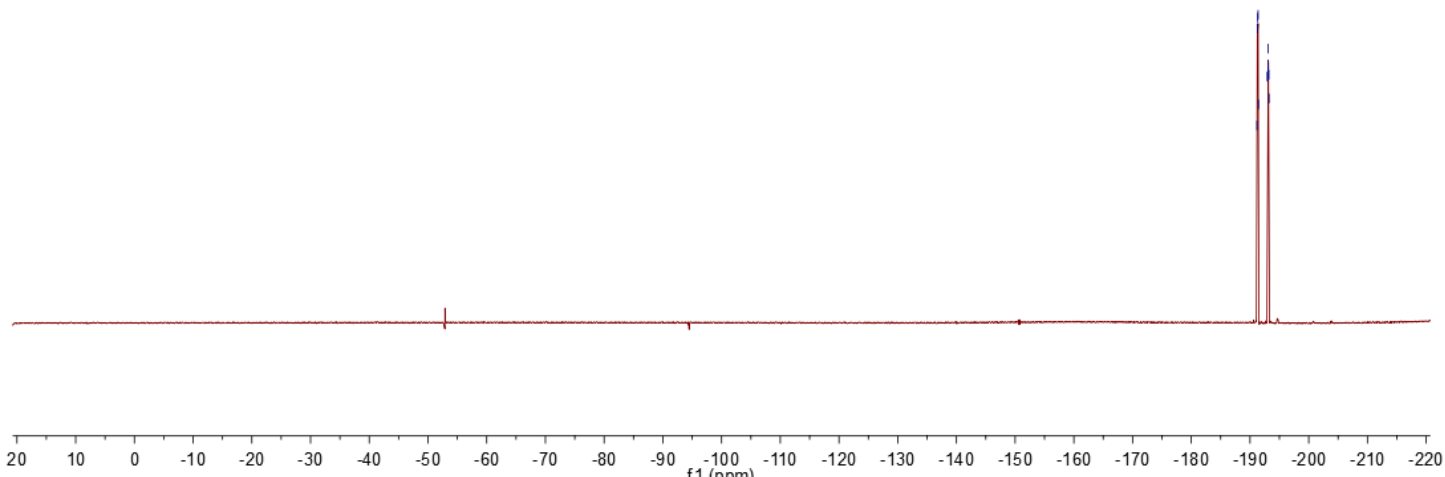




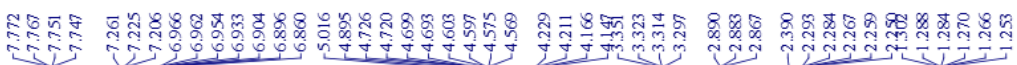

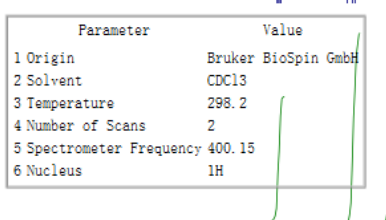

EtOOCFHC

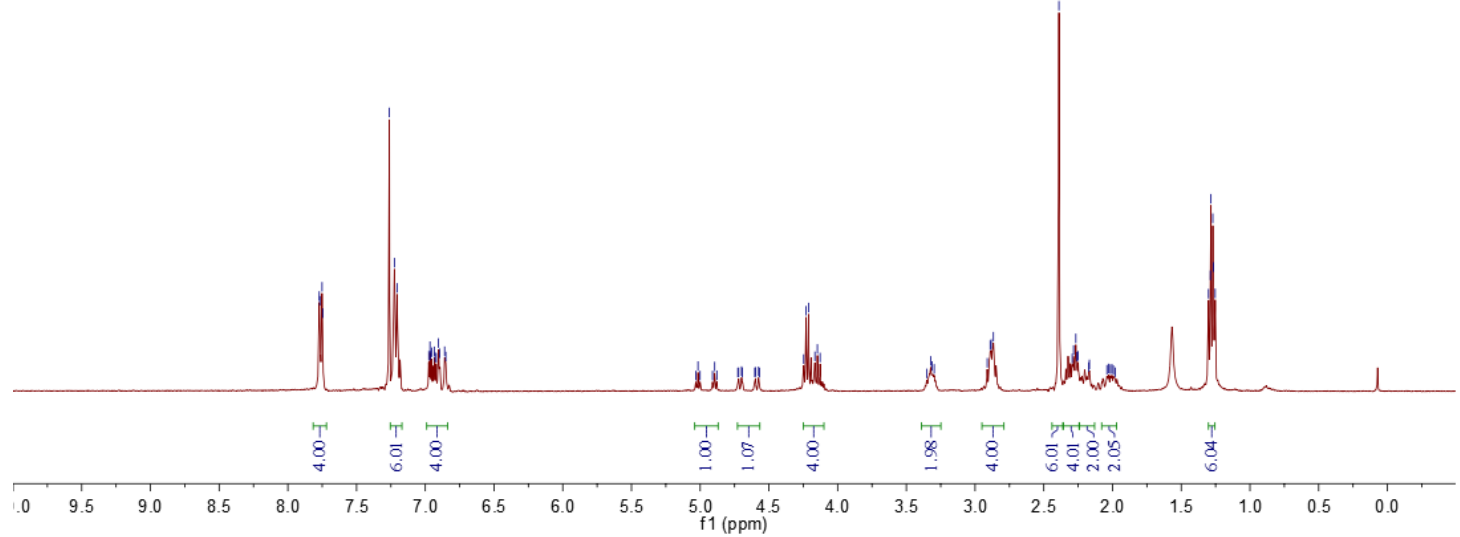

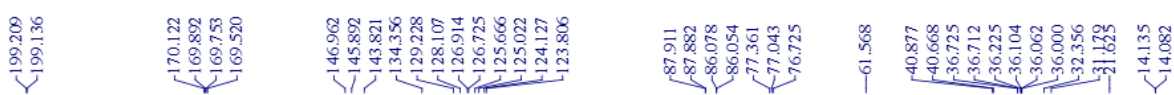

\begin{tabular}{|ll|}
\hline \multicolumn{1}{|c|}{ Parameter } & \multicolumn{1}{c|}{ Value } \\
1 Origin & Bruker BioSpin GmbH \\
2 Solvent & CDC13 \\
3 Temperature & 298.1 \\
4 Number of Scans & 1024 \\
5 Spectrometer Frequency & 100.62 \\
6 Nucleus & $13 C$ \\
\hline
\end{tabular}

EtOOCFHC

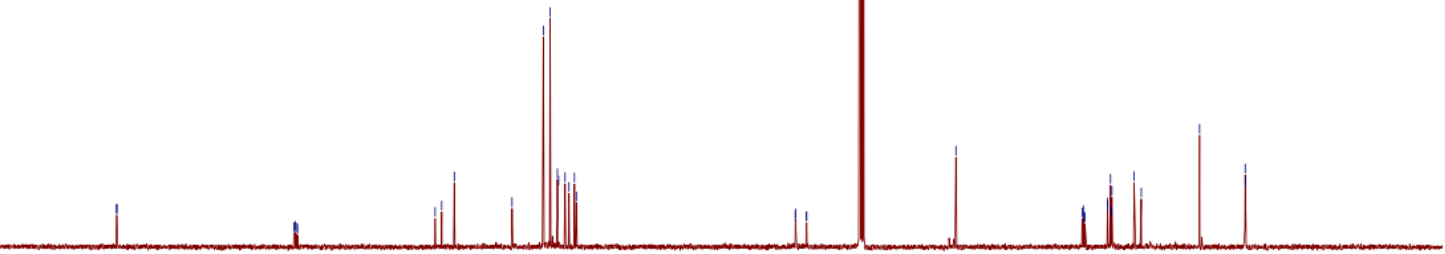

$\begin{array}{lllllllllllllllllllllllllllllll}210 & 200 & 190 & 180 & 170 & 160 & 150 & 140 & 130 & 120 & 110 & 100 & 90 & 80 & 70 & 60 & 50 & 40 & 30 & 20 & 10 & 0 & -10\end{array}$ 

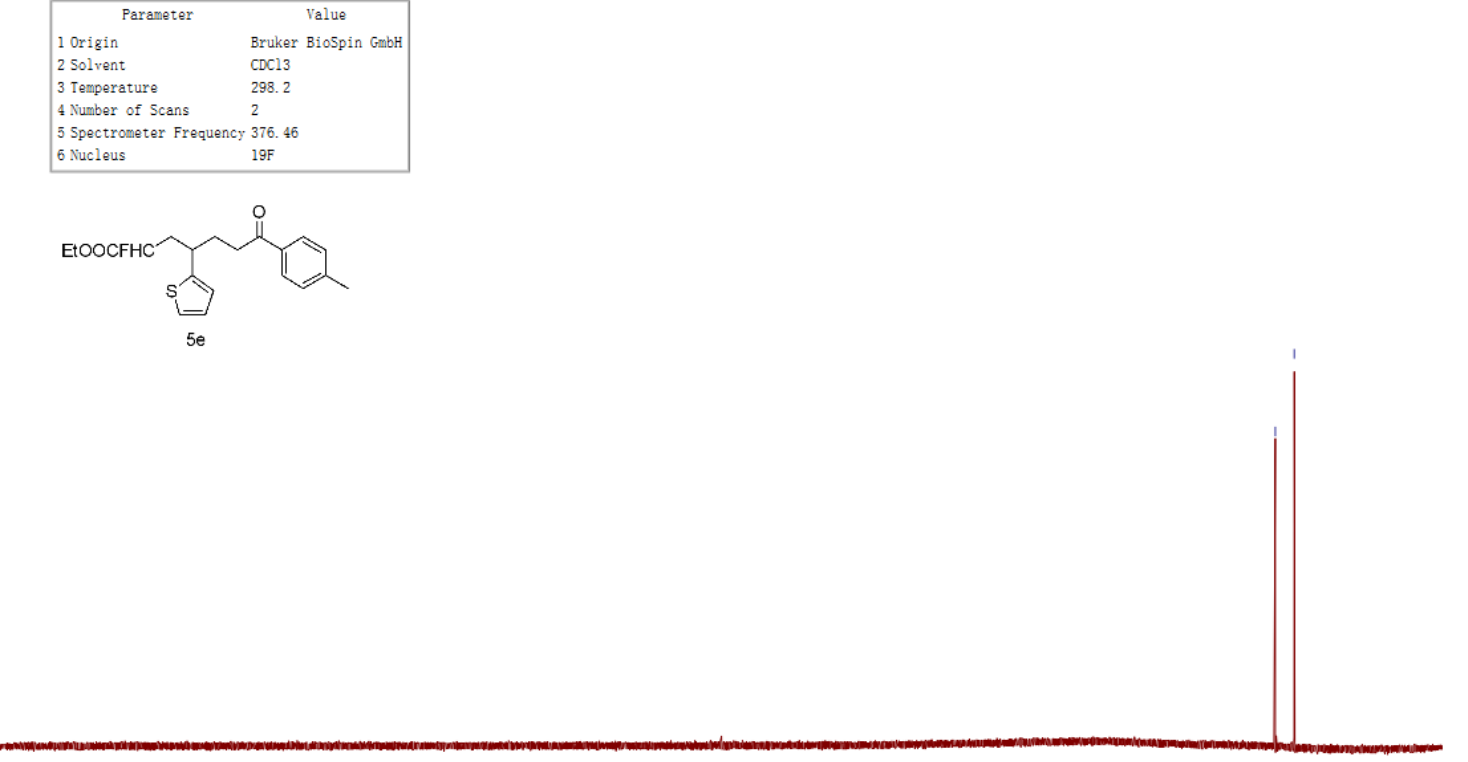

$\begin{array}{lllllllllllllllllllllllllllll}10 & 0 & -10 & -20 & -30 & -40 & -50 & -60 & -70 & -80 & -90 & -100 & -110 & -120 & -130 & -140 & -150 & -160 & -170 & -180 & -190 & -200 & -210\end{array}$

\section{DFT studies}

\section{a. Computational methods}

All structures were optimized using the M06- $2 \mathrm{X}^{1-3}$ functional, which demonstrates excellent performance for main group elements. The $6-31+\mathrm{G}(\mathrm{d})^{4}$ basis set was employed for geometry optimization, in which solvent effect was considered using SMD method ${ }^{5}$ with DMA as the solvent. Vibrational analyses were performed on all optimized geometries to ensure that the optimized structures correspond to local minima (no imaginary frequency) or transition states (one imaginary frequency). In addition, intrinsic reaction coordinate (IRC) calculations were carried out to verify that the transition states connect with appropriate reactants and products. ${ }^{6}$ The Gibbs free energies for all species were obtained at $298.15 \mathrm{~K}$ and $1 \mathrm{~atm}$ at their respective optimized structures. Single-point energy calculations at larger basis set were performed at the M06-2X/6-311++G(d,p)-SMD level of theory. The solution-phase Gibbs free energy was determined by adding the solvation single-point energy and the thermal correction to the Gibbs free energy obtained from vibrational frequency calculation. Unless otherwise specified, the solution-phase Gibbs free energy was used in the discussion. The Gaussian 09 suite of programs ${ }^{7}$ was used throughout. 


\section{b. References}

1. Zhao, Y.; Truhlar, D. G. Theor. Chem. Acc. 2008, 120, 215-241.

2. Walker, M.; Harvey, A. J.; Sen, A.; Dessent, C. E. J. Phys. Chem. A 2013, 117, 12590-12600.

3. Zhao, Y.; Truhlar, D. G. Acc. Chem. Res. 2008, 41 ,157-167.

4. Laurent, A. D.; Jacquemin , D.; Int. J. Quantum. Chem. 2013, 113, 2019-2039.

5. Marenich, A. V.; Cramer, C. J.; Truhlar, D. G. J. Phys. Chem. B 2009, 113, 6378-6396.

6. Fukui, K. Acc. Chem. Res., 1981, 14, 363-368.

7. Frisch, M. J.; Trucks, G. W.; Schlegel, H. B.; Scuseria, G. E.; Robb, M. A.; Cheeseman, J. R.; Scalmani, G.; Barone, V.; Mennucci, B.; Petersson, G. A.; Nakatsuji, H.; Caricato, M.; Li, X.; Hratchian, H. P.; Izmaylov, A. F.; Bloino, J.; Zheng, G.; Sonnenberg, J. L.; Hada, M.; Ehara, M.; Toyota, K.; Fukuda, R.; Hasegawa, J.; Ishida, M.; Nakajima, T.; Honda, Y.; Kitao, O.; Nakai, H.; Vreven, T.; Montgomery, J. A.; Peralta, J. E.; Ogliaro, F.; Bearpark, M.; Heyd, J. J.; Brothers, E.; Kudin, K. N.; Staroverov, V. N.; Kobayashi, R.; Normand, J.; Raghavachari, K.; Rendell, A.; Burant, J. C.; Iyengar, S. S.; Tomasi, J.; Cossi, M.; Rega, N.; Millam, N. J.; Klene, M.; Knox, J. E.; Cross, J. B.; Bakken, V.; Adamo, C.; Jaramillo, J.; Gomperts, R.; Stratmann, R. E.; Yazyev, O.; Austin, A. J.; Cammi, R.; Pomelli, C.; Ochterski, J. W.; Martin, R. L.; Morokuma, K.; Zakrzewski, V. G.; Voth, G. A.; Salvador, P.; Dannenberg, J. J.; Dapprich, S.; Daniels, A. D.; Farkas, O.; Foresman, J. B.; Ortiz, J. V.; Cioslowski, J.; Fox, D. J.; Gaussian 09, Revision C.01, Gaussian, Inc., Wallingford CT, 2010.

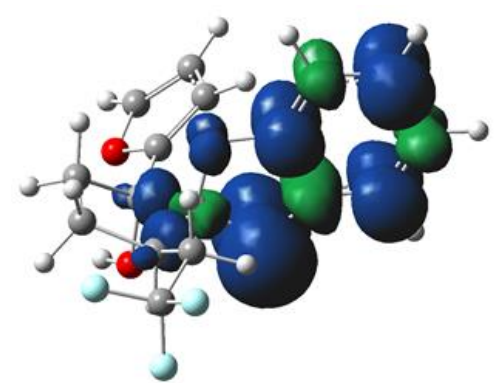

1ab-INT2a

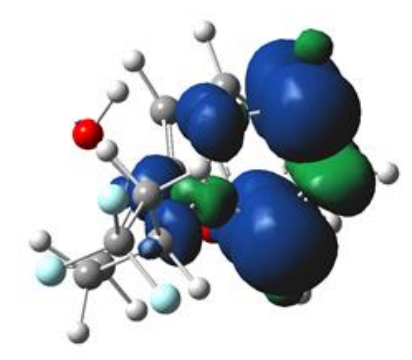

1ab-INT2b

Figure S1. The spin density distribution for 1ab-INT2a and 1ab-INT2b. 


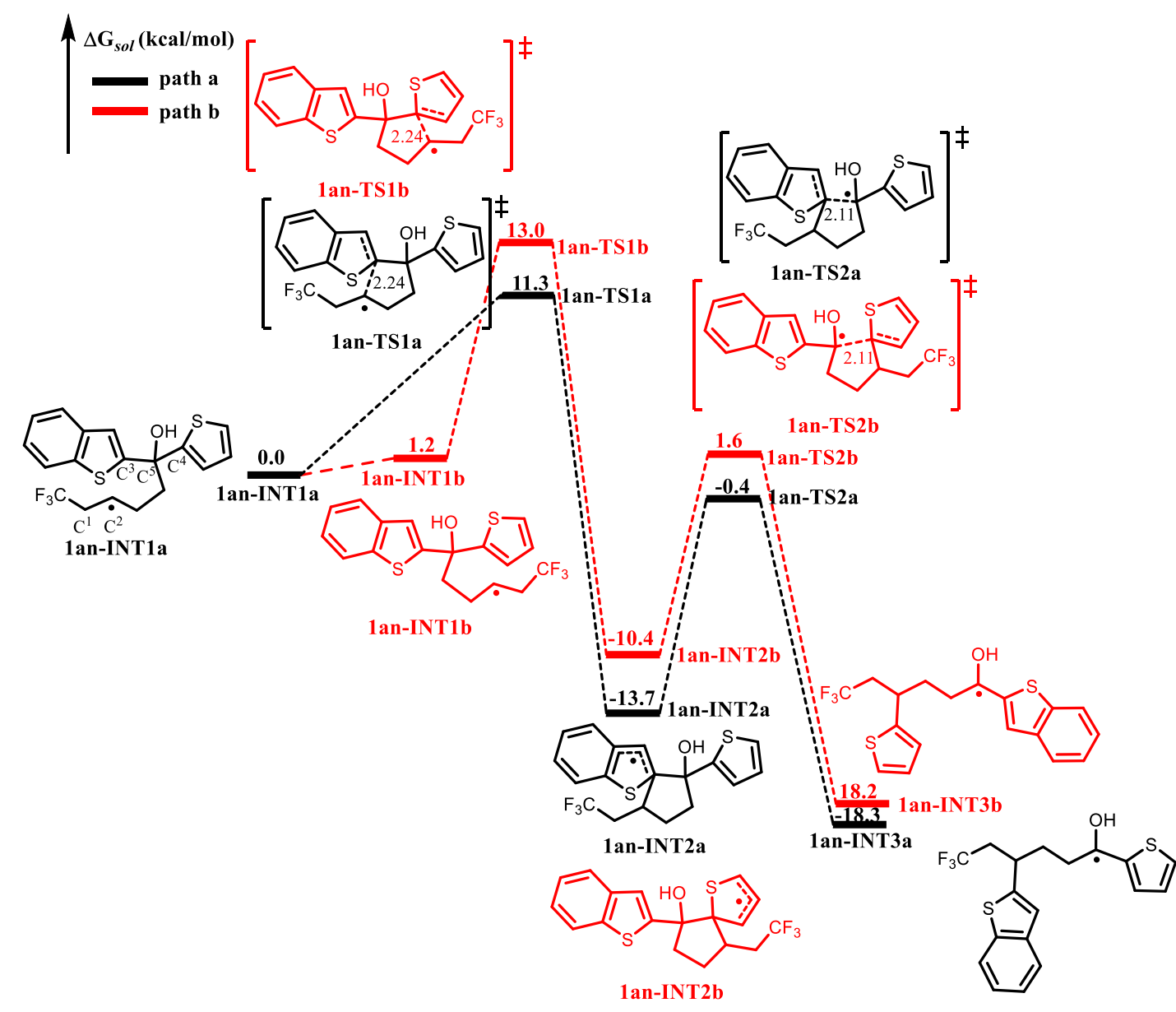

Figure S2. Energy profile (in $\mathrm{kcal} / \mathrm{mol}$ ) for migration of heterocycle of 1an. Bond lengths are shown in $\AA$. 


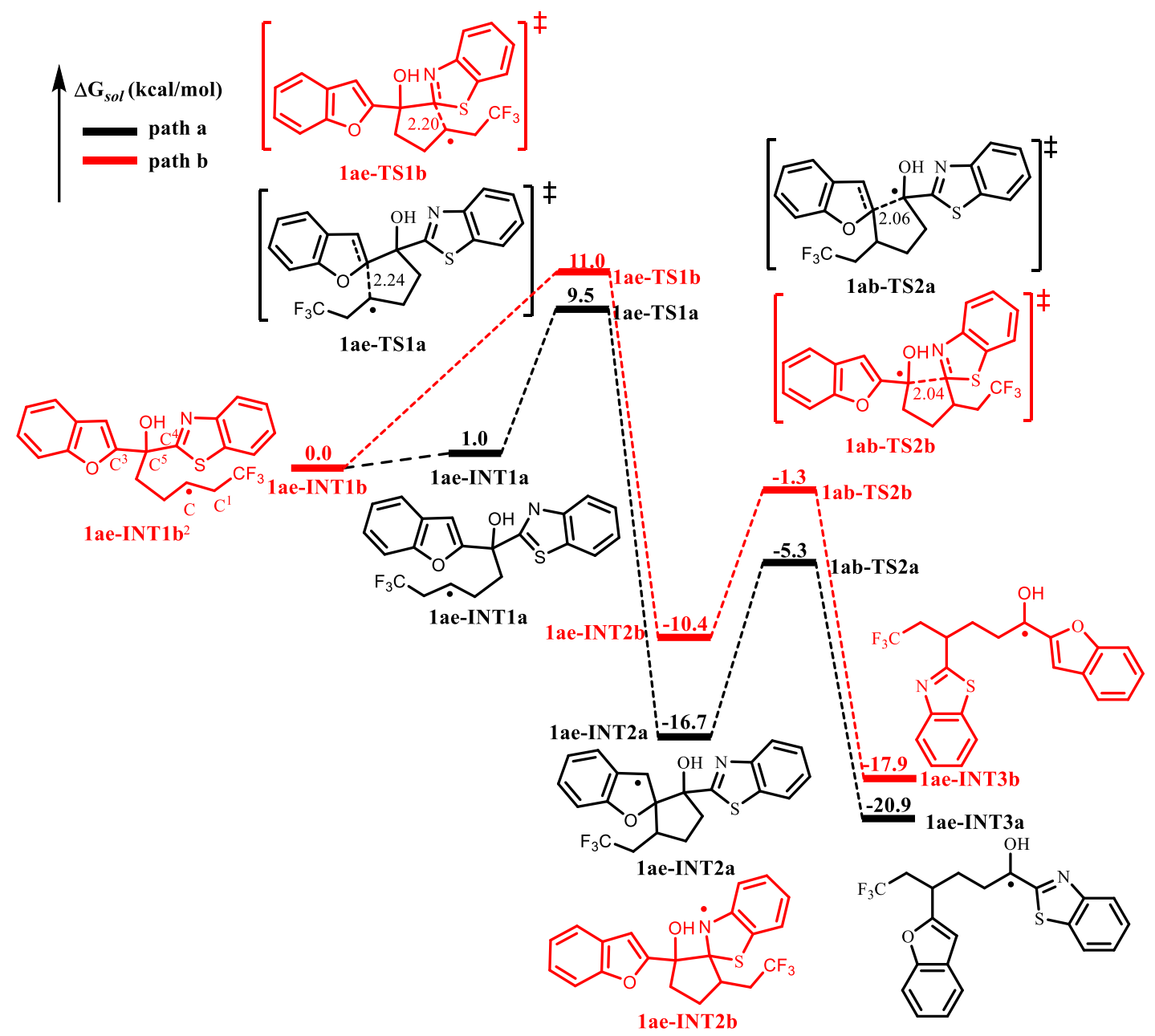

Figure S3. Energy profile (in kcal/mol) for migration of heterocycle of 1ae. Bond lengths are shown in $\AA$. 


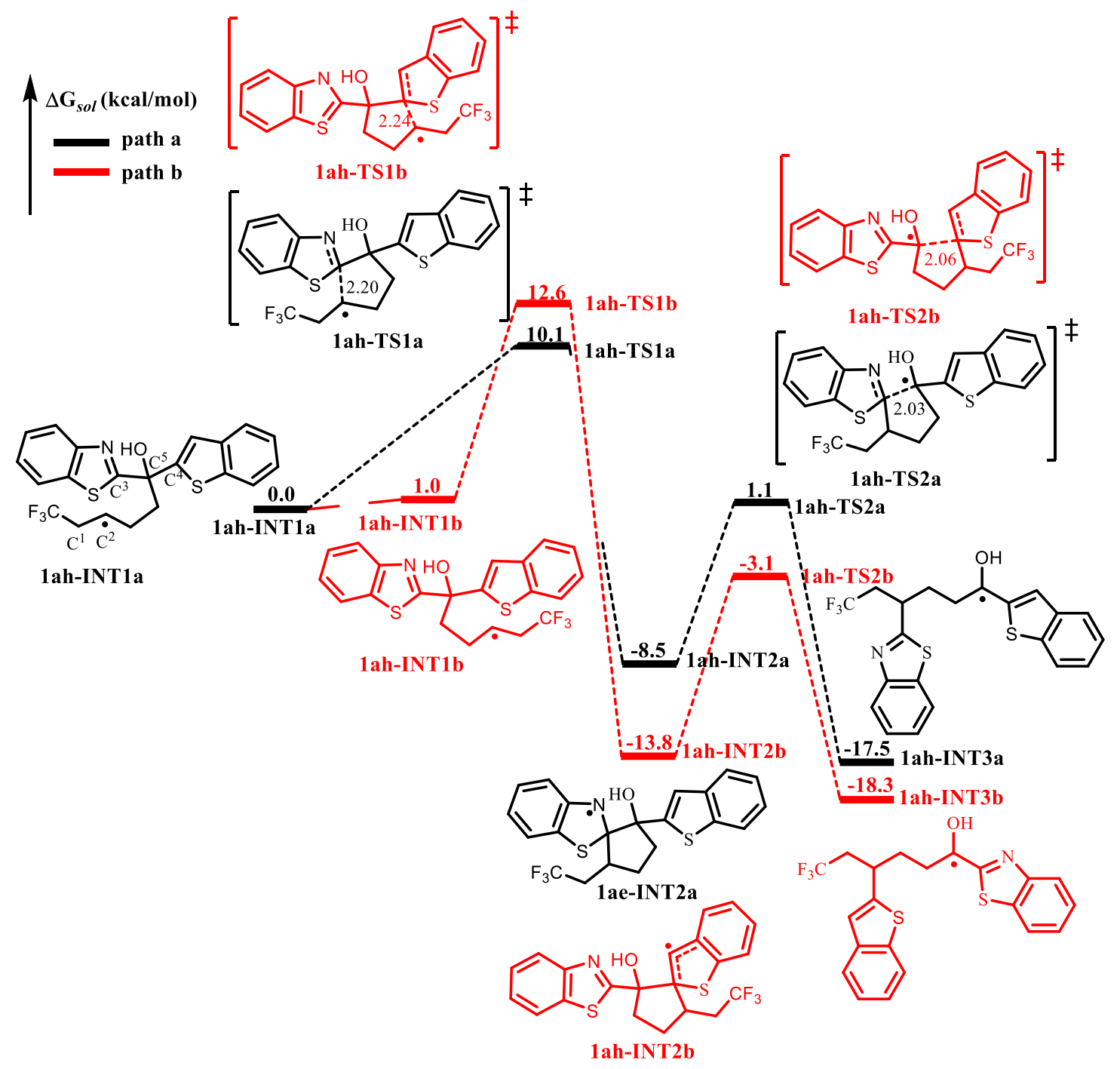

Figure S4. Energy profile (in kcal/mol) for migration of heterocycle of 1ah. Bond lengths are shown in $\AA$. 


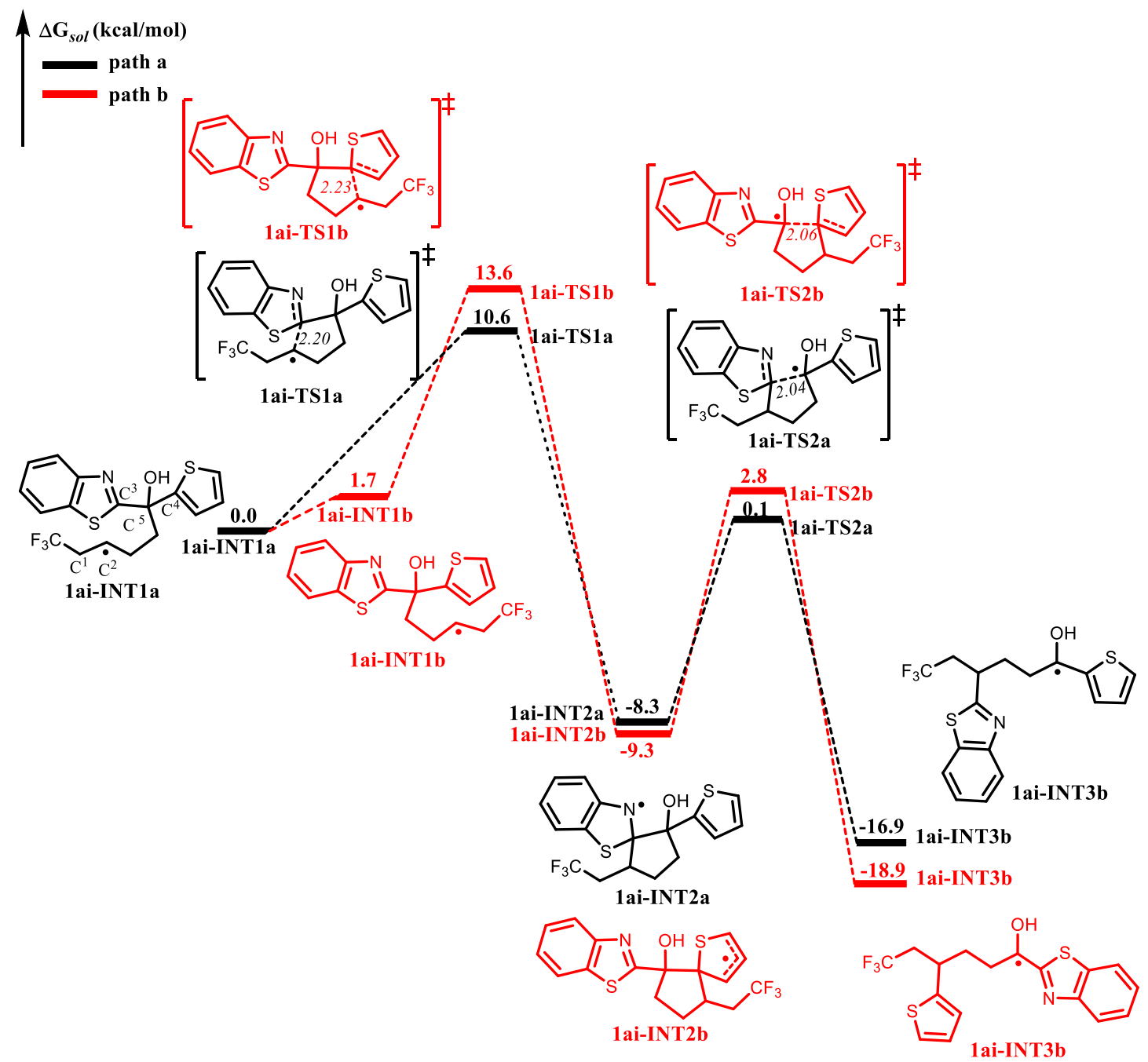

Figure S5. Energy profile (in $\mathrm{kcal} / \mathrm{mol}$ ) for migration of heterocycle of 1ai. Bond lengths are shown in $\AA$. 


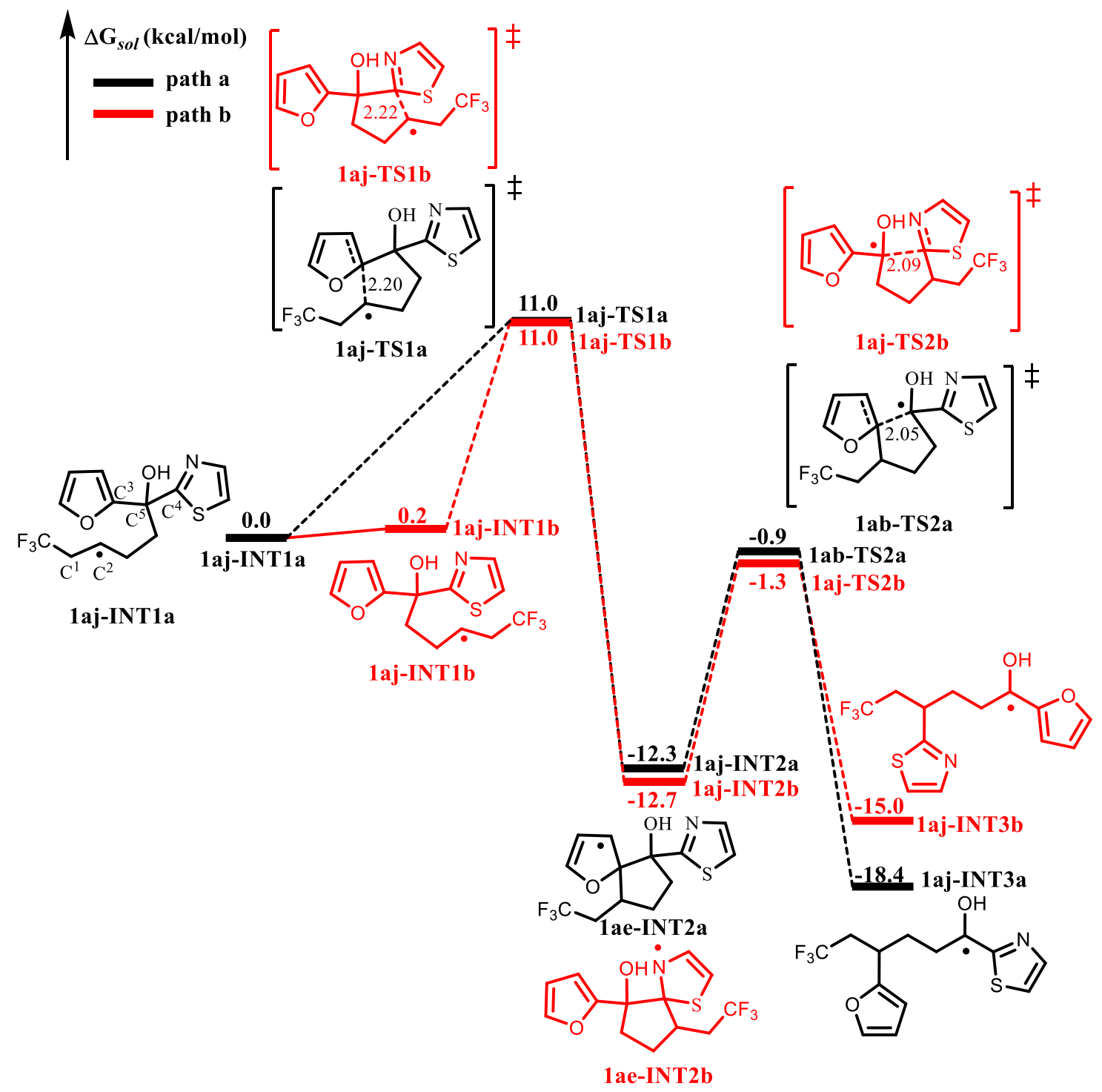

Figure S6. Energy profile (in kcal/mol) for migration of heterocycle of 1aj. Bond lengths are shown in $\AA$. 


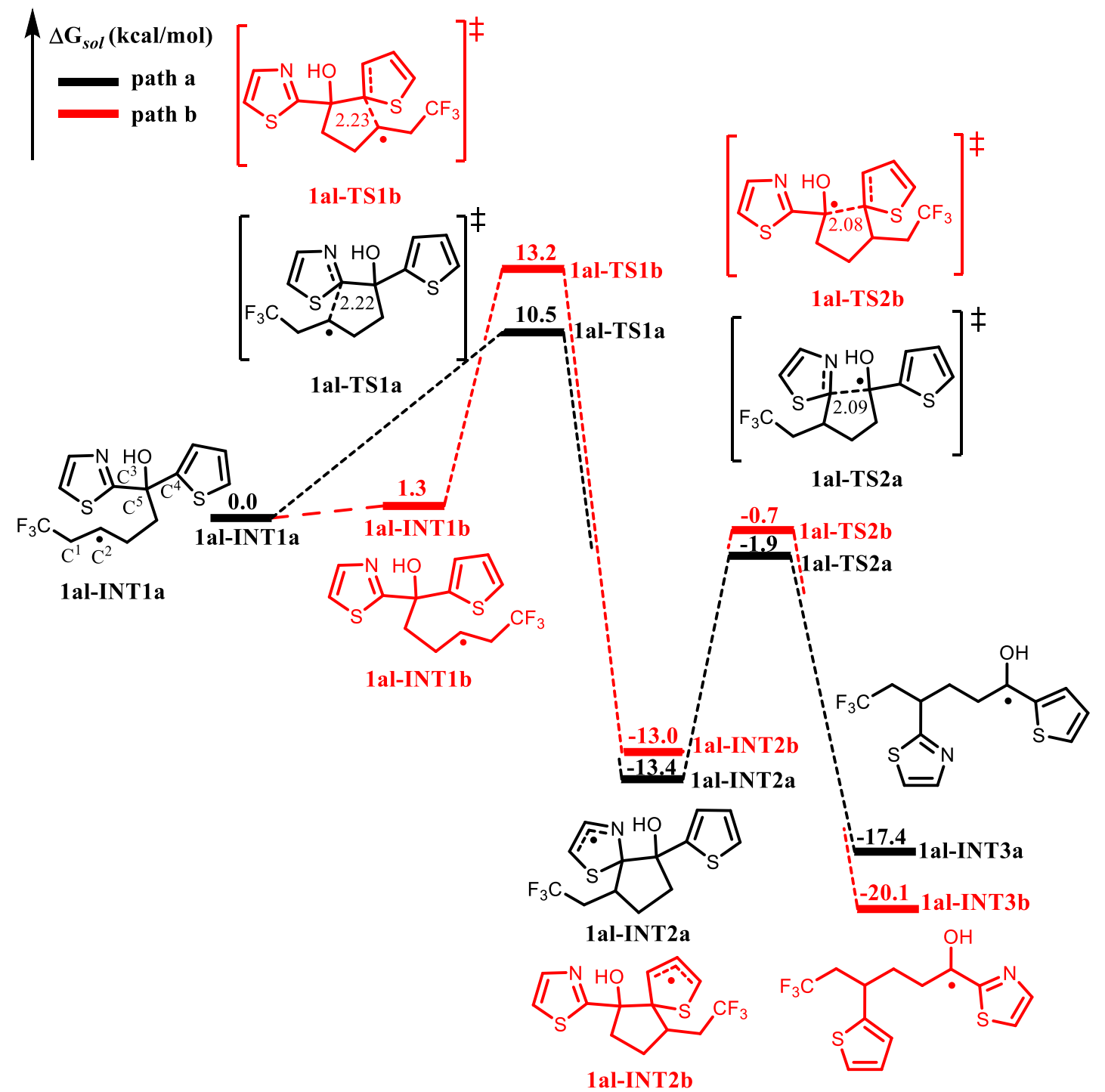

Figure S7. Energy profile (in $\mathrm{kcal} / \mathrm{mol}$ ) for migration of heterocycle of 1al. Bond lengths are shown in $\AA$. 


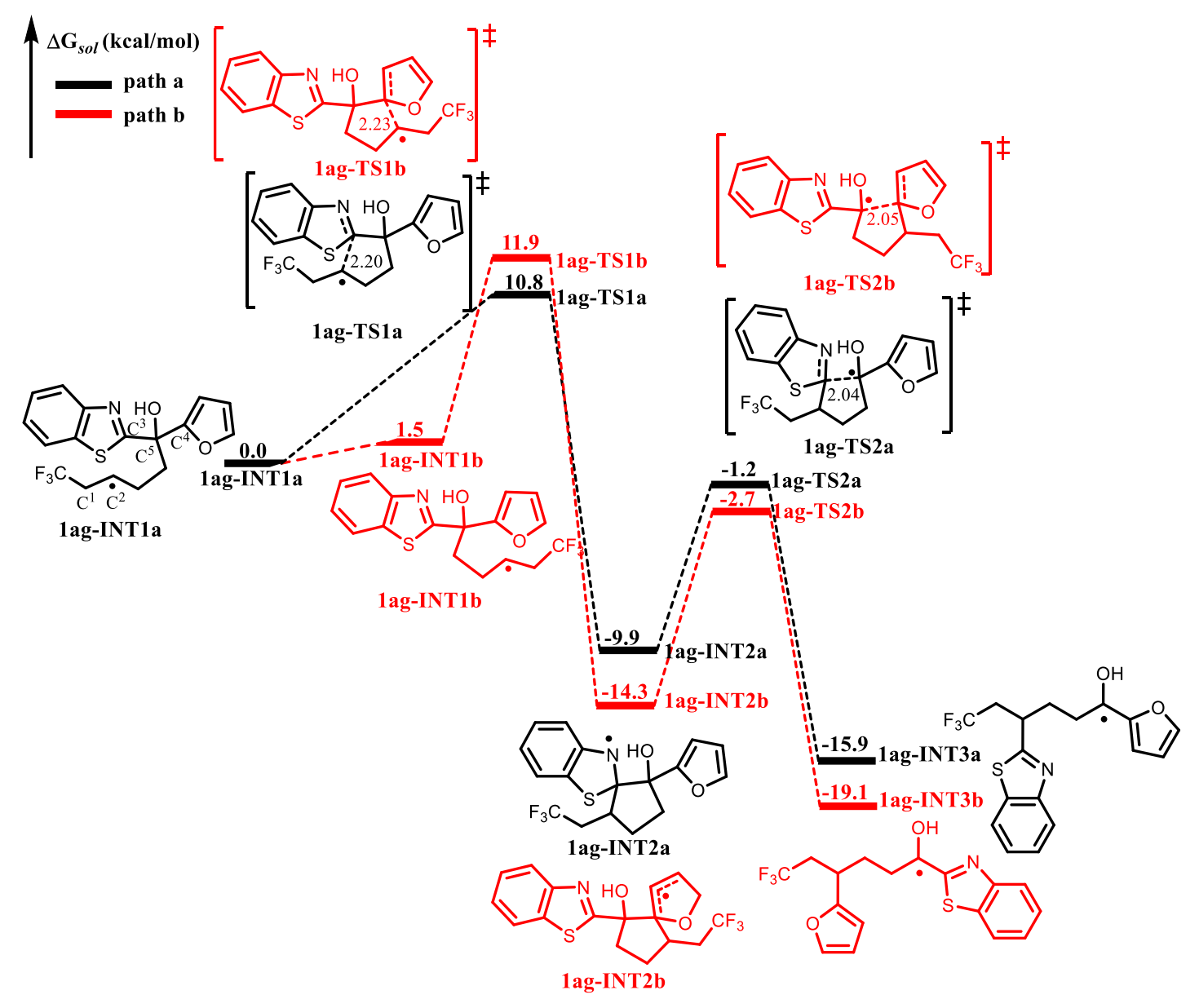

Figure S8. Energy profile (in $\mathrm{kcal} / \mathrm{mol}$ ) for migration of heterocycle of 1ag. Bond lengths are shown in $\AA$. 


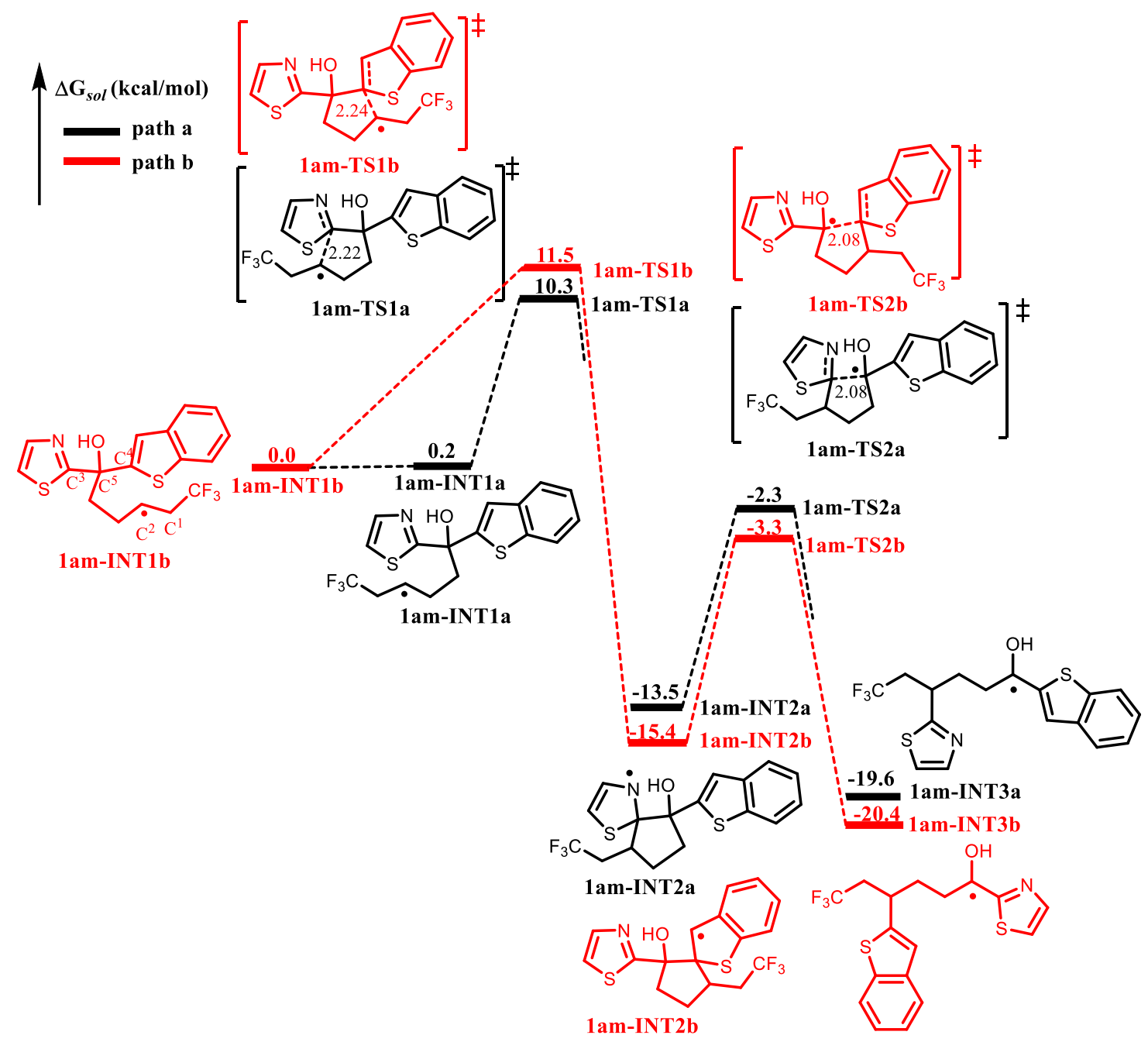

Figure S9. Energy profile (in $\mathrm{kcal} / \mathrm{mol}$ ) for migration of heterocycle of 1am. Bond lengths are shown in $\AA$.

Table S2 Comparisons between calculated and experimental $\Delta \Delta G^{\ddagger}$ values

\begin{tabular}{|c|c|c|c|}
\hline Compound & Experimental Ratio & $\begin{array}{c}\text { Experimental } \Delta \Delta G^{\ddagger} \\
(\mathrm{kcal} / \mathrm{mol})\end{array}$ & $\begin{array}{c}\text { Calculated } \Delta \Delta G^{\ddagger} \\
(\mathrm{kcal} / \mathrm{mol})\end{array}$ \\
\hline 1ab & $>19 / 1$ & -1.7 & -0.7 \\
\hline 1ae & $10 / 1$ & -1.36 & -1.5 \\
\hline 1ag & $10 / 1$ & -1.36 & -1.1 \\
\hline 1ah & $5 / 3$ & -0.30 & -2.5 \\
\hline 1ai & $4 / 1$ & -0.82 & -3.0 \\
\hline 1aj & $1 / 1$ & 0 & 0 \\
\hline 1al & $4 / 1$ & -0.82 & -2.7 \\
\hline 1am & $4 / 3$ & -0.17 & -1.2 \\
\hline 1an & $3 / 1$ & -0.65 & -1.7 \\
\hline
\end{tabular}




\section{Cartesian Coordinates and Energies}

\begin{tabular}{|c|c|c|c|c|c|}
\hline \multirow{2}{*}{$\begin{array}{l}\text { Center } \\
\text { Number }\end{array}$} & \multirow{2}{*}{$\begin{array}{l}\text { Atomic } \\
\text { Number }\end{array}$} & \multirow{2}{*}{$\begin{array}{c}\text { Atomic } \\
\text { Type }\end{array}$} & \multicolumn{3}{|c|}{ Coordinates (Angstroms) } \\
\hline & & & X & $\mathrm{Y}$ & Z \\
\hline 1 & 6 & 0 & -1.465546 & -1.070022 & 0.901781 \\
\hline 2 & 6 & 0 & -0.380912 & -2.066624 & 1. 167266 \\
\hline 3 & 6 & 0 & 0.886639 & -1.904823 & 0.308711 \\
\hline 4 & 6 & 0 & 1. 770224 & -0.702046 & 0.703273 \\
\hline 5 & 1 & 0 & -1.865282 & -0.474032 & 1. 715534 \\
\hline 6 & 1 & 0 & -0.761681 & -3.082073 & 0.974329 \\
\hline 7 & 1 & 0 & -0.102517 & -2.033470 & 2. 225376 \\
\hline 8 & 1 & 0 & 1. 502366 & -2.806682 & 0.408900 \\
\hline 9 & 1 & 0 & 0.630002 & -1.816726 & -0.753290 \\
\hline 10 & 6 & 0 & -2.166402 & -1.006081 & -0.418501 \\
\hline 11 & 1 & 0 & -2.423732 & 0.024139 & -0.693981 \\
\hline 12 & 1 & 0 & -1.563301 & -1.433921 & -1.227089 \\
\hline 13 & 6 & 0 & -3.468783 & -1.770193 & -0.408265 \\
\hline 14 & 9 & 0 & -4.105103 & -1.713341 & -1.598191 \\
\hline 15 & 9 & 0 & -3.299912 & -3.080569 & -0.128247 \\
\hline 16 & 9 & 0 & -4.338466 & -1.297084 & 0.510143 \\
\hline 17 & 8 & 0 & 2. 171335 & -0.795564 & 2. 067470 \\
\hline 18 & 1 & 0 & 2. 658475 & -1.629589 & 2. 179296 \\
\hline 19 & 6 & 0 & 1. 012562 & 0.589663 & 0.607296 \\
\hline 20 & 6 & 0 & -0.073548 & 2. 526083 & 0.837946 \\
\hline 21 & 6 & 0 & -0.256188 & 2. 019247 & -0.457341 \\
\hline 22 & 6 & 0 & -1.014948 & 2. 647114 & -1.437504 \\
\hline 23 & 6 & 0 & -0.690717 & 3. 737699 & 1. 182476 \\
\hline 24 & 6 & 0 & -1.458636 & 4. 383703 & 0.220082 \\
\hline 25 & 6 & 0 & -1.619352 & 3.846866 & -1.071681 \\
\hline 26 & 1 & 0 & -1.129830 & 2. 220230 & -2.429264 \\
\hline 27 & 1 & 0 & -2.226929 & 4. 378364 & -1.798312 \\
\hline 28 & 1 & 0 & -1.946864 & 5. 322222 & 0.466634 \\
\hline 29 & 1 & 0 & -0.570950 & 4. 158912 & 2. 176673 \\
\hline 30 & 6 & 0 & 4. 744323 & -0.219889 & -1.506071 \\
\hline 31 & 6 & 0 & 4. 808148 & -1.494491 & -1.043409 \\
\hline 32 & 6 & 0 & 2. 983752 & -0.630054 & -0.187907 \\
\hline 33 & 8 & 0 & 0.416292 & 0.840702 & -0.598905 \\
\hline 34 & 6 & 0 & 0.771603 & 1. 566240 & 1. 512500 \\
\hline 35 & 1 & 0 & 1. 128877 & 1. 590777 & 2.532195 \\
\hline 36 & 1 & 0 & 5. 454280 & 0.260709 & -2.165231 \\
\hline 37 & 1 & 0 & 5.507606 & -2.306187 & -1.182700 \\
\hline 38 & 6 & 0 & 3. 550790 & 0.348505 & -0.943418 \\
\hline 39 & 1 & 0 & 3. 169623 & 1. 351122 & -1.085950 \\
\hline 40 & 8 & 0 & 3. 741515 & -1.758917 & -0.243547 \\
\hline
\end{tabular}

Zero-point correction=

0. 308362 (Hartree/Particle)

Thermal correction to Energy=

0.329508

Thermal correction to Enthalpy=

0.330452

Thermal correction to Gibbs Free Energy=

Sum of electronic and zero-point Energies=

Sum of electronic and thermal Energies=

0.255194

$-1219.958034$

$-1219.936889$

$-1219.935945$

$-1220.011202$

Sum of electronic and thermal Free Energies

M06 $-2 \mathrm{x} / 6-311++\mathrm{G}(\mathrm{d}, \mathrm{p}) / \mathrm{SMD} / / \mathrm{M} 06-2 \mathrm{x} / 6-31+\mathrm{G}(\mathrm{d}) / \mathrm{SMD} \quad$ energy $=-1220.58508050$

1ab-INT1b

\begin{tabular}{rrrrrr} 
Center & Atomic & Atomic & \multicolumn{3}{c}{ Coordinates (Angstroms) } \\
Number & Number & Type & X & Y & $Z$ \\
-1 & 6 & 0 & -2.638678 & -0.570672 & 1.192543 \\
2 & 6 & 0 & -1.671460 & -1.706652 & 1.161485 \\
3 & 6 & 0 & -0.179356 & -1.308068 & 1.132237 \\
4 & 6 & 0 & 0.232364 & -0.493097 & -0.105447 \\
5 & 1 & 0 & -2.815836 & -0.045800 & 2.126975 \\
6 & 1 & 0 & -1.876167 & -2.344236 & 0.294051 \\
7 & 1 & 0 & -1.805977 & -2.325351 & 2.057690 \\
8 & 1 & 0 & 0.081754 & -0.736823 & 2.029721
\end{tabular}




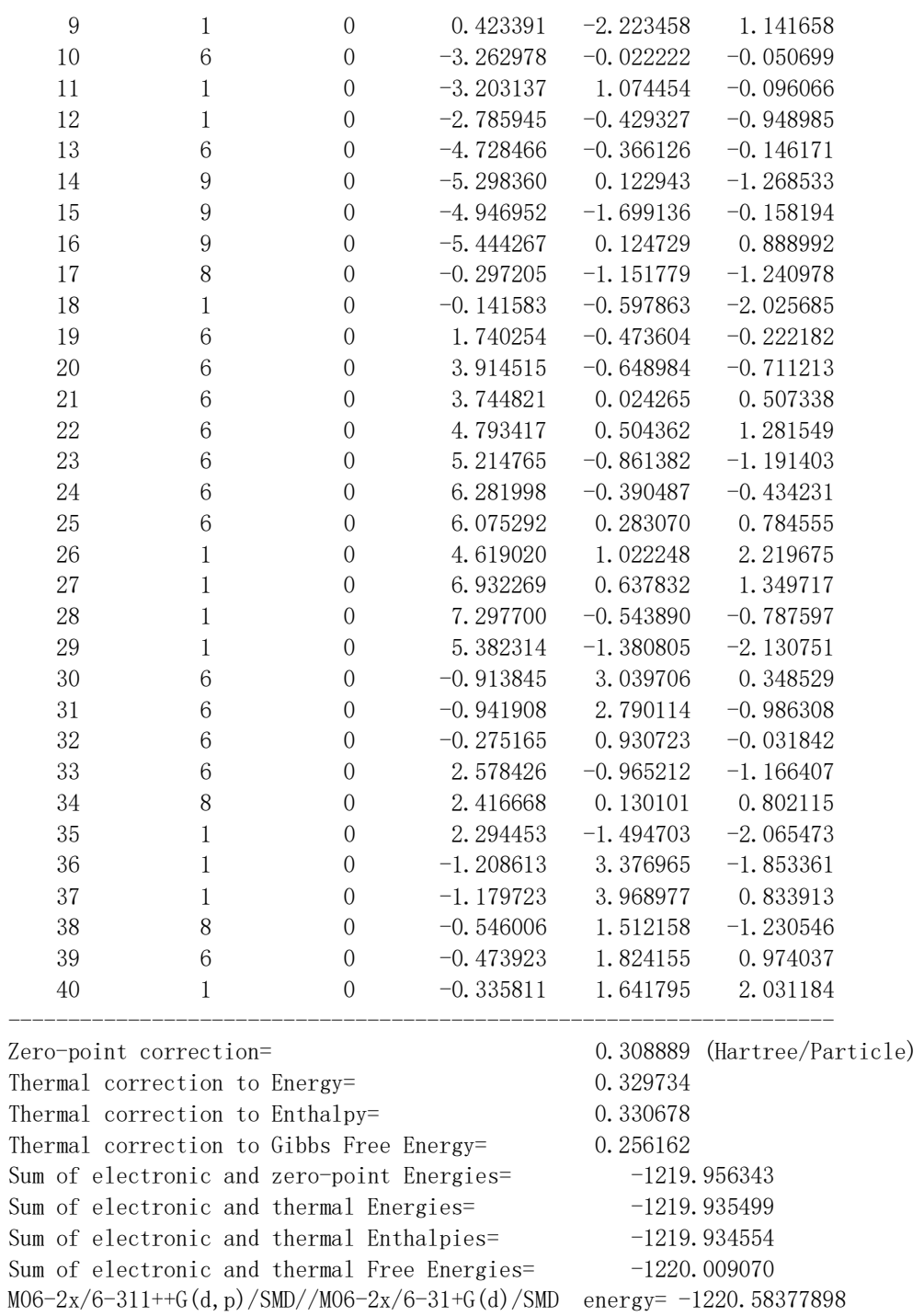

1ab-TS1a

\begin{tabular}{rrrrrr} 
Center & Atomic & Atomic & \multicolumn{3}{c}{ Coordinates (Angstroms) } \\
Number & Number & Type & X & Y & $Z$ \\
-1 & 6 & 0 & -1.144876 & -1.386450 & 0.509222 \\
2 & 6 & 0 & -0.413674 & -2.595953 & -0.013719 \\
3 & 6 & 0 & 0.966825 & -2.187835 & -0.558712 \\
4 & 6 & 0 & 1.540327 & -1.125891 & 0.383415 \\
5 & 1 & 0 & -1.356502 & -1.359966 & 1.575415 \\
6 & 1 & 0 & -0.982512 & -3.086713 & -0.813186 \\
7 & 1 & 0 & -0.284023 & -3.323505 & 0.792553 \\
8 & 1 & 0 & 1.644126 & -3.046069 & -0.629661 \\
9 & 1 & 0 & 0.878051 & -1.753040 & -1.560896 \\
10 & 6 & 0 & -2.149633 & -0.685903 & -0.358746 \\
11 & 1 & 0 & -2.264728 & 0.368035 & -0.073996 \\
12 & 1 & 0 & -1.870583 & -0.728682 & -1.417209 \\
13 & 6 & 0 & -3.529563 & -1.286647 & -0.244541 \\
14 & 9 & 0 & -4.421680 & -0.666105 & -1.047011 \\
15 & 9 & 0 & -3.564191 & -2.594451 & -0.578945 \\
16 & 9 & 0 & -4.025458 & -1.204091 & 1.008777 \\
17 & 8 & 0 & 1.618504 & -1.621209 & 1.717774 \\
18 & 1 & 0 & 2.162005 & -2.427327 & 1.706922 \\
19 & 6 & 0 & 0.576286 & 0.039626 & 0.458164 \\
20 & 6 & 0 & -0.151293 & 2.126598 & 0.915109 \\
21 & 6 & 0 & -0.130106 & 1.910645 & -0.477225 \\
22 & 6 & 0 & -0.554015 & 2.843319 & -1.412015 \\
23 & 6 & 0 & -0.620485 & 3.361844 & 1.397055
\end{tabular}




$\begin{array}{rrrrrr}24 & 6 & 0 & -1.044368 & 4.313226 & 0.476491 \\ 25 & 6 & 0 & -1.013649 & 4.060292 & -0.908090 \\ 26 & 1 & 0 & -0.526847 & 2.632785 & -2.476787 \\ 27 & 1 & 0 & -1.353745 & 4.826569 & -1.598430 \\ 28 & 1 & 0 & -1.409654 & 5.273309 & 0.830053 \\ 29 & 1 & 0 & -0.650679 & 3.564191 & 2.463967 \\ 30 & 6 & 0 & 4.807020 & 0.364167 & -0.644462 \\ 31 & 6 & 0 & 4.989592 & -0.980203 & -0.594569 \\ 32 & 6 & 0 & 2.889108 & -0.633790 & -0.066980 \\ 33 & 8 & 0 & 0.351837 & 0.666571 & -0.755531 \\ 34 & 6 & 0 & 0.364038 & 0.928219 & 1.502467 \\ 35 & 1 & 0 & 0.518844 & 0.710829 & 2.550198 \\ 36 & 1 & 0 & 5.552873 & 1.105247 & -0.897330 \\ 37 & 1 & 0 & 5.836891 & -1.626625 & -0.773237 \\ 38 & 6 & 0 & 3.431951 & 0.592145 & -0.296142 \\ 39 & 1 & 0 & 2.920206 & 1.543536 & -0.229526 \\ 40 & 8 & 0 & 3.830576 & -1.599762 & -0.249065\end{array}$

Zero-point correction=

0. 308174 (Hartree/Particle)

Thermal correction to Energy=

0.328240

Thermal correction to Enthalpy=

Thermal correction to Gibbs Free Energy=

Sum of electronic and zero-point Energies=

Sum of electronic and thermal Energies=

0.329185

0. 257227

$-1219.944300$

$-1219.924234$

$-1219.923290$

Sum of electronic and thermal Free Energies=

$-1219.995247$

M06 $-2 \mathrm{x} / 6-311++\mathrm{G}(\mathrm{d}, \mathrm{p}) / \mathrm{SMD} / / \mathrm{M} 06-2 \mathrm{x} / 6-31+\mathrm{G}(\mathrm{d}) / \mathrm{SMD} \quad$ energy $=-1220.57082855$

$1 \mathrm{ab}-\mathrm{TS} 1 \mathrm{~b}$

\begin{tabular}{|c|c|c|c|c|c|}
\hline \multirow{2}{*}{$\begin{array}{l}\text { Center } \\
\text { Number }\end{array}$} & \multirow{2}{*}{$\begin{array}{l}\text { Atomic } \\
\text { Number }\end{array}$} & \multirow{2}{*}{$\begin{array}{c}\text { Atomic } \\
\text { Type }\end{array}$} & \multicolumn{3}{|c|}{ Coordinates (Angstroms) } \\
\hline & & & $\mathrm{X}$ & Y & Z \\
\hline 1 & 6 & 0 & 2. 321604 & -0.162265 & -0.729902 \\
\hline 2 & 6 & 0 & 1. 797325 & -1.561130 & -0.917771 \\
\hline 3 & 6 & 0 & 0.263259 & -1.526775 & -0.961606 \\
\hline 4 & 6 & 0 & -0.199641 & -0.643339 & 0.197089 \\
\hline 5 & 1 & 0 & 2. 440459 & 0.432854 & -1.633718 \\
\hline 6 & 1 & 0 & 2. 117737 & -2.198452 & -0.087879 \\
\hline 7 & 1 & 0 & 2. 179128 & -2.006820 & -1.845363 \\
\hline 8 & 1 & 0 & -0.088088 & -1.084355 & -1.900781 \\
\hline 9 & 1 & 0 & -0.177625 & -2.524881 & -0.870197 \\
\hline 10 & 6 & 0 & 3. 307832 & 0.135573 & 0.361214 \\
\hline 11 & 1 & 0 & 3. 362483 & 1. 209917 & 0.572989 \\
\hline 12 & 1 & 0 & 3. 043857 & -0.386124 & 1. 288044 \\
\hline 13 & 6 & 0 & 4. 712940 & -0.287658 & 0.009262 \\
\hline 14 & 9 & 0 & 5. 588188 & -0.028843 & 1. 006060 \\
\hline 15 & 9 & 0 & 4. 813559 & -1.609515 & -0.249914 \\
\hline 16 & 9 & 0 & 5. 186179 & 0.347621 & -1.084970 \\
\hline 17 & 8 & 0 & 0.274807 & -1.229889 & 1. 392171 \\
\hline 18 & 1 & 0 & 0.217941 & -0.573124 & 2. 107411 \\
\hline 19 & 6 & 0 & -1.702140 & -0.543693 & 0.266676 \\
\hline 20 & 6 & 0 & -3.886974 & -0.505094 & 0.743463 \\
\hline 21 & 6 & 0 & -3.673656 & -0.104698 & -0.583615 \\
\hline 22 & 6 & 0 & -4.690114 & 0.257090 & -1.458320 \\
\hline 23 & 6 & 0 & -5.199827 & -0.547477 & 1. 234396 \\
\hline 24 & 6 & 0 & -6.235487 & -0.188374 & 0.378508 \\
\hline 25 & 6 & 0 & -5.985286 & 0.208072 & -0.948938 \\
\hline 26 & 1 & 0 & -4.481741 & 0.559871 & -2.479843 \\
\hline 27 & 1 & 0 & -6.818627 & 0.481231 & -1.589674 \\
\hline 28 & 1 & 0 & -7.260079 & -0.213627 & 0.738345 \\
\hline 29 & 1 & 0 & -5.401121 & -0.852653 & 2. 257407 \\
\hline 30 & 6 & 0 & 0.532062 & 2. 964835 & -0.337206 \\
\hline 31 & 6 & 0 & 0.899931 & 2. 686500 & 0.947296 \\
\hline 32 & 6 & 0 & 0.420157 & 0.740425 & 0.017705 \\
\hline 33 & 6 & 0 & -2.572822 & -0.788784 & 1. 276027 \\
\hline 34 & 8 & 0 & -2.340270 & -0.130682 & -0.871450 \\
\hline 35 & 1 & 0 & -2.322866 & -1.135231 & 2.269319 \\
\hline 36 & 1 & 0 & 1. 253293 & 3. 291277 & 1. 770147 \\
\hline 37 & 1 & 0 & 0.525048 & 3. 943587 & -0.798193 \\
\hline 38 & 8 & 0 & 0.776961 & 1. 353179 & 1. 194381 \\
\hline
\end{tabular}




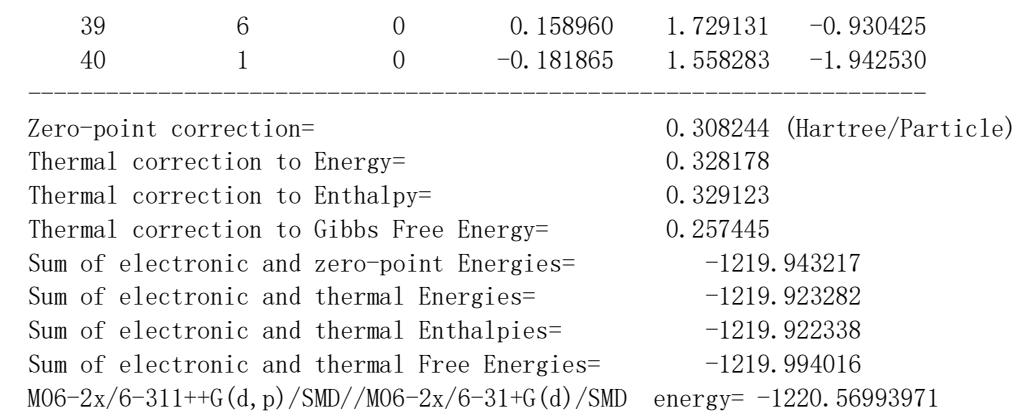

1ab-INT2a

\begin{tabular}{|c|c|c|c|c|c|}
\hline \multirow{2}{*}{$\begin{array}{l}\text { Center } \\
\text { Number }\end{array}$} & \multirow{2}{*}{$\begin{array}{l}\text { Atomic } \\
\text { Number }\end{array}$} & \multirow{2}{*}{$\begin{array}{l}\text { Atomic } \\
\text { Type }\end{array}$} & \multicolumn{3}{|c|}{ Coordinates (Angstroms) } \\
\hline & & & X & Y & Z \\
\hline 1 & 6 & 0 & -1.659125 & -0.615180 & $0.17366^{\circ}$ \\
\hline 2 & 6 & 0 & -1.763176 & -1.803895 & $-0.81400^{\prime}$ \\
\hline 3 & 6 & 0 & -0.336360 & -2.385663 & -0.95045 \\
\hline 4 & 6 & 0 & 0.485677 & -1.732598 & 0.15837 \\
\hline 5 & 1 & 0 & -1.941030 & -0.946213 & 1. 17914 \\
\hline 6 & 1 & 0 & -2.130097 & -1.462398 & -1.78689 \\
\hline 7 & 1 & 0 & -2.470281 & -2.550268 & -0.44385 \\
\hline 8 & 1 & 0 & -0.297655 & -3.473335 & -0.84743 \\
\hline 9 & 1 & 0 & 0.094220 & -2.122046 & -1.91994 \\
\hline 10 & 6 & 0 & -2.477097 & 0.620925 & -0.19266 \\
\hline 11 & 1 & 0 & -2.190250 & 1. 478338 & 0.42768 \\
\hline 12 & 1 & 0 & -2.334469 & 0.893682 & -1.242648 \\
\hline 13 & 6 & 0 & -3.951237 & 0.424825 & 0.01833 \\
\hline 14 & 9 & 0 & -4.657402 & 1.526405 & -0.31642 \\
\hline 15 & 9 & 0 & -4.461490 & -0.588693 & -0.71443 \\
\hline 16 & 9 & 0 & -4.262274 & 0.151937 & 1. 30458 \\
\hline 17 & 8 & 0 & 0.203570 & -2.422454 & 1. 365268 \\
\hline 18 & 1 & 0 & 0.772597 & -2.063598 & 2.068218 \\
\hline 19 & 6 & 0 & -0.155564 & -0.297185 & 0.24542 \\
\hline 20 & 6 & 0 & 1. 106390 & 1. 590906 & 0.81637 \\
\hline 21 & 6 & 0 & 1. 026494 & 1. 454554 & -0.59653 \\
\hline 22 & 6 & 0 & 1. 669213 & 2. 307630 & -1.47306 \\
\hline 23 & 6 & 0 & 1. 871851 & 2. 648932 & 1. 35394 \\
\hline 24 & 6 & 0 & 2. 523145 & 3. 512099 & 0.480218 \\
\hline 25 & 6 & 0 & 2. 426313 & 3. 346526 & -0.91179 \\
\hline 26 & 1 & 0 & 1. 589656 & 2. 174872 & -2.54771 \\
\hline 27 & 1 & 0 & 2. 947791 & 4. 035620 & -1.56961 \\
\hline 28 & 1 & 0 & 3. 119750 & 4. 328265 & 0.87753 \\
\hline 29 & 1 & 0 & 1.948429 & 2. 777366 & 2. 429877 \\
\hline 30 & 6 & 0 & 4. 092411 & -1.488824 & -0.75209 \\
\hline 31 & 6 & 0 & 4. 030665 & -1.477636 & 0.60440 \\
\hline 32 & 6 & 0 & 1.959760 & -1.683558 & -0.09746 \\
\hline 33 & 8 & 0 & 0.237868 & 0.410966 & -0.95538 \\
\hline 34 & 6 & 0 & 0.338587 & 0.552658 & 1. 36685 \\
\hline 35 & 1 & 0 & 0.150889 & 0.350417 & 2. 41406 \\
\hline 36 & 1 & 0 & 4. 987553 & -1.416821 & -1.35473 \\
\hline 37 & 1 & 0 & 4. 773004 & -1.410678 & 1. 38678 \\
\hline 38 & 6 & 0 & 2. 738212 & -1.624051 & -1.21234 \\
\hline 39 & 1 & 0 & 2. 394969 & -1.658549 & -2.23739 \\
\hline 40 & 8 & 0 & 2. 740869 & -1.594050 & 1. 01513 \\
\hline
\end{tabular}

Zero-point correction=

0. 310506 (Hartree/Particle)

Thermal correction to Energy=

0.330458

Thermal correction to Enthalpy=

0.331403

Thermal correction to Gibbs Free Energy=

Sum of electronic and zero-point Energies=

Sum of electronic and thermal Energies=

0.259153

$-1219.988899$

$-1219.968947$

$-1219.968003$

$-1220.040253$

Sum of electronic and thermal Free Energies=

energy $=-1220.61752237$

\section{1ab-INT2b}




\begin{tabular}{|c|c|c|c|c|c|}
\hline \multirow{2}{*}{$\begin{array}{l}\text { Center } \\
\text { Number }\end{array}$} & \multirow{2}{*}{$\begin{array}{l}\text { Atomic } \\
\text { Number }\end{array}$} & \multirow{2}{*}{$\begin{array}{c}\text { Atomic } \\
\text { Type }\end{array}$} & \multicolumn{3}{|c|}{ Coordinates (Angstroms) } \\
\hline & & & X & Y & Z \\
\hline 1 & 6 & 0 & 1. 427413 & -0.200382 & 0.199522 \\
\hline 2 & 6 & 0 & 1. 327711 & -0.295613 & 1. 741368 \\
\hline 3 & 6 & 0 & 0.414405 & 0.865339 & 2. 195016 \\
\hline 4 & 6 & 0 & -0.164196 & 1.502901 & 0.914372 \\
\hline 5 & 1 & 0 & 0.688274 & -0.867499 & -0.260087 \\
\hline 6 & 1 & 0 & 2. 320111 & -0.194322 & 2. 191883 \\
\hline 7 & 1 & 0 & 0.931300 & -1.268760 & 2. 041354 \\
\hline 8 & 1 & 0 & -0.378978 & 0.549882 & 2. 877522 \\
\hline 9 & 1 & 0 & 1. 001055 & 1. 636341 & 2. 704776 \\
\hline 10 & 6 & 0 & 2. 798564 & -0.506795 & -0.397356 \\
\hline 11 & 1 & 0 & 2. 819784 & -0.257916 & -1.464905 \\
\hline 12 & 1 & 0 & 3. 586594 & 0.062492 & 0.105352 \\
\hline 13 & 6 & 0 & 3. 168685 & -1.958986 & -0.294436 \\
\hline 14 & 9 & 0 & 4. 361479 & -2.214807 & -0.873985 \\
\hline 15 & 9 & 0 & 3. 268391 & -2.392289 & 0.980994 \\
\hline 16 & 9 & 0 & 2. 272335 & -2.770121 & -0.897714 \\
\hline 17 & 8 & 0 & -0.435567 & 2. 879797 & 1. 024324 \\
\hline 18 & 1 & 0 & 0.421274 & 3. 329515 & 1. 126295 \\
\hline 19 & 6 & 0 & -1.455672 & 0.871489 & 0.492100 \\
\hline 20 & 6 & 0 & -3.472773 & 0.285128 & -0.276293 \\
\hline 21 & 6 & 0 & -2.806282 & -0.837531 & 0.236204 \\
\hline 22 & 6 & 0 & -3.341162 & -2.119586 & 0.251445 \\
\hline 23 & 6 & 0 & -4.760023 & 0. 121260 & -0.809067 \\
\hline 24 & 6 & 0 & -5.317431 & -1.152517 & -0.806501 \\
\hline 25 & 6 & 0 & -4.618940 & -2.257886 & -0.283787 \\
\hline 26 & 1 & 0 & -2.790358 & -2.962049 & 0.658376 \\
\hline 27 & 1 & 0 & -5.085495 & -3.238578 & -0.297760 \\
\hline 28 & 1 & 0 & -6.312577 & -1.301702 & -1.215762 \\
\hline 29 & 1 & 0 & -5.305322 & 0.968756 & -1.214759 \\
\hline 30 & 6 & 0 & 1. 530689 & 2. 538841 & -1.987240 \\
\hline 31 & 6 & 0 & 2. 334281 & 2. 888360 & -0.915406 \\
\hline 32 & 6 & 0 & 0.970178 & 1. 229850 & -0.140562 \\
\hline 33 & 6 & 0 & -2.560455 & 1. 391366 & -0.094727 \\
\hline 34 & 8 & 0 & -1.577873 & -0.477297 & 0.707627 \\
\hline 35 & 1 & 0 & -2.708862 & 2. 428252 & -0.362292 \\
\hline 36 & 1 & 0 & 3. 121062 & 3. 622525 & -0.808194 \\
\hline 37 & 1 & 0 & 1. 586985 & 2. 987275 & -2.971343 \\
\hline 38 & 8 & 0 & 2. 035746 & 2. 158618 & 0.197671 \\
\hline 39 & 6 & 0 & 0.672553 & 1. 534302 & -1.571153 \\
\hline 40 & 1 & 0 & -0.078390 & 1. 014563 & -2.152078 \\
\hline
\end{tabular}

Zero-point correction=

Thermal correction to Energy=

0.310216 (Hartree/Particle)

Thermal correction to Enthalpy=

0. 330119

0.331063

Thermal correction to Gibbs Free Energy=

Sum of electronic and zero-point Energies=

Sum of electronic and thermal Energies=

0.259036

$-1219.984221$

$-1219.964319$

$-1219.963375$

Sum of electronic and thermal Enthalpies=
Sum of electronic and thermal Free Energies

$-1220.035401$

M06 $-2 \mathrm{x} / 6-311++G(\mathrm{~d}, \mathrm{p}) / \mathrm{SMD} / /$ M06 $-2 \mathrm{x} / 6-31+\mathrm{G}(\mathrm{d}) / \mathrm{SMD} \quad$ energy $=-1220.61253297$

$1 \mathrm{ab}-\mathrm{TS} 2 \mathrm{a}$

\begin{tabular}{rrrrrr} 
Center & Atomic & Atomic & \multicolumn{3}{c}{ Coordinates (Angstroms) } \\
Number & Number & Type & X & Y & $Z$ \\
-1 & 6 & 0 & -1.768695 & -0.309785 & 0.193528 \\
2 & 6 & 0 & -1.932226 & -1.365574 & -0.915527 \\
3 & 6 & 0 & -0.636682 & -2.207487 & -1.024038 \\
4 & 6 & 0 & 0.304295 & -1.880719 & 0.097269 \\
5 & 1 & 0 & -1.995515 & -0.764279 & 1.164015 \\
6 & 1 & 0 & -2.129181 & -0.871286 & -1.872804 \\
7 & 1 & 0 & -2.797613 & -1.997353 & -0.698904 \\
8 & 1 & 0 & -0.859068 & -3.281297 & -1.001695 \\
9 & 1 & 0 & -0.131725 & -2.001062 & -1.971093 \\
10 & 6 & 0 & -2.656380 & 0.929489 & 0.016421 \\
11 & 1 & 0 & -2.470634 & 1.641964 & 0.826510 \\
12 & 1 & 0 & -2.453261 & 1.430177 & -0.936081
\end{tabular}




\begin{tabular}{|c|c|c|c|c|c|}
\hline 13 & 6 & 0 & -4.129106 & 0.624967 & 0.048443 \\
\hline 14 & 9 & 0 & -4.868511 & 1. 754971 & 0.080049 \\
\hline 15 & 9 & 0 & -4.557099 & -0.071741 & -1.025948 \\
\hline 16 & 9 & 0 & -4.488331 & -0.095272 & 1. 133314 \\
\hline 17 & 8 & 0 & -0.077719 & -2.435956 & 1. 295877 \\
\hline 18 & 1 & 0 & 0.570467 & -2.193646 & 1. 981214 \\
\hline 19 & 6 & 0 & -0.323448 & 0.110463 & 0.278092 \\
\hline 20 & 6 & 0 & 1. 469204 & 1. 375286 & 0.844968 \\
\hline 21 & 6 & 0 & 1. 302987 & 1. 361691 & -0.557023 \\
\hline 22 & 6 & 0 & 2. 172933 & 1. 981012 & -1.441397 \\
\hline 23 & 6 & 0 & 2. 589784 & 2. 036360 & 1. 381564 \\
\hline 24 & 6 & 0 & 3. 477263 & 2. 657551 & 0.509108 \\
\hline 25 & 6 & 0 & 3. 273205 & 2. 634405 & -0.883095 \\
\hline 26 & 1 & 0 & 2. 009721 & 1. 943662 & -2.514409 \\
\hline 27 & 1 & 0 & 3. 985483 & 3. 129829 & -1.536593 \\
\hline 28 & 1 & 0 & 4. 348508 & 3. 169924 & 0.907847 \\
\hline 29 & 1 & 0 & 2. 756188 & 2. 058546 & 2. 455190 \\
\hline 30 & 6 & 0 & 3. 829074 & -1.323026 & -0.761047 \\
\hline 31 & 6 & 0 & 3. 771798 & -1.448344 & 0.593286 \\
\hline 32 & 6 & 0 & 1. 722092 & -1.775170 & -0.136548 \\
\hline 33 & 8 & 0 & 0.183447 & 0.665934 & -0.896995 \\
\hline 34 & 6 & 0 & 0.368002 & 0.640084 & 1. 375101 \\
\hline 35 & 1 & 0 & 0.146170 & 0.431727 & 2. 414203 \\
\hline 36 & 1 & 0 & 4. 712715 & -1.105510 & -1.345929 \\
\hline 37 & 1 & 0 & 4. 510369 & -1.392223 & 1. 380101 \\
\hline 38 & 6 & 0 & 2. 500491 & -1.536446 & -1.243751 \\
\hline 39 & 1 & 0 & 2. 158606 & -1.491466 & -2.268797 \\
\hline 40 & 8 & 0 & 2. 501358 & -1.733462 & 0.985914 \\
\hline
\end{tabular}

Zero-point correction=

0. 309261 (Hartree/Particle)

Thermal correction to Energy=

0.329068

Thermal correction to Enthalpy=

0.330012

Thermal correction to Gibbs Free Energy=

Sum of electronic and zero-point Energies=

Sum of electronic and thermal Energies=

0.259476

$-1219.967336$

$-1219.947529$

$-1219.946584$

Sum of electronic and thermal Free Energies=

$-1220.017121$

M06 $-2 x / 6-311++G(d, p) / S M D / / M 06-2 x / 6-31+G(d) / S M D \quad$ energy $=-1220.59543794$

$1 \mathrm{ab}-\mathrm{TS} 2 \mathrm{~b}$

\begin{tabular}{rrrrrr} 
Center & Atomic & Atomic & \multicolumn{2}{c}{ Coordinates (Angstroms) } \\
Number & Number & Type & X & \multicolumn{1}{c}{ Y } & $Z$ \\
-1 & 6 & 0 & 1.839923 & -0.176509 & 0.083468 \\
2 & 6 & 0 & 1.976076 & 0.006947 & 1.596908 \\
3 & 6 & 0 & 0.545695 & 0.015404 & 2.140888 \\
4 & 6 & 0 & -0.329396 & 0.912876 & 1.290969 \\
5 & 1 & 0 & 1.372773 & -1.150518 & -0.113297 \\
6 & 1 & 0 & 2.493896 & 0.951402 & 1.814293 \\
7 & 1 & 0 & 2.557074 & -0.796718 & 2.056649 \\
8 & 1 & 0 & 0.149322 & -1.004399 & 2.107793 \\
9 & 1 & 0 & 0.506570 & 0.357431 & 3.182245 \\
10 & 6 & 0 & 3.150411 & -0.087914 & -0.709641 \\
11 & 1 & 0 & 2.945389 & -0.201709 & -1.779327 \\
12 & 1 & 0 & 3.642377 & 0.878117 & -0.557228 \\
13 & 6 & 0 & 4.152851 & -1.150053 & -0.350420 \\
14 & 9 & 0 & 5.174752 & -1.186031 & -1.233085 \\
15 & 9 & 0 & 4.715578 & -0.966189 & 0.863494 \\
16 & 9 & 0 & 3.618975 & -2.390419 & -0.335790 \\
17 & 8 & 0 & -0.367845 & 2.247331 & 1.631268 \\
18 & 1 & 0 & 0.529058 & 2.616354 & 1.551680 \\
19 & 6 & 0 & -1.646682 & 0.462944 & 0.897862 \\
20 & 6 & 0 & -3.724968 & 0.162500 & 0.108785 \\
21 & 6 & 0 & -3.048970 & -1.067550 & 0.178180 \\
22 & 6 & 0 & -3.610925 & -2.283286 & -0.185363 \\
23 & 6 & 0 & -5.048480 & 0.177451 & -0.358411 \\
24 & 6 & 0 & -5.631749 & -1.028599 & -0.733425 \\
25 & 6 & 0 & -4.925122 & -2.242600 & -0.648874 \\
26 & 1 & 0 & -3.053534 & -3.212206 & -0.112162 \\
27 & 1 & 0 & -5.411862 & -3.166075 & -0.948779
\end{tabular}




$\begin{array}{rrrrrr}28 & 1 & 0 & -6.654789 & -1.036884 & -1.098817 \\ 29 & 1 & 0 & -5.602522 & 1.109588 & -0.425716 \\ 30 & 6 & 0 & -0.107353 & 2.089530 & -2.077705 \\ 31 & 6 & 0 & 0.696371 & 2.896445 & -1.317269 \\ 32 & 6 & 0 & 0.869499 & 0.861804 & -0.435005 \\ 33 & 6 & 0 & -2.784413 & 1.147581 & 0.578065 \\ 34 & 8 & 0 & -1.786090 & -0.882670 & 0.657738 \\ 35 & 1 & 0 & -2.927082 & 2.215762 & 0.663818 \\ 36 & 1 & 0 & 0.903515 & 3.957317 & -1.328323 \\ 37 & 1 & 0 & -0.727135 & 2.413994 & -2.903370 \\ 38 & 8 & 0 & 1.358167 & 2.164401 & -0.378192 \\ 39 & 6 & 0 & 0.043955 & 0.777193 & -1.578722 \\ 40 & 1 & 0 & -0.440989 & -0.125185 & -1.929273\end{array}$

Zero-point correction=

0. 308902 (Hartree/Particle)

Thermal correction to Energy=

0.328722

Thermal correction to Enthalpy=

Thermal correction to Gibbs Free Energy=

Sum of electronic and zero-point Energies=

Sum of electronic and thermal Energies=

0.329666

0.257575

$-1219.963768$

$-1219.943947$

$-1219.943003$

Sum of electronic and thermal Free Energies=

$-1220.015094$

M06 $-2 x / 6-311++G(d, p) / S M D / / M 06-2 x / 6-31+G(d) / S M D \quad$ energy $=-1220.59101317$

1ab-INT3a

\begin{tabular}{|c|c|c|c|c|c|}
\hline \multirow{2}{*}{$\begin{array}{l}\text { Center } \\
\text { Number }\end{array}$} & \multirow{2}{*}{$\begin{array}{l}\text { Atomic } \\
\text { Number }\end{array}$} & \multirow{2}{*}{$\begin{array}{c}\text { Atomic } \\
\text { Type }\end{array}$} & \multicolumn{3}{|c|}{ Coordinates（Angstroms） } \\
\hline & & & X & Y & Z \\
\hline 1 & 6 & 0 & -1.393886 & -0.309300 & -0.079443 \\
\hline 2 & 6 & 0 & -1.587736 & 0.288236 & 1. 326248 \\
\hline 3 & 6 & 0 & -0.438865 & 1. 209489 & 1. 764748 \\
\hline 4 & 6 & 0 & -0.193282 & 2. 332654 & 0.817959 \\
\hline 5 & 1 & 0 & -1.456431 & 0.494642 & -0.822822 \\
\hline 6 & 1 & 0 & -1.689142 & -0.520868 & 2. 058956 \\
\hline 7 & 1 & 0 & -2.522584 & 0.855835 & 1. 342687 \\
\hline 8 & 1 & 0 & -0.688980 & 1. 615357 & 2. 756133 \\
\hline 9 & 1 & 0 & 0.488619 & 0.638687 & 1. 881881 \\
\hline 10 & 6 & 0 & -2.446845 & -1.377674 & -0.430349 \\
\hline 11 & 1 & 0 & -2.248551 & -1.772608 & -1.431643 \\
\hline 12 & 1 & 0 & -2.419088 & -2.212368 & 0.278116 \\
\hline 13 & 6 & 0 & -3.862753 & -0.867307 & -0.450897 \\
\hline 14 & 9 & 0 & -4.699549 & -1.763503 & -1.018001 \\
\hline 15 & 9 & 0 & -4.364716 & -0.617556 & 0.777429 \\
\hline 16 & 9 & 0 & -3.994198 & 0.277005 & -1.155095 \\
\hline 17 & 8 & 0 & -1.295586 & 3. 061582 & 0.496565 \\
\hline 18 & 1 & 0 & -1.051757 & 3. 781182 & -0.112336 \\
\hline 19 & 6 & 0 & -0.032614 & -0.906242 & -0.236556 \\
\hline 20 & 6 & 0 & 2. 056281 & -1.546953 & -0.736503 \\
\hline 21 & 6 & 0 & 1. 590862 & -2.249565 & 0.385567 \\
\hline 22 & 6 & 0 & 2. 341522 & -3.195213 & 1. 072838 \\
\hline 23 & 6 & 0 & 3. 355447 & -1.796899 & -1.201374 \\
\hline 24 & 6 & 0 & 4. 127186 & -2.738133 & -0.528250 \\
\hline 25 & 6 & 0 & 3. 627778 & -3.428644 & 0.592240 \\
\hline 26 & 1 & 0 & 1. 944282 & -3.719041 & 1. 936966 \\
\hline 27 & 1 & 0 & 4. 257676 & -4.157765 & 1. 093561 \\
\hline 28 & 1 & 0 & 5. 136493 & -2.947047 & -0.871430 \\
\hline 29 & 1 & 0 & 3. 747502 & -1.267755 & -2.065622 \\
\hline 30 & 6 & 0 & 3. 190899 & 2. 998305 & -0.337765 \\
\hline 31 & 6 & 0 & 2. 398404 & 3. 947899 & -0.913315 \\
\hline 32 & 6 & 0 & 1. 055641 & 2. 688816 & 0.316066 \\
\hline 33 & 8 & 0 & 0.318425 & -1.862094 & 0.686158 \\
\hline 34 & 6 & 0 & 0.967605 & -0.678202 & -1.121683 \\
\hline 35 & 1 & 0 & 0.942827 & 0.036430 & -1.934355 \\
\hline 36 & 1 & 0 & 4. 260220 & 2. 901258 & -0.471663 \\
\hline 37 & 1 & 0 & 2. 589135 & 4. 773992 & -1.582704 \\
\hline 38 & 6 & 0 & 2. 349031 & 2. 177178 & 0.460296 \\
\hline 39 & 1 & 0 & 2. 634028 & 1. 323436 & 1. 059706 \\
\hline 40 & 8 & 0 & 1. 098896 & 3. 778318 & -0.526896 \\
\hline
\end{tabular}


Thermal correction to Energy=

0.330798

Thermal correction to Enthalpy=

0.331743

Thermal correction to Gibbs Free Energy=

Sum of electronic and zero-point Energies=

Sum of electronic and thermal Energies=

0.256749

$-1219.987201$

$-1219.966439$

$-1219.965495$

$-1220.040488$

Sum of electronic and thermal Free Energies=

M06 $-2 \mathrm{x} / 6-311++G(\mathrm{~d}, \mathrm{p}) / \mathrm{SMD} / /$ M06 $-2 \mathrm{x} / 6-31+\mathrm{G}(\mathrm{d}) / \mathrm{SMD} \quad$ energy $=-1220.61628550$

1ab-INT3b

\begin{tabular}{|c|c|c|c|c|c|}
\hline \multirow{2}{*}{$\begin{array}{l}\text { Center } \\
\text { Number }\end{array}$} & \multirow{2}{*}{$\begin{array}{l}\text { Atomic } \\
\text { Number }\end{array}$} & \multirow{2}{*}{$\begin{array}{c}\text { Atomic } \\
\text { Type }\end{array}$} & \multicolumn{3}{|c|}{ Coordinates (Angstroms) } \\
\hline & & & X & $\mathrm{Y}$ & Z \\
\hline 1 & 6 & 0 & -2.046720 & -0.324595 & -0.204452 \\
\hline 2 & 6 & 0 & -2.020009 & 1. 168114 & -0.579054 \\
\hline 3 & 6 & 0 & -0.690658 & 1. 625500 & -1.211644 \\
\hline 4 & 6 & 0 & 0.411474 & 1. 892066 & -0.240197 \\
\hline 5 & 1 & 0 & -1.951773 & -0.912389 & -1.125597 \\
\hline 6 & 1 & 0 & -2.263524 & 1. 770864 & 0.306844 \\
\hline 7 & 1 & 0 & -2.812439 & 1. 360572 & -1.307798 \\
\hline 8 & 1 & 0 & -0.354252 & 0.880863 & -1.939430 \\
\hline 9 & 1 & 0 & -0.882768 & 2. 553859 & -1.770493 \\
\hline 10 & 6 & 0 & -3.349696 & -0.739737 & 0.506657 \\
\hline 11 & 1 & 0 & -3.312620 & -1.808791 & 0.738045 \\
\hline 12 & 1 & 0 & -3.478338 & -0.192802 & 1. 446710 \\
\hline 13 & 6 & 0 & -4.604523 & -0.526124 & -0.296620 \\
\hline 14 & 9 & 0 & -5.658309 & -1.162024 & 0.260578 \\
\hline 15 & 9 & 0 & -4.967366 & 0.770945 & -0.397733 \\
\hline 16 & 9 & 0 & -4.503140 & -0.991387 & -1.560014 \\
\hline 17 & 8 & 0 & 0.249697 & 2. 866816 & 0.690799 \\
\hline 18 & 1 & 0 & -0.653406 & 3. 224659 & 0.643217 \\
\hline 19 & 6 & 0 & 1. 659185 & 1. 275433 & -0.230824 \\
\hline 20 & 6 & 0 & 3. 734111 & 0.485579 & 0.171349 \\
\hline 21 & 6 & 0 & 3. 147394 & -0.197104 & -0.915737 \\
\hline 22 & 6 & 0 & 3.770465 & -1.220548 & -1.611387 \\
\hline 23 & 6 & 0 & 5. 025917 & 0. 108460 & 0.580043 \\
\hline 24 & 6 & 0 & 5. 667661 & -0.919368 & -0.104927 \\
\hline 25 & 6 & 0 & 5. 052836 & -1.576607 & -1.185055 \\
\hline 26 & 1 & 0 & 3. 281536 & -1.718986 & -2.443096 \\
\hline 27 & 1 & 0 & 5. 581120 & -2.375316 & -1.697439 \\
\hline 28 & 1 & 0 & 6. 665395 & -1.222498 & 0.200467 \\
\hline 29 & 1 & 0 & 5. 510115 & 0.609054 & 1. 413976 \\
\hline 30 & 6 & 0 & 0.920127 & -1.547743 & 1. 685969 \\
\hline 31 & 6 & 0 & 0.358022 & -0.577194 & 2. 453175 \\
\hline 32 & 6 & 0 & -0.889219 & -0.722796 & 0.654290 \\
\hline 33 & 6 & 0 & 2. 765299 & 1. 443305 & 0.600689 \\
\hline 34 & 8 & 0 & 1. 904615 & 0.292824 & -1.172812 \\
\hline 35 & 1 & 0 & 2. 837302 & 2. 141887 & 1. 422694 \\
\hline 36 & 1 & 0 & 0.607927 & -0.147765 & 3. 412909 \\
\hline 37 & 1 & 0 & 1. 810608 & -2.116508 & 1. 919639 \\
\hline 38 & 8 & 0 & -0.748153 & -0.075498 & 1. 844948 \\
\hline 39 & 6 & 0 & 0. 101812 & -1.643936 & 0.510001 \\
\hline 40 & 1 & 0 & 0.244302 & -2.293881 & -0.343269 \\
\hline
\end{tabular}

Zero-point correction $=$

Thermal correction to Energy=

0.310223 (Hartree/Particle)

Thermal correction to Enthalpy=

0.330883

Thermal correction to Gibbs Free Energy=

Sum of electronic and zero-point Energies=

Sum of electronic and thermal Energies=

Sum of electronic and thermal Enthalpies=

Sum of electronic and thermal Free Energies=

0.331827

0.258274

$-1219.987543$

$-1219.966883$

$-1219.965939$

$-1220.039492$

M06 $-2 X / 6-311++G(d, p) / S M D / / M 06-2 X / 6-31+G(d) / S M D \quad$ energy $=-1220.61711733$

1an-INT1a

\begin{tabular}{cccccc} 
Center & Atomic & Atomic & \multicolumn{3}{c}{ Coordinates } \\
Number & Number & Type & X & Y & Z \\
-1 & 6 & 0 & -1.881741 & -0.904310 & 0.780705
\end{tabular}




\begin{tabular}{|c|c|c|c|c|c|}
\hline 2 & 6 & 0 & -0.922482 & -1.912225 & 1. 331734 \\
\hline 3 & 6 & 0 & 0.396347 & -2.069437 & 0.549394 \\
\hline 4 & 6 & 0 & 1. 426133 & -0.957430 & 0.819492 \\
\hline 5 & 1 & 0 & -1.401411 & -2.903678 & 1. 332375 \\
\hline 6 & 1 & 0 & -0.691783 & -1.675808 & 2. 375060 \\
\hline 7 & 1 & 0 & 0.866579 & -3.015507 & 0.837195 \\
\hline 8 & 1 & 0 & 0. 197688 & -2.120567 & -0.527468 \\
\hline 9 & 6 & 0 & -2.562807 & -1.112045 & -0.534539 \\
\hline 10 & 1 & 0 & -2.702204 & -0.168509 & -1.075735 \\
\hline 11 & 1 & 0 & -2.002779 & -1.796624 & -1.182304 \\
\hline 12 & 6 & 0 & -3.941145 & -1.708276 & -0.375834 \\
\hline 13 & 9 & 0 & -4.555182 & -1.901612 & -1.563086 \\
\hline 14 & 9 & 0 & -3.919376 & -2.909220 & 0.241897 \\
\hline 15 & 9 & 0 & -4.762981 & -0.923321 & 0.353581 \\
\hline 16 & 6 & 0 & 0.850910 & 0.410838 & 0.500145 \\
\hline 17 & 6 & 0 & -0.069933 & 2. 543299 & 0.735271 \\
\hline 18 & 6 & 0 & -0.191402 & 2. 366516 & -0.659435 \\
\hline 19 & 6 & 0 & -0.756110 & 3. 348105 & -1.480779 \\
\hline 20 & 6 & 0 & -0.534733 & 3. 737336 & 1. 313775 \\
\hline 21 & 6 & 0 & -1.098762 & 4. 711981 & 0.504145 \\
\hline 22 & 6 & 0 & -1.208651 & 4. 518471 & -0.885290 \\
\hline 23 & 1 & 0 & -0.840901 & 3. 199741 & -2.553788 \\
\hline 24 & 1 & 0 & -1.653558 & 5. 293691 & -1.502618 \\
\hline 25 & 1 & 0 & -1.461305 & 5. 636029 & 0.945246 \\
\hline 26 & 1 & 0 & -0.449768 & 3. 886422 & 2. 387160 \\
\hline 27 & 16 & 0 & 0.457182 & 0.821833 & -1.159004 \\
\hline 28 & 6 & 0 & 4. 373062 & -2.174148 & -1.282680 \\
\hline 29 & 6 & 0 & 4. 976970 & -0.994633 & -0.959617 \\
\hline 30 & 6 & 0 & 3. 061202 & -2.291057 & -0.722765 \\
\hline 31 & 6 & 0 & 2. 690578 & -1.196558 & 0.008744 \\
\hline 32 & 1 & 0 & 2. 427847 & -3.159768 & -0.865494 \\
\hline 33 & 8 & 0 & 1. 714026 & -1.036941 & 2. 212517 \\
\hline 34 & 1 & 0 & 2. 405858 & -0.390360 & 2. 435834 \\
\hline 35 & 1 & 0 & -2.200362 & -0.064506 & 1. 389025 \\
\hline 36 & 6 & 0 & 0.534590 & 1. 398105 & 1. 374051 \\
\hline 37 & 1 & 0 & 0.683324 & 1. 321664 & 2. 446257 \\
\hline 38 & 1 & 0 & 5. 959944 & -0.641267 & -1.246669 \\
\hline 39 & 1 & 0 & 4. 839486 & -2.936846 & -1.896833 \\
\hline 40 & 16 & 0 & 3. 955119 & 0.000143 & 0.009753 \\
\hline
\end{tabular}

Zero-point correction=

Thermal correction to Energy=

0.301685 (Hartree/Particle)

Thermal correction to Enthalpy=

Thermal correction to Gibbs Free Energy=

Sum of electronic and zero-point Energies $=$

Sum of electronic and thermal Energies=

0.323911

0.324855

0.246035

$-1865.900677$

$-1865.878451$

$-1865.877506$

$-1865.956326$

Sum of electronic and thermal Free Energies=

nergy $=-1866.53838340$

1an-INT1b

\begin{tabular}{rrrrrr} 
Center & Atomic & Atomic & \multicolumn{3}{c}{ Coordinates (Angstroms) } \\
Number & Number & Type & X & Y & $Z$ \\
--1 & 6 & 0 & 2.774474 & -0.818260 & -1.112931 \\
2 & 6 & 0 & 1.757773 & -1.895154 & -0.936154 \\
3 & 6 & 0 & 0.284031 & -1.443069 & -1.021533 \\
4 & 6 & 0 & -0.180570 & -0.526512 & 0.127895 \\
5 & 1 & 0 & 2.923322 & -0.386643 & -2.098961 \\
6 & 1 & 0 & 1.912925 & -2.402969 & 0.022258 \\
7 & 1 & 0 & 1.887661 & -2.650078 & -1.723599 \\
8 & 1 & 0 & 0.093109 & -0.942942 & -1.977660 \\
9 & 1 & 0 & -0.351659 & -2.334873 & -0.990315 \\
10 & 6 & 0 & 3.516880 & -0.216380 & 0.035374 \\
11 & 1 & 0 & 3.503453 & 0.882760 & 0.007672 \\
12 & 1 & 0 & 3.099336 & -0.539298 & 0.995399 \\
13 & 6 & 0 & 4.971339 & -0.617261 & 0.042735 \\
14 & 9 & 0 & 5.647819 & -0.072590 & 1.076897 \\
15 & 9 & 0 & 5.139032 & -1.954109 & 0.139860 \\
16 & 9 & 0 & 5.619626 & -0.235683 & -1.079125
\end{tabular}




\begin{tabular}{|c|c|c|c|c|c|}
\hline 17 & 8 & 0 & 0.199937 & -1.182673 & 1. 332216 \\
\hline 18 & 1 & 0 & -0.345135 & -0.847470 & 2. 065690 \\
\hline 19 & 6 & 0 & -1.699146 & -0.407555 & 0.089853 \\
\hline 20 & 6 & 0 & -3.958058 & -0.899238 & 0.431270 \\
\hline 21 & 6 & 0 & -4.065214 & 0.214489 & -0.427879 \\
\hline 22 & 6 & 0 & -5.308234 & 0.715121 & -0.832020 \\
\hline 23 & 6 & 0 & -5.131441 & -1.521236 & 0.894181 \\
\hline 24 & 6 & 0 & -6.364265 & -1.029483 & 0.494406 \\
\hline 25 & 6 & 0 & -6.452188 & 0.082596 & -0.364458 \\
\hline 26 & 1 & 0 & -5.378220 & 1. 574030 & -1.493710 \\
\hline 27 & 1 & 0 & -7.427860 & 0.452186 & -0.666762 \\
\hline 28 & 1 & 0 & -7.274839 & -1.505573 & 0.846671 \\
\hline 29 & 1 & 0 & -5.062587 & -2.379623 & 1. 557428 \\
\hline 30 & 16 & 0 & -2.489965 & 0.839067 & -0.856443 \\
\hline 31 & 6 & 0 & 1. 256598 & 2. 905427 & -0.720657 \\
\hline 32 & 6 & 0 & 1. 362232 & 3. 095771 & 0.627443 \\
\hline 33 & 6 & 0 & 0.730495 & 1. 617652 & -1.052099 \\
\hline 34 & 6 & 0 & 0.454858 & 0.853250 & 0.049193 \\
\hline 35 & 1 & 0 & 0.575593 & 1. 279308 & -2.071630 \\
\hline 36 & 6 & 0 & -2.582596 & -1.234776 & 0.705799 \\
\hline 37 & 1 & 0 & -2.286341 & -2.074259 & 1. 327269 \\
\hline 38 & 1 & 0 & 1. 739771 & 3. 961453 & 1. 157948 \\
\hline 39 & 1 & 0 & 1. 550991 & 3. 647156 & -1.455464 \\
\hline 40 & 16 & 0 & 0.826271 & 1. 713269 & 1. 507621 \\
\hline
\end{tabular}

Zero-point correction=

0. 302003 (Hartree/Particle)

Thermal correction to Energy=

0.323920

Thermal correction to Enthalpy=

0.324865

Thermal correction to Gibbs Free Energy=

Sum of electronic and zero-point Energies=

Sum of electronic and thermal Energies=

0.246998

$-1865.899635$

$-1865.877717$

$-1865.876773$

$-1865.954640$

Sum of electronic and thermal Free Energies=

ergy $=-1866.53746428$

1an-TS1a

\begin{tabular}{|c|c|c|c|c|c|}
\hline \multirow{2}{*}{$\begin{array}{l}\text { Center } \\
\text { Number }\end{array}$} & \multirow{2}{*}{$\begin{array}{l}\text { Atomic } \\
\text { Number }\end{array}$} & \multirow{2}{*}{$\begin{array}{l}\text { Atomic } \\
\text { Type }\end{array}$} & \multicolumn{3}{|c|}{ Coordinates (Angstroms) } \\
\hline & & & X & Y & Z \\
\hline 1 & 6 & 0 & -1.433475 & -1.405197 & 0.410560 \\
\hline 2 & 6 & 0 & -0.816435 & -2.693774 & -0.065854 \\
\hline 3 & 6 & 0 & 0.645805 & -2.477009 & -0.508485 \\
\hline 4 & 6 & 0 & 1. 252978 & -1.381705 & 0.364685 \\
\hline 5 & 1 & 0 & -1.385019 & -3.115054 & -0.902986 \\
\hline 6 & 1 & 0 & -0.834715 & -3.425261 & 0.748286 \\
\hline 7 & 1 & 0 & 1. 224743 & -3.400306 & -0.418968 \\
\hline 8 & 1 & 0 & 0.688315 & -2.158911 & -1.556743 \\
\hline 9 & 6 & 0 & -2.487044 & -0.704589 & -0.392942 \\
\hline 10 & 1 & 0 & -2.493818 & 0.374116 & -0.184098 \\
\hline 11 & 1 & 0 & -2.344118 & -0.848009 & -1.469262 \\
\hline 12 & 6 & 0 & -3.875881 & -1.197640 & -0.069612 \\
\hline 13 & 9 & 0 & -4.822994 & -0.560018 & -0.791078 \\
\hline 14 & 9 & 0 & -4.031582 & -2.516337 & -0.317741 \\
\hline 15 & 9 & 0 & -4.202027 & -1.016575 & 1. 228041 \\
\hline 16 & 6 & 0 & 0.395376 & -0.116063 & 0.227721 \\
\hline 17 & 6 & 0 & -0.179015 & 2. 122207 & 0.717099 \\
\hline 18 & 6 & 0 & -0.184916 & 2. 167870 & -0.698073 \\
\hline 19 & 6 & 0 & -0.545972 & 3. 321135 & -1.396209 \\
\hline 20 & 6 & 0 & -0.532737 & 3. 283423 & 1. 433566 \\
\hline 21 & 6 & 0 & -0.881680 & 4. 433452 & 0.741655 \\
\hline 22 & 6 & 0 & -0.890589 & 4. 454140 & -0.664925 \\
\hline 23 & 1 & 0 & -0.552869 & 3. 337801 & -2.482698 \\
\hline 24 & 1 & 0 & -1.168003 & 5. 364132 & -1.189142 \\
\hline 25 & 1 & 0 & -1.154771 & 5. 330102 & 1. 290935 \\
\hline 26 & 1 & 0 & -0.531253 & 3. 266419 & 2.520475 \\
\hline 27 & 16 & 0 & 0.293672 & 0.629737 & -1.383323 \\
\hline 28 & 6 & 0 & 4. 881211 & -1.311854 & -0.794877 \\
\hline 29 & 6 & 0 & 4. 989902 & -0.131841 & -0.118958 \\
\hline 30 & 6 & 0 & 3. 562950 & -1.863012 & -0.724516 \\
\hline 31 & 6 & 0 & 2. 694292 & -1.089336 & -0.004970 \\
\hline
\end{tabular}




$\begin{array}{rrrrrr}32 & 1 & 0 & 3.281247 & -2.799664 & -1.193451 \\ 33 & 8 & 0 & 1.172938 & -1.858895 & 1.705314 \\ 34 & 1 & 0 & 1.684745 & -1.269345 & 2.285286 \\ 35 & 1 & 0 & -1.523979 & -1.283646 & 1.486789 \\ 36 & 6 & 0 & 0.209823 & 0.839728 & 1.217797 \\ 37 & 1 & 0 & 0.284018 & 0.616349 & 2.277947 \\ 38 & 1 & 0 & 5.861833 & 0.501487 & -0.007946 \\ 39 & 1 & 0 & 5.705080 & -1.777702 & -1.324651 \\ 40 & 16 & 0 & 3.491136 & 0.338726 & 0.593494\end{array}$

Zero-point correction=

0. 301705 (Hartree/Particle)

Thermal correction to Energy=

0.322831

Thermal correction to Enthalpy=

Thermal correction to Gibbs Free Energy=

Sum of electronic and zero-point Energies=

Sum of electronic and thermal Energies=

Sum of electronic and thermal Enthalpies=

Sum of electronic and thermal Free Energies=

0.323775

0.249207

$-1865.885946$

$-1865.864820$

$-1865.863876$

$-1865.938445$

M06 $-2 X / 6-311++G(d, p) / S M D / / M 06-2 X / 6-31+G(d) / S M D \quad$ energy $=-1866.52357888$

1an-TS1b

\begin{tabular}{|c|c|c|c|c|c|}
\hline \multirow{2}{*}{$\begin{array}{l}\text { Center } \\
\text { Number }\end{array}$} & \multirow{2}{*}{$\begin{array}{l}\text { Atomic } \\
\text { Number }\end{array}$} & \multirow{2}{*}{$\begin{array}{l}\text { Atomic } \\
\text { Type }\end{array}$} & \multicolumn{3}{|c|}{ Coordinates（Angstroms） } \\
\hline & & & X & $\mathrm{Y}$ & Z \\
\hline 1 & 6 & 0 & 2. 520475 & -0.227826 & -0.719227 \\
\hline 2 & 6 & 0 & 1. 909143 & -1.562182 & -1.050838 \\
\hline 3 & 6 & 0 & 0.388915 & -1.410818 & -1.188625 \\
\hline 4 & 6 & 0 & -0.089806 & -0.572572 & 0.001321 \\
\hline 5 & 1 & 0 & 2. 717384 & 0.430556 & -1.563243 \\
\hline 6 & 1 & 0 & 2. 124716 & -2.281382 & -0.254797 \\
\hline 7 & 1 & 0 & 2. 325584 & -1.964642 & -1.983695 \\
\hline 8 & 1 & 0 & 0.126421 & -0.891337 & -2.117513 \\
\hline 9 & 1 & 0 & -0.121624 & -2.379084 & -1.185074 \\
\hline 10 & 6 & 0 & 3. 508662 & -0.115305 & 0.404090 \\
\hline 11 & 1 & 0 & 3. 677266 & 0.930608 & 0.684977 \\
\hline 12 & 1 & 0 & 3. 168357 & -0.662212 & 1. 291196 \\
\hline 13 & 6 & 0 & 4. 863689 & -0.672633 & 0.042404 \\
\hline 14 & 9 & 0 & 5. 752133 & -0.548657 & 1. 053114 \\
\hline 15 & 9 & 0 & 4. 825467 & -1.986619 & -0.267484 \\
\hline 16 & 9 & 0 & 5. 413029 & -0.049657 & -1.022712 \\
\hline 17 & 8 & 0 & 0.310338 & -1.285612 & 1. 168223 \\
\hline 18 & 1 & 0 & -0.213300 & -0.969664 & 1. 925123 \\
\hline 19 & 6 & 0 & -1.603787 & -0.439005 & 0.007545 \\
\hline 20 & 6 & 0 & -3.846304 & -1.017023 & 0.342799 \\
\hline 21 & 6 & 0 & -3.995598 & 0.218324 & -0.320586 \\
\hline 22 & 6 & 0 & -5.257711 & 0.755798 & -0.599808 \\
\hline 23 & 6 & 0 & -4.995761 & -1.729487 & 0.730271 \\
\hline 24 & 6 & 0 & -6.247025 & -1.201694 & 0.453153 \\
\hline 25 & 6 & 0 & -6.377398 & 0. 034968 & -0.207751 \\
\hline 26 & 1 & 0 & -5.360373 & 1. 709110 & -1.110981 \\
\hline 27 & 1 & 0 & -7.367337 & 0.431207 & -0.415184 \\
\hline 28 & 1 & 0 & -7.139623 & -1.746392 & 0.747388 \\
\hline 29 & 1 & 0 & -4.893735 & -2.683753 & 1. 240645 \\
\hline 30 & 16 & 0 & -2.444380 & 0.926804 & -0.704036 \\
\hline 31 & 6 & 0 & 0.833426 & 3. 048517 & -0.637584 \\
\hline 32 & 6 & 0 & 1. 090015 & 3. 126110 & 0.708861 \\
\hline 33 & 6 & 0 & 0.511805 & 1. 739901 & -1.065012 \\
\hline 34 & 6 & 0 & 0.634690 & 0.774836 & -0.050805 \\
\hline 35 & 1 & 0 & 0.280490 & 1. 479017 & -2.093088 \\
\hline 36 & 6 & 0 & -2.458693 & -1.365767 & 0.515292 \\
\hline 37 & 1 & 0 & -2.127849 & -2.284087 & 0.990508 \\
\hline 38 & 1 & 0 & 1. 379192 & 3. 995934 & 1.286167 \\
\hline 39 & 1 & 0 & 0.875249 & 3. 907649 & -1.299369 \\
\hline 40 & 16 & 0 & 0.933030 & 1. 587129 & 1. 482279 \\
\hline \multicolumn{4}{|c|}{ Zero-point correction $=$} & \multicolumn{2}{|c|}{ 0.302115 (Hartree/Particle) } \\
\hline \multicolumn{4}{|c|}{ Thermal correction to Energy= } & \multicolumn{2}{|c|}{0.322950} \\
\hline \multicolumn{4}{|c|}{ Thermal correction to Enthalpy= } & \multicolumn{2}{|l|}{0.323895} \\
\hline \multicolumn{4}{|c|}{ Thermal correction to Gibbs Free Energy= } & \multicolumn{2}{|c|}{0.250120} \\
\hline \multicolumn{4}{|c|}{ Sum of electronic and zero-point Energies= } & \multicolumn{2}{|c|}{-1865.884302} \\
\hline
\end{tabular}


1an-INT2a

\begin{tabular}{|c|c|c|c|c|c|}
\hline \multirow{2}{*}{$\begin{array}{l}\text { Center } \\
\text { Number }\end{array}$} & \multirow{2}{*}{$\begin{array}{l}\text { Atomic } \\
\text { Number }\end{array}$} & \multirow{2}{*}{$\begin{array}{l}\text { Atomic } \\
\text { Type }\end{array}$} & \multicolumn{3}{|c|}{ Coordinates (Angstroms) } \\
\hline & & & X & Y & Z \\
\hline 1 & 6 & 0 & -1.671743 & -0.810732 & 0.158879 \\
\hline 2 & 6 & 0 & -1.667561 & -2.143065 & -0.622924 \\
\hline 3 & 6 & 0 & -0.193886 & -2.606184 & -0.676520 \\
\hline 4 & 6 & 0 & 0.565040 & -1.689270 & 0.288661 \\
\hline 5 & 1 & 0 & -2.059831 & -1.994762 & -1.635125 \\
\hline 6 & 1 & 0 & -2.307015 & -2.880317 & -0.131699 \\
\hline 7 & 1 & 0 & -0.057761 & -3.651182 & -0.386554 \\
\hline 8 & 1 & 0 & 0.210874 & -2.484862 & -1.685954 \\
\hline 9 & 6 & 0 & -2.675002 & 0.236161 & -0.317664 \\
\hline 10 & 1 & 0 & -2.453097 & 1. 218386 & 0.117085 \\
\hline 11 & 1 & 0 & -2.669112 & 0.334810 & -1.407268 \\
\hline 12 & 6 & 0 & -4.084897 & -0.091642 & 0.084740 \\
\hline 13 & 9 & 0 & -4.961036 & 0.834410 & -0.360944 \\
\hline 14 & 9 & 0 & -4.510263 & -1.278848 & -0.399332 \\
\hline 15 & 9 & 0 & -4.246492 & -0.152388 & 1. 424621 \\
\hline 16 & 6 & 0 & -0.195255 & -0.321130 & 0.121866 \\
\hline 17 & 6 & 0 & 0.578301 & 1. 951183 & 0.643782 \\
\hline 18 & 6 & 0 & 0.710270 & 1. 971220 & -0.774412 \\
\hline 19 & 6 & 0 & 1. 166971 & 3. 097367 & -1.444735 \\
\hline 20 & 6 & 0 & 0.925495 & 3. 113586 & 1. 373586 \\
\hline 21 & 6 & 0 & 1. 381108 & 4. 236734 & 0.700127 \\
\hline 22 & 6 & 0 & 1. 502700 & 4. 233097 & -0.699207 \\
\hline 23 & 1 & 0 & 1. 263893 & 3. 101528 & -2.527003 \\
\hline 24 & 1 & 0 & 1. 861122 & 5. 120456 & -1.212598 \\
\hline 25 & 1 & 0 & 1. 647433 & 5. 129352 & 1. 258967 \\
\hline 26 & 1 & 0 & 0.831303 & 3. 111760 & 2. 456361 \\
\hline 27 & 16 & 0 & 0.244029 & 0.443761 & -1.513455 \\
\hline 28 & 6 & 0 & 4. 208944 & -2.276504 & -0.600100 \\
\hline 29 & 6 & 0 & 4. 500843 & -1.196035 & 0.179513 \\
\hline 30 & 6 & 0 & 2. 804479 & -2.538052 & -0.666186 \\
\hline 31 & 6 & 0 & 2. 052987 & -1.647425 & 0.050234 \\
\hline 32 & 1 & 0 & 2. 373426 & -3.361775 & -1.225735 \\
\hline 33 & 8 & 0 & 0.289564 & -2.190228 & 1. 598853 \\
\hline 34 & 1 & 0 & 0.801681 & -1.679860 & 2. 249839 \\
\hline 35 & 1 & 0 & -1.866757 & -1.019942 & 1. 216683 \\
\hline 36 & 6 & 0 & 0.111128 & 0.723557 & 1. 142086 \\
\hline 37 & 1 & 0 & -0.093108 & 0.543606 & 2. 193842 \\
\hline 38 & 1 & 0 & 5. 470919 & -0.766247 & 0.398521 \\
\hline 39 & 1 & 0 & 4. 960977 & -2.868287 & -1.110758 \\
\hline 40 & 16 & 0 & 3. 075291 & -0.469799 & 0.826649 \\
\hline
\end{tabular}

Zero-point correction=

Thermal correction to Energy=

Thermal correction to Enthalpy=

Thermal correction to Gibbs Free Energy=

Sum of electronic and zero-point Energies=

Sum of electronic and thermal Energies=

Sum of electronic and thermal Enthalpies=

Sum of electronic and thermal Free Energies=

M06 $-2 X / 6-311++G(d, p) / S M D / / M 06-2 X / 6-31+G(d) / S M D \quad$ energy $=-1866.56645911$

lan-INT2b

\begin{tabular}{cccccr} 
Center & Atomic & Atomic & \multicolumn{3}{c}{ Coordinates (Angstroms) } \\
Number & Number & Type & X & Y & $Z$ \\
---1 & 6 & 0 & -2.321535 & -0.324016 & 0.377378 \\
1 & 6 & 0 & -2.176164 & -1.733430 & -0.239966 \\
2 & 6 & 0 & -0.674965 & -2.012390 & -0.215599 \\
3 & 6 & 0 & -0.072506 & -0.672717 & -0.632550 \\
4 & 1 & 0 & -2.515296 & -0.427313 & 1.450513
\end{tabular}

0. 304138 (Hartree/Particle)

0.325083

0.326028

0.252346

$-1865.927079$

$-1865.906133$

$-1865.905189$

$-1865.978870$ 


\begin{tabular}{|c|c|c|c|c|c|}
\hline 6 & 1 & 0 & -2.534466 & -1.734173 & -1.273530 \\
\hline 7 & 1 & 0 & -2.748736 & -2.478990 & 0.317841 \\
\hline 8 & 1 & 0 & -0.353922 & -2.267081 & 0.801805 \\
\hline 9 & 1 & 0 & -0.361901 & -2.815134 & -0.889597 \\
\hline 10 & 6 & 0 & -3.461544 & 0.502300 & -0.217540 \\
\hline 11 & 1 & 0 & -3.495869 & 1. 499745 & 0.231002 \\
\hline 12 & 1 & 0 & -3.354683 & 0.616419 & -1.302524 \\
\hline 13 & 6 & 0 & -4.817171 & -0.104184 & 0.027383 \\
\hline 14 & 9 & 0 & -5.806544 & 0.719123 & -0.382887 \\
\hline 15 & 9 & 0 & -5.013055 & -1.273774 & -0.617398 \\
\hline 16 & 9 & 0 & -5.046553 & -0.352106 & 1. 335397 \\
\hline 17 & 8 & 0 & -0.429494 & -0.503971 & -1.995325 \\
\hline 18 & 1 & 0 & -0.113933 & 0.364183 & -2.300369 \\
\hline 19 & 6 & 0 & 1. 422880 & -0.544045 & -0.454977 \\
\hline 20 & 6 & 0 & 3. 622590 & 0.231233 & -0.657756 \\
\hline 21 & 6 & 0 & 3. 794730 & -0.757890 & 0.332862 \\
\hline 22 & 6 & 0 & 5. 045974 & -1.021115 & 0.902501 \\
\hline 23 & 6 & 0 & 4. 739577 & 0.973280 & -1.082534 \\
\hline 24 & 6 & 0 & 5. 979963 & 0.717932 & -0.519002 \\
\hline 25 & 6 & 0 & 6. 132523 & -0.274225 & 0.468395 \\
\hline 26 & 1 & 0 & 5. 164711 & -1.785514 & 1. 665362 \\
\hline 27 & 1 & 0 & 7. 112981 & -0.458575 & 0.898227 \\
\hline 28 & 1 & 0 & 6. 846305 & 1. 288799 & -0.840698 \\
\hline 29 & 1 & 0 & 4. 621763 & 1. 739031 & -1.844981 \\
\hline 30 & 16 & 0 & 2. 282633 & -1.548172 & 0.700362 \\
\hline 31 & 6 & 0 & 0.269846 & 1. 802843 & 1. 843951 \\
\hline 32 & 6 & 0 & 0.086833 & 2. 706007 & 0.799197 \\
\hline 33 & 6 & 0 & -0.274402 & 0.557955 & 1. 616405 \\
\hline 34 & 6 & 0 & -0.896685 & 0.349948 & 0.262987 \\
\hline 35 & 1 & 0 & -0.262709 & -0.258606 & 2. 331944 \\
\hline 36 & 6 & 0 & 2. 250426 & 0.325242 & -1.091514 \\
\hline 37 & 1 & 0 & 1.928882 & 1. 031119 & -1.851845 \\
\hline 38 & 1 & 0 & 0.405135 & 3. 740458 & 0.757629 \\
\hline 39 & 1 & 0 & 0.785683 & 2. 075670 & 2. 759786 \\
\hline 40 & 16 & 0 & -0.832311 & 2. 031058 & -0.517969 \\
\hline
\end{tabular}

Zero-point correction=

Thermal correction to Energy=

0. 304406 (Hartree/Particle)

Thermal correction to Enthalpy=

Thermal correction to Gibbs Free Energy=

Sum of electronic and zero-point Energies=

Sum of electronic and thermal Energies=

0.325157

0.326102

0. 251958

$-1865.920886$

$-1865.900135$

$-1865.899191$

$-1865.973334$

Sum of electronic and thermal Free Energies=

M06-2X/6-311++G (d, p) /SMD//M06-2X/6-31+G (d)/SMD energy= -1866.56089691

1an-TS2a

\begin{tabular}{rrrrrr} 
Center & Atomic & Atomic & \multicolumn{3}{c}{ Coordinates (Angstroms) } \\
Number & Number & Type & X & Y & $Z$ \\
-1 & 6 & 0 & -1.964523 & -0.291737 & 0.138706 \\
2 & 6 & 0 & -2.147100 & -1.497137 & -0.804778 \\
3 & 6 & 0 & -0.817332 & -2.281014 & -0.914063 \\
4 & 6 & 0 & 0.156501 & -1.807691 & 0.127778 \\
5 & 1 & 0 & -2.451487 & -1.149183 & -1.799307 \\
6 & 1 & 0 & -2.952038 & -2.136920 & -0.434312 \\
7 & 1 & 0 & -0.979671 & -3.358191 & -0.781852 \\
8 & 1 & 0 & -0.373755 & -2.144952 & -1.903148 \\
9 & 6 & 0 & -2.936200 & 0.864933 & -0.130884 \\
10 & 1 & 0 & -2.689645 & 1.719591 & 0.507954 \\
11 & 1 & 0 & -2.891495 & 1.193575 & -1.174677 \\
12 & 6 & 0 & -4.372460 & 0.521244 & 0.155932 \\
13 & 9 & 0 & -5.174337 & 1.601314 & 0.034050 \\
14 & 9 & 0 & -4.875999 & -0.414358 & -0.677476 \\
15 & 9 & 0 & -4.555988 & 0.049746 & 1.408158 \\
16 & 6 & 0 & -0.525137 & 0.188355 & 0.083198 \\
17 & 6 & 0 & 1.274684 & 1.575094 & 0.754520 \\
18 & 6 & 0 & 1.397951 & 1.664258 & -0.656321 \\
19 & 6 & 0 & 2.459259 & 2.336526 & -1.260998 \\
20 & 6 & 0 & 2.249058 & 2.206379 & 1.556767
\end{tabular}




\begin{tabular}{|c|c|c|c|c|c|}
\hline 21 & 6 & 0 & 3. 300837 & 2. 881036 & 0.956007 \\
\hline 22 & 6 & 0 & 3. 410386 & 2. 945444 & -0.445096 \\
\hline 23 & 1 & 0 & 2. 544615 & 2. 385518 & -2.343418 \\
\hline 24 & 1 & 0 & 4. 244443 & 3. 473689 & -0.897950 \\
\hline 25 & 1 & 0 & 4. 054676 & 3. 361534 & 1. 573547 \\
\hline 26 & 1 & 0 & 2. 173173 & 2. 148383 & 2. 639832 \\
\hline 27 & 16 & 0 & 0.074783 & 0.848230 & -1.465746 \\
\hline 28 & 6 & 0 & 3. 659505 & -1.513316 & -1.174606 \\
\hline 29 & 6 & 0 & 4. 052080 & -1.539660 & 0.136612 \\
\hline 30 & 6 & 0 & 2. 253562 & -1.657614 & -1.335027 \\
\hline 31 & 6 & 0 & 1. 581639 & -1.788999 & -0.132963 \\
\hline 32 & 1 & 0 & 1. 757274 & -1.650334 & -2.298420 \\
\hline 33 & 8 & 0 & -0.235330 & -2.207601 & 1. 388916 \\
\hline 34 & 1 & 0 & 0.199045 & -1.660854 & 2. 070152 \\
\hline 35 & 1 & 0 & -2.115605 & -0.624013 & 1. 171877 \\
\hline 36 & 6 & 0 & 0.134360 & 0.818605 & 1. 152084 \\
\hline 37 & 1 & 0 & -0.168035 & 0.681192 & 2. 187431 \\
\hline 38 & 1 & 0 & 5. 054894 & -1.471859 & 0.540213 \\
\hline 39 & 1 & 0 & 4. 353752 & -1.399342 & -2.000469 \\
\hline 40 & 16 & 0 & 2. 711281 & -1.759829 & 1. 197461 \\
\hline
\end{tabular}

Zero-point correction $=$

0. 302639 (Hartree/Particle)

Thermal correction to Energy=

0. 323523

Thermal correction to Enthalpy=

0.324467

Thermal correction to Gibbs Free Energy=

Sum of electronic and zero-point Energies=

Sum of electronic and thermal Energies=

0.251284

$-1865.905485$

$-1865.884601$

$-1865.883657$

Sum of electronic and thermal Enthalpies=

Sum of electronic and thermal Free Energies=

$-1865.956840$

M06 $-2 \mathrm{X} / 6-311++\mathrm{G}(\mathrm{d}, \mathrm{p}) / \mathrm{SMD} / / \mathrm{M} 06-2 \mathrm{X} / 6-31+\mathrm{G}(\mathrm{d}) / \mathrm{SMD} \quad$ energy $=-1866.54428161$

\section{$1 \mathrm{an}-\mathrm{TS} 2 \mathrm{~b}$}

\begin{tabular}{|c|c|c|c|c|c|}
\hline \multirow{2}{*}{$\begin{array}{l}\text { Center } \\
\text { Number }\end{array}$} & \multirow{2}{*}{$\begin{array}{l}\text { Atomic } \\
\text { Number }\end{array}$} & \multirow{2}{*}{$\begin{array}{c}\text { Atomic } \\
\text { Type }\end{array}$} & \multicolumn{3}{|c|}{ Coordinates（Angstroms） } \\
\hline & & & $\mathrm{X}$ & Y & Z \\
\hline 1 & 6 & 0 & 2. 269592 & -0.314405 & -0.236787 \\
\hline 2 & 6 & 0 & 2.222229 & -1.404159 & 0.843320 \\
\hline 3 & 6 & 0 & 0.773446 & -1.879112 & 0.895959 \\
\hline 4 & 6 & 0 & -0.075210 & -0.662102 & 1. 130027 \\
\hline 5 & 1 & 0 & 2. 246994 & -0.824718 & -1.207946 \\
\hline 6 & 1 & 0 & 2. 523073 & -1.000910 & 1.816842 \\
\hline 7 & 1 & 0 & 2.898946 & -2.227238 & 0.596699 \\
\hline 8 & 1 & 0 & 0.502794 & -2.351274 & -0.056150 \\
\hline 9 & 1 & 0 & 0.609599 & -2.614426 & 1.694515 \\
\hline 10 & 6 & 0 & 3. 529550 & 0.558379 & -0.202152 \\
\hline 11 & 1 & 0 & 3. 492558 & 1. 309662 & -0.998069 \\
\hline 12 & 1 & 0 & 3. 632578 & 1. 079750 & 0.755920 \\
\hline 13 & 6 & 0 & 4. 805004 & -0.214796 & -0.406406 \\
\hline 14 & 9 & 0 & 5. 859981 & 0.609721 & -0.586150 \\
\hline 15 & 9 & 0 & 5. 124794 & -1.005925 & 0.639913 \\
\hline 16 & 9 & 0 & 4. 763198 & -1.018958 & -1.490435 \\
\hline 17 & 8 & 0 & 0.205922 & -0.134941 & 2. 368886 \\
\hline 18 & 1 & 0 & -0.174930 & 0.758377 & 2. 445986 \\
\hline 19 & 6 & 0 & -1.473967 & -0.600124 & 0.737191 \\
\hline 20 & 6 & 0 & -3.699383 & 0.119149 & 0.561706 \\
\hline 21 & 6 & 0 & -3.654236 & -0.827439 & -0.485801 \\
\hline 22 & 6 & 0 & -4.771022 & -1.104356 & -1.279586 \\
\hline 23 & 6 & 0 & -4.904761 & 0.804379 & 0.805820 \\
\hline 24 & 6 & 0 & -6.013478 & 0.535311 & 0.018136 \\
\hline 25 & 6 & 0 & -5.948602 & -0.414171 & -1.019016 \\
\hline 26 & 1 & 0 & -4.720631 & -1.838296 & -2.079302 \\
\hline 27 & 1 & 0 & -6.828973 & -0.611955 & -1.623739 \\
\hline 28 & 1 & 0 & -6.945527 & 1. 062107 & 0.202218 \\
\hline 29 & 1 & 0 & -4.956195 & 1. 536336 & 1. 607874 \\
\hline 30 & 16 & 0 & -2.072923 & -1.561950 & -0.605624 \\
\hline 31 & 6 & 0 & -0.504999 & 1. 864673 & -1.528008 \\
\hline 32 & 6 & 0 & -0.261799 & 2. 739049 & -0.494397 \\
\hline 33 & 6 & 0 & 0.254719 & 0.685164 & -1.437713 \\
\hline 34 & 6 & 0 & 0.992061 & 0.543742 & -0.229469 \\
\hline 35 & 1 & 0 & 0.258180 & -0.095682 & -2.191515 \\
\hline
\end{tabular}




\begin{tabular}{|c|c|c|c|c|c|}
\hline 36 & 6 & 0 & -2.440259 & 0.229103 & 1. 245179 \\
\hline 37 & 1 & 0 & -2.288128 & 0.903776 & 2. 082694 \\
\hline 38 & 1 & 0 & -0.711220 & 3. 708627 & -0.315989 \\
\hline 39 & 1 & 0 & -1.199330 & 2. 081533 & -2.333834 \\
\hline 40 & 16 & 0 & 0.928408 & 2. 123863 & 0.610296 \\
\hline \multicolumn{4}{|c|}{ Zero-point correction= } & \multicolumn{2}{|c|}{ 0.302583 (Hartree/Particle) } \\
\hline \multicolumn{4}{|c|}{ Thermal correction to Energy= } & \multicolumn{2}{|c|}{0.323279} \\
\hline \multicolumn{4}{|c|}{ Thermal correction to Enthalpy= } & \multicolumn{2}{|l|}{0.324224} \\
\hline \multicolumn{4}{|c|}{ Thermal correction to Gibbs Free Energy= } & \multicolumn{2}{|l|}{0.251345} \\
\hline \multicolumn{4}{|c|}{ Sum of electronic and zero-point Energies= } & \multicolumn{2}{|c|}{-1865.902481} \\
\hline \multicolumn{4}{|c|}{ Sum of electronic and thermal Energies $=$} & \multicolumn{2}{|c|}{-1865.881784} \\
\hline \multicolumn{4}{|c|}{ Sum of electronic and thermal Enthalpies= } & \multicolumn{2}{|c|}{-1865.880840} \\
\hline \multicolumn{4}{|c|}{ Sum of electronic and thermal Free Energies= } & \multicolumn{2}{|c|}{-1865.953719} \\
\hline \multicolumn{4}{|c|}{ M06-2X/6-311++G $(d, p) / S M D / / M 06-2 X / 6-31+G(d) / S M D$} & \multicolumn{2}{|c|}{ energy $=-1866.54109660$} \\
\hline
\end{tabular}

1an-INT3a

\begin{tabular}{|c|c|c|c|c|c|}
\hline \multirow{2}{*}{$\begin{array}{l}\text { Center } \\
\text { Number }\end{array}$} & \multirow{2}{*}{$\begin{array}{l}\text { Atomic } \\
\text { Number }\end{array}$} & \multirow{2}{*}{$\begin{array}{l}\text { Atomic } \\
\text { Type }\end{array}$} & \multicolumn{3}{|c|}{ Coordinates (Angstroms) } \\
\hline & & & X & $\mathrm{Y}$ & Z \\
\hline 1 & 6 & 0 & -1.606051 & -0.340378 & -0.033550 \\
\hline 2 & 6 & 0 & -1.894693 & 0.264100 & 1. 352716 \\
\hline 3 & 6 & 0 & -0.755894 & 1. 147939 & 1. 887938 \\
\hline 4 & 6 & 0 & -0.360012 & 2. 225660 & 0.931906 \\
\hline 5 & 1 & 0 & -2.085670 & -0.537670 & 2. 078007 \\
\hline 6 & 1 & 0 & -2.808653 & 0.862112 & 1. 294162 \\
\hline 7 & 1 & 0 & -1.093359 & 1. 607605 & 2. 827666 \\
\hline 8 & 1 & 0 & 0.117751 & 0.537219 & 2. 127506 \\
\hline 9 & 6 & 0 & -2.691841 & -1.330092 & -0.494864 \\
\hline 10 & 1 & 0 & -2.434114 & -1.718074 & -1.485174 \\
\hline 11 & 1 & 0 & -2.779769 & -2.180969 & 0.189946 \\
\hline 12 & 6 & 0 & -4.069540 & -0.733295 & -0.615210 \\
\hline 13 & 9 & 0 & -4.899857 & -1.548700 & -1.300832 \\
\hline 14 & 9 & 0 & -4.666059 & -0.514169 & 0.576328 \\
\hline 15 & 9 & 0 & -4.073782 & 0.449438 & -1.265726 \\
\hline 16 & 6 & 0 & -0.259577 & -1.012153 & -0.096244 \\
\hline 17 & 6 & 0 & 1. 948959 & -1.502002 & -0.699552 \\
\hline 18 & 6 & 0 & 1. 754957 & -2.449816 & 0.327278 \\
\hline 19 & 6 & 0 & 2. 767771 & -3.333808 & 0.714240 \\
\hline 20 & 6 & 0 & 3. 197627 & -1.437800 & -1.342276 \\
\hline 21 & 6 & 0 & 4. 206602 & -2.308769 & -0.958560 \\
\hline 22 & 6 & 0 & 3. 992136 & -3.252648 & 0.063015 \\
\hline 23 & 1 & 0 & 2. 605950 & -4.060966 & 1. 505212 \\
\hline 24 & 1 & 0 & 4. 794403 & -3.926725 & 0.349246 \\
\hline 25 & 1 & 0 & 5. 174232 & -2.263671 & -1.450160 \\
\hline 26 & 1 & 0 & 3. 361761 & -0.706691 & -2.130163 \\
\hline 27 & 16 & 0 & 0.143176 & -2.331687 & 0.997086 \\
\hline 28 & 6 & 0 & 3. 257649 & 2. 243820 & 0.151127 \\
\hline 29 & 6 & 0 & 2. 996117 & 3. 289639 & -0.700333 \\
\hline 30 & 6 & 0 & 2. 120807 & 1. 764130 & 0.832210 \\
\hline 31 & 6 & 0 & 0.937376 & 2. 457705 & 0.492681 \\
\hline 32 & 1 & 0 & 2. 142469 & 0.936994 & 1.532759 \\
\hline 33 & 8 & 0 & -1.420712 & 2. 976288 & 0.532596 \\
\hline 34 & 1 & 0 & -1.168170 & 3. 682331 & -0.086582 \\
\hline 35 & 1 & 0 & -1.561181 & 0.476425 & -0.765799 \\
\hline 36 & 6 & 0 & 0.772009 & -0.697177 & -0.917644 \\
\hline 37 & 1 & 0 & 0.720470 & 0.105131 & -1.648980 \\
\hline 38 & 1 & 0 & 3. 684125 & 3.841425 & -1.328062 \\
\hline 39 & 1 & 0 & 4. 252802 & 1.830200 & 0.281171 \\
\hline 40 & 16 & 0 & 1. 313920 & 3. 722660 & -0.685132 \\
\hline Zero-poi & correct & & & 0.302940 & (Hartree/Particle) \\
\hline Thermal & rrection & Energy $=$ & & 0.325012 & \\
\hline Thermal & rrection & Enthalpy= & & 0.325956 & \\
\hline Therma1 & rrection & Gibbs Fre & ergy $=$ & 0.247642 & \\
\hline Sum of $\mathrm{e}$ & ctronic & zero-poin & ergies= & -1865 & 29629 \\
\hline Sum of $e$ & ctronic & thermal E & ies $=$ & -1865 & 07557 \\
\hline Sum of $\mathrm{e}$ & ctronic & thermal E & 1pies= & -1865. & 06613 \\
\hline Sum of e & ctronic & thermal $\mathrm{F}$ & Energies= & -1865 & 84927 \\
\hline M06-2X/6 & $11++G(d, 1$ & $\mathrm{SMD} / / \mathrm{M} 06-2$ & $31+G(d) / S M D$ & energy $=-1$ & 66.56912268 \\
\hline
\end{tabular}


1an-INT3b

\begin{tabular}{|c|c|c|c|c|c|}
\hline \multirow{2}{*}{$\begin{array}{l}\text { Center } \\
\text { Number }\end{array}$} & \multirow{2}{*}{$\begin{array}{l}\text { Atomic } \\
\text { Number }\end{array}$} & \multirow{2}{*}{$\begin{array}{l}\text { Atomic } \\
\text { Type }\end{array}$} & \multicolumn{3}{|c|}{ Coordinates (Angstroms) } \\
\hline & & & X & $\mathrm{Y}$ & Z \\
\hline 1 & 6 & 0 & 2. 311004 & -0.027456 & -0.405567 \\
\hline 2 & 6 & 0 & 2. 188813 & -1.202430 & 0.582275 \\
\hline 3 & 6 & 0 & 0.850205 & -1.959390 & 0.493433 \\
\hline 4 & 6 & 0 & -0.270056 & -1.306664 & 1. 235383 \\
\hline 5 & 1 & 0 & 2. 269387 & -0.437820 & -1.422927 \\
\hline 6 & 1 & 0 & 2. 348627 & -0.848550 & 1. 608665 \\
\hline 7 & 1 & 0 & 2. 986152 & -1.919356 & 0.366227 \\
\hline 8 & 1 & 0 & 0.576943 & -2.098757 & -0.558376 \\
\hline 9 & 1 & 0 & 0.998607 & -2.962832 & 0.917333 \\
\hline 10 & 6 & 0 & 3. 639640 & 0.738201 & -0.256557 \\
\hline 11 & 1 & 0 & 3. 667952 & 1. 558320 & -0.980932 \\
\hline 12 & 1 & 0 & 3. 744905 & 1. 168587 & 0.745934 \\
\hline 13 & 6 & 0 & 4. 875873 & -0.086477 & -0.501157 \\
\hline 14 & 9 & 0 & 5. 968052 & 0.695124 & -0.648569 \\
\hline 15 & 9 & 0 & 5. 164932 & -0.937655 & 0.506997 \\
\hline 16 & 9 & 0 & 4. 791794 & -0.837253 & -1.619977 \\
\hline 17 & 8 & 0 & 0.036829 & -1.077634 & 2. 534919 \\
\hline 18 & 1 & 0 & -0.685129 & -0.612252 & 2. 991651 \\
\hline 19 & 6 & 0 & -1.530456 & -0.990192 & 0.732800 \\
\hline 20 & 6 & 0 & -3.723777 & -0.129036 & 0.562935 \\
\hline 21 & 6 & 0 & -3.565263 & -0.682159 & -0.732979 \\
\hline 22 & 6 & 0 & -4.570557 & -0.604445 & -1.695770 \\
\hline 23 & 6 & 0 & -4.937318 & 0.521368 & 0.872256 \\
\hline 24 & 6 & 0 & -5.937605 & 0.600094 & -0.085380 \\
\hline 25 & 6 & 0 & -5.759870 & 0.040860 & -1.363277 \\
\hline 26 & 1 & 0 & -4.430964 & -1.035071 & -2.683673 \\
\hline 27 & 1 & 0 & -6.554514 & 0.111630 & -2.100291 \\
\hline 28 & 1 & 0 & -6.871688 & 1. 100976 & 0.153638 \\
\hline 29 & 1 & 0 & -5.078119 & 0.954766 & 1. 859253 \\
\hline 30 & 16 & 0 & -1.996914 & -1.438739 & -0.920936 \\
\hline 31 & 6 & 0 & -0.742043 & 2. 176045 & -0.890961 \\
\hline 32 & 6 & 0 & -0.539127 & 2. 628088 & 0.380341 \\
\hline 33 & 6 & 0 & 0.241540 & 1. 217441 & -1.291459 \\
\hline 34 & 6 & 0 & 1. 164731 & 0.947084 & -0.320258 \\
\hline 35 & 1 & 0 & 0.255559 & 0.728677 & -2.260684 \\
\hline 36 & 6 & 0 & -2.573807 & -0.317651 & 1. 383440 \\
\hline 37 & 1 & 0 & -2.519599 & 0.067960 & 2. 397444 \\
\hline 38 & 1 & 0 & -1.120747 & 3. 351039 & 0.939712 \\
\hline 39 & 1 & 0 & -1.562762 & 2. 505889 & -1.519781 \\
\hline 40 & 16 & 0 & 0.848551 & 1. 896629 & 1. 103711 \\
\hline
\end{tabular}

Zero-point correction=

0. 303302 (Hartree/Particle)

Thermal correction to Energy=

0.325129

Thermal correction to Enthalpy=

0.326073

Thermal correction to Gibbs Free Energy=

Sum of electronic and zero-point Energies=

Sum of electronic and thermal Energies=

0.249399

$-1865.930884$

$-1865.909056$

$-1865.908112$

$-1865.984786$

Sum of electronic and thermal Free Energies

energy $=-1866.57068570$

1ae-INT1a

\begin{tabular}{cccccr} 
Center & Atomic & Atomic & \multicolumn{3}{c}{ Coordinates (Angstroms) } \\
Number & Number & Type & X & Y & $Z$ \\
--1 & 6 & 0 & -2.471493 & -1.252692 & 0.810410 \\
1 & 6 & 0 & -1.358777 & -2.200737 & 1.126418 \\
2 & 6 & 0 & -0.007133 & -1.857687 & 0.472858 \\
3 & 6 & 0 & 0.680115 & -0.624236 & 1.100246 \\
4 & 1 & 0 & -2.960839 & -0.697031 & 1.603368 \\
5 & 1 & 0 & -1.626824 & -3.209201 & 0.774828 \\
6 & 1 & 0 & -1.219859 & -2.268278 & 2.209553 \\
7 & 1 & 0 & 0.682081 & -2.700699 & 0.598890 \\
8 & 1 & 0 & -0.132119 & -1.696679 & -0.604513
\end{tabular}




\begin{tabular}{|c|c|c|c|c|c|}
\hline 10 & 6 & 0 & -3.040454 & -1.158659 & -0.569595 \\
\hline 11 & 1 & 0 & -3.255535 & -0.121828 & -0.858052 \\
\hline 12 & 1 & 0 & -2.365579 & -1.588040 & -1.318527 \\
\hline 13 & 6 & 0 & -4.348414 & -1.902318 & -0.692374 \\
\hline 14 & 9 & 0 & -4.862112 & -1.832563 & -1.939287 \\
\hline 15 & 9 & 0 & -4.228589 & -3.215788 & -0.402179 \\
\hline 16 & 9 & 0 & -5.296523 & -1.416679 & 0.137563 \\
\hline 17 & 8 & 0 & 0.845498 & -0.809279 & 2. 486748 \\
\hline 18 & 1 & 0 & 1. 794398 & -0.965778 & 2. 652396 \\
\hline 19 & 6 & 0 & -0.139152 & 0.623907 & 0.911423 \\
\hline 20 & 6 & 0 & -1.348969 & 2. 497409 & 0.994466 \\
\hline 21 & 6 & 0 & -1.318163 & 2. 001341 & -0.317222 \\
\hline 22 & 6 & 0 & -1.961403 & 2. 602218 & -1.391763 \\
\hline 23 & 6 & 0 & -2.073625 & 3. 669408 & 1. 256023 \\
\hline 24 & 6 & 0 & -2.728988 & 4. 289021 & 0.197771 \\
\hline 25 & 6 & 0 & -2.674540 & 3. 763856 & -1.107504 \\
\hline 26 & 1 & 0 & -1.911087 & 2. 184589 & -2.392686 \\
\hline 27 & 1 & 0 & -3.199207 & 4. 274120 & -1.909941 \\
\hline 28 & 1 & 0 & -3.296338 & 5. 197581 & 0.378361 \\
\hline 29 & 1 & 0 & -2.119782 & 4. 081050 & 2. 260266 \\
\hline 30 & 6 & 0 & 4. 008024 & -0.114978 & -0.966321 \\
\hline 31 & 6 & 0 & 4. 263362 & -0.509879 & 0.360359 \\
\hline 32 & 6 & 0 & 5. 581333 & -0.687094 & 0.799283 \\
\hline 33 & 6 & 0 & 6. 613928 & -0.464959 & -0.099995 \\
\hline 34 & 6 & 0 & 6. 348468 & -0.069263 & -1.423483 \\
\hline 35 & 6 & 0 & 5. 046438 & 0.111787 & -1.873397 \\
\hline 36 & 6 & 0 & 2. 053767 & -0.446405 & 0.461854 \\
\hline 37 & 1 & 0 & 5. 773147 & -0.990930 & 1. 824342 \\
\hline 38 & 1 & 0 & 7. 642928 & -0.596991 & 0. 220926 \\
\hline 39 & 1 & 0 & 7. 174760 & 0.098974 & -2.107864 \\
\hline 40 & 1 & 0 & 4. 845684 & 0.418025 & -2.895603 \\
\hline 41 & 7 & 0 & 3. 125280 & -0.688842 & 1. 137111 \\
\hline 42 & 16 & 0 & 2. 284683 & 0.032956 & -1.207735 \\
\hline 43 & 8 & 0 & -0.572562 & 0.858198 & -0.366927 \\
\hline 44 & 6 & 0 & -0.558354 & 1. 574234 & 1. 778243 \\
\hline 45 & 1 & 0 & -0.352007 & 1. 603986 & 2. 838601 \\
\hline
\end{tabular}

Zero-point correction=

0. 340896 (Hartree/Particle)

Thermal correction to Energy=

0.364880

Thermal correction to Enthalpy=

Thermal correction to Gibbs Free Energy=

Sum of electronic and zero-point Energies=

Sum of electronic and thermal Energies=

0.365824

0.283756

$-1712.540773$

$-1712.516790$

$-1712.515846$

Sum of electronic and thermal Free Energies=

$-1712.597913$

M06 $-2 X / 6-311++G(d, p) / S M D / / M 06-2 X / 6-31+G(d) / S M D \quad$ energy $=-1713.24435767$

1ae-INT1b

\begin{tabular}{rrrrrr} 
Center & Atomic & Atomic & \multicolumn{3}{c}{ Coordinates (Angstroms) } \\
Number & Number & Type & X & Y & $Z$ \\
-1 & 6 & 0 & 2.004004 & -1.345438 & -0.720715 \\
2 & 6 & 0 & 1.179758 & -2.283583 & 0.101127 \\
3 & 6 & 0 & -0.333008 & -2.051408 & -0.026734 \\
4 & 6 & 0 & -0.853347 & -0.889177 & 0.853253 \\
5 & 1 & 0 & 1.767130 & -1.235867 & -1.775690 \\
6 & 1 & 0 & 1.480568 & -2.227179 & 1.155677 \\
7 & 1 & 0 & 1.377320 & -3.321281 & -0.211738 \\
8 & 1 & 0 & -0.596135 & -1.865365 & -1.074354 \\
9 & 1 & 0 & -0.882345 & -2.941818 & 0.298421 \\
10 & 6 & 0 & 3.298009 & -0.784714 & -0.227400 \\
11 & 1 & 0 & 3.568349 & 0.137879 & -0.754047 \\
12 & 1 & 0 & 3.257640 & -0.571207 & 0.848434 \\
13 & 6 & 0 & 4.450639 & -1.740909 & -0.426856 \\
14 & 9 & 0 & 5.618913 & -1.238726 & 0.026167 \\
15 & 9 & 0 & 4.262949 & -2.913577 & 0.216608 \\
16 & 9 & 0 & 4.646981 & -2.050606 & -1.726367 \\
17 & 8 & 0 & -0.681626 & -1.202227 & 2.216495 \\
18 & 1 & 0 & 0.077879 & -0.685865 & 2.545068 \\
19 & 6 & 0 & -2.328059 & -0.685213 & 0.635257
\end{tabular}




\begin{tabular}{rrrrrr}
20 & 6 & 0 & -4.540431 & -0.386274 & 0.682141 \\
21 & 6 & 0 & -4.079188 & -0.359691 & -0.641634 \\
22 & 6 & 0 & -4.898246 & -0.171354 & -1.747326 \\
23 & 6 & 0 & -5.911451 & -0.216272 & 0.923414 \\
24 & 6 & 0 & -6.753267 & -0.025473 & -0.166548 \\
25 & 6 & 0 & -6.254805 & -0.002984 & -1.483144 \\
26 & 1 & 0 & -4.499849 & -0.157240 & -2.757001 \\
27 & 1 & 0 & -6.941661 & 0.148677 & -2.310626 \\
28 & 1 & 0 & -7.818464 & 0.109650 & -0.002379 \\
29 & 1 & 0 & -6.303224 & -0.232118 & 1.936423 \\
30 & 6 & 0 & 1.011383 & 2.341947 & -0.427314 \\
31 & 6 & 0 & 1.481977 & 1.921669 & 0.830971 \\
32 & 6 & 0 & 2.543120 & 2.596956 & 1.446657 \\
33 & 6 & 0 & 3.108554 & 3.679968 & 0.789753 \\
34 & 6 & 0 & 2.628084 & 4.096205 & -0.465396 \\
35 & 6 & 0 & 1.574458 & 3.437802 & -1.087368 \\
36 & 6 & 0 & -0.086376 & 0.390323 & 0.543354 \\
37 & 1 & 0 & 2.906125 & 2.263760 & 2.414857 \\
38 & 1 & 0 & 3.934732 & 4.214911 & 1.248599 \\
39 & 1 & 0 & 3.088074 & 4.947091 & -0.959248 \\
40 & 1 & 1.206139 & 3.761603 & -2.056155 \\
41 & 7 & 0 & 0.830672 & 0.809223 & 1.348481 \\
42 & 16 & 0 & -0.304874 & 1.311193 & -0.931140 \\
43 & 6 & 0 & -3.366172 & -0.601323 & 1.498925 \\
44 & 8 & 0 & -2.726539 & -0.548946 & -0.668565 \\
45 & 1 & 0 & -3.307107 & -0.680058 & 2.575027 \\
----------------------------------------------------------- \\
\hline $0-50$
\end{tabular}

Zero-point correction=

0.340731 (Hartree/Particle)

Thermal correction to Energy=

0.364670

Thermal correction to Enthalpy=

0.365614

Thermal correction to Gibbs Free Energy=

Sum of electronic and zero-point Energies=

Sum of electronic and thermal Energies=

0.283912

$-1712.542462$

$-1712.518523$

$-1712.517579$

$-1712.599281$

Sum of electronic and thermal Free Energies=

rgy $=-1713.24607058$

1ae-TS1a

\begin{tabular}{|c|c|c|c|c|c|}
\hline \multirow{2}{*}{$\begin{array}{l}\text { Center } \\
\text { Number }\end{array}$} & \multirow{2}{*}{$\begin{array}{l}\text { Atomic } \\
\text { Number }\end{array}$} & \multirow{2}{*}{$\begin{array}{l}\text { Atomic } \\
\text { Type }\end{array}$} & \multicolumn{3}{|c|}{ Coordinates (Angstroms) } \\
\hline & & & X & $\mathrm{Y}$ & Z \\
\hline 1 & 6 & 0 & -2.218952 & -1.425770 & 0.407679 \\
\hline 2 & 6 & 0 & -1.510504 & -2.573890 & -0.265255 \\
\hline 3 & 6 & 0 & -0.080311 & -2.166917 & -0.672304 \\
\hline 4 & 6 & 0 & 0.457615 & -1.259214 & 0.438448 \\
\hline 5 & 1 & 0 & -2.431046 & -1.531936 & 1. 468866 \\
\hline 6 & 1 & 0 & -2.054895 & -2.904617 & -1.157809 \\
\hline 7 & 1 & 0 & -1.453701 & -3.420276 & 0.424569 \\
\hline 8 & 1 & 0 & 0.574157 & -3.036216 & -0.792980 \\
\hline 9 & 1 & 0 & -0.089605 & -1.614537 & -1.618640 \\
\hline 10 & 6 & 0 & -3.190819 & -0.576892 & -0.357481 \\
\hline 11 & 1 & 0 & -3.293597 & 0.419303 & 0.092567 \\
\hline 12 & 1 & 0 & -2.888992 & -0.455309 & -1.403524 \\
\hline 13 & 6 & 0 & -4.582374 & -1.161015 & -0.372654 \\
\hline 14 & 9 & 0 & -5.447195 & -0.392676 & -1.069853 \\
\hline 15 & 9 & 0 & -4.629391 & -2.388966 & -0.932623 \\
\hline 16 & 9 & 0 & -5.102941 & -1.290892 & 0.866119 \\
\hline 17 & 8 & 0 & 0.430346 & -1.932818 & 1. 675803 \\
\hline 18 & 1 & 0 & 1. 354126 & -2.087747 & 1. 948404 \\
\hline 19 & 6 & 0 & -0.454612 & -0.051899 & 0.564034 \\
\hline 20 & 6 & 0 & -1.092490 & 2. 037246 & 1. 126786 \\
\hline 21 & 6 & 0 & -1.028268 & 1. 910827 & -0.274438 \\
\hline 22 & 6 & 0 & -1.365034 & 2. 921505 & -1.161489 \\
\hline 23 & 6 & 0 & -1.516165 & 3. 262206 & 1. 671844 \\
\hline 24 & 6 & 0 & -1.851206 & 4. 292570 & 0.800897 \\
\hline 25 & 6 & 0 & -1.778703 & 4. 127512 & -0.595154 \\
\hline 26 & 1 & 0 & -1.306676 & 2. 777434 & -2.236012 \\
\hline 27 & 1 & 0 & -2.049029 & 4. 954433 & -1.245169 \\
\hline 28 & 1 & 0 & -2.179349 & 5. 246855 & 1. 203018 \\
\hline 29 & 1 & 0 & -1.578597 & 3. 397096 & 2. 747866 \\
\hline
\end{tabular}




\begin{tabular}{|c|c|c|c|c|c|}
\hline 30 & 6 & 0 & 3. 982411 & 0.093223 & -0.714084 \\
\hline 31 & 6 & 0 & 4. 078659 & -0.748420 & 0.409740 \\
\hline 32 & 6 & 0 & 5. 329785 & -1.025296 & 0.974956 \\
\hline 33 & 6 & 0 & 6. 456335 & -0.454439 & 0.401079 \\
\hline 34 & 6 & 0 & 6. 349815 & 0.385452 & -0.722459 \\
\hline 35 & 6 & 0 & 5. 115526 & 0.671029 & -1.292865 \\
\hline 36 & 6 & 0 & 1. 881323 & -0.811187 & 0.146514 \\
\hline 37 & 1 & 0 & 5. 398253 & -1.674828 & 1. 842885 \\
\hline 38 & 1 & 0 & 7. 435629 & -0.657253 & 0.824362 \\
\hline 39 & 1 & 0 & 7. 247170 & 0.819715 & -1.153327 \\
\hline 40 & 1 & 0 & 5. 036024 & 1. 321143 & -2.158941 \\
\hline 41 & 7 & 0 & 2. 862623 & -1.241505 & 0.865906 \\
\hline 42 & 16 & 0 & 2. 306835 & 0.259651 & -1.175074 \\
\hline 43 & 8 & 0 & -0.603442 & 0.659514 & -0.615520 \\
\hline 44 & 6 & 0 & -0.665628 & 0.776173 & 1. 654191 \\
\hline 45 & 1 & 0 & -0.565533 & 0.485259 & 2. 690739 \\
\hline
\end{tabular}

Zero-point correction=

0.340350 (Hartree/Particle)

Thermal correction to Energy=

0. 363461

Thermal correction to Enthalpy=

0.364405

Thermal correction to Gibbs Free Energy=

Sum of electronic and zero-point Energies $=$

Sum of electronic and thermal Energies=

0.283714

$-1712.527855$

$-1712.504744$

$-1712.503800$

Sum of electronic and thermal Enthalpies=
Sum of electronic and thermal Free Energies

$-1712.584491$

M06 $-2 \mathrm{X} / 6-311++\mathrm{G}(\mathrm{d}, \mathrm{p}) / \mathrm{SMD} / / \mathrm{M} 06-2 \mathrm{X} / 6-31+\mathrm{G}(\mathrm{d}) / \mathrm{SMD} \quad$ energy $=-1713.23071780$

$1 a e^{-T S} 1 b$

\begin{tabular}{|c|c|c|c|c|c|}
\hline \multirow{2}{*}{$\begin{array}{l}\text { Center } \\
\text { Number }\end{array}$} & \multirow{2}{*}{$\begin{array}{l}\text { Atomic } \\
\text { Number }\end{array}$} & \multirow{2}{*}{$\begin{array}{c}\text { Atomic } \\
\text { Type }\end{array}$} & \multicolumn{3}{|c|}{ Coordinates (Angstroms) } \\
\hline & & & X & Y & Z \\
\hline 1 & 6 & 0 & 1.977556 & -1.202969 & -0.527121 \\
\hline 2 & 6 & 0 & 1. 378796 & -2.546448 & -0.233044 \\
\hline 3 & 6 & 0 & -0.143376 & -2.421937 & -0.306278 \\
\hline 4 & 6 & 0 & -0.532103 & -1.246875 & 0.591955 \\
\hline 5 & 1 & 0 & 1.951421 & -0.894467 & -1.570640 \\
\hline 6 & 1 & 0 & 1.678497 & -2.891705 & 0.760745 \\
\hline 7 & 1 & 0 & 1.728118 & -3.284422 & -0.967828 \\
\hline 8 & 1 & 0 & -0.461616 & -2.216038 & -1.334846 \\
\hline 9 & 1 & 0 & -0.653391 & -3.329903 & 0.030555 \\
\hline 10 & 6 & 0 & 3. 159881 & -0.702680 & 0.249721 \\
\hline 11 & 1 & 0 & 3. 310491 & 0.371878 & 0.096056 \\
\hline 12 & 1 & 0 & 3. 041584 & -0.889152 & 1. 322398 \\
\hline 13 & 6 & 0 & 4. 441162 & -1.375767 & -0.177038 \\
\hline 14 & 9 & 0 & 5. 507698 & -0.907702 & 0.503527 \\
\hline 15 & 9 & 0 & 4. 418168 & -2.711032 & 0.020625 \\
\hline 16 & 9 & 0 & 4. 711296 & -1.190858 & -1.486422 \\
\hline 17 & 8 & 0 & -0.194334 & -1.567254 & 1.921230 \\
\hline 18 & 1 & 0 & 0.168713 & -0.760777 & 2. 335330 \\
\hline 19 & 6 & 0 & -2.006264 & -0.943595 & 0.527484 \\
\hline 20 & 6 & 0 & -4.155801 & -0.403005 & 0.814719 \\
\hline 21 & 6 & 0 & -3.891450 & -0.628399 & -0.544274 \\
\hline 22 & 6 & 0 & -4.845398 & -0.526318 & -1.548857 \\
\hline 23 & 6 & 0 & -5.457908 & -0.052803 & 1. 200862 \\
\hline 24 & 6 & 0 & -6.430579 & 0.058378 & 0.213572 \\
\hline 25 & 6 & 0 & -6.129631 & -0.174656 & -1.142047 \\
\hline 26 & 1 & 0 & -4.598950 & -0.710350 & -2.590032 \\
\hline 27 & 1 & 0 & -6.914602 & -0.078850 & -1.886607 \\
\hline 28 & 1 & 0 & -7.445158 & 0.330431 & 0.490421 \\
\hline 29 & 1 & 0 & -5.698230 & 0.127996 & 2. 244757 \\
\hline 30 & 6 & 0 & 0.685363 & 2. 307982 & -0.619763 \\
\hline 31 & 6 & 0 & 1. 089952 & 2. 020170 & 0.705176 \\
\hline 32 & 6 & 0 & 1. 752708 & 3. 004345 & 1. 458743 \\
\hline 33 & 6 & 0 & 1. 997304 & 4. 238956 & 0.877987 \\
\hline 34 & 6 & 0 & 1. 592896 & 4. 510831 & -0.442230 \\
\hline 35 & 6 & 0 & 0.935249 & 3. 550793 & -1.204210 \\
\hline 36 & 6 & 0 & 0.280001 & 0.004944 & 0.182175 \\
\hline 37 & 1 & 0 & 2. 063465 & 2. 780722 & 2. 475432 \\
\hline 38 & 1 & 0 & 2. 510918 & 5. 008084 & 1. 447316 \\
\hline 39 & 1 & 0 & 1. 796574 & 5. 485588 & -0.875543 \\
\hline
\end{tabular}




\begin{tabular}{|c|c|c|c|c|c|}
\hline 40 & 1 & 0 & 0.624308 & 3. 765114 & -2.222325 \\
\hline 41 & 7 & 0 & 0.782142 & 0.758023 & 1. 148232 \\
\hline 42 & 16 & 0 & -0.139136 & 0.928401 & -1.297286 \\
\hline 43 & 6 & 0 & -2.897791 & -0.618832 & 1. 493902 \\
\hline 44 & 8 & 0 & -2.580204 & -0.964595 & -0.715905 \\
\hline 45 & 1 & 0 & -2.692528 & -0.546292 & 2.552986 \\
\hline \multicolumn{4}{|c|}{ Zero-point correction= } & \multicolumn{2}{|c|}{ 0. 341057 (Hartree/Partic1e) } \\
\hline \multicolumn{4}{|c|}{ Thermal correction to Energy= } & \multicolumn{2}{|c|}{0.363873} \\
\hline \multicolumn{4}{|c|}{ Thermal correction to Enthalpy= } & \multicolumn{2}{|l|}{0.364817} \\
\hline \multirow{2}{*}{\multicolumn{4}{|c|}{$\begin{array}{l}\text { Thermal correction to Gibbs Free Energy= } \\
\text { Sum of electronic and zero-point Energies= }\end{array}$}} & \multicolumn{2}{|l|}{0.286618} \\
\hline & & & ergies $=$ & \multicolumn{2}{|c|}{-1712.527791} \\
\hline \multicolumn{4}{|c|}{ Sum of electronic and thermal Energies $=$} & \multicolumn{2}{|c|}{-1712.504975} \\
\hline \multicolumn{4}{|c|}{ Sum of electronic and thermal Enthalpies= } & \multicolumn{2}{|c|}{-1712.504031} \\
\hline \multicolumn{4}{|c|}{ Sum of electronic and thermal Free Energies= } & \multirow{2}{*}{\multicolumn{2}{|c|}{-1712.582229}} \\
\hline \multicolumn{4}{|c|}{ M06-2X/6-311++G (d, p) /SMD//M06-2X/6-31+G (d) /SMD } & & \\
\hline
\end{tabular}

\section{1ae-INT2a}

\begin{tabular}{|c|c|c|c|c|c|}
\hline \multirow{2}{*}{$\begin{array}{l}\text { Center } \\
\text { Number }\end{array}$} & \multirow{2}{*}{$\begin{array}{l}\text { Atomic } \\
\text { Number }\end{array}$} & \multirow{2}{*}{$\begin{array}{c}\text { Atomic } \\
\text { Type }\end{array}$} & \multicolumn{3}{|c|}{ Coordinates (Angstroms) } \\
\hline & & & $\mathrm{x}$ & Y & Z \\
\hline 1 & 6 & 0 & -2.422037 & -0.781136 & 0.213274 \\
\hline 2 & 6 & 0 & -2.329334 & -1.993228 & -0.746863 \\
\hline 3 & 6 & 0 & -0.825413 & -2.326475 & -0.884062 \\
\hline 4 & 6 & 0 & -0.136409 & -1.548762 & 0.236657 \\
\hline 5 & 1 & 0 & -2.660779 & -1.130660 & 1. 223586 \\
\hline 6 & 1 & 0 & -2.754224 & -1.746161 & -1.724400 \\
\hline 7 & 1 & 0 & -2.893760 & -2.840183 & -0.349665 \\
\hline 8 & 1 & 0 & -0.600659 & -3.392214 & -0.793555 \\
\hline 9 & 1 & 0 & -0.452589 & -1.983167 & -1.853895 \\
\hline 10 & 6 & 0 & -3.415329 & 0.306773 & -0.188513 \\
\hline 11 & 1 & 0 & -3.261045 & 1. 215919 & 0.404576 \\
\hline 12 & 1 & 0 & -3.314265 & 0.565076 & -1.246849 \\
\hline 13 & 6 & 0 & -4.843053 & -0.102633 & 0.035678 \\
\hline 14 & 9 & 0 & -5.707847 & 0.866119 & -0.334532 \\
\hline 15 & 9 & 0 & -5.193003 & -1.207921 & -0.657281 \\
\hline 16 & 9 & 0 & -5.108511 & -0.372976 & 1. 332583 \\
\hline 17 & 8 & 0 & -0.326175 & -2.246547 & 1. 447380 \\
\hline 18 & 1 & 0 & 0.361313 & -1.946938 & 2. 071032 \\
\hline 19 & 6 & 0 & -0.986506 & -0.226361 & 0.295845 \\
\hline 20 & 6 & 0 & -0.124660 & 1. 875017 & 0.867655 \\
\hline 21 & 6 & 0 & -0.160197 & 1.722392 & -0.544774 \\
\hline 22 & 6 & 0 & 0.292680 & 2. 689694 & -1.420390 \\
\hline 23 & 6 & 0 & 0.391804 & 3. 074098 & 1. 406959 \\
\hline 24 & 6 & 0 & 0.849522 & 4. 054419 & 0.534698 \\
\hline 25 & 6 & 0 & 0.803476 & 3. 868495 & -0.857536 \\
\hline 26 & 1 & 0 & 0.254951 & 2. 538313 & -2.494862 \\
\hline 27 & 1 & 0 & 1. 170946 & 4. 651843 & -1.513837 \\
\hline 28 & 1 & 0 & 1. 252319 & 4. 981087 & 0.933181 \\
\hline 29 & 1 & 0 & 0.428908 & 3. 218768 & 2. 482838 \\
\hline 30 & 6 & 0 & 3. 590361 & -0.679442 & -0.693783 \\
\hline 31 & 6 & 0 & 3. 424004 & -0.903211 & 0.687028 \\
\hline 32 & 6 & 0 & 4. 520366 & -0.788526 & 1. 552417 \\
\hline 33 & 6 & 0 & 5. 754881 & -0.451778 & 1. 018348 \\
\hline 34 & 6 & 0 & 5.910191 & -0.229876 & -0.362735 \\
\hline 35 & 6 & 0 & 4. 834515 & -0.340614 & -1.234255 \\
\hline 36 & 6 & 0 & 1. 333757 & -1.260400 & 0.050683 \\
\hline 37 & 1 & 0 & 4. 387690 & -0.962496 & 2. 616426 \\
\hline 38 & 1 & 0 & 6. 615639 & -0.357212 & 1. 673596 \\
\hline 39 & 1 & 0 & 6. 887843 & 0.032748 & -0.756056 \\
\hline 40 & 1 & 0 & 4. 957850 & -0.168546 & -2.299311 \\
\hline 41 & 7 & 0 & 2. 131099 & -1.230838 & 1. 068346 \\
\hline 42 & 16 & 0 & 2. 059910 & -0.892672 & -1.500348 \\
\hline 43 & 8 & 0 & -0.695974 & 0.527053 & -0.906943 \\
\hline 44 & 6 & 0 & -0.660641 & 0.700028 & 1. 417194 \\
\hline 45 & 1 & 0 & -0.806827 & 0.460547 & 2. 462763 \\
\hline
\end{tabular}

Zero-point correction=

Thermal correction to Energy=

Thermal correction to Enthalpy=
0.342913 (Hartree/Particle)

0.365778

0. 366722 
Thermal correction to Gibbs Free Energy=

Sum of electronic and zero-point Energies=

Sum of electronic and thermal Energies=

Sum of electronic and thermal Enthalpies=

Sum of electronic and thermal Free Energies=

M06 $-2 X / 6-311++G(d, p) / S M D / / M 06-2 X / 6-31+G(d) / S M D \quad$ energy $=-1713.27674949$

$1 \mathrm{ae}-\mathrm{INT} 2 \mathrm{~b}$
0.287919

$-1712.571999$

$-1712.549134$

$-1712.548190$

$-1712.626993$

\begin{tabular}{|c|c|c|c|c|c|}
\hline \multirow{2}{*}{$\begin{array}{l}\text { Center } \\
\text { Number }\end{array}$} & \multirow{2}{*}{$\begin{array}{l}\text { Atomic } \\
\text { Number }\end{array}$} & \multirow{2}{*}{$\begin{array}{c}\text { Atomic } \\
\text { Type }\end{array}$} & \multicolumn{3}{|c|}{ Coordinates (Angstroms) } \\
\hline & & & $\mathrm{X}$ & Y & Z \\
\hline 1 & 6 & 0 & 0.498163 & -1.235809 & 0.397220 \\
\hline 2 & 6 & 0 & 0.073765 & -1.637323 & 1. 831455 \\
\hline 3 & 6 & 0 & -0.348961 & -0.338740 & 2. 551572 \\
\hline 4 & 6 & 0 & -0.372176 & 0.775310 & 1. 483771 \\
\hline 5 & 1 & 0 & -0.349161 & -1.367354 & -0.284332 \\
\hline 6 & 1 & 0 & 0.913440 & -2.104469 & 2. 354784 \\
\hline 7 & 1 & 0 & -0.739044 & -2.366490 & 1. 794987 \\
\hline 8 & 1 & 0 & -1.315226 & -0.419410 & 3. 055705 \\
\hline 9 & 1 & 0 & 0.396708 & -0.055473 & 3. 300251 \\
\hline 10 & 6 & 0 & 1. 704670 & -1.987360 & -0.160476 \\
\hline 11 & 1 & 0 & 2. 086293 & -1.505172 & -1.068476 \\
\hline 12 & 1 & 0 & 2. 515574 & -2.029047 & 0.573475 \\
\hline 13 & 6 & 0 & 1. 375084 & -3.401687 & -0.546239 \\
\hline 14 & 9 & 0 & 0.422611 & -3.471071 & -1.501539 \\
\hline 15 & 9 & 0 & 2. 452031 & -4.051833 & -1.035551 \\
\hline 16 & 9 & 0 & 0.923649 & -4.141161 & 0.489741 \\
\hline 17 & 8 & 0 & -0.104709 & 2. 061331 & 1. 980960 \\
\hline 18 & 1 & 0 & 0.806665 & 2. 049789 & 2. 324788 \\
\hline 19 & 6 & 0 & -1.707775 & 0.875675 & 0.810006 \\
\hline 20 & 6 & 0 & -3.639059 & 1. 415889 & -0.179728 \\
\hline 21 & 6 & 0 & -3.562209 & 0.027541 & 0.002271 \\
\hline 22 & 6 & 0 & -4.556201 & -0.863169 & -0.383862 \\
\hline 23 & 6 & 0 & -4.783664 & 1.956535 & -0.784569 \\
\hline 24 & 6 & 0 & -5.792904 & 1. 086187 & -1.179531 \\
\hline 25 & 6 & 0 & -5.681636 & -0.303952 & -0.982417 \\
\hline 26 & 1 & 0 & -4.457373 & -1.932386 & -0.223774 \\
\hline 27 & 1 & 0 & -6.490578 & -0.954021 & -1.302822 \\
\hline 28 & 1 & 0 & -6.687846 & 1. 483183 & -1.650051 \\
\hline 29 & 1 & 0 & -4.876221 & 3. 027628 & -0.940459 \\
\hline 30 & 6 & 0 & 2. 373794 & 1. 641901 & -0.936228 \\
\hline 31 & 6 & 0 & 2. 861148 & 1. 227980 & 0.353853 \\
\hline 32 & 6 & 0 & 4. 192198 & 1. 577449 & 0.730642 \\
\hline 33 & 6 & 0 & 4. 974636 & 2. 291691 & -0.147734 \\
\hline 34 & 6 & 0 & 4. 474647 & 2. 684985 & -1.411511 \\
\hline 35 & 6 & 0 & 3. 177988 & 2. 365829 & -1.810404 \\
\hline 36 & 6 & 0 & 0.762848 & 0.287869 & 0.499909 \\
\hline 37 & 1 & 0 & 4. 559742 & 1. 265416 & 1. 703804 \\
\hline 38 & 1 & 0 & 5. 990342 & 2. 559911 & 0.126944 \\
\hline 39 & 1 & 0 & 5. 113137 & 3. 248843 & -2.084819 \\
\hline 40 & 1 & 0 & 2. 806799 & 2. 678883 & -2.781643 \\
\hline 41 & 7 & 0 & 2. 026840 & 0.540816 & 1. 124475 \\
\hline 42 & 16 & 0 & 0.728697 & 1. 137526 & -1.153027 \\
\hline 43 & 6 & 0 & -2.407396 & 1. 944419 & 0.359323 \\
\hline 44 & 8 & 0 & -2.385167 & -0.300428 & 0.608719 \\
\hline 45 & 1 & 0 & -2.087660 & 2. 976090 & 0.403523 \\
\hline
\end{tabular}

Zero-point correction $=$

Thermal correction to Energy=

0. 343729 (Hartree/Particle)

Thermal correction to Enthalpy=

0.366455

0.367399

Thermal correction to Gibbs Free Energy=

Sum of electronic and zero-point Energies=

Sum of electronic and thermal Energies=

Sum of electronic and thermal Enthalpies=

0.289113

$-1712.562288$

$-1712.539563$

$-1712.538618$

$-1712.616905$

Sum of electronic and thermal Free Energies=

energy $=-1713.26779148$

$1 \mathrm{ae}-\mathrm{TS} 2 \mathrm{a}$

Center Atomic Atomic Coordinates (Angstroms) 


\begin{tabular}{|c|c|c|c|c|c|}
\hline Number & Number & Type & $X$ & Y & Z \\
\hline 1 & 6 & 0 & -2.565685 & -0.255978 & 0.350307 \\
\hline 2 & 6 & 0 & -2.458467 & -1.605042 & -0.365625 \\
\hline 3 & 6 & 0 & -1.414098 & -2.431665 & 0.403146 \\
\hline 4 & 6 & 0 & -0.385620 & -1.536324 & 1. 059271 \\
\hline 5 & 1 & 0 & -2.940272 & -0.410119 & 1. 370565 \\
\hline 6 & 1 & 0 & -2.139811 & -1.439168 & -1.401825 \\
\hline 7 & 1 & 0 & -3.415435 & -2.130889 & -0.394025 \\
\hline 8 & 1 & 0 & -1.903405 & -2.987648 & 1. 209153 \\
\hline 9 & 1 & 0 & -0.935600 & -3.168229 & -0.251540 \\
\hline 10 & 6 & 0 & -3.442367 & 0.787080 & -0.355261 \\
\hline 11 & 1 & 0 & -3.413007 & 1. 732778 & 0.195793 \\
\hline 12 & 1 & 0 & -3.089600 & 0.972668 & -1.374919 \\
\hline 13 & 6 & 0 & -4.891488 & 0.398983 & -0.458558 \\
\hline 14 & 9 & 0 & -5.646489 & 1. 425656 & -0.906154 \\
\hline 15 & 9 & 0 & -5.113095 & -0.624945 & -1.310420 \\
\hline 16 & 9 & 0 & -5.418445 & 0.026531 & 0.727556 \\
\hline 17 & 8 & 0 & -0.482738 & -1.537512 & 2. 427309 \\
\hline 18 & 1 & 0 & 0.287319 & -1.048950 & 2. 778722 \\
\hline 19 & 6 & 0 & -1.160939 & 0.283557 & 0.489482 \\
\hline 20 & 6 & 0 & 0.426849 & 1. 875846 & 0.824069 \\
\hline 21 & 6 & 0 & 0.465324 & 1. 414787 & -0.511571 \\
\hline 22 & 6 & 0 & 1. 438584 & 1. 787786 & -1.425288 \\
\hline 23 & 6 & 0 & 1. 419252 & 2. 778048 & 1. 256240 \\
\hline 24 & 6 & 0 & 2. 400241 & 3. 164795 & 0.352107 \\
\hline 25 & 6 & 0 & 2. 414846 & 2. 676611 & -0.969355 \\
\hline 26 & 1 & 0 & 1. 444791 & 1. 397080 & -2.438565 \\
\hline 27 & 1 & 0 & 3. 202928 & 2. 993155 & -1.646680 \\
\hline 28 & 1 & 0 & 3. 180665 & 3. 850565 & 0.670037 \\
\hline 29 & 1 & 0 & 1. 422547 & 3. 147587 & 2. 277829 \\
\hline 30 & 6 & 0 & 3. 029836 & -1.194535 & -0.747046 \\
\hline 31 & 6 & 0 & 3. 079114 & -0.726076 & 0.584243 \\
\hline 32 & 6 & 0 & 4. 248603 & -0.116882 & 1. 063421 \\
\hline 33 & 6 & 0 & 5. 331306 & 0.016834 & 0.206704 \\
\hline 34 & 6 & 0 & 5. 268949 & -0.453182 & -1.118042 \\
\hline 35 & 6 & 0 & 4. 121940 & -1.067378 & -1.608284 \\
\hline 36 & 6 & 0 & 0.978772 & -1.432891 & 0.569570 \\
\hline 37 & 1 & 0 & 4. 281918 & 0.249690 & 2. 085752 \\
\hline 38 & 1 & 0 & 6. 240302 & 0.494366 & 0.560853 \\
\hline 39 & 1 & 0 & 6. 129221 & -0.334684 & -1.770299 \\
\hline 40 & 1 & 0 & 4. 074383 & -1.426255 & -2.632254 \\
\hline 41 & 7 & 0 & 1. 910830 & -0.884171 & 1. 302876 \\
\hline 42 & 16 & 0 & 1. 454723 & -1.870765 & -1.067296 \\
\hline 43 & 8 & 0 & -0.554023 & 0.545645 & -0.745220 \\
\hline 44 & 6 & 0 & -0.667653 & 1. 207333 & 1. 434334 \\
\hline 45 & 1 & 0 & -1.005756 & 1. 289012 & 2. 459299 \\
\hline
\end{tabular}

Zero-point correction=

0. 342020 (Hartree/Particle)

Thermal correction to Energy=

0.364520

Thermal correction to Enthalpy=

0.365464

0.287600

$-1712.553401$

$-1712.530901$

$-1712.529957$

$-1712.607821$

Sum of electronic and thermal Enthalpies=

Sum of electronic and thermal Free Energies=

ergy $=-1713.25828409$

1ae-TS2b

\begin{tabular}{rrrrrr} 
Center & Atomic & Atomic & \multicolumn{3}{c}{ Coordinates (Angstroms) } \\
Number & Number & Type & X & Y & $Z$ \\
---1 & 6 & 0 & 2.177916 & -0.665584 & -0.012935 \\
1 & 6 & 0 & 2.332351 & -1.430413 & 1.304376 \\
2 & 6 & 0 & 0.934186 & -1.953717 & 1.619358 \\
3 & 6 & 0 & -0.020866 & -0.784939 & 1.572443 \\
4 & 1 & 0 & 1.955648 & -1.398849 & -0.798650 \\
5 & 1 & 0 & 2.689667 & -0.757581 & 2.095101 \\
6 & 1 & 0 & 3.050905 & -2.248805 & 1.212297 \\
7 & 1 & 0 & 0.643602 & -2.701684 & 0.874504 \\
8 & & 0 & & &
\end{tabular}




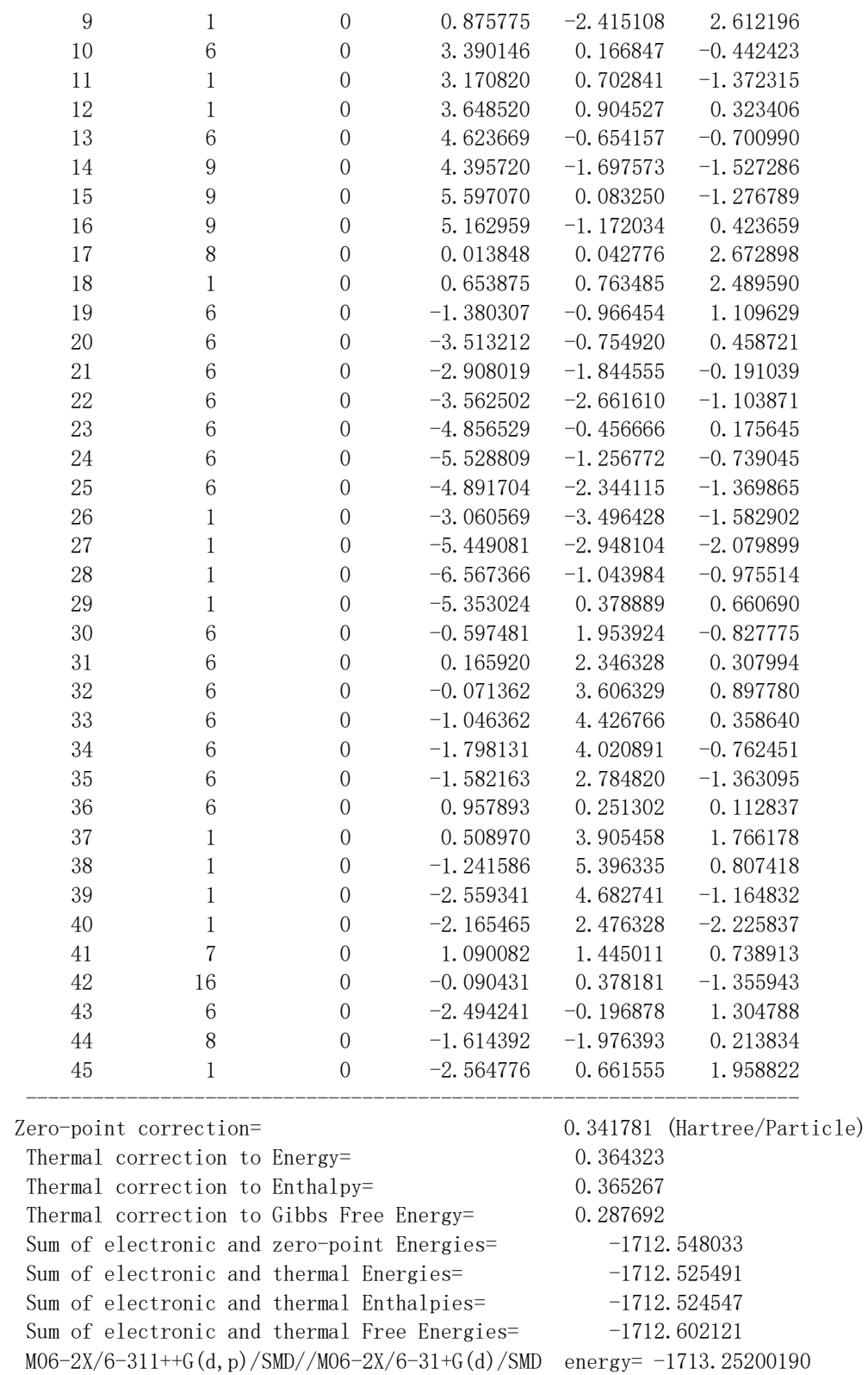

\section{1ae-INT3a}

\begin{tabular}{rrrrrr} 
Center & Atomic & Atomic & \multicolumn{3}{c}{ Coordinates (Angstroms) } \\
Number & Number & Type & $X$ & \multicolumn{1}{c}{ X } & \multicolumn{1}{c}{$Z$} \\
-1 & 6 & 0 & -2.532603 & 0.043780 & 0.332032 \\
2 & 6 & 0 & -2.274304 & -1.369022 & -0.226184 \\
3 & 6 & 0 & -1.548425 & -2.320227 & 0.748078 \\
4 & 6 & 0 & -0.291615 & -1.791526 & 1.351807 \\
5 & 1 & 0 & -2.935018 & -0.027909 & 1.349788 \\
6 & 1 & 0 & -1.715564 & -1.281002 & -1.165441 \\
7 & 1 & 0 & -3.225338 & -1.848755 & -0.470770 \\
8 & 1 & 0 & -2.221401 & -2.571114 & 1.574240 \\
9 & 1 & 0 & -1.334664 & -3.254481 & 0.212666 \\
10 & 6 & 0 & -3.520318 & 0.855272 & -0.531566 \\
11 & 1 & 0 & -3.596573 & 1.871678 & -0.133040 \\
12 & 1 & 0 & -3.177284 & 0.923051 & -1.569265 \\
13 & 6 & 0 & -4.926898 & 0.316279 & -0.566649 \\
14 & 9 & 0 & -5.787855 & 1.235712 & -1.053972 \\
15 & 9 & 0 & -5.072848 & -0.775543 & -1.347754 \\
16 & 9 & 0 & -5.385958 & -0.027499 & 0.655161 \\
17 & 8 & 0 & -0.356934 & -1.477854 & 2.654856 \\
18 & 1 & 0 & 0.499444 & -1.074451 & 2.910852
\end{tabular}




$\begin{array}{rrrrrr}19 & 6 & 0 & -1.259807 & 0.828174 & 0.424666 \\ 20 & 6 & 0 & 0.584757 & 2.014724 & 0.886488 \\ 21 & 6 & 0 & 0.592713 & 1.611493 & -0.457629 \\ 22 & 6 & 0 & 1.626078 & 1.884951 & -1.345521 \\ 23 & 6 & 0 & 1.682449 & 2.735721 & 1.380266 \\ 24 & 6 & 0 & 2.730098 & 3.021090 & 0.511134 \\ 25 & 6 & 0 & 2.703933 & 2.602812 & -0.833702 \\ 26 & 1 & 0 & 1.594768 & 1.545013 & -2.376584 \\ 27 & 1 & 0 & 3.543999 & 2.836353 & -1.482028 \\ 28 & 1 & 0 & 3.592171 & 3.572875 & 0.875882 \\ 29 & 1 & 0 & 1.715214 & 3.057635 & 2.417520 \\ 30 & 6 & 0 & 2.828511 & -1.309790 & -0.838344 \\ 31 & 6 & 0 & 3.028214 & -0.864764 & 0.491140 \\ 32 & 6 & 0 & 4.261363 & -0.290151 & 0.844791 \\ 33 & 6 & 0 & 5.248367 & -0.165639 & -0.122239 \\ 34 & 6 & 0 & 5.032213 & -0.607471 & -1.439501 \\ 35 & 6 & 0 & 3.821550 & -1.187402 & -1.809519 \\ 36 & 6 & 0 & 0.927885 & -1.570273 & 0.695373 \\ 37 & 1 & 0 & 4.416550 & 0.056318 & 1.863044 \\ 38 & 1 & 0 & 6.202024 & 0.283605 & 0.140101 \\ 39 & 1 & 0 & 5.818990 & -0.498057 & -2.180176 \\ 40 & 1 & 0 & 3.655252 & -1.527661 & -2.827603 \\ 41 & 7 & 0 & 1.957338 & -1.022498 & 1.334406 \\ 42 & 16 & 0 & 1.215737 & -1.962991 & -1.006352 \\ 43 & 8 & 0 & -0.539941 & 0.910497 & -0.741543 \\ 44 & 6 & 0 & -0.645355 & 1.491942 & 1.435208 \\ 45 & 1 & 0 & -1.003623 & 1.567224 & 2.453413 \\ -------------------------------------------------\end{array}$

Zero-point correction=

Thermal correction to Energy=

0. 343713 (Hartree/Particle)

Thermal correction to Enthalpy=

0. 366927

Thermal correction to Gibbs Free Energy=

Sum of electronic and zero-point Energies=

Sum of electronic and thermal Energies=

0.367871

0. 289278

$-1712.577788$

$-1712.554573$

$-1712.553629$

$-1712.632222$

Sum of electronic and thermal Free Energies=

M06 $-2 X / 6-311++G(d, p) / S M D / / M 06-2 X / 6-31+G(d) / S M D \quad$ energy $=-1713.28479092$

1ae-INT3b

\begin{tabular}{rrrrrr} 
Center & Atomic & Atomic & \multicolumn{3}{c}{ Coordinates (Angstroms) } \\
Number & Number & Type & $\mathrm{X}$ & $\mathrm{Y}$ & $\mathrm{Z}$ \\
-1 & 6 & 0 & 2.348859 & -0.371100 & -0.215165 \\
2 & 6 & 0 & 2.424983 & -0.993941 & 1.190412 \\
3 & 6 & 0 & 1.155779 & -1.759367 & 1.608838 \\
4 & 6 & 0 & -0.031708 & -0.897860 & 1.896330 \\
5 & 1 & 0 & 2.223618 & -1.176884 & -0.948229 \\
6 & 1 & 0 & 2.671637 & -0.213380 & 1.922116 \\
7 & 1 & 0 & 3.253836 & -1.706906 & 1.207415 \\
8 & 1 & 0 & 0.895737 & -2.489653 & 0.835634 \\
9 & 1 & 0 & 1.399623 & -2.325279 & 2.519733 \\
10 & 6 & 0 & 3.598718 & 0.447103 & -0.588923 \\
11 & 1 & 0 & 3.476802 & 0.868784 & -1.591820 \\
12 & 1 & 0 & 3.743333 & 1.273694 & 0.114139 \\
13 & 6 & 0 & 4.879287 & -0.344831 & -0.615441 \\
14 & 9 & 0 & 4.763708 & -1.502990 & -1.298569 \\
15 & 9 & 0 & 5.871814 & 0.352304 & -1.208182 \\
16 & 9 & 0 & 5.335295 & -0.670157 & 0.612577 \\
17 & 8 & 0 & 0.089537 & 0.100043 & 2.820730 \\
18 & 1 & 0 & 0.742795 & 0.752234 & 2.492791 \\
19 & 6 & 0 & -1.287158 & -1.034766 & 1.314716 \\
20 & 6 & 0 & -3.378099 & -0.790762 & 0.499369 \\
21 & 6 & 0 & -2.751238 & -1.915053 & -0.078549 \\
22 & 6 & 0 & -3.350787 & -2.726968 & -1.028057 \\
23 & 6 & 0 & -4.680748 & -0.458263 & 0.085717 \\
24 & 6 & 0 & -5.297644 & -1.258904 & -0.869592 \\
25 & 6 & 0 & -4.645658 & -2.378220 & -1.418773 \\
26 & 1 & 0 & -2.834851 & -3.585293 & -1.447454 \\
27 & 1 & 0 & -5.156068 & -2.982432 & -2.163095 \\
28 & 1 & 0 & -6.303518 & -1.017093 & -1.201289
\end{tabular}




$\begin{array}{rrrrrr}29 & 1 & 0 & -5.188921 & 0.406098 & 0.504416 \\ 30 & 6 & 0 & -0.921417 & 1.687398 & -0.941102 \\ 31 & 6 & 0 & -0.228342 & 2.202661 & 0.172612 \\ 32 & 6 & 0 & -0.769561 & 3.271138 & 0.899690 \\ 33 & 6 & 0 & -1.991982 & 3.793213 & 0.502843 \\ 34 & 6 & 0 & -2.676783 & 3.268921 & -0.609291 \\ 35 & 6 & 0 & -2.150270 & 2.215158 & -1.346114 \\ 36 & 6 & 0 & 1.164822 & 0.556815 & -0.328972 \\ 37 & 1 & 0 & -0.234989 & 3.661682 & 1.761416 \\ 38 & 1 & 0 & -2.430448 & 4.616639 & 1.058907 \\ 39 & 1 & 0 & -3.634401 & 3.692843 & -0.897388 \\ 40 & 1 & 0 & -2.684074 & 1.806099 & -2.199141 \\ 41 & 7 & 0 & 0.954748 & 1.544367 & 0.481442 \\ 42 & 16 & 0 & -0.029546 & 0.343429 & -1.594874 \\ 43 & 6 & 0 & -2.428193 & -0.236693 & 1.408473 \\ 44 & 8 & 0 & -1.496801 & -2.075463 & 0.425998 \\ 45 & 1 & 0 & -2.527008 & 0.650295 & 2.019362\end{array}$

Zero-point correction $=$

0. 342308 (Hartree/Particle)

Thermal correction to Energy=

0.365779

Thermal correction to Enthalpy=

0.366723

Thermal correction to Gibbs Free Energy=

Sum of electronic and zero-point Energies=

Sum of electronic and thermal Energies=

0. 287056

$-1712.572019$

$-1712.548548$

$-1712.547603$

Sum of electronic and thermal Free Energies=

$-1712.627270$

M06 $-2 X / 6-311++G(d, p) / S M D / / M 06-2 X / 6-31+G(d) / S M D \quad$ energy $=-1713.27772722$

1ah-INT1a

\begin{tabular}{|c|c|c|c|c|c|}
\hline \multirow{2}{*}{$\begin{array}{l}\text { Center } \\
\text { Number }\end{array}$} & \multirow{2}{*}{$\begin{array}{l}\text { Atomic } \\
\text { Number }\end{array}$} & \multirow{2}{*}{$\begin{array}{l}\text { Atomic } \\
\text { Type }\end{array}$} & \multicolumn{3}{|c|}{ Coordinates (Angstroms) } \\
\hline & & & X & Y & Z \\
\hline 1 & 6 & 0 & 2. 274809 & -1.325543 & -0.708213 \\
\hline 2 & 6 & 0 & 1.394323 & -2.270762 & 0.044866 \\
\hline 3 & 6 & 0 & -0.107152 & -2.034656 & -0.175394 \\
\hline 4 & 6 & 0 & -0.685797 & -0.875314 & 0.670126 \\
\hline 5 & 1 & 0 & 2. 124977 & -1.216742 & -1.778982 \\
\hline 6 & 1 & 0 & 1. 625566 & -2.231153 & 1. 117160 \\
\hline 7 & 1 & 0 & 1. 608336 & -3.304438 & -0.269894 \\
\hline 8 & 1 & 0 & -0.290924 & -1.851918 & -1.242380 \\
\hline 9 & 1 & 0 & -0.675136 & -2.928054 & 0.106443 \\
\hline 10 & 6 & 0 & 3.518315 & -0.755987 & -0.106799 \\
\hline 11 & 1 & 0 & 3. 848585 & 0.146335 & -0.633726 \\
\hline 12 & 1 & 0 & 3. 369549 & -0.505360 & 0.951347 \\
\hline 13 & 6 & 0 & 4. 677182 & -1.724285 & -0.153399 \\
\hline 14 & 9 & 0 & 5. 797567 & -1.208770 & 0.395630 \\
\hline 15 & 9 & 0 & 4. 418426 & -2.871265 & 0.511225 \\
\hline 16 & 9 & 0 & 5. 000434 & -2.082977 & -1.414326 \\
\hline 17 & 8 & 0 & -0.546021 & -1.175220 & 2. 042615 \\
\hline 18 & 1 & 0 & 0.213718 & -0.665273 & 2. 380867 \\
\hline 19 & 6 & 0 & -2.166886 & -0.677708 & 0.403586 \\
\hline 20 & 6 & 0 & -4.418961 & -0.174440 & 0.754869 \\
\hline 21 & 6 & 0 & -4.433751 & -0.442689 & -0.629754 \\
\hline 22 & 6 & 0 & -5.609553 & -0.367049 & -1.384866 \\
\hline 23 & 6 & 0 & -5.621495 & 0.178236 & 1. 393626 \\
\hline 24 & 6 & 0 & -6.788445 & 0.257189 & 0.649961 \\
\hline 25 & 6 & 0 & -6.782506 & -0.014015 & -0.731617 \\
\hline 26 & 1 & 0 & -5.607383 & -0.578352 & -2.450585 \\
\hline 27 & 1 & 0 & -7.708159 & 0.052696 & -1.296080 \\
\hline 28 & 1 & 0 & -7.720419 & 0.530323 & 1. 136553 \\
\hline 29 & 1 & 0 & -5.625217 & 0.386728 & 2. 460337 \\
\hline 30 & 6 & 0 & 1. 208354 & 2. 362180 & -0.560720 \\
\hline 31 & 6 & 0 & 1. 626141 & 1. 959850 & 0.721779 \\
\hline 32 & 6 & 0 & 2. 653836 & 2. 651252 & 1. 374999 \\
\hline 33 & 6 & 0 & 3. 239620 & 3. 730877 & 0.730063 \\
\hline 34 & 6 & 0 & 2. 811908 & 4. 128209 & -0.549827 \\
\hline 35 & 6 & 0 & 1. 791213 & 3. 454163 & -1.209223 \\
\hline 36 & 6 & 0 & 0.082934 & 0.411322 & 0.385641 \\
\hline 37 & 1 & 0 & 2. 976686 & 2. 332471 & 2. 362132 \\
\hline 38 & 1 & 0 & 4. 040936 & 4. 277671 & 1. 218100 \\
\hline
\end{tabular}




$\begin{array}{rrrrrr}39 & 1 & 0 & 3.286816 & 4.976668 & -1.033605 \\ 40 & 1 & 0 & 1.462940 & 3.763646 & -2.196863 \\ 41 & 7 & 0 & 0.961919 & 0.847653 & 1.223069 \\ 42 & 16 & 0 & -0.080294 & 1.317098 & -1.106296 \\ 43 & 6 & 0 & -3.099676 & -0.317557 & 1.319332 \\ 44 & 1 & 0 & -2.868761 & -0.159953 & 2.367064 \\ 45 & 16 & 0 & -2.842312 & -0.880199 & -1.204158\end{array}$

Zero-point correction=

0.337566 (Hartree/Particle)

Thermal correction to Energy=

0.362073

Thermal correction to Enthalpy=

0.363017

Thermal correction to Gibbs Free Energy=

Sum of electronic and zero-point Energies=

Sum of electronic and thermal Energies=

Sum of electronic and thermal Enthalpies=

Sum of electronic and thermal Free Energies=

0.279578

$-2035.512562$

$-2035.488055$

$-2035.487111$

$-2035.570550$

M06 $-2 X / 6-311++G(d, p) / S M D / / M 06-2 X / 6-31+G(d) / S M D \quad$ energy $=-2036.22176158$

1ah-INT1b

\begin{tabular}{|c|c|c|c|c|c|}
\hline \multirow{2}{*}{$\begin{array}{l}\text { Center } \\
\text { Number }\end{array}$} & \multirow{2}{*}{$\begin{array}{l}\text { Atomic } \\
\text { Number }\end{array}$} & \multirow{2}{*}{$\begin{array}{c}\text { Atomic } \\
\text { Type }\end{array}$} & \multicolumn{3}{|c|}{ Coordinates (Angstroms) } \\
\hline & & & X & Y & Z \\
\hline 1 & 6 & 0 & -2.574223 & -1.058632 & 0.814174 \\
\hline 2 & 6 & 0 & -1.537964 & -1.897249 & 1. 493868 \\
\hline 3 & 6 & 0 & -0.134898 & -1.846817 & 0.858276 \\
\hline 4 & 6 & 0 & 0.644356 & -0.561855 & 1. 179639 \\
\hline 5 & 1 & 0 & -3.062517 & -0.252455 & 1. 350800 \\
\hline 6 & 1 & 0 & -1.850400 & -2.952214 & 1. 470896 \\
\hline 7 & 1 & 0 & -1.459725 & -1.615116 & 2.548379 \\
\hline 8 & 1 & 0 & 0.463008 & -2.685796 & 1. 231052 \\
\hline 9 & 1 & 0 & -0.218443 & -1.964199 & -0.230307 \\
\hline 10 & 6 & 0 & -3.084721 & -1.404044 & -0.548620 \\
\hline 11 & 1 & 0 & -3.307107 & -0.508107 & -1.140354 \\
\hline 12 & 1 & 0 & -2.369449 & -2.015359 & -1.111492 \\
\hline 13 & 6 & 0 & -4.369452 & -2.195781 & -0.490580 \\
\hline 14 & 9 & 0 & -4.827540 & -2.519769 & -1.718843 \\
\hline 15 & 9 & 0 & -4.233004 & -3.357904 & 0. 184039 \\
\hline 16 & 9 & 0 & -5.365342 & -1.519113 & 0.120746 \\
\hline 17 & 8 & 0 & 0.773029 & -0.487017 & 2. 581637 \\
\hline 18 & 1 & 0 & 1. 540177 & 0.082448 & 2. 777135 \\
\hline 19 & 6 & 0 & -0.068807 & 0.683294 & 0.670904 \\
\hline 20 & 6 & 0 & -1.286466 & 2. 674172 & 0.657989 \\
\hline 21 & 6 & 0 & -1.232382 & 2. 401694 & -0.725024 \\
\hline 22 & 6 & 0 & -1.829493 & 3. 243722 & -1.669164 \\
\hline 23 & 6 & 0 & -1.967346 & 3. 822968 & 1. 098670 \\
\hline 24 & 6 & 0 & -2.564958 & 4. 658989 & 0.167643 \\
\hline 25 & 6 & 0 & -2.496551 & 4. 371204 & -1.208403 \\
\hline 26 & 1 & 0 & -1.776082 & 3. 021705 & -2.731424 \\
\hline 27 & 1 & 0 & -2.971359 & 5. 039113 & -1.921405 \\
\hline 28 & 1 & 0 & -3.093315 & 5. 547397 & 0.501534 \\
\hline 29 & 1 & 0 & -2.019187 & 4. 045833 & 2. 161303 \\
\hline 30 & 6 & 0 & 4. 069592 & -0.864780 & -0.774085 \\
\hline 31 & 6 & 0 & 4. 203101 & -0.133740 & 0.422387 \\
\hline 32 & 6 & 0 & 5. 449191 & 0.395391 & 0.783250 \\
\hline 33 & 6 & 0 & 6. 529241 & 0.183012 & -0.060415 \\
\hline 34 & 6 & 0 & 6. 384142 & -0.548978 & -1.253277 \\
\hline 35 & 6 & 0 & 5. 156683 & -1.082645 & -1.624810 \\
\hline 36 & 6 & 0 & 2. 047198 & -0.602060 & 0.575271 \\
\hline 37 & 1 & 0 & 5. 549405 & 0.958639 & 1. 706577 \\
\hline 38 & 1 & 0 & 7. 502608 & 0.586654 & 0. 202078 \\
\hline 39 & 1 & 0 & 7. 246092 & -0.701614 & -1.896059 \\
\hline 40 & 1 & 0 & 5. 047193 & -1.648492 & -2.545008 \\
\hline 41 & 7 & 0 & 3. 031615 & -0.005333 & 1. 156325 \\
\hline 42 & 16 & 0 & 2. 419929 & -1.407391 & -0.937398 \\
\hline 43 & 6 & 0 & -0.604084 & 1. 667072 & 1. 434461 \\
\hline 44 & 1 & 0 & -0.554010 & 1. 672507 & 2. 517810 \\
\hline 45 & 16 & 0 & -0.334454 & 0.936389 & -1.045432 \\
\hline
\end{tabular}

Zero-point correction=

0. 337618 (Hartree/Particle)

Thermal correction to Energy=

0. 362246 
Thermal correction to Enthalpy=

Thermal correction to Gibbs Free Energy=

Sum of electronic and zero-point Energies=

Sum of electronic and thermal Energies=

Sum of electronic and thermal Enthalpies=

Sum of electronic and thermal Free Energies=
0.363190

0. 278521

$-2035.510236$

$-2035.485608$

$-2035.484664$

$-2035.569333$

ergy $=-2036.21910921$

1ah-TS1a

\begin{tabular}{|c|c|c|c|c|c|}
\hline \multirow{2}{*}{$\begin{array}{l}\text { Center } \\
\text { Number }\end{array}$} & \multirow{2}{*}{$\begin{array}{l}\text { Atomic } \\
\text { Number }\end{array}$} & \multirow{2}{*}{$\begin{array}{l}\text { Atomic } \\
\text { Type }\end{array}$} & \multicolumn{3}{|c|}{ Coordinates (Angstroms) } \\
\hline & & & X & Y & Z \\
\hline 1 & 6 & 0 & 2.271596 & -1.132829 & -0.499442 \\
\hline 2 & 6 & 0 & 1. 677895 & -2.505518 & -0.356981 \\
\hline 3 & 6 & 0 & 0.157668 & -2.411725 & -0.515566 \\
\hline 4 & 6 & 0 & -0.312078 & -1.268921 & 0.382662 \\
\hline 5 & 1 & 0 & 2. 389801 & -0.766809 & -1.517439 \\
\hline 6 & 1 & 0 & 1. 917213 & -2.923106 & 0.624656 \\
\hline 7 & 1 & 0 & 2. 089777 & -3.176642 & -1.121717 \\
\hline 8 & 1 & 0 & -0.097093 & -2.192536 & -1.561165 \\
\hline 9 & 1 & 0 & -0.341256 & -3.344675 & -0.235255 \\
\hline 10 & 6 & 0 & 3. 312073 & -0.652314 & 0.469225 \\
\hline 11 & 1 & 0 & 3. 442065 & 0.433851 & 0.408206 \\
\hline 12 & 1 & 0 & 3. 050305 & -0.915299 & 1. 499743 \\
\hline 13 & 6 & 0 & 4. 666379 & -1.256901 & 0.190753 \\
\hline 14 & 9 & 0 & 5. 602706 & -0.810968 & 1. 053668 \\
\hline 15 & 9 & 0 & 4. 666844 & -2.603233 & 0. 288164 \\
\hline 16 & 9 & 0 & 5. 117745 & -0.966337 & -1.047578 \\
\hline 17 & 8 & 0 & 0.034127 & -1.597710 & 1. 711498 \\
\hline 18 & 1 & 0 & 0.280531 & -0.769163 & 2. 165111 \\
\hline 19 & 6 & 0 & -1.804496 & -0.992008 & 0.295696 \\
\hline 20 & 6 & 0 & -3.927478 & -0.184845 & 0.842607 \\
\hline 21 & 6 & 0 & -4.192691 & -0.763198 & -0.416879 \\
\hline 22 & 6 & 0 & -5.471672 & -0.740687 & -0.984747 \\
\hline 23 & 6 & 0 & -4.978758 & 0.433475 & 1. 543758 \\
\hline 24 & 6 & 0 & -6.246177 & 0.460944 & 0.983692 \\
\hline 25 & 6 & 0 & -6.491562 & -0.123318 & -0.273726 \\
\hline 26 & 1 & 0 & -5.662702 & -1.191976 & -1.954384 \\
\hline 27 & 1 & 0 & -7.492395 & -0.091852 & -0.694658 \\
\hline 28 & 1 & 0 & -7.062093 & 0.938109 & 1. 518916 \\
\hline 29 & 1 & 0 & -4.788753 & 0.883654 & 2. 514720 \\
\hline 30 & 6 & 0 & 0.769298 & 2. 319519 & -0.835456 \\
\hline 31 & 6 & 0 & 1. 074076 & 2. 098772 & 0.528545 \\
\hline 32 & 6 & 0 & 1. 588172 & 3. 151604 & 1. 304602 \\
\hline 33 & 6 & 0 & 1. 786227 & 4. 387224 & 0.708034 \\
\hline 34 & 6 & 0 & 1. 482294 & 4. 592017 & -0.650493 \\
\hline 35 & 6 & 0 & 0.974024 & 3. 562511 & -1.436162 \\
\hline 36 & 6 & 0 & 0.460909 & 0.014914 & 0.008330 \\
\hline 37 & 1 & 0 & 1. 822511 & 2. 979503 & 2. 351363 \\
\hline 38 & 1 & 0 & 2. 183903 & 5. 209597 & 1. 295418 \\
\hline 39 & 1 & 0 & 1. 645900 & 5.569076 & -1.095451 \\
\hline 40 & 1 & 0 & 0.739616 & 3. 724705 & -2.484015 \\
\hline 41 & 7 & 0 & 0.822690 & 0.828797 & 0.986242 \\
\hline 42 & 16 & 0 & 0.127068 & 0.857993 & -1.538622 \\
\hline 43 & 6 & 0 & -2.544682 & -0.332639 & 1. 221999 \\
\hline 44 & 1 & 0 & -2.136564 & 0.046333 & 2. 154365 \\
\hline 45 & 16 & 0 & -2.752341 & -1.479512 & -1.097320 \\
\hline
\end{tabular}

Zero-point correction=

0.337686 (Hartree/Particle)

Thermal correction to Energy=

0.361192

Thermal correction to Enthalpy=

0.362136

0.280983

$-2035.498233$

$-2035.474726$

$-2035.473782$

$-2035.554935$

Sum of electronic and thermal Energies=

Sum of electronic and thermal Enthalpies=

Sum of electronic and thermal Free Energies=

energy $=-2036.20708195$

1ah-TS1b 


\begin{tabular}{|c|c|c|c|c|c|}
\hline \multirow{2}{*}{$\begin{array}{l}\text { Center } \\
\text { Number }\end{array}$} & \multirow{2}{*}{$\begin{array}{l}\text { Atomic } \\
\text { Number }\end{array}$} & \multirow{2}{*}{$\begin{array}{l}\text { Atomic } \\
\text { Type }\end{array}$} & \multicolumn{3}{|c|}{ Coordinates (Angstroms) } \\
\hline & & & X & Y & Z \\
\hline 1 & 6 & 0 & -2.224481 & -1.341252 & 0.523815 \\
\hline 2 & 6 & 0 & -1.574698 & -2.660906 & 0.196684 \\
\hline 3 & 6 & 0 & -0.107466 & -2.451338 & -0.226789 \\
\hline 4 & 6 & 0 & 0.451021 & -1.298373 & 0.597993 \\
\hline 5 & 1 & 0 & -2.393325 & -1.134504 & 1. 577609 \\
\hline 6 & 1 & 0 & -2.110567 & -3.175444 & -0.608992 \\
\hline 7 & 1 & 0 & -1.598347 & -3.307905 & 1. 078682 \\
\hline 8 & 1 & 0 & 0.491402 & -3.354716 & -0.075234 \\
\hline 9 & 1 & 0 & -0.060495 & -2.190986 & -1.292654 \\
\hline 10 & 6 & 0 & -3.225442 & -0.727213 & -0.407879 \\
\hline 11 & 1 & 0 & -3.259689 & 0.364795 & -0.294167 \\
\hline 12 & 1 & 0 & -3.003344 & -0.959079 & -1.454979 \\
\hline 13 & 6 & 0 & -4.629335 & -1.213848 & -0.143851 \\
\hline 14 & 9 & 0 & -5.521260 & -0.679549 & -1.005790 \\
\hline 15 & 9 & 0 & -4.747287 & -2.554401 & -0.256889 \\
\hline 16 & 9 & 0 & -5.062655 & -0.898993 & 1. 095182 \\
\hline 17 & 8 & 0 & 0.363934 & -1.662439 & 1. 958527 \\
\hline 18 & 1 & 0 & 0.966603 & -1.082059 & 2. 458971 \\
\hline 19 & 6 & 0 & -0.401536 & -0.042573 & 0.351533 \\
\hline 20 & 6 & 0 & -1.020656 & 2. 204823 & 0.724843 \\
\hline 21 & 6 & 0 & -0.972338 & 2. 192947 & -0.690205 \\
\hline 22 & 6 & 0 & -1.323392 & 3. 309067 & -1.450346 \\
\hline 23 & 6 & 0 & -1.420853 & 3. 388899 & 1. 377691 \\
\hline 24 & 6 & 0 & -1.760609 & 4. 502352 & 0.624834 \\
\hline 25 & 6 & 0 & -1.714935 & 4. 464835 & -0.780936 \\
\hline 26 & 1 & 0 & -1.287615 & 3. 280838 & -2.536000 \\
\hline 27 & 1 & 0 & -1.986432 & 5. 347352 & -1.352943 \\
\hline 28 & 1 & 0 & -2.069549 & 5. 415994 & 1. 124808 \\
\hline 29 & 1 & 0 & -1.460937 & 3. 417501 & 2. 463531 \\
\hline 30 & 6 & 0 & 4. 143784 & -0.656328 & -0.653252 \\
\hline 31 & 6 & 0 & 3. 932127 & -0.173183 & 0.653018 \\
\hline 32 & 6 & 0 & 4. 975808 & 0.457094 & 1. 343721 \\
\hline 33 & 6 & 0 & 6. 203566 & 0.590089 & 0.712916 \\
\hline 34 & 6 & 0 & 6. 403674 & 0.104149 & -0.592411 \\
\hline 35 & 6 & 0 & 5. 380417 & -0.523987 & -1.290885 \\
\hline 36 & 6 & 0 & 1. 907457 & -0.980903 & 0.280933 \\
\hline 37 & 1 & 0 & 4. 808616 & 0.828696 & 2. 350662 \\
\hline 38 & 1 & 0 & 7. 023853 & 1. 077040 & 1. 231809 \\
\hline 39 & 1 & 0 & 7. 374550 & 0.221542 & -1.064719 \\
\hline 40 & 1 & 0 & 5. 537519 & -0.897703 & -2.298196 \\
\hline 41 & 7 & 0 & 2. 650433 & -0.376105 & 1. 145012 \\
\hline 42 & 16 & 0 & 2. 675770 & -1.392355 & -1.239725 \\
\hline 43 & 6 & 0 & -0.631441 & 0.952506 & 1. 293109 \\
\hline 44 & 1 & 0 & -0.587605 & 0. 769742 & 2. 362030 \\
\hline 45 & 16 & 0 & -0.439043 & 0.637760 & -1.292354 \\
\hline
\end{tabular}

Zero-point correction=

0. 337738 (Hartree/Particle)

Thermal correction to Energy=

0.361219

0.362163

Thermal correction to Enthalpy=

0.281861

$-2035.494778$

$-2035.471296$

$-2035.470352$

$-2035.550654$

Sum of electronic and thermal Energies=

Sum of electronic and thermal Enthalpies=

Sum of electronic and thermal Free Energies=

nergy $=-2036.20401390$

1ah-INT2a

\begin{tabular}{ccccrr} 
Center & Atomic & Atomic & \multicolumn{3}{c}{ Coordinates (Angstroms) } \\
Number & Number & Type & X & Y & Z \\
-1 & 6 & 0 & -2.529004 & -0.505146 & 0.408890 \\
2 & 6 & 0 & -2.447537 & -2.055081 & 0.417831 \\
3 & 6 & 0 & -0.955181 & -2.400039 & 0.458927 \\
4 & 6 & 0 & -0.320405 & -1.293846 & -0.374547 \\
5 & 1 & 0 & -2.849059 & -0.142364 & 1.389540 \\
6 & 1 & 0 & -2.886371 & -2.454065 & -0.500229 \\
7 & 1 & 0 & -2.991992 & -2.482064 & 1.263400
\end{tabular}




\begin{tabular}{|c|c|c|c|c|c|}
\hline 8 & 1 & 0 & -0.572221 & -2.363369 & 1. 486388 \\
\hline 9 & 1 & 0 & -0.729742 & -3.387933 & 0.045952 \\
\hline 10 & 6 & 0 & -3.498730 & 0.060041 & -0.632701 \\
\hline 11 & 1 & 0 & -3.403401 & 1. 147535 & -0.705047 \\
\hline 12 & 1 & 0 & -3.317147 & -0.368105 & -1.623634 \\
\hline 13 & 6 & 0 & -4.936935 & -0.206484 & -0.283138 \\
\hline 14 & 9 & 0 & -5.286340 & 0.324569 & 0.909235 \\
\hline 15 & 9 & 0 & -5.775700 & 0.328570 & -1.196680 \\
\hline 16 & 9 & 0 & -5.244668 & -1.519150 & -0.212716 \\
\hline 17 & 8 & 0 & -0.769006 & -1.484532 & -1.706086 \\
\hline 18 & 1 & 0 & -0.685061 & -0.631475 & -2.170054 \\
\hline 19 & 6 & 0 & 1. 185538 & -1.186815 & -0.332813 \\
\hline 20 & 6 & 0 & 3. 367813 & -0.513398 & -0.839000 \\
\hline 21 & 6 & 0 & 3. 618782 & -1.414004 & 0.217830 \\
\hline 22 & 6 & 0 & 4. 917585 & -1.673433 & 0.671473 \\
\hline 23 & 6 & 0 & 4. 452448 & 0.141003 & -1.451222 \\
\hline 24 & 6 & 0 & 5. 739405 & -0.111740 & -1.003497 \\
\hline 25 & 6 & 0 & 5. 970734 & -1.014442 & 0.052409 \\
\hline 26 & 1 & 0 & 5. 097664 & -2.369469 & 1. 486046 \\
\hline 27 & 1 & 0 & 6. 987165 & -1.199036 & 0.388210 \\
\hline 28 & 1 & 0 & 6. 581658 & 0.390195 & -1.471042 \\
\hline 29 & 1 & 0 & 4. 272414 & 0.836828 & -2.266752 \\
\hline 30 & 6 & 0 & 0.326893 & 1. 966177 & 1. 026022 \\
\hline 31 & 6 & 0 & -0.138041 & 2. 047282 & -0.333619 \\
\hline 32 & 6 & 0 & 0.255392 & 3. 163604 & -1.128906 \\
\hline 33 & 6 & 0 & 1. 068262 & 4. 128826 & -0.578985 \\
\hline 34 & 6 & 0 & 1. 514382 & 4. 026726 & 0.759679 \\
\hline 35 & 6 & 0 & 1. 147686 & 2. 951211 & 1. 566232 \\
\hline 36 & 6 & 0 & -1.060251 & 0.000348 & 0.171617 \\
\hline 37 & 1 & 0 & -0.091056 & 3. 223294 & -2.156540 \\
\hline 38 & 1 & 0 & 1. 377651 & 4. 980636 & -1.177183 \\
\hline 39 & 1 & 0 & 2. 157255 & 4. 800980 & 1. 167384 \\
\hline 40 & 1 & 0 & 1. 498565 & 2. 885186 & 2. 591665 \\
\hline 41 & 7 & 0 & -0.901284 & 1. 054340 & -0.774438 \\
\hline 42 & 16 & 0 & -0.299314 & 0.545350 & 1.793739 \\
\hline 43 & 6 & 0 & 1. 960820 & -0.409441 & -1.133702 \\
\hline 44 & 1 & 0 & 1. 569217 & 0.229158 & -1.920756 \\
\hline 45 & 16 & 0 & 2. 134810 & -2.108728 & 0.818209 \\
\hline
\end{tabular}

Zero-point correction=

0.340639 (Hartree/Particle)

Thermal correction to Energy=

0.363820

Thermal correction to Enthalpy=

Thermal correction to Gibbs Free Energy=

Sum of electronic and zero-point Energies=

Sum of electronic and thermal Energies=

Sum of electronic and thermal Enthalpies=

Sum of electronic and thermal Free Energies=

0.364764

0.285812

$-2035.530280$

$-2035.507099$

$-2035.506155$

$-2035.585107$

M06 $-2 X / 6-311++G(d, p) / S M D / / M 06-2 X / 6-31+G(d) / S M D \quad$ energy $=-2036.24153287$

1ah-INT2b

\begin{tabular}{rrrrrr} 
Center & Atomic & Atomic & \multicolumn{3}{c}{ Coordinates (Angstroms) } \\
Number & Number & Type & X & Y & $Z$ \\
-1 & 6 & 0 & -2.440877 & -0.732607 & 0.254305 \\
2 & 6 & 0 & -2.349902 & -2.120896 & -0.418402 \\
3 & 6 & 0 & -0.854167 & -2.514834 & -0.394448 \\
4 & 6 & 0 & -0.167026 & -1.469225 & 0.482422 \\
5 & 1 & 0 & -2.634337 & -0.868530 & 1.324722 \\
6 & 1 & 0 & -2.715242 & -2.070838 & -1.449923 \\
7 & 1 & 0 & -2.968581 & -2.849710 & 0.110053 \\
8 & 1 & 0 & -0.672053 & -3.515788 & 0.005066 \\
9 & 1 & 0 & -0.443515 & -2.480988 & -1.409568 \\
10 & 6 & 0 & -3.501023 & 0.209479 & -0.310356 \\
11 & 1 & 0 & -3.351967 & 1.230687 & 0.060048 \\
12 & 1 & 0 & -3.477753 & 0.235581 & -1.404098 \\
13 & 6 & 0 & -4.895382 & -0.184800 & 0.087147 \\
14 & 9 & 0 & -5.817586 & 0.662366 & -0.417964 \\
15 & 9 & 0 & -5.238426 & -1.418533 & -0.342924 \\
16 & 9 & 0 & -5.076824 & -0.188907 & 1.425647 \\
17 & 8 & 0 & -0.374748 & -1.842482 & 1.833222
\end{tabular}




$\begin{array}{rrrrrr}18 & 1 & 0 & 0.180947 & -1.264205 & 2.387405 \\ 19 & 6 & 0 & -1.001950 & -0.157280 & 0.188930 \\ 20 & 6 & 0 & -0.112712 & 2.098799 & 0.560138 \\ 21 & 6 & 0 & 0.075491 & 1.993987 & -0.847537 \\ 22 & 6 & 0 & 0.680152 & 3.005650 & -1.578782 \\ 23 & 6 & 0 & 0.327114 & 3.274245 & 1.214810 \\ 24 & 6 & 0 & 0.931082 & 4.284561 & 0.481068 \\ 25 & 6 & 0 & 1.110183 & 4.155270 & -0.905510 \\ 26 & 1 & 0 & 0.818391 & 2.912156 & -2.652531 \\ 27 & 1 & 0 & 1.586849 & 4.954044 & -1.465910 \\ 28 & 1 & 0 & 1.272066 & 5.185106 & 0.983708 \\ 29 & 1 & 0 & 0.190304 & 3.370229 & 2.288725 \\ 30 & 6 & 0 & 3.663024 & -1.272077 & -0.437896 \\ 31 & 6 & 0 & 3.358344 & -0.567082 & 0.743446 \\ 32 & 6 & 0 & 4.373641 & 0.098735 & 1.443662 \\ 33 & 6 & 0 & 5.667397 & 0.044615 & 0.947643 \\ 34 & 6 & 0 & 5.961095 & -0.662989 & -0.232925 \\ 35 & 6 & 0 & 4.967048 & -1.328316 & -0.938975 \\ 36 & 6 & 0 & 1.319013 & -1.281621 & 0.265838 \\ 37 & 1 & 0 & 4.133100 & 0.642371 & 2.352990 \\ 38 & 1 & 0 & 6.467150 & 0.555360 & 1.475720 \\ 39 & 1 & 0 & 6.982679 & -0.690279 & -0.600414 \\ 40 & 1 & 0 & 5.197293 & -1.872857 & -1.849896 \\ 41 & 7 & 0 & 2.021650 & -0.603404 & 1.111638 \\ 42 & 16 & 0 & 2.206218 & -1.985966 & -1.072918 \\ 43 & 6 & 0 & -0.730385 & 0.970211 & 1.124990 \\ 44 & 1 & 0 & -0.983759 & 0.885817 & 2.177489 \\ 45 & 16 & 0 & -0.570005 & 0.488982 & -1.498399 \\ -----------------------------------------1\end{array}$

Zero-point correction=

0. 339899 (Hartree/Particle)

Thermal correction to Energy=

0.363437

Thermal correction to Enthalpy=

0.364381

Thermal correction to Gibbs Free Energy=

Sum of electronic and zero-point Energies=

Sum of electronic and thermal Energies=

Sum of electronic and thermal Enthalpies=

Sum of electronic and thermal Free Energies=

0.283376

$-2035.536835$

$-2035.513297$

$-2035.512353$

$-2035.593359$

M06 $-2 X / 6-311++G(d, p) / S M D / / M 06-2 X / 6-31+G(d) / S M D \quad$ energy $=-2036.24759889$

1ah-TS2a

\begin{tabular}{rrrrrr} 
Center & Atomic & Atomic & \multicolumn{2}{c}{ Coordinates (Angstroms) } \\
Number & Number & Type & X & \multicolumn{1}{c}{ Y } & $Z$ \\
-1 & 6 & 0 & 2.534469 & -0.046365 & -0.683510 \\
2 & 6 & 0 & 2.352122 & -1.553697 & -0.904408 \\
3 & 6 & 0 & 1.588865 & -2.110046 & 0.309892 \\
4 & 6 & 0 & 0.634449 & -1.058173 & 0.845624 \\
5 & 1 & 0 & 2.875534 & 0.428993 & -1.610363 \\
6 & 1 & 0 & 3.298700 & -2.075376 & -1.061002 \\
7 & 1 & 0 & 1.758976 & -1.702732 & -1.815434 \\
8 & 1 & 0 & 1.067118 & -3.036020 & 0.047367 \\
9 & 1 & 0 & 2.286554 & -2.350190 & 1.118839 \\
10 & 6 & 0 & 3.515901 & 0.341463 & 0.430859 \\
11 & 1 & 0 & 3.508486 & 1.427650 & 0.559748 \\
12 & 1 & 0 & 3.256540 & -0.112286 & 1.391265 \\
13 & 6 & 0 & 4.939387 & -0.043908 & 0.135707 \\
14 & 9 & 0 & 5.345920 & 0.333283 & -1.095285 \\
15 & 9 & 0 & 5.793609 & 0.529091 & 1.011246 \\
16 & 9 & 0 & 5.162945 & -1.374041 & 0.216739 \\
17 & 8 & 0 & 1.030279 & -0.579190 & 2.078540 \\
18 & 1 & 0 & 0.850049 & 0.383630 & 2.106759 \\
19 & 6 & 0 & -0.813161 & -1.205964 & 0.704227 \\
20 & 6 & 0 & -3.093073 & -0.695674 & 0.859827 \\
21 & 6 & 0 & -3.121719 & -1.706274 & -0.127342 \\
22 & 6 & 0 & -4.318282 & -2.116752 & -0.723897 \\
23 & 6 & 0 & -4.298594 & -0.075997 & 1.240313 \\
24 & 6 & 0 & -5.484135 & -0.473590 & 0.643383 \\
25 & 6 & 0 & -5.494077 & -1.490110 & -0.331294 \\
26 & 1 & 0 & -4.328802 & -2.897858 & -1.479035 \\
27 & 1 & 0 & -6.434215 & -1.788115 & -0.786500
\end{tabular}




$\begin{array}{rrrrrr}28 & 1 & 0 & -6.418155 & 0.002095 & 0.928280 \\ 29 & 1 & 0 & -4.287089 & 0.707873 & 1.993611 \\ 30 & 6 & 0 & -0.920298 & 1.739225 & -1.030832 \\ 31 & 6 & 0 & -0.262204 & 2.168369 & 0.155345 \\ 32 & 6 & 0 & -0.859142 & 3.169118 & 0.951946 \\ 33 & 6 & 0 & -2.079930 & 3.694406 & 0.566670 \\ 34 & 6 & 0 & -2.725195 & 3.248475 & -0.604768 \\ 35 & 6 & 0 & -2.151871 & 2.271038 & -1.412897 \\ 36 & 6 & 0 & 1.134859 & 0.488701 & -0.372005 \\ 37 & 1 & 0 & -0.358273 & 3.495717 & 1.859207 \\ 38 & 1 & 0 & -2.554041 & 4.457254 & 1.177425 \\ 39 & 1 & 0 & -3.685925 & 3.672484 & -0.881748 \\ 40 & 1 & 0 & -2.655174 & 1.926454 & -2.311731 \\ 41 & 7 & 0 & 0.909268 & 1.540719 & 0.446967 \\ 42 & 16 & 0 & 0.027249 & 0.509796 & -1.808435 \\ 43 & 6 & 0 & -1.760538 & -0.437174 & 1.326039 \\ 44 & 1 & 0 & -1.530581 & 0.307662 & 2.081642 \\ 45 & 16 & 0 & -1.520129 & -2.324558 & -0.450502 \\ --------------------------------------------------\end{array}$

Zero-point correction=

0.338668 (Hartree/Particle)

Thermal correction to Energy=

0. 361745

Thermal correction to Enthalpy=

Thermal correction to Gibbs Free Energy=

Sum of electronic and zero-point Energies=

Sum of electronic and thermal Energies=

0.362689

0.284003

$-2035.514377$

$-2035.491300$

$-2035.490355$

Sum of electronic and thermal Free Energies=

$-2035.569041$

M06-2X/6-311++G (d, p) /SMD//M06-2X/6-31+G(d) /SMD energy $=-2036.22440640$

$1 \mathrm{ah}-\mathrm{TS} 2 \mathrm{~b}$

\begin{tabular}{|c|c|c|c|c|c|}
\hline \multirow{2}{*}{$\begin{array}{l}\text { Center } \\
\text { Number }\end{array}$} & \multirow{2}{*}{$\begin{array}{l}\text { Atomic } \\
\text { Number }\end{array}$} & \multirow{2}{*}{$\begin{array}{c}\text { Atomic } \\
\text { Type }\end{array}$} & \multicolumn{3}{|c|}{ Coordinates (Angstroms) } \\
\hline & & & X & $\mathrm{Y}$ & Z \\
\hline 1 & 6 & 0 & -2.627895 & -0.291197 & 0.238683 \\
\hline 2 & 6 & 0 & -2.733297 & -1.617204 & -0.542598 \\
\hline 3 & 6 & 0 & -1.357600 & -2.328369 & -0.523414 \\
\hline 4 & 6 & 0 & -0.448044 & -1.643266 & 0.455471 \\
\hline 5 & 1 & 0 & -2.767302 & -0.496335 & 1. 305910 \\
\hline 6 & 1 & 0 & -3.032653 & -1.420836 & -1.578845 \\
\hline 7 & 1 & 0 & -3.508766 & -2.248500 & -0.101863 \\
\hline 8 & 1 & 0 & -1.453597 & -3.384609 & -0.247090 \\
\hline 9 & 1 & 0 & -0.902659 & -2.294511 & -1.518108 \\
\hline 10 & 6 & 0 & -3.652497 & 0.770627 & -0.178325 \\
\hline 11 & 1 & 0 & -3.466412 & 1. 703822 & 0.363617 \\
\hline 12 & 1 & 0 & -3.602002 & 0.982453 & -1.251700 \\
\hline 13 & 6 & 0 & -5.074218 & 0.379913 & 0.119931 \\
\hline 14 & 9 & 0 & -5.926843 & 1. 400695 & -0.114651 \\
\hline 15 & 9 & 0 & -5.511928 & -0.654635 & -0.629311 \\
\hline 16 & 9 & 0 & -5.257640 & 0.017986 & 1. 407828 \\
\hline 17 & 8 & 0 & -0.795571 & -1.880901 & 1. 757487 \\
\hline 18 & 1 & 0 & -0.075085 & -1.541059 & 2. 324512 \\
\hline 19 & 6 & 0 & -1.210884 & 0.241281 & 0.121963 \\
\hline 20 & 6 & 0 & 0.548610 & 1. 749861 & 0.603672 \\
\hline 21 & 6 & 0 & 0.679945 & 1. 644077 & -0.806356 \\
\hline 22 & 6 & 0 & 1. 770356 & 2. 186272 & -1.484056 \\
\hline 23 & 6 & 0 & 1. 538632 & 2. 452477 & 1. 324447 \\
\hline 24 & 6 & 0 & 2. 618614 & 2. 998805 & 0.648848 \\
\hline 25 & 6 & 0 & 2. 739983 & 2. 864004 & -0.746663 \\
\hline 26 & 1 & 0 & 1. 869022 & 2. 076828 & -2.560929 \\
\hline 27 & 1 & 0 & 3. 600076 & 3. 288601 & -1.256406 \\
\hline 28 & 1 & 0 & 3. 390100 & 3. 526142 & 1. 203179 \\
\hline 29 & 1 & 0 & 1. 456986 & 2. 537949 & 2. 405206 \\
\hline 30 & 6 & 0 & 3. 275284 & -1.144401 & -0.535963 \\
\hline 31 & 6 & 0 & 3. 039747 & -0.900905 & 0.834791 \\
\hline 32 & 6 & 0 & 4. 084221 & -0.431370 & 1. 646812 \\
\hline 33 & 6 & 0 & 5. 329025 & -0.216236 & 1. 075235 \\
\hline 34 & 6 & 0 & 5.551474 & -0.465101 & -0.292180 \\
\hline 35 & 6 & 0 & 4. 531308 & -0.933324 & -1.111436 \\
\hline 36 & 6 & 0 & 0.987991 & -1.510957 & 0.258126 \\
\hline 37 & 1 & 0 & 3. 899042 & -0.235958 & 2. 699492 \\
\hline
\end{tabular}




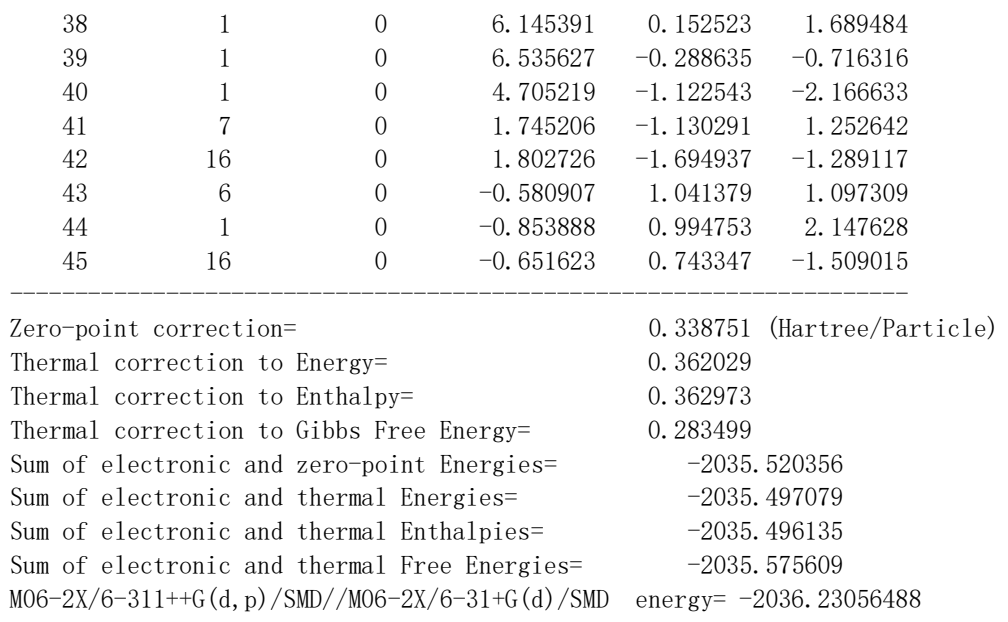

\section{1ah-INT3a}

\begin{tabular}{|c|c|c|c|c|c|}
\hline \multirow{2}{*}{$\begin{array}{l}\text { Center } \\
\text { Number }\end{array}$} & \multirow{2}{*}{$\begin{array}{l}\text { Atomic } \\
\text { Number }\end{array}$} & \multirow{2}{*}{$\begin{array}{c}\text { Atomic } \\
\text { Type }\end{array}$} & \multicolumn{3}{|c|}{ Coordinates (Angstroms) } \\
\hline & & & $\mathrm{x}$ & Y & Z \\
\hline 1 & 6 & 0 & 2. 420063 & 0.133473 & -0.824619 \\
\hline 2 & 6 & 0 & 2. 195506 & -1.398103 & -0.840629 \\
\hline 3 & 6 & 0 & 1.840780 & -2.059752 & 0.511714 \\
\hline 4 & 6 & 0 & 0.746385 & -1.363959 & 1. 254401 \\
\hline 5 & 1 & 0 & 2.800668 & 0.395236 & -1.820711 \\
\hline 6 & 1 & 0 & 3. 098409 & -1.879763 & -1.229402 \\
\hline 7 & 1 & 0 & 1. 402888 & -1.613196 & -1.566551 \\
\hline 8 & 1 & 0 & 1. 569223 & -3.103039 & 0.308777 \\
\hline 9 & 1 & 0 & 2. 722525 & -2.081878 & 1. 160000 \\
\hline 10 & 6 & 0 & 3. 432237 & 0.634792 & 0.213233 \\
\hline 11 & 1 & 0 & 3. 512313 & 1.724160 & 0.151172 \\
\hline 12 & 1 & 0 & 3. 129189 & 0.377601 & 1. 232046 \\
\hline 13 & 6 & 0 & 4. 821964 & 0. 098320 & 0.006686 \\
\hline 14 & 9 & 0 & 5. 263124 & 0.255040 & -1.260142 \\
\hline 15 & 9 & 0 & 5. 711782 & 0.730581 & 0.801299 \\
\hline 16 & 9 & 0 & 4. 941044 & -1.217431 & 0.287638 \\
\hline 17 & 8 & 0 & 1.183206 & -0.562271 & 2. 260579 \\
\hline 18 & 1 & 0 & 0.616988 & 0.229605 & 2. 337123 \\
\hline 19 & 6 & 0 & -0.610664 & -1.409728 & 0.936987 \\
\hline 20 & 6 & 0 & -2.904158 & -0.865351 & 0.866376 \\
\hline 21 & 6 & 0 & -2.825758 & -1.785624 & -0.208869 \\
\hline 22 & 6 & 0 & -3.937118 & -2.101041 & -0.990079 \\
\hline 23 & 6 & 0 & -4.145959 & -0.258036 & 1. 145928 \\
\hline 24 & 6 & 0 & -5.250405 & -0.565901 & 0.365834 \\
\hline 25 & 6 & 0 & -5.149801 & -1.481242 & -0.697294 \\
\hline 26 & 1 & 0 & -3.860067 & -2.809054 & -1.810847 \\
\hline 27 & 1 & 0 & -6.025345 & -1.709209 & -1.298260 \\
\hline 28 & 1 & 0 & -6.205942 & -0.093386 & 0.575912 \\
\hline 29 & 1 & 0 & -4.223042 & 0.452953 & 1. 964959 \\
\hline 30 & 6 & 0 & -1.151987 & 1. 776865 & -0.965380 \\
\hline 31 & 6 & 0 & -0.473231 & 2. 161928 & 0.207468 \\
\hline 32 & 6 & 0 & -1.116330 & 2. 973144 & 1. 151317 \\
\hline 33 & 6 & 0 & -2.421019 & 3. 377204 & 0.904758 \\
\hline 34 & 6 & 0 & -3.088896 & 2. 986016 & -0.269827 \\
\hline 35 & 6 & 0 & -2.464855 & 2. 183719 & -1.217930 \\
\hline 36 & 6 & 0 & 1. 119793 & 0.887339 & -0.658979 \\
\hline 37 & 1 & 0 & -0.593227 & 3. 261785 & 2. 059080 \\
\hline 38 & 1 & 0 & -2.936279 & 4. 001059 & 1. 629310 \\
\hline 39 & 1 & 0 & -4.112620 & 3. 308428 & -0.437311 \\
\hline 40 & 1 & 0 & -2.986743 & 1. 874717 & -2.119212 \\
\hline 41 & 7 & 0 & 0.808286 & 1. 642753 & 0.343454 \\
\hline 42 & 16 & 0 & -0.112572 & 0.753835 & -1.914288 \\
\hline 43 & 6 & 0 & -1.648766 & -0.671037 & 1. 513761 \\
\hline 44 & 1 & 0 & -1.523012 & -0.002373 & 2. 360038 \\
\hline 45 & 16 & 0 & -1.204916 & -2.426749 & -0.390290 \\
\hline
\end{tabular}


Thermal correction to Energy=

0.363574

Thermal correction to Enthalpy=

0. 364519

Thermal correction to Gibbs Free Energy=

Sum of electronic and zero-point Energies=

Sum of electronic and thermal Energies=

0.285381

$-2035.542760$

$-2035.519026$

$-2035.518082$

Sum of electronic and thermal Enthalpies=

$-2035.597219$

M06 $-2 X / 6-311++G(d, p) / S M D / / M 06-2 X / 6-31+G(d) / S M D \quad$ energy $=-2036.25541385$

\section{1ah-INT3b}

\begin{tabular}{|c|c|c|c|c|c|}
\hline \multirow{2}{*}{$\begin{array}{l}\text { Center } \\
\text { Number }\end{array}$} & \multirow{2}{*}{$\begin{array}{l}\text { Atomic } \\
\text { Number }\end{array}$} & \multirow{2}{*}{$\begin{array}{l}\text { Atomic } \\
\text { Type }\end{array}$} & \multicolumn{3}{|c|}{ Coordinates (Angstroms) } \\
\hline & & & X & $\mathrm{Y}$ & Z \\
\hline 1 & 6 & 0 & 2. 494133 & -0.087922 & -0.146069 \\
\hline 2 & 6 & 0 & 2. 665250 & -0.977073 & 1. 106370 \\
\hline 3 & 6 & 0 & 1. 382359 & -1.645089 & 1. 647169 \\
\hline 4 & 6 & 0 & 0.462615 & -2.147628 & 0.592169 \\
\hline 5 & 1 & 0 & 2. 415723 & -0.742943 & -1.021682 \\
\hline 6 & 1 & 0 & 3. 094733 & -0.386627 & 1. 925186 \\
\hline 7 & 1 & 0 & 3. 395842 & -1.754550 & 0.864487 \\
\hline 8 & 1 & 0 & 1. 682671 & -2.474843 & 2. 301553 \\
\hline 9 & 1 & 0 & 0.832699 & -0.937177 & 2.275020 \\
\hline 10 & 6 & 0 & 3. 700057 & 0.845345 & -0.364996 \\
\hline 11 & 1 & 0 & 3. 532781 & 1. 453587 & -1.259360 \\
\hline 12 & 1 & 0 & 3. 842624 & 1. 521843 & 0.485184 \\
\hline 13 & 6 & 0 & 5. 010469 & 0.134794 & -0.577063 \\
\hline 14 & 9 & 0 & 5.968709 & 0.989992 & -0.995259 \\
\hline 15 & 9 & 0 & 5. 494878 & -0.448222 & 0.540028 \\
\hline 16 & 9 & 0 & 4. 932272 & -0.837541 & -1.510073 \\
\hline 17 & 8 & 0 & 1. 015199 & -2.905523 & -0.367801 \\
\hline 18 & 1 & 0 & 0.320718 & -3.114677 & -1.027349 \\
\hline 19 & 6 & 0 & 1. 228162 & 0.728037 & -0.159633 \\
\hline 20 & 6 & 0 & -0.865350 & 1. 537450 & -0.825789 \\
\hline 21 & 6 & 0 & -0.679969 & 2. 271131 & 0.364905 \\
\hline 22 & 6 & 0 & -1.654847 & 3. 151615 & 0.844867 \\
\hline 23 & 6 & 0 & -2.060748 & 1. 696691 & -1.549285 \\
\hline 24 & 6 & 0 & -3.030453 & 2. 568004 & -1.076956 \\
\hline 25 & 6 & 0 & -2.829208 & 3. 290074 & 0.114557 \\
\hline 26 & 1 & 0 & -1.503316 & 3. 707468 & 1. 766226 \\
\hline 27 & 1 & 0 & -3.602486 & 3.964771 & 0.471066 \\
\hline 28 & 1 & 0 & -3.959942 & 2. 690519 & -1.626242 \\
\hline 29 & 1 & 0 & -2.217006 & 1. 131492 & -2.465206 \\
\hline 30 & 6 & 0 & -3.166101 & -0.901352 & 0.611055 \\
\hline 31 & 6 & 0 & -2.871411 & -1.673447 & -0.539160 \\
\hline 32 & 6 & 0 & -3.854193 & -1.821765 & -1.533177 \\
\hline 33 & 6 & 0 & -5.085007 & -1.204234 & -1.361361 \\
\hline 34 & 6 & 0 & -5.362520 & -0.444567 & -0.211047 \\
\hline 35 & 6 & 0 & -4.406344 & -0.289408 & 0.788534 \\
\hline 36 & 6 & 0 & -0.899860 & -1.833917 & 0.475587 \\
\hline 37 & 1 & 0 & -3.633548 & -2.408243 & -2.420863 \\
\hline 38 & 1 & 0 & -5.847665 & -1.308873 & -2.127918 \\
\hline 39 & 1 & 0 & -6.332988 & 0.030046 & -0.099720 \\
\hline 40 & 1 & 0 & -4.616734 & 0.303238 & 1. 674470 \\
\hline 41 & 7 & 0 & -1.602674 & -2.198142 & -0.590491 \\
\hline 42 & 16 & 0 & -1.773739 & -0.855616 & 1. 662680 \\
\hline 43 & 6 & 0 & 0.251643 & 0.669922 & -1.100063 \\
\hline 44 & 1 & 0 & 0.297009 & 0.002234 & -1.956407 \\
\hline 45 & 16 & 0 & 0.852564 & 1. 881608 & 1. 112410 \\
\hline \multicolumn{4}{|c|}{ Zero-point correction $=$} & \multicolumn{2}{|c|}{ 0. 340692 (Hartree/Particle) } \\
\hline \multicolumn{4}{|c|}{ Thermal correction to Energy= } & \multicolumn{2}{|c|}{0.363571} \\
\hline \multicolumn{4}{|c|}{ Thermal correction to Enthalpy $=$} & \multicolumn{2}{|l|}{0.364515} \\
\hline \multicolumn{4}{|c|}{ Thermal correction to Gibbs Free Energy= } & \multicolumn{2}{|l|}{0.287793} \\
\hline \multicolumn{4}{|c|}{ Sum of electronic and zero-point Energies= } & \multicolumn{2}{|c|}{-2035.546868} \\
\hline \multicolumn{4}{|c|}{ Sum of electronic and thermal Energies= } & \multicolumn{2}{|c|}{-2035.523990} \\
\hline \multicolumn{4}{|c|}{ Sum of electronic and thermal Enthalpies= } & \multicolumn{2}{|c|}{-2035.523046} \\
\hline Sum of e & ctronic & thermal $\mathrm{F}$ & Energies= & -2035 & 99768 \\
\hline M06-2X/6 & $11++G(d$, & $\mathrm{SMD} / / \mathrm{M} 06-2$ & $31+G(d) / S M D$ & $\operatorname{rgy}=-2$ & 36. 2591754 \\
\hline
\end{tabular}

1ai-INT1a 


\begin{tabular}{|c|c|c|c|c|c|}
\hline \multirow{2}{*}{$\begin{array}{l}\text { Center } \\
\text { Number }\end{array}$} & \multirow{2}{*}{$\begin{array}{l}\text { Atomic } \\
\text { Number }\end{array}$} & \multirow{2}{*}{$\begin{array}{c}\text { Atomic } \\
\text { Type }\end{array}$} & \multicolumn{3}{|c|}{ Coordinates (Angstroms) } \\
\hline & & & X & Y & Z \\
\hline 1 & 6 & 0 & 1. 405317 & -1.327167 & -0.707548 \\
\hline 2 & 6 & 0 & 0.522029 & -2.263940 & 0.052841 \\
\hline 3 & 6 & 0 & -0.978702 & -2.026678 & -0.172400 \\
\hline 4 & 6 & 0 & -1.562905 & -0.865872 & 0.666845 \\
\hline 5 & 1 & 0 & 1. 250650 & -1.220774 & -1.777901 \\
\hline 6 & 1 & 0 & 0.753573 & -2.215688 & 1. 124938 \\
\hline 7 & 1 & 0 & 0.734868 & -3.300845 & -0.252176 \\
\hline 8 & 1 & 0 & -1.156833 & -1.846004 & -1.240767 \\
\hline 9 & 1 & 0 & -1.547569 & -2.919447 & 0.109185 \\
\hline 10 & 6 & 0 & 2. 656721 & -0.765775 & -0.114913 \\
\hline 11 & 1 & 0 & 2. 988523 & 0.135140 & -0.643381 \\
\hline 12 & 1 & 0 & 2. 517433 & -0.515270 & 0.944514 \\
\hline 13 & 6 & 0 & 3. 809410 & -1.740835 & -0.171319 \\
\hline 14 & 9 & 0 & 4. 936909 & -1.232636 & 0.369950 \\
\hline 15 & 9 & 0 & 3.548779 & -2.887072 & 0.493858 \\
\hline 16 & 9 & 0 & 4. 121124 & -2.099878 & -1.435050 \\
\hline 17 & 8 & 0 & -1.449005 & -1.169027 & 2. 043031 \\
\hline 18 & 1 & 0 & -0.666650 & -0.697792 & 2. 385173 \\
\hline 19 & 6 & 0 & -3.034941 & -0.646143 & 0.376085 \\
\hline 20 & 6 & 0 & -5.259880 & -0.007327 & 0.653726 \\
\hline 21 & 6 & 0 & -5.281901 & -0.391256 & -0.656025 \\
\hline 22 & 6 & 0 & 0.358767 & 2. 361493 & -0.539603 \\
\hline 23 & 6 & 0 & 0.779680 & 1. 946058 & 0.738092 \\
\hline 24 & 6 & 0 & 1. 815962 & 2. 624668 & 1. 390998 \\
\hline 25 & 6 & 0 & 2. 407204 & 3. 704681 & 0.751416 \\
\hline 26 & 6 & 0 & 1. 976485 & 4. 114982 & -0.523213 \\
\hline 27 & 6 & 0 & 0.947442 & 3.453538 & -1.182564 \\
\hline 28 & 6 & 0 & -0.777100 & 0.412931 & 0.398191 \\
\hline 29 & 1 & 0 & 2. 141442 & 2. 295968 & 2. 374036 \\
\hline 30 & 1 & 0 & 3. 215068 & 4. 241463 & 1. 239777 \\
\hline 31 & 1 & 0 & 2. 455326 & 4. 963565 & -1.002901 \\
\hline 32 & 1 & 0 & 0.616719 & 3. 773114 & -2.166180 \\
\hline 33 & 7 & 0 & 0.109053 & 0.835171 & 1. 234962 \\
\hline 34 & 16 & 0 & -0.941282 & 1. 331268 & -1.086067 \\
\hline 35 & 6 & 0 & -3.967104 & -0.151673 & 1. 245720 \\
\hline 36 & 1 & 0 & -3.733399 & 0.095067 & 2.275425 \\
\hline 37 & 16 & 0 & -3.736918 & -0.947778 & -1.182244 \\
\hline 38 & 1 & 0 & -6.131733 & 0.362424 & 1. 182542 \\
\hline 39 & 1 & 0 & -6.117758 & -0.394579 & -1.345091 \\
\hline
\end{tabular}

Zero-point correction=

Thermal correction to Energy=

0.289942 (Hartree/Particle)

Thermal correction to Enthalpy=

0.311842

Thermal correction to Gibbs Free Energy=

Sum of electronic and zero-point Energies=

Sum of electronic and thermal Energies=

0.312787

0.234749

$-1881.963594$

$-1881.941693$

$-1881.940749$

Sum of electronic and thermal Enthalpies=
Sum of electronic and thermal Free Energies

$-1882.018786$

M06 $-2 X / 6-311++G(d, p) / S M D / / M 06-2 X / 6-31+G(d) / S M D \quad$ energy $=-1882.59366560$

\section{1ai-INT1b}

\begin{tabular}{rrrrrr} 
Center & Atomic & Atomic & \multicolumn{3}{c}{ Coordinates (Angstroms) } \\
Number & Number & Type & X & Y & $Z$ \\
\hline 1 & 6 & 0 & -2.745649 & -0.537597 & -0.594353 \\
2 & 6 & 0 & -1.985749 & -1.510043 & 0.248076 \\
3 & 6 & 0 & -0.476120 & -1.524333 & -0.037658 \\
4 & 6 & 0 & 0.289073 & -0.405030 & 0.687928 \\
5 & 1 & 0 & -2.585735 & -0.544986 & -1.669195 \\
6 & 1 & 0 & -2.361486 & -2.530183 & 0.069018 \\
7 & 1 & 0 & -2.159101 & -1.305503 & 1.312153 \\
8 & 1 & 0 & -0.036416 & -2.473629 & 0.287610 \\
9 & 1 & 0 & -0.314959 & -1.439674 & -1.120589 \\
10 & 6 & 0 & -3.907214 & 0.233682 & -0.059702 \\
11 & 1 & 0 & -3.740930 & 0.535985 & 0.981769 \\
12 & 1 & 0 & -4.106069 & 1.132929 & -0.653290
\end{tabular}




\begin{tabular}{|c|c|c|c|c|c|}
\hline 13 & 6 & 0 & -5.185231 & -0.572101 & -0.066104 \\
\hline 14 & 9 & 0 & -6.231678 & 0.117911 & 0.436036 \\
\hline 15 & 9 & 0 & -5.545871 & -0.960650 & -1.308075 \\
\hline 16 & 9 & 0 & -5.085456 & -1.701309 & 0.668889 \\
\hline 17 & 8 & 0 & 0.147828 & -0.643835 & 2. 072416 \\
\hline 18 & 1 & 0 & 0.855675 & -0.153175 & 2. 529084 \\
\hline 19 & 6 & 0 & -0.268196 & 0.972706 & 0.358725 \\
\hline 20 & 6 & 0 & -1.508953 & 2.938146 & 0.540268 \\
\hline 21 & 6 & 0 & -1.079137 & 3. 044233 & -0.749870 \\
\hline 22 & 6 & 0 & 4. 004752 & -0.585054 & -0.650954 \\
\hline 23 & 6 & 0 & 3. 941983 & -0.129431 & 0.680242 \\
\hline 24 & 6 & 0 & 5. 119828 & 0.208792 & 1. 359690 \\
\hline 25 & 6 & 0 & 6. 329389 & 0.085230 & 0.692455 \\
\hline 26 & 6 & 0 & 6. 379999 & -0.371052 & -0.637556 \\
\hline 27 & 6 & 0 & 5. 222302 & -0.712712 & -1.325068 \\
\hline 28 & 6 & 0 & 1. 777348 & -0.439667 & 0.350004 \\
\hline 29 & 1 & 0 & 5. 068654 & 0.559579 & 2. 386383 \\
\hline 30 & 1 & 0 & 7. 252405 & 0.343589 & 1.203005 \\
\hline 31 & 1 & 0 & 7. 340042 & -0.459489 & -1.137537 \\
\hline 32 & 1 & 0 & 5. 263868 & -1.066386 & -2.350822 \\
\hline 33 & 7 & 0 & 2. 660354 & -0.058775 & 1. 209226 \\
\hline 34 & 16 & 0 & 2. 393280 & -0.943692 & -1.213564 \\
\hline 35 & 6 & 0 & -1.041609 & 1. 745666 & 1. 178401 \\
\hline 36 & 1 & 0 & -1.288663 & 1. 458661 & 2. 194449 \\
\hline 37 & 16 & 0 & -0.082223 & 1. 708220 & -1.202623 \\
\hline 38 & 1 & 0 & -2.137535 & 3. 678540 & 1. 023684 \\
\hline 39 & 1 & 0 & -1.265618 & 3. 841224 & -1.459412 \\
\hline \multicolumn{4}{|c|}{ Zero-point correction= } & \multicolumn{2}{|c|}{ 0. 290163 (Hartree/Particle) } \\
\hline \multirow{2}{*}{\multicolumn{4}{|c|}{$\begin{array}{l}\text { Thermal correction to Energy= } \\
\text { Thermal correction to Enthalpy= }\end{array}$}} & \multicolumn{2}{|c|}{0.311987} \\
\hline & & & & \multicolumn{2}{|l|}{0.312931} \\
\hline \multicolumn{4}{|c|}{ Thermal correction to Gibbs Free Energy= } & \multicolumn{2}{|l|}{0.235444} \\
\hline \multicolumn{4}{|c|}{ Sum of electronic and zero-point Energies= } & \multicolumn{2}{|c|}{-1881.961669} \\
\hline \multicolumn{4}{|c|}{ Sum of electronic and thermal Energies $=$} & \multicolumn{2}{|c|}{-1881.939845} \\
\hline \multicolumn{4}{|c|}{ Sum of electronic and thermal Enthalpies $=$} & \multirow{2}{*}{\multicolumn{2}{|c|}{$\begin{array}{l}-1881.938901 \\
-1882016388\end{array}$}} \\
\hline \multicolumn{4}{|c|}{ Sum of electronic and thermal Free Energies= } & & \\
\hline \multicolumn{4}{|c|}{ M06-2X/6-311++G (d, p) /SMD //M06-2X/6-31+G (d) /SMD } & \multicolumn{2}{|c|}{$\begin{array}{l}-1882.016388 \\
\mathrm{rgy}=-1882.5915\end{array}$} \\
\hline
\end{tabular}

1ai-TS1a

\begin{tabular}{rrrrrr} 
Center & Atomic & Atomic & \multicolumn{3}{c}{ Coordinates (Angstroms) } \\
Number & Number & Type & $X$ & Y & \multicolumn{1}{c}{ Z } \\
-1 & 6 & 0 & 1.444367 & -1.120057 & -0.543483 \\
2 & 6 & 0 & 0.828980 & -2.490239 & -0.482278 \\
3 & 6 & 0 & -0.696071 & -2.365937 & -0.563167 \\
4 & 6 & 0 & -1.107047 & -1.266558 & 0.413783 \\
5 & 1 & 0 & 1.556005 & -0.686572 & -1.535267 \\
6 & 1 & 0 & 1.103758 & -2.985803 & 0.452838 \\
7 & 1 & 0 & 1.194193 & -3.106630 & -1.313661 \\
8 & 1 & 0 & -0.997597 & -2.087843 & -1.581997 \\
9 & 1 & 0 & -1.193968 & -3.307205 & -0.309855 \\
10 & 6 & 0 & 2.510006 & -0.727649 & 0.438084 \\
11 & 1 & 0 & 2.635079 & 0.360087 & 0.478831 \\
12 & 1 & 0 & 2.279767 & -1.091343 & 1.445220 \\
13 & 6 & 0 & 3.856910 & -1.294164 & 0.059649 \\
14 & 9 & 0 & 4.818932 & -0.937897 & 0.935895 \\
15 & 9 & 0 & 3.861282 & -2.643442 & 0.014755 \\
16 & 9 & 0 & 4.271218 & -0.873815 & -1.154543 \\
17 & 8 & 0 & -0.688530 & -1.664678 & 1.704406 \\
18 & 1 & 0 & -0.460473 & -0.856515 & 2.201373 \\
19 & 6 & 0 & -2.596272 & -0.968745 & 0.418656 \\
20 & 6 & 0 & -4.662240 & -0.095848 & 1.061731 \\
21 & 6 & 0 & -5.012771 & -0.707994 & -0.107595 \\
22 & 6 & 0 & -0.052655 & 2.347314 & -0.738839 \\
23 & 6 & 0 & 0.303731 & 2.089823 & 0.606323 \\
24 & 6 & 0 & 0.853819 & 3.119951 & 1.387868 \\
25 & 6 & 0 & 1.035513 & 4.369930 & 0.815970 \\
26 & 6 & 0 & 0.680097 & 4.611187 & -0.523682 \\
27 & 6 & 0 & 0.135864 & 3.604407 & -1.314839 \\
28 & 6 & 0 & -0.339293 & 0.023345 & 0.057656
\end{tabular}




$\begin{array}{rrrrrr}29 & 1 & 0 & 1.127808 & 2.920085 & 2.419968 \\ 30 & 1 & 0 & 1.460691 & 5.174910 & 1.408152 \\ 31 & 1 & 0 & 0.831729 & 5.598605 & -0.949632 \\ 32 & 1 & 0 & -0.137614 & 3.794782 & -2.348387 \\ 33 & 7 & 0 & 0.064027 & 0.808970 & 1.041538 \\ 34 & 16 & 0 & -0.726942 & 0.906533 & -1.454628 \\ 35 & 6 & 0 & -3.272969 & -0.243120 & 1.360685 \\ 36 & 1 & 0 & -2.795368 & 0.176030 & 2.241435 \\ 37 & 16 & 0 & -3.661291 & -1.477785 & -0.851444 \\ 38 & 1 & 0 & -5.365455 & 0.439811 & 1.690290 \\ 39 & 1 & 0 & -5.989487 & -0.765247 & -0.572266\end{array}$

Zero-point correction=

0.290115 (Hartree/Particle)

Thermal correction to Energy=

0.310999

Thermal correction to Enthalpy=

0.311944

Thermal correction to Gibbs Free Energy=

Sum of electronic and zero-point Energies=

Sum of electronic and thermal Energies=

0.236794

$-1881.949114$

$-1881.928229$

$-1881.927285$

-1882.002435
nergy $=-1882.57875537$

Sum of electronic and thermal Free Energies=

M06 $-2 X / 6-311++G(d, p) / S M D / / M 06-2 X / 6-31+G(d) / S M D \quad$ energy $=-1882.57875537$

\section{$1 \mathrm{ai}-\mathrm{TS} 1 \mathrm{~b}$}

\begin{tabular}{|c|c|c|c|c|c|}
\hline \multirow{2}{*}{$\begin{array}{l}\text { Center } \\
\text { Number }\end{array}$} & \multirow{2}{*}{$\begin{array}{l}\text { Atomic } \\
\text { Number }\end{array}$} & \multirow{2}{*}{$\begin{array}{c}\text { Atomic } \\
\text { Type }\end{array}$} & \multicolumn{3}{|c|}{ Coordinates (Angstroms) } \\
\hline & & & X & Y & Z \\
\hline 1 & 6 & 0 & -2.535633 & -0.307092 & -0.502131 \\
\hline 2 & 6 & 0 & -2.035550 & -1.724952 & -0.556558 \\
\hline 3 & 6 & 0 & -0.516196 & -1.723668 & -0.761497 \\
\hline 4 & 6 & 0 & 0.059775 & -0.703476 & 0.215388 \\
\hline 5 & 1 & 0 & -2.703552 & 0.185411 & -1.458495 \\
\hline 6 & 1 & 0 & -2.514554 & -2.281402 & -1.372455 \\
\hline 7 & 1 & 0 & -2.270710 & -2.242192 & 0.378941 \\
\hline 8 & 1 & 0 & -0.077621 & -2.711159 & -0.586702 \\
\hline 9 & 1 & 0 & -0.277391 & -1.420921 & -1.790260 \\
\hline 10 & 6 & 0 & -3.474141 & 0.104910 & 0.593550 \\
\hline 11 & 1 & 0 & -3.171257 & -0.323585 & 1. 555887 \\
\hline 12 & 1 & 0 & -3.513951 & 1. 195374 & 0.697001 \\
\hline 13 & 6 & 0 & -4.890986 & -0.345820 & 0.341905 \\
\hline 14 & 9 & 0 & -5.725046 & 0.016308 & 1. 341137 \\
\hline 15 & 9 & 0 & -5.407516 & 0.175892 & -0.791809 \\
\hline 16 & 9 & 0 & -5.001843 & -1.687056 & 0.224383 \\
\hline 17 & 8 & 0 & -0.278623 & -1.130649 & 1. 519359 \\
\hline 18 & 1 & 0 & 0.304503 & -0.660655 & 2. 143236 \\
\hline 19 & 6 & 0 & -0.580921 & 0.669528 & -0.041523 \\
\hline 20 & 6 & 0 & -0.968166 & 2. 933628 & 0.478470 \\
\hline 21 & 6 & 0 & -0.675199 & 3. 047185 & -0.856144 \\
\hline 22 & 6 & 0 & 3. 949200 & -0.535195 & -0.452926 \\
\hline 23 & 6 & 0 & 3. 612495 & -0.089464 & 0.840372 \\
\hline 24 & 6 & 0 & 4. 617095 & 0.351727 & 1. 711901 \\
\hline 25 & 6 & 0 & 5. 931727 & 0.337916 & 1. 270494 \\
\hline 26 & 6 & 0 & 6. 256645 & -0.109037 & -0.023465 \\
\hline 27 & 6 & 0 & 5. 273340 & -0.550549 & -0.899773 \\
\hline 28 & 6 & 0 & 1. 575634 & -0.582851 & 0.134603 \\
\hline 29 & 1 & 0 & 4. 353538 & 0.694818 & 2. 708246 \\
\hline 30 & 1 & 0 & 6. 723631 & 0.677190 & 1.931637 \\
\hline 31 & 1 & 0 & 7. 293926 & -0.109813 & -0.344979 \\
\hline 32 & 1 & 0 & 5. 527124 & -0.894580 & -1.897887 \\
\hline 33 & 7 & 0 & 2. 256396 & -0.131332 & 1. 133090 \\
\hline 34 & 16 & 0 & 2. 499583 & -1.027920 & -1.287606 \\
\hline 35 & 6 & 0 & -0.848881 & 1. 608531 & 0.962700 \\
\hline 36 & 1 & 0 & -1.024768 & 1. 324225 & 1. 994627 \\
\hline 37 & 16 & 0 & -0.221445 & 1. 526830 & -1.547387 \\
\hline 38 & 1 & 0 & -1.258179 & 3. 778999 & 1. 094048 \\
\hline 39 & 1 & 0 & -0.698537 & 3. 936006 & -1.475052 \\
\hline
\end{tabular}

Zero-point correction=

0.290214 (Hartree/Particle)

Thermal correction to Energy=

0.310937

Thermal correction to Enthalpy=

0.311881

Thermal correction to Gibbs Free Energy=

0. 238246 
Sum of electronic and zero-point Energies= Sum of electronic and thermal Energies= Sum of electronic and thermal Enthalpies= Sum of electronic and thermal Free Energies=

$-1881.945600$ $-1881.924877$ $-1881.923933$ $-1881.997568$ M06 $-2 X / 6-311++G(d, p) / S M D / / M 06-2 X / 6-31+G(d) / S M D \quad$ energy $=-1882.57551037$

\section{1ai-INT2a}

\begin{tabular}{|c|c|c|c|c|c|}
\hline \multirow{2}{*}{$\begin{array}{l}\text { Center } \\
\text { Number }\end{array}$} & \multirow{2}{*}{$\begin{array}{l}\text { Atomic } \\
\text { Number }\end{array}$} & \multirow{2}{*}{$\begin{array}{c}\text { Atomic } \\
\text { Type }\end{array}$} & \multicolumn{3}{|c|}{ Coordinates (Angstroms) } \\
\hline & & & X & Y & Z \\
\hline 1 & 6 & 0 & 1. 830080 & -0.328021 & -0.460816 \\
\hline 2 & 6 & 0 & 1. 960231 & -1.873465 & -0.535394 \\
\hline 3 & 6 & 0 & 0.530003 & -2.423643 & -0.495257 \\
\hline 4 & 6 & 0 & -0.198883 & -1.438854 & 0.410389 \\
\hline 5 & 1 & 0 & 2. 049093 & 0.114294 & -1.436508 \\
\hline 6 & 1 & 0 & 2. 514835 & -2.240815 & 0.331809 \\
\hline 7 & 1 & 0 & 2. 496512 & -2.188878 & -1.433598 \\
\hline 8 & 1 & 0 & 0.078658 & -2.418932 & -1.495258 \\
\hline 9 & 1 & 0 & 0.472230 & -3.442631 & -0.100805 \\
\hline 10 & 6 & 0 & 2. 764069 & 0.325957 & 0.561413 \\
\hline 11 & 1 & 0 & 2. 519139 & 1. 384059 & 0.692350 \\
\hline 12 & 1 & 0 & 2. 697536 & -0.165664 & 1. 536980 \\
\hline 13 & 6 & 0 & 4. 204484 & 0.284641 & 0.131332 \\
\hline 14 & 9 & 0 & 5. 006915 & 0.896249 & 1. 029300 \\
\hline 15 & 9 & 0 & 4. 690786 & -0.965958 & -0.017741 \\
\hline 16 & 9 & 0 & 4. 410726 & 0.909384 & -1.049247 \\
\hline 17 & 8 & 0 & 0.362765 & -1.592759 & 1. 705218 \\
\hline 18 & 1 & 0 & 0.178346 & -0.776222 & 2. 204178 \\
\hline 19 & 6 & 0 & -1.701838 & -1.550515 & 0.467455 \\
\hline 20 & 6 & 0 & -3.919953 & -1.222510 & 1. 125199 \\
\hline 21 & 6 & 0 & -4.101276 & -2.097474 & 0.092539 \\
\hline 22 & 6 & 0 & -1.351386 & 1. 740572 & -0.881426 \\
\hline 23 & 6 & 0 & -0.838412 & 1. 856297 & 0.458253 \\
\hline 24 & 6 & 0 & -1.331963 & 2. 900175 & 1. 295157 \\
\hline 25 & 6 & 0 & -2.281912 & 3. 766107 & 0.802661 \\
\hline 26 & 6 & 0 & -2.770782 & 3. 633253 & -0.518194 \\
\hline 27 & 6 & 0 & -2.310654 & 2. 625631 & -1.363574 \\
\hline 28 & 6 & 0 & 0.319194 & -0.042233 & -0.137034 \\
\hline 29 & 1 & 0 & -0.949726 & 2. 984929 & 2. 308244 \\
\hline 30 & 1 & 0 & -2.667510 & 4. 561800 & 1. 433018 \\
\hline 31 & 1 & 0 & -3.521125 & 4. 329113 & -0.881130 \\
\hline 32 & 1 & 0 & -2.695860 & 2. 534900 & -2.374651 \\
\hline 33 & 7 & 0 & 0.064843 & 0.961909 & 0.841632 \\
\hline 34 & 16 & 0 & -0.591540 & 0.421339 & -1.707391 \\
\hline 35 & 6 & 0 & -2.542079 & -0.911803 & 1. 341419 \\
\hline 36 & 1 & 0 & -2.195858 & -0.240886 & 2. 122249 \\
\hline 37 & 16 & 0 & -2.602123 & -2.554215 & -0.623447 \\
\hline 38 & 1 & 0 & -4.733905 & -0.814088 & 1. 714468 \\
\hline 39 & 1 & 0 & -5.029151 & -2.508017 & -0.286658 \\
\hline
\end{tabular}

Zero-point correction=

0. 293000 (Hartree/Particle)

Thermal correction to Energy=

313572

0.314516

0.241008

$-1881.981223$

$-1881.960651$

$-1881.959707$

$-1882.033215$

Sum of electronic and thermal Enthalpies=

Sum of electronic and thermal Free Energies=

ergy $=-1882.61317129$

\section{1ai-INT2b}

\begin{tabular}{cccccr} 
Center & Atomic & Atomic & \multicolumn{3}{c}{ Coordinates (Angstroms) } \\
Number & Number & Type & X & Y & $Z$ \\
-----1 & 6 & 0 & -2.368923 & -0.161530 & -0.392584 \\
1 & 6 & 0 & -2.192854 & -1.703129 & -0.351235 \\
2 & 6 & 0 & -0.685820 & -1.954810 & -0.456621 \\
3 & 6 & 0 & -0.096499 & -0.780476 & 0.311208 \\
4 & 1 & 0 & -2.756963 & 0.144034 & -1.368602 \\
5 & & 0 & & &
\end{tabular}




\begin{tabular}{|c|c|c|c|c|c|}
\hline 6 & 1 & 0 & -2.751511 & -2.197471 & -1.149581 \\
\hline 7 & 1 & 0 & -2.557822 & -2.094819 & 0.602492 \\
\hline 8 & 1 & 0 & -0.373408 & -2.911965 & -0.029546 \\
\hline 9 & 1 & 0 & -0.356113 & -1.919627 & -1.502846 \\
\hline 10 & 6 & 0 & -3.336053 & 0.368941 & 0.670285 \\
\hline 11 & 1 & 0 & -3.073743 & 0.004142 & 1. 669111 \\
\hline 12 & 1 & 0 & -3.333855 & 1. 463487 & 0.689055 \\
\hline 13 & 6 & 0 & -4.763530 & -0.025165 & 0.405916 \\
\hline 14 & 9 & 0 & -5.600647 & 0.498303 & 1. 328023 \\
\hline 15 & 9 & 0 & -5.204998 & 0.407476 & -0.795770 \\
\hline 16 & 9 & 0 & -4.972123 & -1.358754 & 0.422510 \\
\hline 17 & 8 & 0 & -0.446362 & -0.955255 & 1. 673234 \\
\hline 18 & 1 & 0 & 0.086265 & -0.327391 & 2. 194477 \\
\hline 19 & 6 & 0 & -0.924597 & 0.451166 & -0.244139 \\
\hline 20 & 6 & 0 & -0.081179 & 2. 721653 & 0.008026 \\
\hline 21 & 6 & 0 & 0.277212 & 2. 498277 & -1.320243 \\
\hline 22 & 6 & 0 & 3. 796053 & -0.644090 & -0.288907 \\
\hline 23 & 6 & 0 & 3. 405667 & 0.097871 & 0.843446 \\
\hline 24 & 6 & 0 & 4. 369942 & 0.770146 & 1. 606768 \\
\hline 25 & 6 & 0 & 5. 699146 & 0.686115 & 1. 220898 \\
\hline 26 & 6 & 0 & 6. 078429 & -0.057987 & 0.088168 \\
\hline 27 & 6 & 0 & 5. 135946 & -0.730432 & -0.679104 \\
\hline 28 & 6 & 0 & 1. 402369 & -0.606262 & 0.219540 \\
\hline 29 & 1 & 0 & 4. 064461 & 1. 341745 & 2. 478591 \\
\hline 30 & 1 & 0 & 6. 460182 & 1. 201411 & 1. 799411 \\
\hline 31 & 1 & 0 & 7. 126317 & -0.108642 & -0.192814 \\
\hline 32 & 1 & 0 & 5. 431909 & -1.303368 & -1.552759 \\
\hline 33 & 7 & 0 & 2. 042770 & 0.087820 & 1. 101533 \\
\hline 34 & 16 & 0 & 2. 386838 & -1.359261 & -1.021942 \\
\hline 35 & 6 & 0 & -0.758745 & 1. 678338 & 0.601195 \\
\hline 36 & 1 & 0 & -1.105091 & 1. 689871 & 1. 629130 \\
\hline 37 & 16 & 0 & -0.279759 & 0.962138 & -1.912371 \\
\hline 38 & 1 & 0 & 0.160066 & 3. 647391 & 0.521959 \\
\hline 39 & 1 & 0 & 0.798072 & 3. 177480 & -1.984232 \\
\hline
\end{tabular}

Zero-point correction $=$

0. 292628 (Hartree/Particle)

Thermal correction to Energy=

0.313320

Thermal correction to Enthalpy=

Thermal correction to Gibbs Free Energy=

Sum of electronic and zero-point Energies=

Sum of electronic and thermal Energies=

Sum of electronic and thermal Enthalpies=

0.314264

0.240213

$-1881.981939$

$-1881.961248$

$-1881.960303$

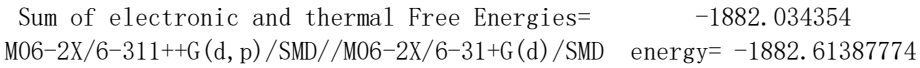

1ai-TS2a

\begin{tabular}{rrrrrr} 
Center & Atomic & Atomic & \multicolumn{3}{c}{ Coordinates (Angstroms) } \\
Number & Number & Type & $X$ & Y & $Z$ \\
-1 & 6 & 0 & 1.699316 & -0.358853 & 0.025850 \\
2 & 6 & 0 & 1.934514 & -1.119790 & 1.331729 \\
3 & 6 & 0 & 0.722315 & -2.036393 & 1.465562 \\
4 & 6 & 0 & -0.546971 & -1.230757 & 1.251497 \\
5 & 1 & 0 & 1.706889 & -1.085575 & -0.797787 \\
6 & 1 & 0 & 2.002547 & -0.416336 & 2.172000 \\
7 & 1 & 0 & 2.860193 & -1.700129 & 1.305368 \\
8 & 1 & 0 & 0.801060 & -2.827145 & 0.710860 \\
9 & 1 & 0 & 0.664145 & -2.518565 & 2.447800 \\
10 & 6 & 0 & 2.703306 & 0.756088 & -0.282519 \\
11 & 1 & 0 & 2.435168 & 1.257863 & -1.218627 \\
12 & 1 & 0 & 2.709885 & 1.504338 & 0.516431 \\
13 & 6 & 0 & 4.118130 & 0.271888 & -0.444767 \\
14 & 9 & 0 & 4.919396 & 1.239910 & -0.939791 \\
15 & 9 & 0 & 4.682364 & -0.120020 & 0.718009 \\
16 & 9 & 0 & 4.221705 & -0.777091 & -1.288835 \\
17 & 8 & 0 & -1.007793 & -0.566815 & 2.365686 \\
18 & 1 & 0 & -0.437148 & 0.214408 & 2.512306 \\
19 & 6 & 0 & -1.661817 & -1.815424 & 0.517435 \\
20 & 6 & 0 & -3.795075 & -2.062204 & -0.400532 \\
21 & 6 & 0 & -3.104262 & -2.991559 & -1.129988
\end{tabular}




\begin{tabular}{|c|c|c|c|c|c|}
\hline 22 & 6 & 0 & -1.458087 & 1. 770207 & -0.783666 \\
\hline 23 & 6 & 0 & -0.914770 & 2. 096405 & 0.491249 \\
\hline 24 & 6 & 0 & -1.447034 & 3. 188367 & 1. 209952 \\
\hline 25 & 6 & 0 & -2.489492 & 3. 912199 & 0.658159 \\
\hline 26 & 6 & 0 & -3.020970 & 3. 572224 & -0.601932 \\
\hline 27 & 6 & 0 & -2.513026 & 2. 500709 & -1.330735 \\
\hline 28 & 6 & 0 & 0.292714 & 0. 234787 & 0.109933 \\
\hline 29 & 1 & 0 & -1.032211 & 3. 437392 & 2. 182865 \\
\hline 30 & 1 & 0 & -2.909506 & 4. 752172 & 1. 203700 \\
\hline 31 & 1 & 0 & -3.840699 & 4. 154463 & -1.012436 \\
\hline 32 & 1 & 0 & -2.927262 & 2. 242676 & -2.301067 \\
\hline 33 & 7 & 0 & 0.110456 & 1. 311140 & 0.914523 \\
\hline 34 & 16 & 0 & -0.605238 & 0.410174 & -1.451328 \\
\hline 35 & 6 & 0 & -2.970646 & -1.383708 & 0.539780 \\
\hline 36 & 1 & 0 & -3.313633 & -0.598825 & 1. 204767 \\
\hline 37 & 16 & 0 & -1.444395 & -3.062741 & -0.673241 \\
\hline 38 & 1 & 0 & -4.855302 & -1.872696 & -0.528924 \\
\hline 39 & 1 & 0 & -3.480902 & -3.656726 & -1.897765 \\
\hline
\end{tabular}

Zero-point correction=

Thermal correction to Energy=

0.291296 (Hartree/Particle)

Thermal correction to Enthalpy=

0.311721

0.312666

Thermal correction to Gibbs Free Energy=

Sum of electronic and zero-point Energies $=$

Sum of electronic and thermal Energies=

0.239923

$-1881.968651$

$-1881.948225$

$-1881.947281$

Sum of electronic and thermal Enthalpies=

Sum of electronic and thermal Free Energies=

$-1882.020024$

M06-2X/6-311++G (d, p) /SMD//M06-2X/6-31+G (d)/SMD energy $=-1882.59875004$

\section{1ai-TS2b}

\begin{tabular}{|c|c|c|c|c|c|}
\hline \multirow{2}{*}{$\begin{array}{l}\text { Center } \\
\text { Number }\end{array}$} & \multirow{2}{*}{$\begin{array}{l}\text { Atomic } \\
\text { Number }\end{array}$} & \multirow{2}{*}{$\begin{array}{c}\text { Atomic } \\
\text { Type }\end{array}$} & \multicolumn{3}{|c|}{ Coordinates (Angstroms) } \\
\hline & & & $\mathrm{x}$ & Y & Z \\
\hline 1 & 6 & 0 & -2.396652 & -0.061331 & -0.408851 \\
\hline 2 & 6 & 0 & -2.259808 & -1.605555 & -0.376488 \\
\hline 3 & 6 & 0 & -0.789583 & -1.955362 & -0.586302 \\
\hline 4 & 6 & 0 & -0.021405 & -1.074860 & 0.351970 \\
\hline 5 & 1 & 0 & -2.744756 & 0.225943 & -1.407237 \\
\hline 6 & 1 & 0 & -2.894316 & -2.070368 & -1.135278 \\
\hline 7 & 1 & 0 & -2.578012 & -1.987207 & 0.598402 \\
\hline 8 & 1 & 0 & -0.591294 & -3.013782 & -0.374826 \\
\hline 9 & 1 & 0 & -0.479273 & -1.753753 & -1.619175 \\
\hline 10 & 6 & 0 & -3.421375 & 0.467005 & 0.603192 \\
\hline 11 & 1 & 0 & -3.198005 & 0.112130 & 1. 615282 \\
\hline 12 & 1 & 0 & -3.423891 & 1. 561493 & 0.614550 \\
\hline 13 & 6 & 0 & -4.834643 & 0.056698 & 0.288924 \\
\hline 14 & 9 & 0 & -5.711688 & 0.608735 & 1. 155329 \\
\hline 15 & 9 & 0 & -5.223210 & 0.445051 & -0.945303 \\
\hline 16 & 9 & 0 & -5.040370 & -1.275989 & 0.344929 \\
\hline 17 & 8 & 0 & -0.421736 & -1.235263 & 1. 653559 \\
\hline 18 & 1 & 0 & 0.238656 & -0.795252 & 2. 223197 \\
\hline 19 & 6 & 0 & -1.034575 & 0.632649 & -0.205588 \\
\hline 20 & 6 & 0 & 0.325479 & 2. 427453 & 0.523098 \\
\hline 21 & 6 & 0 & 0.688528 & 2. 381212 & -0.807225 \\
\hline 22 & 6 & 0 & 3. 732766 & -0.506900 & -0.464500 \\
\hline 23 & 6 & 0 & 3. 416569 & -0.173914 & 0.870900 \\
\hline 24 & 6 & 0 & 4. 419405 & 0.326727 & 1. 715919 \\
\hline 25 & 6 & 0 & 5. 702845 & 0.479546 & 1.213210 \\
\hline 26 & 6 & 0 & 6. 005018 & 0.141453 & -0.118782 \\
\hline 27 & 6 & 0 & 5. 025585 & -0.354168 & -0.971489 \\
\hline 28 & 6 & 0 & 1. 403108 & -0.833659 & 0.217131 \\
\hline 29 & 1 & 0 & 4. 176401 & 0.586024 & 2. 742562 \\
\hline 30 & 1 & 0 & 6. 488064 & 0.866549 & 1. 856170 \\
\hline 31 & 1 & 0 & 7. 018215 & 0.268844 & -0.488476 \\
\hline 32 & 1 & 0 & 5. 259709 & -0.613959 & -1.999720 \\
\hline 33 & 7 & 0 & 2. 099038 & -0.377405 & 1. 227166 \\
\hline 34 & 16 & 0 & 2. 303516 & -1.100987 & -1.269492 \\
\hline 35 & 6 & 0 & -0.695385 & 1. 521815 & 0.852604 \\
\hline 36 & 1 & 0 & -1.117430 & 1. 425481 & 1. 847390 \\
\hline 37 & 16 & 0 & -0.274311 & 1. 253100 & -1.692982 \\
\hline
\end{tabular}




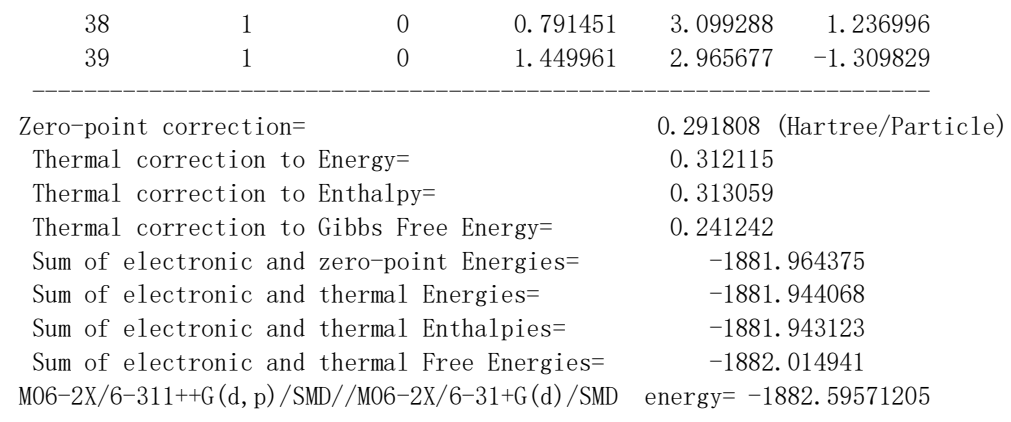

1ai-INT3a

\begin{tabular}{|c|c|c|c|c|c|}
\hline \multirow{2}{*}{$\begin{array}{l}\text { Center } \\
\text { Number }\end{array}$} & \multirow{2}{*}{$\begin{array}{l}\text { Atomic } \\
\text { Number }\end{array}$} & \multirow{2}{*}{$\begin{array}{c}\text { Atomic } \\
\text { Type }\end{array}$} & \multicolumn{3}{|c|}{ Coordinates (Angstroms) } \\
\hline & & & $\mathrm{X}$ & Y & Z \\
\hline 1 & 6 & 0 & 1. 937828 & -0.153623 & -0.257803 \\
\hline 2 & 6 & 0 & 1. 921722 & -1.168039 & 0.900572 \\
\hline 3 & 6 & 0 & 0.718545 & -2.126791 & 0.880580 \\
\hline 4 & 6 & 0 & -0.601885 & -1.518208 & 1. 245682 \\
\hline 5 & 1 & 0 & 2. 013820 & -0.705083 & -1.202441 \\
\hline 6 & 1 & 0 & 1.981965 & -0.632635 & 1. 857063 \\
\hline 7 & 1 & 0 & 2. 824351 & -1.781764 & 0.834768 \\
\hline 8 & 1 & 0 & 0.654081 & -2.593493 & -0.109555 \\
\hline 9 & 1 & 0 & 0.931518 & -2.935249 & 1. 596127 \\
\hline 10 & 6 & 0 & 3. 102987 & 0.849290 & -0.175122 \\
\hline 11 & 1 & 0 & 3. 056593 & 1. 539844 & -1.023339 \\
\hline 12 & 1 & 0 & 3. 042881 & 1. 436894 & 0.746583 \\
\hline 13 & 6 & 0 & 4. 471100 & 0.220990 & -0.214431 \\
\hline 14 & 9 & 0 & 5. 427704 & 1. 148387 & -0.430733 \\
\hline 15 & 9 & 0 & 4. 809020 & -0.401355 & 0.934776 \\
\hline 16 & 9 & 0 & 4. 597866 & -0.696334 & -1.196171 \\
\hline 17 & 8 & 0 & -0.672255 & -0.797870 & 2. 404361 \\
\hline 18 & 1 & 0 & -0.058464 & -0.037378 & 2. 342137 \\
\hline 19 & 6 & 0 & -1.805077 & -1.711022 & 0.569049 \\
\hline 20 & 6 & 0 & -4.060758 & -1.486161 & -0.057192 \\
\hline 21 & 6 & 0 & -3.627157 & -2.352115 & -1.031588 \\
\hline 22 & 6 & 0 & -1.486783 & 1. 685424 & -0.842039 \\
\hline 23 & 6 & 0 & -0.997441 & 1. 912953 & 0.459984 \\
\hline 24 & 6 & 0 & -1.757033 & 2. 656793 & 1. 373064 \\
\hline 25 & 6 & 0 & -2.987039 & 3. 151863 & 0.966369 \\
\hline 26 & 6 & 0 & -3.465782 & 2. 919876 & -0.336623 \\
\hline 27 & 6 & 0 & -2.723037 & 2. 189656 & -1.255889 \\
\hline 28 & 6 & 0 & 0.660525 & 0.647525 & -0.281926 \\
\hline 29 & 1 & 0 & -1.378994 & 2. 823274 & 2. 378165 \\
\hline 30 & 1 & 0 & -3.592156 & 3. 725192 & 1. 662523 \\
\hline 31 & 1 & 0 & -4.433087 & 3. 316784 & -0.630507 \\
\hline 32 & 1 & 0 & -3.097359 & 2. 008537 & -2.259214 \\
\hline 33 & 7 & 0 & 0.227067 & 1. 320237 & 0.736155 \\
\hline 34 & 16 & 0 & -0.344695 & 0.703096 & -1.715584 \\
\hline 35 & 6 & 0 & -3.050461 & -1.120148 & 0.858975 \\
\hline 36 & 1 & 0 & -3.186358 & -0.438034 & 1. 690375 \\
\hline 37 & 16 & 0 & -1.949453 & -2.748324 & -0.846893 \\
\hline 38 & 1 & 0 & -5.081773 & -1.121429 & -0.006749 \\
\hline 39 & 1 & 0 & -4.194906 & -2.786387 & -1.845118 \\
\hline
\end{tabular}

Zero-point correction=

0.291556 (Hartree/Particle)

Thermal correction to Energy=

0.313033

Thermal correction to Enthalpy=

0. 313978

0.238003

$-1881.992336$

$-1881.970858$

$-1881.969914$

$-1882.045889$

Sum of electronic and thermal Enthalpies=

Sum of electronic and thermal Free Energies=

nergy $=-1882.62379002$

\section{1ai-INT3b}

Center Atomic Atomic Coordinates (Angstroms)




\begin{tabular}{|c|c|c|c|c|c|}
\hline Number & Number & Type & $X$ & Y & Z \\
\hline 1 & 6 & 0 & -2.094632 & 0.053004 & 0.106299 \\
\hline 2 & 6 & 0 & -2.132617 & -0.436839 & -1.352571 \\
\hline 3 & 6 & 0 & -0.745320 & -0.633360 & -1.988050 \\
\hline 4 & 6 & 0 & 0.180928 & -1.415776 & -1.126871 \\
\hline 5 & 1 & 0 & -1.694247 & -0.754315 & 0.732722 \\
\hline 6 & 1 & 0 & -2.696723 & 0.271997 & -1.972596 \\
\hline 7 & 1 & 0 & -2.672872 & -1.387190 & -1.388845 \\
\hline 8 & 1 & 0 & -0.874049 & -1.152578 & -2.947662 \\
\hline 9 & 1 & 0 & -0.290319 & 0.336397 & -2.211723 \\
\hline 10 & 6 & 0 & -3.494533 & 0.409411 & 0.642874 \\
\hline 11 & 1 & 0 & -3.409393 & 0.750306 & 1. 679223 \\
\hline 12 & 1 & 0 & -3.954128 & 1. 213749 & 0.057404 \\
\hline 13 & 6 & 0 & -4.476199 & -0.732771 & 0.652511 \\
\hline 14 & 9 & 0 & -5.573696 & -0.429912 & 1. 379622 \\
\hline 15 & 9 & 0 & -4.927305 & -1.067711 & -0.575558 \\
\hline 16 & 9 & 0 & -3.962487 & -1.860116 & 1. 188873 \\
\hline 17 & 8 & 0 & -0.288264 & -2.590859 & -0.679444 \\
\hline 18 & 1 & 0 & 0.400674 & -2.999427 & -0.114473 \\
\hline 19 & 6 & 0 & -1.171478 & 1. 224471 & 0.326692 \\
\hline 20 & 6 & 0 & 0.492964 & 2. 591712 & 1. 239257 \\
\hline 21 & 6 & 0 & 0.000603 & 3. 426237 & 0.279623 \\
\hline 22 & 6 & 0 & 3. 635466 & 0.034622 & -0.292873 \\
\hline 23 & 6 & 0 & 3. 403142 & -1.203829 & 0.354767 \\
\hline 24 & 6 & 0 & 4. 395621 & -1.732897 & 1. 198313 \\
\hline 25 & 6 & 0 & 5.577472 & -1.027791 & 1. 372631 \\
\hline 26 & 6 & 0 & 5. 793151 & 0.199693 & 0.721568 \\
\hline 27 & 6 & 0 & 4. 824536 & 0.742340 & -0.117891 \\
\hline 28 & 6 & 0 & 1. 470778 & -1.034232 & -0.733525 \\
\hline 29 & 1 & 0 & 4. 222563 & -2.681542 & 1. 698833 \\
\hline 30 & 1 & 0 & 6. 349372 & -1.429369 & 2. 022910 \\
\hline 31 & 1 & 0 & 6. 726650 & 0.733414 & 0.873523 \\
\hline 32 & 1 & 0 & 4. 991435 & 1. 690387 & -0.621155 \\
\hline 33 & 7 & 0 & 2. 189244 & -1.788905 & 0.092351 \\
\hline 34 & 16 & 0 & 2. 254566 & 0.456172 & -1.276098 \\
\hline 35 & 6 & 0 & -0.179834 & 1. 329178 & 1. 262461 \\
\hline 36 & 1 & 0 & 0.066211 & 0.519066 & 1. 943508 \\
\hline 37 & 16 & 0 & -1.287183 & 2. 688221 & -0.605269 \\
\hline 38 & 1 & 0 & 1. 310801 & 2. 857192 & 1. 900897 \\
\hline 39 & 1 & 0 & 0.318405 & 4. 431454 & 0.030686 \\
\hline
\end{tabular}

Zero-point correction=

Thermal correction to Energy=

0.292835 (Hartree/Particle)

Thermal correction to Enthalpy=

0.314198

0.315142

0.238685

$-1881.995317$

$-1881.973955$

$-1881.973010$

$-1882.049468$

Sum of electronic and zero-point Energies

Sum of electronic and thermal Energies=

Sum of electronic and thermal Enthalpies=

Sum of electronic and thermal Free Energies=

nergy $=-1882.62770206$

1aj-INT1a

\begin{tabular}{rrrrrr} 
Center & Atomic & Atomic & \multicolumn{3}{c}{ Coordinates (Angstroms) } \\
Number & Number & Type & X & Y & $Z$ \\
-1 & 6 & 0 & -1.807766 & -0.234740 & -0.711929 \\
2 & 6 & 0 & -1.049077 & -1.467572 & -0.340800 \\
3 & 6 & 0 & 0.463081 & -1.364980 & -0.591150 \\
4 & 6 & 0 & 1.189138 & -0.501360 & 0.461062 \\
5 & 1 & 0 & -1.417463 & -2.323366 & -0.928909 \\
6 & 1 & 0 & -1.226510 & -1.718603 & 0.711850 \\
7 & 1 & 0 & 0.918903 & -2.360977 & -0.553753 \\
8 & 1 & 0 & 0.648165 & -0.953964 & -1.591436 \\
9 & 6 & 0 & -2.896179 & 0.316599 & 0.149269 \\
10 & 1 & 0 & -2.664698 & 0.190781 & 1.214231 \\
11 & 1 & 0 & -3.067707 & 1.381325 & -0.043822 \\
12 & 6 & 0 & -4.219994 & -0.373659 & -0.080977 \\
13 & 9 & 0 & -5.196227 & 0.109897 & 0.716829 \\
14 & 9 & 0 & -4.661168 & -0.235278 & -1.349983
\end{tabular}




\begin{tabular}{rrrrrr}
15 & 9 & 0 & -4.157889 & -1.702339 & 0.154123 \\
16 & 8 & 0 & 0.956530 & -1.045553 & 1.742164 \\
17 & 1 & 0 & 1.824494 & -1.198612 & 2.160312 \\
18 & 6 & 0 & 0.672515 & 0.915111 & 0.461862 \\
19 & 6 & 0 & -0.241679 & 2.927457 & 0.775449 \\
20 & 6 & 0 & 0.142246 & 2.797878 & -0.520715 \\
21 & 6 & 0 & 4.970864 & -0.367214 & -0.624975 \\
22 & 6 & 0 & 4.828902 & -0.868441 & 0.631224 \\
23 & 6 & 0 & 2.689603 & -0.508052 & 0.194189 \\
24 & 7 & 0 & 3.529672 & -0.945141 & 1.084828 \\
25 & 16 & 0 & 3.424888 & 0.036194 & -1.277129 \\
26 & 8 & 0 & 0.711592 & 1.578062 & -0.721858 \\
27 & 6 & 0 & 0.109596 & 1.693366 & 1.423988 \\
28 & 1 & 0 & -0.053151 & 1.414229 & 2.455370 \\
29 & 1 & 0 & 5.638380 & -1.198511 & 1.271078 \\
30 & 1 & 0 & 5.879162 & -0.209279 & -1.192351 \\
31 & 1 & 0 & -0.717809 & 3.791252 & 1.219511 \\
32 & 1 & 0 & 0.100058 & 3.448354 & -1.382553 \\
33 & 1 & 0 & -1.682646 & 0.177312 & -1.709421 \\
\hline
\end{tabular}

Zero-point correction=

0.245908 (Hartree/Particle)

Thermal correction to Energy=

0.264685

Thermal correction to Enthalpy=

0.265629

Thermal correction to Gibbs Free Energy=

Sum of electronic and zero-point Energies=

Sum of electronic and thermal Energies=

0. 194347

$-1405.440343$

$-1405.421567$

$-1405.420622$

Sum of electronic and thermal Enthalpies=

$-1405.491904$

M06 $-2 X / 6-311++G(d, p) / S M D / / M 06-2 X / 6-31+G(d) / S M D \quad$ energy $=-1405.98586990$

laj-INT1b

\begin{tabular}{|c|c|c|c|c|c|}
\hline \multirow{2}{*}{$\begin{array}{l}\text { Center } \\
\text { Number }\end{array}$} & \multirow{2}{*}{$\begin{array}{l}\text { Atomic } \\
\text { Number }\end{array}$} & \multirow{2}{*}{$\begin{array}{l}\text { Atomic } \\
\text { Type }\end{array}$} & \multicolumn{3}{|c|}{ Coordinates (Angstroms) } \\
\hline & & & X & $\mathrm{Y}$ & Z \\
\hline 1 & 6 & 0 & 1. 624557 & -0.369823 & -0.731863 \\
\hline 2 & 6 & 0 & 0.921542 & -1.535392 & -0.113752 \\
\hline 3 & 6 & 0 & -0.587455 & -1.570148 & -0.393537 \\
\hline 4 & 6 & 0 & -1.406711 & -0.643557 & 0.534450 \\
\hline 5 & 1 & 0 & 1. 469498 & -0.171962 & -1.789141 \\
\hline 6 & 1 & 0 & 1. 100159 & -1.553415 & 0.969704 \\
\hline 7 & 1 & 0 & 1. 349275 & -2.473422 & -0.502825 \\
\hline 8 & 1 & 0 & -0.782451 & -1.304295 & -1.439399 \\
\hline 9 & 1 & 0 & -0.978657 & -2.581043 & -0.233272 \\
\hline 10 & 6 & 0 & 2. 748665 & 0.331972 & -0.042185 \\
\hline 11 & 1 & 0 & 2. 934370 & 1. 320947 & -0.474579 \\
\hline 12 & 1 & 0 & 2. 546103 & 0.453474 & 1.029710 \\
\hline 13 & 6 & 0 & 4. 051343 & -0.427268 & -0.141772 \\
\hline 14 & 9 & 0 & 5. 067845 & 0.216214 & 0.470829 \\
\hline 15 & 9 & 0 & 3. 979176 & -1.652180 & 0.423033 \\
\hline 16 & 9 & 0 & 4. 439683 & -0.623274 & -1.420189 \\
\hline 17 & 8 & 0 & -1.300737 & -1.085863 & 1. 871091 \\
\hline 18 & 1 & 0 & -0.692955 & -0.479486 & 2. 333633 \\
\hline 19 & 6 & 0 & -2.866989 & -0.698583 & 0.175020 \\
\hline 20 & 6 & 0 & -5.088617 & -0.821785 & -0.019196 \\
\hline 21 & 6 & 0 & -4.540063 & -0.565077 & -1.233561 \\
\hline 22 & 6 & 0 & -0.132440 & 3. 018966 & -0.115352 \\
\hline 23 & 6 & 0 & 0.285070 & 2. 550009 & 1. 091255 \\
\hline 24 & 6 & 0 & -0.886557 & 0.785812 & 0.456016 \\
\hline 25 & 7 & 0 & -0.148365 & 1. 279710 & 1. 405348 \\
\hline 26 & 16 & 0 & -1.127655 & 1. 843468 & -0.895245 \\
\hline 27 & 6 & 0 & -3.992261 & -0.908607 & 0.907245 \\
\hline 28 & 8 & 0 & -3.184210 & -0.491726 & -1.129918 \\
\hline 29 & 1 & 0 & -4.034273 & -1.101607 & 1. 969542 \\
\hline 30 & 1 & 0 & -6.141844 & -0.938451 & 0.197200 \\
\hline 31 & 1 & 0 & -4.946513 & -0.419051 & -2.223936 \\
\hline 32 & 1 & 0 & 0.911490 & 3. 088878 & 1. 792284 \\
\hline 33 & 1 & 0 & 0.065568 & 3. 980733 & -0.571498 \\
\hline
\end{tabular}

Zero-point correction=

0.245959 (Hartree/Particle)

Thermal correction to Energy=

0.264666 
Thermal correction to Enthalpy=

Thermal correction to Gibbs Free Energy=

Sum of electronic and zero-point Energies=

Sum of electronic and thermal Energies=

Sum of electronic and thermal Enthalpies=

Sum of electronic and thermal Free Energies=
0.265610

0.195351

$-1405.441001$

$-1405.422294$

$-1405.421349$

$-1405.491609$

energy $=-1405.98661451$

\section{1aj-TS1a}

\begin{tabular}{|c|c|c|c|c|c|}
\hline \multirow{2}{*}{$\begin{array}{l}\text { Center } \\
\text { Number }\end{array}$} & \multirow{2}{*}{$\begin{array}{l}\text { Atomic } \\
\text { Number }\end{array}$} & \multirow{2}{*}{$\begin{array}{c}\text { Atomic } \\
\text { Type }\end{array}$} & \multicolumn{3}{|c|}{ Coordinates (Angstroms) } \\
\hline & & & X & Y & Z \\
\hline 1 & 6 & 0 & -1.579273 & -0.024865 & -0.568237 \\
\hline 2 & 6 & 0 & -1.086371 & -1.364740 & -1.046905 \\
\hline 3 & 6 & 0 & 0.445321 & -1.345970 & -1.149288 \\
\hline 4 & 6 & 0 & 0.958976 & -0.673955 & 0.129245 \\
\hline 5 & 1 & 0 & -1.516436 & -1.620803 & -2.024396 \\
\hline 6 & 1 & 0 & -1.387302 & -2.144740 & -0.340314 \\
\hline 7 & 1 & 0 & 0.867973 & -2.351985 & -1.238357 \\
\hline 8 & 1 & 0 & 0.766851 & -0.761365 & -2.019498 \\
\hline 9 & 6 & 0 & -2.586850 & 0.044587 & 0.541235 \\
\hline 10 & 1 & 0 & -2.341693 & -0.660558 & 1. 343788 \\
\hline 11 & 1 & 0 & -2.636550 & 1. 051985 & 0.970345 \\
\hline 12 & 6 & 0 & -3.984638 & -0.286957 & 0.082967 \\
\hline 13 & 9 & 0 & -4.884071 & -0.212922 & 1. 089203 \\
\hline 14 & 9 & 0 & -4.426596 & 0.550428 & -0.880916 \\
\hline 15 & 9 & 0 & -4.088818 & -1.534101 & -0.425692 \\
\hline 16 & 8 & 0 & 0.514505 & -1.402976 & 1. 254109 \\
\hline 17 & 1 & 0 & 1. 302741 & -1.669275 & 1. 763227 \\
\hline 18 & 6 & 0 & 0.347416 & 0.713804 & 0.220047 \\
\hline 19 & 6 & 0 & 0.049141 & 2. 839124 & 0.898805 \\
\hline 20 & 6 & 0 & 0.407577 & 2. 823097 & -0.418644 \\
\hline 21 & 6 & 0 & 4. 859863 & -0.253557 & -0.151373 \\
\hline 22 & 6 & 0 & 4. 514867 & -1.045183 & 0.899132 \\
\hline 23 & 6 & 0 & 2. 475909 & -0.595426 & 0.162400 \\
\hline 24 & 7 & 0 & 3. 160034 & -1.233382 & 1. 066360 \\
\hline 25 & 16 & 0 & 3. 442283 & 0.296521 & -0.967099 \\
\hline 26 & 8 & 0 & 0.659172 & 1. 551133 & -0.827483 \\
\hline 27 & 6 & 0 & 0.071496 & 1. 488476 & 1. 343338 \\
\hline 28 & 1 & 0 & -0.152306 & 1. 109510 & 2. 330826 \\
\hline 29 & 1 & 0 & 5. 207989 & -1.517071 & 1. 585132 \\
\hline 30 & 1 & 0 & 5. 848400 & 0.042466 & -0.478981 \\
\hline 31 & 1 & 0 & -0.194667 & 3. 719379 & 1. 478877 \\
\hline 32 & 1 & 0 & 0.536709 & 3. 596733 & -1.162251 \\
\hline 33 & 1 & 0 & -1.653124 & 0.768561 & -1.312024 \\
\hline
\end{tabular}

Zero-point correction=

0. 245972 (Hartree/Particle)

Thermal correction to Energy=

0.263592

Thermal correction to Enthalpy=

0.264536

Thermal correction to Gibbs Free Energy=

Sum of electronic and zero-point Energies=

Sum of electronic and thermal Energies=

0. 197974

$-1405.426612$

$-1405.408991$

$-1405.408047$

$-1405.474609$

Sum of electronic and thermal Free Energies=

nergy $=-1405.97199016$

$1 a j-T S 1 b$

\begin{tabular}{cccccr} 
Center & Atomic & Atomic & \multicolumn{3}{c}{ Coordinates (Angstroms) } \\
Number & Number & Type & X & Y & Z \\
-1 & 6 & 0 & 1.381621 & -0.334489 & -0.537499 \\
2 & 6 & 0 & 0.918531 & -1.761439 & -0.512746 \\
3 & 6 & 0 & -0.600532 & -1.784438 & -0.687475 \\
4 & 6 & 0 & -1.188074 & -0.826226 & 0.349735 \\
5 & 1 & 0 & 1.398001 & 0.138959 & -1.517816 \\
6 & 1 & 0 & 1.190078 & -2.234752 & 0.435616 \\
7 & 1 & 0 & 1.394636 & -2.331670 & -1.322018 \\
8 & 1 & 0 & -0.875738 & -1.443383 & -1.692703 \\
9 & 1 & 0 & -1.020719 & -2.785223 & -0.545137
\end{tabular}




\begin{tabular}{|c|c|c|c|c|c|}
\hline 10 & 6 & 0 & 2. 437825 & 0.145378 & 0.413120 \\
\hline 11 & 1 & 0 & 2. 456654 & 1. 239759 & 0.466940 \\
\hline 12 & 1 & 0 & 2. 277830 & -0.249781 & 1. 421964 \\
\hline 13 & 6 & 0 & 3. 820997 & -0.282369 & -0.010906 \\
\hline 14 & 9 & 0 & 4. 770815 & 0.160633 & 0.839502 \\
\hline 15 & 9 & 0 & 3. 955752 & -1.624688 & -0.067762 \\
\hline 16 & 9 & 0 & 4. 155924 & 0.183825 & -1.232949 \\
\hline 17 & 8 & 0 & -0.894655 & -1.326484 & 1. 635943 \\
\hline 18 & 1 & 0 & -0.674701 & -0.563134 & 2. 203098 \\
\hline 19 & 6 & 0 & -2.682642 & -0.691235 & 0.214712 \\
\hline 20 & 6 & 0 & -4.900147 & -0.460478 & 0.380327 \\
\hline 21 & 6 & 0 & -4.534008 & -0.421609 & -0.926356 \\
\hline 22 & 6 & 0 & -0.329336 & 2. 975236 & -0.148581 \\
\hline 23 & 6 & 0 & 0.001383 & 2. 534582 & 1. 104352 \\
\hline 24 & 6 & 0 & -0.510443 & 0.555394 & 0.215526 \\
\hline 25 & 7 & 0 & -0.164729 & 1. 202017 & 1. 327550 \\
\hline 26 & 16 & 0 & -0.911401 & 1. 666151 & -1.108964 \\
\hline 27 & 6 & 0 & -3.686758 & -0.637096 & 1. 130631 \\
\hline 28 & 8 & 0 & -3.185936 & -0.564801 & -1.040893 \\
\hline 29 & 1 & 0 & -3.578698 & -0.718444 & 2. 203329 \\
\hline 30 & 1 & 0 & -5.906868 & -0.379877 & 0.767295 \\
\hline 31 & 1 & 0 & -5.078363 & -0.314574 & -1.853665 \\
\hline 32 & 1 & 0 & 0.364058 & 3. 170929 & 1. 904207 \\
\hline 33 & 1 & 0 & -0.278841 & 3. 983750 & -0.539102 \\
\hline
\end{tabular}

Zero-point correction=

0. 246198 (Hartree/Particle)

Thermal correction to Energy=

0.263854

Thermal correction to Enthalpy=

0.264798

Thermal correction to Gibbs Free Energy=

Sum of electronic and zero-point Energies=

Sum of electronic and thermal Energies=

0.197842

$-1405.426287$

$-1405.408632$

$-1405.407687$

$-1405.474643$

Sum of electronic and thermal Free Energies=

ergy $=-1405.97189612$

1aj-INT2a

\begin{tabular}{rrrrrr} 
Center & Atomic & Atomic & \multicolumn{2}{c}{ Coordinates (Angstroms) } \\
Number & Number & Type & X & \multicolumn{1}{c}{ Y } \\
-1 & 6 & 0 & -1.427655 & -0.013884 & -0.477702 \\
2 & 6 & 0 & -1.244060 & -1.532254 & -0.742105 \\
3 & 6 & 0 & 0.271112 & -1.766452 & -0.771267 \\
4 & 6 & 0 & 0.782960 & -0.780339 & 0.270576 \\
5 & 1 & 0 & -1.730929 & -1.848274 & -1.667819 \\
6 & 1 & 0 & -1.683350 & -2.108140 & 0.077888 \\
7 & 1 & 0 & 0.561259 & -2.792184 & -0.526860 \\
8 & 1 & 0 & 0.685784 & -1.510262 & -1.752913 \\
9 & 6 & 0 & -2.482002 & 0.303483 & 0.584644 \\
10 & 1 & 0 & -2.302830 & -0.261770 & 1.505454 \\
11 & 1 & 0 & -2.480721 & 1.370738 & 0.828903 \\
12 & 6 & 0 & -3.880579 & -0.007421 & 0.128788 \\
13 & 9 & 0 & -4.795399 & 0.330044 & 1.064179 \\
14 & 9 & 0 & -4.219623 & 0.665549 & -0.992957 \\
15 & 9 & 0 & -4.085505 & -1.313940 & -0.143134 \\
16 & 8 & 0 & 0.336460 & -1.215491 & 1.539239 \\
17 & 1 & 0 & 0.897144 & -0.780771 & 2.207830 \\
18 & 6 & 0 & -0.016340 & 0.529481 & -0.094804 \\
19 & 6 & 0 & 0.888934 & 2.633412 & 0.332536 \\
20 & 6 & 0 & 1.164568 & 2.265496 & -0.973038 \\
21 & 6 & 0 & 4.645436 & -0.192488 & -0.054663 \\
22 & 6 & 0 & 4.227039 & -0.088250 & 1.237104 \\
23 & 6 & 0 & 2.271004 & -0.554558 & 0.312966 \\
24 & 7 & 0 & 2.881635 & -0.297266 & 1.435451 \\
25 & 16 & 0 & 3.308039 & -0.563863 & -1.075172 \\
26 & 8 & 0 & 0.592467 & 1.074953 & -1.291849 \\
27 & 6 & 0 & 0.125079 & 1.628894 & 0.908107 \\
28 & 1 & 0 & -0.252240 & 1.584394 & 1.921167 \\
29 & 1 & 0 & 4.863595 & 0.136861 & 2.084621 \\
30 & 1 & 0 & 5.645195 & -0.075041 & -0.452968 \\
31 & 1 & 0 & 1.217949 & 3.554172 & 0.798093
\end{tabular}




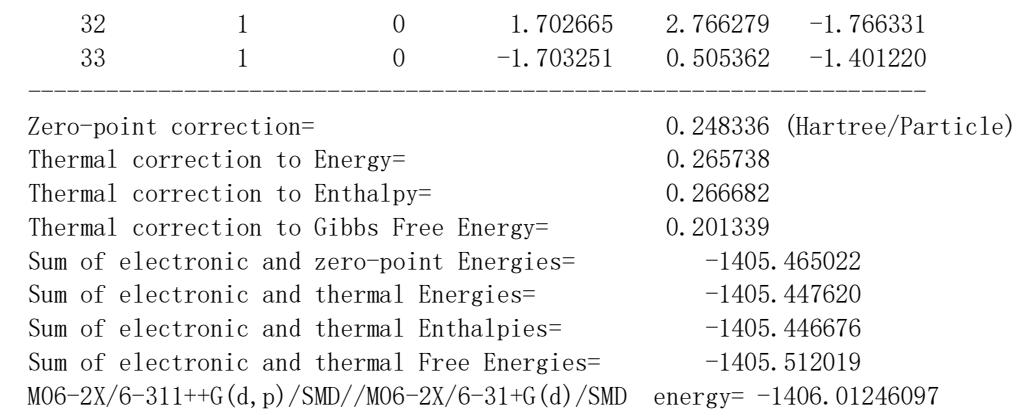

$1 a j-I N T 2 b$

\begin{tabular}{|c|c|c|c|c|c|}
\hline \multirow{2}{*}{$\begin{array}{l}\text { Center } \\
\text { Number }\end{array}$} & \multirow{2}{*}{$\begin{array}{l}\text { Atomic } \\
\text { Number }\end{array}$} & \multirow{2}{*}{$\begin{array}{c}\text { Atomic } \\
\text { Type }\end{array}$} & \multicolumn{3}{|c|}{ Coordinates (Angstroms) } \\
\hline & & & $\mathrm{X}$ & Y & Z \\
\hline 1 & 6 & 0 & 0.785840 & -0.186263 & 0.272878 \\
\hline 2 & 6 & 0 & 0.622139 & -0.778754 & 1. 694634 \\
\hline 3 & 6 & 0 & -0.790058 & -0.382111 & 2. 179206 \\
\hline 4 & 6 & 0 & -1.504884 & 0.267932 & 0.975186 \\
\hline 5 & 1 & 0 & 0.508423 & -0.942313 & -0.470574 \\
\hline 6 & 1 & 0 & 1. 382762 & -0.363746 & 2. 363042 \\
\hline 7 & 1 & 0 & 0.758101 & -1.862617 & 1. 672220 \\
\hline 8 & 1 & 0 & -1.365257 & -1.225491 & 2. 569051 \\
\hline 9 & 1 & 0 & -0.726320 & 0.373641 & 2. 967522 \\
\hline 10 & 6 & 0 & 2. 177884 & 0.344392 & -0.059494 \\
\hline 11 & 1 & 0 & 2. 166259 & 0.923770 & -0.990488 \\
\hline 12 & 1 & 0 & 2. 556957 & 0.987583 & 0.740654 \\
\hline 13 & 6 & 0 & 3. 182495 & -0.754181 & -0.262201 \\
\hline 14 & 9 & 0 & 4. 397023 & -0.266595 & -0.593512 \\
\hline 15 & 9 & 0 & 3. 366118 & -1.513693 & 0.839054 \\
\hline 16 & 9 & 0 & 2. 831550 & -1.604558 & -1.251357 \\
\hline 17 & 8 & 0 & -2.468592 & 1.231182 & 1. 322344 \\
\hline 18 & 1 & 0 & -1.991657 & 1.967183 & 1. 747128 \\
\hline 19 & 6 & 0 & -2.225194 & -0.744815 & 0.136153 \\
\hline 20 & 6 & 0 & -3.536965 & -1.986401 & -1.183659 \\
\hline 21 & 6 & 0 & -2.497546 & -2.737459 & -0.736835 \\
\hline 22 & 6 & 0 & -0.202779 & 3. 040912 & -1.160848 \\
\hline 23 & 6 & 0 & 0.173814 & 3. 163163 & 0.189591 \\
\hline 24 & 6 & 0 & -0.290568 & 0.922124 & 0.215466 \\
\hline 25 & 7 & 0 & 0.127181 & 2. 090208 & 0.941948 \\
\hline 26 & 16 & 0 & -0.699108 & 1. 444325 & -1.526258 \\
\hline 27 & 6 & 0 & -3.359148 & -0.680652 & -0.613127 \\
\hline 28 & 8 & 0 & -1.694083 & -1.995764 & 0.070348 \\
\hline 29 & 1 & 0 & -3.987349 & 0.189703 & -0.741417 \\
\hline 30 & 1 & 0 & -4.334436 & -2.316835 & -1.835213 \\
\hline 31 & 1 & 0 & -2.197311 & -3.764601 & -0.886492 \\
\hline 32 & 1 & 0 & 0.487997 & 4. 111799 & 0.618016 \\
\hline 33 & 1 & 0 & -0.223695 & 3. 834230 & -1.897858 \\
\hline
\end{tabular}

Zero-point correction=

0.249090 (Hartree/Particle)

Thermal correction to Energy=

0.266661

Thermal correction to Enthalpy=

0.267605

Thermal correction to Gibbs Free Energy=

Sum of electronic and zero-point Energies=

Sum of electronic and thermal Energies=

Sum of electronic and thermal Enthalpies=

Sum of electronic and thermal Free Energies=

0.201294

$-1405.465550$

$-1405.447980$

$-1405.447036$

$-1405.513347$

M06 $-2 X / 6-311++G(d, p) / S M D / / M 06-2 X / 6-31+G(d) / S M D \quad$ energy $=-1406.01312683$

\section{1aj-TS2a}

\begin{tabular}{rrrrrr} 
Center & Atomic & Atomic & \multicolumn{3}{c}{ Coordinates (Angstroms) } \\
Number & Number & Type & X & Y & Z \\
-1 & 6 & 0 & -1.437613 & -0.044671 & -0.382610 \\
2 & 6 & 0 & -1.304369 & -1.557599 & -0.117760 \\
3 & 6 & 0 & 0.168979 & -1.906618 & -0.302094 \\
4 & 6 & 0 & 0.941033 & -0.948741 & 0.557023
\end{tabular}




\begin{tabular}{|c|c|c|c|c|c|}
\hline 5 & 1 & 0 & -1.943775 & -2.132518 & -0.792994 \\
\hline 6 & 1 & 0 & -1.607533 & -1.790804 & 0.908625 \\
\hline 7 & 1 & 0 & 0.385336 & -2.942631 & -0.011692 \\
\hline 8 & 1 & 0 & 0. 462879 & -1.780786 & -1.351550 \\
\hline 9 & 6 & 0 & -2.620784 & 0.608766 & 0.341056 \\
\hline 10 & 1 & 0 & -2.536416 & 0.485389 & 1. 426295 \\
\hline 11 & 1 & 0 & -2.659228 & 1. 679859 & 0.119677 \\
\hline 12 & 6 & 0 & -3.957810 & 0.047439 & -0.060253 \\
\hline 13 & 9 & 0 & -4.972754 & 0.765204 & 0.469155 \\
\hline 14 & 9 & 0 & -4.144510 & 0.054122 & -1.397961 \\
\hline 15 & 9 & 0 & -4.152353 & -1.226509 & 0.341974 \\
\hline 16 & 8 & 0 & 0.634610 & -1.090396 & 1. 889482 \\
\hline 17 & 1 & 0 & 1. 297375 & -0.584549 & 2. 397542 \\
\hline 18 & 6 & 0 & -0.141060 & 0.690941 & -0.043267 \\
\hline 19 & 6 & 0 & 1. 104410 & 2. 512203 & 0.492546 \\
\hline 20 & 6 & 0 & 1. 354058 & 2. 142078 & -0.804870 \\
\hline 21 & 6 & 0 & 4. 553650 & -0.099896 & -0.530162 \\
\hline 22 & 6 & 0 & 4. 346789 & 0.153705 & 0.794509 \\
\hline 23 & 6 & 0 & 2. 331423 & -0.632162 & 0.313340 \\
\hline 24 & 7 & 0 & 3. 100412 & -0.151889 & 1. 272514 \\
\hline 25 & 16 & 0 & 3. 125589 & -0.771273 & -1.232990 \\
\hline 26 & 8 & 0 & 0.544351 & 1. 121750 & -1.175858 \\
\hline 27 & 6 & 0 & 0.100285 & 1. 648057 & 0.972753 \\
\hline 28 & 1 & 0 & -0.342362 & 1. 640031 & 1. 959899 \\
\hline 29 & 1 & 0 & 5. 089838 & 0.567939 & 1. 466754 \\
\hline 30 & 1 & 0 & 5. 451647 & 0.053268 & -1.114719 \\
\hline 31 & 1 & 0 & 1. 594637 & 3. 315824 & 1. 026405 \\
\hline 32 & 1 & 0 & 2. 025939 & 2. 523235 & -1.561624 \\
\hline 33 & 1 & 0 & -1.569723 & 0.102129 & -1.461128 \\
\hline \multicolumn{4}{|c|}{ Zero-point correction $=$} & 0.247500 & (Hartree/Particle) \\
\hline \multicolumn{4}{|c|}{ Thermal correction to Energy= } & 0.264675 & \\
\hline \multicolumn{4}{|c|}{ Thermal correction to Enthalpy= } & 0.265619 & \\
\hline \multicolumn{4}{|c|}{ Thermal correction to Gibbs Free Energy= } & 0.200621 & \\
\hline \multicolumn{4}{|c|}{ Sum of electronic and zero-point Energies= } & \multicolumn{2}{|c|}{-1405.447177} \\
\hline \multicolumn{4}{|c|}{ Sum of electronic and thermal Energies= } & \multicolumn{2}{|c|}{-1405.430003} \\
\hline \multicolumn{4}{|c|}{ Sum of electronic and thermal Enthalpies= } & \multicolumn{2}{|c|}{-1405.429059} \\
\hline \multirow{2}{*}{\multicolumn{4}{|c|}{ Sum of electronic and thermal Free Energies $=$}} & \multicolumn{2}{|c|}{-1405.494057} \\
\hline & & & & energy $=-1$ & 405. 99362112 \\
\hline
\end{tabular}

1aj-TS2b

\begin{tabular}{rrrrrr} 
Center & Atomic & Atomic & \multicolumn{3}{c}{ Coordinates (Angstroms) } \\
Number & Number & Type & \multicolumn{1}{c}{ X } & \multicolumn{1}{c}{ Y } & \multicolumn{1}{c}{$Z$} \\
\hline 1 & 6 & 0 & 1.177852 & -0.248350 & -0.086089 \\
2 & 6 & 0 & 1.137718 & -1.423634 & 0.893672 \\
3 & 6 & 0 & -0.274196 & -1.990774 & 0.778529 \\
4 & 6 & 0 & -1.270015 & -0.869575 & 0.960128 \\
5 & 1 & 0 & 1.060357 & -0.652500 & -1.099877 \\
6 & 1 & 0 & 1.342050 & -1.071820 & 1.913650 \\
7 & 1 & 0 & 1.885448 & -2.181596 & 0.645802 \\
8 & 1 & 0 & -0.404292 & -2.443874 & -0.209724 \\
9 & 1 & 0 & -0.472270 & -2.759702 & 1.535106 \\
10 & 6 & 0 & 2.448861 & 0.607276 & -0.039787 \\
11 & 1 & 0 & 2.381720 & 1.420596 & -0.770716 \\
12 & 1 & 0 & 2.585686 & 1.049806 & 0.951902 \\
13 & 6 & 0 & 3.704467 & -0.156198 & -0.360394 \\
14 & 9 & 0 & 4.755360 & 0.671033 & -0.549332 \\
15 & 9 & 0 & 4.072924 & -1.008521 & 0.620246 \\
16 & 9 & 0 & 3.595975 & -0.895982 & -1.485076 \\
17 & 8 & 0 & -1.493980 & -0.476959 & 2.258885 \\
18 & 1 & 0 & -0.826533 & 0.207921 & 2.482630 \\
19 & 6 & 0 & -2.495150 & -0.854800 & 0.193703 \\
20 & 6 & 0 & -4.472021 & -0.448411 & -0.779322 \\
21 & 6 & 0 & -3.677500 & -1.162953 & -1.624295 \\
22 & 6 & 0 & -1.357521 & 2.691016 & -0.169261 \\
23 & 6 & 0 & -0.810258 & 2.542618 & 1.085925 \\
24 & 6 & 0 & -0.033311 & 0.636794 & 0.198103 \\
25 & 7 & 0 & -0.010580 & 1.469252 & 1.270811 \\
26 & 16 & 0 & -0.842340 & 1.412370 & -1.198168
\end{tabular}




$\begin{array}{rrrrrr}27 & 6 & 0 & -3.704412 & -0.240386 & 0.407267 \\ 28 & 8 & 0 & -2.478770 & -1.420152 & -1.044745 \\ 29 & 1 & 0 & -3.993048 & 0.294244 & 1.301513 \\ 30 & 1 & 0 & -5.481519 & -0.115828 & -0.979319 \\ 31 & 1 & 0 & -3.817474 & -1.560975 & -2.619216 \\ 32 & 1 & 0 & -0.975793 & 3.236187 & 1.904229 \\ 33 & 1 & 0 & -2.016259 & 3.477228 & -0.515725\end{array}$

Zero-point correction=

0.247028 (Hartree/Particle)

Thermal correction to Energy=

0.264381

Thermal correction to Enthalpy=

0.265325

Thermal correction to Gibbs Free Energy=

Sum of electronic and zero-point Energies=

Sum of electronic and thermal Energies=

Sum of electronic and thermal Enthalpies=

Sum of electronic and thermal Free Energies=

0.199325

$-1405.446894$

$-1405.429541$

$-1405.428597$

$-1405.494597$

M06 $-2 X / 6-311++G(d, p) / S M D / / M 06-2 X / 6-31+G(d) / S M D \quad$ energy $=-1405.99290055$

1aj-INT3a

\begin{tabular}{|c|c|c|c|c|c|}
\hline \multirow{2}{*}{$\begin{array}{l}\text { Center } \\
\text { Number }\end{array}$} & \multirow{2}{*}{$\begin{array}{l}\text { Atomic } \\
\text { Number }\end{array}$} & \multirow{2}{*}{$\begin{array}{l}\text { Atomic } \\
\text { Type }\end{array}$} & \multicolumn{3}{|c|}{ Coordinates (Angstroms) } \\
\hline & & & $\mathrm{X}$ & Y & Z \\
\hline 1 & 6 & 0 & -1.517677 & 0.166696 & -0.343905 \\
\hline 2 & 6 & 0 & -1.250106 & -1.263414 & 0.167371 \\
\hline 3 & 6 & 0 & 0.142387 & -1.806578 & -0.192128 \\
\hline 4 & 6 & 0 & 1. 240566 & -1.276593 & 0.664445 \\
\hline 5 & 1 & 0 & -1.991067 & -1.937353 & -0.272549 \\
\hline 6 & 1 & 0 & -1.387122 & -1.296283 & 1. 254787 \\
\hline 7 & 1 & 0 & 0.122087 & -2.899893 & -0.076510 \\
\hline 8 & 1 & 0 & 0.366267 & -1.606631 & -1.246689 \\
\hline 9 & 6 & 0 & -2.842312 & 0.744883 & 0.182578 \\
\hline 10 & 1 & 0 & -2.821992 & 0.845265 & 1. 273145 \\
\hline 11 & 1 & 0 & -3.005321 & 1. 738274 & -0.246374 \\
\hline 12 & 6 & 0 & -4.062921 & -0.068022 & -0.157056 \\
\hline 13 & 9 & 0 & -5.196831 & 0.625884 & 0.082692 \\
\hline 14 & 9 & 0 & -4.105052 & -0.428508 & -1.457633 \\
\hline 15 & 9 & 0 & -4.169307 & -1.208798 & 0.557827 \\
\hline 16 & 8 & 0 & 1. 020134 & -1.342041 & 1. 989829 \\
\hline 17 & 1 & 0 & 1. 805416 & -0.969538 & 2. 441335 \\
\hline 18 & 6 & 0 & -0.415375 & 1. 118296 & 0.013860 \\
\hline 19 & 6 & 0 & 1. 135322 & 2. 500754 & 0.855150 \\
\hline 20 & 6 & 0 & 1. 406629 & 2. 251588 & -0.453142 \\
\hline 21 & 6 & 0 & 4. 511762 & -0.021709 & -0.875712 \\
\hline 22 & 6 & 0 & 4. 513597 & 0. 067908 & 0.491109 \\
\hline 23 & 6 & 0 & 2. 484689 & -0.802681 & 0.241619 \\
\hline 24 & 7 & 0 & 3. 397012 & -0.372030 & 1. 128332 \\
\hline 25 & 16 & 0 & 3. 022026 & -0.707971 & -1.429490 \\
\hline 26 & 8 & 0 & 0.466200 & 1. 423725 & -0.976845 \\
\hline 27 & 6 & 0 & -0.057429 & 1. 762099 & 1. 160511 \\
\hline 28 & 1 & 0 & -0.567701 & 1. 700968 & 2. 112947 \\
\hline 29 & 1 & 0 & 5. 340738 & 0.460596 & 1. 073050 \\
\hline 30 & 1 & 0 & 5. 292020 & 0.261837 & -1.569915 \\
\hline 31 & 1 & 0 & 1. 711476 & 3. 127940 & 1.522196 \\
\hline 32 & 1 & 0 & 2. 181726 & 2. 581647 & -1.130262 \\
\hline 33 & 1 & 0 & -1.562793 & 0.134871 & -1.439684 \\
\hline
\end{tabular}

Zero-point correction=

0.248752 (Hartree/Particle)

Thermal correction to Energy=

0.266867

Thermal correction to Enthalpy=

0.267811

0.200023

$-1405.472771$

$-1405.454656$

$-1405.453711$

$-1405.521499$

Sum of electronic and thermal Enthalpies=

Sum of electronic and thermal Free Energies=

ergy $=-1406.02081038$

\section{1aj-INT3b}

$\begin{array}{llccc}\text { Center } & \text { Atomic } & \text { Atomic } & \text { Coordinates (Angstroms) } \\ \text { Number } & \text { Number } & \text { Type } & X & \text { Y Z }\end{array}$




\begin{tabular}{|c|c|c|c|c|c|}
\hline 1 & 6 & 0 & 1. 260035 & -0.036341 & -0.166953 \\
\hline 2 & 6 & 0 & 1. 093798 & -1.324417 & 0.654702 \\
\hline 3 & 6 & 0 & -0.233918 & -2.058845 & 0.399001 \\
\hline 4 & 6 & 0 & -1.451547 & -1.347708 & 0.892230 \\
\hline 5 & 1 & 0 & 1. 182898 & -0.287763 & -1.231880 \\
\hline 6 & 1 & 0 & 1. 213688 & -1.093041 & 1. 721333 \\
\hline 7 & 1 & 0 & 1.901146 & -2.013909 & 0.393242 \\
\hline 8 & 1 & 0 & -0.338173 & -2.258925 & -0.672427 \\
\hline 9 & 1 & 0 & -0.171590 & -3.033961 & 0.904809 \\
\hline 10 & 6 & 0 & 2. 610210 & 0.666651 & 0.074634 \\
\hline 11 & 1 & 0 & 2. 659481 & 1. 584803 & -0.519465 \\
\hline 12 & 1 & 0 & 2. 722213 & 0.938042 & 1. 129582 \\
\hline 13 & 6 & 0 & 3. 818365 & -0.147179 & -0.308257 \\
\hline 14 & 9 & 0 & 4. 929009 & 0.619618 & -0.356494 \\
\hline 15 & 9 & 0 & 4. 094366 & -1.144716 & 0.558322 \\
\hline 16 & 9 & 0 & 3. 696374 & -0.720554 & -1.523845 \\
\hline 17 & 8 & 0 & -1.522540 & -1.017087 & 2. 218910 \\
\hline 18 & 1 & 0 & -0.840731 & -0.337306 & 2. 403360 \\
\hline 19 & 6 & 0 & -2.569037 & -1.032459 & 0.118964 \\
\hline 20 & 6 & 0 & -4.491114 & -0.288993 & -0.795837 \\
\hline 21 & 6 & 0 & -3.742718 & -0.925125 & -1.744379 \\
\hline 22 & 6 & 0 & -1.592687 & 2. 637922 & 0.148795 \\
\hline 23 & 6 & 0 & -1.118639 & 2. 286265 & 1. 375568 \\
\hline 24 & 6 & 0 & 0.179547 & 0.965601 & 0.159226 \\
\hline 25 & 7 & 0 & -0.111876 & 1. 345007 & 1. 373607 \\
\hline 26 & 16 & 0 & -0.751337 & 1. 760168 & -1.070444 \\
\hline 27 & 6 & 0 & -3.752465 & -0.351781 & 0.415838 \\
\hline 28 & 8 & 0 & -2.581822 & -1.388355 & -1.208541 \\
\hline 29 & 1 & 0 & -4.021712 & 0.052094 & 1. 381434 \\
\hline 30 & 1 & 0 & -5.458152 & 0.170037 & -0.953514 \\
\hline 31 & 1 & 0 & -3.894340 & -1.130077 & -2.794497 \\
\hline 32 & 1 & 0 & -1.476246 & 2. 681179 & 2. 319449 \\
\hline 33 & 1 & 0 & -2.375778 & 3. 343965 & -0.095557 \\
\hline
\end{tabular}

Zero-point correction=

0. 247808 (Hartree/Particle)

Thermal correction to Energy=

0.265997

Thermal correction to Enthalpy=

Thermal correction to Gibbs Free Energy=

Sum of electronic and zero-point Energies=

Sum of electronic and thermal Energies=

Sum of electronic and thermal Enthalpies=

0.266941

0. 199065

$-1405.466730$

$-1405.448541$

$-1405.447597$

$-1405.515473$

Sum of electronic and thermal Free Energies=

nergy $=-1406.01456737$

1al-INT1a

\begin{tabular}{rrrrrr} 
Center & Atomic & Atomic & \multicolumn{3}{c}{ Coordinates (Angstroms) } \\
Number & Number & Type & $X$ & Y & $Z$ \\
--1 & 6 & 0 & -1.888144 & -0.600689 & 0.998264 \\
1 & 6 & 0 & -0.950227 & -1.749393 & 0.831307 \\
2 & 6 & 0 & 0.547589 & -1.395773 & 0.929923 \\
3 & 6 & 0 & 1.064645 & -0.482441 & -0.193602 \\
4 & 1 & 0 & -2.054574 & -0.190781 & 1.990153 \\
5 & 1 & 0 & -1.126286 & -2.240127 & -0.131643 \\
6 & 1 & 0 & -1.142116 & -2.494471 & 1.615020 \\
7 & 1 & 0 & 0.775835 & -0.935951 & 1.899831 \\
8 & 1 & 0 & 1.111373 & -2.334861 & 0.886205 \\
9 & 6 & 0 & -2.640067 & -0.002528 & -0.146221 \\
10 & 1 & 0 & -2.728246 & 1.088198 & -0.056330 \\
11 & 1 & 0 & -2.161752 & -0.231128 & -1.105504 \\
12 & 6 & 0 & -4.051221 & -0.532591 & -0.235201 \\
13 & 9 & 0 & -4.732751 & -0.000754 & -1.272409 \\
14 & 9 & 0 & -4.088831 & -1.872529 & -0.399944 \\
15 & 9 & 0 & -4.776387 & -0.267599 & 0.872783 \\
16 & 8 & 0 & 0.662693 & -1.074520 & -1.418978 \\
17 & 1 & 0 & 0.679683 & -0.377407 & -2.099917 \\
18 & 6 & 0 & 2.582874 & -0.350829 & -0.157361 \\
19 & 6 & 0 & 5.028393 & -0.819718 & -0.124660 \\
20 & 6 & 0 & 4.750868 & 0.483757 & -0.417907 \\
21 & & & & &
\end{tabular}




\begin{tabular}{rrrrrr}
22 & 6 & 0 & -0.242708 & 3.181250 & 0.411641 \\
23 & 6 & 0 & -0.339106 & 2.825962 & -0.898388 \\
24 & 6 & 0 & 0.478970 & 0.922975 & -0.121912 \\
25 & 1 & 0 & -0.712326 & 3.460612 & -1.693367 \\
26 & 1 & 0 & -0.511511 & 4.119409 & 0.880348 \\
27 & 7 & 0 & 0.078380 & 1.545113 & -1.190378 \\
28 & 16 & 0 & 0.397885 & 1.870000 & 1.330665 \\
29 & 6 & 0 & 3.346705 & 0.750465 & -0.439924 \\
30 & 16 & 0 & 3.591564 & -1.736232 & 0.130666 \\
31 & 1 & 0 & 2.929532 & 1.728256 & -0.660899 \\
32 & 1 & 0 & 5.996198 & -1.297975 & -0.032613 \\
33 & 1 & 0 & 5.513087 & 1.231576 & -0.607711 \\
\hline
\end{tabular}

Zero-point correction=

Thermal correction to Energy=

0.242624 (Hartree/Particle)

Thermal correction to Enthalpy=

Thermal correction to Gibbs Free Energy=

Sum of electronic and zero-point Energies $=$

Sum of electronic and thermal Energies=

0.262812

0.190438

$-1728.410829$

$-1728.391584$

$-1728.390640$

$-1728.463014$

Sum of electronic and thermal Free Energies=

energy $=-1728.96171682$

\section{1a1-INT1b}

\begin{tabular}{|c|c|c|c|c|c|}
\hline \multirow{2}{*}{$\begin{array}{l}\text { Center } \\
\text { Number }\end{array}$} & \multirow{2}{*}{$\begin{array}{l}\text { Atomic } \\
\text { Number }\end{array}$} & \multirow{2}{*}{$\begin{array}{l}\text { Atomic } \\
\text { Type }\end{array}$} & \multicolumn{3}{|c|}{ Coordinates (Angstroms) } \\
\hline & & & X & Y & Z \\
\hline 1 & 6 & 0 & -1.994835 & -0.284226 & 0.694569 \\
\hline 2 & 6 & 0 & -1.118316 & -1.484483 & 0.842774 \\
\hline 3 & 6 & 0 & 0.238599 & -1.416674 & 0.111473 \\
\hline 4 & 6 & 0 & 1. 242553 & -0.419303 & 0.738001 \\
\hline 5 & 1 & 0 & -1.638704 & -2.366000 & 0.442048 \\
\hline 6 & 1 & 0 & -0.924050 & -1.678568 & 1. 901942 \\
\hline 7 & 1 & 0 & 0.704132 & -2.408878 & 0.155008 \\
\hline 8 & 1 & 0 & 0.094030 & -1.180264 & -0.951653 \\
\hline 9 & 6 & 0 & -2.811579 & -0.072358 & -0.540175 \\
\hline 10 & 1 & 0 & -2.902649 & 0.989188 & -0.797010 \\
\hline 11 & 1 & 0 & -2.385842 & -0.598832 & -1.403238 \\
\hline 12 & 6 & 0 & -4.222986 & -0.585600 & -0.379811 \\
\hline 13 & 9 & 0 & -4.964853 & -0.400396 & -1.492910 \\
\hline 14 & 9 & 0 & -4.268066 & -1.907654 & -0.107510 \\
\hline 15 & 9 & 0 & -4.883908 & 0. 028979 & 0.624647 \\
\hline 16 & 6 & 0 & 0.829729 & 1. 026626 & 0.563526 \\
\hline 17 & 6 & 0 & 0.398660 & 3. 270444 & 1.028266 \\
\hline 18 & 6 & 0 & 0.049641 & 3. 228892 & -0.289888 \\
\hline 19 & 6 & 0 & 4. 647242 & -0.955082 & -1.185605 \\
\hline 20 & 6 & 0 & 4. 583160 & -1.693476 & -0.044916 \\
\hline 21 & 6 & 0 & 2. 618558 & -0.686762 & 0. 125685 \\
\hline 22 & 8 & 0 & 1. 345979 & -0.649675 & 2. 130305 \\
\hline 23 & 1 & 0 & 2. 029798 & -1.333744 & 2. 255777 \\
\hline 24 & 1 & 0 & -2.074648 & 0.443085 & 1. 495586 \\
\hline 25 & 7 & 0 & 3. 427377 & -1.536304 & 0.686785 \\
\hline 26 & 16 & 0 & 3. 216034 & -0.007248 & -1.354006 \\
\hline 27 & 1 & 0 & 5. 353706 & -2.367016 & 0.310799 \\
\hline 28 & 1 & 0 & 5. 445171 & -0.904341 & -1.915689 \\
\hline 29 & 16 & 0 & 0.254798 & 1. 648199 & -0.952447 \\
\hline 30 & 6 & 0 & 0.851532 & 2. 004598 & 1. 517516 \\
\hline 31 & 1 & 0 & 1. 168082 & 1. 818294 & 2. 537038 \\
\hline 32 & 1 & 0 & 0.337277 & 4. 168410 & 1. 633653 \\
\hline 33 & 1 & 0 & -0.319384 & 4. 031025 & -0.917483 \\
\hline \multicolumn{4}{|c|}{ Zero-point correction= } & \multicolumn{2}{|c|}{0.242455 (Hartree/Particle) } \\
\hline \multicolumn{4}{|c|}{ Thermal correction to Energy= } & \multicolumn{2}{|c|}{0.261791} \\
\hline \multicolumn{4}{|c|}{ Thermal correction to Enthalpy $=$} & \multicolumn{2}{|l|}{0.262736} \\
\hline \multicolumn{4}{|c|}{ Thermal correction to Gibbs Free Energy= } & \multicolumn{2}{|l|}{0.190165} \\
\hline \multicolumn{4}{|c|}{ Sum of electronic and zero-point Energies= } & \multicolumn{2}{|c|}{-1728.408826} \\
\hline \multicolumn{4}{|c|}{ Sum of electronic and thermal Energies $=$} & \multicolumn{2}{|c|}{-1728.389490} \\
\hline \multicolumn{4}{|c|}{ Sum of electronic and thermal Enthalpies= } & \multicolumn{2}{|c|}{-1728.388545} \\
\hline \multicolumn{4}{|c|}{$\begin{array}{l}\text { Sum of electronic and thermal Free Energies }= \\
\text { M06 }-2 X / 6-311++G(d, p) / S M D / / M 06-2 X / 6-31+G(d) / S M D\end{array}$} & \multicolumn{2}{|c|}{-1728.461116} \\
\hline
\end{tabular}


1al-TS1a

\begin{tabular}{|c|c|c|c|c|c|}
\hline \multirow{2}{*}{$\begin{array}{l}\text { Center } \\
\text { Number }\end{array}$} & \multirow{2}{*}{$\begin{array}{l}\text { Atomic } \\
\text { Number }\end{array}$} & \multirow{2}{*}{$\begin{array}{c}\text { Atomic } \\
\text { Type }\end{array}$} & \multicolumn{3}{|c|}{ Coordinates (Angstroms) } \\
\hline & & & X & Y & Z \\
\hline 1 & 6 & 0 & -1.625914 & -0.239337 & 0.618681 \\
\hline 2 & 6 & 0 & -1.036776 & -1.594551 & 0.903401 \\
\hline 3 & 6 & 0 & 0.491995 & -1.493691 & 1. 005973 \\
\hline 4 & 6 & 0 & 0.966150 & -0.598964 & -0.137894 \\
\hline 5 & 1 & 0 & -1.843483 & 0.388002 & 1. 480795 \\
\hline 6 & 1 & 0 & -1.294375 & -2.287563 & 0.097645 \\
\hline 7 & 1 & 0 & -1.441273 & -1.999637 & 1. 839165 \\
\hline 8 & 1 & 0 & 0.796336 & -1.048947 & 1. 961523 \\
\hline 9 & 1 & 0 & 0.953507 & -2.484843 & 0.940091 \\
\hline 10 & 6 & 0 & -2.556276 & -0.053831 & -0.543627 \\
\hline 11 & 1 & 0 & -2.624975 & 1. 000785 & -0.833369 \\
\hline 12 & 1 & 0 & -2.226678 & -0.633253 & -1.412538 \\
\hline 13 & 6 & 0 & -3.962134 & -0.495376 & -0.221794 \\
\hline 14 & 9 & 0 & -4.800089 & -0.314876 & -1.264677 \\
\hline 15 & 9 & 0 & -4.038309 & -1.802959 & 0.106812 \\
\hline 16 & 9 & 0 & -4.493566 & 0.186226 & 0.815887 \\
\hline 17 & 8 & 0 & 0.534689 & -1.216669 & -1.342291 \\
\hline 18 & 1 & 0 & 0.524568 & -0.526219 & -2.030525 \\
\hline 19 & 6 & 0 & 2. 469212 & -0.395315 & -0.173494 \\
\hline 20 & 6 & 0 & 4. 932256 & -0.754977 & -0.189855 \\
\hline 21 & 6 & 0 & 4. 583749 & 0.496425 & -0.609475 \\
\hline 22 & 6 & 0 & -0.003031 & 3. 156872 & 0.399469 \\
\hline 23 & 6 & 0 & -0.257513 & 2. 744918 & -0.881251 \\
\hline 24 & 6 & 0 & 0.254085 & 0.754594 & -0.025806 \\
\hline 25 & 1 & 0 & -0.591189 & 3. 398373 & -1.680030 \\
\hline 26 & 1 & 0 & -0.101217 & 4. 152355 & 0.813733 \\
\hline 27 & 7 & 0 & -0.049172 & 1. 422684 & -1.135703 \\
\hline 28 & 16 & 0 & 0.540490 & 1. 830946 & 1. 357978 \\
\hline 29 & 6 & 0 & 3. 169221 & 0.700289 & -0.602974 \\
\hline 30 & 16 & 0 & 3. 548787 & -1.697545 & 0.219633 \\
\hline 31 & 1 & 0 & 2. 696239 & 1. 630580 & -0.904380 \\
\hline 32 & 1 & 0 & 5. 924527 & -1.177323 & -0.086418 \\
\hline 33 & 1 & 0 & 5. 303801 & 1. 249986 & -0.909552 \\
\hline
\end{tabular}

Zero-point correction=

Thermal correction to Energy=

0.243058 (Hartree/Particle)

Thermal correction to Enthalpy=

0.261163

Thermal correction to Gibbs Free Energy=

Sum of electronic and zero-point Energies=

Sum of electronic and thermal Energies=

0.262107

0.194385

$-1728.398102$

$-1728.379997$

$-1728.379053$

$-1728.446776$

Sum of electronic and thermal Free Energies=

ergy $=-1728.94897920$

1a1-TS1b

\begin{tabular}{rrrrrr} 
Center & Atomic & Atomic & \multicolumn{3}{c}{ Coordinates (Angstroms) } \\
Number & Number & Type & X & Y & $Z$ \\
-1 & 6 & 0 & -1.552871 & -0.703144 & 0.161440 \\
2 & 6 & 0 & -1.000049 & -1.724495 & -0.801493 \\
3 & 6 & 0 & 0.528177 & -1.550985 & -0.990831 \\
4 & 6 & 0 & 1.071800 & -0.800450 & 0.231325 \\
5 & 1 & 0 & -1.498098 & -1.645035 & -1.773938 \\
6 & 1 & 0 & -1.197359 & -2.730427 & -0.414197 \\
7 & 1 & 0 & 1.041955 & -2.513081 & -1.083849 \\
8 & 1 & 0 & 0.747157 & -0.977735 & -1.900719 \\
9 & 6 & 0 & -2.630555 & 0.250268 & -0.256802 \\
10 & 1 & 0 & -2.601783 & 1.170427 & 0.343508 \\
11 & 1 & 0 & -2.547126 & 0.522292 & -1.314167 \\
12 & 6 & 0 & -4.011067 & -0.328075 & -0.071859 \\
13 & 9 & 0 & -4.979891 & 0.525229 & -0.469340 \\
14 & 9 & 0 & -4.195241 & -1.466729 & -0.775458 \\
15 & 9 & 0 & -4.279434 & -0.632585 & 1.216080 \\
16 & 6 & 0 & 0.305458 & 0.509994 & 0.382358
\end{tabular}




$\begin{array}{rrrrrr}17 & 6 & 0 & -0.157161 & 2.587700 & 1.380827 \\ 18 & 6 & 0 & -0.176083 & 2.954522 & 0.059627 \\ 19 & 6 & 0 & 4.906749 & -0.074351 & -0.350856 \\ 20 & 6 & 0 & 4.703660 & -1.153712 & 0.450716 \\ 21 & 6 & 0 & 2.580790 & -0.606378 & 0.120476 \\ 22 & 8 & 0 & 0.822459 & -1.555256 & 1.404006 \\ 23 & 1 & 0 & 1.630360 & -2.069443 & 1.588252 \\ 24 & 1 & 0 & -1.583988 & -0.998779 & 1.206916 \\ 25 & 7 & 0 & 3.383134 & -1.446343 & 0.707726 \\ 26 & 16 & 0 & 3.392851 & 0.620514 & -0.800073 \\ 27 & 1 & 0 & 5.481627 & -1.770553 & 0.884490 \\ 28 & 1 & 0 & 5.842842 & 0.352320 & -0.687837 \\ 29 & 16 & 0 & 0.207183 & 1.625862 & -0.980339 \\ 30 & 6 & 0 & 0.169334 & 1.223333 & 1.576070 \\ 31 & 1 & 0 & 0.262659 & 0.742729 & 2.543451 \\ 32 & 1 & 0 & -0.368018 & 3.281213 & 2.188467 \\ 33 & 1 & 0 & -0.410382 & 3.921923 & -0.367428 \\ & 1 & & & \end{array}$

Zero-point correction=

0. 242576 (Hartree/Particle)

Thermal correction to Energy=

0.260769

Thermal correction to Enthalpy=

0. 261713

Thermal correction to Gibbs Free Energy=

Sum of electronic and zero-point Energies=

Sum of electronic and thermal Energies=

0.193703

$-1728.393183$

$-1728.374990$

$-1728.374046$

Sum of electronic and thermal Free Energies=

$-1728.442057$

M06 $-2 X / 6-311++G(d, p) / S M D / / M 06-2 X / 6-31+G(d) / S M D \quad$ energy $=-1728.94390465$

1a1-INT2a

\begin{tabular}{|c|c|c|c|c|c|}
\hline \multirow{2}{*}{$\begin{array}{l}\text { Center } \\
\text { Number }\end{array}$} & \multirow{2}{*}{$\begin{array}{l}\text { Atomic } \\
\text { Number }\end{array}$} & \multirow{2}{*}{$\begin{array}{l}\text { Atomic } \\
\text { Type }\end{array}$} & \multicolumn{3}{|c|}{ Coordinates (Angstroms) } \\
\hline & & & $\mathrm{X}$ & Y & Z \\
\hline 1 & 6 & 0 & 0.912852 & -0.181409 & 0.264096 \\
\hline 2 & 6 & 0 & 0.711121 & -0.768568 & 1. 684269 \\
\hline 3 & 6 & 0 & -0.618045 & -0.188830 & 2. 218989 \\
\hline 4 & 6 & 0 & -1.302912 & 0.525861 & 1. 034084 \\
\hline 5 & 1 & 0 & 0.553764 & -0.895167 & -0.486310 \\
\hline 6 & 1 & 0 & 1.539506 & -0.472801 & 2. 334813 \\
\hline 7 & 1 & 0 & 0.702877 & -1.861866 & 1. 648942 \\
\hline 8 & 1 & 0 & -1.275255 & -0.940638 & 2. 664437 \\
\hline 9 & 1 & 0 & -0.417242 & 0.566689 & 2. 983918 \\
\hline 10 & 6 & 0 & 2. 347509 & 0.207026 & -0.084455 \\
\hline 11 & 1 & 0 & 2. 381711 & 0.796435 & -1.008594 \\
\hline 12 & 1 & 0 & 2. 801925 & 0.797764 & 0.716678 \\
\hline 13 & 6 & 0 & 3. 230128 & -0.986520 & -0.316974 \\
\hline 14 & 9 & 0 & 4. 486410 & -0.621986 & -0.649798 \\
\hline 15 & 9 & 0 & 3. 340920 & -1.782950 & 0.767861 \\
\hline 16 & 9 & 0 & 2. 785051 & -1.775536 & -1.319175 \\
\hline 17 & 8 & 0 & -2.117774 & 1. 606851 & 1. 423014 \\
\hline 18 & 1 & 0 & -1.529771 & 2. 269444 & 1. 829584 \\
\hline 19 & 6 & 0 & -2.183792 & -0.385315 & 0.213508 \\
\hline 20 & 6 & 0 & -3.281883 & -2.300336 & -0.939092 \\
\hline 21 & 6 & 0 & -3.895967 & -1.109318 & -1.200536 \\
\hline 22 & 6 & 0 & 0.267497 & 3. 116185 & -1.169893 \\
\hline 23 & 6 & 0 & 0.685805 & 3. 203508 & 0.170994 \\
\hline 24 & 6 & 0 & -0.043613 & 1. 033961 & 0.229845 \\
\hline 25 & 1 & 0 & 1. 121869 & 4. 110206 & 0.583160 \\
\hline 26 & 1 & 0 & 0.324736 & 3. 900332 & -1.914725 \\
\hline 27 & 7 & 0 & 0.524430 & 2. 150807 & 0.935657 \\
\hline 28 & 16 & 0 & -0.431107 & 1. 590133 & -1.505187 \\
\hline 29 & 6 & 0 & -3.265505 & -0.011889 & -0.538140 \\
\hline 30 & 16 & 0 & -1.938473 & -2.102509 & 0.122102 \\
\hline 31 & 1 & 0 & -3.601922 & 1. 016522 & -0.605491 \\
\hline 32 & 1 & 0 & -3.540313 & -3.287512 & -1.302506 \\
\hline 33 & 1 & 0 & -4.768010 & -1.013732 & -1.838424 \\
\hline
\end{tabular}

Zero-point correction=

0.245768 (Hartree/Particle)

Thermal correction to Energy=

0.263696

Thermal correction to Enthalpy=

0.264641

Thermal correction to Gibbs Free Energy=

0.197335 
Sum of electronic and zero-point Energies= Sum of electronic and thermal Energies= Sum of electronic and thermal Enthalpies= Sum of electronic and thermal Free Energies=

$-1728.437131$

$-1728.419202$

$-1728.418258$

$-1728.485563$

M06 $-2 X / 6-311++G(d, p) / S M D / / M 06-2 X / 6-31+G(d) / S M D \quad$ energy $=-1728.99003400$

1al-INT2b

\begin{tabular}{|c|c|c|c|c|c|}
\hline \multirow{2}{*}{$\begin{array}{l}\text { Center } \\
\text { Number }\end{array}$} & \multirow{2}{*}{$\begin{array}{l}\text { Atomic } \\
\text { Number }\end{array}$} & \multirow{2}{*}{$\begin{array}{c}\text { Atomic } \\
\text { Type }\end{array}$} & \multicolumn{3}{|c|}{ Coordinates (Angstroms) } \\
\hline & & & X & Y & Z \\
\hline 1 & 6 & 0 & -1.430346 & -0.376150 & 0.201604 \\
\hline 2 & 6 & 0 & -1.178843 & -1.654291 & -0.628621 \\
\hline 3 & 6 & 0 & 0.346842 & -1.908959 & -0.573542 \\
\hline 4 & 6 & 0 & 0.899287 & -0.892405 & 0.426797 \\
\hline 5 & 1 & 0 & -1.504154 & -1.503989 & -1.664168 \\
\hline 6 & 1 & 0 & -1.746376 & -2.497814 & -0.229024 \\
\hline 7 & 1 & 0 & 0.611120 & -2.920797 & -0.255722 \\
\hline 8 & 1 & 0 & 0.791625 & -1.745635 & -1.561367 \\
\hline 9 & 6 & 0 & -2.581916 & 0.508179 & -0.268105 \\
\hline 10 & 1 & 0 & -2.557055 & 1. 477689 & 0.243593 \\
\hline 11 & 1 & 0 & -2.540987 & 0.689169 & -1.346827 \\
\hline 12 & 6 & 0 & -3.929077 & -0.091919 & 0.020217 \\
\hline 13 & 9 & 0 & -4.933303 & 0.727112 & -0.359692 \\
\hline 14 & 9 & 0 & -4.133237 & -1.262941 & -0.621757 \\
\hline 15 & 9 & 0 & -4.121730 & -0.344303 & 1. 333167 \\
\hline 16 & 6 & 0 & -0.061286 & 0. 350780 & 0.230765 \\
\hline 17 & 6 & 0 & 0.530408 & 2. 635975 & 0.800129 \\
\hline 18 & 6 & 0 & 0.718562 & 2. 690899 & -0.580353 \\
\hline 19 & 6 & 0 & 4. 699590 & -0.180910 & -0.277211 \\
\hline 20 & 6 & 0 & 4. 346346 & 0.151022 & 0.995741 \\
\hline 21 & 6 & 0 & 2. 367700 & -0.557578 & 0.299554 \\
\hline 22 & 8 & 0 & 0.679952 & -1.409845 & 1. 728242 \\
\hline 23 & 1 & 0 & 1. 227347 & -0.887424 & 2. 342981 \\
\hline 24 & 1 & 0 & -1.615204 & -0.659081 & 1. 244064 \\
\hline 25 & 7 & 0 & 3. 026864 & -0.071080 & 1. 313218 \\
\hline 26 & 16 & 0 & 3. 330983 & -0.794511 & -1.121868 \\
\hline 27 & 1 & 0 & 5. 015654 & 0.558988 & 1. 743907 \\
\hline 28 & 1 & 0 & 5. 667894 & -0.098396 & -0.754025 \\
\hline 29 & 16 & 0 & 0.306311 & 1. 200597 & -1.379343 \\
\hline 30 & 6 & 0 & 0.087294 & 1. 417277 & 1. 267863 \\
\hline 31 & 1 & 0 & -0.133762 & 1. 200606 & 2. 308308 \\
\hline 32 & 1 & 0 & 0.716591 & 3. 494313 & 1. 438857 \\
\hline 33 & 1 & 0 & 1. 050023 & 3. 540965 & -1.164149 \\
\hline
\end{tabular}

Zero-point correction=

Thermal correction to Energy=

0. 244926 (Hartree/Particle)

Thermal correction to Enthalpy=

0.263088

0.264032

0.196554

$-1728.435823$

$-1728.417662$

$-1728.416717$

$-1728.484195$

Sum of electronic and thermal Enthalpies=

Sum of electronic and thermal Free Energies

nergy $=-1728.98848068$

1a1-TS2a

\begin{tabular}{rrrrrr} 
Center & Atomic & Atomic & \multicolumn{3}{c}{ Coordinates (Angstroms) } \\
Number & Number & Type & X & Y & $Z$ \\
-1 & 6 & 0 & 1.318106 & -0.237330 & 0.017182 \\
2 & 6 & 0 & 1.263967 & -1.151848 & 1.243073 \\
3 & 6 & 0 & -0.170540 & -1.669311 & 1.289315 \\
4 & 6 & 0 & -1.129958 & -0.501592 & 1.193736 \\
5 & 1 & 0 & 1.139377 & -0.853962 & -0.873884 \\
6 & 1 & 0 & 1.512343 & -0.582769 & 2.148756 \\
7 & 1 & 0 & 1.973938 & -1.979044 & 1.164173 \\
8 & 1 & 0 & -0.321008 & -2.356832 & 0.448869 \\
9 & 1 & 0 & -0.384328 & -2.220341 & 2.212793 \\
10 & 6 & 0 & 2.628116 & 0.536608 & -0.166335 \\
11 & 1 & 0 & 2.559548 & 1.195520 & -1.038839
\end{tabular}




$\begin{array}{rrrrrr}12 & 1 & 0 & 2.839029 & 1.155655 & 0.711351 \\ 13 & 6 & 0 & 3.829290 & -0.340948 & -0.388974 \\ 14 & 9 & 0 & 4.907166 & 0.381875 & -0.763476 \\ 15 & 9 & 0 & 4.203906 & -1.022917 & 0.715041 \\ 16 & 9 & 0 & 3.633995 & -1.263072 & -1.355746 \\ 17 & 8 & 0 & -1.327950 & 0.182561 & 2.371122 \\ 18 & 1 & 0 & -0.588400 & 0.818086 & 2.479027 \\ 19 & 6 & 0 & -2.371567 & -0.628901 & 0.448047 \\ 20 & 6 & 0 & -4.118274 & -1.126747 & -1.253444 \\ 21 & 6 & 0 & -4.478060 & -0.104851 & -0.417608 \\ 22 & 6 & 0 & -1.006497 & 2.798810 & -0.615678 \\ 23 & 6 & 0 & -0.443849 & 2.852979 & 0.642461 \\ 24 & 6 & 0 & 0.161609 & 0.752340 & 0.136493 \\ 25 & 1 & 0 & -0.536280 & 3.703600 & 1.310326 \\ 26 & 1 & 0 & -1.610575 & 3.553383 & -1.103497 \\ 27 & 7 & 0 & 0.271404 & 1.776354 & 1.026010 \\ 28 & 16 & 0 & -0.620980 & 1.306689 & -1.376022 \\ 29 & 6 & 0 & -3.479243 & 0.185623 & 0.553691 \\ 30 & 16 & 0 & -2.561363 & -1.757078 & -0.861846 \\ 31 & 1 & 0 & -3.555393 & 0.967280 & 1.301093 \\ 32 & 1 & 0 & -4.686357 & -1.560572 & -2.067483 \\ 33 & 1 & 0 & -5.424843 & 0.419283 & -0.491967 \\ ------------------------------------------------\end{array}$

Zero-point correction $=$

0.243727 (Hartree/Particle)

Thermal correction to Energy=

0.261560

Thermal correction to Enthalpy=

0.262505

Thermal correction to Gibbs Free Energy=

Sum of electronic and zero-point Energies=

Sum of electronic and thermal Energies=

0.194974

$-1728.418253$

$-1728.400420$

$-1728.399476$

$-1728.467007$

Sum of electronic and thermal Free Energies=

ergy $=-1728.96932491$

1al-TS2b

\begin{tabular}{|c|c|c|c|c|c|}
\hline \multirow{2}{*}{$\begin{array}{l}\text { Center } \\
\text { Number }\end{array}$} & \multirow{2}{*}{$\begin{array}{l}\text { Atomic } \\
\text { Number }\end{array}$} & \multirow{2}{*}{$\begin{array}{c}\text { Atomic } \\
\text { Type }\end{array}$} & \multicolumn{3}{|c|}{ Coordinates (Angstroms) } \\
\hline & & & X & $\mathrm{Y}$ & Z \\
\hline 1 & 6 & 0 & -1.470513 & -0.224339 & 0.185115 \\
\hline 2 & 6 & 0 & -1.306863 & -1.375409 & -0.826847 \\
\hline 3 & 6 & 0 & 0.178124 & -1.808601 & -0.858138 \\
\hline 4 & 6 & 0 & 0.916695 & -1.185405 & 0.291929 \\
\hline 5 & 1 & 0 & -1.618500 & -1.048369 & -1.825950 \\
\hline 6 & 1 & 0 & -1.955468 & -2.210729 & -0.549917 \\
\hline 7 & 1 & 0 & 0.282387 & -2.898720 & -0.805550 \\
\hline 8 & 1 & 0 & 0.644469 & -1.485580 & -1.794440 \\
\hline 9 & 6 & 0 & -2.675351 & 0.685455 & -0.080572 \\
\hline 10 & 1 & 0 & -2.689889 & 1. 506362 & 0.644215 \\
\hline 11 & 1 & 0 & -2.642642 & 1. 117479 & -1.086523 \\
\hline 12 & 6 & 0 & -3.998935 & -0.019246 & 0.046182 \\
\hline 13 & 9 & 0 & -5.030470 & 0.851959 & 0.002612 \\
\hline 14 & 9 & 0 & -4.223867 & -0.915349 & -0.938799 \\
\hline 15 & 9 & 0 & -4.122492 & -0.696667 & 1. 208031 \\
\hline 16 & 6 & 0 & -0.180474 & 0.575995 & 0.249016 \\
\hline 17 & 6 & 0 & 1. 147223 & 2. 339233 & 1. 090280 \\
\hline 18 & 6 & 0 & 1. 313214 & 2. 540787 & -0.262064 \\
\hline 19 & 6 & 0 & 4. 593118 & -0.119829 & -0.299468 \\
\hline 20 & 6 & 0 & 4. 293386 & -0.219377 & 1. 028006 \\
\hline 21 & 6 & 0 & 2. 320763 & -0.823961 & 0.218443 \\
\hline 22 & 8 & 0 & 0.561335 & -1.717739 & 1. 503192 \\
\hline 23 & 1 & 0 & 1. 215153 & -1.413950 & 2. 163150 \\
\hline 24 & 1 & 0 & -1.586430 & -0.651364 & 1. 187241 \\
\hline 25 & 7 & 0 & 3. 016635 & -0.619769 & 1. 318527 \\
\hline 26 & 16 & 0 & 3. 218726 & -0.552754 & -1.248393 \\
\hline 27 & 1 & 0 & 4. 984393 & -0.007339 & 1. 835902 \\
\hline 28 & 1 & 0 & 5.527360 & 0.171757 & -0.761652 \\
\hline 29 & 16 & 0 & 0.327774 & 1. 470410 & -1.202787 \\
\hline 30 & 6 & 0 & 0.250728 & 1. 296919 & 1. 389794 \\
\hline 31 & 1 & 0 & -0.024655 & 0.992675 & 2. 395120 \\
\hline 32 & 1 & 0 & 1. 664887 & 2. 929224 & 1. 840118 \\
\hline 33 & 1 & 0 & 1. 952584 & 3.263545 & -0.754727 \\
\hline
\end{tabular}


Zero-point correction=

Thermal correction to Energy=

Thermal correction to Enthalpy=

Thermal correction to Gibbs Free Energy=

Sum of electronic and zero-point Energies=

Sum of electronic and thermal Energies=

Sum of electronic and thermal Enthalpies=

Sum of electronic and thermal Free Energies=

M06 $-2 X / 6-311++G(d, p) / S M D / / M 06-2 X / 6-31+G(d) / S M D \quad$ energy $=-1728.96893729$

1a1-INT3a

\begin{tabular}{|c|c|c|c|c|c|}
\hline \multirow{2}{*}{$\begin{array}{l}\text { Center } \\
\text { Number }\end{array}$} & \multirow{2}{*}{$\begin{array}{l}\text { Atomic } \\
\text { Number }\end{array}$} & \multirow{2}{*}{$\begin{array}{c}\text { Atomic } \\
\text { Type }\end{array}$} & \multicolumn{3}{|c|}{ Coordinates (Angstroms) } \\
\hline & & & X & Y & Z \\
\hline 1 & 6 & 0 & 1. 443921 & -0.044108 & -0.126339 \\
\hline 2 & 6 & 0 & 1.231397 & -1.152200 & 0.917594 \\
\hline 3 & 6 & 0 & -0.136722 & -1.847272 & 0.813438 \\
\hline 4 & 6 & 0 & -1.315896 & -1.004683 & 1. 188784 \\
\hline 5 & 1 & 0 & 1. 325643 & -0.478556 & -1.126660 \\
\hline 6 & 1 & 0 & 1. 390970 & -0.742100 & 1.923518 \\
\hline 7 & 1 & 0 & 1.993889 & -1.922678 & 0.774487 \\
\hline 8 & 1 & 0 & -0.258330 & -2.231452 & -0.205850 \\
\hline 9 & 1 & 0 & -0.115165 & -2.722093 & 1. 480078 \\
\hline 10 & 6 & 0 & 2.833435 & 0.616522 & -0.039042 \\
\hline 11 & 1 & 0 & 2.904152 & 1. 423359 & -0.775748 \\
\hline 12 & 1 & 0 & 2. 996544 & 1. 047825 & 0.954186 \\
\hline 13 & 6 & 0 & 3. 989124 & -0.307740 & -0.319531 \\
\hline 14 & 9 & 0 & 5. 131761 & 0.384866 & -0.513619 \\
\hline 15 & 9 & 0 & 4. 245750 & -1.171356 & 0.685809 \\
\hline 16 & 9 & 0 & 3. 801738 & -1.056305 & -1.426804 \\
\hline 17 & 8 & 0 & -1.311049 & -0.427312 & 2. 427132 \\
\hline 18 & 1 & 0 & -0.547473 & 0.184165 & 2. 485309 \\
\hline 19 & 6 & 0 & -2.472377 & -0.832855 & 0.429934 \\
\hline 20 & 6 & 0 & -4.248817 & -0.834913 & -1.341888 \\
\hline 21 & 6 & 0 & -4.570157 & -0.035654 & -0.272131 \\
\hline 22 & 6 & 0 & -1.265158 & 2. 790088 & -0.245289 \\
\hline 23 & 6 & 0 & -0.781828 & 2. 631686 & 1. 017819 \\
\hline 24 & 6 & 0 & 0.423263 & 1. 056382 & 0.031177 \\
\hline 25 & 1 & 0 & -1.098918 & 3. 201417 & 1. 883662 \\
\hline 26 & 1 & 0 & -2.015789 & 3. 485331 & -0.598636 \\
\hline 27 & 7 & 0 & 0.177811 & 1. 654274 & 1. 165226 \\
\hline 28 & 16 & 0 & -0.492701 & 1. 674597 & -1.305484 \\
\hline 29 & 6 & 0 & -3.584007 & -0.026779 & 0.738835 \\
\hline 30 & 16 & 0 & -2.710818 & -1.607608 & -1.134276 \\
\hline 31 & 1 & 0 & -3.644054 & 0.545786 & 1. 656797 \\
\hline 32 & 1 & 0 & -4.826943 & -1.020016 & -2.238590 \\
\hline 33 & 1 & 0 & -5.494810 & 0.530076 & -0.218674 \\
\hline
\end{tabular}

Zero-point correction=

Thermal correction to Energy=

0. 244337 (Hartree/Particle)

Thermal correction to Enthalpy=

Thermal correction to Gibbs Free Energy=

Sum of electronic and zero-point Energies=

Sum of electronic and thermal Energies=

Sum of electronic and thermal Enthalpies=

Sum of electronic and thermal Free Energies=

0.244021 (Hartree/Particle)

0.261903

0.262847

0. 196612

$-1728.417212$

$-1728.399330$

$-1728.398385$

$-1728.464621$ 


$\begin{array}{rrrrrr}7 & 1 & 0 & 0.357432 & -1.273537 & -2.688697 \\ 8 & 1 & 0 & 0.868847 & 0.199081 & -1.867273 \\ 9 & 6 & 0 & -2.733497 & 0.462427 & 0.420282 \\ 10 & 1 & 0 & -2.781371 & 0.871874 & 1.433865 \\ 11 & 1 & 0 & -3.140399 & 1.212286 & -0.267463 \\ 12 & 6 & 0 & -3.680245 & -0.708321 & 0.387517 \\ 13 & 9 & 0 & -4.854498 & -0.408587 & 0.984161 \\ 14 & 9 & 0 & -3.993200 & -1.110739 & -0.863353 \\ 15 & 9 & 0 & -3.194231 & -1.793784 & 1.025834 \\ 16 & 6 & 0 & -0.414109 & 1.339675 & 0.274948 \\ 17 & 6 & 0 & 1.153687 & 2.830915 & 1.163322 \\ 18 & 6 & 0 & 0.690972 & 3.570703 & 0.115629 \\ 19 & 6 & 0 & 4.521245 & -0.434412 & 0.531644 \\ 20 & 6 & 0 & 4.116057 & -1.601764 & 1.122431 \\ 21 & 6 & 0 & 2.342116 & -1.280223 & -0.174895 \\ 22 & 8 & 0 & 0.476950 & -2.699414 & -0.325434 \\ 23 & 1 & 0 & 1.058935 & -3.146198 & 0.323708 \\ 24 & 1 & 0 & -0.919763 & -0.611358 & 0.822052 \\ 25 & 7 & 0 & 2.906701 & -2.087575 & 0.735843 \\ 26 & 16 & 0 & 3.324619 & 0.116364 & -0.590915 \\ 27 & 1 & 0 & 4.696451 & -2.141899 & 1.862774 \\ 28 & 1 & 0 & 5.438046 & 0.118308 & 0.690750 \\ 29 & 16 & 0 & -0.522938 & 2.722892 & -0.775870 \\ 30 & 6 & 0 & 0.519512 & 1.550507 & 1.250580 \\ 31 & 1 & 0 & 0.752244 & 0.804795 & 2.005689 \\ 32 & 1 & 0 & 1.922127 & 3.176949 & 1.846511 \\ 33 & 1 & 0 & 0.989159 & 4.565733 & -0.191732 \\ ----------------------------------------------\end{array}$

Zero-point correction=

Thermal correction to Energy=

0.245092（Hartree/Particle)

Thermal correction to Enthalpy=

0.263894

Thermal correction to Gibbs Free Energy=

Sum of electronic and zero-point Energies=

Sum of electronic and thermal Energies=

0. 264839

0. 193768

$-1728.444101$

$-1728.425298$

$-1728.424354$

$-1728.495424$

Sum of electronic and thermal Free Energies $=$

M06 $-2 X / 6-311++G(d, p) / S M D / / M 06-2 X / 6-31+G(d) / S M D \quad$ energy $=-1728.99705831$

\section{1ag-INT1a}

\begin{tabular}{rrrrrr} 
Center & Atomic & Atomic & \multicolumn{3}{c}{ Coordinates (Angstroms) } \\
Number & Number & Type & $\mathrm{X}$ & $\mathrm{Y}$ & $\mathrm{Z}$ \\
-1 & 6 & 0 & 1.254837 & -1.305769 & -0.773634 \\
2 & 6 & 0 & 0.378914 & -2.286703 & -0.062431 \\
3 & 6 & 0 & -1.122951 & -2.071705 & -0.301676 \\
4 & 6 & 0 & -1.736118 & -0.952422 & 0.573431 \\
5 & 1 & 0 & 1.120568 & -1.168493 & -1.843160 \\
6 & 1 & 0 & 0.592553 & -2.271066 & 1.014457 \\
7 & 1 & 0 & 0.618471 & -3.307635 & -0.400105 \\
8 & 1 & 0 & -1.307396 & -1.851671 & -1.359839 \\
9 & 1 & 0 & -1.678293 & -2.982991 & -0.053838 \\
10 & 6 & 0 & 2.479189 & -0.733461 & -0.136468 \\
11 & 1 & 0 & 2.804656 & 0.186728 & -0.634667 \\
12 & 1 & 0 & 2.308898 & -0.512803 & 0.925177 \\
13 & 6 & 0 & 3.653313 & -1.683078 & -0.187504 \\
14 & 9 & 0 & 4.758339 & -1.163337 & 0.388148 \\
15 & 9 & 0 & 3.402631 & -2.847898 & 0.448627 \\
16 & 9 & 0 & 3.999520 & -2.010036 & -1.451061 \\
17 & 8 & 0 & -1.650995 & -1.301487 & 1.937492 \\
18 & 1 & 0 & -0.916174 & -0.792473 & 2.327231 \\
19 & 6 & 0 & -3.198291 & -0.780226 & 0.263107 \\
20 & 6 & 0 & -5.414159 & -0.528111 & 0.150664 \\
21 & 6 & 0 & -4.888229 & -0.487764 & -1.099758 \\
22 & 6 & 0 & 0.120898 & 2.357229 & -0.494716 \\
23 & 6 & 0 & 0.515935 & 1.921219 & 0.784203 \\
24 & 6 & 0 & 1.510517 & 2.613569 & 1.486302 \\
25 & 6 & 0 & 2.086473 & 3.727755 & 0.893305 \\
26 & 6 & 0 & 1.681248 & 4.158946 & -0.382942 \\
27 & 6 & 0 & 0.693902 & 3.484198 & -1.090462 \\
28 & 6 & 0 & -0.982213 & 0.352729 & 0.355629
\end{tabular}




$\begin{array}{rrrrrr}29 & 1 & 0 & 1.816432 & 2.269283 & 2.470299 \\ 30 & 1 & 0 & 2.862280 & 4.275633 & 1.419943 \\ 31 & 1 & 0 & 2.147546 & 5.034322 & -0.825460 \\ 32 & 1 & 0 & 0.383173 & 3.819447 & -2.075430 \\ 33 & 7 & 0 & -0.136001 & 0.778580 & 1.231474 \\ 34 & 16 & 0 & -1.125260 & 1.300844 & -1.111396 \\ 35 & 6 & 0 & -4.306327 & -0.718370 & 1.047168 \\ 36 & 8 & 0 & -3.536841 & -0.645281 & -1.045763 \\ 37 & 1 & 0 & -4.329603 & -0.800077 & 2.124364 \\ 38 & 1 & 0 & -5.309986 & -0.366972 & -2.087106 \\ 39 & 1 & 0 & -6.460297 & -0.437430 & 0.409586\end{array}$

Zero-point correction=

0. 293464 (Hartree/Particle)

Thermal correction to Energy=

0.314827

Thermal correction to Enthalpy=

Thermal correction to Gibbs Free Energy=

Sum of electronic and zero-point Energies=

Sum of electronic and thermal Energies=

Sum of electronic and thermal Enthalpies=

Sum of electronic and thermal Free Energies=

0.315771

0.239854

$-1558.991764$

$-1558.970402$

$-1558.969458$

$-1559.045374$

M06 $-2 X / 6-311++G(d, p) / S M D / / M 06-2 X / 6-31+G(d) / S M D \quad$ energy $=-1559.61674223$

1ag-INT1b

\begin{tabular}{|c|c|c|c|c|c|}
\hline \multirow{2}{*}{$\begin{array}{l}\text { Center } \\
\text { Number }\end{array}$} & \multirow{2}{*}{$\begin{array}{l}\text { Atomic } \\
\text { Number }\end{array}$} & \multirow{2}{*}{$\begin{array}{l}\text { Atomic } \\
\text { Type }\end{array}$} & \multicolumn{3}{|c|}{ Coordinates (Angstroms) } \\
\hline & & & X & Y & Z \\
\hline 1 & 6 & 0 & -2.905687 & 0.012626 & 0.812382 \\
\hline 2 & 6 & 0 & -1.926250 & -0.855294 & 1. 537169 \\
\hline 3 & 6 & 0 & -0.532452 & -0.951808 & 0.889784 \\
\hline 4 & 6 & 0 & 0.298560 & 0.344729 & 1. 024350 \\
\hline 5 & 1 & 0 & -3.338825 & 0.876800 & 1. 304706 \\
\hline 6 & 1 & 0 & -2.315985 & -1.883778 & 1.589261 \\
\hline 7 & 1 & 0 & -1.811392 & -0.507154 & 2.568037 \\
\hline 8 & 1 & 0 & 0.039367 & -1.748457 & 1. 380804 \\
\hline 9 & 1 & 0 & -0.617848 & -1.219963 & -0.170068 \\
\hline 10 & 6 & 0 & -3.441273 & -0.370990 & -0.530675 \\
\hline 11 & 1 & 0 & -3.600036 & 0.503368 & -1.173096 \\
\hline 12 & 1 & 0 & -2.774342 & -1.064513 & -1.055721 \\
\hline 13 & 6 & 0 & -4.779489 & -1.063060 & -0.429011 \\
\hline 14 & 9 & 0 & -5.271348 & -1.408042 & -1.638365 \\
\hline 15 & 9 & 0 & -4.720342 & -2.200388 & 0.297352 \\
\hline 16 & 9 & 0 & -5.718774 & -0.289796 & 0.157151 \\
\hline 17 & 8 & 0 & 0.419987 & 0.717492 & 2. 378800 \\
\hline 18 & 1 & 0 & 1. 280656 & 0.386865 & 2. 696609 \\
\hline 19 & 6 & 0 & -0.348811 & 1. 499808 & 0.312759 \\
\hline 20 & 6 & 0 & -1.312537 & 3. 365134 & -0.443946 \\
\hline 21 & 6 & 0 & -1.331352 & 2. 417125 & -1.415806 \\
\hline 22 & 6 & 0 & 3. 686511 & -0.417376 & -0.858080 \\
\hline 23 & 6 & 0 & 3. 849623 & -0.406568 & 0.539963 \\
\hline 24 & 6 & 0 & 5. 104975 & -0.669744 & 1. 102100 \\
\hline 25 & 6 & 0 & 6. 168128 & -0.938539 & 0.252297 \\
\hline 26 & 6 & 0 & 5. 995098 & -0.945759 & -1.143689 \\
\hline 27 & 6 & 0 & 4. 756294 & -0.684796 & -1.716359 \\
\hline 28 & 6 & 0 & 1. 692308 & 0.079841 & 0.464030 \\
\hline 29 & 1 & 0 & 5. 225691 & -0.660117 & 2. 181521 \\
\hline 30 & 1 & 0 & 7. 149143 & -1.146118 & 0.669192 \\
\hline 31 & 1 & 0 & 6. 843580 & -1.158999 & -1.787169 \\
\hline 32 & 1 & 0 & 4. 625225 & -0.689863 & -2.794282 \\
\hline 33 & 7 & 0 & 2. 691727 & -0.121998 & 1. 253782 \\
\hline 34 & 16 & 0 & 2. 026679 & -0.047012 & -1.252773 \\
\hline 35 & 8 & 0 & -0.741744 & 1. 274567 & -0.968135 \\
\hline 36 & 6 & 0 & -0.661844 & 2. 766877 & 0.689917 \\
\hline 37 & 1 & 0 & -0.470002 & 3.210805 & 1. 656340 \\
\hline 38 & 1 & 0 & -1.709923 & 4. 368192 & -0.519543 \\
\hline 39 & 1 & 0 & -1.696850 & 2. 393443 & -2.432364 \\
\hline Zero-poir & correct & & & 0.293603 & (Hartree/Particle) \\
\hline Thermal & rrection & Energy $=$ & & 0.314980 & \\
\hline Thermal & rrection & Enthalpy= & & 0.315924 & \\
\hline Thermal & rrection & Gibbs Free & lergy $=$ & 0.240263 & \\
\hline Sum of $\mathrm{e}$ & ctronic & zero-point & hergies= & -1558 & 899988 \\
\hline
\end{tabular}




\begin{tabular}{|c|c|c|c|c|c|}
\hline \multirow{2}{*}{$\begin{array}{l}\text { Center } \\
\text { Number }\end{array}$} & \multirow{2}{*}{$\begin{array}{l}\text { Atomic } \\
\text { Number }\end{array}$} & \multirow{2}{*}{$\begin{array}{l}\text { Atomic } \\
\text { Type }\end{array}$} & \multicolumn{3}{|c|}{ Coordinates (Angstroms) } \\
\hline & & & X & Y & Z \\
\hline 1 & 6 & 0 & 1. 261289 & -1.146786 & -0.523578 \\
\hline 2 & 6 & 0 & 0.736800 & -2.527277 & -0.262348 \\
\hline 3 & 6 & 0 & -0.784012 & -2.500993 & -0.418694 \\
\hline 4 & 6 & 0 & -1.299711 & -1.363508 & 0.462418 \\
\hline 5 & 1 & 0 & 1. 261738 & -0.834068 & -1.566154 \\
\hline 6 & 1 & 0 & 1. 003153 & -2.855724 & 0.746510 \\
\hline 7 & 1 & 0 & 1. 173724 & -3.238675 & -0.976283 \\
\hline 8 & 1 & 0 & -1.058337 & -2.307789 & -1.462538 \\
\hline 9 & 1 & 0 & -1.250751 & -3.443525 & -0.115943 \\
\hline 10 & 6 & 0 & 2. 372280 & -0.574567 & 0.306544 \\
\hline 11 & 1 & 0 & 2. 462165 & 0.507501 & 0.157857 \\
\hline 12 & 1 & 0 & 2. 217630 & -0.769958 & 1. 373128 \\
\hline 13 & 6 & 0 & 3. 711066 & -1.166864 & -0.059604 \\
\hline 14 & 9 & 0 & 4. 715242 & -0.631153 & 0.664735 \\
\hline 15 & 9 & 0 & 3. 762350 & -2.500454 & 0.144198 \\
\hline 16 & 9 & 0 & 4. 027361 & -0.969178 & -1.356743 \\
\hline 17 & 8 & 0 & -1.014214 & -1.676181 & 1. 807743 \\
\hline 18 & 1 & 0 & -0.733599 & -0.851351 & 2. 248085 \\
\hline 19 & 6 & 0 & -2.783292 & -1.148078 & 0.316596 \\
\hline 20 & 6 & 0 & -4.965681 & -0.683458 & 0.448709 \\
\hline 21 & 6 & 0 & -4.636973 & -0.970308 & -0.837108 \\
\hline 22 & 6 & 0 & -0.272233 & 2. 280160 & -0.650434 \\
\hline 23 & 6 & 0 & 0.086160 & 2. 005249 & 0.690420 \\
\hline 24 & 6 & 0 & 0.632791 & 3. 025865 & 1. 486923 \\
\hline 25 & 6 & 0 & 0.809617 & 4. 284304 & 0.932775 \\
\hline 26 & 6 & 0 & 0.451746 & 4. 543659 & -0.403065 \\
\hline 27 & 6 & 0 & -0.090079 & 3. 546788 & -1.208011 \\
\hline 28 & 6 & 0 & -0.545508 & -0.058236 & 0.109661 \\
\hline 29 & 1 & 0 & 0.908637 & 2. 812120 & 2. 515718 \\
\hline 30 & 1 & 0 & 1. 232625 & 5. 082228 & 1.535997 \\
\hline 31 & 1 & 0 & 0.599569 & 5.537731 & -0.814634 \\
\hline 32 & 1 & 0 & -0.365779 & 3. 751599 & -2.238203 \\
\hline 33 & 7 & 0 & -0.147913 & 0.717518 & 1. 105334 \\
\hline 34 & 16 & 0 & -0.949501 & 0.849713 & -1.384358 \\
\hline 35 & 6 & 0 & -3.750695 & -0.799360 & 1. 207091 \\
\hline 36 & 8 & 0 & -3.311277 & -1.259086 & -0.930097 \\
\hline 37 & 1 & 0 & -3.617603 & -0.647232 & 2. 269353 \\
\hline 38 & 1 & 0 & -5.198391 & -1.024434 & -1.758781 \\
\hline 39 & 1 & 0 & -5.949401 & -0.426792 & 0.817618 \\
\hline
\end{tabular}

Zero-point correction $=$

0. 293614 (Hartree/Particle)

Thermal correction to Energy=

0.313927

Thermal correction to Enthalpy=

0.314871

Thermal correction to Gibbs Free Energy=

Sum of electronic and zero-point Energies=

Sum of electronic and thermal Energies=

0.242152

$-1558.977184$

$-1558.956871$

$-1558.955927$

$-1559.028647$

Sum of electronic and thermal Free Energies=

ergy $=-1559.60179817$

lag-TS1b

\begin{tabular}{rrrrrr} 
Center & Atomic & Atomic & \multicolumn{3}{c}{ Coordinates } \\
Number & Number & Type & X & Y & $Z$ \\
\hline--1 & 6 & 0 & -2.525358 & -0.721288 & 0.303274 \\
1 & 6 & 0 & -1.934503 & -1.780329 & -0.594377 \\
2 & 6 & 0 & -0.475929 & -1.432239 & -0.952897 \\
3 & 6 & 0 & 0.149281 & -0.813402 & 0.301403 \\
4 & 1 & 0 & -2.748008 & -1.012822 & 1.327275 \\
5 & 1 & 0 & -2.514496 & -1.885851 & -1.519155
\end{tabular}




\begin{tabular}{|c|c|c|c|c|c|}
\hline 7 & 1 & 0 & -1.949758 & -2.745283 & -0.080201 \\
\hline 8 & 1 & 0 & 0.093997 & -2.315460 & -1.259650 \\
\hline 9 & 1 & 0 & -0.440831 & -0.703878 & -1.771349 \\
\hline 10 & 6 & 0 & -3.422356 & 0.337603 & -0.268028 \\
\hline 11 & 1 & 0 & -3.427724 & 1. 238882 & 0.358630 \\
\hline 12 & 1 & 0 & -3.117099 & 0.624284 & -1.280409 \\
\hline 13 & 6 & 0 & -4.861733 & -0.105461 & -0.359503 \\
\hline 14 & 9 & 0 & -5.657715 & 0.854363 & -0.880848 \\
\hline 15 & 9 & 0 & -5.027146 & -1.197862 & -1.136296 \\
\hline 16 & 9 & 0 & -5.385632 & -0.417372 & 0.845700 \\
\hline 17 & 8 & 0 & 0.063114 & -1.712507 & 1. 384768 \\
\hline 18 & 1 & 0 & 0.966358 & -2.023153 & 1. 582002 \\
\hline 19 & 6 & 0 & -0.649252 & 0.420866 & 0.680548 \\
\hline 20 & 6 & 0 & -1.043039 & 2. 423810 & 1. 636295 \\
\hline 21 & 6 & 0 & -1.012502 & 2. 562893 & 0.278958 \\
\hline 22 & 6 & 0 & 3. 783115 & 0.417915 & -0.625552 \\
\hline 23 & 6 & 0 & 3. 803459 & -0.657749 & 0.281698 \\
\hline 24 & 6 & 0 & 5. 024576 & -1.168553 & 0.739221 \\
\hline 25 & 6 & 0 & 6. 198360 & -0.592241 & 0.276116 \\
\hline 26 & 6 & 0 & 6. 168046 & 0.482040 & -0.631620 \\
\hline 27 & 6 & 0 & 4. 964256 & 1. 001272 & -1.092068 \\
\hline 28 & 6 & 0 & 1. 608463 & -0.452952 & 0.074281 \\
\hline 29 & 1 & 0 & 5. 033840 & -1.997523 & 1. 441201 \\
\hline 30 & 1 & 0 & 7. 155537 & -0.973985 & 0.618705 \\
\hline 31 & 1 & 0 & 7. 101145 & 0.915067 & -0.979858 \\
\hline 32 & 1 & 0 & 4. 944490 & 1. 831878 & -1.791408 \\
\hline 33 & 7 & 0 & 2. 548139 & -1.123068 & 0.652107 \\
\hline 34 & 16 & 0 & 2.128548 & 0.839837 & -0.990691 \\
\hline 35 & 8 & 0 & -0.732142 & 1. 369061 & -0.312438 \\
\hline 36 & 6 & 0 & -0.758498 & 1. 058260 & 1.913243 \\
\hline 37 & 1 & 0 & -0.683670 & 0.576225 & 2. 877971 \\
\hline 38 & 1 & 0 & -1.242820 & 3. 211959 & 2. 350070 \\
\hline 39 & 1 & 0 & -1.176348 & 3. 396477 & -0.389099 \\
\hline \multicolumn{4}{|c|}{ Zero-point correction $=$} & 0.293256 & (Hartree/Particle) \\
\hline \multicolumn{4}{|c|}{ Thermal correction to Energy= } & 0.313641 & \\
\hline \multicolumn{4}{|c|}{ Thermal correction to Enthalpy $=$} & 0.314586 & \\
\hline \multicolumn{4}{|c|}{ Thermal correction to Gibbs Free Energy= } & 0.242023 & \\
\hline \multicolumn{4}{|c|}{ Sum of electronic and zero-point Energies= } & \multicolumn{2}{|c|}{$\begin{array}{l}0.242023 \\
\quad-1558.975873\end{array}$} \\
\hline \multicolumn{4}{|c|}{ Sum of electronic and thermal Energies $=$} & \multicolumn{2}{|c|}{-1558.955488} \\
\hline \multicolumn{4}{|c|}{ Sum of electronic and thermal Enthalpies= } & \multicolumn{2}{|c|}{-1558.954544} \\
\hline \multicolumn{4}{|c|}{ Sum of electronic and thermal Free Energies= } & \multicolumn{2}{|c|}{-1559.027106} \\
\hline M06-2X/6 & $c 0$ & M06-2X & $21+\mathrm{C}(\mathrm{d}) / \mathrm{cu}$ & energy $=-1$ & 559. 59994449 \\
\hline
\end{tabular}

\section{1ag-INT2a}

\begin{tabular}{rrrrrr} 
Center & Atomic & Atomic & \multicolumn{3}{c}{ Coordinates (Angstroms) } \\
Number & Number & Type & \multicolumn{1}{c}{ X } & Y & \multicolumn{1}{c}{$Z$} \\
-1 & 6 & 0 & 0.724806 & 0.935618 & 0.276213 \\
2 & 6 & 0 & 1.488771 & 0.975396 & 1.622607 \\
3 & 6 & 0 & 1.500787 & -0.469345 & 2.167672 \\
4 & 6 & 0 & 0.931238 & -1.367996 & 1.050383 \\
5 & 1 & 0 & 1.440009 & 0.822813 & -0.546612 \\
6 & 1 & 0 & 0.977629 & 1.641459 & 2.324524 \\
7 & 1 & 0 & 2.498942 & 1.364097 & 1.473954 \\
8 & 1 & 0 & 2.493349 & -0.807177 & 2.475335 \\
9 & 1 & 0 & 0.834269 & -0.558028 & 3.030631 \\
10 & 6 & 0 & -0.167984 & 2.141585 & -0.004813 \\
11 & 1 & 0 & -0.834789 & 1.949150 & -0.854045 \\
12 & 1 & 0 & -0.782735 & 2.388723 & 0.866367 \\
13 & 6 & 0 & 0.617666 & 3.371607 & -0.361362 \\
14 & 9 & 0 & -0.187824 & 4.422727 & -0.623303 \\
15 & 9 & 0 & 1.452951 & 3.769552 & 0.622220 \\
16 & 9 & 0 & 1.384626 & 3.196867 & -1.459473 \\
17 & 8 & 0 & 0.286591 & -2.528964 & 1.512022 \\
18 & 1 & 0 & -0.486982 & -2.238810 & 2.028321 \\
19 & 6 & 0 & 1.999397 & -1.840307 & 0.110033 \\
20 & 6 & 0 & 3.386739 & -2.845741 & -1.328351 \\
21 & 6 & 0 & 3.901706 & -1.643483 & -0.962381 \\
22 & 6 & 0 & -2.313972 & -0.842681 & -0.794286
\end{tabular}




$\begin{array}{rrrrrr}23 & 6 & 0 & -2.376448 & -0.448626 & 0.589054 \\ 24 & 6 & 0 & -3.653958 & -0.322691 & 1.211516 \\ 25 & 6 & 0 & -4.789334 & -0.571346 & 0.474410 \\ 26 & 6 & 0 & -4.703454 & -0.952667 & -0.885223 \\ 27 & 6 & 0 & -3.472395 & -1.091754 & -1.523426 \\ 28 & 6 & 0 & -0.085867 & -0.383427 & 0.352018 \\ 29 & 1 & 0 & -3.701036 & -0.027284 & 2.255583 \\ 30 & 1 & 0 & -5.766806 & -0.474614 & 0.937269 \\ 31 & 1 & 0 & -5.615030 & -1.143322 & -1.443535 \\ 32 & 1 & 0 & -3.422664 & -1.390110 & -2.566304 \\ 33 & 7 & 0 & -1.222930 & -0.228986 & 1.208543 \\ 34 & 16 & 0 & -0.664148 & -0.972662 & -1.313776 \\ 35 & 6 & 0 & 2.140490 & -2.975074 & -0.627654 \\ 36 & 8 & 0 & 3.070245 & -1.023067 & -0.084682 \\ 37 & 1 & 0 & 1.441765 & -3.799017 & -0.665729 \\ 38 & 1 & 0 & 4.804122 & -1.106806 & -1.217835 \\ 39 & 1 & 0 & 3.835255 & -3.556047 & -2.009490\end{array}$

Zero-point correction=

0.296471 (Hartree/Particle)

Thermal correction to Energy=

0.316578

Thermal correction to Enthalpy=

0.317522

Thermal correction to Gibbs Free Energy=

Sum of electronic and zero-point Energies=

Sum of electronic and thermal Energies=

0.245779

$-1559.011915$

$-1558.991808$

$-1558.990864$

Sum of electronic and thermal Enthalpies=

$-1559.062607$

M06 $-2 X / 6-311++G(d, p) / S M D / /$ M06 $-2 X / 6-31+G(d) / S M D \quad$ energy $=-1559.63847596$

\section{1ag-INT2b}

\begin{tabular}{|c|c|c|c|c|c|}
\hline \multirow{2}{*}{$\begin{array}{l}\text { Center } \\
\text { Number }\end{array}$} & \multirow{2}{*}{$\begin{array}{l}\text { Atomic } \\
\text { Number }\end{array}$} & \multirow{2}{*}{$\begin{array}{c}\text { Atomic } \\
\text { Type }\end{array}$} & \multicolumn{3}{|c|}{ Coordinates (Angstroms) } \\
\hline & & & X & Y & Z \\
\hline 1 & 6 & 0 & -2.320153 & -0.332793 & 0.250933 \\
\hline 2 & 6 & 0 & -2.177192 & -1.685592 & -0.490314 \\
\hline 3 & 6 & 0 & -0.659900 & -1.970266 & -0.587725 \\
\hline 4 & 6 & 0 & -0.004606 & -0.992077 & 0.386458 \\
\hline 5 & 1 & 0 & -2.549787 & -0.515001 & 1. 306270 \\
\hline 6 & 1 & 0 & -2.617928 & -1.628792 & -1.490224 \\
\hline 7 & 1 & 0 & -2.699363 & -2.477399 & 0.052228 \\
\hline 8 & 1 & 0 & -0.387892 & -2.997456 & -0.331268 \\
\hline 9 & 1 & 0 & -0.305952 & -1.770520 & -1.603847 \\
\hline 10 & 6 & 0 & -3.353556 & 0.632046 & -0.325407 \\
\hline 11 & 1 & 0 & -3.223679 & 1. 637197 & 0.093096 \\
\hline 12 & 1 & 0 & -3.270568 & 0.699741 & -1.414276 \\
\hline 13 & 6 & 0 & -4.764083 & 0.224405 & -0.010570 \\
\hline 14 & 9 & 0 & -5.665236 & 1. 081520 & -0.537100 \\
\hline 15 & 9 & 0 & -5.080641 & -0.999063 & -0.487859 \\
\hline 16 & 9 & 0 & -5.010217 & 0.184662 & 1. 317338 \\
\hline 17 & 8 & 0 & -0.167468 & -1.491230 & 1. 696210 \\
\hline 18 & 1 & 0 & 0.498743 & -1.057910 & 2. 261118 \\
\hline 19 & 6 & 0 & -0.909188 & 0.287095 & 0.235119 \\
\hline 20 & 6 & 0 & -0.134471 & 2. 471660 & 0.432555 \\
\hline 21 & 6 & 0 & -0.155701 & 2. 104317 & -0.902250 \\
\hline 22 & 6 & 0 & 3. 687716 & -0.146098 & -0.692854 \\
\hline 23 & 6 & 0 & 3. 530910 & -0.156460 & 0.706942 \\
\hline 24 & 6 & 0 & 4. 624038 & 0.129307 & 1. 536317 \\
\hline 25 & 6 & 0 & 5.845923 & 0.418824 & 0.948084 \\
\hline 26 & 6 & 0 & 5. 991855 & 0.426002 & -0.451619 \\
\hline 27 & 6 & 0 & 4. 918960 & 0.145003 & -1.287801 \\
\hline 28 & 6 & 0 & 1. 452805 & -0.680319 & 0.149141 \\
\hline 29 & 1 & 0 & 4. 498199 & 0.120419 & 2. 615270 \\
\hline 30 & 1 & 0 & 6. 704067 & 0.643344 & 1. 574624 \\
\hline 31 & 1 & 0 & 6. 959708 & 0.654819 & -0.887993 \\
\hline 32 & 1 & 0 & 5. 035154 & 0.151346 & -2.367423 \\
\hline 33 & 7 & 0 & 2. 250903 & -0.465059 & 1. 143843 \\
\hline 34 & 16 & 0 & 2. 164542 & -0.535086 & -1.445911 \\
\hline 35 & 8 & 0 & -0.648465 & 0.847602 & -1.072546 \\
\hline 36 & 6 & 0 & -0.617388 & 1. 405918 & 1. 177991 \\
\hline 37 & 1 & 0 & -0.753410 & 1. 346158 & 2. 249999 \\
\hline 38 & 1 & 0 & 0.197290 & 3. 434355 & 0.801383 \\
\hline
\end{tabular}


Zero-point correction=

Thermal correction to Energy=

Thermal correction to Enthalpy=

Thermal correction to Gibbs Free Energy=

Sum of electronic and zero-point Energies=

Sum of electronic and thermal Energies=

Sum of electronic and thermal Enthalpies=

Sum of electronic and thermal Free Energies=

M06 $-2 X / 6-311++G(d, p) / S M D / / M 06-2 X / 6-31+G(d) / S M D=$

1ag-TS2a

\begin{tabular}{|c|c|c|c|c|c|}
\hline \multirow{2}{*}{$\begin{array}{l}\text { Center } \\
\text { Number }\end{array}$} & \multirow{2}{*}{$\begin{array}{l}\text { Atomic } \\
\text { Number }\end{array}$} & \multirow{2}{*}{$\begin{array}{c}\text { Atomic } \\
\text { Type }\end{array}$} & \multicolumn{3}{|c|}{ Coordinates (Angstroms) } \\
\hline & & & X & Y & Z \\
\hline 1 & 6 & 0 & 1. 696515 & -0.376992 & -0.090187 \\
\hline 2 & 6 & 0 & 1. 974463 & -1.347058 & 1. 060810 \\
\hline 3 & 6 & 0 & 0.797842 & -2.317861 & 1. 044001 \\
\hline 4 & 6 & 0 & -0.481153 & -1.512458 & 1. 067194 \\
\hline 5 & 1 & 0 & 1. 777832 & -0.940467 & -1.028614 \\
\hline 6 & 1 & 0 & 2. 025791 & -0.802072 & 2. 012714 \\
\hline 7 & 1 & 0 & 2. 923078 & -1.872952 & 0.926024 \\
\hline 8 & 1 & 0 & 0.836570 & -2.924538 & 0.133195 \\
\hline 9 & 1 & 0 & 0.800877 & -2.993714 & 1. 907363 \\
\hline 10 & 6 & 0 & 2. 614608 & 0.847021 & -0.163847 \\
\hline 11 & 1 & 0 & 2. 328666 & 1. 483591 & -1.008320 \\
\hline 12 & 1 & 0 & 2. 542372 & 1. 442156 & 0.751616 \\
\hline 13 & 6 & 0 & 4. 066496 & 0.505519 & -0.354905 \\
\hline 14 & 9 & 0 & 4. 802488 & 1. 606333 & -0.620764 \\
\hline 15 & 9 & 0 & 4. 626316 & -0.062969 & 0.734837 \\
\hline 16 & 9 & 0 & 4. 276042 & -0.350283 & -1.378175 \\
\hline 17 & 8 & 0 & -0.831181 & -1.001665 & 2. 297852 \\
\hline 18 & 1 & 0 & -0.410063 & -0.120582 & 2. 389731 \\
\hline 19 & 6 & 0 & -1.652426 & -1.989723 & 0.364778 \\
\hline 20 & 6 & 0 & -3.652982 & -2.323832 & -0.584321 \\
\hline 21 & 6 & 0 & -2.686542 & -2.971756 & -1.294321 \\
\hline 22 & 6 & 0 & -1.673383 & 1. 476318 & -0.746561 \\
\hline 23 & 6 & 0 & -1.162709 & 1. 770819 & 0.548535 \\
\hline 24 & 6 & 0 & -1.816838 & 2. 733220 & 1. 345941 \\
\hline 25 & 6 & 0 & -2.944449 & 3. 363782 & 0.848902 \\
\hline 26 & 6 & 0 & -3.441637 & 3. 056627 & -0.433088 \\
\hline 27 & 6 & 0 & -2.813080 & 2. 112383 & -1.239209 \\
\hline 28 & 6 & 0 & 0.245520 & 0.093896 & 0.037378 \\
\hline 29 & 1 & 0 & -1.427835 & 2. 957862 & 2. 335342 \\
\hline 30 & 1 & 0 & -3.458208 & 4. 103536 & 1. 455924 \\
\hline 31 & 1 & 0 & -4.329366 & 3.563800 & -0.799395 \\
\hline 32 & 1 & 0 & -3.200287 & 1. 878031 & -2.226604 \\
\hline 33 & 7 & 0 & -0.046013 & 1. 082736 & 0.914837 \\
\hline 34 & 16 & 0 & -0.668871 & 0.281479 & -1.512351 \\
\hline 35 & 6 & 0 & -2.983536 & -1.678980 & 0.498695 \\
\hline 36 & 8 & 0 & -1.471202 & -2.782461 & -0.726176 \\
\hline 37 & 1 & 0 & -3.413836 & -1.059200 & 1. 273428 \\
\hline 38 & 1 & 0 & -2.705183 & -3.595345 & -2.176877 \\
\hline 39 & 1 & 0 & -4.711192 & -2.313204 & -0.807040 \\
\hline
\end{tabular}

Zero-point correction=

Thermal correction to Energy=

0. 294613 (Hartree/Particle)

Thermal correction to Enthalpy=

Thermal correction to Gibbs Free Energy=

Sum of electronic and zero-point Energies=

Sum of electronic and thermal Energies=

Sum of electronic and thermal Enthalpies=

Sum of electronic and thermal Free Energies=

0.314568

0.315512

0.243837

$-1558.997662$

$-1558.977707$

$-1558.976763$

$-1559.048438$

M06 $-2 X / 6-311++G(d, p) / S M D / / M 06-2 X / 6-31+G(d) / S M D \quad$ energy $=-1559.62270284$

\section{1ag-TS2b}

\begin{tabular}{llclc} 
Center & Atomic & Atomic & \multicolumn{3}{c}{ Coordinates (Angstroms) } \\
Number & Number & Type & $X$ & Y Z
\end{tabular}




\begin{tabular}{|c|c|c|c|c|c|}
\hline 1 & 6 & 0 & -2.319838 & -0.084142 & 0.267497 \\
\hline 2 & 6 & 0 & -2.249797 & -1.503933 & -0.324589 \\
\hline 3 & 6 & 0 & -0.796074 & -2.020092 & -0.220553 \\
\hline 4 & 6 & 0 & 0.029750 & -1.098252 & 0.632949 \\
\hline 5 & 1 & 0 & -2.465748 & -0.150144 & 1. 351100 \\
\hline 6 & 1 & 0 & -2.558425 & -1.488053 & -1.375012 \\
\hline 7 & 1 & 0 & -2.944301 & -2.162175 & 0.203479 \\
\hline 8 & 1 & 0 & -0.755642 & -3.030830 & 0.201772 \\
\hline 9 & 1 & 0 & -0.352446 & -2.070119 & -1.219243 \\
\hline 10 & 6 & 0 & -3.433646 & 0.791201 & -0.320575 \\
\hline 11 & 1 & 0 & -3.397499 & 1. 792953 & 0.119805 \\
\hline 12 & 1 & 0 & -3.331023 & 0.888381 & -1.406445 \\
\hline 13 & 6 & 0 & -4.815585 & 0.260054 & -0.054412 \\
\hline 14 & 9 & 0 & -5.767645 & 1. 140440 & -0.432480 \\
\hline 15 & 9 & 0 & -5.085288 & -0.887778 & -0.712193 \\
\hline 16 & 9 & 0 & -5.030886 & 0.004003 & 1. 254163 \\
\hline 17 & 8 & 0 & -0.249470 & -1.207795 & 1.972609 \\
\hline 18 & 1 & 0 & 0.443744 & -0.720603 & 2. 459215 \\
\hline 19 & 6 & 0 & -0.983388 & 0.596551 & 0.093427 \\
\hline 20 & 6 & 0 & 0.411253 & 2. 379276 & -0.004494 \\
\hline 21 & 6 & 0 & 0.310214 & 1.796516 & -1.241061 \\
\hline 22 & 6 & 0 & 3. 630964 & -0.378625 & -0.619776 \\
\hline 23 & 6 & 0 & 3. 458671 & -0.043735 & 0.741666 \\
\hline 24 & 6 & 0 & 4. 527602 & 0.521725 & 1. 453790 \\
\hline 25 & 6 & 0 & 5.731676 & 0.735912 & 0.798312 \\
\hline 26 & 6 & 0 & 5.889795 & 0.396262 & -0.557169 \\
\hline 27 & 6 & 0 & 4. 842397 & -0.164353 & -1.280166 \\
\hline 28 & 6 & 0 & 1. 419600 & -0.823977 & 0.343954 \\
\hline 29 & 1 & 0 & 4. 397016 & 0.781402 & 2. 500677 \\
\hline 30 & 1 & 0 & 6.565797 & 1. 173406 & 1. 339259 \\
\hline 31 & 1 & 0 & 6. 842033 & 0.572722 & -1.048655 \\
\hline 32 & 1 & 0 & 4. 964458 & -0.427287 & -2.326882 \\
\hline 33 & 7 & 0 & 2. 206033 & -0.310648 & 1. 257025 \\
\hline 34 & 16 & 0 & 2. 153163 & -1.068626 & -1.241040 \\
\hline 35 & 8 & 0 & -0.586512 & 0.779991 & -1.225747 \\
\hline 36 & 6 & 0 & -0.460642 & 1. 667383 & 0.847976 \\
\hline 37 & 1 & 0 & -0.646843 & 1. 837792 & 1. 900541 \\
\hline 38 & 1 & 0 & 1. 044564 & 3. 219886 & 0.247209 \\
\hline 39 & 1 & 0 & 0.785982 & 2. 001039 & -2.190308 \\
\hline
\end{tabular}

Zero-point correction=

Thermal correction to Energy=

0.294621 (Hartree/Particle)

Thermal correction to Enthalpy=

0.314777

Thermal correction to Gibbs Free Energy=

Sum of electronic and zero-point Energies=

Sum of electronic and thermal Energies=

Sum of electronic and thermal Enthalpies=

Sum of electronic and thermal Free Energies=

0.315721

0.243650

$-1558.999676$

$-1558.979520$

$-1558.978576$

$-1559.050646$

M06 $-2 X / 6-311++G(d, p) / S M D / / M 06-2 X / 6-31+G(d) / S M D \quad$ energy $=-1559.62486823$

lag-INT3a

\begin{tabular}{rrrrrr} 
Center & Atomic & Atomic & \multicolumn{3}{c}{ Coordinates (Angstroms) } \\
Number & Number & Type & X & Y & $Z$ \\
\hline 1 & 6 & 0 & -1.815553 & 0.146267 & -0.253502 \\
2 & 6 & 0 & -1.902975 & 1.242070 & 0.823761 \\
3 & 6 & 0 & -0.796673 & 2.308431 & 0.730595 \\
4 & 6 & 0 & 0.562811 & 1.827919 & 1.125302 \\
5 & 1 & 0 & -1.941120 & 0.613568 & -1.237511 \\
6 & 1 & 0 & -1.907110 & 0.775848 & 1.817637 \\
7 & 1 & 0 & -2.863570 & 1.753347 & 0.715566 \\
8 & 1 & 0 & -0.760207 & 2.714053 & -0.286130 \\
9 & 1 & 0 & -1.083934 & 3.135700 & 1.396574 \\
10 & 6 & 0 & -2.882117 & -0.952276 & -0.088842 \\
11 & 1 & 0 & -2.752716 & -1.713292 & -0.865202 \\
12 & 1 & 0 & -2.787559 & -1.440779 & 0.886357 \\
13 & 6 & 0 & -4.301791 & -0.464666 & -0.208204 \\
14 & 9 & 0 & -5.163176 & -1.496866 & -0.331204 \\
15 & 9 & 0 & -4.719761 & 0.243101 & 0.862422
\end{tabular}




$\begin{array}{rrrrrr}16 & 9 & 0 & -4.493885 & 0.325079 & -1.285604 \\ 17 & 8 & 0 & 0.713434 & 1.236160 & 2.352088 \\ 18 & 1 & 0 & 0.277556 & 0.358419 & 2.326307 \\ 19 & 6 & 0 & 1.719345 & 1.945742 & 0.355336 \\ 20 & 6 & 0 & 3.749583 & 1.826189 & -0.617734 \\ 21 & 6 & 0 & 2.880540 & 2.489362 & -1.436826 \\ 22 & 6 & 0 & 1.744241 & -1.445922 & -0.751998 \\ 23 & 6 & 0 & 1.286430 & -1.623174 & 0.568731 \\ 24 & 6 & 0 & 2.109232 & -2.247683 & 1.515687 \\ 25 & 6 & 0 & 3.369330 & -2.674375 & 1.123013 \\ 26 & 6 & 0 & 3.815992 & -2.492595 & -0.199068 \\ 27 & 6 & 0 & 3.010280 & -1.881385 & -1.151669 \\ 28 & 6 & 0 & -0.470350 & -0.536129 & -0.228587 \\ 29 & 1 & 0 & 1.755754 & -2.377072 & 2.535089 \\ 30 & 1 & 0 & 4.023253 & -3.154088 & 1.845382 \\ 31 & 1 & 0 & 4.807628 & -2.834160 & -0.481313 \\ 32 & 1 & 0 & 3.359347 & -1.736516 & -2.169929 \\ 33 & 7 & 0 & 0.023109 & -1.105948 & 0.824814 \\ 34 & 16 & 0 & 0.527229 & -0.605161 & -1.668641 \\ 35 & 6 & 0 & 3.020748 & 1.472999 & 0.548533 \\ 36 & 8 & 0 & 1.652823 & 2.583614 & -0.860020 \\ 37 & 1 & 0 & 3.370473 & 0.922785 & 1.411047 \\ 38 & 1 & 0 & 2.982837 & 2.946485 & -2.410685 \\ 39 & 1 & 0 & 4.788850 & 1.615255 & -0.832873 \\ ---------------------------------------19\end{array}$

Zero-point correction=

0.295202 (Hartree/Particle)

Thermal correction to Energy=

0.315987

Thermal correction to Enthalpy=

0. 316931

Thermal correction to Gibbs Free Energy=

Sum of electronic and zero-point Energies=

Sum of electronic and thermal Energies=

0.243525

$-1559.018583$

$-1558.997797$

$-1558.996853$

$-1559.070260$

Sum of electronic and thermal Free Energies=

ergy $=-1559.64567722$

1ag-INT3b

\begin{tabular}{rrrrrr} 
Center & Atomic & Atomic & \multicolumn{2}{c}{ Coordinates (Angstroms) } \\
Number & Number & Type & X & Y & Z \\
-1 & 6 & 0 & 2.201776 & 0.199797 & -0.139785 \\
2 & 6 & 0 & 2.113299 & -0.252238 & 1.329576 \\
3 & 6 & 0 & 0.678200 & -0.259689 & 1.875584 \\
4 & 6 & 0 & -0.244780 & -1.101897 & 1.065575 \\
5 & 1 & 0 & 1.712261 & -0.549381 & -0.773943 \\
6 & 1 & 0 & 2.718946 & 0.410663 & 1.958119 \\
7 & 1 & 0 & 2.532947 & -1.257699 & 1.424778 \\
8 & 1 & 0 & 0.699359 & -0.646420 & 2.904545 \\
9 & 1 & 0 & 0.283551 & 0.761054 & 1.929899 \\
10 & 6 & 0 & 3.651628 & 0.371259 & -0.629401 \\
11 & 1 & 0 & 3.647580 & 0.674045 & -1.680876 \\
12 & 1 & 0 & 4.175543 & 1.142322 & -0.054715 \\
13 & 6 & 0 & 4.487174 & -0.878324 & -0.550754 \\
14 & 9 & 0 & 5.633379 & -0.750206 & -1.254130 \\
15 & 9 & 0 & 4.858285 & -1.200128 & 0.707217 \\
16 & 9 & 0 & 3.853534 & -1.961646 & -1.048606 \\
17 & 8 & 0 & 0.223453 & -2.312153 & 0.723654 \\
18 & 1 & 0 & -0.476029 & -2.777418 & 0.218203 \\
19 & 6 & 0 & 1.458233 & 1.476840 & -0.368502 \\
20 & 6 & 0 & 0.138938 & 3.204728 & -0.912691 \\
21 & 6 & 0 & 1.042662 & 3.597743 & 0.020848 \\
22 & 6 & 0 & -3.767749 & 0.176331 & 0.219753 \\
23 & 6 & 0 & -3.522628 & -1.116442 & -0.303986 \\
24 & 6 & 0 & -4.533949 & -1.763504 & -1.035386 \\
25 & 6 & 0 & -5.746700 & -1.117329 & -1.223746 \\
26 & 6 & 0 & -5.974571 & 0.166345 & -0.696566 \\
27 & 6 & 0 & -4.987270 & 0.825578 & 0.030160 \\
28 & 6 & 0 & -1.550214 & -0.778228 & 0.670496 \\
29 & 1 & 0 & -4.350433 & -2.755270 & -1.439190 \\
30 & 1 & 0 & -6.534027 & -1.610061 & -1.786942 \\
31 & 1 & 0 & -6.932467 & 0.652298 & -0.856586 \\
& & 0 & & &
\end{tabular}




\begin{tabular}{|c|c|c|c|c|c|}
\hline 32 & 1 & 0 & -5.163195 & 1.816922 & 0.437588 \\
\hline 33 & 7 & 0 & -2.279865 & -1.634638 & -0.039167 \\
\hline 34 & 16 & 0 & -2.355717 & 0.744325 & 1. 078675 \\
\hline 35 & 8 & 0 & 1.852153 & 2. 558532 & 0.360151 \\
\hline 36 & 6 & 0 & 0.410521 & 1.816169 & -1.166794 \\
\hline 37 & 1 & 0 & -0.111404 & 1. 152128 & -1.843969 \\
\hline 38 & 1 & 0 & -0.628677 & 3. 819857 & -1.362524 \\
\hline 39 & 1 & 0 & 1. 237523 & 4. 534199 & 0.523933 \\
\hline \multicolumn{4}{|c|}{ Zero-point correction $=$} & \multicolumn{2}{|c|}{ 0.296332（Hartree/Particle) } \\
\hline \multicolumn{4}{|c|}{ Thermal correction to Energy= } & \multicolumn{2}{|c|}{0.317192} \\
\hline \multicolumn{4}{|c|}{ Thermal correction to Enthalpy= } & \multirow{2}{*}{\multicolumn{2}{|c|}{$\begin{array}{l}0.318136 \\
0.243109\end{array}$}} \\
\hline \multicolumn{4}{|c|}{ Thermal correction to Gibbs Free Energy= } & & 0.243109 \\
\hline \multicolumn{4}{|c|}{ Sum of electronic and zero-point Energies $=$} & \multirow{2}{*}{\multicolumn{2}{|c|}{-1559.023430}} \\
\hline \multicolumn{4}{|c|}{ Sum of electronic and thermal Energies $=$} & & \\
\hline \multicolumn{4}{|c|}{ Sum of electronic and thermal Enthalpies= } & \multicolumn{2}{|c|}{$\begin{array}{l}-1559.002570 \\
-1559.001626\end{array}$} \\
\hline \multicolumn{4}{|c|}{ Sum of electronic and thermal Free Energies $=$} & \multicolumn{2}{|c|}{-1559.076653} \\
\hline \multicolumn{4}{|c|}{ M06-2X/6-311++G (d, p) /SMD//M06-2X/6-31+G(d)/SMD } & \multicolumn{2}{|c|}{ energy $=-1559.65050270$} \\
\hline
\end{tabular}

\section{1am-INT1a}

\begin{tabular}{|c|c|c|c|c|c|}
\hline \multirow{2}{*}{$\begin{array}{l}\text { Center } \\
\text { Number }\end{array}$} & \multirow{2}{*}{$\begin{array}{l}\text { Atomic } \\
\text { Number }\end{array}$} & \multirow{2}{*}{$\begin{array}{l}\text { Atomic } \\
\text { Type }\end{array}$} & \multicolumn{3}{|c|}{ Coordinates (Angstroms) } \\
\hline & & & X & Y & Z \\
\hline 1 & 6 & 0 & -2.857796 & -0.536608 & 1. 008211 \\
\hline 2 & 6 & 0 & -1.913735 & -1.692150 & 0.976080 \\
\hline 3 & 6 & 0 & -0.418667 & -1.330109 & 1. 093523 \\
\hline 4 & 6 & 0 & 0.117395 & -0.464167 & -0.058149 \\
\hline 5 & 1 & 0 & -3.090129 & -0.064731 & 1.958249 \\
\hline 6 & 1 & 0 & -2.055464 & -2.264462 & 0.053313 \\
\hline 7 & 1 & 0 & -2.133099 & -2.367180 & 1.813650 \\
\hline 8 & 1 & 0 & -0.211462 & -0.826271 & 2. 046003 \\
\hline 9 & 1 & 0 & 0.146501 & -2.268725 & 1. 108041 \\
\hline 10 & 6 & 0 & -3.532953 & -0.017344 & -0.220108 \\
\hline 11 & 1 & 0 & -3.632825 & 1. 075682 & -0.203909 \\
\hline 12 & 1 & 0 & -2.987915 & -0.299575 & -1.128077 \\
\hline 13 & 6 & 0 & -4.932098 & -0.565438 & -0.370782 \\
\hline 14 & 9 & 0 & -5.545920 & -0.103747 & -1.481120 \\
\hline 15 & 9 & 0 & -4.951766 & -1.913239 & -0.455634 \\
\hline 16 & 9 & 0 & -5.730518 & -0.241245 & 0.669108 \\
\hline 17 & 8 & 0 & -0.288513 & -1.087354 & -1.265408 \\
\hline 18 & 1 & 0 & -0.229811 & -0.419350 & -1.973079 \\
\hline 19 & 6 & 0 & 1.639602 & -0.368677 & -0.026596 \\
\hline 20 & 6 & 0 & 4. 079479 & -0.944359 & -0.037057 \\
\hline 21 & 6 & 0 & 3. 830586 & 0.432210 & -0.213237 \\
\hline 22 & 6 & 0 & 4. 918128 & 1. 310684 & -0.366017 \\
\hline 23 & 6 & 0 & 5. 380696 & -1.459419 & -0.007022 \\
\hline 24 & 6 & 0 & 6. 437798 & -0.572938 & -0.160551 \\
\hline 25 & 6 & 0 & 6. 208275 & 0.804753 & -0.339365 \\
\hline 26 & 1 & 0 & 4. 738662 & 2. 373917 & -0.503073 \\
\hline 27 & 1 & 0 & 7. 053777 & 1. 476322 & -0.457109 \\
\hline 28 & 1 & 0 & 7. 456541 & -0.949380 & -0.141704 \\
\hline 29 & 1 & 0 & 5. 559819 & -2.522082 & 0.131217 \\
\hline 30 & 6 & 0 & -1.136771 & 3. 236726 & 0.421064 \\
\hline 31 & 6 & 0 & -1.188513 & 2. 853866 & -0.884072 \\
\hline 32 & 6 & 0 & -0.439890 & 0.954100 & -0.034301 \\
\hline 33 & 1 & 0 & -1.517401 & 3. 477537 & -1.706811 \\
\hline 34 & 1 & 0 & -1.404417 & 4. 190402 & 0.857881 \\
\hline 35 & 7 & 0 & -0.784920 & 1. 559729 & -1.131285 \\
\hline 36 & 16 & 0 & -0.561362 & 1.934138 & 1. 393204 \\
\hline 37 & 6 & 0 & 2. 419178 & 0.728846 & -0.207786 \\
\hline 38 & 16 & 0 & 2. 590612 & -1.837960 & 0.137808 \\
\hline 39 & 1 & 0 & 2. 031313 & 1.734013 & -0.342736 \\
\hline
\end{tabular}

Zero-point correction=

0.290419 (Hartree/Particle)

Thermal correction to Energy=

0.312252

Thermal correction to Enthalpy=

0.313196

0. 235156

$-1881.959693$

$-1881.937860$

$-1881.936916$

Sum of electronic and thermal Energies=

Sum of electronic and thermal Enthalpies= 
Sum of electronic and thermal Free Energies=

$-1882.014956$

M06 $-2 X / 6-311++G(d, p) / S M D / / M 06-2 X / 6-31+G(d) / S M D \quad$ energy $=-1882.58977130$

1am-INT1b

\begin{tabular}{|c|c|c|c|c|c|}
\hline \multirow{2}{*}{$\begin{array}{l}\text { Center } \\
\text { Number }\end{array}$} & \multirow{2}{*}{$\begin{array}{l}\text { Atomic } \\
\text { Number }\end{array}$} & \multirow{2}{*}{$\begin{array}{l}\text { Atomic } \\
\text { Type }\end{array}$} & \multicolumn{3}{|c|}{ Coordinates (Angstroms) } \\
\hline & & & X & Y & Z \\
\hline 1 & 6 & 0 & -1.799043 & -0.946223 & 0.900374 \\
\hline 2 & 6 & 0 & -0.781984 & -1.897976 & 1. 448478 \\
\hline 3 & 6 & 0 & 0.554343 & -1.950238 & 0.683940 \\
\hline 4 & 6 & 0 & 1. 476512 & -0.752804 & 0.956819 \\
\hline 5 & 1 & 0 & -1.189803 & -2.920367 & 1. 429348 \\
\hline 6 & 1 & 0 & -0.580000 & -1.662067 & 2. 497884 \\
\hline 7 & 1 & 0 & 1. 103442 & -2.850944 & 0.980543 \\
\hline 8 & 1 & 0 & 0.363494 & -2.026211 & -0.394649 \\
\hline 9 & 6 & 0 & -2.427618 & -1.152815 & -0.441146 \\
\hline 10 & 1 & 0 & -2.671550 & -0.199794 & -0.925186 \\
\hline 11 & 1 & 0 & -1.779248 & -1.721180 & -1.118222 \\
\hline 12 & 6 & 0 & -3.725155 & -1.921580 & -0.356229 \\
\hline 13 & 9 & 0 & -4.292073 & -2.100336 & -1.568920 \\
\hline 14 & 9 & 0 & -3.565472 & -3.152940 & 0.175114 \\
\hline 15 & 9 & 0 & -4.647782 & -1.298077 & 0.407170 \\
\hline 16 & 6 & 0 & 0.830990 & 0.566961 & 0.562421 \\
\hline 17 & 6 & 0 & -0.202320 & 2. 653693 & 0.713128 \\
\hline 18 & 6 & 0 & -0.264459 & 2. 440992 & -0.680155 \\
\hline 19 & 6 & 0 & -0.845349 & 3. 374411 & -1.545109 \\
\hline 20 & 6 & 0 & -0.745374 & 3. 836221 & 1. 245694 \\
\hline 21 & 6 & 0 & -1.325269 & 4. 763631 & 0.393122 \\
\hline 22 & 6 & 0 & -1.375027 & 4. 534152 & -0.994364 \\
\hline 23 & 1 & 0 & -0.883555 & 3. 198371 & -2.616495 \\
\hline 24 & 1 & 0 & -1.833374 & 5. 272772 & -1.645715 \\
\hline 25 & 1 & 0 & -1.746982 & 5. 678781 & 0.798750 \\
\hline 26 & 1 & 0 & -0.706364 & 4. 013638 & 2. 317339 \\
\hline 27 & 6 & 0 & 4. 669518 & -1.291077 & -1.288282 \\
\hline 28 & 6 & 0 & 4. 930481 & -0.487714 & -0.220283 \\
\hline 29 & 6 & 0 & 2. 799344 & -0.886586 & 0.207578 \\
\hline 30 & 8 & 0 & 1. 752329 & -0.744291 & 2. 341821 \\
\hline 31 & 1 & 0 & 2. 540582 & -0.187569 & 2. 479986 \\
\hline 32 & 1 & 0 & -2.208151 & -0.166925 & 1. 534310 \\
\hline 33 & 7 & 0 & 3. 863264 & -0.262743 & 0.618746 \\
\hline 34 & 16 & 0 & 3. 025676 & -1.805603 & -1.245142 \\
\hline 35 & 1 & 0 & 5. 889394 & -0.033879 & 0.000191 \\
\hline 36 & 1 & 0 & 5. 339943 & -1.609707 & -2.076130 \\
\hline 37 & 16 & 0 & 0.476906 & 0.919216 & -1.119817 \\
\hline 38 & 6 & 0 & 0.437757 & 1. 557199 & 1. 399852 \\
\hline 39 & 1 & 0 & 0.563226 & 1. 511505 & 2. 476278 \\
\hline
\end{tabular}

Zero-point correction $=$

0. 290246 (Hartree/Particle)

Thermal correction to Energy=

0.312174

Thermal correction to Enthalpy=

0.313118

Thermal correction to Gibbs Free Energy=

Sum of electronic and zero-point Energies=

Sum of electronic and thermal Energies=

0.235174

$-1881.959948$

$-1881.938020$

$-1881.937076$

Sum of electronic and thermal Enthalpies=

Sum of electronic and thermal Free Energies=

$-1882.015020$

M06 $-2 X / 6-311++G(d, p) / S M D / / M 06-2 X / 6-31+G(d) / S M D \quad$ energy $=-1882.59006166$

1am-TS1a

\begin{tabular}{rrrrrr} 
Center & Atomic & Atomic & \multicolumn{3}{c}{ Coordinates (Angstroms) } \\
Number & Number & Type & X & Y & $Z$ \\
\hline 1 & 6 & 0 & -2.560479 & -0.256634 & 0.584792 \\
2 & 6 & 0 & -2.003556 & -1.634210 & 0.822878 \\
3 & 6 & 0 & -0.478880 & -1.566351 & 0.989317 \\
4 & 6 & 0 & 0.053456 & -0.623831 & -0.088616 \\
5 & 1 & 0 & -2.801639 & 0.330211 & 1.468768 \\
6 & 1 & 0 & -2.239909 & -2.281968 & -0.025861 \\
7 & 1 & 0 & -2.450325 & -2.078813 & 1.720643 \\
8 & 1 & 0 & -0.203465 & -1.176370 & 1.977267
\end{tabular}




\begin{tabular}{|c|c|c|c|c|c|}
\hline 9 & 1 & 0 & -0.032732 & -2.561691 & 0.890533 \\
\hline 10 & 6 & 0 & -3.444353 & 0.001414 & -0.599800 \\
\hline 11 & 1 & 0 & -3.489315 & 1. 070137 & -0.837297 \\
\hline 12 & 1 & 0 & -3.090361 & -0.537983 & -1.484758 \\
\hline 13 & 6 & 0 & -4.866422 & -0.437205 & -0.351871 \\
\hline 14 & 9 & 0 & -5.664421 & -0.192153 & -1.412603 \\
\hline 15 & 9 & 0 & -4.968311 & -1.759242 & -0.095211 \\
\hline 16 & 9 & 0 & -5.427480 & 0.195683 & 0.700896 \\
\hline 17 & 8 & 0 & -0.330840 & -1.172780 & -1.339900 \\
\hline 18 & 1 & 0 & -0.307861 & -0.446556 & -1.990267 \\
\hline 19 & 6 & 0 & 1. 561958 & -0.449585 & -0.055937 \\
\hline 20 & 6 & 0 & 4. 026768 & -0.893098 & 0.017012 \\
\hline 21 & 6 & 0 & 3. 708496 & 0.434620 & -0.337921 \\
\hline 22 & 6 & 0 & 4. 751961 & 1. 342270 & -0.595152 \\
\hline 23 & 6 & 0 & 5. 352653 & -1.329596 & 0.122068 \\
\hline 24 & 6 & 0 & 6. 364558 & -0.415887 & -0.138299 \\
\hline 25 & 6 & 0 & 6. 065949 & 0.913187 & -0.495062 \\
\hline 26 & 1 & 0 & 4. 520132 & 2. 368303 & -0.868942 \\
\hline 27 & 1 & 0 & 6. 877209 & 1. 607948 & -0.692656 \\
\hline 28 & 1 & 0 & 7. 401112 & -0.732287 & -0.064614 \\
\hline 29 & 1 & 0 & 5.584917 & -2.354324 & 0.398358 \\
\hline 30 & 6 & 0 & -0.884635 & 3. 114358 & 0.601423 \\
\hline 31 & 6 & 0 & -1.091178 & 2. 772347 & -0.708271 \\
\hline 32 & 6 & 0 & -0.641323 & 0.734180 & 0.063957 \\
\hline 33 & 1 & 0 & -1.383487 & 3. 470744 & -1.484834 \\
\hline 34 & 1 & 0 & -0.988322 & 4. 088260 & 1. 063020 \\
\hline 35 & 7 & 0 & -0.888501 & 1. 461983 & -1.022100 \\
\hline 36 & 16 & 0 & -0.397331 & 1.733698 & 1.511464 \\
\hline 37 & 6 & 0 & 2. 284099 & 0.654988 & -0.375593 \\
\hline 38 & 16 & 0 & 2. 583380 & -1.833391 & 0.299114 \\
\hline 39 & 1 & 0 & 1.840982 & 1. 613437 & -0.631994 \\
\hline \multicolumn{4}{|c|}{ Zero-point correction= } & 0.290645 & (Hartree/Particle) \\
\hline \multicolumn{4}{|c|}{ Thermal correction to Energy= } & 0.311405 & \\
\hline \multicolumn{4}{|c|}{ Thermal correction to Enthalpy= } & 0.312349 & \\
\hline \multicolumn{4}{|c|}{ Thermal correction to Gibbs Free Energy= } & 0.238698 & \\
\hline \multicolumn{4}{|c|}{ Sum of electronic and zero-point Energies= } & \multicolumn{2}{|c|}{-1881.947158} \\
\hline \multicolumn{4}{|c|}{ Sum of electronic and thermal Energies $=$} & \multicolumn{2}{|c|}{-1881.926399} \\
\hline \multicolumn{4}{|c|}{ Sum of electronic and thermal Enthalpies= } & \multicolumn{2}{|c|}{-1881.925455} \\
\hline \multirow{2}{*}{\multicolumn{4}{|c|}{ Sum of electronic and thermal Free Energies= }} & \multicolumn{2}{|c|}{-1881.999106} \\
\hline & & & & 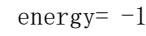 & \\
\hline
\end{tabular}

\section{1am-TS1b}

\begin{tabular}{rrrrrr} 
Center & Atomic & Atomic & \multicolumn{3}{c}{ Coordinates (Angstroms) } \\
Number & Number & Type & X & Y & $Z$ \\
-1 & 6 & 0 & -1.323121 & -1.418232 & 0.486703 \\
2 & 6 & 0 & -0.585468 & -2.675023 & 0.102830 \\
3 & 6 & 0 & 0.866881 & -2.350603 & -0.300176 \\
4 & 6 & 0 & 1.341209 & -1.185323 & 0.559159 \\
5 & 1 & 0 & -1.083017 & -3.186063 & -0.728992 \\
6 & 1 & 0 & -0.570684 & -3.362836 & 0.953946 \\
7 & 1 & 0 & 1.525823 & -3.214133 & -0.167512 \\
8 & 1 & 0 & 0.904384 & -2.058346 & -1.357947 \\
9 & 6 & 0 & -2.380012 & -0.843403 & -0.407064 \\
10 & 1 & 0 & -2.501976 & 0.235227 & -0.238198 \\
11 & 1 & 0 & -2.149683 & -1.004811 & -1.465539 \\
12 & 6 & 0 & -3.736009 & -1.457958 & -0.161316 \\
13 & 9 & 0 & -4.679070 & -0.951229 & -0.984620 \\
14 & 9 & 0 & -3.744003 & -2.795148 & -0.351557 \\
15 & 9 & 0 & -4.179766 & -1.252649 & 1.097111 \\
16 & 6 & 0 & 0.400056 & 0.011669 & 0.345336 \\
17 & 6 & 0 & -0.390032 & 2.194765 & 0.772951 \\
18 & 6 & 0 & -0.344503 & 2.220664 & -0.642166 \\
19 & 6 & 0 & -0.784787 & 3.323540 & -1.374589 \\
20 & 6 & 0 & -0.882306 & 3.326869 & 1.454242 \\
21 & 6 & 0 & -1.311398 & 4.427812 & 0.728854 \\
22 & 6 & 0 & -1.265510 & 4.428156 & -0.677279 \\
23 & 1 & 0 & -0.750437 & 3.324444 & -2.460616 \\
24 & 1 & 0 & -1.607876 & 5.299651 & -1.227706
\end{tabular}




$\begin{array}{rrrrrr}25 & 1 & 0 & -1.691761 & 5.301369 & 1.250725 \\ 26 & 1 & 0 & -0.922695 & 3.325730 & 2.540487 \\ 27 & 6 & 0 & 4.994169 & -0.271314 & -0.588736 \\ 28 & 6 & 0 & 4.724229 & 0.194859 & 0.661949 \\ 29 & 6 & 0 & 2.772674 & -0.763118 & 0.261662 \\ 30 & 8 & 0 & 1.272398 & -1.592778 & 1.910330 \\ 31 & 1 & 0 & 1.827700 & -0.982526 & 2.429498 \\ 32 & 1 & 0 & -1.489593 & -1.263265 & 1.549650 \\ 33 & 7 & 0 & 3.462806 & -0.088307 & 1.133221 \\ 34 & 16 & 0 & 3.622720 & -1.110907 & -1.207799 \\ 35 & 1 & 0 & 5.415284 & 0.751434 & 1.283795 \\ 36 & 1 & 0 & 5.905488 & -0.175899 & -1.165053 \\ 37 & 16 & 0 & 0.307241 & 0.726139 & -1.281685 \\ 38 & 6 & 0 & 0.097145 & 0.963188 & 1.310463 \\ 39 & 1 & 0 & 0.157076 & 0.758634 & 2.374672\end{array}$

Zero-point correction=

0.290323 (Hartree/Particle)

Thermal correction to Energy=

0.311105

Thermal correction to Enthalpy=

Thermal correction to Gibbs Free Energy=

Sum of electronic and zero-point Energies=

Sum of electronic and thermal Energies=

0. 312049

0.238391

$-1881.944721$

$-1881.923939$

$-1881.922995$

Sum of electronic and thermal Free Energies=

$-1881.996653$

M06 $-2 X / 6-311++G(d, p) / S M D / / M 06-2 X / 6-31+G(d) / S M D \quad$ energy $=-1882.57492295$

1am-INT2a

\begin{tabular}{|c|c|c|c|c|c|}
\hline \multirow{2}{*}{$\begin{array}{l}\text { Center } \\
\text { Number }\end{array}$} & \multirow{2}{*}{$\begin{array}{l}\text { Atomic } \\
\text { Number }\end{array}$} & \multirow{2}{*}{$\begin{array}{c}\text { Atomic } \\
\text { Type }\end{array}$} & \multicolumn{3}{|c|}{ Coordinates (Angstroms) } \\
\hline & & & X & $\mathrm{Y}$ & Z \\
\hline 1 & 6 & 0 & 1. 643043 & -0.278409 & 0.273019 \\
\hline 2 & 6 & 0 & 1. 571978 & -0.551287 & 1. 797285 \\
\hline 3 & 6 & 0 & 0.615671 & 0.505843 & 2. 394795 \\
\hline 4 & 6 & 0 & -0.046974 & 1. 233901 & 1. 204940 \\
\hline 5 & 1 & 0 & 0.940218 & -0.933975 & -0.253607 \\
\hline 6 & 1 & 0 & 2. 565953 & -0.452752 & 2. 243934 \\
\hline 7 & 1 & 0 & 1. 232765 & -1.573458 & 1. 988241 \\
\hline 8 & 1 & 0 & -0.133121 & 0.085387 & 3. 071529 \\
\hline 9 & 1 & 0 & 1. 184732 & 1. 251937 & 2. 956576 \\
\hline 10 & 6 & 0 & 3. 023832 & -0.448015 & -0.356323 \\
\hline 11 & 1 & 0 & 3. 048717 & -0.033242 & -1.371244 \\
\hline 12 & 1 & 0 & 3. 794309 & 0.052124 & 0.238120 \\
\hline 13 & 6 & 0 & 3. 424294 & -1.890363 & -0.485978 \\
\hline 14 & 9 & 0 & 4. 641131 & -2.021542 & -1.055214 \\
\hline 15 & 9 & 0 & 3. 489191 & -2.530433 & 0.701458 \\
\hline 16 & 9 & 0 & 2.567684 & -2.602023 & -1.250067 \\
\hline 17 & 8 & 0 & -0.394000 & 2. 569193 & 1. 484051 \\
\hline 18 & 1 & 0 & 0.439542 & 3. 047285 & 1. 647639 \\
\hline 19 & 6 & 0 & -1.316959 & 0.566317 & 0.730363 \\
\hline 20 & 6 & 0 & -3.150292 & -1.043976 & 0.157716 \\
\hline 21 & 6 & 0 & -3.446769 & 0.289108 & -0.195134 \\
\hline 22 & 6 & 0 & -4.681123 & 0.572894 & -0.807797 \\
\hline 23 & 6 & 0 & -4.047263 & -2.090446 & -0.085515 \\
\hline 24 & 6 & 0 & -5.257694 & -1.784428 & -0.691941 \\
\hline 25 & 6 & 0 & -5.572896 & -0.459749 & -1.051261 \\
\hline 26 & 1 & 0 & -4.923844 & 1. 595524 & -1.084975 \\
\hline 27 & 1 & 0 & -6.526982 & -0.248124 & -1.525521 \\
\hline 28 & 1 & 0 & -5.970739 & -2.579432 & -0.890583 \\
\hline 29 & 1 & 0 & -3.804988 & -3.112467 & 0.192256 \\
\hline 30 & 6 & 0 & 1. 735245 & 2. 791894 & -1.698718 \\
\hline 31 & 6 & 0 & 2. 431097 & 2. 930637 & -0.483256 \\
\hline 32 & 6 & 0 & 1. 108833 & 1. 166478 & 0.132563 \\
\hline 33 & 1 & 0 & 3. 198043 & 3. 687853 & -0.339873 \\
\hline 34 & 1 & 0 & 1. 873451 & 3. 389568 & -2.591275 \\
\hline 35 & 7 & 0 & 2. 120323 & 2. 120914 & 0.499779 \\
\hline 36 & 16 & 0 & 0.551494 & 1. 559777 & -1.601437 \\
\hline 37 & 6 & 0 & -2.374667 & 1. 188562 & 0.149314 \\
\hline 38 & 16 & 0 & -1.579152 & -1.162389 & 0.908124 \\
\hline 39 & 1 & 0 & -2.403972 & 2. 257821 & -0.029098 \\
\hline
\end{tabular}


Zero-point correction $=$

Thermal correction to Energy=

Thermal correction to Enthalpy=

Thermal correction to Gibbs Free Energy=

Sum of electronic and zero-point Energies=

Sum of electronic and thermal Energies=

Sum of electronic and thermal Enthalpies=

Sum of electronic and thermal Free Energies=
0. 293495 (Hartree/Particle)

0.314071

0.315015

0.241967

$-1881.985904$

$-1881.965328$

$-1881.964384$

$-1882.037432$

ergy $=-1882.61829397$

$1 \mathrm{am}-\mathrm{INT} 2 \mathrm{~b}$

\begin{tabular}{|c|c|c|c|c|c|}
\hline \multirow{2}{*}{$\begin{array}{l}\text { Center } \\
\text { Number }\end{array}$} & \multirow{2}{*}{$\begin{array}{l}\text { Atomic } \\
\text { Number }\end{array}$} & \multirow{2}{*}{$\begin{array}{c}\text { Atomic } \\
\text { Type }\end{array}$} & \multicolumn{3}{|c|}{ Coordinates (Angstroms) } \\
\hline & & & X & Y & Z \\
\hline 1 & 6 & 0 & -1.832826 & -0.559567 & 0.195220 \\
\hline 2 & 6 & 0 & -1.893839 & -1.911397 & -0.553225 \\
\hline 3 & 6 & 0 & -0.462288 & -2.497404 & -0.504851 \\
\hline 4 & 6 & 0 & 0.313200 & -1.597064 & 0.456040 \\
\hline 5 & 1 & 0 & -2.212004 & -1.759503 & -1.590704 \\
\hline 6 & 1 & 0 & -2.619958 & -2.580635 & -0.086203 \\
\hline 7 & 1 & 0 & -0.424665 & -3.532296 & -0.155382 \\
\hline 8 & 1 & 0 & -0.006152 & -2.465115 & -1.500282 \\
\hline 9 & 6 & 0 & -2.751833 & 0.536496 & -0.337643 \\
\hline 10 & 1 & 0 & -2.487570 & 1. 509111 & 0.094706 \\
\hline 11 & 1 & 0 & -2.695418 & 0.619933 & -1.427243 \\
\hline 12 & 6 & 0 & -4.194447 & 0.296923 & 0.008398 \\
\hline 13 & 9 & 0 & -4.993035 & 1. 276606 & -0.467045 \\
\hline 14 & 9 & 0 & -4.673813 & -0.860249 & -0.497597 \\
\hline 15 & 9 & 0 & -4.411531 & 0.243600 & 1. 340540 \\
\hline 16 & 6 & 0 & -0.333167 & -0.171628 & 0. 192276 \\
\hline 17 & 6 & 0 & 0.907224 & 1. 881318 & 0.688091 \\
\hline 18 & 6 & 0 & 1. 116686 & 1. 803919 & -0.719127 \\
\hline 19 & 6 & 0 & 1. 923876 & 2. 714081 & -1.385822 \\
\hline 20 & 6 & 0 & 1. 533826 & 2. 926906 & 1. 407854 \\
\hline 21 & 6 & 0 & 2. 338068 & 3. 836855 & 0.737862 \\
\hline 22 & 6 & 0 & 2. 536592 & 3. 734095 & -0.648344 \\
\hline 23 & 1 & 0 & 2. 078393 & 2. 642326 & -2.459042 \\
\hline 24 & 1 & 0 & 3. 171743 & 4. 452485 & -1.158149 \\
\hline 25 & 1 & 0 & 2. 822855 & 4. 636532 & 1. 290484 \\
\hline 26 & 1 & 0 & 1. 382332 & 3. 001020 & 2. 481500 \\
\hline 27 & 6 & 0 & 4. 177408 & -1.787042 & -0.231549 \\
\hline 28 & 6 & 0 & 3. 903955 & -1.354382 & 1. 031009 \\
\hline 29 & 6 & 0 & 1. 819002 & -1.613285 & 0.332441 \\
\hline 30 & 8 & 0 & -0.023929 & -1.988833 & 1. 774988 \\
\hline 31 & 1 & 0 & 0.639007 & -1.596687 & 2. 372647 \\
\hline 32 & 1 & 0 & -2.071653 & -0.727065 & 1. 251435 \\
\hline 33 & 7 & 0 & 2. 567510 & -1.264654 & 1. 341149 \\
\hline 34 & 16 & 0 & 2. 708014 & -2.092048 & -1.075035 \\
\hline 35 & 1 & 0 & 4. 645281 & -1.091057 & 1. 776083 \\
\hline 36 & 1 & 0 & 5. 140788 & -1.938151 & -0.701747 \\
\hline 37 & 16 & 0 & 0.211880 & 0.483936 & -1.456100 \\
\hline 38 & 6 & 0 & 0.075482 & 0.862316 & 1. 183674 \\
\hline 39 & 1 & 0 & -0.218529 & 0.775688 & 2. 225197 \\
\hline
\end{tabular}

Zero-point correction=

0.292380 (Hartree/Particle)

Thermal correction to Energy=

0.313227

Thermal correction to Enthalpy=

0.314171

0.239088

$-1881.986899$

$-1881.966052$

$-1881.965108$

$-1882.040191$

Sum of electronic and thermal Enthalpies=

Sum of electronic and thermal Free Energies=

M06 $-2 X / 6-311++G(d, p) / S M D / / M 06-2 X / 6-31+G(d) / S M D \quad$ energy $=-1882.61846023$

$1 \mathrm{am}-\mathrm{TS} 2 \mathrm{a}$

\begin{tabular}{cccccc} 
Center & Atomic & Atomic & \multicolumn{3}{c}{ Coordinates (Angstroms) } \\
Number & Number & Type & X & \multicolumn{2}{c}{ Y } \\
\hline---1 & 6 & 0 & -2.131059 & -0.239025 & -0.056028
\end{tabular}




$\begin{array}{rrrrrr}18 & 6 & 0 & 1.471126 & 1.540589 & -0.697653 \\ 19 & 6 & 0 & 2.586404 & 2.080313 & -1.336505 \\ 20 & 6 & 0 & 2.274872 & 2.312393 & 1.466708 \\ 21 & 6 & 0 & 3.378257 & 2.858658 & 0.829716 \\ 22 & 6 & 0 & 3.538083 & 2.742746 & -0.563367 \\ 23 & 1 & 0 & 2.712654 & 1.988178 & -2.412091 \\ 24 & 1 & 0 & 4.413404 & 3.170704 & -1.043366 \\ 25 & 1 & 0 & 4.134812 & 3.376777 & 1.412449 \\ 26 & 1 & 0 & 2.159658 & 2.390226 & 2.544917 \\ 27 & 6 & 0 & 3.901041 & -1.566041 & -0.335998 \\ 28 & 6 & 0 & 3.651687 & -1.352218 & 0.989389 \\ 29 & 6 & 0 & 1.566985 & -1.715508 & 0.333855 \\ 30 & 8 & 0 & -0.285218 & -1.960688 & 1.772346 \\ 31 & 1 & 0 & 0.444285 & -1.691507 & 2.365045 \\ 32 & 1 & 0 & -2.144059 & -0.448490 & 1.273526 \\ 33 & 7 & 0 & 2.338344 & -1.442504 & 1.365236 \\ 34 & 16 & 0 & 2.428973 & -1.908278 & -1.164768 \\ 35 & 1 & 0 & 4.406978 & -1.122168 & 1.732205 \\ 36 & 1 & 0 & 4.849968 & -1.550440 & -0.856407 \\ 37 & 16 & 0 & 0.138216 & 0.686659 & -1.452699 \\ 38 & 6 & 0 & 0.125239 & 0.961914 & 1.155063 \\ 39 & 1 & 0 & -0.189480 & 0.920182 & 2.193821 \\ ----------------------------------------\end{array}$

Zero-point correction $=$

Thermal correction to Energy=

0.291342 (Hartree/Particle)

Thermal correction to Enthalpy=

0.311999

Thermal correction to Gibbs Free Energy=

Sum of electronic and zero-point Energies=

Sum of electronic and thermal Energies=

0.312943

0.240038

$-1881.969073$

$-1881.948416$

$-1881.947471$

$-1882.020376$

Sum of electronic and thermal Free Energies=

rgy $=-1882.60026250$

1am-INT3a

\begin{tabular}{|c|c|c|c|c|c|}
\hline \multirow{2}{*}{$\begin{array}{l}\text { Center } \\
\text { Number }\end{array}$} & \multirow{2}{*}{$\begin{array}{l}\text { Atomic } \\
\text { Number }\end{array}$} & \multirow{2}{*}{$\begin{array}{c}\text { Atomic } \\
\text { Type }\end{array}$} & \multicolumn{3}{|c|}{ Coordinates (Angstroms) } \\
\hline & & & X & $\mathrm{Y}$ & Z \\
\hline 1 & 6 & 0 & 2. 203307 & -0.073057 & -0.149060 \\
\hline 2 & 6 & 0 & 2. 200319 & -0.707704 & 1. 250691 \\
\hline 3 & 6 & 0 & 0.857013 & -1.351004 & 1. 638097 \\
\hline 4 & 6 & 0 & -0.290840 & -0.404267 & 1. 795921 \\
\hline 5 & 1 & 0 & 1. 898762 & -0.833023 & -0.879394 \\
\hline 6 & 1 & 0 & 2. 512439 & 0.039566 & 1.991934 \\
\hline 7 & 1 & 0 & 2. 949118 & -1.503748 & 1. 281468 \\
\hline 8 & 1 & 0 & 0.610976 & -2.117956 & 0.895465 \\
\hline 9 & 1 & 0 & 1. 002592 & -1.872146 & 2.595421 \\
\hline 10 & 6 & 0 & 3. 581795 & 0.479391 & -0.562140 \\
\hline 11 & 1 & 0 & 3. 519295 & 0.911269 & -1.566140 \\
\hline 12 & 1 & 0 & 3. 906589 & 1. 265689 & 0.127326 \\
\hline 13 & 6 & 0 & 4. 681265 & -0.549330 & -0.612993 \\
\hline 14 & 9 & 0 & 5. 760288 & -0.080647 & -1.275229 \\
\hline 15 & 9 & 0 & 5. 126993 & -0.920788 & 0.606083 \\
\hline 16 & 9 & 0 & 4. 307526 & -1.682832 & -1.243186 \\
\hline 17 & 8 & 0 & -0.172029 & 0.588032 & 2. 722452 \\
\hline 18 & 1 & 0 & 0.633988 & 1. 109110 & 2. 528495 \\
\hline 19 & 6 & 0 & -1.514426 & -0.486882 & 1. 135470 \\
\hline 20 & 6 & 0 & -3.444639 & -1.101136 & -0.370042 \\
\hline 21 & 6 & 0 & -3.673973 & 0.082512 & 0.376897 \\
\hline 22 & 6 & 0 & -4.896970 & 0.763815 & 0.199573 \\
\hline 23 & 6 & 0 & -4.389765 & -1.603682 & -1.263094 \\
\hline 24 & 6 & 0 & -5.588696 & -0.911067 & -1.418483 \\
\hline 25 & 6 & 0 & -5.837226 & 0.265682 & -0.689571 \\
\hline 26 & 1 & 0 & -5.091437 & 1. 672879 & 0.762979 \\
\hline 27 & 1 & 0 & -6.778912 & 0.790129 & -0.826032 \\
\hline 28 & 1 & 0 & -6.337131 & -1.286346 & -2.110348 \\
\hline 29 & 1 & 0 & -4.197930 & -2.512674 & -1.826929 \\
\hline 30 & 6 & 0 & -0.463796 & 2. 725404 & -0.821710 \\
\hline 31 & 6 & 0 & 0.214147 & 2. 965110 & 0.334656 \\
\hline 32 & 6 & 0 & 1. 228591 & 1. 077475 & -0.226781 \\
\hline 33 & 1 & 0 & 0.045618 & 3. 809249 & 0.993213 \\
\hline
\end{tabular}




\begin{tabular}{|c|c|c|c|c|c|}
\hline 34 & 1 & 0 & -1.255253 & 3. 309141 & -1.274251 \\
\hline 35 & 7 & 0 & 1. 174214 & 2. 032589 & 0.660493 \\
\hline 36 & 16 & 0 & 0.114324 & 1.272483 & -1.542761 \\
\hline 37 & 6 & 0 & -2.578984 & 0.415085 & 1. 226436 \\
\hline 38 & 16 & 0 & -1.876058 & -1.795038 & -0.008317 \\
\hline 39 & 1 & 0 & -2.550234 & 1. 287692 & 1.868747 \\
\hline \multicolumn{4}{|c|}{ Zero-point correction $=$} & \multicolumn{2}{|c|}{ 0.291646 (Hartree/Particle) } \\
\hline \multicolumn{4}{|c|}{ Thermal correction to Energy= } & \multicolumn{2}{|c|}{0.313187} \\
\hline \multicolumn{4}{|c|}{ Thermal correction to Enthalpy $=$} & \multicolumn{2}{|l|}{0.314132} \\
\hline \multicolumn{4}{|c|}{ Thermal correction to Gibbs Free Energy= } & \multicolumn{2}{|l|}{0.237304} \\
\hline \multicolumn{4}{|c|}{ Sum of electronic and zero-point Energies= } & \multicolumn{2}{|c|}{-1881.991799} \\
\hline \multicolumn{4}{|c|}{ Sum of electronic and thermal Energies $=$} & \multicolumn{2}{|c|}{-1881.970257} \\
\hline \multicolumn{4}{|c|}{ Sum of electronic and thermal Enthalpies $=$} & \multicolumn{2}{|c|}{-1881.969313} \\
\hline \multicolumn{4}{|c|}{ Sum of electronic and thermal Free Energies= } & \multicolumn{2}{|c|}{-1882.046141} \\
\hline M06-2X/6 & 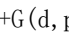 & & $-31+G(d) / S M$ & energy= & 82.623481 \\
\hline
\end{tabular}

\section{$1 \mathrm{am}-\mathrm{INT} 3 \mathrm{~b}$}

\begin{tabular}{|c|c|c|c|c|c|}
\hline \multirow{2}{*}{$\begin{array}{l}\text { Center } \\
\text { Number }\end{array}$} & \multirow{2}{*}{$\begin{array}{l}\text { Atomic } \\
\text { Number }\end{array}$} & \multirow{2}{*}{$\begin{array}{c}\text { Atomic } \\
\text { Type }\end{array}$} & \multicolumn{3}{|c|}{ Coordinates (Angstroms) } \\
\hline & & & $\mathrm{X}$ & Y & Z \\
\hline 1 & 6 & 0 & -1.766421 & 0.110634 & 0.070414 \\
\hline 2 & 6 & 0 & -2.023671 & -0.735208 & -1.192948 \\
\hline 3 & 6 & 0 & -0.852600 & -1.643121 & -1.618256 \\
\hline 4 & 6 & 0 & -0.225778 & -2.371123 & -0.482946 \\
\hline 5 & 1 & 0 & -2.269789 & -0.081048 & -2.039288 \\
\hline 6 & 1 & 0 & -2.904405 & -1.358456 & -1.011589 \\
\hline 7 & 1 & 0 & -1.229912 & -2.364470 & -2.356006 \\
\hline 8 & 1 & 0 & -0.082127 & -1.052087 & -2.123055 \\
\hline 9 & 6 & 0 & -2.844476 & 1. 190455 & 0.285015 \\
\hline 10 & 1 & 0 & -2.619382 & 1.752615 & 1. 196603 \\
\hline 11 & 1 & 0 & -2.874259 & 1. 898030 & -0.551231 \\
\hline 12 & 6 & 0 & -4.242716 & 0.656434 & 0.450600 \\
\hline 13 & 9 & 0 & -5.083815 & 1. 613497 & 0.899036 \\
\hline 14 & 9 & 0 & -4.783171 & 0.195550 & -0.697613 \\
\hline 15 & 9 & 0 & -4.312119 & -0.358369 & 1. 338172 \\
\hline 16 & 6 & 0 & -0.409789 & 0.765310 & 0.099897 \\
\hline 17 & 6 & 0 & 1.728085 & 1. 393506 & 0.818447 \\
\hline 18 & 6 & 0 & 1. 666227 & 2. 077446 & -0.413862 \\
\hline 19 & 6 & 0 & 2. 740033 & 2. 836573 & -0.889984 \\
\hline 20 & 6 & 0 & 2. 903299 & 1. 474133 & 1. 585661 \\
\hline 21 & 6 & 0 & 3. 972343 & 2. 222963 & 1. 116422 \\
\hline 22 & 6 & 0 & 3. 890724 & 2. 901348 & -0.114252 \\
\hline 23 & 1 & 0 & 2. 680226 & 3. 357095 & -1.841872 \\
\hline 24 & 1 & 0 & 4. 738651 & 3. 482893 & -0.464817 \\
\hline 25 & 1 & 0 & 4. 885080 & 2. 287060 & 1.702105 \\
\hline 26 & 1 & 0 & 2. 967082 & 0.944405 & 2. 533073 \\
\hline 27 & 6 & 0 & 3. 518416 & -1.956537 & 0.172483 \\
\hline 28 & 6 & 0 & 2. 906964 & -2.706099 & 1. 141967 \\
\hline 29 & 6 & 0 & 1. 127664 & -2.343655 & -0.135134 \\
\hline 30 & 8 & 0 & -1.065532 & -3.074335 & 0.299670 \\
\hline 31 & 1 & 0 & -0.542084 & -3.467650 & 1. 028360 \\
\hline 32 & 1 & 0 & -1.782824 & -0.557027 & 0.940757 \\
\hline 33 & 7 & 0 & 1. 575561 & -2.935737 & 0.980082 \\
\hline 34 & 16 & 0 & 2. 374643 & -1.500176 & -1.042506 \\
\hline 35 & 1 & 0 & 3. 417008 & -3.113498 & 2. 008380 \\
\hline 36 & 1 & 0 & 4. 557534 & -1.661178 & 0.106008 \\
\hline 37 & 16 & 0 & 0.134906 & 1. 801089 & -1.211636 \\
\hline 38 & 6 & 0 & 0.519003 & 0.654627 & 1. 082642 \\
\hline 39 & 1 & 0 & 0.369847 & 0.041303 & 1. 967563 \\
\hline
\end{tabular}

Zero-point correction=

0.293028 (Hartree/Particle)

Thermal correction to Energy=

0.314331

Thermal correction to Enthalpy=

0.315275

0. 239931

$-1881.994538$

$-1881.973236$

$-1881.972291$

$-1882.047636$

Sum of electronic and zero-point Energies=

Sum of electronic and thermal Energies=

Sum of electronic and thermal Free Energies=

energy $=-1882.62729466$ 
1an-INT1a

\begin{tabular}{|c|c|c|c|c|c|}
\hline \multirow{2}{*}{$\begin{array}{l}\text { Center } \\
\text { Number }\end{array}$} & \multirow{2}{*}{$\begin{array}{l}\text { Atomic } \\
\text { Number }\end{array}$} & \multirow{2}{*}{$\begin{array}{l}\text { Atomic } \\
\text { Type }\end{array}$} & \multicolumn{3}{|c|}{ Coordinates (Angstroms) } \\
\hline & & & X & $\mathrm{Y}$ & Z \\
\hline 1 & 6 & 0 & -1.881741 & -0.904310 & 0.780705 \\
\hline 2 & 6 & 0 & -0.922482 & -1.912225 & 1. 331734 \\
\hline 3 & 6 & 0 & 0.396347 & -2.069437 & 0.549394 \\
\hline 4 & 6 & 0 & 1. 426133 & -0.957430 & 0.819492 \\
\hline 5 & 1 & 0 & -1.401411 & -2.903678 & 1. 332375 \\
\hline 6 & 1 & 0 & -0.691783 & -1.675808 & 2. 375060 \\
\hline 7 & 1 & 0 & 0.866579 & -3.015507 & 0.837195 \\
\hline 8 & 1 & 0 & 0.197688 & -2.120567 & -0.527468 \\
\hline 9 & 6 & 0 & -2.562807 & -1.112045 & -0.534539 \\
\hline 10 & 1 & 0 & -2.702204 & -0.168509 & -1.075735 \\
\hline 11 & 1 & 0 & -2.002779 & -1.796624 & -1.182304 \\
\hline 12 & 6 & 0 & -3.941145 & -1.708276 & -0.375834 \\
\hline 13 & 9 & 0 & -4.555182 & -1.901612 & -1.563086 \\
\hline 14 & 9 & 0 & -3.919376 & -2.909220 & 0.241897 \\
\hline 15 & 9 & 0 & -4.762981 & -0.923321 & 0.353581 \\
\hline 16 & 6 & 0 & 0.850910 & 0.410838 & 0.500145 \\
\hline 17 & 6 & 0 & -0.069933 & 2. 543299 & 0.735271 \\
\hline 18 & 6 & 0 & -0.191402 & 2. 366516 & -0.659435 \\
\hline 19 & 6 & 0 & -0.756110 & 3. 348105 & -1.480779 \\
\hline 20 & 6 & 0 & -0.534733 & 3. 737336 & 1. 313775 \\
\hline 21 & 6 & 0 & -1.098762 & 4. 711981 & 0.504145 \\
\hline 22 & 6 & 0 & -1.208651 & 4. 518471 & -0.885290 \\
\hline 23 & 1 & 0 & -0.840901 & 3. 199741 & -2.553788 \\
\hline 24 & 1 & 0 & -1.653558 & 5. 293691 & -1.502618 \\
\hline 25 & 1 & 0 & -1.461305 & 5. 636029 & 0.945246 \\
\hline 26 & 1 & 0 & -0.449768 & 3. 886422 & 2. 387160 \\
\hline 27 & 16 & 0 & 0.457182 & 0.821833 & -1.159004 \\
\hline 28 & 6 & 0 & 4. 373062 & -2.174148 & -1.282680 \\
\hline 29 & 6 & 0 & 4. 976970 & -0.994633 & -0.959617 \\
\hline 30 & 6 & 0 & 3. 061202 & -2.291057 & -0.722765 \\
\hline 31 & 6 & 0 & 2. 690578 & -1.196558 & 0.008744 \\
\hline 32 & 1 & 0 & 2. 427847 & -3.159768 & -0.865494 \\
\hline 33 & 8 & 0 & 1. 714026 & -1.036941 & 2. 212517 \\
\hline 34 & 1 & 0 & 2. 405858 & -0.390360 & 2. 435834 \\
\hline 35 & 1 & 0 & -2.200362 & -0.064506 & 1. 389025 \\
\hline 36 & 6 & 0 & 0.534590 & 1. 398105 & 1. 374051 \\
\hline 37 & 1 & 0 & 0.683324 & 1. 321664 & 2. 446257 \\
\hline 38 & 1 & 0 & 5. 959944 & -0.641267 & -1.246669 \\
\hline 39 & 1 & 0 & 4. 839486 & -2.936846 & -1.896833 \\
\hline 40 & 16 & 0 & 3. 955119 & 0.000143 & 0.009753 \\
\hline
\end{tabular}

Zero-point correction=

0. 301685 (Hartree/Particle)

Thermal correction to Energy=

0.323911

Thermal correction to Enthalpy=

0.324855

Thermal correction to Gibbs Free Energy=

Sum of electronic and zero-point Energies=

Sum of electronic and thermal Energies=

0.246035

$-1865.900677$

$-1865.878451$

$-1865.877506$

$-1865.956326$

Sum of electronic and thermal Free Energies

M06 $-2 X / 6-311++G(d, p) / S M D / / M 06-2 X / 6-31+G(d) / S M D \quad$ energy $=-1866.53838340$

1 an-INT1b

\begin{tabular}{cccccr} 
Center & $\begin{array}{c}\text { Atomic } \\
\text { Number }\end{array}$ & Number & Atomic & \multicolumn{3}{c}{ Coordinates (Angstroms) } \\
- & Type & X & Y & $Z$ \\
1 & 6 & 0 & 2.774474 & -0.818260 & -1.112931 \\
2 & 6 & 0 & 1.757773 & -1.895154 & -0.936154 \\
3 & 6 & 0 & 0.284031 & -1.443069 & -1.021533 \\
4 & 6 & 0 & -0.180570 & -0.526512 & 0.127895 \\
5 & 1 & 0 & 2.923322 & -0.386643 & -2.098961 \\
6 & 1 & 0 & 1.912925 & -2.402969 & 0.022258 \\
7 & 1 & 0 & 1.887661 & -2.650078 & -1.723599 \\
8 & 1 & 0 & 0.093109 & -0.942942 & -1.977660 \\
9 & 1 & 0 & -0.351659 & -2.334873 & -0.990315
\end{tabular}




\begin{tabular}{|c|c|c|c|c|c|}
\hline 10 & 6 & 0 & 3. 516880 & -0.216380 & 0.035374 \\
\hline 11 & 1 & 0 & 3. 503453 & 0.882760 & 0.007672 \\
\hline 12 & 1 & 0 & 3. 099336 & -0.539298 & 0.995399 \\
\hline 13 & 6 & 0 & 4. 971339 & -0.617261 & 0.042735 \\
\hline 14 & 9 & 0 & 5.647819 & -0.072590 & 1. 076897 \\
\hline 15 & 9 & 0 & 5. 139032 & -1.954109 & 0.139860 \\
\hline 16 & 9 & 0 & 5. 619626 & -0.235683 & -1.079125 \\
\hline 17 & 8 & 0 & 0.199937 & -1.182673 & 1. 332216 \\
\hline 18 & 1 & 0 & -0.345135 & -0.847470 & 2. 065690 \\
\hline 19 & 6 & 0 & -1.699146 & -0.407555 & 0.089853 \\
\hline 20 & 6 & 0 & -3.958058 & -0.899238 & 0.431270 \\
\hline 21 & 6 & 0 & -4.065214 & 0.214489 & -0.427879 \\
\hline 22 & 6 & 0 & -5.308234 & 0.715121 & -0.832020 \\
\hline 23 & 6 & 0 & -5.131441 & -1.521236 & 0.894181 \\
\hline 24 & 6 & 0 & -6.364265 & -1.029483 & 0.494406 \\
\hline 25 & 6 & 0 & -6.452188 & 0.082596 & -0.364458 \\
\hline 26 & 1 & 0 & -5.378220 & 1. 574030 & -1.493710 \\
\hline 27 & 1 & 0 & -7.427860 & 0.452186 & -0.666762 \\
\hline 28 & 1 & 0 & -7.274839 & -1.505573 & 0.846671 \\
\hline 29 & 1 & 0 & -5.062587 & -2.379623 & 1.557428 \\
\hline 30 & 16 & 0 & -2.489965 & 0.839067 & -0.856443 \\
\hline 31 & 6 & 0 & 1. 256598 & 2. 905427 & -0.720657 \\
\hline 32 & 6 & 0 & 1. 362232 & 3. 095771 & 0.627443 \\
\hline 33 & 6 & 0 & 0.730495 & 1. 617652 & -1.052099 \\
\hline 34 & 6 & 0 & 0.454858 & 0.853250 & 0.049193 \\
\hline 35 & 1 & 0 & 0.575593 & 1. 279308 & -2.071630 \\
\hline 36 & 6 & 0 & -2.582596 & -1.234776 & 0.705799 \\
\hline 37 & 1 & 0 & -2.286341 & -2.074259 & 1. 327269 \\
\hline 38 & 1 & 0 & 1. 739771 & 3. 961453 & 1. 157948 \\
\hline 39 & 1 & 0 & 1. 550991 & 3. 647156 & -1.455464 \\
\hline 40 & 16 & 0 & 0.826271 & 1. 713269 & 1.507621 \\
\hline \multicolumn{4}{|c|}{ Zero-point correction $=$} & \multicolumn{2}{|c|}{ 0.302003 (Hartree/Particle) } \\
\hline \multirow{2}{*}{\multicolumn{4}{|c|}{$\begin{array}{l}\text { Thermal correction to Energy= } \\
\text { Thermal correction to Enthalpy= }\end{array}$}} & \multicolumn{2}{|c|}{0.323920} \\
\hline & & & & \multicolumn{2}{|l|}{0.324865} \\
\hline \multicolumn{4}{|c|}{ Thermal correction to Gibbs Free Energy= } & \multicolumn{2}{|c|}{0.246998} \\
\hline \multicolumn{4}{|c|}{ Sum of electronic and zero-point Energies= } & \multicolumn{2}{|c|}{-1865.899635} \\
\hline \multicolumn{4}{|c|}{ Sum of electronic and thermal Energies= } & \multicolumn{2}{|c|}{-1865.877717} \\
\hline \multicolumn{4}{|c|}{ Sum of electronic and thermal Enthalpies= } & \multicolumn{2}{|c|}{-1865.876773} \\
\hline \multicolumn{4}{|c|}{ Sum of electronic and thermal Free Energies= } & \multirow{2}{*}{\multicolumn{2}{|c|}{-1865.954640}} \\
\hline \multicolumn{4}{|c|}{$106-2 \mathrm{X} / 6-311++\mathrm{G}(\mathrm{d}, \mathrm{p}) / \mathrm{SMD} / / \mathrm{M} 06-2 \mathrm{X} / 6-31+\mathrm{G}(\mathrm{d}) / \mathrm{SMD}$} & & \\
\hline
\end{tabular}

1an-TS1a

\begin{tabular}{|c|c|c|c|c|c|}
\hline \multirow{2}{*}{$\begin{array}{l}\text { Center } \\
\text { Number }\end{array}$} & \multirow{2}{*}{$\begin{array}{l}\text { Atomic } \\
\text { Number }\end{array}$} & \multirow{2}{*}{$\begin{array}{c}\text { Atomic } \\
\text { Type }\end{array}$} & \multicolumn{3}{|c|}{ Coordinates (Angstroms) } \\
\hline & & & $\mathrm{X}$ & Y & Z \\
\hline 1 & 6 & 0 & -1.433475 & -1.405197 & 0.410560 \\
\hline 2 & 6 & 0 & -0.816435 & -2.693774 & -0.065854 \\
\hline 3 & 6 & 0 & 0.645805 & -2.477009 & -0.508485 \\
\hline 4 & 6 & 0 & 1.252978 & -1.381705 & 0.364685 \\
\hline 5 & 1 & 0 & -1.385019 & -3.115054 & -0.902986 \\
\hline 6 & 1 & 0 & -0.834715 & -3.425261 & 0.748286 \\
\hline 7 & 1 & 0 & 1.224743 & -3.400306 & -0.418968 \\
\hline 8 & 1 & 0 & 0.688315 & -2.158911 & -1.556743 \\
\hline 9 & 6 & 0 & -2.487044 & -0.704589 & -0.392942 \\
\hline 10 & 1 & 0 & -2.493818 & 0.374116 & -0.184098 \\
\hline 11 & 1 & 0 & -2.344118 & -0.848009 & -1.469262 \\
\hline 12 & 6 & 0 & -3.875881 & -1.197640 & -0.069612 \\
\hline 13 & 9 & 0 & -4.822994 & -0.560018 & -0.791078 \\
\hline 14 & 9 & 0 & -4.031582 & -2.516337 & -0.317741 \\
\hline 15 & 9 & 0 & -4.202027 & -1.016575 & 1. 228041 \\
\hline 16 & 6 & 0 & 0.395376 & -0.116063 & 0.227721 \\
\hline 17 & 6 & 0 & -0.179015 & 2. 122207 & 0.717099 \\
\hline 18 & 6 & 0 & -0.184916 & 2. 167870 & -0.698073 \\
\hline 19 & 6 & 0 & -0.545972 & 3. 321135 & -1.396209 \\
\hline 20 & 6 & 0 & -0.532737 & 3. 283423 & 1. 433566 \\
\hline 21 & 6 & 0 & -0.881680 & 4. 433452 & 0.741655 \\
\hline 22 & 6 & 0 & -0.890589 & 4. 454140 & -0.664925 \\
\hline 23 & 1 & 0 & -0.552869 & 3. 337801 & -2.482698 \\
\hline 24 & 1 & 0 & -1.168003 & 5. 364132 & -1.189142 \\
\hline
\end{tabular}




$\begin{array}{rrrrrr}25 & 1 & 0 & -1.154771 & 5.330102 & 1.290935 \\ 26 & 1 & 0 & -0.531253 & 3.266419 & 2.520475 \\ 27 & 16 & 0 & 0.293672 & 0.629737 & -1.383323 \\ 28 & 6 & 0 & 4.881211 & -1.311854 & -0.794877 \\ 29 & 6 & 0 & 4.989902 & -0.131841 & -0.118958 \\ 30 & 6 & 0 & 3.562950 & -1.863012 & -0.724516 \\ 31 & 6 & 0 & 2.694292 & -1.089336 & -0.004970 \\ 32 & 1 & 0 & 3.281247 & -2.799664 & -1.193451 \\ 33 & 8 & 0 & 1.172938 & -1.858895 & 1.705314 \\ 34 & 1 & 0 & 1.684745 & -1.269345 & 2.285286 \\ 35 & 1 & 0 & -1.523979 & -1.283646 & 1.486789 \\ 36 & 6 & 0 & 0.209823 & 0.839728 & 1.217797 \\ 37 & 1 & 0 & 0.284018 & 0.616349 & 2.277947 \\ 38 & 1 & 0 & 5.861833 & 0.501487 & -0.007946 \\ 39 & 1 & 0 & 5.705080 & -1.777702 & -1.324651 \\ 40 & 16 & 0 & 3.491136 & 0.338726 & 0.593494 \\ -------------------------------------------------------------\end{array}$

Zero-point correction=

Thermal correction to Energy=

0. 301705 (Hartree/Particle)

Thermal correction to Enthalpy=

0.32283

Thermal correction to Gibbs Free Energy=

Sum of electronic and zero-point Energies=

Sum of electronic and thermal Energies=

Sum of electronic and thermal Enthalpies=

Sum of electronic and thermal Free Energies=

0.323775

0. 249207

$-1865.885946$

$-1865.864820$

$-1865.863876$

$-1865.938445$

M06 $-2 \mathrm{X} / 6-311++\mathrm{G}(\mathrm{d}, \mathrm{p}) / \mathrm{SMD} / / \mathrm{M} 06-2 \mathrm{X} / 6-31+\mathrm{G}(\mathrm{d}) / \mathrm{SMD} \quad$ energy $=-1866.52357888$

\section{1an-TS1b}

\begin{tabular}{|c|c|c|c|c|c|}
\hline \multirow{2}{*}{$\begin{array}{l}\text { Center } \\
\text { Number }\end{array}$} & \multirow{2}{*}{$\begin{array}{l}\text { Atomic } \\
\text { Number }\end{array}$} & \multirow{2}{*}{$\begin{array}{c}\text { Atomic } \\
\text { Type }\end{array}$} & \multicolumn{3}{|c|}{ Coordinates (Angstroms) } \\
\hline & & & $\mathrm{x}$ & Y & Z \\
\hline 1 & 6 & 0 & 2. 520475 & -0.227826 & -0.719227 \\
\hline 2 & 6 & 0 & 1.909143 & -1.562182 & -1.050838 \\
\hline 3 & 6 & 0 & 0.388915 & -1.410818 & -1.188625 \\
\hline 4 & 6 & 0 & -0.089806 & -0.572572 & 0.001321 \\
\hline 5 & 1 & 0 & 2. 717384 & 0.430556 & -1.563243 \\
\hline 6 & 1 & 0 & 2. 124716 & -2.281382 & -0.254797 \\
\hline 7 & 1 & 0 & 2. 325584 & -1.964642 & -1.983695 \\
\hline 8 & 1 & 0 & 0.126421 & -0.891337 & -2.117513 \\
\hline 9 & 1 & 0 & -0.121624 & -2.379084 & -1.185074 \\
\hline 10 & 6 & 0 & 3. 508662 & -0.115305 & 0.404090 \\
\hline 11 & 1 & 0 & 3. 677266 & 0.930608 & 0.684977 \\
\hline 12 & 1 & 0 & 3. 168357 & -0.662212 & 1. 291196 \\
\hline 13 & 6 & 0 & 4. 863689 & -0.672633 & 0.042404 \\
\hline 14 & 9 & 0 & 5.752133 & -0.548657 & 1. 053114 \\
\hline 15 & 9 & 0 & 4. 825467 & -1.986619 & -0.267484 \\
\hline 16 & 9 & 0 & 5. 413029 & -0.049657 & -1.022712 \\
\hline 17 & 8 & 0 & 0.310338 & -1.285612 & 1. 168223 \\
\hline 18 & 1 & 0 & -0.213300 & -0.969664 & 1. 925123 \\
\hline 19 & 6 & 0 & -1.603787 & -0.439005 & 0.007545 \\
\hline 20 & 6 & 0 & -3.846304 & -1.017023 & 0. 342799 \\
\hline 21 & 6 & 0 & -3.995598 & 0.218324 & -0.320586 \\
\hline 22 & 6 & 0 & -5.257711 & 0.755798 & -0.599808 \\
\hline 23 & 6 & 0 & -4.995761 & -1.729487 & 0.730271 \\
\hline 24 & 6 & 0 & -6.247025 & -1.201694 & 0.453153 \\
\hline 25 & 6 & 0 & -6.377398 & 0.034968 & -0.207751 \\
\hline 26 & 1 & 0 & -5.360373 & 1. 709110 & -1.110981 \\
\hline 27 & 1 & 0 & -7.367337 & 0.431207 & -0.415184 \\
\hline 28 & 1 & 0 & -7.139623 & -1.746392 & 0.747388 \\
\hline 29 & 1 & 0 & -4.893735 & -2.683753 & 1. 240645 \\
\hline 30 & 16 & 0 & -2.444380 & 0.926804 & -0.704036 \\
\hline 31 & 6 & 0 & 0.833426 & 3. 048517 & -0.637584 \\
\hline 32 & 6 & 0 & 1. 090015 & 3. 126110 & 0.708861 \\
\hline 33 & 6 & 0 & 0.511805 & 1. 739901 & -1.065012 \\
\hline 34 & 6 & 0 & 0.634690 & 0.774836 & -0.050805 \\
\hline 35 & 1 & 0 & 0.280490 & 1. 479017 & -2.093088 \\
\hline 36 & 6 & 0 & -2.458693 & -1.365767 & 0.515292 \\
\hline 37 & 1 & 0 & -2.127849 & -2.284087 & 0.990508 \\
\hline 38 & 1 & 0 & 1. 379192 & 3. 995934 & 1. 286167 \\
\hline 39 & 1 & 0 & 0.875249 & 3. 907649 & -1.299369 \\
\hline
\end{tabular}




\begin{tabular}{|c|c|c|c|c|c|}
\hline 40 & 16 & 0 & 0.933030 & 1. 587129 & 1. 482279 \\
\hline \multicolumn{4}{|c|}{ Zero-point correction $=$} & \multicolumn{2}{|c|}{ 0. 302115 (Hartree/Particle) } \\
\hline \multicolumn{4}{|c|}{ Thermal correction to Energy= } & \multicolumn{2}{|c|}{0.322950} \\
\hline \multicolumn{4}{|c|}{ Thermal correction to Enthalpy $=$} & \multicolumn{2}{|l|}{0.323895} \\
\hline \multicolumn{4}{|c|}{ Thermal correction to Gibbs Free Energy= } & \multicolumn{2}{|l|}{0.250120} \\
\hline \multicolumn{4}{|c|}{ Sum of electronic and zero-point Energies= } & \multicolumn{2}{|c|}{-1865.884302} \\
\hline \multicolumn{4}{|c|}{ Sum of electronic and thermal Energies $=$} & \multicolumn{2}{|c|}{-1865.863467} \\
\hline \multicolumn{4}{|c|}{ Sum of electronic and thermal Enthalpies= } & \multicolumn{2}{|c|}{-1865.862522} \\
\hline \multicolumn{4}{|c|}{ Sum of electronic and thermal Free Energies= } & \multicolumn{2}{|c|}{-1865.936297} \\
\hline M06-2X/6- & $1++G(d, p$ & D//M06-2X & $1+G(d) / S M D$ & energy $=-18$ & 66. 52173813 \\
\hline 1an-INT2a & & & & & \\
\hline Center & Atomic & Atomic & Coorc & dinates (Ang & troms) \\
\hline Number & Number & Type & X & Y & Z \\
\hline 1 & 6 & 0 & -1.671743 & -0.810732 & 0.158879 \\
\hline 2 & 6 & 0 & -1.667561 & -2.143065 & -0.622924 \\
\hline 3 & 6 & 0 & -0.193886 & -2.606184 & -0.676520 \\
\hline 4 & 6 & 0 & 0.565040 & -1.689270 & 0.288661 \\
\hline 5 & 1 & 0 & -2.059831 & -1.994762 & -1.635125 \\
\hline 6 & 1 & 0 & -2.307015 & -2.880317 & -0.131699 \\
\hline 7 & 1 & 0 & -0.057761 & -3.651182 & -0.386554 \\
\hline 8 & 1 & 0 & 0.210874 & -2.484862 & -1.685954 \\
\hline 9 & 6 & 0 & -2.675002 & 0.236161 & -0.317664 \\
\hline 10 & 1 & 0 & -2.453097 & 1. 218386 & 0.117085 \\
\hline 11 & 1 & 0 & -2.669112 & 0.334810 & -1.407268 \\
\hline 12 & 6 & 0 & -4.084897 & -0.091642 & 0.084740 \\
\hline 13 & 9 & 0 & -4.961036 & 0.834410 & -0.360944 \\
\hline 14 & 9 & 0 & -4.510263 & -1.278848 & -0.399332 \\
\hline 15 & 9 & 0 & -4.246492 & -0.152388 & 1. 424621 \\
\hline 16 & 6 & 0 & -0.195255 & -0.321130 & 0.121866 \\
\hline 17 & 6 & 0 & 0.578301 & 1.951183 & 0.643782 \\
\hline 18 & 6 & 0 & 0.710270 & 1. 971220 & -0.774412 \\
\hline 19 & 6 & 0 & 1. 166971 & 3. 097367 & -1.444735 \\
\hline 20 & 6 & 0 & 0.925495 & 3. 113586 & 1. 373586 \\
\hline 21 & 6 & 0 & 1. 381108 & 4. 236734 & 0.700127 \\
\hline 22 & 6 & 0 & 1. 502700 & 4. 233097 & -0.699207 \\
\hline 23 & 1 & 0 & 1. 263893 & 3. 101528 & -2.527003 \\
\hline 24 & 1 & 0 & 1. 861122 & 5. 120456 & -1.212598 \\
\hline 25 & 1 & 0 & 1. 647433 & 5. 129352 & 1. 258967 \\
\hline 26 & 1 & 0 & 0.831303 & 3. 111760 & 2. 456361 \\
\hline 27 & 16 & 0 & 0.244029 & 0.443761 & -1.513455 \\
\hline 28 & 6 & 0 & 4. 208944 & -2.276504 & -0.600100 \\
\hline 29 & 6 & 0 & 4. 500843 & -1.196035 & 0.179513 \\
\hline 30 & 6 & 0 & 2. 804479 & -2.538052 & -0.666186 \\
\hline 31 & 6 & 0 & 2. 052987 & -1.647425 & 0.050234 \\
\hline 32 & 1 & 0 & 2. 373426 & -3.361775 & -1.225735 \\
\hline 33 & 8 & 0 & 0.289564 & -2.190228 & 1. 598853 \\
\hline 34 & 1 & 0 & 0.801681 & -1.679860 & 2. 249839 \\
\hline 35 & 1 & 0 & -1.866757 & -1.019942 & 1. 216683 \\
\hline 36 & 6 & 0 & 0.111128 & 0.723557 & 1. 142086 \\
\hline 37 & 1 & 0 & -0.093108 & 0.543606 & 2. 193842 \\
\hline 38 & 1 & 0 & 5. 470919 & -0.766247 & 0.398521 \\
\hline 39 & 1 & 0 & 4. 960977 & -2.868287 & -1.110758 \\
\hline 40 & 16 & 0 & 3. 075291 & -0.469799 & 0.826649 \\
\hline Zero-poi & correct & & & 0.304138 & (Hartree/Particle) \\
\hline Thermal & rrection & Energy= & & 0.325083 & \\
\hline Thermal & rrection & Enthalpy= & & 0.326028 & \\
\hline Thermal & rrection & Gibbs Fre & ergy $=$ & 0.252346 & \\
\hline Sum of $\mathrm{e}$ & ctronic & zero-poir & ergies= & -1865 . & 27079 \\
\hline Sum of $\mathrm{e}$ & ctronic & thermal $\mathrm{E}$ & ies $=$ & -1865 & 06133 \\
\hline Sum of $\mathrm{e}$ & ctronic & thermal $\mathrm{E}$ & 1 pies $=$ & -1865. & 05189 \\
\hline Sum of e & ctronic & thermal $\mathrm{F}$ & Energies= & -1865 & 978870 \\
\hline
\end{tabular}

lan-INT2b

Center Atomic Atomic Coordinates (Angstroms) 


\begin{tabular}{|c|c|c|c|c|c|}
\hline Number & Number & Type & X & Y & Z \\
\hline 1 & 6 & 0 & -2.321535 & -0.324016 & 0.377378 \\
\hline 2 & 6 & 0 & -2.176164 & -1.733430 & -0.239966 \\
\hline 3 & 6 & 0 & -0.674965 & -2.012390 & -0.215599 \\
\hline 4 & 6 & 0 & -0.072506 & -0.672717 & -0.632550 \\
\hline 5 & 1 & 0 & -2.515296 & -0.427313 & 1. 450513 \\
\hline 6 & 1 & 0 & -2.534466 & -1.734173 & -1.273530 \\
\hline 7 & 1 & 0 & -2.748736 & -2.478990 & 0. 317841 \\
\hline 8 & 1 & 0 & -0.353922 & -2.267081 & 0.801805 \\
\hline 9 & 1 & 0 & -0.361901 & -2.815134 & -0.889597 \\
\hline 10 & 6 & 0 & -3.461544 & 0.502300 & -0.217540 \\
\hline 11 & 1 & 0 & -3.495869 & 1. 499745 & 0.231002 \\
\hline 12 & 1 & 0 & -3.354683 & 0.616419 & -1.302524 \\
\hline 13 & 6 & 0 & -4.817171 & -0.104184 & 0.027383 \\
\hline 14 & 9 & 0 & -5.806544 & 0.719123 & -0.382887 \\
\hline 15 & 9 & 0 & -5.013055 & -1.273774 & -0.617398 \\
\hline 16 & 9 & 0 & -5.046553 & -0.352106 & 1. 335397 \\
\hline 17 & 8 & 0 & -0.429494 & -0.503971 & -1.995325 \\
\hline 18 & 1 & 0 & -0.113933 & 0.364183 & -2.300369 \\
\hline 19 & 6 & 0 & 1. 422880 & -0.544045 & -0.454977 \\
\hline 20 & 6 & 0 & 3. 622590 & 0.231233 & -0.657756 \\
\hline 21 & 6 & 0 & 3. 794730 & -0.757890 & 0.332862 \\
\hline 22 & 6 & 0 & 5. 045974 & -1.021115 & 0.902501 \\
\hline 23 & 6 & 0 & 4. 739577 & 0.973280 & -1.082534 \\
\hline 24 & 6 & 0 & 5. 979963 & 0.717932 & -0.519002 \\
\hline 25 & 6 & 0 & 6. 132523 & -0.274225 & 0.468395 \\
\hline 26 & 1 & 0 & 5. 164711 & -1.785514 & 1. 665362 \\
\hline 27 & 1 & 0 & 7. 112981 & -0.458575 & 0.898227 \\
\hline 28 & 1 & 0 & 6.846305 & 1. 288799 & -0.840698 \\
\hline 29 & 1 & 0 & 4. 621763 & 1. 739031 & -1.844981 \\
\hline 30 & 16 & 0 & 2. 282633 & -1.548172 & 0.700362 \\
\hline 31 & 6 & 0 & 0.269846 & 1. 802843 & 1. 843951 \\
\hline 32 & 6 & 0 & 0.086833 & 2. 706007 & 0.799197 \\
\hline 33 & 6 & 0 & -0.274402 & 0.557955 & 1. 616405 \\
\hline 34 & 6 & 0 & -0.896685 & 0.349948 & 0.262987 \\
\hline 35 & 1 & 0 & -0.262709 & -0.258606 & 2. 331944 \\
\hline 36 & 6 & 0 & 2. 250426 & 0.325242 & -1.091514 \\
\hline 37 & 1 & 0 & 1. 928882 & 1. 031119 & -1.851845 \\
\hline 38 & 1 & 0 & 0.405135 & 3. 740458 & 0.757629 \\
\hline 39 & 1 & 0 & 0.785683 & 2. 075670 & 2. 759786 \\
\hline 40 & 16 & 0 & -0.832311 & 2. 031058 & -0.517969 \\
\hline
\end{tabular}

Zero-point correction=

0. 304406 (Hartree/Particle)

Thermal correction to Energy=

0.325157

Thermal correction to Enthalpy=

0.326102

0.251958

$-1865.920886$

$-1865.900135$

$-1865.899191$

$-1865.973334$

Sum of electronic and thermal Energies=

Sum of electronic and thermal Enthalpies=

Sum of electronic and thermal Free Energies=

M06 $-2 X / 6-311++G(d, p) / S M D / / M 06-2 X / 6-31+G(d) / S M D \quad$ energy $=-1866.56089691$

1an-TS2a

\begin{tabular}{|c|c|c|c|c|c|}
\hline \multirow{2}{*}{$\begin{array}{l}\text { Center } \\
\text { Number }\end{array}$} & \multirow{2}{*}{$\begin{array}{l}\text { Atomic } \\
\text { Number }\end{array}$} & \multirow{2}{*}{$\begin{array}{l}\text { Atomic } \\
\text { Type }\end{array}$} & \multicolumn{3}{|c|}{ Coordinates (Angstroms) } \\
\hline & & & X & Y & Z \\
\hline 1 & 6 & 0 & -1.964523 & -0.291737 & 0.138706 \\
\hline 2 & 6 & 0 & -2.147100 & -1.497137 & -0.804778 \\
\hline 3 & 6 & 0 & -0.817332 & -2.281014 & -0.914063 \\
\hline 4 & 6 & 0 & 0.156501 & -1.807691 & 0.127778 \\
\hline 5 & 1 & 0 & -2.451487 & -1.149183 & -1.799307 \\
\hline 6 & 1 & 0 & -2.952038 & -2.136920 & -0.434312 \\
\hline 7 & 1 & 0 & -0.979671 & -3.358191 & -0.781852 \\
\hline 8 & 1 & 0 & -0.373755 & -2.144952 & -1.903148 \\
\hline 9 & 6 & 0 & -2.936200 & 0.864933 & -0.130884 \\
\hline 10 & 1 & 0 & -2.689645 & 1. 719591 & 0.507954 \\
\hline 11 & 1 & 0 & -2.891495 & 1. 193575 & -1.174677 \\
\hline 12 & 6 & 0 & -4.372460 & 0.521244 & 0. 155932 \\
\hline 13 & 9 & 0 & -5.174337 & 1. 601314 & 0.034050 \\
\hline
\end{tabular}




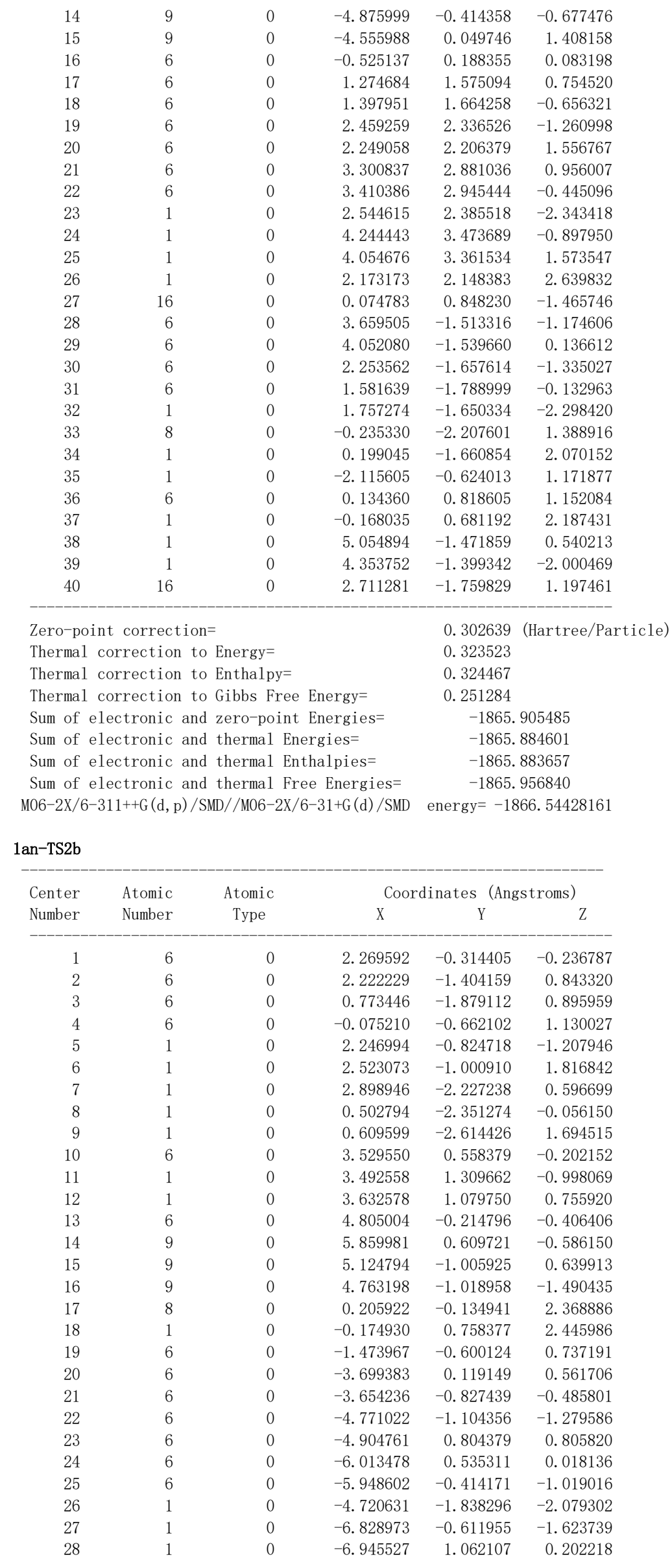




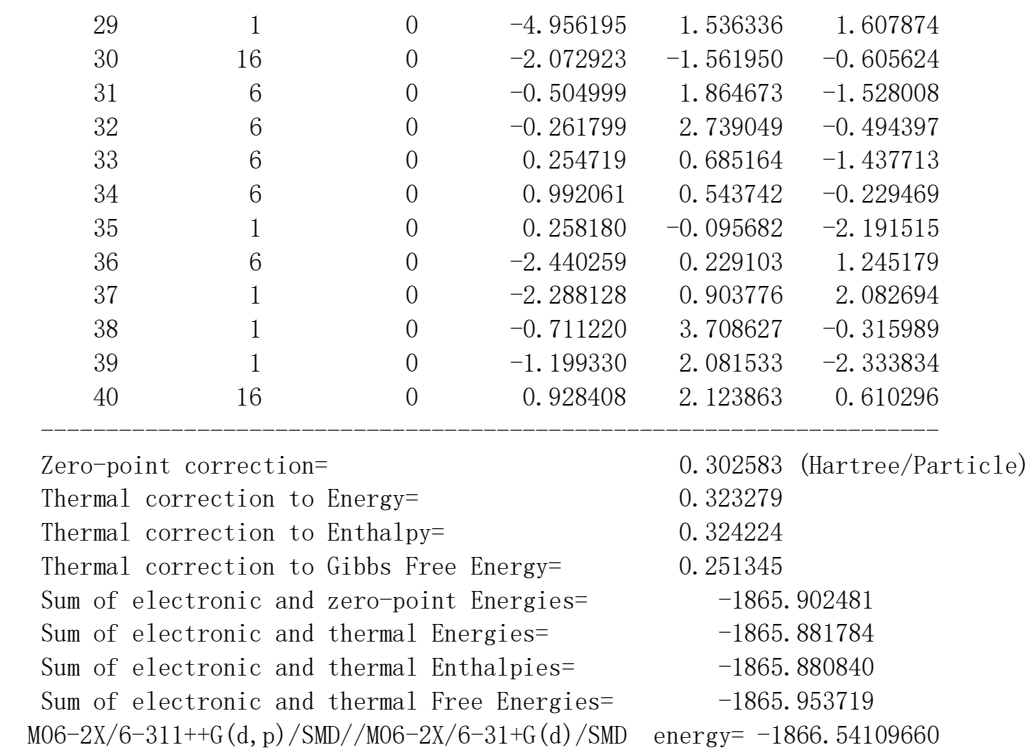

\section{1an-INT3a}

\begin{tabular}{|c|c|c|c|c|c|}
\hline \multirow{2}{*}{$\begin{array}{l}\text { Center } \\
\text { Number }\end{array}$} & \multirow{2}{*}{$\begin{array}{l}\text { Atomic } \\
\text { Number }\end{array}$} & \multirow{2}{*}{$\begin{array}{l}\text { Atomic } \\
\text { Type }\end{array}$} & \multicolumn{3}{|c|}{ Coordinates (Angstroms) } \\
\hline & & & $\mathrm{X}$ & Y & Z \\
\hline 1 & 6 & 0 & -1.606051 & -0.340378 & -0.033550 \\
\hline 2 & 6 & 0 & -1.894693 & 0.264100 & 1. 352716 \\
\hline 3 & 6 & 0 & -0.755894 & 1. 147939 & 1. 887938 \\
\hline 4 & 6 & 0 & -0.360012 & 2.225660 & 0.931906 \\
\hline 5 & 1 & 0 & -2.085670 & -0.537670 & 2. 078007 \\
\hline 6 & 1 & 0 & -2.808653 & 0.862112 & 1. 294162 \\
\hline 7 & 1 & 0 & -1.093359 & 1. 607605 & 2.827666 \\
\hline 8 & 1 & 0 & 0.117751 & 0.537219 & 2. 127506 \\
\hline 9 & 6 & 0 & -2.691841 & -1.330092 & -0.494864 \\
\hline 10 & 1 & 0 & -2.434114 & -1.718074 & -1.485174 \\
\hline 11 & 1 & 0 & -2.779769 & -2.180969 & 0. 189946 \\
\hline 12 & 6 & 0 & -4.069540 & -0.733295 & -0.615210 \\
\hline 13 & 9 & 0 & -4.899857 & -1.548700 & -1.300832 \\
\hline 14 & 9 & 0 & -4.666059 & -0.514169 & 0.576328 \\
\hline 15 & 9 & 0 & -4.073782 & 0.449438 & -1.265726 \\
\hline 16 & 6 & 0 & -0.259577 & -1.012153 & -0.096244 \\
\hline 17 & 6 & 0 & 1. 948959 & -1.502002 & -0.699552 \\
\hline 18 & 6 & 0 & 1. 754957 & -2.449816 & 0.327278 \\
\hline 19 & 6 & 0 & 2. 767771 & -3.333808 & 0.714240 \\
\hline 20 & 6 & 0 & 3. 197627 & -1.437800 & -1.342276 \\
\hline 21 & 6 & 0 & 4. 206602 & -2.308769 & -0.958560 \\
\hline 22 & 6 & 0 & 3. 992136 & -3.252648 & 0.063015 \\
\hline 23 & 1 & 0 & 2. 605950 & -4.060966 & 1. 505212 \\
\hline 24 & 1 & 0 & 4. 794403 & -3.926725 & 0.349246 \\
\hline 25 & 1 & 0 & 5. 174232 & -2.263671 & -1.450160 \\
\hline 26 & 1 & 0 & 3. 361761 & -0.706691 & -2.130163 \\
\hline 27 & 16 & 0 & 0.143176 & -2.331687 & 0.997086 \\
\hline 28 & 6 & 0 & 3. 257649 & 2. 243820 & 0.151127 \\
\hline 29 & 6 & 0 & 2. 996117 & 3. 289639 & -0.700333 \\
\hline 30 & 6 & 0 & 2. 120807 & 1. 764130 & 0.832210 \\
\hline 31 & 6 & 0 & 0.937376 & 2. 457705 & 0.492681 \\
\hline 32 & 1 & 0 & 2. 142469 & 0.936994 & 1. 532759 \\
\hline 33 & 8 & 0 & -1.420712 & 2. 976288 & 0.532596 \\
\hline 34 & 1 & 0 & -1.168170 & 3. 682331 & -0.086582 \\
\hline 35 & 1 & 0 & -1.561181 & 0.476425 & -0.765799 \\
\hline 36 & 6 & 0 & 0.772009 & -0.697177 & -0.917644 \\
\hline 37 & 1 & 0 & 0.720470 & 0.105131 & -1.648980 \\
\hline 38 & 1 & 0 & 3. 684125 & 3. 841425 & -1.328062 \\
\hline 39 & 1 & 0 & 4. 252802 & 1. 830200 & 0.281171 \\
\hline 40 & 16 & 0 & 1. 313920 & 3. 722660 & -0.685132 \\
\hline
\end{tabular}

Zero-point correction=

0. 302940 (Hartree/Particle)

Thermal correction to Energy=

0.325012 
Thermal correction to Enthalpy=

Thermal correction to Gibbs Free Energy=

Sum of electronic and zero-point Energies=

Sum of electronic and thermal Energies=

Sum of electronic and thermal Enthalpies=

Sum of electronic and thermal Free Energies=

M06-2X/6-311++G (d, p) /SMD//M06-2X/6-31+G(d) /SMD

\section{1an-INT3b}

\begin{tabular}{|c|c|c|c|c|c|}
\hline \multirow{2}{*}{$\begin{array}{l}\text { Center } \\
\text { Number }\end{array}$} & \multirow{2}{*}{$\begin{array}{l}\text { Atomic } \\
\text { Number }\end{array}$} & \multirow{2}{*}{$\begin{array}{l}\text { Atomic } \\
\text { Type }\end{array}$} & \multicolumn{3}{|c|}{ Coordinates (Angstroms) } \\
\hline & & & X & $\mathrm{Y}$ & Z \\
\hline 1 & 6 & 0 & 2. 311004 & -0.027456 & -0.405567 \\
\hline 2 & 6 & 0 & 2. 188813 & -1.202430 & 0.582275 \\
\hline 3 & 6 & 0 & 0.850205 & -1.959390 & 0.493433 \\
\hline 4 & 6 & 0 & -0.270056 & -1.306664 & 1. 235383 \\
\hline 5 & 1 & 0 & 2. 269387 & -0.437820 & -1.422927 \\
\hline 6 & 1 & 0 & 2. 348627 & -0.848550 & 1. 608665 \\
\hline 7 & 1 & 0 & 2. 986152 & -1.919356 & 0.366227 \\
\hline 8 & 1 & 0 & 0.576943 & -2.098757 & -0.558376 \\
\hline 9 & 1 & 0 & 0.998607 & -2.962832 & 0.917333 \\
\hline 10 & 6 & 0 & 3. 639640 & 0.738201 & -0.256557 \\
\hline 11 & 1 & 0 & 3. 667952 & 1. 558320 & -0.980932 \\
\hline 12 & 1 & 0 & 3. 744905 & 1. 168587 & 0.745934 \\
\hline 13 & 6 & 0 & 4. 875873 & -0.086477 & -0.501157 \\
\hline 14 & 9 & 0 & 5. 968052 & 0.695124 & -0.648569 \\
\hline 15 & 9 & 0 & 5. 164932 & -0.937655 & 0.506997 \\
\hline 16 & 9 & 0 & 4. 791794 & -0.837253 & -1.619977 \\
\hline 17 & 8 & 0 & 0.036829 & -1.077634 & 2. 534919 \\
\hline 18 & 1 & 0 & -0.685129 & -0.612252 & 2. 991651 \\
\hline 19 & 6 & 0 & -1.530456 & -0.990192 & 0.732800 \\
\hline 20 & 6 & 0 & -3.723777 & -0.129036 & 0.562935 \\
\hline 21 & 6 & 0 & -3.565263 & -0.682159 & -0.732979 \\
\hline 22 & 6 & 0 & -4.570557 & -0.604445 & -1.695770 \\
\hline 23 & 6 & 0 & -4.937318 & 0.521368 & 0.872256 \\
\hline 24 & 6 & 0 & -5.937605 & 0.600094 & -0.085380 \\
\hline 25 & 6 & 0 & -5.759870 & 0.040860 & -1.363277 \\
\hline 26 & 1 & 0 & -4.430964 & -1.035071 & -2.683673 \\
\hline 27 & 1 & 0 & -6.554514 & 0.111630 & -2.100291 \\
\hline 28 & 1 & 0 & -6.871688 & 1. 100976 & 0.153638 \\
\hline 29 & 1 & 0 & -5.078119 & 0.954766 & 1. 859253 \\
\hline 30 & 16 & 0 & -1.996914 & -1.438739 & -0.920936 \\
\hline 31 & 6 & 0 & -0.742043 & 2. 176045 & -0.890961 \\
\hline 32 & 6 & 0 & -0.539127 & 2. 628088 & 0. 380341 \\
\hline 33 & 6 & 0 & 0.241540 & 1. 217441 & -1.291459 \\
\hline 34 & 6 & 0 & 1. 164731 & 0.947084 & -0.320258 \\
\hline 35 & 1 & 0 & 0.255559 & 0.728677 & -2.260684 \\
\hline 36 & 6 & 0 & -2.573807 & -0.317651 & 1. 383440 \\
\hline 37 & 1 & 0 & -2.519599 & 0.067960 & 2. 397444 \\
\hline 38 & 1 & 0 & -1.120747 & 3. 351039 & 0.939712 \\
\hline 39 & 1 & 0 & -1.562762 & 2. 505889 & -1.519781 \\
\hline 40 & 16 & 0 & 0.848551 & 1. 896629 & 1. 103711 \\
\hline
\end{tabular}

Zero-point correction=

Thermal correction to Energy=

0.303302 (Hartree/Particle)

Thermal correction to Enthalpy=

Thermal correction to Gibbs Free Energy=

Sum of electronic and zero-point Energies=

Sum of electronic and thermal Energies=

Sum of electronic and thermal Enthalpies=

Sum of electronic and thermal Free Energies=

0.325129

0.326073

0.249399

$-1865.930884$

$-1865.909056$

$-1865.908112$

$-1865.984786$

M06 $-2 X / 6-311++G(d, p) / S M D / / M 06-2 X / 6-31+G(d) / S M D \quad$ energy $=-1866.57068570$ 


\section{Mechanism studies}

\section{a. Emission quenching experiments (Stern-Volmer Studies)}

Emission intensities were recorded using a FLS980 (Edinburgh Instrument, UK) luminescence spectrophotometer. All Mes-Acr- $\mathrm{ClO}_{4}$ solutions were excited at $430 \mathrm{~nm}$ and the emission intensity was collected at $574 \mathrm{~nm}$. In a typical experiment, to a $3 \cdot 10^{-6} \mathrm{M}$ solution of Mes-Acr- $\mathrm{ClO}_{4}$ in $\mathrm{MeCN}$ was added the appropriate amount of a quencher (DMA or 1a) in a screw-top quartz cuvette. After degassing the sample with a stream of $\mathrm{N}_{2}$ for 10 minutes, the emission of the sample was collected.

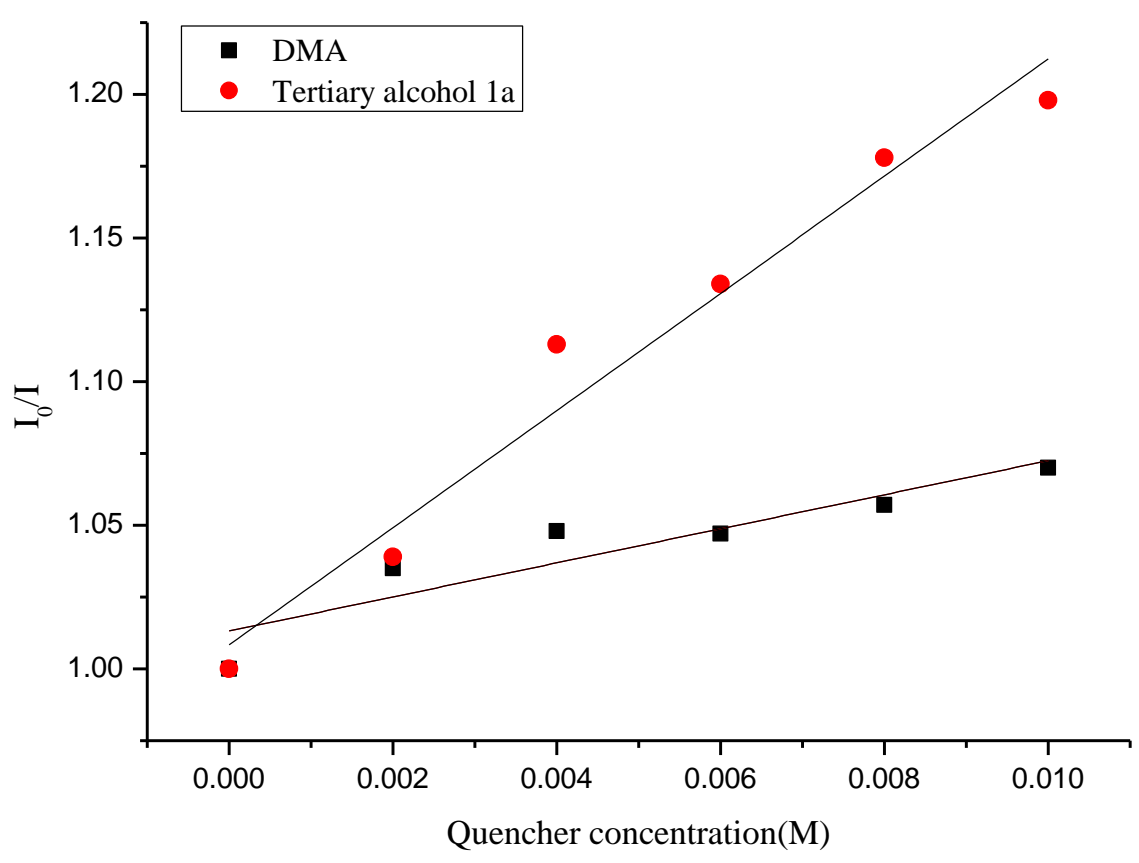

Figure S10. Emission quenching experiments

\section{b. Absorption studies}

Solutions of different complexes were introduced to a $1 \mathrm{~cm}$ path length quartz cuvette equipped with a Teflon ${ }^{\circledR}$ septum and analyzed using an Agilent Cary 5000 spectrophotometer.

For the solutions of DMA in MeCN: DMA $(0.0006 \mathrm{mmol})$ were dissolved in MeCN $(2 \mathrm{~mL})$. The mixtures were stirred for $5 \mathrm{~min}$, then transformed to $1 \mathrm{~cm}$ path length quartz cuvettes, sealed with Teflon ${ }^{\circledR}$ septa and degassed with a stream of argon for 10 minutes.

For the solutions of Togni's reagent in MeCN: Togni's reagent $(0.0006 \mathrm{mmol})$ were dissolved in $\mathrm{MeCN}(2 \mathrm{~mL})$. The mixtures were stirred for $5 \mathrm{~min}$, then transformed to $1 \mathrm{~cm}$ path length quartz cuvettes, sealed with Teflon ${ }^{\circledR}$ septa and degassed with a stream of argon for 10 minutes.

For the solutions of DMA and Togni's reagent in MeCN: DMA $(0.0006 \mathrm{mmol})$ and Togni's reagent $(0.0006 \mathrm{mmol})$ were dissolved in $\mathrm{MeCN}(2 \mathrm{~mL})$. The mixtures were stirred for $5 \mathrm{~min}$, then transformed to $1 \mathrm{~cm}$ path length quartz cuvettes, sealed with Teflon ${ }^{\circledR}$ septa and degassed with a stream of argon for 10 minutes. 


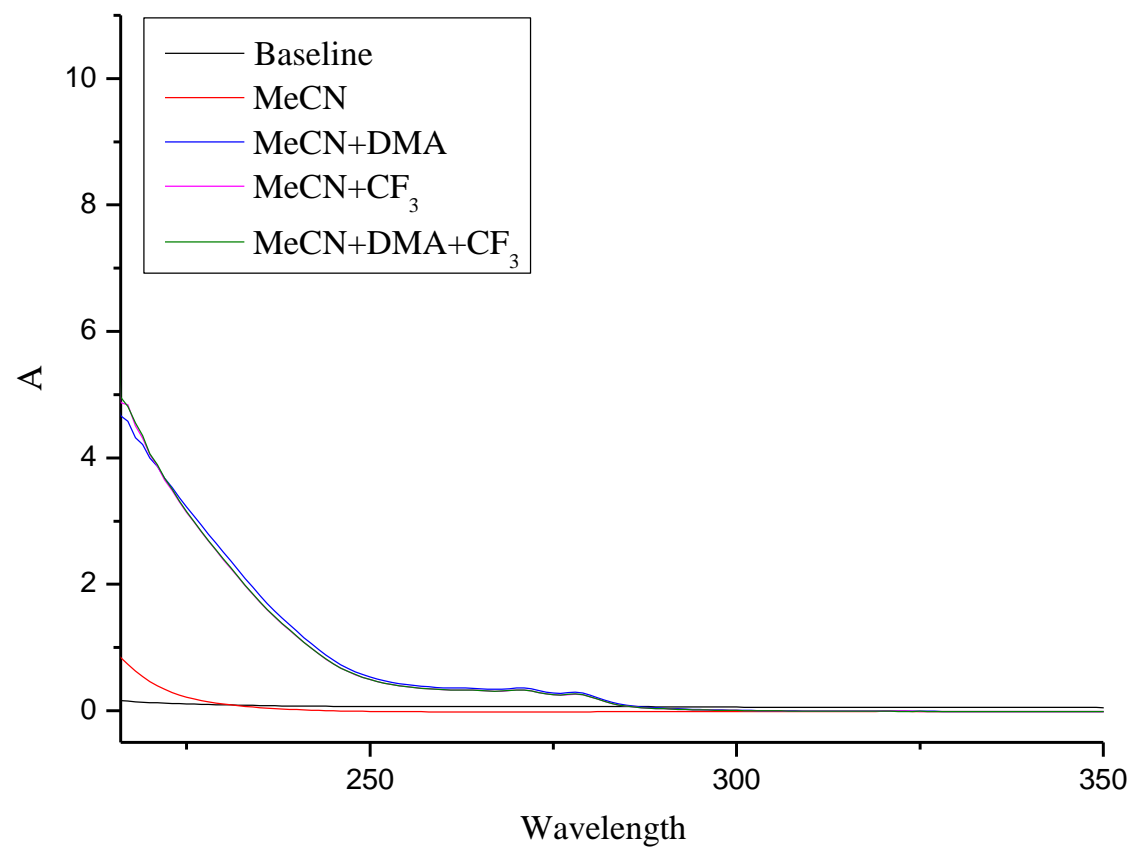

Figure S11. Absorption spectra of DMA, Togni's reagent, and the mixture of DMA and Togni's reagent.

\section{c. Proposed mechanism}<smiles>O=C1OI(C(F)(F)F)c2ccccc21</smiles>

Togni's reagent II

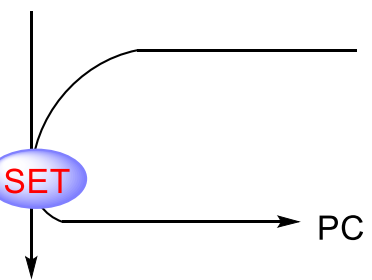

$\mathrm{PC}^{\cdot-}$<smiles></smiles><smiles>[X]C(=P)CCC(CC)C1=CCCC1</smiles>

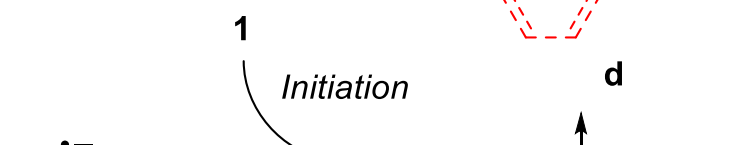

$P C$

hv
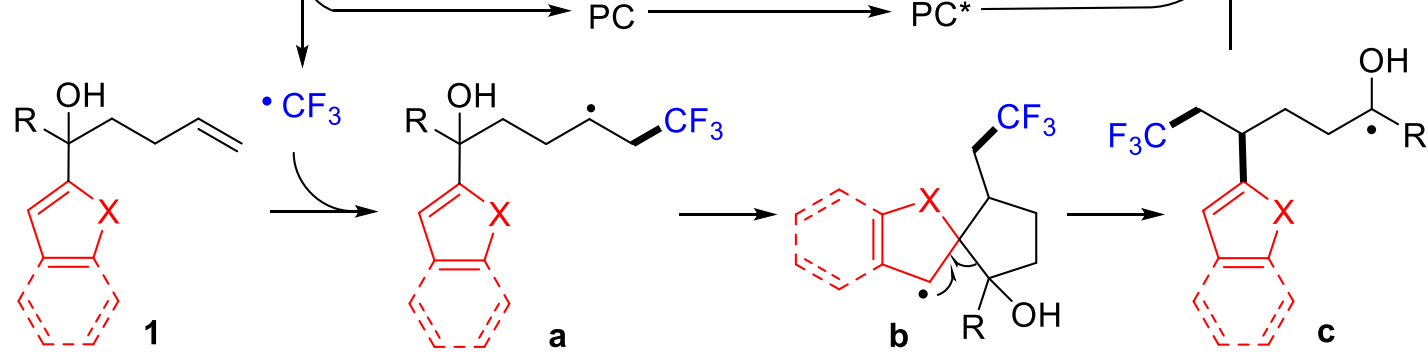

Figure S12. Proposed mechanism. 